Portland State University

PDXScholar

1996

\title{
The Development and Evaluation of Aggregation Methods for Group Pairwise Comparison Judgments
}

Sida Zhou

Portland State University

Follow this and additional works at: https://pdxscholar.library.pdx.edu/open_access_etds Let us know how access to this document benefits you.

\section{Recommended Citation}

Zhou, Sida, "The Development and Evaluation of Aggregation Methods for Group Pairwise Comparison Judgments" (1996). Dissertations and Theses. Paper 1222.

https://doi.org/10.15760/etd.1221

This Dissertation is brought to you for free and open access. It has been accepted for inclusion in Dissertations and Theses by an authorized administrator of PDXScholar. Please contact us if we can make this document more accessible: pdxscholar@pdx.edu. 
THE DEVELOPMENT AND EVALUATION OF AGGREGATION METHODS FOR GROUP PAIRWISE COMPARISON JUDGMENTS

by

SIDA ZHOU

A dissertation submitted in partial fulfillment of the requirements for the degree of

DOCTOR OF PHILOSOPHY

in

SYSTEMS SCIENCE: ENGINEERING MANAGEMENT

Portland State University

1996 
UMI Number: 9623540

\section{UMI Microform 9623540}

Copyright 1996, by UMI Company. All rights reserved.

This microform edition is protected against unauthorized copying under Title 17, United States Code.

\section{UMI \\ 300 North Zeeb Road \\ Ann Arbor, MI 48103}




\section{DISSERTATION APPROVAL}

The abstract and dissertation of Sida Zhou for the Doctor of Philosophy in Systems

Science: Engineering Management were presented December 1, 1995, and accepted by the dissertation committee and the doctoral program.

COMMITTEE APPROVALS:

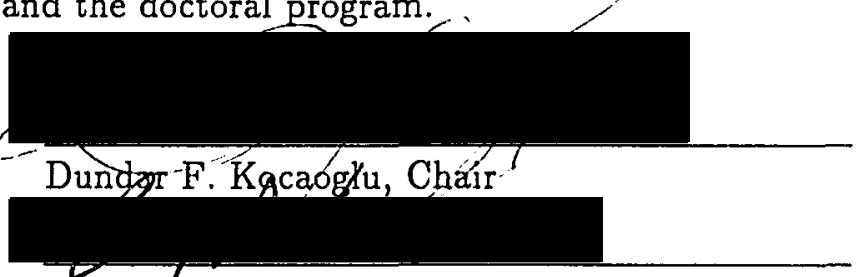

Barry F. And $\not$ rson

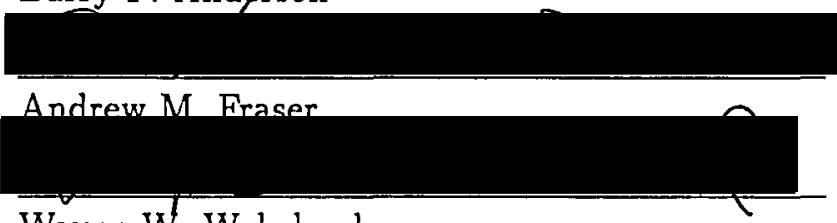

Wayne W. Wakeland

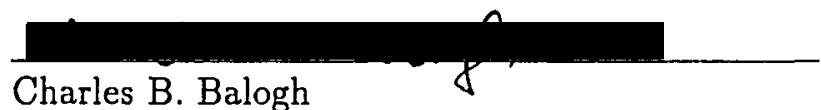

Representative of the Office of Graduate Studies

DOCTORAL PROGRAM APPROVAL:

Beatrice T. Oshika, Director

Systems Science Ph.D. Program

by on in Gexece 1996 


\begin{abstract}
An abstract of the dissertation of Sida Zhou for the Doctor of Philosophy in Systems Science: Engineering Management presented December 1, 1995.

Title: THE DEVELOPMENT AND EVALUATION OF AGGREGATION METHODS FOR GROUP PAIRWISE COMPARISON JUDGMENTS
\end{abstract}

The basic problem of decision making is to choose the best alternative from a set of competing alternatives that are evaluated under conflicting criteria. In general, the process is to evaluate decision elements by quantifying the subjective judgments. The Analytic Hierarchy Process (AHP) provides us with a comprehensive framework for solving such problems. As pointed out by Saaty, AHP "enables us to cope with the intuitive, the rational, and the irrational, all at the same time, when we make multicriteria and multiactor decisions". Furthermore, in most organizations decisions are made collectively, regardless of whether the organization is public or private. It is sometimes difficult to achieve consensus among group members, or for all members of a group to meet.

The purpose of this dissertation was two-fold: First, we developed a new aggregation method - Minimum Distance Method (MDM) - to support group decision process and to help the decision makers achieve consensus under the framework of AHP. Second, we evaluated the performance of aggregation methods by using accuracy and group disagreement criteria. The evaluations were performed through simulation and empirical tests. 
MDM

- employs the general distance concept, which is very appealing to the compromise nature of a group decision making.

- preserves all of the characteristics of the functional equations approach proposed by Aczel and Saaty.

- is based on a goal programming model, which is easy to solve by using a commercial software such as LINDO.

- provides the weighted membership capability for participants.

- allows for sensitivity analysis to investigate the effect of importance levels of decision makers in the group.

The conclusions include the following:

- Simulation and empirical tests show that the two most important factors in the aggregation of pairwise comparison judgments are the probability distribution of error terms and the aggregation method.

- Selection of the appropriate aggregation method can result in significant improvements in decision quality.

- The MDM outperforms the other aggregation methods when the pairwise comparison judgments have large variances. 
- Some of the prioritization methods, such as EV[AA'], EV[A'A], arithmetic and geometric mean of EV[AA'] and $\mathrm{EV}\left[\mathrm{A}^{\prime} \mathrm{A}\right]$, can be dropped from consideration due to their poor performance. 


\section{ACKNOWLEDGMENTS}

I would like to acknowledge the following individuals without whose support and encouragement this study might not have come to fruition:

First and foremost, my love and gratitude go to my wife, Chunping Guo. Her patience, caring and supporting helped me to reach a goal that often seemed elusive and distant.

To Dundar Kocaoglu, Chairman of my Committee, I give special thanks for providing advice and guidance, keeping me focused and picking up several downs throughout the length of the study. He spent countless hours walking with me through many rough roads and every corner of this study.

To Barry Anderson, Andrew Fraser, Wayne Wakeland and Charles Balogh, members of my committee, I am especially grateful to them who reviewed the dissertation, made many valuable suggestions and served in my committee.

I am indebted to Ann White, a friend and colleague, who helped with detailed editorial work of the dissertation and made numerous helpful suggestions.

To Dawn Kuenle and Marion Cole-Crow of the System Science and Engineering Management Program staff, my thanks for many ways they have helped me with all the procedures needed to finish my study.

To Clarice (keli) Zhou, my daughter, who has been a big part of my academic career at Portland State University. Some day, when she understand what all this has meant, I hope she is proud of her dad, and I hope she is inspired to be willing to think hard about a problem and work hard to make a difference. 
Finally, to my friends and colleagues who encouraged me along the way. I owe a debt of gratitude. 


\section{Contents}

List of Figures $\quad v$

List of Tables $\quad x$

1 INTRODUCTION 1

1.1 Objectives of This Dissertation $\ldots \ldots \ldots \ldots \ldots$

1.2 A Judgment Aggregating Method . . . . . . . . . . . . . . . 3

1.3 A Simulation and Empirical Test of Methods for Aggregating Judg-

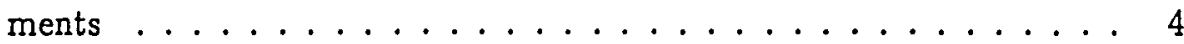

1.4 Dissertation Outline .................. 5

2 BACKGROUND AND LITERATURE REVIEW 6

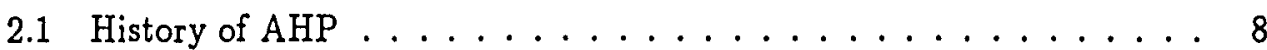

2.2 AHP and Its Procedures $\ldots \ldots \ldots \ldots \ldots$

2.2 .1 The AHP ..................... 10

2.2 .2 AHP Procedures . . . . . . . . . . . . . . . . 14

2.3 Characteristics of Group Decision Making . . . . . . . . . . . 19

2.3.1 Boundary of the Group . . . . . . . . . . . . . . 21

2.3.2 Information Handling Capability . . . . . . . . . . 22

2.3 .3 Tension and Conflict . . . . . . . . . . . . 23

2.3 .4 Resistance . . . . . . . . . . . . . . . 24

2.3.5 Explicit-Implicit. . . . . . . . . . . . . . . 24

2.3.6 Normative and Localized Behavior . . . . . . . . . . . 25

2.4 Techniques for Group Decision Making . . . . . . . . . . . . 26

2.4 .1 Brainstorming . . . . . . . . . . . . . 27

2.4 .2 Nominal Group Technique (NGT) . . . . . . . . . . . . 29

2.4 .3 Surveys . . . . . . . . . . . . . . . . . 29

2.4 .4 Delphi Technique . . . . . . . . . . . . . 31

2.4 .5 Structure Modeling . . . . . . . . . . . . . . . 32

2.5 AHP for Group Decision Making . . . . . . . . . . . . . . 36

2.6 Areas of Research in AHP . . . . . . . . . . . . . . 39

2.6 .1 Hierarchic Structure . . . . . . . . . . . . . . 40

2.6 .2 Incomplete Comparison $\ldots \ldots \ldots \ldots \ldots 4$

2.6 .3 Consistency . . . . . . . . . . . . . . 44 
2.6.4 Relationship of the AHP to Utility Theory . . . . . . . . . 45

2.6 .5 Uncertainty in AHP . . . . . . . . . . . . . 46

2.6.6 Analysis of Sensitivity of Reciprocal Matrices . . . . . . . 48

2.6.7 The method to Derive the Priority Vector . . . . . . . . . 49

2.6.8 Comparison of Prioritization Methods . . . . . . . . . 50

2.6.9 Group Judgments and Consensus . . . . . . . . . . . 52

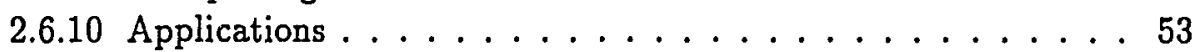

2.7 Summary . . . . . . . . . . . . . . . . . 53

3 METHODS OF AGGREGATING JUDGMENTS FOR PAIRWISE COMPARISONS $\quad 54$

3.1 Definition of Aggregation Problem . . . . . . . . . . . 56

3.1.1 Representation of Pairwise Comparison Matrix for Group Judgment Aggregation ................. 58

3.1.2 Representation of Priority Vector for Group Judgment Aggre-

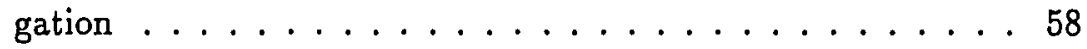

3.1 .3 Aggregation Approaches . . . . . . . . . . . . . . . 59

3.2 Existing Methods for Aggregating Pairwise Comparison Judgments . 61

3.2 .1 Geometric Mean . . . . . . . . . . . . . . . . . 61 61

3.2.2 Weighted Geometric Mean . . . . . . . . . . . . 63

3.2 .3 Arithmetic Mean . . . . . . . . . . . . . . . . . . 65

3.3 The Minimum Distance Method for Aggregating Pairwise Comparison Judgments . . . . . . . . . . . . . . . . . 66

3.3.1 Distance as Accuracy Measurement . . . . . . . . . . . . . 67

3.3.2 Distance as Group Disagreement . . . . . . . . . . . . 67

3.3.3 The Minimum Distance Method for Pairwise Comparison Matrix 71

3.3.4 The Minimum Distance Method for Priority Vectors . . . . . . 76

3.3.5 The Weighted Membership in the Minimum Distance Method 78

3.4 The Sensitivity and Reliability of the Minimum Distance Method . . 80

3.5 Numerical Examples of the Minimum Distance Method . . . . . . . 81

3.5.1 MDM Operated on Pairwise Comparison Matrices . . . . . . . 82

3.5.2 Weighted Membership and Sensitivity Analysis . . . . . . 85

3.5.3 MDM Operated on Priority Vectors . . . . . . . . . . . . 89

4 COMPARISON STUDY AND SIMULATION PROCEDURES 92

4.1 Objectives and Considerations . . . . . . . . . . . . 95

4.1 .1 Objectives of Comparison Study . . . . . . . . . . . . . 96

4.1.2 Considerations for Comparison Study . . . . . . . . . . . 97

4.2 Input Data Generation and The Perturbation Method . . . . . . . . 102

4.2.1 Characteristics of Actual Judgments . . . . . . . . . . . 102

4.2.2 Input Data Generation for Simulation . . . . . . . . . . . 103

4.2.3 Generation of Perturbation Distributions . . . . . . . . . . 106 
4.2.4 Generation of Uniform Distribution Input Data . . . . . . . 108

4.2.5 Generation of Lognormal Distribution Input Data . . . . . . . 109

4.2.6 Generation of Gamma Distribution Input Data . . . . . . . 111

4.3 Performance Measurements . . . . . . . . . . . . . . . . . . 112

4.3.1 The Accuracy Measurements $\left(d_{1}\right) \ldots \ldots . \ldots . \ldots 112$

4.3.2 The Disagreement Measurements $\left(d_{2}\right) \ldots \ldots \ldots \ldots 114$

4.4 The Simulation Approach . . . . . . . . . . . . . . . 115

4.4.1 Data Generation Procedures . . . . . . . . . . . . 117

4.4 .2 Simulation Control Factors . . . . . . . . . . . . . . 118

4.4.3 Simulation Procedures . . . . . . . . . . . . . . . 119

4.5 The Empirical Approach . . . . . . . . . . . . . . . . . . . 124

5 SIMULATION RESULTS AND DISCUSSIONS 131

5.1 Simulation Set Up . . . . . . . . . . . . . . . . . 132

5.2 Aggregation Methods vs. Type of Input Data . . . . . . . . . . . 135

5.3 Prioritization Methods vs. Aggregation Methods . . . . . . . . . . . 147

5.4 Aggregation Methods vs. Number of Decision Makers . . . . . . . . 150

5.5 Analysis of the empirical test of the aggregation methods . . . . . . 168

5.6 Summary of the Analysis . . . . . . . . . . . . . . . . 177

6 CONCLUSIONS $\quad 182$

6.1 Main Results . . . . . . . . . . . . . . . . . . 183

6.2 Contributions . . . . . . . . . . . . . . . . . 185

6.3 Suggested Future Work . . . . . . . . . . . . . . . . 186

A The Prioritization Methods in AHP 188

A.1 The (Right) Eigenvalue Method . . . . . . . . . . . . . . . . . . 189

A.2 The Mean Transformation Method . . . . . . . . . . . . . . . . 190

A.3 Row Geometric Mean (or the Logarithmic Least Squares) Method . . 191

A.4 The Column Geometric Mean Method . . . . . . . . . . . . . . . 191

A.5 The Harmonic Mean (the left eigenvector) method . . . . . . . . . . . 191

A.6 The Simple Row Average . . . . . . . . . . . . . . . . . . . . . . . . . . . 192

A.7 Ordinary Least Squares . . . . . . . . . . . . . . . . . . . . 192

A.8 Constant Sum Method . . . . . . . . . . . . . . . . . . 192

A.9 Column-Row Sums Method . . . . . . . . . . . . . . . 194

B The Mean and Standard Deviation of Accuracy Measurement from Simulation

C The Mean and Standard Deviation of Group Disagreement Measurement from Simulation

D Paired Comparison Data for Empirical Test 270 
E The Results of Empirical Test for Accuracy and Group Disagreement

278

Bibliography

284 


\section{List of Figures}

2.1 The standard form of decision schema in the analytic hierarchy process: a hierarchy with $k$ levels. . . . . . . . . . . . . . . 15

2.2 Functional Representation of Interpretive Structural Modeling . . . . 34

4.1 The procedures of comparison study for both simulation and empirical test . . . . . . . . . . . . . . . . . . 98

4.2 The flow diagram of simulation study for aggregation method operated on pairwise comparison matrices. . . . . . . . . . . . 122

4.3 The flow diagram of simulation study for aggregation method operated on priority vectors. . . . . . . . . . . . . . . . . 123

4.4 The flow diagram of empirical test for aggregation method operated on priority vectors. . . . . . . . . . . . . . . . . . 127

4.5 The flow diagram of empirical test for aggregation method operated on priority vectors. . . . . . . . . . . . . . . . 128

5.1 The Mean Accuracy of Geometric Mean Method Operated on Final Priority Vector $(\mathrm{N}=8, \mathrm{M}=3) \ldots \ldots \ldots \ldots$

5.2 The Mean Accuracy of Geometric Mean Method Operated on Pairwise Comparison Matrix $(\mathrm{N}=8, \mathrm{M}=3) \ldots \ldots . \ldots 139$

5.3 The Mean Accuracy of Arithmetic Mean Method Operated on Final Priority Vector $(N=8, M=3) \ldots \ldots \ldots \ldots$

5.4 The Mean Accuracy of Minimum Distance Method Operated on Pairwise Comparison Matrix $(\mathrm{N}=8, \mathrm{M}=3) \ldots \ldots \ldots . \ldots 140$

5.5 The Mean Accuracy of Minimum Distance Method Operated on Final

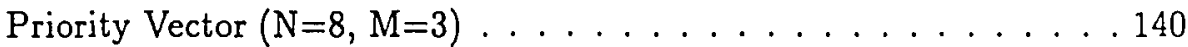

5.6 The Mean Disagreement of Geometric Mean Method Operated on Final Priority Vector $(\mathrm{N}=12, \mathrm{M}=7) \ldots \ldots . . \ldots . . . \ldots 141$

5.7 The Mean Disagreement of Geometric Mean Method Operated on Pairwise Comparison Matrix $(\mathrm{N}=12, \mathrm{M}=7) \ldots \ldots \ldots . \ldots 141$

5.8 The Mean Disagreement of Arithmetic Mean Method Operated on Final Priority Vector $(\mathrm{N}=12, \mathrm{M}=7) \ldots \ldots \ldots \ldots$

5.9 The Mean Disagreement of Minimum Distance Method Operated on Pairwise Comparison Matrix $(\mathrm{N}=12, \mathrm{M}=7) \ldots \ldots . . \ldots 142$

5.10 The Mean Disagreement of Minimum Distance Method Operated on Final Priority Vector $(\mathrm{N}=12, \mathrm{M}=7) \ldots \ldots \ldots . \ldots 143$ 
5.11 The Mean Accuracy of Uniform Distribution for All Aggregation Methods $(\mathrm{N}=8, \mathrm{M}=9) \ldots \ldots \ldots \ldots . \ldots \ldots$

5.12 The Mean Accuracy of Lognormal Distribution for All Aggregation Methods $(N=8, M=9) \ldots \ldots \ldots$. . . . . . . . . . . . 144

5.13 The Mean Accuracy of Gamma Distribution for All Aggregation Methods $(N=8, M=9) \ldots \ldots \ldots \ldots \ldots$. . . . . . . . . . . . . . . . . . . . . .

5.14 The Mean Disagreement of Uniform Distribution for All Aggregation Methods $(\mathrm{N}=12, \mathrm{M}=7) \ldots \ldots \ldots \ldots \ldots$

5.15 The Mean Disagreement of Lognormal Distribution for All Aggregation Methods $(\mathrm{N}=12, \mathrm{M}=7) \ldots \ldots \ldots \ldots$

5.16 The Mean Disagreement of Gamma Distribution for All Aggregation Methods $(\mathrm{N}=12, \mathrm{M}=7) \ldots \ldots \ldots \ldots$

5.17 The Mean Accuracy of Geometric Mean Method with Uniform Distribution for $M=3,5,7,9(\mathrm{~N}=10)$, The Geometric Mean Method Operates on Final Priority Vector . . . . . . . . . . . . . . . . . 152

5.18 The Mean Accuracy of Geometric Mean Method with Lognormal Distribution for $M=3,5,7,9(N=10)$, The Geometric Mean Method Operates on Final Priority Vector . . . . . . . . . . . . . . . 153

5.19 The Mean Accuracy of Geometric Mean Method with Gamma Distribution for $\mathrm{M}=3,5,7,9(\mathrm{~N}=10)$, The Geometric Mean Method Operates on Final Priority Vector . . . . . . . . . . . . . . . 153

5.20 The Mean Accuracy of Geometric Mean Method with Uniform Distribution for $\mathrm{M}=3,5,7,9(\mathrm{~N}=10)$, The Geometric Mean Method Operates on Pairwise Comparison Matrix . . . . . . . . . . . . . . . 154

5.21 The Mean Accuracy of Geometric Mean Method with Lognormal Distribution for $M=3,5,7,9(\mathrm{~N}=10)$, The Geometric Mean Method Operates on Pairwise Comparison Matrix . . . . . . . . . . . . . . 154

5.22 The Mean Accuracy of Geometric Mean Method with Gamma Distribution for $M=3,5,7,9(\mathrm{~N}=10)$, The Geometric Mean Method Operates on Pairwise Comparison Matrix . . . . . . . . . . . 155

5.23 The Mean Accuracy of Arithmetic Mean Method with Uniform Distribution for $\mathrm{M}=3,5,7,9(\mathrm{~N}=10)$, The Arithmetic Mean Method Operates on Final Priority Vector . . . . . . . . . . . . . . . 155

5.24 The Mean Accuracy of Arithmetic Mean Method with Lognormal Distribution for $M=3,5,7,9(\mathrm{~N}=10)$, The Arithmetic Mean Method Operates on Final Priority Vector . . . . . . . . . . . . . . . 156

5.25 The Mean Accuracy of Arithmetic Mean Method with Gamma Distribution for $M=3,5,7,9(\mathrm{~N}=10)$, The Arithmetic Mean Method Operates on Final Priority Vector . . . . . . . . . . . . . 156

5.26 The Mean Accuracy of Minimum Distance Method with Uniform Distribution for $\mathrm{M}=3,5,7,9(\mathrm{~N}=10)$, The Minimum Distance Method Operates on Final Priority Vector . . . . . . . . . . . 157 
5.27 The Mean Accuracy of Minimum Distance Method with Lognormal Distribution for $\mathrm{M}=3,5,7,9(\mathrm{~N}=10)$, The Minimum Distance Method Operates on Final Priority Vector . . . . . . . . . . . . 157

5.28 The Mean Accuracy of Minimum Distance Method with Gamma Distribution for $\mathrm{M}=3,5,7,9(\mathrm{~N}=10)$, The Minimum Distance Method Operates on Final Priority Vector . . . . . . . . . . . . . 158

5.29 The Mean Accuracy of Minimum Distance Method with Uniform Distribution for $\mathrm{M}=3,5,7,9(\mathrm{~N}=10)$, The Minimum Distance Method Operates on Pairwise Comparison Matrix . . . . . . . . . . . 158

5.30 The Mean Accuracy of Minimum Distance Method with Lognormal Distribution for $\mathrm{M}=3,5,7,9(\mathrm{~N}=10)$, The Minimum Distance Method Operates on Pairwise Comparison Matrix . . . . . . . . . . 159

5.31 The Mean Accuracy of Minimum Distance Method with Gamma Distribution for $M=3,5,7,9(N=10)$, The Minimum Distance Method Operates on Pairwise Comparison Matrix . . . . . . . . . . . . . . 159

5.32 The Mean Disagreement of Geometric Mean Method with Uniform Distribution for $\mathrm{M}=3,5,7,9(\mathrm{~N}=10)$, The Geometric Mean Method Operates on Final Priority Vector . . . . . . . . . . . . . . . 160

5.33 The Mean Disagreement of Geometric Mean Method with Lognormal Distribution for $\mathrm{M}=3,5,7,9(\mathrm{~N}=10)$, The Geometric Mean Method Operates on Final Priority Vector . . . . . . . . . . . . . . . 160

5.34 The Mean Disagreement of Geometric Mean Method with Gamma Distribution for $\mathrm{M}=3,5,7,9(\mathrm{~N}=10)$, The Geometric Mean Method Operates on Final Priority Vector . . . . . . . . . . . . . . . 161

5.35 The Mean Disagreement of Geometric Mean Method with Uniform Distribution for $\mathrm{M}=3,5,7,9(\mathrm{~N}=10)$, The Geometric Mean Method Operates on Pairwise Comparison Matrix . . . . . . . . . . . . 162

5.36 The Mean Disagreement of Geometric Mean Method with Lognormal Distribution for $M=3,5,7,9(N=10)$, The Geometric Mean Method Operates on Pairwise Comparison Matrix . . . . . . . . . . . . . 162

5.37 The Mean Disagreement of Geometric Mean Method with Gamma Distribution for $M=3,5,7,9(N=10)$, The Geometric Mean Method Operates on Pairwise Comparison Matrix . . . . . . . . . . 163

5.38 The Mean Disagreement of Arithmetic Mean Method with Uniform Distribution for $M=3,5,7,9(N=10)$, The Arithmetic Mean Method Operates on Final Priority Vector . . . . . . . . . . . . . . 163

5.39 The Mean Disagreement of Arithmetic Mean Method with Lognormal Distribution for $M=3,5,7,9(N=10)$, The Arithmetic Mean Method Operates on Final Priority Vector . . . . . . . . . . . . . . . . . 164

5.40 The Mean Disagreement of Arithmetic Mean Method with Gamma Distribution for $M=3,5,7,9(\mathrm{~N}=10)$, The Arithmetic Mean Method Operates on Final Priority Vector . . . . . . . . . . . . . 164 
5.41 The Mean Disagreement of Minimum Distance Method with Uniform Distribution for $\mathrm{M}=3,5,7,9(\mathrm{~N}=10)$, The Minimum Distance Method Operates on Final Priority Vector . . . . . . . . . . . . 165

5.42 The Mean Disagreement of Minimum Distance Method with Lognormal Distribution for $\mathrm{M}=3,5,7,9(\mathrm{~N}=10)$, The Minimum Distance Method Operates on Final Priority Vector . . . . . . . . . . . . 165

5.43 The Mean Disagreement of Minimum Distance Method with Ganma Distribution for $M=3,5,7,9(N=10)$, The Minimum Distance Method Operates on Final Priority Vector . . . . . . . . . . . . 166

5.44 The Mean Disagreement of Minimum Distance Method with Uniform Distribution for $M=3,5,7,9(\mathrm{~N}=10)$, The Minimum Distance Method Operates on Pairwise Comparison Matrix . . . . . . . 166

5.45 The Mean Disagreement of Minimum Distance Method with Lognormal Distribution for $\mathrm{M}=3,5,7,9(\mathrm{~N}=10)$, The Minimum Distance Method Operates on Pairwise Comparison Matrix . . . . . . . . . . 167

5.46 The Mean Disagreement of Minimum Distance Method with Gamma Distribution for $M=3,5,7,9(\mathrm{~N}=10)$, The Minimum Distance Method Operates on Pairwise Comparison Matrix . . . . . . . . . . 167

5.47 The Mean Accuracy of Aggregation Method for Category One Empirical Data . . . . . . . . . . . . . . . . . . . 170

5.48 The Mean Accuracy of Aggregation Method for Category Two Empirical Data . . . . . . . . . . . . . . . . 170

5.49 The Mean Accuracy of Aggregation Method for Category Three Empirical Data . . . . . . . . . . . . . . . . . . 171

5.50 The Mean Accuracy of Aggregation Method for Category Four Empirical Data . . . . . . . . . . . . . . . . . . . 171

5.51 The Mean Accuracy of Aggregation Method for Category Five Empirical Data . . . . . . . . . . . . . . . . . . . . . 172

5.52 The Mean Accuracy of Aggregation Method for Category Six Empirical Data . . . . . . . . . . . . . . . . . . . . . . . . 172

5.53 The Mean Accuracy of Aggregation Method for Category Seven Empirical Data . . . . . . . . . . . . . . . . . . . . . 173

5.54 The Mean Disagreement of Aggregation Method for Category One Empirical Data . . . . . . . . . . . . . . . . 173

5.55 The Mean Disagreement of Aggregation Method for Category Two Empirical Data . . . . . . . . . . . . . . . . . . . . . 174

5.56 The Mean Disagreement of Aggregation Method for Category Three Empirical Data . . . . . . . . . . . . . . . . . . . . . 174

5.57 The Mean Disagreement of Aggregation Method for Category Four Empirical Data . . . . . . . . . . . . . . . . . . . . 175

5.58 The Mean Disagreement of Aggregation Method for Category Five Empirical Data . . . . . . . . . . . . . . . . . . 175 
5.59 The Mean Disagreement of Aggregation Method for Category Six Empirical Data . . . . . . . . . . . . . . . . . . 176

5.60 The Mean Disagreement of Aggregation Method for Category Seven

Empirical Data . . . . . . . . . . . . . . . . . 176 


\section{List of Tables}

2.1 Pairwise Comparison of Two Elements . . . . . . . . . . . . 17

4.1 The List of Judgment Aggregation Methods . . . . . . . . . . . . . 96

4.2 Abbreviation for Judgment Prioritization Methods . . . . . . . . . . 101

4.3 The Estimation Categories . . . . . . . . . . . . . . . . . . . . . 129

4.4 The Estimation Categories (Continued) . . . . . . . . . . . . . 130

5.1 The prioritization methods with good mean accuracy and good mean disagreement over different input data type for all the aggregation methods . . . . . . . . . . . . . . . . . . . . . . 148

5.2 The comparison of prioritization methods for accuracy . . . . . . 178

5.3 The comparison of prioritization methods for group disagreement . . 179

5.4 The comparison of aggregation methods for accuracy . . . . . . . 180

5.5 The comparison of aggregation methods for group disagreement . . . 181

B.1 Mean and Standard Deviation of Accuracy Measurement $\left(d_{1}\right)$ from Simulation (With $N=8, M=3$, scale $[1 / 9,9], T=500$ ) $\ldots 197$

B.2 Mean and Standard Deviation of Accuracy Measurement $\left(d_{1}\right)$ from Simulation (With $N=8, M=3$, scale $[1 / 9,9], T=500$ ) [Continued] 198

B.3 Mean and Standard Deviation of Accuracy Measurement $\left(d_{1}\right)$ from Simulation (With $N=8, M=3$, scale $[1 / 9,9], T=500$ ) [continued] 199

B.4 Mean and Standard Deviation of Accuracy Measurement $\left(d_{1}\right)$ from Simulation (With $N=8, M=5$, scale $[1 / 9,9], T=500$ ) $\ldots 200$

B.5 Mean and Standard Deviation of Accuracy Measurement $\left(d_{1}\right)$ from Simulation (With $N=8, M=5$, scale $[1 / 9,9], T=500$ ) [Continued] 201

B.6 Mean and Standard Deviation of Accuracy Measurement $\left(d_{1}\right)$ from Simulation (With $N=8, M=5$, scale $[1 / 9,9], T=500$ ) [Continued] 202

B.7 Mean and Standard Deviation of Accuracy Measurement $\left(d_{1}\right)$ from Simulation (With $N=8, M=7$, scale $[1 / 9,9], T=500$ ) $\ldots \ldots 203$

B.8 Mean and Standard Deviation of Accuracy Measurement $\left(d_{1}\right)$ from Simulation (With $\mathrm{N}=8, \mathrm{M}=7$, scale [1/9,9], $\mathrm{T}=500$ ) [Continued] 204

B.9 Mean and Standard Deviation of Accuracy Measurement $\left(d_{1}\right)$ from Simulation (With $N=8, M=7$, scale $[1 / 9,9], T=500$ ) [Continued] 205

B.10 Mean and Standard Deviation of Accuracy Measurement $\left(d_{1}\right)$ from Simulation (With $N=8, M=9$, scale $[1 / 9,9], T=500$ ) $\ldots . .206$ 
B.11 Mean and Standard Deviation of Accuracy Measurement $\left(d_{1}\right)$ from Simulation (With $N=8, M=9$, scale $[1 / 9,9], T=500$ ) [Continued] 207 B.12 Mean and Standard Deviation of Accuracy Measurement $\left(d_{1}\right)$ from Simulation (With $N=8, M=9$, scale $[1 / 9,9], T=500$ ) [Continued] 208

B.13 Mean and Standard Deviation of Accuracy Measurement $\left(d_{1}\right)$ from Simulation (With $N=10, M=3$, scale $[1 / 9,9], T=500$ ) $\ldots 209$

B.14 Mean and Standard Deviation of Accuracy Measurement $\left(d_{1}\right)$ from Simulation (With $N=10, M=3$, scale $[1 / 9,9], T=500$ ) [Continued] 210

B.15 Mean and Standard Deviation of Accuracy Measurement $\left(d_{1}\right)$ from Simulation (With $N=10, M=3$, scale $[1 / 9,9], T=500$ ) [Continued] 211

B.16 Mean and Standard Deviation of Accuracy Measurement $\left(d_{1}\right)$ from Simulation (With $N=10, M=5$, scale $[1 / 9,9], T=500$ ) $\ldots 212$

B.17 Mean and Standard Deviation of Accuracy Measurement $\left(d_{1}\right)$ from Simulation (With $N=10, M=5$, scale $[1 / 9,9], T=500$ ) [Continued] 213

B.18 Mean and Standard Deviation of Accuracy Measurement $\left(d_{1}\right)$ from Simulation (With $N=10, M=5$, scale $[1 / 9,9], T=500$ ) [Continued] 214

B.19 Mean and Standard Deviation of Accuracy Measurement $\left(d_{1}\right)$ from Simulation (With $\mathrm{N}=10, \mathrm{M}=7$, scale $[1 / 9,9], \mathrm{T}=500$ ) $\ldots 215$

B.20 Mean and Standard Deviation of Accuracy Measurement $\left(d_{1}\right)$ from Simulation (With $N=10, M=7$, scale $[1 / 9,9], T=500$ ) [Continued] 216

B.21 Mean and Standard Deviation of Accuracy Measurement $\left(d_{1}\right)$ from Simulation (With $N=10, M=7$, scale $[1 / 9,9], T=500$ ) [Continued] 217

B.22 Mean and Standard Deviation of Accuracy Measurement $\left(d_{1}\right)$ from Simulation (With $N=10, M=9$, scale $[1 / 9,9], T=500) \ldots 218$

B.23 Mean and Standard Deviation of Accuracy Measurement $\left(d_{1}\right)$ from Simulation (With $N=10, M=9$, scale $[1 / 9,9], T=500$ ) [Continued] 219

B.24 Mean and Standard Deviation of Accuracy Measurement $\left(d_{1}\right)$ from Simulation (With $N=10, M=9$, scale $[1 / 9,9], T=500$ ) [Continued] 220

B.25 Mean and Standard Deviation of Accuracy Measurement $\left(d_{1}\right)$ from Simulation (With $N=12, M=3$, scale $[1 / 9,9], T=500) \ldots 221$

B.26 Mean and Standard Deviation of Accuracy Measurement $\left(d_{1}\right)$ from Simulation (With $N=12, M=3$, scale $[1 / 9,9], T=500$ ) [Continued] 222

B.27 Mean and Standard Deviation of Accuracy Measurement $\left(d_{1}\right)$ from Simulation (With $\mathrm{N}=12, \mathrm{M}=3$, scale $[1 / 9,9], \mathrm{T}=500$ ) [Continued] 223

B.28 Mean and Standard Deviation of Accuracy Measurement $\left(d_{1}\right)$ from Simulation (With $N=12, M=5$, scale $[1 / 9,9], T=500) \ldots 224$

B.29 Mean and Standard Deviation of Accuracy Measurement $\left(d_{1}\right)$ from Simulation (With $N=12, M=5$, scale $[1 / 9,9], T=500$ ) [Continued] 225

B.30 Mean and Standard Deviation of Accuracy Measurement $\left(d_{1}\right)$ from Simulation (With $N=12, M=5$, scale $[1 / 9,9], T=500$ ) [Continued] 226

B.31 Mean and Standard Deviation of Accuracy Measurement $\left(d_{1}\right)$ from Simulation (With $N=12, M=7$, scale $[1 / 9,9], T=500$ ) $\ldots 227$ 
B.32 Mean and Standard Deviation of Accuracy Measurement $\left(d_{1}\right)$ from Simulation (With $N=12, M=7$, scale $[1 / 9,9], T=500$ ) [Continued] 228

B.33 Mean and Standard Deviation of Accuracy Measurement $\left(d_{1}\right)$ from Simulation (With $N=12, M=7$, scale $[1 / 9,9], T=500$ ) [Continued] 229

B.34 Mean and Standard Deviation of Accuracy Measurement $\left(d_{1}\right)$ from Simulation (With $N=12, M=9$, scale $[1 / 9,9], T=500) \ldots 230$

B.35 Mean and Standard Deviation of Accuracy Measurement $\left(d_{1}\right)$ from Simulation (With $N=12, M=9$, scale $[1 / 9,9], T=500$ ) [Continued] 231

B.36 Mean and Standard Deviation of Accuracy Measurement $\left(d_{1}\right)$ from Simulation (With $N=12, M=9$, scale $[1 / 9,9], T=500$ ) [Continued] 232

C.1 Mean and Standard Deviation of Group Disagreement $\left(d_{2}\right)$ from Simulation (With $N=8, M=3$, scale $[1 / 9,9], T=500) \ldots 234$

C.2 Mean and Standard Deviation of Group Disagreement $\left(d_{2}\right)$ from Simulation (With $\mathrm{N}=8, \mathrm{M}=3$, scale $[1 / 9,9], \mathrm{T}=500$ ) [Continued] . . 235

C.3 Mean and Standard Deviation of Group Disagreement $\left(d_{2}\right)$ from Simulation (With $\mathrm{N}=8, \mathrm{M}=3$, scale $[1 / 9,9], \mathrm{T}=500$ ) [Continued] . . 236

C.4 Mean and Standard Deviation of Group Disagreement $\left(d_{2}\right)$ from Simulation (With $\mathrm{N}=8, \mathrm{M}=5$, scale $[1 / 9,9], \mathrm{T}=500$ ) $\ldots . . .237$

C.5 Mean and Standard Deviation of Group Disagreement $\left(d_{2}\right)$ from Simulation (With $\mathrm{N}=8, \mathrm{M}=5$, scale $[1 / 9,9], \mathrm{T}=500$ ) [Continued] . . 238

C. 6 Mean and Standard Deviation of Group Disagreement $\left(d_{2}\right)$ from Simulation (With $\mathrm{N}=8, \mathrm{M}=5$, scale $[1 / 9,9], \mathrm{T}=500$ ) [Continued] . . 239

C.7 Mean and Standard Deviation of Group Disagreement $\left(d_{2}\right)$ from Simulation (With $\mathrm{N}=8, \mathrm{M}=7$, scale $[1 / 9,9], \mathrm{T}=500$ ) . . . . . 240

C.8 Mean and Standard Deviation of Group Disagreement $\left(d_{2}\right)$ from Simulation (With $\mathrm{N}=8, \mathrm{M}=7$, scale $[1 / 9,9], \mathrm{T}=500$ ) [Continued] . . 241

C.9 Mean and Standard Deviation of Group Disagreement $\left(d_{2}\right)$ from Simulation (With $\mathrm{N}=8, \mathrm{M}=7$, scale $[1 / 9,9], \mathrm{T}=500$ ) [Continued] . . 242

C.10 Mean and Standard Deviation of Group Disagreement $\left(d_{2}\right)$ from Simulation (With $N=8, M=9$, scale $[1 / 9,9], T=500) \ldots 243$

C.11 Mean and Standard Deviation of Group Disagreement $\left(d_{2}\right)$ from Simulation (With $\mathrm{N}=8, \mathrm{M}=9$, scale $[1 / 9,9], \mathrm{T}=500$ ) [Continued] . . 244

C.12 Mean and Standard Deviation of Group Disagreement $\left(d_{2}\right)$ from Simulation (With $N=8, M=9$, scale $[1 / 9,9], T=500$ ) [Continued] . . 245

C.13 Mean and Standard Deviation of Group Disagreement $\left(d_{2}\right)$ from Simulation (With $\mathrm{N}=10, \mathrm{M}=3$, scale $[1 / 9,9], \mathrm{T}=500$ ) $\ldots 246$

C.14 Mean and Standard Deviation of Group Disagreement $\left(d_{2}\right)$ from Simulation (With $\mathrm{N}=10, \mathrm{M}=3$, scale $[1 / 9,9], \mathrm{T}=500$ ) [Continued] .247

C.15 Mean and Standard Deviation of Group Disagreement $\left(d_{2}\right)$ from Simulation (With $\mathrm{N}=10, \mathrm{M}=3$, scale $[1 / 9,9], \mathrm{T}=500$ ) [Continued] .248

C.16 Mean and Standard Deviation of Group Disagreement $\left(d_{2}\right)$ from Simulation (With $\mathrm{N}=10, \mathrm{M}=5$, scale $[1 / 9,9], \mathrm{T}=500$ ) . . . . . 249 
C.17 Mean and Standard Deviation of Group Disagreement $\left(d_{2}\right)$ from Simulation (With $\mathrm{N}=10, \mathrm{M}=5$, scale $[1 / 9,9], \mathrm{T}=500$ ) [Continued] . 250

C.18 Mean and Standard Deviation of Group Disagreement $\left(d_{2}\right)$ from Simulation (With $\mathrm{N}=10, \mathrm{M}=5$, scale $[1 / 9,9], \mathrm{T}=500$ ) [Continued] . 251

C.19 Mean and Standard Deviation of Group Disagreement $\left(d_{2}\right)$ from Simulation (With $\mathrm{N}=10, \mathrm{M}=7$, scale $[1 / 9,9], \mathrm{T}=500$ ) $\ldots . . .252$

C.20 Mean and Standard Deviation of Group Disagreement $\left(d_{2}\right)$ from Simulation (With $\mathrm{N}=10, \mathrm{M}=7$, scale $[1 / 9,9], \mathrm{T}=500$ ) [Continued] . 253

C.21 Mean and Standard Deviation of Group Disagreement $\left(d_{2}\right)$ from Simulation (With $\mathrm{N}=10, \mathrm{M}=7$, scale $[1 / 9,9], \mathrm{T}=500$ ) [Continued] . 254

C.22 Mean and Standard Deviation of Group Disagreement $\left(d_{2}\right)$ from Simulation (With $\mathrm{N}=10, \mathrm{M}=9$, scale $[1 / 9,9], \mathrm{T}=500$ ) $\ldots \ldots 255$

C.23 Mean and Standard Deviation of Group Disagreement $\left(d_{2}\right)$ from Simulation (With $\mathrm{N}=10, \mathrm{M}=9$, scale $[1 / 9,9], \mathrm{T}=500$ ) [Continued] . 256

C.24 Mean and Standard Deviation of Group Disagreement $\left(d_{2}\right)$ from Simulation (With $\mathrm{N}=10, \mathrm{M}=9$, scale $[1 / 9,9], \mathrm{T}=500$ ) [Continued] .257

C.25 Mean and Standard Deviation of Group Disagreement $\left(d_{2}\right)$ from Simulation (With $\mathrm{N}=12, \mathrm{M}=3$, scale $[1 / 9,9], \mathrm{T}=500$ ) $\ldots \ldots 258$

C.26 Mean and Standard Deviation of Group Disagreement $\left(d_{2}\right)$ from Simulation (With $\mathrm{N}=12, \mathrm{M}=3$, scale $[1 / 9,9], \mathrm{T}=500$ ) [Continued] . 259

C.27 Mean and Standard Deviation of Group Disagreement $\left(d_{2}\right)$ from Simulation (With $\mathrm{N}=12, \mathrm{M}=3$, scale $[1 / 9,9], \mathrm{T}=500$ ) [Continued] . 260

C.28 Mean and Standard Deviation of Group Disagreement $\left(d_{2}\right)$ from Simulation (With $\mathrm{N}=12, \mathrm{M}=5$, scale $[1 / 9,9], \mathrm{T}=500$ ) . . . . 261

C.29 Mean and Standard Deviation of Group Disagreement $\left(d_{2}\right)$ from Simulation (With $\mathrm{N}=12, \mathrm{M}=5$, scale $[1 / 9,9], \mathrm{T}=500$ ) [Continued] .262

C.30 Mean and Standard Deviation of Group Disagreement $\left(d_{2}\right)$ from Simulation (With $\mathrm{N}=12, \mathrm{M}=5$, scale $[1 / 9,9], \mathrm{T}=500$ ) [Continued] .263

C.31 Mean and Standard Deviation of Group Disagreement $\left(d_{2}\right)$ from Simulation (With $\mathrm{N}=12, \mathrm{M}=7$, scale $[1 / 9,9], \mathrm{T}=500$ ) . . . . . 264

C.32 Mean and Standard Deviation of Group Disagreement $\left(d_{2}\right)$ from Simulation (With $\mathrm{N}=12, \mathrm{M}=7$, scale $[1 / 9,9], \mathrm{T}=500$ ) [Continued] . 265

C.33 Mean and Standard Deviation of Group Disagreement $\left(d_{2}\right)$ from Simulation (With $\mathrm{N}=12, \mathrm{M}=7$, scale $[1 / 9,9], \mathrm{T}=500$ ) [Continued] . 266

C.34 Mean and Standard Deviation of Group Disagreement $\left(d_{2}\right)$ from Simulation (With $N=12, M=9$, scale $[1 / 9,9], T=500$ ) $\ldots 267$

C.35 Mean and Standard Deviation of Group Disagreement $\left(d_{2}\right)$ from Simulation (With $\mathrm{N}=12, \mathrm{M}=9$, scale $[1 / 9,9], \mathrm{T}=500$ ) [Continued] .268

C.36 Mean and Standard Deviation of Group Disagreement $\left(d_{2}\right)$ from Simulation (With $\mathrm{N}=12, \mathrm{M}=9$, scale $[1 / 9,9], \mathrm{T}=500$ ) [Continued] .269

D.1 Category 1 - Lengths of Straight Lines . . . . . . . . . . . 271

D.2 Category 2 - Air Distance between Pittsburgh and Other Cities . . 272 
D.3 Category $3-$ Number of Times Football Teams Have Won the Super Bowl . . . . . . . . . . . . . . . . . . . 273

D.4 Category 4 - Metropolitan Area Population in 1980 . . . . . . . . 274

D.5 Category 5 - Annual Number of Air Passengers in Airports . . . . 275

D.6 Category 6 - Number of Professionals in Major Occupation in the Unite State . . . . . . . . . . . . . . . . . 276

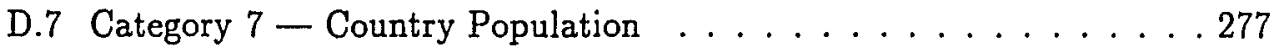

E.1 The Results of Empirical Test for Accuracy and Group Disaggrement with Geometric Mean Operated on Pairwise Comparison Matrix . . . 279

E.2 The Results of Empirical Test for Accuracy and Group Disaggrement with Arithmetic Mean Operated on Priority Vector . . . . . . . . . 280

E.3 The Results of Empirical Test for Accuracy and Group Disaggrement with Geometric Mean Operated on Priority Vector . . . . . . . . . 281

E.4 The Results of Empirical Test for Accuracy and Group Disaggrement with MDM Operated on Priority Vector . . . . . . . . . . . . . 282

E.5 The Results of Empirical Test for Accuracy and Group Disaggrement with MDM Operated on Pairwise Comparison Matrix . . . . . . . . 283 


\section{Chapter 1}

\section{INTRODUCTION}

\subsection{Objectives of This Dissertation}

Decision making is the process of selecting a possible course of action from all available alternatives. In almost all such selections, the multiplicity of criteria for judging the alternatives is pervasive. This decision making domain encompasses so many forms of problems that no single decision making procedure can possibly be sufficient. In fact, formal decision making methods are so numerous and diverse that they constitute the core of disciplines ranging from statistics, operations research/management science, and decision theory itself.

Despite many forms that decision problems exhibit, one of the fundamental tasks is to provide judgments about relative merits of choices that are available. For example, the grocery shopper chooses a preferred package, presumably considering such factors as price, flavor, packaging, and quantity. Businesses establish budget priorities; personnel departments evaluate potential employees; and corporate managers make program planning and program evaluations. All of these decisions can 
be described as fundamental comparison tasks. The pairwise comparisons technique is a technique used in judgment quantification for the evaluation of important relationships among decision elements. The process of qualifying judgments by using the pairwise comparison technique includes evaluating the importance of the relationship between a pair of decision elements. This is done for each pair, one at a time, without the distraction of the other elements. When all comparisons are completed, the results are expressed on a ratio scale as a reciprocal matrix via the pairwise comparison matrix. Then, by evaluating the reciprocal matrix in some representative way, the relative contribution of decision elements to the problem objective can be evaluated in the form of a normalized vector, and the methods that quantify the relative merits of each decision elements are called prioritization methods.

Within the above described process of qualifying judgments, this dissertation focuses on the methods for group judgment aggregation and the characteristics of judgment aggregation methods. Therefore, the following two objectives will be achieved:

1. to develop a new method for aggregating the judgments for group decision making, and

2. to make a comparison study of the aggregating methods.

These objectives form separate chapters in this dissertation, but they are linked together under the framework of the Hierarchical Decision Model (HDM) [1] via the Analytic Hierarchy Process (AHP) [2] [3]. The framework of AHP is discussed 
in detail in the next chapter. In the following sections, these two objectives are explained, and their expected research results are described.

\section{$1.2 \quad$ A Judgment Aggregating Method}

Aggregation of judgments is a critical aspect of the judgment quantification process for group decision making. In the typical situation, $m$ individuals ${ }^{1}$ provide quantifiable judgments such as pairwise comparison judgments. After all the information is considered and all efforts at changing each other's opinion are exhausted, either a consensus is reached or different judgments have to be aggregated. This is done either by a systematic group decision procedure, bringing consensus among the individuals, or by an aggregating method external to the decision makers. The focus of Chapter 3 in this dissertation is on the external method for aggregating the pairwise comparison judgments. Several aggregating methods are also reviewed in section 3.2, which include simple average and geometric mean.

A new aggregation approach is proposed in Chapter 3 . This new method is based on the following concepts:

1. general distance concept that developed by Yu [4] and Cook et al.[5]

2. the group disagreement can be expressed as a distance function of individual judgments v.s. the aggregated group judgments

In this new aggregation method, we treat the aggregated group judgments in the form of weighted geometric mean of the individual's judgment. In this approach,

\footnotetext{
${ }^{1}$ In this dissertation, individual, person, estimator and decision maker are used interchangeably. All mean the same that a human makes a pairwise comparison judgment in a decision situation
} 
the absolute distance appears to be the adequate distance function, which is also supported by the work of Cook et al. [5]. The objective is to find the weights of the weighted geometric mean which minimize the group disagreement in terms of distance function. We call this approach the Minimum Distance Method (MDM). The aggregation method leads itself to a goal programming formulation, which can be solved by using commercial software such as LINDO. The simulation and empirical test explained in Chapter 4 and 5 reveal that this new approach gives the best results in terms of accuracy when the variance of the judgments is high.

\subsection{A Simulation and Empirical Test of Methods for Aggregating Judgments}

The arithmetic mean and geometric mean methods have been used for judgment aggregations for a long time. Aczel and Saaty's $[6,7,8]$ contribution have been to provide a mathematical justification for geometric mean approach. However, very little has been done to test the different approaches by researchers. In Chapters 4 and 5, a simulation and empirical test are designed and conducted to evaluate the performance of aggregation methods which will be discussed in Chapter 3. Aggregation methods under study include the geometric mean, arithmetic mean and MDM proposed in this dissertation. The performance is evaluated by two criteria:

1. accuracy measurement, which is proposed to measure how close the aggregated group judgments is to the "real" value.

2. group disagreement, which is used to measure the deviation between the group members' judgments and the aggregated group judgments. 
Due to the need of transferring the pairwise comparison matrix to priority vector, the prioritization methods (see Appendix A for detail) are also involved in the simulation and empirical test. Fifteen prioritization methods are tested in this dissertation. As the result, the simulation and empirical test not only answer which aggregation method has good results in terms of measurements, but also determines the prioritization methods for which the aggregation method produces the best results in terms of measurements. The simulation uses various distributions for an error term in generating input data for the pairwise comparison matrix.

\subsection{Dissertation Outline}

Chapter 2 of this dissertation presents a literature review. Chapter 3, 4 and 5 explain the concepts and research questions involved in each of the two objectives presented in this chapter, and answer those questions in detail. Each chapter presents background information and a literature review on its discussed objective, then describes the proposed approach and analyzes the results. Chapter 5 discusses conclusions and the main results of this dissertation. Suggestions for future work are also included in Chapter 5. Appendix A presents background information on prioritization methods. Appendix B - E contains the data used in the dissertation. 


\section{Chapter 2}

\section{BACKGROUND AND LITERATURE REVIEW}

Applied decision analysis is concerned with the study of techniques to aid decision makers faced with complex decision problems, i.e. problems that challenge or exhaust the decision maker's capability to comprehend the consequences of any action he ${ }^{1}$ may take to solve them. Today's decision makers and problem solvers in government, business and industry - in any area of our society - encounter a variety of problems. "These problems are highly complex, often interdisciplinary or transdisciplinary, with social, economic, political, and emotional factors intertwined with more quantifiable factors of physical technology [9]". When attempting to solve a problem, all important factors of the problem should be considered, which in turn requires the decision makers to exercise the judgments on matters with more important consequences and complexity as a group. Moreover, decision makers are increasingly being called upon to make important judgment in unfamiliar circumstances. At the same time, decision support system (DSS) and group decision support system (GDSS) are emerging as very interesting tools to help and support the complexity of the individual and group decision process. As pointed out by

\footnotetext{
${ }^{1}$ Third singular is used to denote both genders in this dissertation. This approach is taken to avoid the inconvenience of using terms such as "he/she" and "his/her".
} 
DeSanctis and Gallupe, "A GDSS is an interactive, computer-based system that facilitates the solution of unstructured problems by a set of decision makers together as a group" [10]. In general, there is a need "for better support of deliberation and judgment to enable more structured problem solving and decision making"[11].

In this dissertation, there are two objectives. One focus is to develop methoc's to combine the group judgments for the group decision process, and adaptable for incorporation into any group decision support system (GDSS). Another objective is to evaluate the performance of the methods developed in this dissertation and other existing methods proposed in the literature. All of the work presented in this dissertation is under the decision analysis framework of the Analytic Hierarchy Process (AHP). The details of work are presented in Chapters 3, 4 and 5. In this chapter, the background information and literature research are presented, covering the following items:

- History of AHP

- AHP and its procedure

- Characteristics of group decision making

- Techniques of group decision making

- The research areas of AHP 


\section{$2.1 \quad$ History of AHP}

AHP, as a general theory of measurement, had its beginnings in the fall of 1971 while Saaty was working on problems of contingency planning for the Department of Defense [3]. The application maturity of the theory came with the Sudan Transport Study in 1973, which Saaty was directing $[12,13]$. Its theoretical enrichment was happening all along the way, with greatest intensity between 1974 and $1980[3,14$, $15,2,16,17,18,19]$. During this period, the theoretical works are focused on the foundation of the AHP paradigm, broadly speaking, rest upon two concepts:

- a theory of measurement and prioritization, known as eigenvector prioritization.

- a theory of hierarchical composition.

Ever since Saaty's development of the Analytic Hierarchy Process (AHP) in the 1970s, the research area have been greatly extended since 1980s. The most significant advance in the AHP include establishing the axiomatic foundation of AHP [20] and the relationship between priority theory (AHP) and utility theory $[21,22]$. Other research areas include:

- Prioritization Method deals with translating qualitative judgment in pairwise comparison matrix into priority vector $[23,24,25,26,27,28]$.

- Incomplete Pairwise Comparison deals with incomplete judgments $[29,30,31$, 32]. 
- The Composition Principle deals with approaches for combining the priority vectors through the hierarchy $[15,33,31]$

- Group Judgment and Consensus deals with the approaches for aggregating judgment for group decision making $[6,7,8]$.

All of those research areas will be further reviewed in the section 2.6.

\section{$2.2 \quad \mathrm{AHP}$ and Its Procedures}

As Saaty [3] points out, complex decision problems generally require systematic structuring and decomposition before the rudiments of the problem are understood an dealt with decisively. Ideally, the analysis of complex problems should incorporate both the qualitative and quantitative aspects of the problem into a framework capable of generating priorities for the proposed solution strategies. The Analytic Hierarchy Process (AHP) is a method that can be used to establish measures in both the physical and social domains. It has become increasingly popular in diverse areas of application. As its name indicates, this decision method is characteristically analytic, i.e. its basic philosophy stresses the decomposition and recomposition of complex problems as a fundamental solution approach.

The AHP is a general theory of measurement. It is used to derive ratio scales and choices for multi-criteria decision problems. The building-block of the AHP is pairwise comparison, which is used to derive the preferences of decision makers. Pairwise comparisons may be taken from actual measurements or from a funda- 
mental scale which reflects the relative strength of preferences and feelings. In its general form, the AHP is a nonlinear framework for carrying out both deductive and inductive thinking. It takes multiple factors into consideration simultaneously and allows for dependence, for feedback, and for making numerical tradeoffs to arrive at an aggregation or conclusion.

In order to put research objectives in perspective, this section begins with a background description of AHP to discuss foundations and axioms involved in the AHP and is followed by application procedures for multi-criteria decision problems.

\subsubsection{The AHP}

The AHP is a problem-solving framework. It is a systematic procedure for representing the elements of multi-criteria decision problems. It organizes the basic rationality by breaking down a problem into its smaller constituent parts, which Saaty believes better fits the human cognitive style because of the way it decomposes and synthesizes the decision problems and then calls for only simple pairwise comparison judgments to develop priorities in each level of the hierarchy. Three principles guide one in problem solving using the AHP [28].

Principle of Decomposition: It calls for structuring the hierarchy to capture the essential elements of the multi-criteria decision problem. The hierarchy is constructed in such a way with the elements at a level being "independent" from those at succeeding levels, working downward from the focus in the top level, to criteria bearing on the focus in the second level, followed by subcriteria in the third 
level, and so on, from the more general to the more particular and definite. The hierarchical structure also can start from the bottom from particular alternatives and move up to more general objectives and goals. Saaty [20] makes a distinction between two types of relationships or dependence among the elements of hierarchy, which he calls functional and structural. The former is the familiar contextual dependence of elements on the other elements in performing their function, whereas the latter is the dependence of the priority of elements on the priority and number of other elements. Absolute measurement, sometimes called scoring, is used when it is desired to ignore such structural dependence among elements, while relative measurement is used otherwise.

Principle of Comparative Judgments: It calls for setting up a matrix to carry out pairwise comparisons of the relative importance of elements in some given level with respect to a shared criterion or property in the level above. In the case where no quantitative measurement exists, the judgment is made by the individual or group of individuals who are engaged in solving the decision problem. The scale for entering judgments is mentioned in Step 2 of section 2.1.2. The process could be started either at the bottom level and move upward or at the top level and move downward. An entry of each matrix belongs to a fundamental scale employed in the comparisons. These are used to generate a derived ratio scale.

Principle of aggregating the priorities: In the AHP, priorities are synthesized from the second level down by multiplying local priorities by the priority of their corresponding criterion in the level above and then adding them together for each element in a level according to the criteria it affects. This gives the composite 
or global priority of that element, which in turn is used to weigh the local priorities of the elements in the level below compared to each other with it as the criterion, and so on to the bottom level. When a group uses the AHP, its judgments should be combined.

Keeping these principles in mind, Saaty [20] proposes four axioms on which the AHP is based. The theory of the AHP is derived from these axioms. The axioms are as follows:

Axiom 1: (Reciprocal Comparison). The decision maker must be able to make comparisons and state the strength of his preferences. The intensity of these preferences must satisfy the reciprocal condition: If $\mathrm{A}$ is $\mathrm{x}$ times more preferred than $B$, then $B$ is $1 / x$ times more preferred than $A$.

Whenever we make a paired comparison we need to consider both members of the pair to judge their relative values. For example, if one ball is judged to be four times larger than another, then the other one is automatically one fourth as large as the first because it participated in making the first judgment. The comparison matrices that we considered are formed by making paired reciprocal comparisons, and this is a powerful means of solving multi-criteria problems, which is the basis of the AHP.

An important aspect of the AHP is the idea of consistency. If one has a scale for properties possessed by some objects, and the properties are measured by the scale, then their relative weights with respect to those properties are fixed. In this case, there is no judgmental inconsistency. But when comparing with respect to a property for which there is no established scale or measure, we are trying to derive a 
scale through comparing the objects two at a time. Since the objects may be involved in more than one comparison and we have no standard scale, and the objects are assigned relative values as a matter of judgment, inconsistencies may well occur. There are several consistency measurements are presented in the literature, which will be discussed in section 2.4.0.

Axiom 2: (Homogeneity). The preferences are represented by means of a bounded scale.

Homogeneity is essential for meaningful comparisons, as the mind tends to make large errors when comparing widely disparate elements. For example, we cannot compare a mouse with an elephant according to size. When the disparity is great, elements should be placed in separate clusters of comparable size, or at different levels altogether.

Axiom 3: (Independence). When expressing preferences, criteria are assumed to be independent of the properties of the alternatives.

Axiom 4: (Expectations). For the purpose of making a decision, the hierarchical structure is assumed to be complete.

This axiom simply says that the decision makers who have reasons for their beliefs should make sure that their ideas are adequately represented in the model. All alternatives, criteria and expectations (explicit and implicit) can be and should be represented in the hierarchy. It neither assumes rationality of the process nor that the process can only accommodate a rational outlook. People often have expectations that are irrational. 
The relaxation of Axiom 1 indicates that the question used to elicit the judgments or paired comparisons is not clearly or correctly stated. If Axiom 2 is not satisfied, then the elements being compared are not homogeneous and clusters may need to be formed. Axiom 3 implies that the weights of criteria must be independent of the alternatives considered. A way to deal with a violation of this axiom is to use a generalization of the AHP known as the supermatrix approach. Finally, if Axiom 4 is not satisfied, then the decision maker is not using all the criteria and/or all the alternatives available or necessary to meet his reasonable expectations and hence the decision is incomplete.

\subsubsection{AHP Procedures}

Decision applications of the AHP are carried out in four steps $[58,22]$ :

Step 1: Setting up the decision hierarchy by breaking down the decision problem into a hierarchy of interrelated decision elements.

Step 2: Collecting input data by pairwise comparisons of decision elements.

Step 3: Using "scaling" methods to estimate the relative weights of decision elements.

Step 4: Aggregating the relative weights of decision elements to arrive at a set of ratings for the decision alternatives (or outcomes).

In Step 1, which is perhaps the most important aspect of the AHP, the decision analyst should break down the decision problem into a hierarchy of interrelated 
elements $[2,12,16,14,3]$. At the top of the hierarchy lies the most macro decision objective, such as the objective to maximize the wealth of the shareholder. The lower levels of the hierarchy contain attributes that increase at the lower levels of the hierarchy. The last levels of the hierarchy contain decision alternatives or selection choices. The decision schema, hence, has a standard form as depicted in Fig. $2.1[34]$.

Level 1

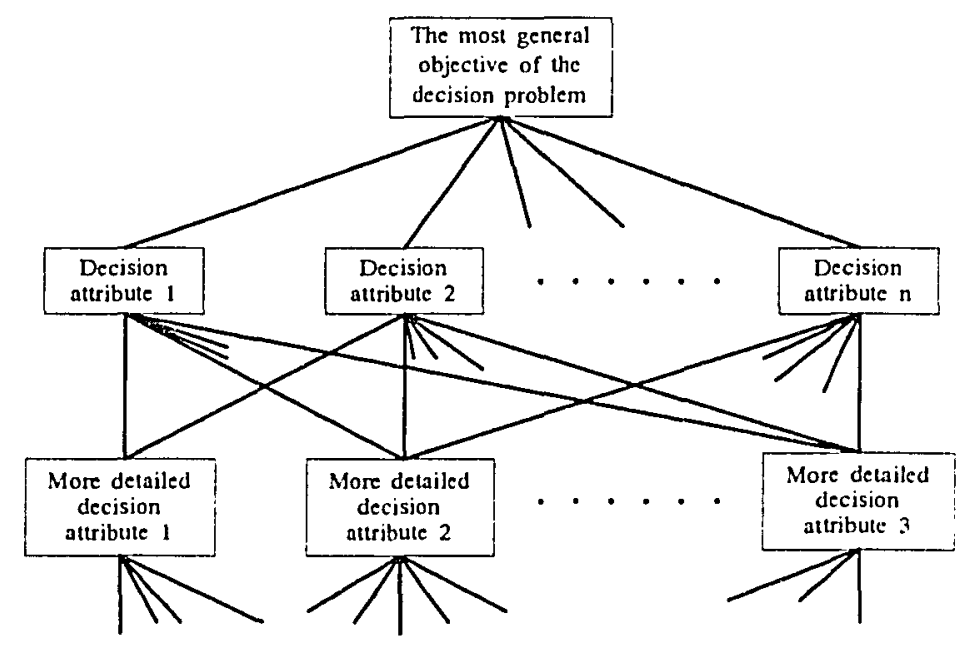

Level 2

Level 3
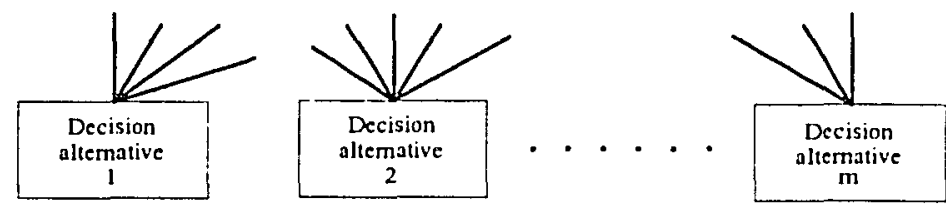

Figure 2.1: The standard form of decision schema in the analytic hierarchy process: a hierarchy with $\mathrm{k}$ levels.

For example, Kocaoglu's MOGSA [1] is a hierarchical model using the Mission, Objective, Goals, Strategies, and Actions levels envisioned by the decision maker in the decision process. MOGSA can be used as a general guideline for forming a 
hierarchy. This approach has been satisfactorily used by Shipley [35] for strategic planning of the engineering school in a university. Forman et al. [11] also provide a list of typical hierarchical structures:

- Goal, criteria, alternatives

- Goal, criteria, subcriteria, alternatives

- Goal, scenarios, criteria, (subcriteria), alternatives

- Goal, actors, criteria, (subcriteria), alternatives

- Goal, ..., subcriteria, levels of intensities (many alternatives)

In setting up the decision hierarchy, the number of levels depends on the degree of details that the analyst requires to solve the problem. Since each level entails pairwise comparisons of its elements, Saaty [3] suggests that the number of elements at each level be limited to a maximum of nine. This constraint, however, is not a necessary condition of the method and has not been adhered to in all applications.

In Step 2, the input data for the problem consists of matrices of pairwise comparisons of elements of one level that contribute to achieving the objectives of the next higher level. For example, in a project selection application, project 2 may be twice as important as project 1 in terms of profit. The input matrix in this case would look like Table 2.1:

The value 2 in row 2 and column 1 of the above matrix indicates that project 2 is twice as important as project 1 in achieving the objective of the next higher level: 
Table 2.1: Pairwise Comparison of Two Elements

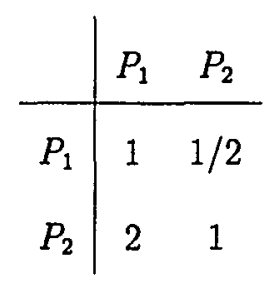

in this case, profitability. In row 1 , column 2 , the value of $1 / 2$ indicates the relative importance of project 1 compared to project 2 . When compared with itself, each element of the input matrix is always equal to one, and the lower triangle elements of the matrix are the reciprocals of upper triangle elements. Thus, pairwise comparison data are collected for only half of the matrix elements, excluding diagonal elements.

One may argue that it is possible to assign weights directly to the elements of a level. For example, instead of obtaining pairwise weights, one may directly assign relative weights of $2 / 3$ and $1 / 3$ to project 1 and project 2 for their role in making a profit. The argument in AHP is that such a direct assignment of weights is too abstract for the evaluator and results in inaccuracies. Pairwise comparisons, on the other hand, give the evaluator a basis on which to reveal his or her preference by comparing two elements. The evaluator has the option of expressing preferences between the two as equally preferred, weakly preferred, strongly preferred, or absolutely preferred, which would be translated into pairwise weights of $1,3,5,7$ and 9 , respectively, with $2,4,6,8$ as intermediate values. We can also use the Constant-Sum Measurement for the same purpose. A total of 100 points are distributed between the two elements to express the respondent's judgment about the 
ratio of one element to the other; one element of the pair is given the integer value (I) from 1 to 99 , and the other element has the value $(100-\mathfrak{I})$. For example, if one element is three times as important as the other, 75 and 25 are distributed, respectively.

In Step 3, the AHP takes as input the above pairwise comparison matrix and produces the relative weights of elements at each level as output. The argument for the solution methodology is as follows $[36,37]$ : If the evaluator could know the actual relative weights $\left(v_{j \downarrow}\right.$ of $\mathrm{n}$ elements $(j=1, \cdots, n)$, which is at one level of the hierarchy with respect to one level higher, the matrix of pairwise comparisons would be $A_{\tau}=\left(v_{j t} / v_{k t}\right)(i, j=1, \cdots, n)$. In this case, the relative weights could be trivially obtained from each one of $\mathrm{n}$ rows of matrix $\mathrm{A}$, where $V_{\mathbb{I}}=\left(v_{11}, \cdots, v_{n t}\right)$ is the vector of actual relative weights, and $\mathrm{n}$ is the number of elements.

AHP posits that the evaluator does not know $V$ and, therefore, is not able to produce the pairwise relative weights of matrix $A_{\mathbb{T}}$ accurately. Thus, the observed pairwise comparison matrix $A$ contains inconsistencies. The estimation of $V_{\mathfrak{T}}$ (denoted as $V$ ) could be obtained from

$$
V=f(A)
$$

where $A$ is the observed matrix of pairwise comparisons, $f()$ indicates the estimation method used. (A number of estimation methods exist. For a detailed review of them please see Appendix A.) An important concern is the difficulty to satisfy the consistency conditions. It is not unusual for an evaluator to be inconsistent in expressing his judgments, especially if he is dealing with fuzzy concepts such as 
quality, attractiveness, evolvability, etc. Inconsistency can also be caused by the limited scale that evaluators used to elicit their judgments.

In Step 4, it aggregates relative weights of various levels obtained from Step 3 in order to produce a vector of composite weights which serve as ratings of decision alternatives (or selection choices) in achieving the most general objective of the problem. The composite relative weight vector of elements at kth level with respect to that of the first level may be computed from

$$
C[1, k]=\prod_{i=2}^{k} B_{i}
$$

where $[1, k]$ is the matrix of composite weights of elements at level $\mathrm{k}$ with respect to the elements on level 1 , and $B_{i}$ is the $n_{i-1}$ by $n_{i}$ matrix with rows consisting of estimated $V$ vectors, $n_{i}$ representing the number of elements at level i [38]. At the top level, $k$, where we usually have one element, such as the mission, $[1, k]$ is reduced to a vector of composite weights. We also noticed that this approach of aggregation will cause the rank reversal problem. Barzilai and Golany [39] proposed a axiomatic framework for deriving consistent weight ratios from pairwise comparison matrices and aggregating weights and comparison matrices. If a multiplicative aggregation rule is used and normalized vectors are replaced with weight-ratio matrices, and the rank reversal problem can be avoided.

\subsection{Characteristics of Group Decision Making}

There are three major reasons for people to make a decision as a group. First, the decision problems that modern businesses and governments are confronted with 
different types and complexities, those complexities range from a lack of complete information, conflicts among objectives or interests, linkages between problems, and the cost nature of commitments in resolving complex problems [40]. Second, "in society, decisions often affect groups of people instead of isolated individuals. However, the group decision making is usually understood to be the reduction of many different individual preference (interests) to a single choice, either by conflict or by compromise[9]." Third, the information handling capability of human being is limited by his knowledge, experiences, and even his very nature.

Characteristics of the group process are reviewed in this section. Carefully handling those characteristics during the group decision process will help us to improve the individual and group performance as a whole. The characteristics being reviewed include these factors:
a. boundary of the group
b. information aspects
c. tension and conflict among group members
d. resistance nature of human beings
e. explicit-implicit nature of the problem description
f. normative and localized behavior of group processing 


\subsubsection{Boundary of the Group}

The boundary of the group is defined as certain restrictions applied on such things as: who the group's members are, what the entry and exit requirements are, and how much commitment the members have to the group. For example, formal organizational groups may have quite impermeable boundaries, allowing inside only people of particular rank or those who are deemed by the group's leader to be relevant to the problem. In particular, the following considerations should be taken into account for identifying the boundary of the group:

- The size of the group is only mildly approximated by the numerical count of bodies in attendance at meeting. The most important factor of all is that the willingness and ability of each member, singly and collectively, to commit his or her resources and energy to the problem of the group, and its maintenance determine the effective size of the group [41].

- The task environment is also the group's boundary. The group manifest purpose determines what problems it is supposed to deal with. For example, engineering managers consider problems of engineering [41].

- The value and belief system of the group may also be considered as part of its boundary. A group of engineering managers might view a problem in one way, while a group of marketing managers might see the same situation quite differently - the differences arising from their different professional experiences. The effectiveness of a group coping with its task environment is often 
made difficult by the fact that people with similar backgrounds, personalities, or roles are likely to define a problem in only one way and missing possible alternatives $[41,40]$.

\subsubsection{Information Handling Capability}

The limited capability of handling the information for individuals is one of the most important factors for people forming groups to deal with complex decision problems. It is generally impossible for any decision maker involved to construct a comprehensive model of the decision situation with all relevant parameters and their relationships. With only limited information available, no formulation of a complex problem can be assumed automatically to contain all possible solutions to the problem. However, "today's decision makers and problem solvers in government, business, industry, and education - in any area of our society - are confronted with a variety of problems. These problems are highly complex, often interdisciplinary or transdisciplinary, with social, economic, political, and emotional factors intertwined with more quantifiable factors of physical technology [9]". Therefore, when attempting to solve a complex decision problem, all important factors of the problem should be considered, which in turn requires us to make decisions as a group to enhance the capability to handle all necessary information. Furthermore, it is not the case that two different participants in a problem have the same information available to them. In fact, the information available to two participants will generally be different, unless there has been complete and continuing commu- 
nication between them. Each participant's perception of the problem in which he is involved is based on the information available to him and depends on the nature of his motivations and spheres of competence, experience and judgment.

\subsubsection{Tension and Conflict}

"Complex decision problems are often concerned with situations in which a number of objectives must be pursued simultaneously and in which it is necessary to consider all of these objectives in choosing a policy or course of action. In most situations, those objectives are conflict with each other [40]". In such cases, the adoption of a course of action that allows maximum achievement of one objective may result in less progress toward satisfying others. Consequently, for every potential decision there are sources of tension and conflict. First, whenever the decision involves a choice between alternatives, there is a loss and gain of factors that must be weighed. There are further potential conflicts as a result of disagreement among individual participants, as well as from the implication any decision will have upon the group as a whole. Second, natural tension and conflict are created after the individual or group makes a decision. This stems from being faced with having to live with the decision that has just been made and thus having to continually justify it in the mind of the group and in the mind of others.

It appears natural, therefore, that tension and points of conflict exist within decision making groups. The question becomes one of whether or not the sources of tension are clearly recognized and dealt with in the most constructive manner 
possible. All too often the greatest sources of tension and conflict are completely avoided, denied or ignored. If the actual sources of tension are not uncovered and dealt with, it is highly likely that they will be diffused into other areas of the group's experience.

\subsubsection{Resistance}

The individual attempts to bring his or her life into state of equilibrium in which he is able to predict events and reduce conflict. To change this relatively stable, steady state results in a need to change accustomed patterns of behavior and creates, at least temporarily, discomfort and tension. However, problem solving and eventual decision making often lead to innovation, alternative courses of action, and a disruption of a group's or individual's state of equilibrium. It is evident that unless individuals feel personally secure and relatively unthreatened within the problem solving group, they will tend to respond with their own characteristic patterns of defense [40]. The frustration which often arises from working with a decision making group results from an inability to understand and accept as perfectly natural many of the resistances that develop during the decision making process.

\subsubsection{Explicit-Implicit}

Explicit problems of the group dominate the implicit functions. By dominate we mean that when issues are made explicit by the group, they are treated as legitimate topics for discussion and come under the self-conscious control of the members. 
Hoffman [41] points out "As the members define the decision problem and suggest solutions, they develop implicit norms about turn taking, dominance relationships, etc." Other issues that also affect the group are kept at an implicit level, where their interpretation is more ambiguous. But it is not unusual for decisions to be made by implicit criteria that are not discussed, especially if the power relationships in the group are clearly understood.

\subsubsection{Normative and Localized Behavior}

Hoffman [41] points out another dimension of the group process, i.e the normative and localized behavior. The norms are a set of guidelines developed to regulate the behaviors of group members to replace the need for direct interpersonal control. There are two extreme points of this normative and localized behavior dimension. At the localized extreme of the dimension, each person behaves somewhat idiosyncratically, reflecting his or her personality, external role, or even temporary mood. At other points along the dimension are such phenomena as stereotypes and coalition formation, in which the norms for some subset of the group are different than they are for others.

The norms not only exist in the concerning participation, expressions of emotionality, and etc, but also in the procedures by which a group solves a problem. The various techniques that have been invented to facilitate problem solving, which will be discussed next section, such as brainstorming and Delphi method, have explicitly stated rules to which the members must conform. In addition to the explicitly 
stated norms, there is an implicit one too, which usually define the general character of a group meeting. It is noticed that the concept of the norm may be quite different. The events that define the norm will be interpreted differently according to the motives and perceptions of each individual. Therefore, norms often lead to dysfunctional consequences for groups and are difficult to change.

\subsection{Techniques for Group Decision Making}

Group decision making under multiple criteria includes such diverse and interconnected fields as preference analysis, utility theory, social choice theory, committee decision theory, theory of voting, general game theory, expert evaluation analysis, aggregation of qualitative factors, and economic equilibrium theory. With the focus of expert judgment aggregation in this dissertation, the techniques for expert judgment and group participation is the object of this review.

The problem of group decision making can be broadly classified into two categories: experts' judgment and group participation. The expert judgment process entails making a decision by inventing a new alternative. Specifically, it is concerned with forecasting and involves constructing supplemental objects which may be new designs or technical solutions. On the other hand, the group participation process entails groups which have common interests, such as a community or an organization, making a decision. The techniques used for expert judgment and group participation focus on the method of generating/pooling ideas and the method of systematic structuring, which are classified by Hwang and Lin [9]. 
The idea generation methods are for producing a large quantity of ideas. The methods of stimulating are brainstorming and brainwriting and its variations, and the Nominal Group Technique (NGT). In general, brainstorming refers to verbal generation of ideas while brainwriting involves silent, written idea generation. NGT is a combination of brainwriting, discussion and voting techniques to generate a solution. On the other hand, polling of experts' options can be used to produce a quick sense of the prospects in a particular subject area. A critical concern of this method is identification of experts. Experts may be certified by a variety of means - educational degree, professional memberships, peer recognization, and even selfproclamation. Two type of experts can be identified as potentially useful in the problem solving. The first belongs to the representatives of subpopulation whose attitudes or actions influence the research topics we are concerned with. The methods of surveys and Delphi will be reviewed which use these types of experts. The second type of expert has extensive special knowledge and experience about the research topic we are concerned with. The methods of conferences and Successive Additive Numeration use these experts. More detailed descriptions of the above mentioned techniques are presented in the following sections.

\subsubsection{Brainstorming}

Osborn's [42] attempts to improve the creativity of his advertising staff evolved into the brainstorming method. Fundamental to its use is the "principle of deferred judgment" - the postponement of evaluation during the period of idea generation. 
The value of this method is two-fold: First, the members' efforts are concentrated on developing a roster of possible solutions, then on their evaluation. In this way no solution can acquire enough positive valence to pass the adoption threshold nor enough negative valence to drop below the rejection threshold before many alternatives have been proposed and described. Second, by having a procedure - a task norm - that permits only the proposing of alternatives, the members feel secure in searching for new ideas without fear that their current favorite will be discarded. Members can be proactive rather than defensive in their approach to problems [41]. There are four basic rules used to guide a brainstorming session [9]:

1. Criticism is ruled out

2. Free-wheeling is welcomed

3. Quantity is wanted

4. Combination and improvement are sought

Usually, the brainstorming group consists of members, a leader, a secretary, and a blackboard. The leader should remind the group of the problem at hand and the rules for brainstorming. The recording secretary should sit next to the leader so that he is in the direct line of conversation between him and the others. The ideas should be taken down reportorially - not word by word. To achieve good success in free-wheeling, only people of equal status should be invited to participate. The brainwriting method is developed to avoid negative effects of brainstorming sessions or group meetings so that the influence of opinion-leaders, some group members, 
and restraints against free-wheeling speaking is eliminated [9].

\subsubsection{Nominal Group Technique (NGT)}

This method [69], which combines elements of brainwriting, brainstorming, and the voting technique, adds another dimension to the separation of idea generation and idea evaluation. Studies of brainstorming groups show a tendency to limit solution proposals to particular directions. The NGT attempts to release the total creativity of the group in two ways [41]. First, group members are required to develop solutions to the problem individually, without consulting each other. In this way, each member's perspective on the problem enters the group's problemsolving efforts uncontaminated by the other's points of view. Second, each member is required to contribute one solution to the group in turn or to pass his or her turn. This procedure continues until all solution possibilities have been exhausted. In this way, every member's idea has a chance to enter the group's deliberations without having to fight its way in. The principal advantage of NGT over brainstorming in the solution proposal stage is its defense against the participation and influence biases that derive from the personalities or statutes of the members.

\subsubsection{Surveys}

This is a method to poll a group of experts about their opinions. Surveys are useful when a group of appropriate respondents can be identified and when interaction among the respondents is not a necessary consideration. Surveys may be formal 
or informal. In general, there are three important forms:
A. Face-to-face interviews
B. Telephone interviews
C. Mail questionnaires

Survey techniques usually involve several stages as identified in [9], which are:

1. Planning stage, which involves setting the goals for the survey and advising a general strategy to obtain and analyze the data.

2. Research design stage, which is a prearranged program for collecting and analyzing the information needed to satisfy the study objectives at the lowest possible cost.

3. Sampling, which is the process of choosing certain people in the population to represent the whole. At this stage the researcher must carefully define the population to be studied.

4. Questionnaire design, which is a process of translating the broad objectives of the study into questions that will obtain the necessary information. At the same time the form of survey is also laid out.

5. Editing and coding, which is designed to translate the information recorded in the questionnaires into a form suitable for statistical analysis.

6. Preparation for analysis, which is a process to identify and correct any errors in above mentioned stages. 
7. Analysis and reporting, which is a stage of presentation and interpretation of simple distributions and cross tabulations of information collected in the survey.

\subsubsection{Delphi Technique}

The Delphi Technique [43] was designed primarily for noninteracting groups, which can be viewed as a modification of the brainwriting and survey technique. In this method, a panel is used with members in communication remotely through several rounds of questionnaires transmitted in writing. However, besides its obvious advantages for a group whose members are geographically distant, one of its principal objectives is to minimize the effects of status differences on the decision-making process. Delphi is an expert opinion survey with three special features - anonymous response, iteration and controlled feedback, and statistical group response. In its simplest form, the method asks each member of the group to make an independent and anonymous judgment on a predefined problem. This judgment is then averaged, giving each person's judgment equal weight. The members are then told what the average and the distribution of judgments were and are asked to vote again. Reasons for different votes may be included in the report. This process may be repeated again if necessary to promote consensus.

The principal advantages of the Delphi Method are two related ones. First, the anonymity of votes and their equal weight prevent the higher status members from having undue weight on the decision. On the assumption that all members 
of the group have relevant information, the intrusion of maintenance factors on the decision is then reduced. The second advantage is that there is an explicit, easily understood mechanism for making a final decision, which avoids the biases of the implicit valence adoption process [41].

By avoiding any discussion of the problem among the members, however, the Delphi Technique runs two risks. The first is a lack of understanding of the problem and of the final decision. There is an implicit demand for conformity to the majority created by the noninteractive process of collecting judgments. It is difficult for a group to adopt a truly creative solution to a problem through the Delphi Technique since the ideas of the minority are not usually clarified [41].

\subsubsection{Structure Modeling}

Systematic structuring analysis employs interaction matrices, graphs, intent structures, signal flow graphs, etc, to identify a structure within a system of related elements. The purpose of the systematic structuring process is to transform unclear, poorly articulated mental models of systiems into visible, well-defined models useiul for many applications. There are two such models for this purpose.

Interpretive Structure Modeling (ISM): This approach "is intended for use when it is desired to utilize systematic and logical thinking to approach a complex issue and then to communicate the results of that thinking to others [44]." The objective is to expedite the process of creating a digraph, which can converted to a structural model. This objective is achieved by the systematic application of 
some notions of graph theory in such a way that theoretical, conceptual and computational leverage is exploited to efficiently construct a directed graph, or network representation, of complex pattern of contextual relationship among a set of element with the aid of computer.

The mathematical basis for ISM is found in theory of sets, relations, and directed graphs. Warfield [45] has presented comprehensive techniques for identification of the structure in a system. In general, the process of ISM is based upon the oneon-one correspondence between a binary matrix and a graphical representation of a directed network. The fundamental concepts of the process are an "element set" and a "contextual relation." The element set is identified within some situational context, and the contextual relation is selected as a possible statement of relationship among the elements in a manner that is contextually significant for the purposes of enquiry. The elements correspond to the nodes on a network model, and the presence of the relation between any two elements is denoted by a directed line (or link) connecting those two elements (nodes). In the equivalent binary matrix representation, the elements are the contents of the index set for the rows and columns of the matrix, and the presence of the relation directed from element $i$ to element $\mathrm{j}$ is indicated by placing a 1 in the corresponding intersection of row $\mathrm{i}$ and column j.

Fig. 2.2 is a representation of the principle operations of ISM when implemented in man/machine interactive mode as depicted by Malone [44]. "People are assumed to make observations in the real world and to draw upon their own knowledge and attitudes to identify pertinent concepts and relationships. The embedding opera- 
tion is performed jointly by man and machine. The computer is supplied with an appropriate list of elements and the definition of a pertinent relation. A systematic sequence of queries is then generated, and a binary matrix representation of the system is assembled from responses provided by a person or group of persons. When the matrix model is completed, computer operations are performed in order to partition the elements into natural hierarchical levels and to establish a minimal set of linkages which captures the entire pattern of the relation. The multilevel directed graph which results can be inspected and interpretive symbols introduced according to the context, to produce an interpretive structural model. This process can be iterative until the creators are satisfied."

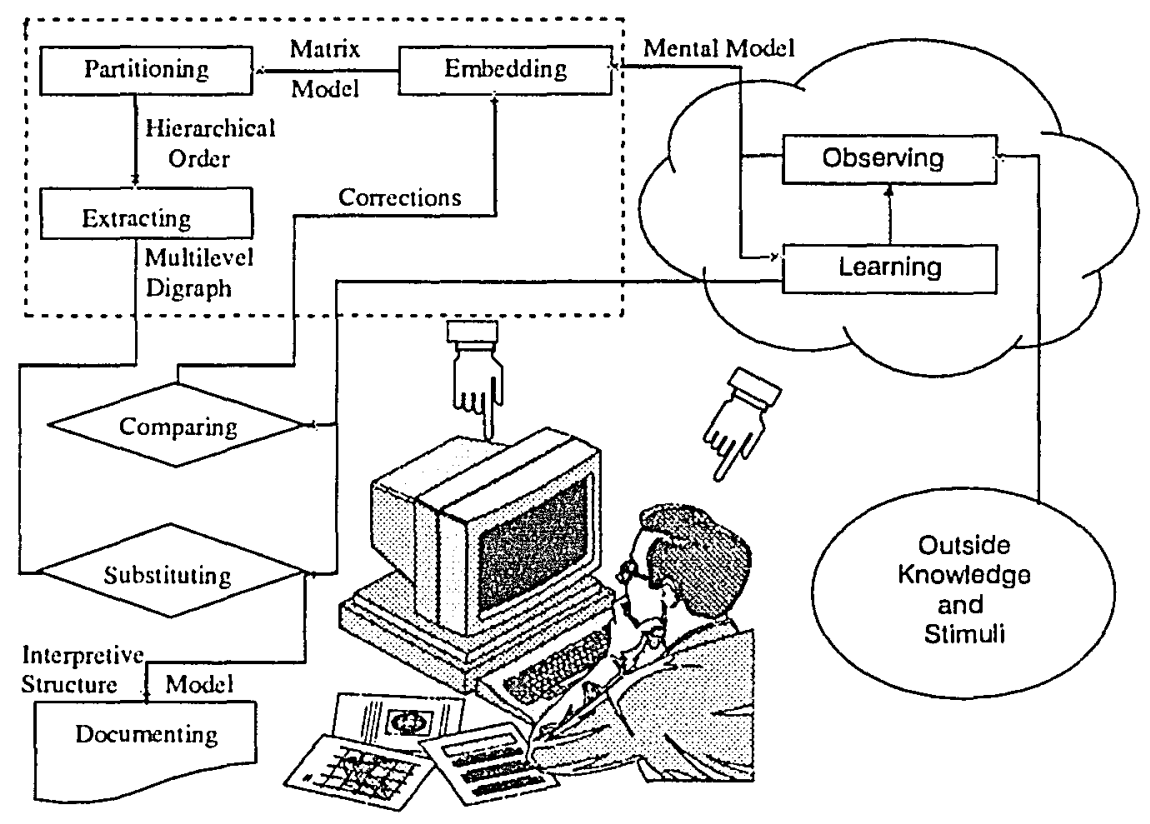

Figure 2.2: Functional Representation of Interpretive Structural Modeling 
There are several advantages of using ISM as pointed out by Warfield [45] and Malone [46]:

- ISM operates without such a priori knowledge of the structure. The process is initiated by specifying an element set and the a transitive relational statement.

- No knowledge of the underlying mathematics of the process is required of the user. He simply must process enough knowledge of the context to answer the queries of the computer.

- The process is systematic efficiency; computer is programmed to handle all possible pairwise interactions of elements either through asking questions of user or using transitive inference based on the responses of user.

Cognitive Map: This method is a mathematical model of a person's belief system and is designed to capture the structure of causal assertions of a person with respect to a particular policy domain, and generate the consequences that follow from this structure. A cognitive map contains only two basic types of elements: concepts and causal beliefs. The concepts are treated as variables, and the causal beliefs are treated as the relationship between the variables. The concepts that a person uses are represented as points, the causal links between these points should represent the relationships. This gives a graphical representation of the causal assertions of a person as a graph of points and arrows. The policy alternatives, all of the various causes and effects, the goals, and the ultimate utility of the decision maker can all be thought of as concept variables and represented as points in the cognitive 
map. As pointed out by Hwang and Lin [9], the real power of this approach is when a cognitive map is pictured in graph form; it is relatively easy to see how each of the concepts and causal relationships relate to each other, and to see the overall structure of the whole set of portrayed assertions. Three methods for deriving a cognitive map are proposed by Hwang and Lin [9]. First, the cognitive map can be derived from existing documents, which has the advantage of being both unobtrusive and fully able to employ the concepts used by the decision maker himself. Second, the questionnaire method employs a questionnaire sent to a panel of judges who are in a position to make an informed estimation of causal links, which has the advantage of allowing aggregation of individual opinions and results in a much wider range of information than researchers can select for documentary analysis. The third method is to use an open- ended probing interview. It has the advantage of allowing the researcher to interact actively with the source of his data.

\subsection{AHP for Group Decision Making}

From the above discussion of techniques for group decision making, researchers share the task of addressing two major issues [47]. One is the processing of information. When we speak of decision making, whether with reference to individuals, groups, organizations, governments, or any other entity, of necessity we speak of information processing. This includes collecting and evaluating information, forging alternative courses of action, and selecting one as preferred. The study of groups as decision makers, however, entails a second focus: the social-psychological dynamic 
of behavior. All attempts to understand group decision making must address both issues.

There is a strong mutuality of influence between information-handling activities and social psychological forces. How information is acquired and evaluated can limit the nature of social interaction among group members. For example, the procedure for reaching planning decisions in groups called the Nominal Group Technique (NGT) imposes strict guidelines concerning how information is to be managed, and these guidelines in turn limit the ways in which social influence can take place among group members.

Different perspectives on group decision making, then, ultimately address the interrelationship of information-processing activities and the dynamics of behavior in small groups in order to understand and improve group decision making. The interaction of social behavior and information processing is handled by introducing the interventions into the group decision process [47]. The techniques for improving group decision making have build-in mechanisms for the interventions. Especially, those group decision methods are for generating and polling ideas, even for the problem structure.

Interventions to improve group decision making can be regarded as being of two types their primary target as pointed out by Guzzo [47], which are the action of group decision making and inputs to group decision making. The first type has as its target direct changes in the behavior of decision- making group members. These changes could be brought about by the creation of new patterns of social interaction, or by the establishment of specific procedures of task accomplishment: 
requiring groups to adhere to a sequence of steps such as defining the problem, generating alternatives, and then evaluating and choosing among alternatives for example. Thus, such interventions can affect either or both the social-psychological influences residing in a group and the processes of manipulating and utilizing information.

The second type, input-oriented interventions, also seeks to change behavior in groups, but it attempts to do this indirectly rather than directly. Inputs to a group decision include the distribution of abilities and vested interests among group members, the nature of available information, group size, the reward structure under which a group exits, and the time pressures for decision making. Thus without explicitly specifying new patterns of behavior for group members, it is possible to intervene to arrange inputs and circumstances such that effective decision making will be more likely. As with action-oriented interventions, the consequences of inputoriented interventions can affect information processing and social-psychological factors in a group.

The Analytic Hierarchy Process is a compensatory methodology for structure, evaluation and choice. The AHP improves the decision process by structure intervention. Problems have to be addressed in the hierarchy structure fashion with its unique pairwise comparison evaluation phase to facilitate the choice, which is also an intervention. At the same time, we open the question of how to structure the hierarchy and what should be included in the method of idea generating and polling method, which have been reviewed in above section. It is obvious that AHP can serve a basis for group decision making and allows us to integrate all the other idea 
generating/polling methods to facilitate comprehensive decision making process.

There are several advantages in this due to the structured nature of AHP as pointed out by Dyer and Forman [11].

1. AHP helps to structure a group decision so that the discussion centers on objectives rather than on alternatives.

2. AHP analysis involves structured discussion. Every topic and factors relevant to the decision are addressed in turn. Individual group members with information, knowledge and expertise relative to a specific factor are naturally presented with the opportunity to make their views known; strong members of the group cannot continuously bring the conversation back to their area of expertise.

3. Because the analysis is structured, discussion continues until all available and pertinent information has been considered, and a consensus choice of the alternative most likely to achieve the organizations' stated objectives is achieved.

In the above sections, AHP procedures, group characteristics and the techniques for group decision making have been reviewed. In the following section, a overall picture of the AHP research area will be presented.

\subsection{Areas of Research in AHP}

Research has been conducted in various areas of AHP. Some of the topics on which research concentrates are: 
1. Hierarchy Structure

2. Incomplete Comparison

3. Consistency Analysis

4. Relationship of the AHP to Utility Theory

5. Uncertainty in AHP

6. Analysis of Sensitivity of Reciprocal Matrices

7. The Method to Estimate the Underlying Scale

8. Comparison of Estimation Methods

9. Group Judgments and Consensus

10. Applications

The above research areas will be reviewed briefly in order to present the whole picture for AHP research areas. Although some of the areas do not have a direct impact on the proposed dissertation, all of them are generalized in the following discussion to give a complete picture of the field. Detailed review of those areas, on which this proposed dissertation focuses, will be presented in their corresponding chapters.

\subsubsection{Hierarchic Structure}

A hierarchy is a simple structure used to represent the simplest type of functional (contextual or semantic) dependence of one level or component of a system on 
another in a sequential manner. A hierarchy represents a linear chain of interactions. One result of this approach is to assume the functional independence of an upper part, component or cluster from its lower parts. This often does not imply its structural independence from the lower parts, which involves information on the number of elements, their measurements, etc. But there is a more general way to structure a problem involving functional dependence. It allows for feedback between components. It is a network system of which a hierarchy is a special case. Saaty [33] has provided a theory for the priorities of a network system. This network can be used to identify relationships among components using one's own thoughts, relatively free of rules. It is especially suited for modeling dependence relations.

The sensitivity analysis of the structure, called the backward process $[19,38]$, could be seen as an extension of the forward process. In such an analysis, one may fix the desired outcome and change the structure of the hierarchy to observe how the desired outcome may be achieved. The formulation of the decision structure may also be extended to time-dependent and dynamic structures [15]. This aspect, although of high value to real and complicated systems, is yet to be developed into an operational method. This process can be described as both forward and backward, with both hierarchical structure being evaluated. The hierarchical structure of the backward process is compared to the structure of the forward process. If they are the same or almost the same, then the process is stopped. However, if the structure in the backward process is not the same as the forward process, then they are combined to form a censuses structure.

Khorramshahgol [48] proposed a systematic approach for identifying criteria and 
objectives, which is of paramount importance to a decision-making process and is the basis for a sound decision. The approach uses the Delphi method and integrates it with the AHP. It assists the decision maker(s) in systematically identifying the organizational objectives and then setting priorities among them.

\subsubsection{Incomplete Comparison}

The standard mode of questioning in the AHP requires the decision maker to complete a sequence of positive reciprocal matrices by answering $n(n-1) / 2$ questions for each matrix, each entry being an approximation to the ratio of the weights of the $\mathrm{n}$ items being compared. If $\mathrm{n}$ is large, these comparisons can become an onerous task. Thus, one would be likely to find a method in which the decision maker could complete less than $n(n-1) / 2$ comparisons but still answer enough comparisons in order to derive a meaningful measure of the alternatives' relative weights. Harker [29, 30] has presented two methods, which can be classified as the Incomplete Pairwise Comparison (IPC), to deal with the incomplete comparisons. One is in the context of an iterative scheme for the elicitation of the pairwise comparison matrix $A$, which is based upon the approximation of missing elements of pairwise comparison matrix A with data available from the completed comparisons. This approximation of $a_{j k}$ is formed by taking the geometric mean of the intensity of all paths in the directed graph associated with the partially completed matrix A, which connects the alternatives $\mathrm{j}$ and $\mathrm{k}$. This approximation scheme in some sense mimics what the decision makers would have to perform if they were forced to complete a given 
comparison. Another is a more natural approach to dealing with the missing entries $a_{j k}$. Instead of approximating the missing entry $a_{j k}$, which is itself an approximation of the ratio $v_{j} / v_{k}$, it $\left(a_{j k}\right)$ is set to be equal to $v_{j} / v_{k}$. The necessary theory to deal with this situation in which some $a_{j k}$ s take on the functional form $v_{j} / v_{k}$ is also developed and found consistent with Saaty's eigenvalue method. This way, the questioning process can be substantially shortened by ordering the questions in decreasing informational value and by stopping the process when the added value of questions decreases below a certain level.

Weiss et al. [31] discussed a number of design issues involved in the implementation of AHP for large-scale systems. Specifically, the paper describes the use of incomplete experimental designs for simplifying data-collection tasks for group decision making. The idea behind this approach is to segment the hierarchy into more manageable parts by using the method of balanced incomplete block designs (BIBD), and to allow each member in the decision group to make a relatively small number of pairwise comparisons. The individuals' weights are then aggregated by using the geometric mean. One of the specific BIBD designs was proposed by $\mathrm{Ra}[23]$ to develop a shortcut for pairwise comparisons. Ra's method is called "chainwise paired comparisons".

Millet and Harker [32] proposed further opportunities for effort reduction through globally effective allocation of questions. Global efficiency means that the process goes beyond efficiency and effectiveness for the whole hierarchy. The first motivating concept behind the proposed technique is the utilization of the current node global weight as a major input to the effort allocation process. This approach requires more 
effort from the DM when making comparisons for a node that has an overall high impact on the final priorities. Contrasted with this approach, the IPC technique can lead the decision maker (DM) to spend time on ineffectual comparisons under a node with a negligible global weight. A second idea is that a node with a very low global weight compared to its peers at the same level can be frozen. The questioning process for such a node and for all the nodes below it can be completely avoided, allowing attention to be focused on substantial branches of the hierarchy. A third opportunity for effect reduction is found in cases where the DM wants only to identify the best $n$ out of $m$ alternatives. As the approximate relative weights of the alternatives begin to unfold, we propose to cease elicitation of ratios for clearly inferior alternatives.

\subsubsection{Consistency}

The AHP does not require that judgments be consistent or even transitive. The degree of consistency of the judgment can be measured, which is a distinguishing characteristic of the AHP. Several consistency measurements have been developed, and these measurements are associated with certain methods to estimate the underlying scale. Besides the measurement itself, some researches are focused on developing some procedures to adjust the inconsistent judgments.

The relationship between rank preservation and consistency has been studied by Saaty and Vargus [49]. Three methods of deriving ratio estimates are examined: the eigenvalue, the logarithmic least squares, and the least squares methods. It is shown 
that only the principal eigenvector directly deals with the question of inconsistency and captures the rank order inherent in the inconsistent data.

\subsubsection{Relationship of the AHP to Utility Theory}

There is a basic distinction between the utility theory and AHP. The former quantifies the intensity of preferences through probability distributions. In AHP, however, the preferences are defined based on the set of consequences. No probability measures are involved. Other important distinctions are pointed out by Vargas [21]. First, AHP deals with pairwise comparisons, providing a method to elicit judgments of individuals and to synthesize them into priorities that represent the relative attractiveness of the consequences according to criteria. Second, AHP is a group decision-making methodology. Judgments of individuals can be fused into a single judgment through compromises or through synthesis criteria, which we will discuss in detail in Chapters 3,4 and 5. Third, AHP can deal with several levels of complexity. Fourth, AHP is a true measurement theory in the sense that when there are scales associated with the consequences, the AHP can reproduce known results. Utility theory, on the other hand, can only be used for individual decision makers and cannot be used to estimate numerical values from existing scales. Also, it cannot deal with more than two levels of complexity. Besides knowing those distinctions, it is most important to understand the relationships between the reciprocal property and preference relations. This is explored by Vargas [21] with and without the axiom of transitivity. 
The relationship between the AHP and the additive value function has been studied by Kamenetzky [22]. He concluded that the measure of preference obtained by applying the AHP to the multicriteria decision-making problem under certainty satisfies the definition of an additive value function. The comparison of the AHP and the standard method of building an additive value function seems to indicate that the AHP may provide a useful tool in evaluating unidimensional value functions, but it seems less rigorous than the standard method with respect to the aggregation of unidimensional value functions into an overall measure of preference. A procedure that attempts to combine features of both methods has been proposed. For building the unidimensional value functions, this procedure relies on the AHP. For determining the weighing constants, it combines elements of both the AHP and the standard method.

\subsubsection{Uncertainty in AHP}

Dennis [50] developed an approach to modeling the assignment of priorities under uncertainty in hierarchically structured multicriteria decision problems. The theoretical results indicate that the analysis of uncertainty in complex decision problems is distributionally invariant to the associated hierarchy, both in depth and in nodal ramifications. Since the properties of the underlying probability distribution (i.e. the Dirichlet distribution) are well known, it is not difficult to conduct the probabilistic analysis of these problems within the AHP decision framework.

The uncertainty in the relative weights of a pairwise comparison matrix in the 
AHP is caused by the uncertainty in our decision judgments and in many cases cannot be avoided. In Zahir's [51] study, it is explicitly shown how such uncertainty can be incorporated within the framework of AHP and how the resulting uncertainties in the relative priorities of the decision alternatives can be computed. The required algorithm and the computational procedures are also developed and illustrated with examples. Uncertainty is introduced as a fundamental concept independent of the concept of consistency with a view to extending the AHP as a decision analysis procedure.

The standard application of the AHP assumes that all alternatives are known and available to the decision maker at the time of the evaluation. Weiss [31] relaxes that assumption and models the situation where alternatives become available to the decision maker sequentially, and an accept/reject decision must be made before other alternatives become available. Once an alternative is accepted, no other alternatives are evaluated by the decision maker. Uncertainty about the value of future alternatives and the number of alternatives is included. It is well-known that AHP is an alternative dependent. That is, the relative weights and the final rankings that are given to alternatives are functions of the set of alternatives given to the decision maker. This fact complicates the situation in the current application since the problem is not merely to decide upon the set of alternatives to include in the hierarchy, but rather how to evaluate a set of potential, and yet unknown, alternatives. A technique similar to the classic "secretary problem" of operations research was presented, and this technique involves prioritizing criteria of possible alternatives before the alternatives become available, scoring the alternatives and 
then comparing the score of an alternative with an easily computed (through a dynamic programming recursive process) critical value.

\subsubsection{Analysis of Sensitivity of Reciprocal Matrices}

As in any decision process, decision makers are interested in the sensitivity of the outcomes. In AHP, researchers focus on analyzing the sensitivity of priorities when the entries of A are perturbed. Vargas [49] has developed a method based on the Hadamard product of Matrices to analyze the sensitivity of reciprocal matrices. It has been proven that these types of matrices can be decomposed into the Hadamard Product of a consistent matrix and an inconsistent matrix. The consistent matrix has the same principal eigenvector as the original matrix, and the inconsistent matrix has the same principal eigenvalue as the original one. This decomposition can be used in the analysis of sensitivity to compute the principle eigenvector of a perturbed reciprocal matrix.

Saaty and Vargas [52] investigated the effect of uncertainty in judgment on the stability of the rank order of alternatives. The uncertainty experienced by decision makers in making comparisons is measured by associating with each judgment an interval of numerical values. The approach leads to estimating the probability that an alternative or project exchanges rank with other projects. These probabilities are then used to calculate the probability that the project would change rank at all. The priority of importance of each project is combined with the probability that it does not change rank to obtain the final ranking. Vargas [53] developed 
a method to estimate the average opinion (or core) of a group of people. The method elicits judgments from a smaller group of individuals rather than the total population. What we obtain is a scattering of values around a core value that is being estimated. Some of those values will be closer to the core and others will lie away from it. The method allows us, given the density of concentration of the judgments, to use to a greater extent those values closer to the core.The method generates a surface which is more like a probability distribution that can be used to estimate the core without treating the data as if it were direct estimates of it. The shape of the relevant distribution corresponding to a Dirichlet distribution. It has been proven that the only distribution of judgments which yields this type of result is the gamma distribution. Under the assumption of total consistency, if the judgments are gamma distributed, the principal right-eigenvector of the resulting reciprocal matrix of pairwise comparisons is Dirichlet distributed. If the assumption of consistency is relaxed, then the hypothesis that the principal right-eigenvector follows a Dirichlet distribution is accepted if inconsistency is $10 \%$ or less.

\subsubsection{The method to Derive the Priority Vector}

There are several methods to derive priority vectors from matrices of pairwise comparisons including the eigenvector method [3], the logarithmic least squares methods $[27,54]$, the least squares methods [26], the constant-sum method [24] and the column-row sums method [55]. A detailed review of these methods and others are given in Appendix A. 


\subsubsection{Comparison of Prioritization Methods}

The focus of this research is to develop a set of criteria to decide which method is the "best" one.

Fichtner [25] proposed an axiomatic approach to decide which method is the best one. Invariance principles are motivated and formulated as axioms. There are four axioms involved: correctness in the consistent case, comparison order invariance, smoothness and power invariance. The only method which fulfills all these axioms uses the geometric row means. It is often called Logarithmic Least Squares Method (LLSM). However, only one axiom would have to be replaced in order to get the widely used Right Eigenvector.

Saaty and Vargas [56] compared three methods - the eigenvalue, logarithmic least squares, and least squares methods - used to derive estimates of ratio scales from a positive reciprocal matrix. The criteria for comparison are the measurement of consistency, dual solutions, and rank preservation. It is shown that the eigenvalue procedure, which is metric- free, leads to a structural index for measuring inconsistency, has two separate dual interpretations, and is the only method that guarantees rank preservation under inconsistency conditions.

Zahedi [38] used a simulation analysis to investigate the statistical accuracy and rank preservation capability of the AHP estimation methods. The methods under study consist of the eigenvalue, mean transformation, row geometric mean, column geometric mean, harmonic mean and simple row average. The methods are compared under three distributions for error terms - gamma, lognormal and 
uniform - and under two types of input matrices of various sizes. There are several findings:

1. The most important factors in the estimation of relative weights comprise the probability distribution of error terms and the type of input matrix.

2. While analysts do not control the probability distribution of error terms, they can improve the estimation by collecting data for the upper and lower triangles of the input matrix.

3. The column geometric and simple row average could be dropped from the list of estimators because they generally show the highest degree of sensitivity toward the underlying distribution of error terms and exhibit, in some cases, very poor accuracy and rank statistics.

4. In the computation of the eigenvalue method, the "size" criterion performs exactly as well as the "convergence" criterion, and has the additional advantage of computational efficiency, which becomes crucial for cases with a large number of elements.

5. Of the four methods (excluding the column geometric and the simple row average), no method dominates others in all statistics. The mean transformation method, however, is the most robust toward the underlying distribution and type and size of input matrix. Hence, in absence of knowledge of the distribution of error terms, the mean transformation is recommended. 
6. When an alternative has a relative weight close to zero for an attribute, the symmetric type of input matrix is inappropriate because the performance of all methods deteriorates as the pairwise scores become very small or very large. The full input type does not exhibit extensive sensitivity to the extreme values, and hence constitutes the better choice.

Ra [55] also proposed a logical inference approach to select the best method. As selection criteria, three cases of inconsistent judgments - risky choice, rank preservation, and symmetry - have been designed. The major methods have been used to obtain subjective values for sets of decision elements with known values. Two methods - the column-row sums method and the logarithmic least squares method - are shown to give robust results in all cases.

\subsubsection{Group Judgments and Consensus}

Synthesizing judgments is often an important part of the AHP. Aczel and Saaty $[6,7,8]$ have proposed a functional approach to synthesize the judgments. There are several conditions which are reasonable to require for this approach: (1) separability and unanimity conditions, (2) reciprocal property, (3) homogeneity condition, (4) power conditions. Under these conditions the geometric mean is the functional form. For a more detailed review, see Chapter 3. 


\subsubsection{Applications}

The areas in which AHP is applied are diverse and numerous. The papers range from economic/management, political and social problems to technological problems. Detailed references can be found in Vargas [57].

\subsection{Summary}

In this chapter, we have reviewed broad areas of methods and techniques for group decision making. In the subsequent chapters, we will focus on two objectives: developing new approaches for aggregating group judgments and analyzing the performance of aggregation methods. The two above improvements are important to the AHP. 


\section{Chapter 3}

\section{METHODS OF AGGREGATING JUDGMENTS FOR PAIRWISE COMPARISONS}

In many decision problems, the consequences of an action may impact several individuals or groups of individuals in different ways. Each of these individuals or groups may have different preferences for the consequences. For example, the new product development program in a corporation affects the top management group, engineering department, finance department, marketing department, personnel department and so on. As another example, setting of new occupational health and safety standards affects workers, stockholders, and consumers, etc. The individual, agency, or group responsible for a complex decision may feel that the decision should reflect the preferences of all those who are affected. However, moving from a single decision maker to a multiple decision maker introduces a great deal of complexity into analysis as we reviewed in Chapter 2. The problem is no longer the selection of the most preferred alternative among the nondominated solutions according to one individual's preference structure. The analysis must be extended to account for the conflicts among different interest groups who have different objectives, goals, criteria, and so on. They usually have disagreements among themselves. The dis- 
agreements come from the differences in their subjective evaluations of the decision problems, caused by the differences in knowledge and/or the differences in personal or group objectives, goals and criteria.

The group's decision is usually understood to be the reduction of different individual preferences among objects in a given situation to a single collective preference, or group preference. Many researchers have concentrated on the analysis of decisions that are "correct" or "reasonable" from certain points of view. In this dissertation, we are interested in how group choices are made. This approach allows one to treat the group decision problems as a generalized problem of transition from given "individual sets of data or preference" to "group set of data or preference". The individuals involved and their data or preference can vary greatly from situation to situation. Members of a group may use several different techniques to arrive at a final decision. Some use the social choice theory, which is voting, while others use the experts judgment/group participation analysis, which is discussing and guessing at the advantages and disadvantages of the project, while still others may use the game theory approach where each decision maker has his own strategy. In general, three approaches can be used to resolve the differences of preferences among individual members of a group:

1. Consensus can be reached through systematical communication and discussion of each individual's judgments or preferences.

2. External rules, such as voting, can be used to determine the group choices.

3. A combination of methods in itein 1 and 2 can be used. 
Determining the external rules and procedures for aggregating judgments is one of the important issues of the group decision problem. The focus of this chapter is on understanding and developing the external rules for aggregating judgments ${ }^{1}$ under the decision analysis framework of AHP. This chapter is organized as follows: In section 3.1, the definition of aggregating judgments for pairwise comparisons is given. Section 3.1 also provides the notations used throughout the rest of the dissertation. In section 3.2 , the existing rules or functions for aggregating pairwise comparison judgments are reviewed. In section 3.3, the proposed aggregation methods for pairwise comparisons are discussed in detail. Finally, a comprehensive example to demonstrate the presented aggregation methods is illustrated in section 3.5 .

\subsection{Definition of Aggregation Problem}

Definitions and descriptions of group aggregation problems are presented in this section, which will serve as the basis for reviewing existing aggregation methods and developing new aggregation methods.

Suppose in a group decision making situation the group consists of $m$ individuals, and the group decision problem has $n$ elements. If the pairwise comparison matrices are made separately by each individual in the decision group, we should obtain m $n \times n$ pairwise comparison matrices; each pairwise: comparison matrix results in one priority vector. This priority vector consists of $\mathrm{n}$ elements for the priority

\footnotetext{
'Judgments in this dissertation mean the pairwise corriparison judgments, we' also use pairwise comparison judgments and pairwise comparison matrices interchiangablly
} 
weights and is derived by using prioritization methods (see Appendix A for details). Two concepts have been involved. One is the pairwise comparison matrix $(A)$, which is the result of pairwise comparison. Each element in matrix $A$ records the relative preference of one element over another element. The other concept is priority vector, which is derived from matrix $A$ and records the relative weight of one decision element over the n decision elements. With the objective of obtaining the aggregated group priority vector in mind, the aggregation is based on the individual pairwise comparison matrices within a group. Therefore, two distint ways to aggregate the group judgments, i.e. pairwise comparison matrices, are defined as follows:

- Approach A: The aggregation methods are operated on a group of pairwise comparison matrices. An aggregated group pairwise comparison matrix is obtained from the operation. Then, the aggregated group priority vector is derived from the aggregated group pairwise comparison matrix by using the prioritization method.

- Approach B: The aggregation methods are operated on a group of priority vectors. An aggregated priority vector is obtained from the aggregation process. Group priority vectors are derived from the corresponding pairwise comparison matrices by employing the prioritization method.

In the following sections, we will put these two approaches in a mathernatical form. Both approaches are the integrated part of this dissertation. 


\subsubsection{Representation of Pairwise Comparison Matrix for Group Judgment Aggregation}

Suppose there is a decision group of $\mathrm{m}$ persons, each of whom has a pairwise comparison matrix $A_{i}$ defined over n decision elements, where i stands for member $i$ in the group and $i=1, \cdots, m$. Considering the judgments are made separately by each member, the judgments of the group can be represented by a vector of m-components, where each component is an $n \times n$ pairwise comparison matrix. Let $\left\{A_{i}\right\}=\left(A_{1}, A_{2}, \ldots, A_{m}\right)$ be the vector. Each $A_{i}$ can be represented as:

$$
A_{i}=\left[\begin{array}{ccccc}
\left\{a_{11}\right\}_{i} & \left\{a_{12}\right\}_{i} & \ldots & \ldots & \left\{a_{1 n}\right\}_{i} \\
\left\{a_{21}\right\}_{i} & \left\{a_{22}\right\}_{i} & \ldots & \ldots & \left\{a_{2 n}\right\}_{i} \\
\cdot & \cdot & \ldots & \ldots & \cdot \\
\cdot & & & & \\
\cdot & & \ldots & \ldots & \cdot \\
\left\{a_{n 1}\right\}_{i} & \left\{a_{n 2}\right\}_{i} & \ldots & \ldots & \left\{a_{n n}\right\}_{i}
\end{array}\right]
$$

where $\left\{a_{j k}\right\}_{i}$ denotes a pairwise comparison regarding decision elements $\mathrm{j}$ and $\mathrm{k}$ $(j, k=1,2, \ldots, n)$ judged by person $\mathrm{i}$ in the group.

\subsubsection{Representation of Priority Vector for Group Judg- ment Aggregation}

The priority vector is derived from the pairwise comparison matrix by using the prioritization methods listed in Appendix A. In a group decision situation defined in section 3.1.1, each pairwise comparison matrix $A_{i}$ has a corresponding priority vector $V_{i}$. Therefore, priority vectors of a group can also be represented by a vector of m-components. Each component itself is a vector of n-components. Let $V=$ 
$\left(V_{1}, V_{2}, \ldots, V_{m}\right)$ be the vector. $V_{i}$ denotes the priority vector of person $i$ derived from $A_{i} . V_{i}$ can be explicitly expressed as:

$$
V_{i}=f\left(A_{i}\right)=\left(\left\{v_{1}\right\}_{i},\left\{v_{2}\right\}_{i}, \cdots,\left\{v_{n}\right\}_{i}\right)
$$

where $f()$ denotes any of the prioritization methods described in Appendix A. It also represents the prioritization methods which are operated on $A_{i} \cdot\left\{v_{j}\right\}_{i}$ denotes the relative weight for decision element $j$ of person $i$, which is derived from pairwise comparison matrix $A_{i}$.

\subsubsection{Aggregation Approaches}

Now let $\bar{A}$ denote the aggregated group pairwise comparison matrix and $\bar{V}$ denote the aggregated group priority vector. Our objective is to investigate the two aggregation approaches, i.e. $\mathrm{A}$ and $\mathrm{B}$.

Approach A: This approach represents that the aggregation is through the pairwise comparison matrices. Suppose $A_{G}($ ) stands for the aggregation method. This approach can be expressed as $\bar{A}=A_{G}\left(\left\{A_{i}\right\}\right)$ and $\bar{V}=f(\bar{A})$. More specifically, we have

$$
\bar{A}=A_{G}\left(\left\{A_{i}\right\}\right)=A_{G}\left(A_{1}, A_{2}, \ldots, A_{m}\right)=\left[\begin{array}{cccccc}
\bar{a}_{11} & \bar{a}_{12} & \ldots & \ldots & \bar{a}_{1 n} \\
\bar{a}_{21} & \bar{a}_{22} & \ldots & \ldots & \bar{a}_{2 n} \\
\cdot & . & \ldots & \ldots & . \\
. & . & \ldots & \ldots & . \\
. & . & \ldots & \ldots & . \\
\bar{a}_{n 1} & \bar{a}_{n 2} & \ldots & \ldots & \bar{a}_{n n}
\end{array}\right]
$$


and

$$
\bar{V}=f(\bar{A})=\left(\bar{v}_{1}, \bar{v}_{2}, \ldots, \bar{v}_{n}\right)
$$

where $\bar{a}_{j k}$ is the aggregated group pairwise comparison between decision element $j$ and element k. $\bar{v}_{j}$ is the aggregated relative weight for decision element $j$.

Approach B: This is an approach to aggregate the judgments through priority vectors. In section 3.1.2, for each pairwise comparison matrix $\left\{A_{i}\right\}(i=1, \cdots, m)$ in the group, there is a corresponding priority vector $\left\{V_{i}\right\}$. If $V_{G}()$ stands for the aggregation method operating on the priority vectors, then approach $B$ can be expressed as:

$$
V_{i}=f\left(A_{i}\right)=\left(\left\{v_{1}\right\}_{i},\left\{v_{2}\right\}_{i}, \cdots,\left\{v_{n}\right\}_{i}\right)
$$

and

$$
\bar{V}=V_{G}\left(\left\{V_{i}\right\}\right)=V_{G}\left(V_{1}, \cdots, V_{m}\right)=\left(\bar{v}_{1}, \bar{v}_{2}, \ldots, \bar{v}_{n}\right)
$$

where $\left\{v_{j}\right\}_{i}$ is the relative weight of decision element $\mathrm{j}$ for person $\mathrm{i}$ in the group. $\bar{v}_{j}$ is the aggregated relative weight for decision element $j$ for the group.

In summary, the judgment quantification process involves comparisons among decision elements (i.e. alternatives) according to a given criterion. The individual judgments are made by comparing an object, say $C$, with another, say $D$, according to the given criterion. In a group decision process, these individual judgments need to be aggregated into a single judgment. Two general approaches can be conducted for aggregating the pairwise judgments. One is to aggregate $\left\{A_{i}\right\}=\left(A_{1}, A_{2}, \ldots, A_{m}\right)$ 
to $\bar{A}=A_{G}\left(\left\{A_{i}\right\}\right)$ as indicated in expression (3.3), which we call Approach A. Then the aggregated group priority vector is obtained by using the prioritization method, which is $\bar{V}=f(\bar{A})$ as shown in Eqn. (3.4). The other approach is to use the prioritization method on each of $\left\{A_{i}\right\}$ to get $\left\{V_{i}\right\}=\left(\left\{v_{1}\right\}_{i},\left\{v_{2}\right\}_{i}, \cdots,\left\{v_{n}\right\}_{i}\right)$ as indicated in Eqn. (3.5). Then $\left\{V_{i}\right\}=\left(V_{1}, V_{2}, \ldots, V_{m}\right)$ is aggregated to obtain $\bar{V}=V_{G}\left(\left\{V_{i}\right\}\right)$, which we call Approach B. Both of these two different approaches will be carried out in this chapter.

\subsection{Existing Methods for Aggregating Pairwise Comparison Judgments}

There are two existing methods to aggregate pairwise comparison judgments. One is the function equation approach via geometric mean, which was developed by Aczel and Saaty $[6,7,8]$. The other one is the arithmetic mean, which is the commonly used method. Both of these methods are simple and easy to use. As mentioned in section 3.1, there are two distinct approaches to aggregate pairwise comparison judgments for each aggregation method. In the following section, we will review the existing aggregation methods. Specifically, we will focus on how those two approaches can be applied.

\subsubsection{Geometric Mean}

Aczel et al. $[6,7,8]$ proposed a functional equation approach to aggregate the ratio judgments. Let us suppose that the numerical judgments $x_{1}, x_{2}, \ldots, x_{m}$ given by m persons lie in a continuum (interval) $P$ of positive numbers so that $P$ may contain 
$x_{1}, x_{2}, \ldots, x_{m}$ as well as their powers, reciprocals and geometric means, etc. The aggregating function $f()$ will map $P_{m}$ into a proper interval $J$, and $f\left(x_{1}, x_{2}, \ldots, x_{m}\right)$ will be called the result of the aggregation for the judgments $x_{1}, x_{2}, \ldots, x_{m}$. The function $f()$, which should satisfy the Separability condition, Unanimity condition and Reciprocal condition, is the geometric mean as follows:

$$
f\left(x_{1}, x_{2}, \ldots, x_{m}\right)=\left(x_{1} x_{2} \cdots x_{m}\right)^{\frac{1}{m}}
$$

Given expression (3.7), let us apply this equation to the aggregation problem defined in section 3.1. Since $x_{i}$ is a ratio judgment, so is $\left\{a_{j k}\right\}_{i}$. Therefore, Eqn. (3.7) can be directly applied to the aggregation problem.

Approach A: The approach $\mathrm{A}$ is to derive $\bar{A}$ from $\left\{A_{i}\right\}$. By applying expression (3.7) to every element of the pairwise comparison matrix $\left\{A_{i}\right\}$, we will have the following expression:

$$
\bar{A}=\left[\begin{array}{ccccc}
\bar{a}_{11} & \bar{a}_{12} & \ldots & \ldots & \bar{a}_{1 n} \\
\bar{a}_{21} & \bar{a}_{22} & \ldots & \ldots & \bar{a}_{2 n} \\
\cdot & \cdot & \ldots & \ldots & \cdot \\
\cdot & . & \ldots & \ldots & \cdot \\
\cdot & . & \ldots & \ldots & \cdot \\
\bar{a}_{n 1} & \bar{a}_{n 2} & \ldots & \ldots & \bar{a}_{n n}
\end{array}\right]
$$




$$
=\left[\begin{array}{ccccc}
\left(\prod_{i=1}^{m}\left\{a_{11}\right\}_{i}\right)^{\frac{1}{m}} & \left(\prod_{i=1}^{m}\left\{a_{12}\right\}_{i}\right)^{\frac{1}{m}} & \ldots & \ldots & \left(\prod_{i=1}^{m}\left\{a_{1 n}\right\}_{i}\right)^{\frac{1}{m}} \\
\left(\prod_{i=1}^{m}\left\{a_{21}\right\}_{i}\right)^{\frac{1}{m}} & \left(\prod_{i=1}^{m}\left\{a_{22}\right\}_{i}\right)^{\frac{1}{m}} & \ldots & \ldots & \left(\prod_{i=1}^{m}\left\{a_{2 n}\right\}_{i}\right)^{\frac{1}{m}} \\
\cdot & \cdot & \ldots & \ldots & \\
\cdot & \ldots & \ldots & \cdot \\
\cdot & \ldots & \ldots & \ldots & \\
\left(\prod_{i=1}^{m}\left\{a_{n 1}\right\}_{i}\right)^{\frac{1}{m}} & \left(\prod_{i=1}^{m}\left\{a_{n 2}\right\}_{i}\right)^{\frac{1}{m}} & \ldots & \ldots & \left(\prod_{i=1}^{m}\left\{a_{n n}\right\}_{i}\right)^{\frac{1}{m}}
\end{array}\right]
$$

Once $\bar{A}$ is obtained, the priority $\bar{V}$ can be derived from $\bar{V}=f(\bar{A})$. The methods to derive $\bar{V}$ from $\bar{A}$ are summarized in Appendix A. The most often used methods are geometric mean, eigenvector and constant sum method.

Approach B: As an alternative to approach A, the aggregated group priority vector $\bar{V}$ can be obtained from the priority vector of each person in the group. The priority vector for each individual $V_{i}$ of the group is derived from $A_{i}$. Prioritization methods in Appendix A are used for the derivation. Approach B can be summarized as follows:

$$
\begin{aligned}
& V_{i}=f\left(A_{i}\right)=\left(\left\{v_{1}\right\}_{i}, \cdots,\left\{v_{n}\right\}_{i}\right), \quad i=1, \cdots, m \\
& \bar{V}=\left(\bar{v}_{1}, \bar{v}_{2}, \cdots, \bar{v}_{n}\right)=\left(\left(\prod_{i=1}^{m}\left\{v_{1}\right\}_{i}\right)^{\frac{1}{m}},\left(\prod_{i=1}^{m}\left\{v_{2}\right\}_{i}\right)^{\frac{1}{m}}, \cdots,\left(\prod_{i=1}^{m}\left\{v_{n}\right\}_{i}\right)^{\frac{1}{m}}\right)
\end{aligned}
$$

\subsubsection{Weighted Geometric Mean}

If we consider that all judging persons have different weights when the judgments are aggregated, the geometric mean in section 3.2 .1 becomes the weighted geometric mean method. The different weight to a different person stands for the importance, 
or the expertise of that person in the decision problem. Aczel et al. [8] show that a weighted geometric mean is a robust method to aggregate group judgments with a different weight for each person. If we assume $w_{i}$ is the weight for person $i$, the general form of the weighted geometric mean can be expressed as:

$$
f\left(x_{1}, x_{2}, \ldots, x_{m}\right)=x_{1}^{w_{1}} x_{2}^{w_{2}} \cdots x_{m}^{w_{m}}, \quad \sum_{i=1}^{m} w_{i}=1
$$

where $x_{i}$ are the ratio judgments.

Based on the weighted geometric mean concept represented in Eqn. 3.11, the group decision problem defined in section 3.1 can be expressed as follows in terms approach $A$ and $B$, respectively.

Approach A: The aggregated pairwise comparison matrix is generated from $\left\{A_{i}\right\}$. It will be in the following form:

$$
\begin{aligned}
& \bar{A}=\left(\bar{a}_{j k}\right)=\left(\prod_{i=1}^{m}\left(\left\{a_{j k}\right\}_{i}\right)^{w_{i}}\right), \quad \text { and } j, k=1,2, \cdots, n, \sum_{i=1}^{m} w_{i}=1 \\
& \bar{V}=f(\bar{A})=\left(\bar{v}_{1}, \cdots, \bar{v}_{n}\right)
\end{aligned}
$$

Approach B: This is an approach to aggregate the group priority vector from the individual priority vectors $\left\{V_{i}\right\}$. The individual priority vector $V_{i}$ is obtained from the individual pairwise comparison matrix $A_{i}$. The mathematical form for this approach is as follows:

$$
\begin{aligned}
& V_{i}=f\left(A_{i}\right)=\left(\left\{v_{1}\right\}_{i}, \cdots,\left\{v_{n}\right\}_{i}\right), \quad i=1, \cdots, m \\
& \bar{V}=\left(\prod_{i=1}^{m}\left(\left\{v_{1}\right\}_{i}\right)^{w_{i}}, \prod_{i=2}^{m}\left(\left\{v_{2}\right\}_{i}\right)^{w_{i}}, \cdots, \prod_{i=1}^{m}\left(\left\{v_{n}\right\}_{i}\right)^{w_{i}}\right) \\
& \sum_{i=1}^{m} w_{j i}=1, \quad j=1,2, \ldots, n
\end{aligned}
$$




\subsubsection{Arithmetic Mean}

In addition to the geometric mean and weighted geometric mean methods discussed above, the arithmetic mean can also be used to aggregate group judgments. The only difference is that the arithmetic mean can only be applied on the final priority weights, i.e. Approach B. This is because of the reciprocal property of pairwise comparison and $1 / \sum_{i=1}^{m} a_{j k i} \neq \sum_{i=1}^{m} 1 / a_{j k i}$. The arithmetic mean method cannot be used to aggregate the pairwise comparison matrix A. So we have the mathematical form of the arithmetic mean operated on the priority weight as follows:

$$
\begin{aligned}
& V_{i}=f\left(A_{i}\right)=\left(\left\{v_{1}\right\}_{i}, \cdots,\left\{v_{n}\right\}_{i}\right), \quad i=1, \cdots, m \\
& \bar{V}=\left(\bar{v}_{1}, \bar{v}_{2}, \cdots, \bar{v}_{n}\right)=\left(\sum_{i=1}^{m}\left\{v_{1}\right\}_{i}, \sum_{i=1}^{m}\left\{v_{2}\right\}_{i}, \cdots, \sum_{i=1}^{m}\left\{v_{n}\right\}_{i}\right)
\end{aligned}
$$

In the constant sum method (see Appendix A for detail), raw data, which is the original constant-sum pairwise comparison data, can also be used for aggregation purposes by using the arithmetic mean. For example, suppose there are three individuals to compare two elements, say $\mathrm{C}$ and $\mathrm{D}$. Their corresponding judgments for three individuals are $[80,20],[70,30]$ and $[75,25]$, respectively. By using arithmetic mean approach, the aggregated group judgment in constant-sum form is $[75,25]$, which is still $75+25=100$ !

The existing methods of aggregating judgments, which are geometric mean, weighted geometric mean and arithmetic mean, have been reviewed and discussed in this section. Two approaches of aggregating pairwise comparison judgments, i.e. approach $\mathrm{A}$ and approach $\mathrm{B}$, are formed for the existing methods. In the follow- 
ing section, a new method will be proposed. This method is based on the general distance concept.

\subsection{The Minimum Distance Method for Aggre- gating Pairwise Comparison Judgments}

In this section, we will focus on a new approach (i.e. the distance approach). This new approach is based on the following concepts and assumptions:

1. General distance concept as indicated by Yu and Cook in $[4,5]$, respectively.

2. Group disagreement be expressed as a distance function of individual judgments v.s. the aggregated judgments.

3. Aggregated judgments are in the form of weighted geometric mean.

Under the above conditions, the aggregation method leads itself to the formulation of goal programming by using Cook et al's work as illustrated in [5], which is relatively easy to solve by using commercial software such as LINDO.

The distance concept has been used by researchers to aggregate group judgments. Among them are Kemeny and Snell's distance measure to aggregate a set of ordered rankings as indicated in [58], and Yu's general distance approach to solve the group decision problem by using the concept of an ideal solution to describe measurements of compromise in utility space as presented in [4]. The concept of distance between pairwise comparison matrices or between priority vectors is also very appealing for the group decision problem defined in section 3.1 of this chapter. This concept can be applied in several aspects of group decision measurements. 


\subsubsection{Distance as Accuracy Measurement}

Considering $\mathrm{n}$ attributes, all of which are measurable in a common criterion, one would contend that the resultant relative weights of $\mathrm{n}$ attributes are correct at the level of significance of the instrument if the attributes are physically measured with a precise instrument. Let a second estimate of relative weights be provided by an estimator that is less precise, a human evaluator for instance. Taking the first estimate as correct, the distance or disagreement between the two estimates results from errors made by the estimator.

In such a case, distance becomes a measure of accuracy, where the correct measure has been objectively assessed by a precise tool. Suppose $v_{j}$ is the measure from the human and $\left\{v_{\tau}\right\}_{j}$ is the measure from the instrument for attribute $j$ $(j=1, \cdots, n)$, respectively, Then, the differences $\left(v_{j}-\left\{v_{\tau}\right\}_{j}\right)$ represent the assessment error of the human.

\subsubsection{Distance as Group Disagreement}

Another circumstance to consider is the comparison of priority vectors or pairwise comparison matrices supplied by different sources, none of which is sufficiently precise to assure objective correctness. A group of experts (say $m$ of them) might provide estimates regarding the relative value of several program strategies. Each expert's judgments are summarized by a vector of relative weights or pairwise comparison matrices. Since no objective standard for correctness exists, the disagreements between the judgments of the experts cannot be interpreted directly as inaccuracy.

However, some indication of the extent to which the experts agree may be a 
useful guide to make inferences regarding how well the attributes are known. If the experts clearly understand the value of the program strategies with respect to the given criterion, one would expect $d\left(i_{1}, i_{2}\right)$ to be small for any two estimators $i_{1}$ and $i_{2}$, where $d\left(i_{1}, i_{2}\right)$ is the value difference between two estimators $i_{1}$ and $i_{2}$. An example of the criterion may be the increase of the market share during the next five years. However, unless the market is unusually well known and well behaved, the alternative strategies are likely to be evaluated differently from one expert to the next. The resultant significantly nonzero values for the $d\left(i_{1}, i_{2}\right)$ suggest that a summary of the $d\left(i_{1}, i_{2}\right)$ values could provide a useful guide to expert judgment variance. Furthermore, such variance would mean that the experts have different understandings of the available strategies and their effects on market share.

Suppose that there are $m$ experts in the group and their judgments are transformed into an estimate of relative weights or pairwise comparison matrices, then those estimates can be aggregated into group estimates. Furthermore, if all estimators are considered equally important, the group's geometric mean estimate may be defined as the aggregated group priority vector $\bar{V}$ or the aggregated group pairwise comparison matrix $\bar{A}$. The $\bar{V}$ or $\bar{A}$ can also be obtained using any of the methods mentioned in section 3.2. By defining disagreement as an algebraic deviation, we can define a function to represent the deviation of each individual's judgment from the aggregated group priority vector or aggregated group pairwise comparison matrix. An example of such a function would be the combined distance between aggregated group estimates and individual estimates. Such a function is regarded as group disagreement. 
The interpretation of group disagreement depends largely upon the decision making circumstance. Keeney and Raiffa [59] point out that the differences in personal objectives and preferences will lead individuals to assess alternatives differently even though each estimator may have the same level of knowledge. Nevertheless, if a group of experts is exploring a problem area that is independent of personal concerns, then the remaining variance can be attributed primarily to differences in knowledge and understanding about the attributes.

In such circumstances the combined value of the group disagreement (say $D$ ) may allow inferences about completeness of understanding. If complete understanding would lead all the estimators to provide the same relative weights, then $D$ measures the incompleteness of each estimator's level of knowledge. The vector $\bar{V}$ or matrix $\bar{A}$ represents the group's collective judgment regarding the true values $V_{\mathfrak{T}}$ or $A_{\mathfrak{T}}$. If it is important that the group decision be accurate, then one would desire minimum deviation between $\bar{V}$ and $V_{\tau}$ or $\bar{A}$ and $A_{T}$. Since the true value $V_{T}$ and $A_{T}$ cannot be assessed directly, one does not know whether the $\bar{V}$ or $\bar{A}$ is in fact a good approximation. However, information obtained from the disagreement measurement can be used to make inferences on the prudence of trusting $\bar{V}$ and $\bar{A}$.

The premise for choosing the group decision making process is that the group is more likely to be accurate than any given individual estimator would be. In any given instance, one member of a group of estimators may prove to be particularly accurate. However, one cannot generally know the "correct" result when assessing fuzzy attributes. Therefore, no means exist for identifying an individual estimator that would regularly surpass a carefully achieved group consensus. 
When group disagreement $D$ is zero, the group decision represents consensus. In the consensus situation, if the individual estimates are accurate, the group decision will also be accurate. However, good consensus does not mean that the estimates are accurate. In general, the smaller $D$ is, the better the consensus. When disagreement is high, the group decision should be reexamined. Individual estimators whose assessments differ from the group average or aggregated values can explain their rationales, often broadening the group's understanding. Thus, a knowledge of the degree of the group's understanding and of the degree of group disagreement can be used to prompt discussion until the group decision approaches consensus. If the estimators provide accurate information in the discussions, the group decision is likely to approach the best available choice. The group decision may not always prove to be correct; time unveils many uncertainties. However, the group decision can approach the best possible decision given the information available at the time.

Based on the above reasons for the distance concept, minimization of $D$ (i.e. the group disagreements) is proposed as the criterion for deriving $\bar{V}$ and $\bar{A}$. Based on this criterion, an aggregation method, along with the assumption that the aggregated judgments are in the form of weighted geometric mean, is developed in the following sections. The literature indicates that the absolute distance is an adequate distance function (see Cook et al. [5]). The objective of our work is to find the weights of the weighted geometric mean in order to minimize the group disagreement. The aggregation method leads itself to a goal programming formulation, which can be solved by using commercial software such as LINDO. The detailed presentation of this aggregation method is in the following section. 


\subsubsection{The Minimum Distance Method for Pairwise Com- parison Matrix}

In the preceeding section, we have discussed the appealing characteristics of the distance concept for the group judgment aggregation problem defined in section 3.1 of this chapter. We also stated that our objective is to minimize the group disagreement $D$. Therefore, we call this method minimum distance method (MDM). Let us first consider aggregating the pairwise comparison matrix (i.e. Approach A). Specifically, consider that $\mathrm{m}$ group members have provided data for pairwise comparison matrices $\left\{A_{i}\right\}(i=1, \cdots, m)$ regarding $n$ decision elements. Let $A_{i}=$ $\left(\left\{a_{j k}\right\}_{i}\right),(j, k=1,2, \cdots, n) . \bar{A}$ is the matrix of aggregated group estimates. In order to obtain $\bar{A}$, the objective can be expressed as:

Objective: Minimize $D$

and $\mathrm{D}$ is defined as:

$$
D=\sum_{i=1}^{m} d\left(A_{i}, \bar{A}\right)
$$

in which d stands for any distance function between $A_{i}$ and $\bar{A}$, such as the squared distance, absolute distance and so on.

Expression (3.20) requires that we examine the aggregation of pairwise comparison matrices from the view point of a distance measure on the set of pairwise comparison matrices $\left(\left\{A_{i}\right\}\right.$ and $\left.\bar{A}\right)$. The problem is to determine a consensus pairwise comparison matrix $(\bar{A})$ that best agrees with all the group members' pairwise comparison matrices $\left(\left\{A_{i}\right\}\right)$ in terms of distance measure. In the following, we will 
develop the mathematical formula for MDM operated on the pairwise comparison matrices. Let us consider the following two factors.

First, from our literature review in this section we have noticed that the weighted geometric mean method is the extension of the geometric mean when an individual has different weights. Aczel and Saaty [8] demonstrated that weighted geometric mean is a robust method for aggregating group judgment. Therefore, the $\bar{A}$ can be expressed as follows:

$$
\begin{aligned}
& \bar{A}=\left(\bar{a}_{j k}\right)=\left(\prod_{i=1}^{m}\left(\left\{a_{j k}\right\}_{i}\right)^{w_{i}}\right), \quad \text { and } j, k=1,2, \cdots, n \\
& \sum_{i=1}^{m} w_{i}=1, \quad \bar{V}=f(\bar{A})
\end{aligned}
$$

where $w_{i}$ is the weight assigned to person $i$, and $\bar{a}_{j k}$ takes the form of the weighted geometric mean. Therefore, the aggregation problem defined in expressions (3.19), (3.21) and (3.22) becomes to find the optimal $w_{i}$ so that the group disagreement $D$ is minimal. In other words, the aggregated pairwise comparison matrix $\bar{A}$ should satisfy not only the expression (3.19), but also the expressions (3.21) and (3.22). There are two significant advantages in assuming that $\bar{A}$ is in the form of the weighted geometric mean:

- Under the following proposed distance function the weighted geometric mean form allows us to convert the problem defined in expressions (3.19) and (3.20)into a linear programming formulation which can be easily solved.

- The weighted geometric mean form reduces the number of variables to be determined in expression (3.19) from $n(n-1) / 2$ to $\mathrm{m}$. This is because of the 
fact that even though reciprocal matrix $\bar{A}$ contains $n(n-1) / 2$ independent variables or elements $\left(\bar{a}_{j k}\right)$, there are only $\mathrm{m} w_{i}$ representing the weights to be assigned to the $\mathrm{m}$ decision makers.

Second, what kind of distance function of $d\left(A_{\boldsymbol{i}}, \bar{A}\right)$ will be the most suitable form for our aggregation problem? There are at least two criteria:

1. $\bar{A}$ should be unique to satisfy the Eqn. (3.19)

2. $\bar{A}$ should be easy to calculate

Cook and Kress [5] have proven that the unique distance between any two pairwise comparison matrices $\mathrm{A}$ and $\mathrm{B}$ should be in the following form:

$$
d(A, B)=\frac{1}{2} \sum_{j=1}^{n} \sum_{k=1}^{n}\left|\ln \left(\frac{a_{j k}}{b_{j k}}\right)\right|
$$

as long as $A$ and $B$ satisfy three axioms as shown in [5]. In order to better explain the MDM, those three axioms are listed: +

Axiom 1: (metric properties)

1. For any two pairwise comparison matrices $A, d(A, B) \geq 0$ with equality iff $A \equiv B$, where $d()$ stands for the distance between $\mathrm{A}$ and $\mathrm{B}$.

2. $d(A, B)=d(B, A)$

3. $d(A, B)+d(B, C) \geq d(A, C)$

Axiom 2: If $\mathrm{A}, \mathrm{B}$ are two pairwise comparison matrices, and $A=B$ except for one pair $(j, k)$ for which $a_{j k} \neq b_{j k}$, then $d(A, B)=H\left(\frac{a_{j k}}{b_{j k}}\right)$, where $\mathrm{H}$ is a continue function. 
Axiom 3: (scaling axiom) $H\left(C_{0}\right)=1$ for some $C_{0}$. The value $C_{0}$ can be chosen arbitrarily and will be called the base of the distance.

Cook and Kress [5] proved that $H$ has the form of expression (3.23). Axiom 1 and axiom 3 are easy to understand. There are two meanings for axiom 2:

1. If two judgments regarding the odds of favoring one object over another are the same in all cases except for exactly one pair of objects $(j, k)$, the distance between $A$ and $B$ is reduced to the distance relative to $i$ and $j$ only.

2. Since the original data (i.e. $a_{j k}$ ) expressing the extent to which one object is preferred to another is given as a ratio, then the differences between judgments $\left(a_{j k}\right.$ versus $\left.b_{j k}\right)$ should also be expressed as some function $\mathrm{H}$ of the ratio of these judgments.

Reciprocal pairwise comparison matrix $A_{i}$ and $\bar{A}$ always satisfy the above axioms given the distance function in expression (3.23). So expression 3.23 can be used in the aggregation problem defined in expression (3.19). Given the logarithmic form of the distance function (3.23), the nonlinear relationship between $w_{i}$ and $D$ presented in expressions (3.21) and (3.22) becomes linear. There is a significant advantage for our problem in using expression (3.23). Later, we will show another advantage in that the distant function defined in (3.23) can convert our problem into a linear programming formulation.

The distance function presented in expression (3.23) combined with expressions (3.19), (3.21) and (3.22) can now be used to determine the unknown aggregated 
group pairwise comparison matrix $\bar{A}$. A formal definition for the aggregation of group judgments is given below.

Definition: The consensus pairwise comparison matrix $\bar{A}$ is that matrix which minimizes the total absolute distance:

$$
D=\sum_{i=1}^{m} d\left(A_{i}, \bar{A}\right)=\sum_{i=1}^{m} \sum_{j=1}^{n} \sum_{k=1}^{n}\left|\ln \left(\left\{a_{j k}\right\}_{i}\right)-\ln \left(\bar{a}_{j k}\right)\right|
$$

and is subject to the following constraints:

$$
\ln \left(\bar{a}_{j k}\right)=\sum_{i=1}^{m} w_{i} \ln \left(\left\{a_{j k}\right\}_{i}\right), \quad \sum_{i=1}^{m} w_{i}=1, \quad j, k=1, \cdots n .
$$

where $w_{i}$ is the weight assigned to decision maker $\mathrm{i}$.

The aggregation problem defined in expressions (3.24) and (3.25) can be further expanded. Let us make the following transformation:

$$
\left\{P_{j k}\right\}_{i}-\left\{N_{j k}\right\}_{i}=\ln \left(\left\{a_{j k}\right\}_{i}\right)-\ln \left(\bar{a}_{j k}\right)
$$

where $\left\{N_{j k}\right\}_{i} \geq 0,\left\{P_{j k}\right\}_{i} \geq 0$, and $\bar{a}_{j k}$ are the aggregated values. The original problem is now equivalent to the foilowing goal programming problem, and a numerical example of this is presented in section 3.5 .

$$
\begin{gathered}
\operatorname{minimize} \sum_{i=1}^{m} \sum_{j=1}^{n} \sum_{k=1}^{n}\left(\left\{N_{j k}\right\}_{i}+\left\{P_{j k}\right\}_{i}\right) \\
\text { subject to } \sum_{l=1}^{m} w_{l} \ln \left(\left\{a_{j k}\right\}_{l}\right)-\left\{N_{j k}\right\}_{i}+\left\{P_{j k}\right\}_{i}=\ln \left(\left\{a_{j k}\right\}_{i}\right) \\
i=1,2, \cdots, m, \text { and } j, k=1,2, \cdots, n \\
\sum_{i=1}^{m} w_{i}=1 \\
\left\{N_{j k}\right\}_{i},\left\{P_{j k}\right\}_{i}, w_{i} \geq 0 .
\end{gathered}
$$




\subsubsection{The Minimum Distance Method for Priority Vectors}

In the above, the aggregation with the pairwise comparison matrix is discussed. Now we turn to discuss the aggregation with priority vectors, which is Approach $\mathbf{B}$. Consider that $\mathrm{m}$ group members have provided pairwise comparison matrices $\left\{A_{i}\right\}$ $(i=1, \cdots, m)$ regarding $\mathbf{n}$ decision elements. The corresponding priority vector for each member is $V_{i}=\left(\left\{v_{1}\right\}_{i},\left\{v_{2}\right\}_{i}, \cdots,\left\{v_{n}\right\}_{i}\right)$. Let $\bar{V}$ be the aggregated group estimates for the priority vector. Hence, the objective of the aggregation can be represented as:

$$
\text { Objective }: \quad \text { Minimize } D=\text { Minimize } \sum_{i=1}^{m} d\left(V_{i}, \bar{V}\right)
$$

where $d$ can also use the logarithm form as:

$$
d(V, \bar{V})=\sum_{i=1}^{m} \sum_{j=1}^{n} \mid \ln \left(\left\{v_{j}\right\}_{i}-\ln \left(\bar{v}_{i}\right) \mid\right.
$$

and $\bar{V}$ can be expressed in the weighted geometric form:

$$
\bar{V}=\left(\bar{v}_{1}, \bar{v}_{2}, \cdots, \bar{v}_{n}\right)=\left(\prod_{i=1}^{m}\left\{v_{1}\right\}_{i}{ }^{w_{i}}, \prod_{i=1}^{m}\left\{v_{2}\right\}_{i}{ }^{w_{i}}, \cdots, \prod_{i=1}^{m}\left\{v_{n}\right\}_{i}{ }^{w_{i}}\right)
$$

The distance function presented in expression (3.29) combined with expressions (3.30) and (3.31) can now be used to determine the unknown aggregated group priority vector $\bar{V}$. The formal definition for the aggregation of a group priority vector can be expressed as follows:

Definition: The consensus priority vector $\bar{V}$ is the vector which minimizes the total absolute distance:

$$
D=\sum_{i=1}^{m} d\left(V_{i}, \bar{V}\right)=\sum_{i=1}^{m} \sum_{j=1}^{n}\left|\ln \left(\left\{v_{j}\right\}_{i}\right)-\ln \left(\bar{v}_{j}\right)\right|
$$


and subject to the following constraints:

$$
\ln \left(\bar{v}_{j}\right)=\sum_{i=1}^{m} w_{i} \ln \left(\left\{v_{j}\right\}_{i}\right), \quad \sum_{i=1}^{m} w_{i}=1, \quad j, k=1,2, \cdots, n
$$

where $w_{\mathrm{i}}$ is the weight assigned to decision maker $\mathrm{i}$. As in section 3.3.3, the aggregation problem defined above can be further simplified. Let us make the following transformation:

$$
\left\{P_{j}\right\}_{i}-\left\{N_{j}\right\}_{i}=\ln \left(\left\{v_{j}\right\}_{i}\right)-\ln \left(\bar{v}_{j}\right)
$$

where $\left\{N_{j}\right\}_{i} \geq 0,\left\{P_{j}\right\}_{i} \geq 0$, and $\bar{v}_{j}$ are the aggregated values. The original problem is now equivalent to the following goal programming problem, and an example of it is presented in section 3.5:

$$
\begin{gathered}
\text { minimize } \sum_{i=1}^{m} \sum_{j=1}^{n} \sum_{k=1}^{n}\left(\left\{N_{j}\right\}_{i}+\left\{P_{j}\right\}_{i}\right) \\
\text { subject to } \sum_{l=1}^{m} w_{l} \ln \left(\left\{v_{j}\right\}_{l}\right)-\left\{N_{j}\right\}_{i}+\left\{P_{j}\right\}_{i}=\ln \left(\left\{v_{j}\right\}_{i}\right) \\
i=1,2, \cdots, m, \text { and } j=1,2, \cdots, n \\
\sum_{i=1}^{m} w_{i}=1 \\
\left\{N_{j}\right\}_{i},\left\{P_{j}\right\}_{i}, w_{i} \geq 0 .
\end{gathered}
$$




\subsubsection{The Weighted Membership in the Minimum Distance Method}

In the above two sections, we presented two versions of the minimum distance method. One is based on the pairwise comparison matrix (i.e. Approach A); the other is on the final priority vectors of pairwise comparison judgments (i.e. approach B). We should keep in mind that the final priority vector can also transfer to a consistent pairwise comparison matrix. In turn the aggregation for the pairwise comparison matrix can be used to aggregate the final priority vectors. We should notice that the $w_{i}(i=1,2, \cdots, m)$ used above are not the physical values assigned to decision maker, but they are only mathematical and logical integrations of the distance approach. However, it is often desirable to attach a positive weight $\theta_{i}$ to decision maker $i$ in order to reflect their relative importance, rather than weighting them equally as suggested in sections 3.3 .3 and 3.3.4. Therefore, expressions (3.27) and (3.35) become the expressions (3.37) and (3.38) respectively:

$$
\begin{aligned}
& \operatorname{minimize} \sum_{i=1}^{m} \sum_{j=1}^{n} \sum_{k=1}^{n} \theta_{i}\left(\left\{N_{j k}\right\}_{i}+\left\{P_{j k}\right\}_{i}\right) \\
& \operatorname{minimize} \sum_{i=1}^{m} \sum_{j=1}^{n} \theta_{i}\left(\left\{N_{j}\right\}_{i}+\left\{P_{j}\right\}_{i}\right)
\end{aligned}
$$

with their corresponding constraints remaining unchanged. Weights can be introduced in other ways, of course, such as applying them directly to the pairwise comparison matrices or the final priority vectors. This approach would lead to a slightly different linear programming formulation than that given by (3.37) and (3.38) respectively. However, we believe that expressions (3.37) and (3.38) are much simpler solutions than the one which applies the weights directly to the pairwise comparison 
matrices or the final priority vectors. Therefore, the mathematical formation of the latter approach is omitted from this section in order to avoid confusion. In general, they are equivalent to allowing more important members of the decision making group a heavier weight than a member of lesser importance. The importance of the members can be determined by their knowledge, experience and even the status of the member in given organization.

The $\theta_{i}(i=1, \cdots, m)$ in expression (3.37) and (3.38) can be interpreted as weights on the goals in a hierarchical sense. They tend to sway the aggregated pairwise comparison matrix or aggregated priority vector closer to the judgments of the more important members, and away from those of less important members. For example, given a marketing situation, weighting is an important concept in that different consumers and even groups of consumers may need to be weighted according to the relevance of their actions and attitudes in regard to purchasing behavior. Therefore, if a group of consumers gathered to evaluate a particular consumer product, the final results should be weighted according to both advertising and duration of customer exposure to the products in question.

Hence, weighting of decision makers or evaluators, whether they are consumers, committee members, managers or voters, is an important issue. Saaty [3] suggests that the AHP method can be used "to derive priorities for several individuals involved according to the soundness of their judgment," and that "factors affecting judgment may be: relative intelligence (however measured), years of experience, past record, depth of knowledge, experience in related fields, personal involvement in the issue at stake, and so on." This can be done as a subsidiary AHP model 
constructed for evaluating player importance.

\subsection{The Sensitivity and Reliability of the Mini- mum Distance Method}

There is a difficulty with a formulation such as (3.37) and (3.38) due to the fact that in most situations the decision maker would be unsure as to what would constitute a reasonably accurate set of weights $\theta_{i}$. Different values of weights $\theta_{i}$ may lead to a different aggregated pairwise comparison matrix or different final priority vector. Therefore, an important issue is how sensitive is the optimal solution of (3.37) and (3.38) to the judgment of any particular decision maker. To obtain a measure of the reliability (stability) of the aggregated pairwise comparison matrix or aggregated final priority vector, it is necessary to analyze their sensitivity to changes in the parameters $\theta_{i}$ of (3.37) and (3.38). Let us consider the goal programming problem (3.37) again:

$$
\begin{gathered}
\operatorname{minimize} \sum_{i=1}^{m} \sum_{j=1}^{n} \sum_{k=1}^{n} \theta_{i}\left(\left\{N_{j k}\right\}_{i}+\left\{P_{j k}\right\}_{i}\right) \\
\text { subject to } \sum_{i=1}^{m} w_{l} \ln \left(\left\{a_{j k}\right\}_{l}\right)-\left\{N_{j k}\right\}_{i}+\left\{P_{j k}\right\}_{i}=\ln \left(\left\{a_{j k}\right\}_{i}\right) \\
i=1,2, \cdots, m, \text { and } j, k=1,2, \cdots, n . \\
\sum_{i=1}^{m} w_{i}=1, \quad \sum_{i=1}^{m} \theta_{i}=1 \\
\left\{N_{j k}\right\}_{i},\left\{P_{j k}\right\}_{i}, w_{i} \geq 0, \theta_{i} \geq 0 .
\end{gathered}
$$


The above expressions constitute a goal programming model. Sensitivity of such models has been studied in detail [60]. A detailed discussion of sensitivity analysis will not be given in this dissertation. However, its application will be described in section 3.5 .

\subsection{Numerical Examples of the Minimum Dis- tance Method}

In the above section, the proposed aggregation method (MDM) has been discussed in detail. In this section, an example is presented for MDM of aggregating both pairwise comparison matrices and the final priority vectors. The example includes also the sensitivity analysis and will demonstrate how the MDM works. Suppose there are four estimators (say $A, B, C$ and $D$ ) for four decision elements. The corresponding four pairwise comparison matrices obtained from estimator $A$, $B, C$ and $D$ are as follows:

$$
\begin{array}{ll}
A=\left[\begin{array}{cccc}
1 & \frac{1}{2} & \frac{1}{3} & \frac{1}{4} \\
2 & 1 & \frac{2}{3} & \frac{1}{2} \\
3 & \frac{3}{2} & 1 & \frac{3}{4} \\
4 & 2 & \frac{4}{3} & 1
\end{array}\right] \quad B=\left[\begin{array}{cccc}
1 & 2 & \frac{2}{3} & \frac{1}{2} \\
\frac{1}{2} & 1 & \frac{1}{3} & \frac{1}{4} \\
\frac{3}{2} & 3 & 1 & \frac{3}{4} \\
2 & 4 & \frac{4}{3} & 1
\end{array}\right] \\
C=\left[\begin{array}{llll}
1 & \frac{1}{3} & \frac{1}{2} & \frac{1}{4} \\
3 & 1 & \frac{3}{2} & \frac{3}{4} \\
2 & \frac{2}{3} & 1 & \frac{1}{2} \\
4 & \frac{4}{3} & 2 & 1
\end{array}\right] \quad D=\left[\begin{array}{cccc}
1 & \frac{4}{3} & 2 & 4 \\
\frac{3}{4} & 1 & \frac{3}{2} & 3 \\
\frac{1}{2} & \frac{2}{3} & 1 & 2 \\
\frac{1}{4} & \frac{1}{3} & \frac{1}{2} & 1
\end{array}\right]
\end{array}
$$


The priority vectors of above pairwise comparison matrices are:

$$
\begin{array}{ll}
V_{A}=(0.1,0.2,0.3,0.4) & V_{B}=(0.2,0.1,0.3,0.4) \\
V_{C}=(0.1,0.3,0.2,0.4) & V_{D}=(0.4,0.3,0.2,0.1)
\end{array}
$$

\subsubsection{MDM Operated on Pairwise Comparison Matrices}

By applying expressions (3.27) and (3.28), the goal programming model for aggregating the pairwise comparison matrices is constructed as follows:

$$
\text { minimize } \sum_{i \in\{A, B, C, D\}} \sum_{j=1}^{4} \sum_{k=1}^{4}\left(\left\{N_{j k}\right\}_{i}+\left\{P_{j k}\right\}_{i}\right)
$$

where $\left\{N_{j k}\right\}_{i}$ is the negative deviation from the comparison of elements $j$ and $k$ by estimator $i,\left\{P_{j k}\right\}_{i}$ is the positive deviation from the comparison of elements $j$ and $k$ by estimator $i, i=A, \cdots, D$ (estimators), $j$ and $k=1, \cdots, 4$ (the elements being compared. The expressions for constraints are as follows:

$$
\begin{gathered}
\text { subject to } \sum_{l \in\{A, B, C, D\}} w_{l} \ln \left(\left\{a_{j k}\right\}_{l}\right)-\left\{N_{j k}\right\}_{i}+\left\{P_{j k}\right\}_{i}=\ln \left(\left\{a_{j k}\right\}_{i}\right) \\
i \in\{A, B, C, D\}, \text { and } j, k=1,2, \cdots, 4 \\
\sum_{i \in\{A, B, C, D\}} w_{i}=1 \\
\left\{N_{j k}\right\}_{i},\left\{P_{j k}\right\}_{i}, w_{i} \geq 0 .
\end{gathered}
$$

The goal programming defined in expressions (3.44) and (3.45) are in the following form (call it input deck of LINDO) when it is inputted to LINDO: 
MIN $\quad \mathrm{N} 12 \mathrm{~A}+\mathrm{P} 12 \mathrm{~A}+\mathrm{N} 13 \mathrm{~A}+\mathrm{P} 13 \mathrm{~A}+\mathrm{N} 14 \mathrm{~A}+\mathrm{P} 14 \mathrm{~A}+\mathrm{N} 23 \mathrm{~A}+\mathrm{P} 23 \mathrm{~A}+\mathrm{N} 24 \mathrm{~A}+$

$\mathrm{P} 24 \mathrm{~A}+\mathrm{N} 34 \mathrm{~A}+\mathrm{P} 34 \mathrm{~A}+\mathrm{N} 12 \mathrm{~B}+\mathrm{P} 12 \mathrm{~B}+\mathrm{N} 13 \mathrm{~B}+\mathrm{P} 13 \mathrm{~B}+\mathrm{N} 14 \mathrm{~B}+\mathrm{P} 14 \mathrm{~B}+$ $\mathrm{N} 23 \mathrm{~B}+\mathrm{P} 23 \mathrm{~B}+\mathrm{N} 24 \mathrm{~B}+\mathrm{P} 24 \mathrm{~B}+\mathrm{N} 34 \mathrm{~B}+\mathrm{P} 34 \mathrm{~B}+\mathrm{N} 12 \mathrm{C}+\mathrm{P} 12 \mathrm{C}+\mathrm{N} 13 \mathrm{C}+$ $\mathrm{P} 13 \mathrm{C}+\mathrm{N} 14 \mathrm{C}+\mathrm{P} 14 \mathrm{C}+\mathrm{N} 23 \mathrm{C}+\mathrm{P} 23 \mathrm{C}+\mathrm{N} 24 \mathrm{C}+\mathrm{P} 24 \mathrm{C}+\mathrm{N} 34 \mathrm{C}+\mathrm{P} 34 \mathrm{C}+$ $\mathrm{N} 12 \mathrm{D}+\mathrm{P} 12 \mathrm{D}+\mathrm{N} 13 \mathrm{D}+\mathrm{P} 13 \mathrm{D}+\mathrm{N} 14 \mathrm{D}+\mathrm{P} 14 \mathrm{D}+\mathrm{N} 23 \mathrm{D}+\mathrm{P} 23 \mathrm{D}+\mathrm{N} 24 \mathrm{D}+$ P24D+N34D+P34D

\section{SUBJECT TO}

$-0.6931 \mathrm{WA}+0.6931 \mathrm{WB}-1.0986 \mathrm{WC}+0.2876 \mathrm{WD}-\mathrm{N} 12 \mathrm{~A}+\mathrm{P} 12 \mathrm{~A}=-0.6931$ $-0.6931 \mathrm{WA}+0.6931 \mathrm{WB}-1.0986 \mathrm{WC}+0.2876 \mathrm{WD}-\mathrm{N} 12 \mathrm{~B}+\mathrm{P} 12 \mathrm{~B}=0.6931$ $-0.6931 \mathrm{WA}+0.6931 \mathrm{WB}-1.0986 \mathrm{WC}+0.2876 \mathrm{WD}-\mathrm{N} 12 \mathrm{C}+\mathrm{P} 12 \mathrm{C}=-1.0986$ $-0.6931 \mathrm{WA}+0.6931 \mathrm{WB}-1.0986 \mathrm{WC}+0.2876 \mathrm{WD}-\mathrm{N} 12 \mathrm{D}+\mathrm{P} 12 \mathrm{D}=0.2876$ $-1.0986 \mathrm{WA}-0.4055 \mathrm{WB}-0.6931 \mathrm{WC}+0.6931 \mathrm{WD}-\mathrm{N} 13 \mathrm{~A}+\mathrm{P} 13 \mathrm{~A}=-1.0986$ $-1.0986 \mathrm{WA}-0.4055 \mathrm{WB}-0.6931 \mathrm{WC}+0.6931 \mathrm{WD}-\mathrm{N} 13 \mathrm{~B}+\mathrm{P} 13 \mathrm{~B}=-0.4055$ $-1.0986 \mathrm{WA}-0.4055 \mathrm{WB}-0.6931 \mathrm{WC}+0.6931 \mathrm{WD}-\mathrm{N} 13 \mathrm{C}+\mathrm{P} 13 \mathrm{C}=-0.6931$ $-1.0986 \mathrm{WA}-0.4055 \mathrm{WB}-0.6931 \mathrm{WC}+0.6931 \mathrm{WD}-\mathrm{N} 13 \mathrm{D}+\mathrm{P} 13 \mathrm{D}=0.6931$ $-1.3863 W A-0.6931 W B-1.3863 W C+1.3862 W D-N 14 A+P 14 A=-1.3863$ $-1.3863 W A-0.6931 W B-1.3863 W C+1.3862 W D-N 14 B+P 14 B=-0.6931$ $-1.3863 \mathrm{WA}-0.6931 \mathrm{WB}-1.3863 \mathrm{WC}+1.3862 \mathrm{WD}-\mathrm{N} 14 \mathrm{C}+\mathrm{P} 14 \mathrm{C}=-1.3863$ $-1.3863 \mathrm{WA}-0.6931 \mathrm{WB}-1.3863 \mathrm{WC}+1.3862 \mathrm{WD}-\mathrm{N} 14 \mathrm{D}+\mathrm{P} 14 \mathrm{D}=1.3862$ $-0.4055 \mathrm{WA}-1.0986 \mathrm{WB}+0.4055 \mathrm{WC}+0.4055 \mathrm{WD}-\mathrm{N} 23 \mathrm{~A}+\mathrm{P} 23 \mathrm{~A}=-0.4055$ $-0.4055 \mathrm{WA}-1.0986 \mathrm{WB}+0.4055 \mathrm{WC}+0.4055 \mathrm{WD}-\mathrm{N} 23 \mathrm{~B}+\mathrm{P} 23 \mathrm{~B}=-1.0986$ $-0.4055 \mathrm{WA}-1.0986 \mathrm{WB}+0.4055 \mathrm{WC}+0.4055 \mathrm{WD}-\mathrm{N} 23 \mathrm{C}+\mathrm{P} 23 \mathrm{C}=0.4055$ $-0.4055 \mathrm{WA}-1.0986 \mathrm{WB}+0.4055 \mathrm{WC}+0.4055 \mathrm{WD}-\mathrm{N} 23 \mathrm{D}+\mathrm{P} 23 \mathrm{D}=0.4055$ $-0.6931 \mathrm{WA}-1.3863 \mathrm{WB}-0.2877 \mathrm{WC}+1.0986 \mathrm{WD}-\mathrm{N} 24 \mathrm{~A}+\mathrm{P} 24 \mathrm{~A}=-0.6931$ $-0.6931 \mathrm{WA}-1.3863 \mathrm{WB}-0.2877 \mathrm{WC}+1.0986 \mathrm{WD}-\mathrm{N} 24 \mathrm{~B}+\mathrm{P} 24 \mathrm{~B}=-1.3863$ $-0.6931 W A-1.3863 W B-0.2877 W C+1.0986 W D-N 24 C+P 24 C=-0.2877$ $-0.6931 W A-1.3863 W B-0.2877 W C+1.0986 W D-N 24 D+P 24 D=1.0986$ $-0.2877 W A-0.2877 W B-0.6931 W C+0.6931 W D-N 34 A+P 34 A=-0.2877$ $-0.2877 W A-0.2877 W B-0.6931 W C+0.6931 W D-N 34 B+P 34 B=-0.2877$ $-0.2877 W A-0.2877 W B-0.6931 W C+0.6931 W D-N 34 C+P 34 C=-0.6931$ $-0.2877 W A-0.2877 W B-0.6931 W C+0.6931 W D-N 34 D+P 34 D=0.6931$ $W A+W B+W C+W 4=1.0$

END

where $\left\{N_{j k}\right\}_{i},\left\{P_{j k}\right\}_{i}$, and $w_{i}$ show as $N j k i, P j k i$, and $W i$, respectively. We should also notice the principle of $\ln (x)=-\ln (1 / x)$, where $x \geq 0$. The constants in the input deck of LINDO are from $\ln (2)=0.6931, \ln (3)=1.0986, \ln (4)=1.3863$, $\ln (4 / 3)=0.2877$, and $\ln (3 / 2)=0.4055$. 
The goal programming model was solved by LINDO with the following results:

$$
w_{A}=0.5, \quad w_{B}=0.0, \quad w_{C}=0.15, \quad w_{D}=0.35
$$

The corresponding pairwise comparison matrix $\bar{A}$ can now be obtained by using the expression (3.21), which are:

$$
\bar{A}=\left(\bar{a}_{j k}\right)=\left(\prod_{i \in\{A, B, C, D\}}\left(\left\{a_{j k}\right\}_{i}\right)^{w_{i}}\right), \quad \text { and } j, k=1,2, \cdots, 4
$$

Specifically, combining the $w_{i}(i \in\{A, B, C, D\})$ value with the respective pairwise comparison matrices $\left\{A_{i}\right\}$ obtained from the four estimators, the $\bar{A}$, the aggregated pairwise comparison matrix, is obtained as follows:

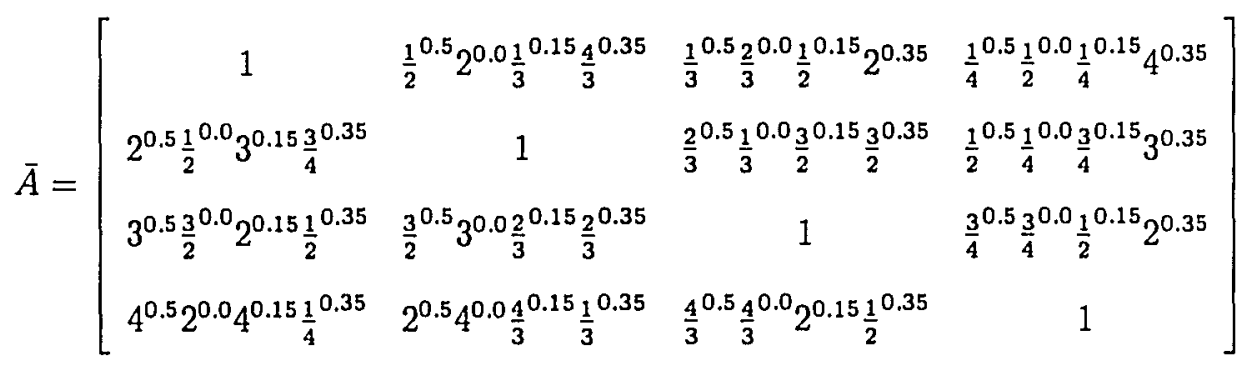

$$
\begin{aligned}
& =\left[\begin{array}{cccc}
1 & 0.5 & 0.5 & 0.375 \\
2 & 1 & 1 & 0.75 \\
2 & 1 & 1 & 0.75 \\
2.667 & 1.333 & 1.333 & 1
\end{array}\right]
\end{aligned}
$$

Using the $\bar{A}$ as the aggregated matrix, we can obtain the aggregated priority vector, $\bar{V}$, which represents the minimum distance criterion. Several methods as 
described in Appendix A can be used for this purpose. For example, using the geometric mean method:

$$
\bar{V}=(0.13,0.26,0.26,0.35)
$$

The individual priority weight are:

$$
v_{1}=0.13, \quad v_{2}=0.26, \quad v_{3}=0.26, \quad v_{4}=0.35
$$

$\bar{V}$, the resulting vector obtained after the aggregation process, is the one which minimizes the distance between the $A_{i}$ ( $i \in\{A, B, C, D\}$ and the aggregated value $(\bar{A})$ in the multi dimensional space.

Note that once $\bar{A}$ has been obtained, the calculation of $\bar{V}$ is not limited to the geometric mean method used above. Any of the priorization methods given in Appendix A can be used for that purpose. The MDM developed in this dissertation is applicable to all of those methods.

\subsubsection{Weighted Membership and Sensitivity Analysis}

The example presented in section 3.5.1 used implicitly equal weights for the four estimators. Different weights can be incorporated into the example as discussed in section 3.3.5. The goal programming model for operating on pairwise comparison matrices is as follows:

$$
\text { minimize } \sum_{i \in\{A, B, C, D\}} \sum_{j=1}^{4} \sum_{k=1}^{4} \theta_{i}\left(\left\{N_{j k}\right\}_{i}+\left\{P_{j k}\right\}_{i}\right)
$$

where $\theta_{i}(i \in\{A, B, C, D\})$ is the weights assigned to the estimator $i$. The weights represents the relative importance of the estimator. We should notice the difference 
of between weight $w_{i}$ and weight $\theta_{i} . w_{i}$ are the mathematical and logical integration of the MDM. The meaning of the variables in expression (3.51) is the same as in section 3.5.1. The expression for constrains are as follows:

$$
\begin{aligned}
& \text { subject to } \sum_{l \in\{A, B, C, D\}} w_{l} \ln \left(\left\{a_{j k}\right\}_{l}\right)-\left\{N_{j k}\right\}_{i}+\left\{P_{j k}\right\}_{i}=\ln \left(\left\{a_{j k}\right\}_{i}\right) \\
& i \in\{A, B, C, D\}, \text { and } j, k=1,2, \cdots, 4 \\
& \quad \sum_{i \in\{A, B, C, D\}} w_{i}=1 \\
& \left\{N_{j k}\right\}_{i},\left\{P_{j k} \hat{\xi}_{i}, w_{i} \geq 0 .\right.
\end{aligned}
$$

Suppose the following weights of $\theta_{i}$ ( $i \in\{A, B, C, D\}$ ) have been assigned to the estimators:

$$
\theta_{A}=0.1, \quad \theta_{B}=0.4, \quad \theta_{C}=0.3, \quad \theta_{D}=0.2,
$$

The input deck of LINDO for goal programming model defined in expressions (3.51) and (3.52) are as follows:

MIN $0.1 \mathrm{~N} 12 \mathrm{~A}+0.1 \mathrm{P} 12 \mathrm{~A}+0.1 \mathrm{~N} 13 \mathrm{~A}+0.1 \mathrm{P} 13 \mathrm{~A}+0.1 \mathrm{~N} 14 \mathrm{~A}+0.1 \mathrm{P} 14 \mathrm{~A}+$

$0.1 \mathrm{~N} 23 \mathrm{~A}+0.1 \mathrm{P} 23 \mathrm{~A}+0.1 \mathrm{~N} 24 \mathrm{~A}+0.1 \mathrm{P} 24 \mathrm{~A}+0.1 \mathrm{~N} 34 \mathrm{~A}+0.1 \mathrm{P} 34 \mathrm{~A}+$

$0.40 \mathrm{~N} 12 \mathrm{~B}+0.40 \mathrm{P} 12 \mathrm{~B}+0.40 \mathrm{~N} 13 \mathrm{~B}+0.40 \mathrm{P} 13 \mathrm{~B}+0.40 \mathrm{~N} 14 \mathrm{~B}+0.40 \mathrm{P} 14 \mathrm{~B}+$

$0.40 \mathrm{~N} 23 \mathrm{~B}+0.40 \mathrm{P} 23 \mathrm{~B}+0.40 \mathrm{~N} 24 \mathrm{~B}+0.40 \mathrm{P} 24 \mathrm{~B}+0.40 \mathrm{~N} 34 \mathrm{~B}+0.40 \mathrm{P} 34 \mathrm{~B}+$

$0.3 \mathrm{~N} 12 \mathrm{C}+0.3 \mathrm{P} 12 \mathrm{C}+0.3 \mathrm{~N} 13 \mathrm{C}+0.3 \mathrm{P} 13 \mathrm{C}+0.3 \mathrm{~N} 14 \mathrm{C}+0.3 \mathrm{P} 14 \mathrm{C}+$

$0.3 \mathrm{~N} 23 \mathrm{C}+0.3 \mathrm{P} 23 \mathrm{C}+0.3 \mathrm{~N} 24 \mathrm{C}+0.3 \mathrm{P} 24 \mathrm{C}+0.3 \mathrm{~N} 34 \mathrm{C}+0.3 \mathrm{P} 34 \mathrm{C}+$

$0.20 \mathrm{~N} 12 \mathrm{D}+0.20 \mathrm{P} 12 \mathrm{D}+0.20 \mathrm{~N} 13 \mathrm{D}+0.20 \mathrm{P} 13 \mathrm{D}+0.20 \mathrm{~N} 14 \mathrm{D}+0.20 \mathrm{P} 14 \mathrm{D}+$

$0.20 \mathrm{~N} 23 \mathrm{D}+0.20 \mathrm{P} 23 \mathrm{D}+0.20 \mathrm{~N} 24 \mathrm{D}+0.20 \mathrm{P} 24 \mathrm{D}+0.20 \mathrm{~N} 34 \mathrm{D}+0.20 \mathrm{P} 34 \mathrm{D}$

SUBJECT TO

$-0.6931 \mathrm{WA}+0.6931 \mathrm{WB}-1.0986 \mathrm{WC}+0.2876 \mathrm{WD}-\mathrm{N} 12 \mathrm{~A}+\mathrm{P} 12 \mathrm{~A}=-0.6931$

$-0.6931 \mathrm{WA}+0.6931 \mathrm{WB}-1.0986 \mathrm{WC}+0.2876 \mathrm{WD}-\mathrm{N} 12 \mathrm{~B}+\mathrm{P} 12 \mathrm{~B}=0.6931$

$-0.6931 \mathrm{WA}+0.6931 \mathrm{WB}-1.0986 \mathrm{WC}+0.2876 \mathrm{WD}-\mathrm{N} 12 \mathrm{C}+\mathrm{P} 12 \mathrm{C}=-1.0986$ 


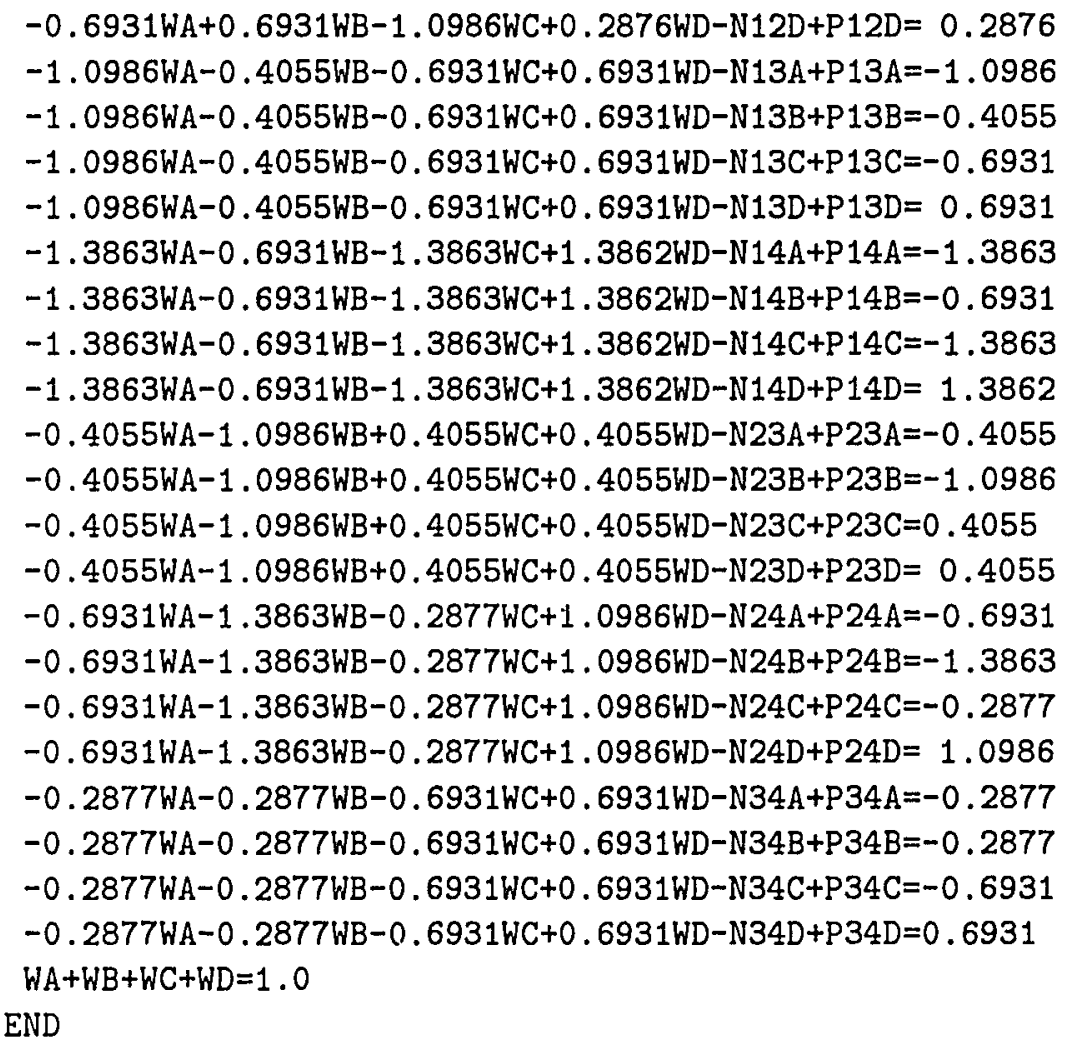

The above goal programming model was solved by LINDO with the following results:

$$
w_{A}=0.1, \quad w_{B}=0.5, \quad w_{C}=0.3, \quad w_{D}=0.1
$$

Due to the introduction of weights $\theta_{i}$ to the original goal programming model as shown in expressions 3.44 and 3.45 , the $w_{i}$ obtained in this section are significantly different from the results of previous section, which are:

$$
w_{A}=0.5, \quad w_{B}=0.0, \quad w_{C}=0.15, \quad w_{D}=0.35
$$


The corresponding aggregated pairwise comparison matrix $\bar{A}$ can now be obtained by using the expression (3.21), which are:

$$
\bar{A}=\left(\bar{a}_{j k}\right)=\left(\prod_{i \in\{A, B, C, D\}}\left(\left\{a_{j k}\right\}_{i}\right)^{w_{i}}\right), \quad \text { and } j, k=1,2, \cdots, 4
$$

Combining the $w_{\mathbf{i}}(i \in\{A, B, C, D\})$ value with the respective pairwise comparison matrices $\left\{A_{i}\right\}$ obtained from the four estimators, the $\bar{A}$, the aggregated pairwise comparison matrix, is obtained as follows:

$$
\begin{aligned}
& \bar{A}=\left[\begin{array}{cccc}
1 & \frac{1}{2}^{0.1} 2^{0.5} \frac{1}{3}^{0.3} \frac{4}{3}^{0.1} & \frac{1}{3}^{0.1} \frac{2}{3}^{0.5} \frac{1}{2}^{0.3} 2^{0.1} & \frac{1}{4}^{0.1} \frac{1}{2}^{0.5} \frac{1}{4}^{0.3} 4^{0.1} \\
2^{0.1 \frac{1}{2}^{0.5} 3^{0.3 \frac{3}{4}^{0.1}}} & 1 & \frac{2}{3}^{0.1} \frac{1}{3}^{0.5} \frac{3}{2}^{0.3} \frac{3}{2}^{0.1} & \frac{1}{2}^{0.1} \frac{1}{4}^{0.5} \frac{3}{4}^{0.3} 3^{0.1} \\
3^{0.1 \frac{3}{2}^{0.5} 2^{0.3 \frac{1}{2}^{0.1}}} & \frac{3}{2}^{0.1} 3^{0.5} \frac{2}{3}^{0.3} \frac{1}{3}^{0.1} & 1 & \frac{3}{4}^{0.1} \frac{3}{4}^{0.5} \frac{1}{2}^{0.3} 2^{0.1} \\
4^{0.1} 2^{0.5} 4^{0.3 \frac{1}{4}^{0.1}} & 2^{0.1} 4^{0.5 \frac{4}{3}^{0.3}} \frac{1}{3}^{0.1} & \frac{4}{3}^{0.1} \frac{4}{3}^{0.5} 2^{0.3} \frac{1}{2}^{0.1} & 1
\end{array}\right] \\
& =\left[\begin{array}{cccc}
1 & 0.98 & 0.64 & 0.47 \\
1.02 & 1 & 0.65 & 0.48 \\
1.56 & 1.54 & 1 & 0.73 \\
2.13 & 2.08 & 1.37 & 1
\end{array}\right]
\end{aligned}
$$

Using $\bar{A}$ as the aggregated matrix, we can now obtain the aggregated priority vector, $\bar{V}$, which represents the minimum distance criterion. Several methods as described in appendix $A$ can be used for this purpose. For example, using the geometric mean method:

$$
\bar{V}=(0.18,0.18,0.27,0.37)
$$


The individual priority weights are:

$$
v_{1}=0.18, \quad v_{2}=0.18, \quad v_{3}=0.27, \quad v_{4}=0.37
$$

$\bar{V}$, the resulting vector obtained after the aggregation process, is the one which minimize the distance between the $A_{i}(i \in\{A, B, C, D\}$ and the aggregated value $(\bar{A})$ in the multi dimensional space.

As we discussed in section 3.4 , sensitivity analysis of weights $\theta_{i}$ is very important. We would like to know in what ranges the changes of $\theta_{i}$ will not alter the original decision, which means that $\bar{V}$ will remain same. The sensitivity analysis was carried out by LINDO. The results are as follows:

$$
\theta_{A}=0.1, \quad 0.4 \leq \theta_{B} \leq 0.6, \quad 0.1 \leq \theta_{C} \leq 0.3, \quad \theta_{D}=0.2
$$

Expression (3.60) tells us that that $\bar{V}$ will keep the same if $\theta_{B}$ takes any value between 0.4 to 0.6 , and $\theta_{C}$ takes any value between 0.1 to 0.3 .

\subsubsection{MDM Operated on Priority Vectors}

In the same way as shown in section 3.5 .1 , the goal programming model for aggregating the priority vector from each decision maker can be obtained by using expressions (3.35) and (3.36), which are presented as follows:

$$
\text { minimize } \sum_{i \in\{A, B, C, D\}} \sum_{j=1}^{4} \sum_{k=1}^{4}\left(\left\{N_{j}\right\}_{i}+\left\{P_{j}\right\}_{i}\right)
$$

where $\left\{N_{j}\right\}_{i}$ is the negative deviation from the relative weight of element $j$ by estimator $i,\left\{P_{j}\right\}_{i}$ is the positive deviation from the relative weight of element $j$ 
by estimator $i, i$ stands for the estimators, which is from $A$ to $D$ in this example, $j$ and $k=1, \cdots, 4$ (the elements being compared). The constraints for the goal programming model are as follows:

$$
\begin{gathered}
\text { subject to } \sum_{l \in\{A, B, C, D\}} w_{l} \ln \left(\left\{v_{j}\right\}_{l}\right)-\left\{N_{j}\right\}_{i}+\left\{P_{j}\right\}_{i}=\ln \left(\left\{v_{j}\right\}_{i}\right) \\
i \in\{A, B, C, D\}, \text { and } j=1,2, \cdots, 4 \\
\sum_{i \in\{A, B, C, D\}} w_{i}=1 \\
\left\{N_{j}\right\}_{i},\left\{P_{j}\right\}_{i}, w_{i} \geq 0 .
\end{gathered}
$$

Given the priority vector of each estimator as presented in expressions (3.42) and (3.43), the input deck of LINDO for the goal programming model defined in expressions (3.61) and (3.62) are presented in the following:

$M I N \quad N 1 A+P 1 A+N 2 A+P 2 A+N 3 A+P 3 A+N 4 A+P 4 A+N 1 B+P 1 B+N 2 B+$
$P 2 B+N 3 B+P 3 B+N 4 B+P 4 B+N 1 C+P 1 C+N 2 C+P 2 C+N 3 C+P 3 C+$
$N 4 C+P 4 C+N 1 D+P 1 D+N 2 D+P 2 D+N 3 D+P 3 D+N 4 D+P 4 D$

SUBJECT TO

$0.0000 \mathrm{WA}+0.6931 \mathrm{WB}+0.0000 \mathrm{WC}+1.3863 \mathrm{WD}-\mathrm{N} 1 \mathrm{~A}+\mathrm{P} 1 \mathrm{~A}=0$

$0.0000 \mathrm{WA}+0.6931 \mathrm{WB}+0.0000 \mathrm{WC}+1.3863 \mathrm{WD}-\mathrm{N} 1 \mathrm{~B}+\mathrm{P} 1 \mathrm{~B}=0.6931$

$0.0000 \mathrm{WA}+0.6931 \mathrm{WB}+0.0000 \mathrm{WC}+1.3863 \mathrm{WD}-\mathrm{N} 1 \mathrm{C}+\mathrm{P} 1 \mathrm{C}=0$

$0.0000 \mathrm{WA}+0.6931 \mathrm{WB}+0.0000 \mathrm{WC}+1.3863 \mathrm{WD}-\mathrm{N} 1 \mathrm{D}+\mathrm{P} 1 \mathrm{D}=1.3863$

$0.6931 W A+0.0000 W B+1.0986 W C+1.0986 W D-N 2 A+P 2 A=0.6931$

$0.6931 \mathrm{WA}+0.0000 \mathrm{WB}+1.0986 \mathrm{WC}+1.0986 \mathrm{WD}-\mathrm{N} 2 \mathrm{~B}+\mathrm{P} 2 \mathrm{~B}=0.0000$

$0.6931 \mathrm{WA}+0.0000 \mathrm{WB}+1.0986 \mathrm{WC}+1.0986 \mathrm{WD}-\mathrm{N} 2 \mathrm{C}+\mathrm{P} 2 \mathrm{C}=1.0986$

$0.6931 W A+0.0000 W B+1.0986 W C+1.0986 W D-N 2 D+P 2 D=1.0986$

$1.0986 \mathrm{WA}+1.0986 \mathrm{WB}+0.6931 \mathrm{WC}+0.6931 \mathrm{WD}-\mathrm{N} 3 \mathrm{~A}+\mathrm{P} 3 \mathrm{~A}=1.0986$

$1.0986 \mathrm{WA}+1.0986 \mathrm{WB}+0.6931 \mathrm{WC}+0.6931 \mathrm{WD}-\mathrm{N} 3 \mathrm{~B}+\mathrm{P} 3 \mathrm{~B}=1.0986$

$1.0986 \mathrm{WA}+1.0986 \mathrm{WB}+0.6931 \mathrm{WC}+0.6931 \mathrm{WD}-\mathrm{N} 3 \mathrm{C}+\mathrm{P} 3 \mathrm{C}=0.6931$

$1.0986 \mathrm{WA}+1.0986 \mathrm{WB}+0.6931 \mathrm{WC}+0.6931 \mathrm{WD}-\mathrm{N} 3 \mathrm{D}+\mathrm{P} 3 \mathrm{D}=0.6931$ 
1.3863WA+1.3863WB+1.3863WC+0.0000WD-N4A+P4A $=1.3863$

1.3863WA+1.3863WB+1.3863WC+0.0000W4-N4B+P4B $=1.3863$

$1.3863 \mathrm{WA}+1.3863 \mathrm{WB}+1.3863 \mathrm{WC}+0.0000 \mathrm{WD}-\mathrm{N} 4 \mathrm{C}+\mathrm{P} 4 \mathrm{C}=1.3863$

1.3863WA+1.3863WB+1.3863WC+0.0000WD-N4D+P4D $=0.0000$

$W A+W B+W 3+W D=1.0$

END

where $\left\{N_{j}\right\}_{i},\left\{P_{j}\right\}_{i}$ and $w_{i}$ show as $\mathrm{Nji}, \mathrm{Pji}$ and $\mathrm{Wi}$, respectively. The values of the constants are from $\ln (1)=0, \ln (2)=0.6931, \ln (3)=1.0986$, and $\ln (4)=1.3863$.

The goal programming model was solved by LINDO, $w_{i}(i \in\{A, B, C, D\})$ are obtained as:

$$
w_{A}=1.0, \quad w_{B}=0.0, \quad w_{C}=0.0, \quad w_{D}=0.0
$$

The corresponding aggregated priority vector $\bar{V}$ can now be obtained by using the expression (3.15), which are:

$$
\bar{V}=\left(\prod_{i \in\{A, B, C, D\}}\left(\left\{v_{1}\right\}_{i}\right)^{w_{i}}, \prod_{i \in\{A, B, C, D\}}\left(\left\{v_{2}\right\}_{i}\right)^{w_{i}}, \cdots, \prod_{i \in\{A, B, C, D\}}\left(\left\{v_{n}\right\}_{i}\right)^{w_{i}}\right)
$$

Combining the values of $w_{A}, w_{B}, w_{C}$ and $w_{D}$ with respective priority vector obtained from four estimators, the $\bar{V}$, aggregated priority vector, is obtained as:

$$
\begin{aligned}
& \bar{V}=\left(0.1^{1.0} 0.2^{0.0} 0.1^{0.0} 0.4^{0.0}, 0.2^{1.0} 0.1^{0.0} 0.3^{0.0} 0.3^{0.0}, 0.3^{1.0} 0.3^{0.0} 0.2^{0.0} 0.2^{0.0},\right. \\
& \left.0.4^{1.0} 0.4^{0.0} 0.4^{0.0} 0.1^{0.0}\right) \\
& =(0.1,0.2,0.3,0.4)
\end{aligned}
$$

$\bar{V}$, the resulting vector obtained after the aggregation process, is the one which minimize the distance the $V_{i}(i \in\{A, B, C, D\})$ and the aggregated value in the multi dimensional space. 


\section{Chapter 4}

\section{COMPARISON STUDY AND SIMULATION PROCEDURES}

The previous chapters investigated the judgment aggregation methodologies from mathematical and logical points of view. Judgment aggregation within the framework of AHP, as one of the most important aspects of a group decision making process, has been discussed in detail by Aczel and Saaty $[6,7,8]$ as well as in this dissertation. Aczel and Saaty's work focuses on the functional equations approach (i.e. the geometric mean approach). Several conditions must be satisfied to use that approach; three conditions (separability, unanimity and reciprocal) have been discussed in Chapter 3. Aczel and Saaty have shown that the only function to satisfy these three conditions is geometric function. We propose an approach in this dissertation, the distance approach, which we have named the Minimum Distance Method (MDM), is based on the Cook et al's distance axiom [5] and weighted geometric mean concept. This new approach not only appeals to the compromising nature of the group decision making process, but it also preserves these conditions that Aczel and Saaty have stipulated. We will carry further the study of aggregation methods by evaluating their performance in this and following chapters. 
The arithmetic mean and geometric mean methods have been used for judgment aggregations for a long time. Aczel and Saaty's contribution has been to provide a mathematical justification for the geometric mean approach. However, based on the literature search, there has been little done regarding the performance of those methods presented so far. It would be possible and important to "test" all present judgment aggregation methods by examining their performance according to certain performance measurements. The "test" of judgment aggregation methods would be valuable, especially when the alternatives and equally "reasonable" methods (arithmetic mean, geometric mean and MDM) has been proposed or practiced.

Two approaches are adopted to study the performance of judgment aggregation methods in this dissertation. One is simulation by which a large number of judgments (i.e pairwise comparison matrices) for group decision situations are created by computer. The decision to use any particular scientific technique in pursuing a problem is determined by a large number of factors. The appropriateness of the method is one consideration, the potential to advance theoretical understanding is another, and economy is yet a third. The reasons for using the simulation are as follows:

- Computer simulation often leads to a more complete expression of a theory than may otherwise be possible. This is primarily because of the ability of the computer program to deal with great complexity, both in terms of its own variables and in terms of its data. A verbally stated theory, or indeed a mathematically presented one, often becomes incomprehensible when it attempts to deal with large numbers of variables and parameters simultaneously. 
- Exploration with the simulation may suggest relationships that can be explored in a real experiment. The net result of this complex interconnection between theory, simulation, and experimentation is an advancement in the theoretical understanding of the process that is all important in research.

- Computer simulation is a model of some real process, the program's activities can be made to parallel the actual process to a greater degree than it possible with other forms of models. This is of benefit even in simple and well-specified theories - it allows the theory to be more easily understood because it is possible to "watch" the process unfold over the course of operating the program.

- An operating computer simulation in many respects provides an ideal experimental subject for research. Once the program is operating correctly, it is a relatively simple matter to run many experimental quickly. It suffers rıone of the practical problems that plague behavioral researchers - it does not need to be fed, housed, or paid; it does not require a massive survey effort, and etc.

- In a computer model, it is easy to represent randomness and to deal with random variables. for example, to make several simulation runs of a program assuming that a variable has different distributions.

But we also notice that there may exist discrepancies between those pairwise comparison matrices generated by computer and the pairwise comparison matrices obtained from actual judgments. It is desirable to "test" the aggregation method 
through the actual judgment data. Then the empirical data (i.e the empirical test approach), which is obtained from groups of students measuring values of seven categories, are used to test the aggregation methods.

This chapter is structured as follows: Section 4.1 presents the objectives of the proposed comparison study. Section 4.4 deals with the simulation approach in general and is followed by section 4.2 and section 4.3 for the analysis of perturbation methods and of performance measurements respectively. Section 4.5 deals with the empirical test approach.

\subsection{Objectives and Considerations}

The existing aggregation methods (arithmetic and geometric mean) were reviewed in section 3.2, and a new method - Minimum Distance Method (MDM)

- was proposed in section 3.3. Due to different assumptions and the underlying input data (i.e. the judgments) distributions, the solutions obtained from different methods will be different. Consequently, evaluation of the performances of those methods is important and necessary for helping us to use those methods. To facilitate the discussion in this chapter, a list of the judgment aggregation methods to be evaluated is given in the Table 4.1. The abbreviation given in this table will be used throughout this and the next chapters. In Table 4.1, one point needs special attention. The geometric mean method and MDM deal with two kinds of data, one is the pairwise comparison matrix, and the other one is the final priority vector. 
Table 4.1: The List of Judgment Aggregation Methods

\begin{tabular}{||c|c|l||}
\hline No. & ABBREVIATION & \multicolumn{1}{|c||}{ DESCRIPTIONS } \\
\hline \hline 1 & A-GE(V) & $\begin{array}{l}\text { The Geometric Mean operates on the } \\
\text { priority vector of group members }\end{array}$ \\
\hline 2 & A-GE(M) & $\begin{array}{l}\text { The Geometric Mean operates on the } \\
\text { pairwise comparison matrix of group members }\end{array}$ \\
\hline 3 & A-AM(V) & $\begin{array}{l}\text { The Arithmetic Mean operates on the } \\
\text { priority vector of group members }\end{array}$ \\
\hline 4 & A-MDM(M) & $\begin{array}{l}\text { The Minimum Distance Method operates on the } \\
\text { pairwise comparison matrix }\end{array}$ \\
\hline 5 & A-MDM(V) & $\begin{array}{l}\text { The Minimum Distance Method operates on the } \\
\text { priority vector of group members }\end{array}$ \\
\hline
\end{tabular}

\subsubsection{Objectives of Comparison Study}

Specifically, the major purpose of this study is to evaluate and contrast judgment aggregation methods so that the characteristics of the aggregation methods can be better understood. The significance of this chapter is: the first goal is to investigate the following issues:

1. How do the aggregation methods function with respect to the different types of input data?

2. What is the relationship between aggregation methods and the number of decision makers?

3. What is the influence of prioritization methods over the aggregation methods or vice versa?

The second goal is to generalize the findings from the comparison of judgment aggregation methods and to develop guidance for the use of aggregation methods. 


\subsubsection{Considerations for Comparison Study}

Simulation and empirical test approaches are employed in the comparison study. Regardless which approaches are used, the comparison study begins with groups of input data (i.e the judgments). The input data is in the form of a pairwise comparison matrix $(A)$. Suppose $\mathrm{m}$ is the size of the group referred to as the number of decision makers, then we use $\left\{A_{i}\right\}$ to represent a group of pairwise comparison matrices, where $i=1, \cdots, m$. Furthermore, suppose $\mathrm{T}$ is the number of groups, then we denote groups of input data as $\left\{A_{i}\right\}_{t}$, where $t=1, \cdots, T$. After the input data are ready, which can be obtained either through computer generations or from real judgments, they are fed into the aggregation process. There are two variations of the aggregation process: one is to aggregate the pairwise comparison matrices $\left\{A_{i}\right\}$ then derive the priority vector from the aggregated pairwise comparison matrix. The other one is to derive the priority veciors of each pairwise comparison matrix in $\left\{A_{i}\right\}$ and then to aggregate priority vectors. Finally, the performance of the aggregation process is evaluated by performance measurements. The above discussed procedure is summarized in Fig.4.1

From Fig. 4.1, the following items should be further discussed in general even though the detailed mathematical descriptions are presented in subsequent sections.

- Input data to the aggregation methods, i.e. what are the judgments

- Performance measurements

- Prioritization methods 


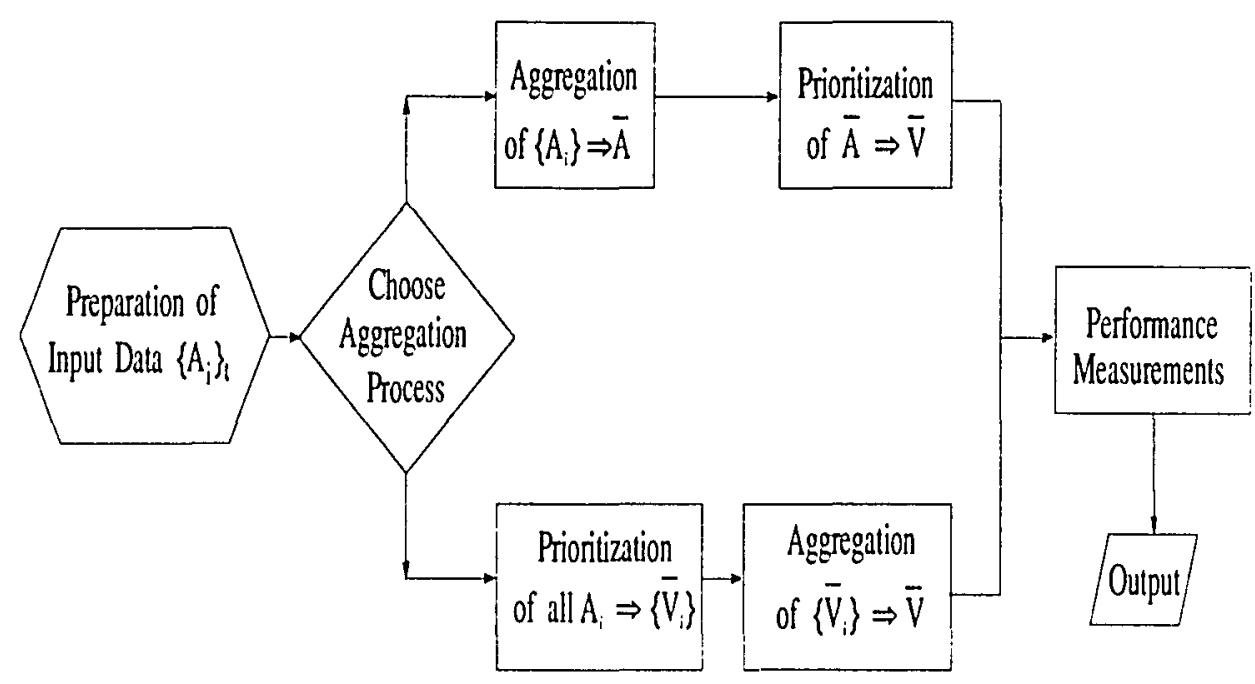

Figure 4.1: The procedures of comparison study for both simulation and empirical test

We will spend this section discussing these items and their underlying relationships in general.

Input data: The ways to get the input data (i.e. the pairwise comparison matrices) for evaluation of aggregation methods are different for simulation study and emperical test. In the simulation approach, a large number of groups of pairwise comparison matrices (i.e. judgments) are generated by using a computer. The data generation procedures are discussed in detail in section 4.4.1. Each group of pairwise comparison matrices consists of $m$ (i.e. number of decision makers) individual pairwise comparison matrix, just as a decision making group has members and each member make a judgment. Therefore, in simulation, the input data generation is the process to mimic the judgments of group decision makers. The empirical testing 
data are actual judgments from a group of students, and details of those data are discussed in section 4.5 .

Performance measurements: In order to compare the aggregated results of simulation and empirical test for aggregation methods, criteria and measurements are needed to gauge the performance of each aggregation method. Two kinds of measurements for performance are proposed in section 4.3. Briefly, one measurement is the accuracy, which is to measure how closely the aggregated group priority vector matches the "real" priority vector. In this study, the "real" priority vectors are known. The other one is the measurement for disagreement, which is designed to measure the deviation between the group members' responses and aggregated group priority vector (response). This is the measurement to indicate the extent to which the group as whole satisfies the aggregated group priority vector.

Prioritization methods: In AHP, the output of group decision making is in the form of a priority vector; therefore, we need to use prioritization methods, which transfer the pairwise comparison matrix into the priority vector. The involvement of prioritization methods greatly complicates the simulation and empirical test process because we have one more dimension to consider for both the simulation and empirical test. Furthermore, the impacts of the prioritization methods on the aggregation methods or vice versa are also the concerns of the comparison study. For example, what is the best combination of prioritization method and aggregation method in order to produce the best aggregation result? Therefore, for each set of pairwise comparison matrices, all the prioritization methods have to be applied, and each of those aggregation results will be subjected to performance evaluation. 
The prioritization methods themselves have been a major research area especially in the past fifteen years. Since the method of paired comparisons was first discussed by Thurstone $[61,62]$ in 1927 , and more recently, the effective use of the reciprocal matrices was demonstrated by Saaty [2] in 1977, there has been an increased interest in the problem of prioritization through ratio scale measurements. To a large extent, the interest in this problem is due to the development of various new prioritization methods and their successful use in experimental and practical situations, especially in the areas of social sciences and management. A large number of techniques has been proposed for prioritization through scaling ratio judgment, ranging from relatively simple averages [37] to more complicated methods, such as the constantsum method $[24,1]$, the column-row sums method [23], the eigenvalue method [2, $3]$, the geometric mean $[63,27,28]$, the least squares $[64,26]$, the weighted least squares [65], and so on. A summary of these techniques is given in Appendix A. An abbreviation of various prioritization techniques is presented in Table $4.2^{1}$. The abbreviations and the identification numbers for prioritization methods in Table 4.2 will be used throughout this and next chapter.

\footnotetext{
${ }^{1}$ In Table 4.2, $\mathrm{A}$ is the pairwise comparison matrix, $\mathrm{A}$ ' is the transpose of matrix $\mathrm{A}$ and $\mathrm{AA}$ ' stand for multiplication of matrix A with matrix $A^{\prime}$
} 
Table 4.2: Abbreviation for Judgment Prioritization Methods

\begin{tabular}{||c|l|l||}
\hline No. & ABBREVIATION & \multicolumn{1}{c|}{ DESCRIPTIONS } \\
\hline \hline 1 & CSM & Constant-Sum Method \\
\hline 2 & R-EV & Right Eigenvector of [A] Matrix \\
\hline 3 & L-EV & Left Eigenvector of [A] Matrix \\
\hline 4 & AM-EV & $\begin{array}{l}\text { Arithmetic Means of Right and Left } \\
\text { Eigenvector of [A] Matrix }\end{array}$ \\
\hline 5 & GM-EV & $\begin{array}{l}\text { Geometric Means of Right and Left } \\
\text { Eigenvector of [A] Matrix }\end{array}$ \\
\hline 6 & EV[AA'] & Eigenvector of [AA'] Matrix \\
\hline 7 & EV[A'A] & Eigenvector of [A'A] Matrix \\
\hline 8 & $\begin{array}{l}\text { AM-EV[AA'] } \\
\text { AND EV[A'A] }\end{array}$ & $\begin{array}{l}\text { Arithmetic Means of Eigenvector of } \\
{[\text { AA'] and [A'A] Matrices }}\end{array}$ \\
\hline 9 & $\begin{array}{l}\text { GE-EV[AA'] } \\
\text { AND EV[A'A] }\end{array}$ & $\begin{array}{l}\text { Geometric Means of Eigenvector of } \\
\text { [AA'] and [A'A] Matrices }\end{array}$ \\
\hline 10 & GM & $\begin{array}{l}\text { Logarithmic Least Squares Method } \\
\text { via row Geometric Means Method }\end{array}$ \\
\hline 11 & C-RSM & $\begin{array}{l}\text { Column-Row Sums Method. } \\
\text { (Normalized geometric means of two } \\
\text { normalized vectors of the inverse } \\
\text { column sums and the row sums) }\end{array}$ \\
\hline 12 & MT & Mean Transformation Method \\
\hline 13 & SAV & $\begin{array}{l}\text { Average of Row Elements of [A] } \\
\text { Matrix }\end{array}$ \\
\hline 14 & NEV & New Eigenweight Method \\
\hline 15 & LSM & Least Squares Method \\
\hline
\end{tabular}




\subsection{Input Data Generation and The Perturbation Method}

An important part of input data generation is how to get quality pairwise comparison matrix $(A)$ for simulation. Quality here means that generated pairwise comparison matrices by computer should be close to the actual judgments. Therefore, in this section, we will first discuss the characteristics of actual judgments in section 4.2.1. In section 4.2.2, input data generation procedures are presented in general. Sections 4.2.3, section 4.2.4, 4.2.5 and 4.2.6 will discuss detailed data generation procedures via probability distributions.

\subsubsection{Characteristics of Actual Judgments}

Suppose we compare $\mathrm{n}$ decision elements, and the pairwise comparison matrix is used to express the ratio judgments in AHP. The matrix of pairwise comparisons shows the extent to which one element is preferred over another in achieving an objective at one level higher in the hierarchy, which has been discussed in Chapter 2 . There are two situations when pairwise comparisons are made, which are consistent and inconsistent cases in terms of the pairwise comparison matrix $(A)$.

Consistent situation: In this situation, the pairwise comparisons are made without measurement errors, i.e. the corresponding pairwise comparison matrix is consistent. Assume that pairwise comparison matrix is denoted as $A$. If $V=$ $\left(v_{1}, v_{2}, \cdots, v_{n}\right)$ is the priority vector of $\mathrm{n}$ decision elements, which is derived from $A$ by using any prioritization methods in Appendix A, then the $n \times n$ square matrix 
(A) of pairwise comparisons should satisfy the following relationship:

$$
A=\left[a_{j k}\right]=\left[v_{j} / v_{k}\right], \quad j, \dot{\kappa}=1, \cdots, n
$$

Inconsistent situation: In this situation, pairwise comparisons are made with measurement errors. The relationship between pairwise comparison matrix $A$ and priority vector denoted in the above expression 4.1 no longer holds true. In general, those measurement errors are largely due to the estimator's perception and knowledge. Consequently, the matrix would be inconsistent. This happens frequently and is not a disaster. Usually, unless the estimator methodically pays attention to building up the judgments from n-1 decision elements, his pairwise comparison matrix is not likely to be consistent. Furthermore, in the case of measurement error, one of the most important things that the decision makers would like to know is how good the pairwise comparison matrix $A$ is. One way to measure the goodness of the pairwise comparison judgments is to use the difference between matrix $A$ and matrix $\left[v_{j l} / v_{k l}\right]$, where $V_{\mathfrak{T}}=\left(v_{1 t}, v_{2 t}, \cdots, v_{n t}\right)$ is the actual priority vector. But in the real world, it is very difficult and even impossible to know the actual priority vector $V_{\pi}$. We only can get the estimation $V$ of priority vector by using various prioritization methods (see Appendix A) from matrix $A$.

\subsubsection{Input Data Generation for Simulation}

Given the nature of both consistency and inconsistency of pairwise comparison judgments, we would like to generate the input data for simulation with the following characteristics:

- Have a "true" priority vector so that the aggregation performance can be 
measured.

- Take the nature of both consistency and inconsistency of pairwise comparison matrices into consideration.

The procedure of input data generation can be described as follows: For each simulated group $(t)(t=1, \cdots, T)$, a corresponding priority vector $V_{t}=\left(v_{1 t}, v_{2 t}, \cdots, v_{n t}\right)$ is generated by computer. We take the $V_{t}$ as the "true" priority vector. Based on $V_{t}$, a consistent matrix $A_{t}$ is constructed by $A_{t}=\left[v_{j t} / v_{k t}\right]$, which is the consistent matrix according Eqn. 4.1. The simulated groups of pairwise comparison matrices $\left\{A_{i}\right\}_{t}(i=1, \cdots, m)$, where $\mathrm{m}$ is the size of the group, are derived from $A_{t}$. Keep in mind the multi-dimensional situation in the simulation. There are $\mathrm{T}$ groups of pairwise comparison matrices, so the $t$ is from 1 to $\mathrm{T}$ through out this chapter. Within each group of pairwise comparison matrices, there are $\mathrm{m}$ individual pairwise comparison matrices.

For any given $t$, we need a mechanism to derive pairwise comparison matrices $\left\{A_{i}\right\}_{t}$ from consistent matrix $A_{t}$. Those pairwise comparison matrices $\left\{A_{i}\right\}_{t}$ should have the characteristics of actual judgments as described in section 4.2.1. Suppose for any given i, $A_{i}$ can be expressed as:

$$
A_{i}=\left[a_{j k}\right]_{i}=\left[\frac{v_{j t}}{v_{k t}} e_{j k}\right]_{i}, \quad j, k=1, \cdots, n
$$

where $e_{j k}$ is called the measurement error term. When $e_{i j}=1$, the $A_{i}$ is equal to $A_{t}$ which is a consistent matrix. When $e_{j k} \neq 1$ and $e_{j k}>1$, the $A_{i}$ is away from consistent matrix $A_{t}$, the magnitude of difference is determined by the value of $e_{j k}$, 
and that is where the measurement error term comes from. Therefore, we have a mechanism to generate $A_{i}$ from $A_{t}$, which is realized by using Eqn. 4.2 with change of $e_{j k}$ value. If $e_{j k}$ are generated in such a way that the mean of $e_{j k}$ is equal to one, we then get a group of matrices $\left\{A_{i}\right\}$ either consistent or inconsistent.

According to the approach of generating $A_{i}$ presented above, all the pairwise comparison matrices in a group are generated from a single pairwise comparison matrix $A_{t}$. Considering the case that the mean of $e_{i j}$ is equal to one, the procedure of data generation described in this section implies that the "true" priority vector of the group should be $V_{t}$. By repeating this procedure, a number of groups of pairwise comparison matrices can be generated.

The multiplicative form for the measurement error was used for perturbating the $A_{t}$ to form matrix $A_{i}$. The reasons for using this form are two-fold. First of all, this form is easy to understood. Second, the multiplicative form for the measurement error was originally proposed by Saaty [3] to derive the inconsistency measure for the pairwise comparison. Zahedi [66] also used the multiplicative form in a simulation study to compare the prioritization methods.

In the above discussion, we have decided on the form of measurement error term for perturbation. Now the focus of input data generation is how to determine the value of $e_{j k}$. The measurement error means that the judgment error or inconsistency occurs when the ratio judgments are made among $\mathrm{n}$ decision elements. It describes "the effect of inconsistency on what is thought to be the psychological process involved in pairwise comparisons of a set of data" [3]. Hence, when different decision makers or groups of decision makers are involved in a decision process, their ratio 
judgments will be different, and so are the error terms. Furthermore, the underlying distribution of judgments and error terms should not only be different but also in a wide variety of types. Consequently, the results from aggregation and prioritization will be different too. Due to the psychologically complex implication of measurement error terms, it is very difficult or even impossible to reproduce the error term distributions by computer. However, in this study we are concerned about the performance of aggregation and prioritization methods. From logical and mathematical points of view, only the typical probability distributions should be used to generate the measurement error terms $\left(e_{j k}\right)$. But the proposed probability distributions should cover a wide variety of distribution types and have non-negativity random variables. There are three typical distributions to satisfy those conditions, i.e. gamma, lognormal and uniform distributions. We also noticed those distributions have been used in other studies as well $[63,3,66]$. Zahedi $[66]$ used these three distributions to generate pairwise comparison matrices. Those matrices were used to study the performance of prioritization methods. The simulation approach presented in this dissertation can be viewed as the extension of Zahedi's approach to a group decision situation.

\subsubsection{Generation of Perturbation Distributions}

Three probability distributions are typically used in the perturbation process. This allows comparison between the simulation results using different probability distributions. In order to make cross comparison meaningful, the input data gener- 
ation process should satisfy the following two conditions.

1. Each element $a_{j k}$ of $A_{i}$ must be generated within a given interval $I$ regardless of probability distributions. $I=\left[\rho\left\{a_{j k}\right\}_{t}, \eta\left\{a_{j k}\right\}_{t}\right]$, where $\rho$ and $\eta$ are constants, $\left\{a_{j k}\right\}_{t}$ is the element of $A_{t} . \rho$ and $\eta$ should be determined so that the interval I will be symmetric to $a_{j k}$. For the purpose of the simulation conducted in this study, $\eta=1.5$ and $\rho=0.5$ are used.

2. The mean of $e_{j k}$ should be equal to one, i.e. $E\left(e_{j k}\right)=1.0$. Equivalently, the mean of $a_{j k}$ should be equal to $\left\{a_{j k}\right\}_{t}$, i.e $E\left(a_{j k}\right)=\left\{a_{j k}\right\}_{t}$. Those relationships are for all the simulated probability distributions.

From the simulation point of view, there are two ways to simulate the measurement errors:

1. To generate the probability distribution of $e_{j k}$ with the mean value of $E\left(e_{j k}\right)=$ 1.0 (note: when $e_{j k}=1$, there are no measurement errors).

2. To create the probability distribution of $a_{j k}$ with the mean value, $E\left(a_{j k}\right)=$ $\left\{a_{j k}\right\}_{t}=v_{j t} / v_{k t}$

These two approaches are equivalent; they generate the data with the same mean and same distribution. In this dissertation, the latter is adopted and the same approach is also discussed in [66].

In the following three sections, we will discuss how to generate a group of pairwise comparison matrices $\left\{A_{i}\right\}$ by using the three probability distributions. The 
significance of the following sections is to demonstrate that under what conditions, those three distributions will result in the same mean and with all or nearly all of $a_{j k}$ generated by computer fall in the given interval $I=\left[\rho\left\{a_{j k}\right\}_{t}, \eta\left\{a_{i k}\right\}_{t}\right]$. This condition is very important for us to comparing the simulation results across those distributions.

\subsubsection{Generation of Uniform Distribution Input Data}

This section describes how to generate a uniform distribution over the interval $I=\left[\rho\left\{a_{j k}\right\}_{t}, \eta\left\{a_{j k}\right\}_{t}\right]$ for a computer simulation program. When we say a distribution over an interval $I=\left\{\rho\left\{a_{j k}\right\}_{t}, \eta\left\{a_{j k}\right\}_{t}\right]$, it means that the points $\left(a_{j k}\right)$ generated by computer fall in the interval $I$ with a given distribution. Two parameters need to be determined to completely specify the uniform distribution, which are expected value $(\mu)$ and variance. With the probability density function $p\left(a_{j k}\right)=1 /\left[(\eta-\rho)\left\{a_{j k}\right\}_{t}\right]$ (suppose $t$ is given), we have

$$
\begin{aligned}
& E\left(a_{j k}{ }^{2}\right)=\int_{\rho\left\{a_{j k}\right\}_{t}}^{\eta\left\{a_{j k}\right\}_{t}} \frac{a_{j k}{ }^{2}}{(\eta-\rho)\left\{a_{j k}\right\}_{t}} d a_{j k}=\left(\eta^{2}+\eta \rho+\rho^{2}\right) \frac{\left\{a_{j k}\right\}_{t}}{3} \\
& \operatorname{Var}\left(a_{j k}\right)=E\left(a_{j k}{ }^{2}\right)-\left(E\left(a_{j k}\right)\right)^{2}=(\eta-\rho)^{2} \frac{\left\{a_{j k}\right\}_{t}}{12} \\
& E\left(a_{j k}\right)=\int_{\rho\left\{a_{j k}\right\}_{t}}^{\eta\left\{a_{j k}\right\}_{t}} \frac{a_{j k}}{(\eta-\rho)\left\{a_{j k}\right\}_{t}} d a_{j k}=\frac{(\eta+\rho)\left\{a_{j k}\right\}_{t}}{2}
\end{aligned}
$$

With $\eta=1.5$ and $\rho=0.5$, from the above equations, we can get $E\left(a_{j k}\right)=\left\{a_{j k}\right\}_{t}$ and $\operatorname{Var}\left(a_{j k}\right)=\left\{a_{j k}\right\}_{t}{ }^{2} / 12=0.08\left\{a_{j k}\right\}_{t}{ }^{2}$. This mean and variance will guarantee that all $a_{j k}$ generated by computer fall into interval $I=\left[0.5\left\{a_{j k}\right\}_{t}, 1.5\left\{a_{j k}\right\}_{t}\right]$. 


\subsubsection{Generation of Lognormal Distribution Input Data}

It is easy to notice that generated $a_{j k}$ s from the computer fall $100 \%$ in the interval I for uniform distribution. However, for lognormal distribution, it is impossible for all simulation observations (i.e. generated $a_{j k} \mathrm{~s}$ ) to fall in the interval $I$. This is due to the nature of lognormal distribution, whose simulation observations can only fall $100 \%$ in the interval $[0, \infty]$. In order to make meaningful comparison of simulation results between uniform and lognormal distribution, the objective is to make interval $I$ contain a substantial portion (say $90 \%$ ) of simulated observations, which are $a_{j k}$ 's. The rest of this section will derive the conditions for lognormal distribution such that $90 \%$ of generated $a_{j k}$ fall in the interval $I=\left[\rho\left\{a_{j k}\right\}_{t}, \eta\left\{a_{j k}\right\}_{t}\right]$.

Suppose $x$ has a normal distribution with expected value (mean) $\mu$ and variance $\sigma^{2}, a_{j k}$ has the lognormal distribution with $a_{j k}=e^{x}$. The objective is to determine $\mu$ and $\sigma^{2}$ such that the interval $I=\left[\rho\left\{a_{j k}\right\}_{t}, \eta\left\{a_{j k}\right\}_{t}\right]$ would contain $95 \%$ simulated $a_{j k}$. The probability density function of $a_{j k}$ is as follows:

$$
p\left(a_{j k}\right)=\frac{1}{a_{j k} \sigma \sqrt{2 \pi}} e^{\frac{\left(l n a_{j k}-\mu\right)^{2}}{2 \sigma^{2}}}
$$

The r-th moment is

$$
E\left(a_{j k}^{r}\right)=\int_{-\infty}^{\infty} p\left(a_{j k}\right) a_{j k}^{r} d a_{j k}^{r}=e^{\left(r \mu+r^{2} \sigma^{2} / 2\right)}
$$

With this $\mathrm{r}$-th moment, the expected value and variance are

$$
\begin{aligned}
& E\left(a_{j k}\right)=e^{\mu+\sigma^{2} / 2} \\
& \operatorname{Var}\left(a_{j k}\right)=e^{2 \mu+2 \sigma^{2}}+e^{2 \mu+\sigma^{2}}
\end{aligned}
$$


As mentioned in section 4.2 .3 , we expect that $E\left(a_{j k}\right)=\left\{a_{j k}\right\}_{t}$. By using this expression, we can get the relationship between $\mu$ and $\left\{a_{j k}\right\}_{t}$ as follows:

$$
\mu=\ln \left\{a_{j k}\right\}_{t}-\frac{\sigma^{2}}{2}
$$

From Eqn.4.10, the normal distribution has mean $\mu=\ln \left\{a_{j k}\right\}_{t}-\frac{\sigma^{2}}{2}$ and a standard deviation of $\sigma$. As one of the characteristics for normal distribution states, $95 \%$ of observations of normal distribution fall in an interval of $\ln \left\{a_{j k}\right\}_{t}-\sigma^{2} / 2 \pm$ $2 \sigma$. Consequently, the corresponding lognormal distribution contains $95 \%$ of its observations in the interval $I=\left[e^{\ln \left\{a_{j k}\right\}_{t}-\sigma^{2} / 2-2 \sigma}, e^{\left.\ln \left\{a_{j k}\right\}_{t}-\sigma^{2} / 2+2 \sigma\right]}\right.$. Furthermore, we expect that interval $I$ equals $\left[\rho\left\{a_{j k}\right\}_{t}, \eta\left\{a_{j k}\right\}_{t}\right]$. Combining these two expressions, lower and upper bounds of interval $I$ should satisfy the following conditions:

$$
e^{\ln \left\{a_{j k}\right\}_{t}-\sigma^{2} / 2-2 \sigma}=\rho\left\{a_{j k}\right\}_{t} \text { and } e^{\ln \left\{a_{j k}\right\}_{t}-\sigma^{2} / 2+2 \sigma}=\eta\left\{a_{j k}\right\}_{t}
$$

The expression in (4.11) is equivalent to following equations:

$$
e^{-\sigma^{2} / 2-2 \sigma}=\rho \quad \text { and } \quad e^{\left.-\sigma^{2} / 2+2 \sigma\right)=\eta}
$$

For any given $\rho$ and $\eta$, the equations in (4.12) can be solved by any approximation method to get $\sigma$. For example, if $\rho=0.5$ and $\eta=1.5$, the approximate solution is $\sigma^{2}=0.05$. From $\mu=\ln \left\{a_{j k}\right\}_{t}-\frac{\sigma^{2}}{2}$, we can get $\mu=\ln \left\{a_{j k}\right\}_{t}-0.0025$. Hence, the interval $I$ contains $100 \%$ of the uniform distribution and about $95 \%$ of the lognormal distribution.

The expected value and variance of generated $a_{j k}$, in case of lognormal distributions, are $E\left(a_{j k}\right)=\left\{a_{j k}\right\}_{t}$, and $\operatorname{Var}\left(a_{j k}\right)=0.05\left(\left\{a_{j k}\right\}_{\ell}\right)^{2}$ for $\rho=0.5$ and $\eta=1.5$. 
The variance of the lognormal distribution, hence, is smaller than that of the uniform distribution in this analysis.

\subsubsection{Generation of Gamma Distribution Input Data}

In the above two sections, we have mathematically derived the expected value and variance of $a_{j k}$, such that $a_{j k}$ will fall in the interval of $I=\left[\rho\left\{a_{j k}\right\}_{t}, \eta\left\{a_{j k}\right\}_{t}\right]$ $100 \%$ and $95 \%$ for uniform and lognormal distributions, respectively. For gamma distribution, the situation is more complex. It is very difficult if not impossible to have an analytical form to express the conditions that the generated $a_{j k}$ fall in the interval $I$. Instead, we offer an explanation originally provided by Zahedi [66] as a justification for the simulation. As we know, the standard gamma distribution with mean equal to 1 becomes an exponential distribution, and the generation of the gamma distribution has been carried out by directly generating $a_{j k}$ in the standard gamma distribution:

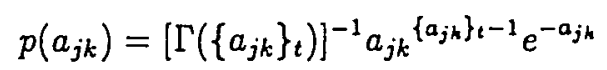

The expected value and variance are equal to the true pairwise comparison value, i.e. $E\left(a_{j k}\right)=\left\{a_{j k}\right\}_{t}$ and $\operatorname{Var}\left(a_{j k}\right)=\left\{a_{j k}\right\}_{t}$.

Due to the equality of mean and variance in the gamma distribution, it is impossible to develop a process similar to that of the lognormal distribution to establish the compatibility of the confidence intervals. As pointed out in [66], "the Chi-square distribution (which is a special form of gamma distribution with a variance twice as large as the standard gamma), shows that the interval $I$ (with $\rho=0.5$ and $\eta=1.5$ ) 
contains more than $80 \%$ of observations in all cases of $a_{j k} \geq 0$. For the standard gamma with half the variance of Chi-square, this percentage should be higher, and thus closer to $95 \%$. The variance of the gamma distribution is higher than that of the lognormal and uniform for all $a_{j k} \leq 12$ and $a_{j k} \leq 12$, respectively."

\subsection{Performance Measurements}

In the previous section, the generation of input data by using the perturbation method has been discussed in detail, which is one of the most important components of simulation. In this section, another important component of simulation, the performance measurements as indicated in section 4.1 .2 , will be discussed. Two measurements will be used to evaluate the performance of aggregation methods. Those measurements deal with two significant aspects of the aggregated group judgment and have been discussed in [1]. The first measurement is the accuracy measurement or discrepancy between the actual (true) and aggregated group judgment value. The second measurement is used to measure the satisfaction of group members with regard to the aggregated group judgment. These two measurements will be discussed in detail in the following sections.

\subsubsection{The Accuracy Measurements $\left(d_{1}\right)$}

Of considerable interest to us is the issue of how closely the group priority vector developed by aggregation methods matches the "true" priority vector. In this simulation study, the "true" priority vector is known due to our simulation design 
discussed in section 4.2.2. Therefore, to test for accuracy we must compare the aggregated group results in simulations with real answers that are known. In general, two statistical forms can be used for validating theoretical results against reality, i.e. root mean square deviation (RMS) and the median absolute deviation about the median (MAD). In this dissertation, RMS is used. This definition of accuracy, which stands for the discrepancy between the "true" priority vector and the aggregated priority vector is attractive for several reasons. First, RMS type measures are found in numerous statistical problems for which a usual objective is the minimization of RMS error. Second, RMS measures have already been adopted for use in measuring the accuracy $[3,1]$. Third, the results are easy to interpret.

To measure RMS discrepancy, we proceed as follows. For any give $\mathrm{t}(t=$ $1, \cdots, T)$, we have two vectors: $V_{t}=\left(v_{1 t}, v_{2 t}, \cdots, v_{n t}\right)$ is the "true" priority vector and $\bar{V}_{t}=\left(\bar{v}_{1 t}, \bar{v}_{2 t}, \cdots, \bar{V}_{n t}\right)$ is the aggregated priority vector. The RMS discrepancy for each $t$ is:

$$
\left\{d_{1}\right\}_{t}=\sqrt{\frac{1}{n} \sum_{j=1}^{n}\left[\bar{v}_{j t}-v_{j t}\right]^{2}}
$$

Where $\mathrm{n}$ is the number of decision elements in the simulation, $d_{1}$ stands for RMS discrepancy. We should notice that in the simulation, there should be many groups of pairwise comparison matrices generated in order to get statistical significance. Suppose there are $\mathrm{T}$ simulation runs, $\mathrm{T}$ is the number of groups as illustrated in section 4.1.2. Given the number of simulation runs, the mean $(E)$ and the standard deviation $(S)$ of $\left\{d_{1}\right\}_{t}$ are used for collective comparisons over different aggregation 
methods. $E$ and $S$ are defined as follows:

$$
\begin{aligned}
& E\left(d_{1}\right)=\frac{1}{T} \sum_{t=1}^{T}\left\{d_{1}\right\}_{t} \\
& S\left(d_{1}\right)=\sqrt{\frac{1}{T-1} \sum_{t=1}^{T}\left(\left\{d_{1}\right\}_{t}-E\left(d_{1}\right)\right)^{2}}
\end{aligned}
$$

\subsubsection{The Disagreement Measurements $\left(d_{2}\right)$}

Another important measurement, which was discussed in [24], used in a comparison study is the disagreement measurement $\left(d_{2}\right)$, which is designed to measure the deviation among the group responses and aggregated priority vector. This measure is used to indicate the degree of alignment or correspondence of the group as a whole to the aggregated priority vector, which means the smaller the $d_{2}$ is, the greater the degree to which the group members are aligned or correspond with the aggregated priority vector. This degree of group lignment or correspondence can also be interpreted as the consensus among group members. In order to define this disagreement measurement, the RMS form of deviation is used for the disagreement measurement.

Considering there are many simulation runs, for each simulation run, $V_{i t}=$ $\left(\left\{v_{1 i}\right\}_{t},\left\{v_{2 i}\right\}_{t}, \cdots,\left\{v_{n i}\right\}_{t}\right)$ is the individual priority vector of given group $t$ with $i$ simulated members $(i=1, \cdots, m), \bar{V}_{t}=\left(\bar{v}_{1 t}, \bar{v}_{2 t}, \cdots, \bar{v}_{n t}\right)$ is the aggregated priority vector of group $t(t=1, \cdots, T)$. By using the RMS concept, we get:

$$
\left\{d_{2 i}\right\}_{t}=\sqrt{\frac{1}{n} \sum_{j=1}^{n}\left(\left\{v_{j i}\right\}_{t}-\bar{v}_{j t}\right)}
$$


where the $\left\{d_{2 j}\right\}_{t}$ is the indication of the degree of satisfaction of group member $i$ over the aggregated priority vector $\bar{V}_{t}$. We also notice that the group disagreement deals with two dimensions of data instead of one dimension as the accuracy measurement did. These two dimensions come from decision elements $\mathrm{j}(1$ to $\mathrm{n}$ ) and number of decision makers $\mathrm{i}$ ( 1 to $\mathrm{m}$ ). Therefore, the group disagreement or the RMS form of two dimensional data can be expressed as:

$$
\left\{d_{2}\right\}_{t}=\sqrt{\frac{1}{m} \sum_{i=1}^{m}\left(\left\{d_{2 i}\right\}_{t}\right)^{2}}
$$

As indicated above, there are $\mathrm{T}$ simulation runs. The mean $E$ and the standard deviation $(S)$ of disagreement measurement $\left(d_{2}\right)$ are also used for collective comparisons over different aggregation methods. $E\left(d_{2}\right)$ and $S\left(d_{2}\right)$ can be expressed as:

$$
\begin{aligned}
& E\left(d_{2}\right)=\frac{1}{T} \sum_{t=1}^{T}\left\{d_{2}\right\}_{t} \\
& E\left(d_{2}\right)=\frac{1}{T} \sum_{t=1}^{T}\left\{d_{2}\right\}_{t} \\
& S\left(d_{2}\right)=\sqrt{\frac{1}{T-1} \sum_{t=1}^{T}\left(\left\{d_{2}\right\}_{t}-E\left(d_{2}\right)\right)^{2}}
\end{aligned}
$$

\subsection{The Simulation Approach}

In the previous section, comparison study procedures in general have been presented. In this section, we will focus on illustrating the simulation approach in detail. The simulation approach uses a computer to generate a large number of groups $(T)$ of pairwise comparison matrices, each group of pairwise comparison matrices consists of $\mathrm{m}$ (number of decision makers) matrices. For the purpose of simulation 
performed in this study, $\mathrm{T}=500$ is used. As presented in section 4.1 .2 , $\mathrm{T}$ groups of pairwise comparison matrices can be denoted as $\left\{A_{i}\right\}_{t}$, where $t=1, \cdots, T$ and $i=1, \cdots, m$. For each group of pairwise comparison matrices $\left(\left\{A_{i}\right\}\right)$, it represents a group decision process with each of $\left\{A_{i}\right\}$ generated from computer. We should also notice that the significant characteristics of actual judgments is the inconsistency inherent in the pairwise comparison matrix, which is due to the fact that each individual has limitations. Those limitations may range from psychological reasons to the scales used for eliciting the judgments and rnaking consistent pairwise comparison among elements. In order to mimic actual judgments, the pairwise comparison matrix in $\left\{A_{i}\right\}_{t}$ has build-in inconsistency. The inconsistency is built by using the perturbation mechanism which has been discussed in detailed in section 4.2. In general, perturbations are realized by introducing measurement errors into each element of the pairwise comparison matrix, and the measurement errors are generated by using certain probability distributions. Each group of pairwise comparison matrix $\left\{A_{i}\right\}$ is subject to the evaluations of judgment aggregation methods, of prioritization methods, and of performance measurements. We also notice that there are $\mathrm{T}$ groups of pairwise comparison matrices involved in the simulation. The performance measure for each judgment aggregation method is in statistical form, i.e. mean and standard deviation. 


\subsubsection{Data Generation Procedures}

The simulation process starts with the generation of $\mathrm{T}$ groups of "true" values of priority vectors $V_{t}=\left(v_{1 t}, v_{2 t}, \cdots, v_{n t}\right),(t=1,2, \cdots, T)$ by a random number generator, where $\mathrm{n}$ is the number of decision elements involved and $\mathrm{T}$ is the number of groups to be simulated. $\mathrm{T}$ also represents simulation runs. Then, for each $V_{t}=$ $\left(v_{1 t}, v_{2 t}, \cdots, v_{n t}\right)$ a consistent pairwise comparison matrices $A_{t},(t=1$ to $T)$, is generated. $A_{t}$ are computed by using $\left\{a_{j k}\right\}_{t}=v_{j t} / v_{k t}$. Each matrix $A_{t},(t=$ $1,2, \cdots, T)$ forms the input to generate $m$ pairwise comparison matrices in a given group $\left\{A_{i}\right\}_{t},(i=1,2, \cdots, m)$, where $\mathrm{m}$ is the supposed number of decision makers in the simulated group. Consequently, $A_{i}$ is the ratio judgment of decision maker i. $A_{i}$ is generâtúc by adding measurement errors to matrix $A_{t}$ according to one of the proposed probability distributions as indicated in section 4.2.3. For each group of $\left\{A_{i}\right\}_{t},(i=1,2, \cdots, m)$, all combinations of aggregation methods with prioritization methods are applied to produce the aggregated group priority vector $\bar{V}_{t}=\left(\bar{v}_{1 t}, \bar{v}_{2 t}, \cdots, \bar{v}_{n t}\right)$.

The aggregated value of group priority vectors $\bar{V}_{t}=\left(\bar{v}_{1 t}, \bar{v}_{2 t}, \cdots, \bar{v}_{n t}\right)$ are subjected to evaluations of performance measurements, which have been discussed in section 4.3. The output of the simulation study consists of two sets of statistics (mean and variance) with respect to the measurements. One measurement is for the accuracy of the aggregated group priority vector against the "true" priority vector. The second measurement is for the group disagreements among simulated group members. 


\subsubsection{Simulation Control Factors}

In summary, there are two important issues associated with the simulation addressed in this chapter. One is the factors that influence the simulation process. The other one is the evaluation of the performance of aggregation methods. It is obvious that a large number of factors are involved in the above discussions. All those factors influence the simulation processes. We call them control factors of the proposed simulation. In general, giving different values of the control factors will result in simulating different decision situations. The interpretation of simulation results are mainly dependent on the control factors. There are seven control factors in this analysis. They are :

1. number of decision elements or alternatives in a given decision problems ( $\mathrm{n}$ ),

2. number of decision makers (m),

3. judgment scales to be used,

4. number of simulation runs $(\mathrm{T})$,

5. prioritization methods which are used to derive the priority vector,

6. probability distribution of the error terms, and

7. performance measurements.

All control factors, except the probability distribution of the error term, are explicit and easy to understand. Probability distribution, which is one of the most 
important parts of this simulation, has been discussed in detail in section 4.2.3. Another important component of this simulation is the performance measurement, which also has been discussed in detail in section 4.3. The detailed steps for the simulation are presented in the following section.

\subsubsection{Simulation Procedures}

Based on above discussions, the procedures, which are used to conduct the simulation comparison study for any given judgment aggregation method, can be summarized in the following steps. These procedures are repeated for each aggregation method.

Step 1: Generate T (number of simulation runs) groups of "true" priority vectors $V_{t}=\left(v_{1 t}, v_{2 t}, \cdots, v_{n t}\right),(t=1,2, \cdot, T)$. Each of those has n elements, which represent the simulated decision elements.

Step 2: For any given $t$, which is from 1 to $\mathrm{T}$, the priority vector $V_{t}=\left(v_{1 t}, v_{2 t}, \cdots, v_{n t}\right)$ generated from Step 1 is converted to a consistent pairwise comparison ma$\operatorname{trix} A_{t}$. The $A_{t}$ is built by using $A_{t}=\left(\left\{a_{j k}\right\}_{t}\right)=\left(v_{j t} / v_{k t}\right)$.

Step 3: For any given $t$, the consistent pairwise comparison matrix $A_{t}$, which is created in Step 2, is used to generate a group of pairwise comparison matrices $\left\{A_{i}\right\}_{t}$, where $i=1, \cdots, m, \mathrm{~m}$ is the number of simulated decision makers. $\left\{A_{i}\right\}_{t}$ are generated from $A_{t}$ by using perturbation methods described in section 4.2.3. There are three distributions to be considered, uniform, 
lognormal and gamma. Further, there are two possible approaches for judgment aggregation as described in Step 4a and Step 4b.

Step 4a: Approach A: judgment aggregation method is operated on the pairwise comparison matrices. In this step, the generated group pairwise comparison matrices $\left\{A_{i}\right\}_{t}$ are aggregated by using one of the aggregation methods. The aggregated group pairwise comparison matrix $\bar{A}_{t}$ (for any given $\mathrm{t}$ ) is then used to derive the group priority vector $\bar{V}_{t}$ (for any given $t$ ). The group priority vector $\bar{V}_{t}$ is derived by employing one of fifteen prioritization methods listed in Table. 4.2.

Step 4b: Approach B: judgment aggregation method is operated on priority vector of each simulated group member. In this approach, priority vectors $\left\{V_{i}\right\}_{t}(i=$ $1, \cdots, m)$ for simulated group members are derived from the corresponding pairwise comparison matrix $\left\{A_{i}\right\}_{t} .\left\{V_{i}\right\}_{t}$ are obtained from one of fifteen prioritization methods listed in Table 4.2. The group priority vector is obtained from aggregating $\left\{V_{i}\right\}_{t}$ by using a given aggregation method.

Step 5: For each aggregated group priority vector from Step 4a or $4 b$, the performance measurements, i.e. the accuracy measurement and group disagreement measurement, are calculated and those results are saved.

Step 6: Go to Step 4a or $4 \mathrm{~b}$ for another combination of the prioritization method and aggregation method. This process is repeated until all combinations are exhausted. Then go to Step 7 . 
Step 7: Go to Step 3 for another probability distribution for error terms until all proposed probability distributions are exhausted. Then go to Step 8 .

Step 8: Go to Step 2 for another "true" priority vector until all (total of $\mathrm{T}$ ) the "true" priority vectors created in Step 1 are exhausted. Then go to Step 10.

Step 9: For each combination of the probability distribution and prioritization method, the mean and standard deviation of the performance measurements over $\mathrm{T}$ simulation runs are calculated for analysis.

Step 10: For a given judgment aggregation method, stop. Or, go to Step 1 for another judgment aggregation method. The process is repeated until all judgment aggregation methods listed in Table. 4.1 are finished.

A detailed flow chart of these steps, which also reflects the computer implementations of the simulation, is presented in Fig. 4.2 and 4.3. The difference of these two figures is that Fig. 4.2 is for the judgment aggregation methods operated on pairwise comparison matrices. Fig. 4.3 is for the judgment aggregation methods operated on the priority vectors. 


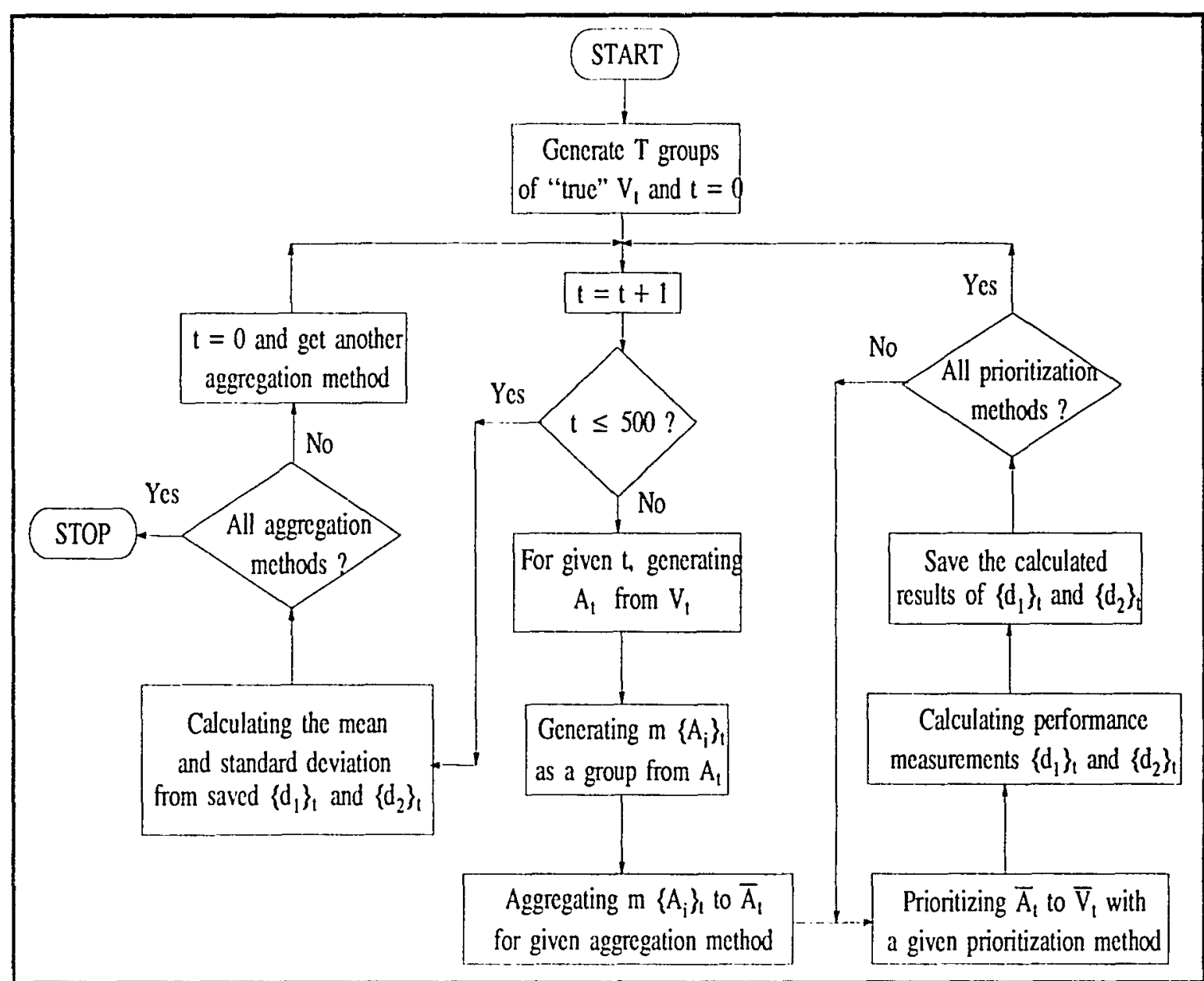




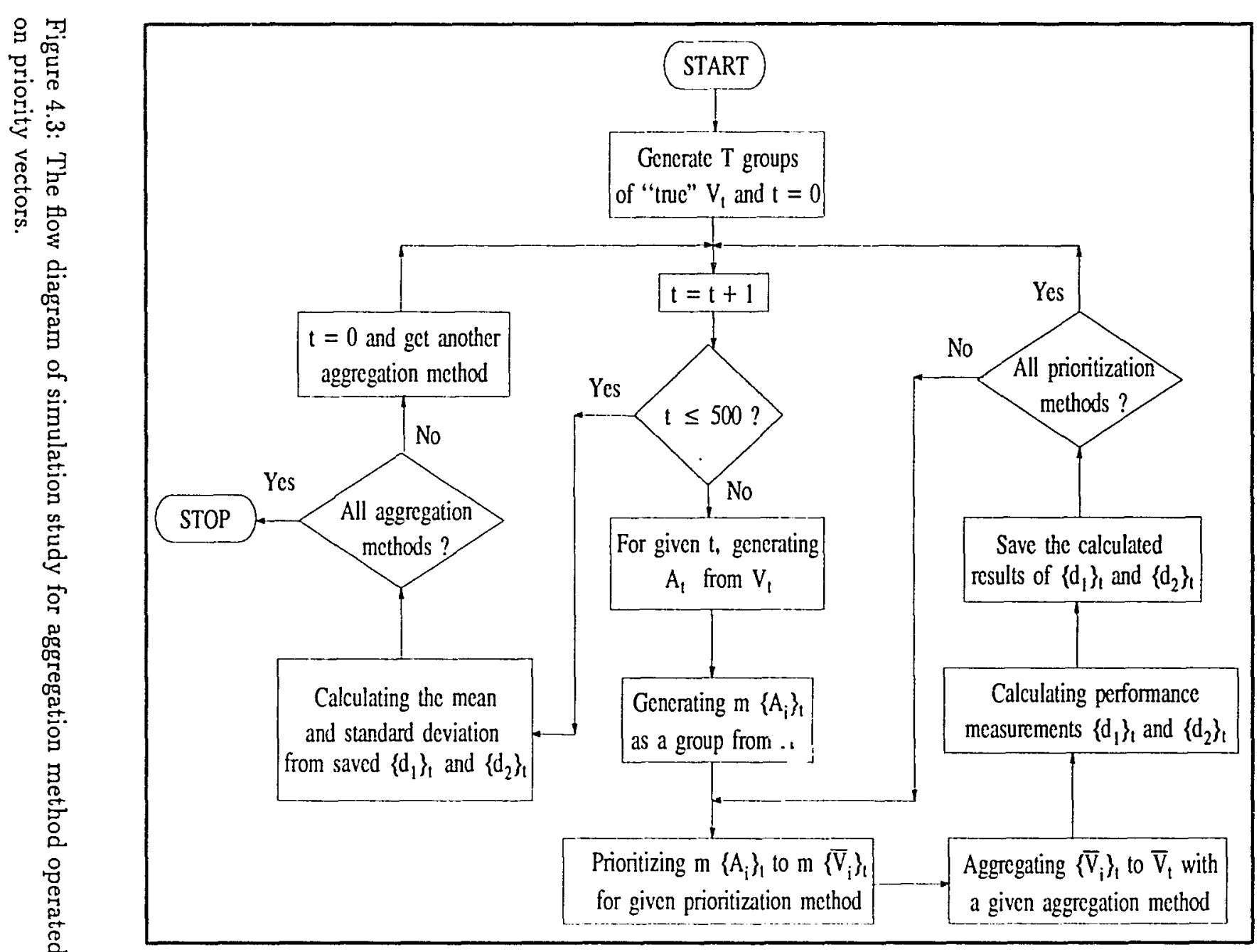




\subsection{The Empirical Approach}

Simulation study is a fast way to reproduce or partially produce the real situation of pairwise comparison judgment. Significant differences may exist between the simulated judgments and real judgments made by real people. The reasons, which may respond to this discrepancy, are due to the limited probability distribution involved in the perturbation mechanism of a simulation and the uncertainty involved in the underlying distribution of the real judgments made by individuals. Consequently, it is desirable to test the aggregation methods by using the actual data from individuals' judgments. In addition to the above discussed simulation, a set of empirical judgment data have also been used to test the aggregation methods. Those data were collected from an IE-204 course at the University of Pittsburgh in 1984, and were also used in [55] to test the appropriateness of prioritization methods. A total of 39 graduate students were asked to estimate values of various elements in seven categories, as summarized in Tables 4.5 and 4.5. All the categories had six judgment elements. Objective values were known but not given to the students, and those values are also presented in Table 4.5 and 4.5. In Appendix D, the data collected for the seven categories are listed.

The procedures to test those seven categories are presented in the following steps, which is similar to the one we have presented for the simulation. There are some differences; the input data is not generated by computer and it is real judgment data. There is only one group for each category; therefore, the performance measurements are not in statistical form. The objective is to calculate the measurements for all 
combinations of aggregation methods and prioritization methods. Then the results are compared with simulation results and among themselves. The procedures to conduct the above mentioned test are summarized as follows:

Step 1: Get a group of actual pairwise comparison matrices from disk $\left\{A_{i}\right\}_{C}$, which is also corresponding to a category, where $i=1, \cdots, m, \mathrm{~m}$ is the number of students in a given category, $\mathrm{C}$ is the number of categories in the empirical test $(C=1, \cdots, 7)$. As we pointed out in the simulation procedure, there are two possible approaches for judgment aggregation indicated in Step 2a or Step $2 b$.

Step 2a: Approach A: judgment aggregation method is operated on the pairwise comparison matrices. In this step, the group pairwise comparison matrices $\left\{A_{i}\right\}_{C}$ are aggregated by using one of aggregation methods. The aggregated group pairwise comparison matrix $\bar{A}_{C}$ (for any given $\mathrm{C}$ ) is then used to derive the group priority vector $\bar{V}_{C}$ (for any given $\mathrm{C}$ ). The group priority vector $\bar{V}_{C}$ is derived by employing one of fifteen prioritization methods listed in Table 4.2.

Step 2b: Approach B: judgment aggregation method is operated on priority vector of each simulated group member. In this approach, priority vectors $\left\{V_{i}\right\}_{C}(i=$ $1, \cdots, m)$ for simulated group members is derived from the corresponding pairwise comparison matrix $\left\{A_{i}\right\}_{C} \cdot\left\{V_{i}\right\}_{C}$ are obtained from one of fifteen prioritization methods listed in Table. 4.2. The group priority vector is obtained from aggregating $\left\{V_{i}\right\}_{C}$ by using a given aggregation method. 
Step 3: For each aggregated group priority vector from Step $2 a$ or $2 b$, the performance measurements, i.e. the accuracy measurement and group disagreement measurement, are calculated.

Step 4: Go to Step 4a or $4 \mathrm{~b}$ for another combination of the prioritization method and aggregation method. This process is repeated until all combinations are exhausted. Then go to Step 5 .

Step 5: For a given judgment aggregation method, stop. Or, go to Step 1 for another judgment aggregation method. The process is repeated until all judgment aggregation methods listed in Table 4.1 are finished.

Modified flow charts of Fig. 4.2 and 4.3 are presented in Fig. 4.4 and 4.5 to represent the above described steps. Those two figures also reflect the computer implementations of empirical tests. The differences of those two figures are that Fig. 4.4 is for the process of judgment aggregation methods operated on the pairwise comparison matrices. Fig. 4.5 is for the case of judgment aggregation methods operated on the priority vectors. 


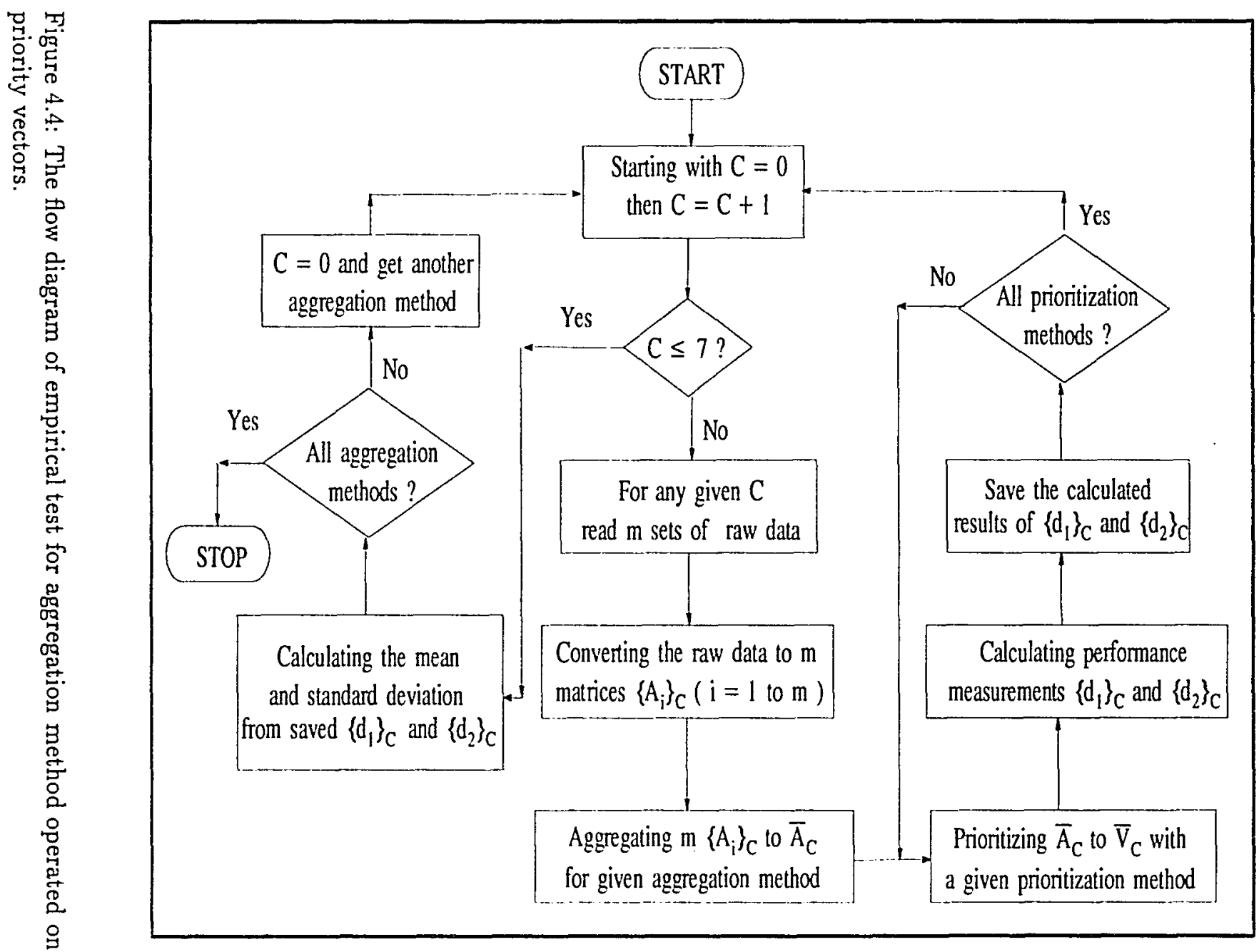




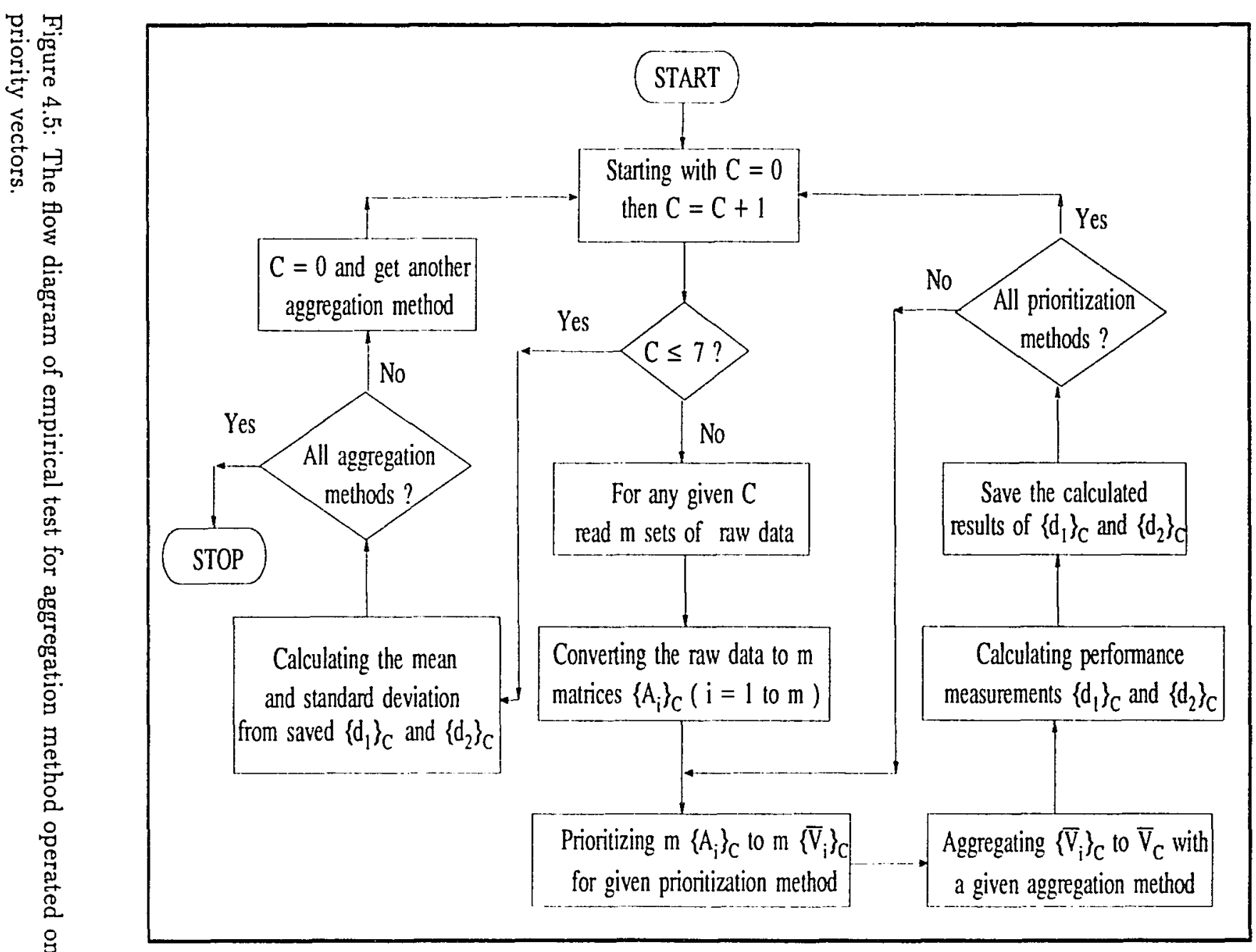


Table 4.3: The Estimation Categories

\begin{tabular}{||c|l|l||}
\hline Category 1 & Lengths of Straight Line (cm) & Actual \\
\hline \hline 1 & & $8(0.18)$ \\
\hline 2 & & $5(0.11)$ \\
\hline 3 & - & $7(0.16)$ \\
\hline 4 & & $9(0.20)$ \\
\hline 5 & & $6(0.13)$ \\
\hline 6 & & $10(0.22)$ \\
\hline \hline Category 2 & Air Distance (miles) & Actual \\
\hline \hline 1 & Between Pittsburgh and Cleveland & $115(0.04)$ \\
\hline 2 & Between Pittsburgh and Detroit & $205(0.07)$ \\
\hline 3 & Between Pittsburgh and Indianapolis & $330(0.11)$ \\
\hline 4 & Between Pittsburgh and Miami & $1010(0.35)$ \\
\hline 5 & Between Pittsburgh and New Orleans & $910(0.32)$ \\
\hline 6 & Between Pittsburgh and New York & $317(0.11)$ \\
\hline \hline Category 3 & Number of Super Bowls Won & Actual \\
\hline \hline 1 & Pittsburgh Steelers & $4(0.29)$ \\
\hline 2 & Dallas Cowboys & $2(0.14)$ \\
\hline 3 & Washington Redskins & $1(0.07)$ \\
\hline 4 & Green Bay Packers & $2(0.14)$ \\
\hline 5 & Oakland Raiders & $3(0.22)$ \\
\hline 6 & Maimi Dolphins & $2(0.14)$ \\
\hline \hline Category 4 & Metropolitan & Actual \\
\hline \hline 1 & Boston & $2,763,357(0.10)$ \\
\hline 2 & Chicago & $7,103,328(0.26)$ \\
\hline 3 & Houston & $2,905,350(0.11)$ \\
\hline 4 & New York & $9,119,737(0.33)$ \\
\hline 5 & Pittsburgh & $2,263,894(0.08)$ \\
\hline 6 & San Francisco & $3,252,751(0.12)$ \\
\hline \hline & continued on next page & \\
\hline \hline & & \\
\hline \hline & & \\
\hline \hline
\end{tabular}


Table 4.4: The Estimation Categories (Continued)

\begin{tabular}{||c|l|l||}
\hline Category 5 & Annual Number of Passengers in Airports & Actual \\
\hline \hline 1 & Atlanta & $37,594,073(0.22)$ \\
\hline 2 & Chicago & $37,992,151(0.22)$ \\
\hline 3 & Dallas/Fort Worth & $25,533,929(0.15)$ \\
\hline 4 & Los Angeles & $32,722,534(0.20)$ \\
\hline 5 & New York JFK & $25,752,719(0.15)$ \\
\hline 6 & Pittsburgh & $10,112,266(0.06)$ \\
\hline \hline Category 6 & Professional in Major Occupations & Actual \\
\hline \hline 1 & Accountants & $1,126,000(0.26)$ \\
\hline 2 & Computer Programmers & $367,000(0.08)$ \\
\hline 3 & Engineers & $1,537,000(0.35)$ \\
\hline 4 & Lawyers and Judges & $581,000(0.13)$ \\
\hline 5 & Life and Physical Scientist & $311,000(0.07)$ \\
\hline 6 & Physicians & $454,000(0.10)$ \\
\hline \hline Category 7 & Country Populations (in Millions) & Actual \\
\hline \hline 1 & Brazil & $124.5(0.05)$ \\
\hline 2 & India & $700.0(0.29)$ \\
\hline 3 & Japan & $118.5(0.05)$ \\
\hline 4 & People's Republic of China & $1020.0(0.41)$ \\
\hline 5 & Unites States & $232.0(0.09)$ \\
\hline 6 & USSR & $269.9(0.11)$ \\
\hline
\end{tabular}




\section{Chapter 5}

\section{SIMULATION RESULTS AND DISCUSSIONS}

In chapter 4, several issues regarding procedures for comparison study and simulations are discussed; among those discussions are:

- Why and how the simulation for aggregation methods were carried out.

- Perturbation method for generating the pairwise comparison matrices for simulation were illustrated.

- Performance measurements for comparing the test results were presented.

We will carry further the study of aggregation methods by looking at simulation and empirical testing results. As we pointed out in the beginning of chapter 4, the purpose of the simulation study is to examine the characteristics of aggregation methods for group decision making and to provide the guidelines for users to apply these methods. In group decision situations, there are several issues we are concerned about such as how many decision makers should be in the decision making group, who should be in the decision making group, and how complex is the decision making task.

Those who are chosen to be in the decision making group determine the judg- 
ments (i.e. the input data to the aggregation process), because the judgments are the reflections of decision makers' knowledge and information. How many decision makers should be in the decision making group usually is determined by the complexity of the decision making task. One measure of complexity is the number of decision elements. In general, the more the decision elements are, the more decision makers are needed because the capability to handle the decision making elements are limited for each individual decision maker. However, the capability limitation for each individual decision maker is not modeled in our simulation process presented in the Chapter 4. On one hand, this capability limitation is not a major issue from the aggregation point of view. On the other hand, modeling the capability limitation process is a very complex task and psychological in nature, which is out the context of this dissertation. Therefore, results are interpreted by the following categories:

1. aggregation methods vs. input data type

2. aggregation methods vs. prioritization methods

3. aggregation methods vs. number of decision makers

\subsection{Simulation Set Up}

The proposed simulation study and empirical test, which have been discussed in Chapter 4, have been carried out by using two kinds of software: the IMSL MATH/LIBRARY and LINDO. The IMSL MATH/LIBRARY is a collection of FORTRAN subroutines and functions useful in research and mathematical analy- 
sis, which includes subroutines as linear systems, eigensystem analysis, optimization and so on. To use any of these routines, a program in FORTRAN must be written to call the IMSL MATH/LIBRARY routine. Two routines were called in our study. One is EVCRG, which is used to calculate the maximum eigenvalue and its corresponding eigenvector. Another is BCLSF, which is used to solve a nonlinear least squares problem in our study. LINDO is an optimization modeling system to deal with linear, nonlinear and integer programming. This program is used to solve the goal programming which we proposed in Chapter 3 for MDM aggregation methods. All the simulation and calculations are conducted on the IBM mainframe 4381.

The simulation results are summarized into two groups according to the performance measurements, which are accuracy and disagreement. The simulation results for accuracy measurement are presented in Appendix B. Appendix C contains the simulation results for group disagreement measurement. All the notations in Appendix $B$, Appendix $C$ and in this chapter are consistent with the notation definition of Table 4.1 and Table 4.2. Table 4.1 defines the abbreviations for aggregation methods, which covers the geometric mean operated on the final priority vector, geometric mean method operated on pairwise comparison matrix, arithmetic mean operated on final priority vector, minimum distance method operated on the final vector and minimum distance method operated on the pairwise comparison matrix. Table 4.2 covers all the notations for prioritization methods. The other notations used in Appendix B, C and in this chapter but not included in Table 4.1 and 4.2 are summarized in the following: UF stands for uniform probability distribution in perturbation process, LN represents lognormal probability distribution, and GA 
stands for gamma probability distribution in the perturbation process.

In simulation, only the selected value of control factors $n$ and $m$ are simulated. This will not lose the generality of this study because for the other values of $n$ and $m$, the behaviors are the same as those which have been simulated. As we also pointed out in Chapter 4, the simulation is very complex due to involving not only aggregation methods but also the prioritization methods. Five aggregation methods (considering data types on which the aggregation methods operated) have been studied. For each aggregation method, there are fifteen prioritization methods to combine with. This significantly increases the time to run the simulation. Due to limited computer resources, only certain $n$ and $m$ are simulated. The size $(n)$ of the input matrices of pairwise comparisons (also referred to as the number of decision elements) in the simulation study is set to be 8,10 and 12 instead of 3 to 15 . The number of decision makers simulated is $3,5,7$ and 9 . The number of simulation runs $(T)$, which is for each combination the aggregation method, prioritization method, number of decision elements, and number of decision makers, is 500 . The judgment scale is $[1 / 9,9]$. We should also notice that the input matrices are formed once in the "symmetric" fashion in which the elements of the upper triangle of a matrix are generated by using $a_{j k}=\left(v_{j} / v_{k}\right) e_{j k}$ and the elements of the lower triangle are computed from $a_{k j}=1 / a_{j k}$. The discussion of the simulation results are presented in the following sections. 


\subsection{Aggregation Methods vs. Type of Input Data}

The relationship between aggregation methods and the type of input data is one of the major concerns of this simulation study. The input data here refers to the judgments made by decision makers, or in our simulation situation the generated pairwise comparison matrices. Three sets of input data have been studied, based on the perturbation distribution utilized uniform, lognormal and garnma. As discussed in Chapter 4. The implication is that when using a uniform perturbation, for example, the probability for decision makers to have the same judgment is equal, which also means that the decision makers have the same information and knowledge about the decision to be made. In this section, the influence of distributions, i.e. the input data type, on group decision making in terms of accuracy and group disagreement measurements are investigated.

Accuracy: The simulation results with respect to the input data type are presented in Fig. 5.1 through Fig. 5.5. In those figures, the horizontal axis represents the prioritization methods in their ID. No. as indicated in Table 4.2. Throughout the whole chapter, all the figures' horizontal axis are the same. The vertical axis represents the mean of accuracy measurement over all the simulation runs. Accuracy is the function of data type as shown in Fig. 5.1 to Fig. 5.5, which means for any given aggregation and prioritization methods, the accuracy is dependent on data type. Lognormal distribution input data type presents better accuracy than both uniform and gamma distribution, and gamma distribution yields the least accuracy. This is under the condition that the simulation data, i.e. the elements $\left(\hat{a}_{j k}\right)$ 
of pairwise comparison matrix, fall in the interval $\left[0.5 a_{j k}, 1.5 a_{j k}\right]$, where $a_{j k}$ is the given "true" value. Fig. 5.1 through Fig. 5.5 only demonstrate the case of $N=8$ and $M=3$ for all the aggregation methods. The result is the same for all other combinations of $N$ and $M$ as well (see Appendix B for detail). The patterns of Fig.5.1 through Fig. 5.5 are the same, which means that functionality of all the aggregation methods for the same data type is the same.

Group disagreement: The simulation results for group disagreement with respect to three different input data types are presented in Fig. 5.6 through Fig. 5.10. As indicated in those figures, group disagreement is also a function of data types, which means for any given aggregation and prioritization methods, the group disagreement is dependent on data type. As with accuracy measurement, lognormal distribution input data type presents better group agreement than both uniform and gamma distribution, and gamma distribution yields the worst group agreement. We should notice that the vertical axis in Fig. 5.6 through Fig. 5.10 represents the mean value of group disagreement measurement over all 500 simulation runs. The patterns demonstrated in Fig. 5.6 through Fig.5.10 can be explained by the same reason as mentioned in the accuracy section above, which is due to the variance $\left(\sigma^{2}\right)$ of input data distributions. The lognormal distribution has smaller $\sigma^{2}$, and the simulation data, i.e. aggregation results, generated by this distribution are closer to their true data; hence, the group disagreement is smaller. The results demonstrated in Fig. 5.6 through Fig. 5.10 are the case of $N=12$ and $M=7$. This result is also true for all other combinations of $N$ and $M$ as well (see Appendix $\mathrm{C}$ for detail). 
Performance within given distribution: The general relationships of aggregation methods with input data types have been presented above. As expected, the higher the variance of input data, i.e. the input pairwise comparison matrices, the worse the aggregation results. Now let us look one step further within each input data distribution at how the aggregation methods are functioning. For uniform input data type, the performance of aggregation methods is shown in Fig. 5.11 and Fig. 5.14 for accuracy and group disagreement measurement, respectively. The MDM approach operated on the final priority vectors gives inferior results compared with other aggregation methods for a given accuracy level. For lognormal input data distribution, simulation results are illustrated in Fig. 5.12 and Fig. 5.15 for accuracy and group disagreement measurement, respectively. The results are the same as the uniform input data type with the MDM operated on the final priority vectors giving the "worst" result. The rest of aggregation methods give almost identical results, and the difference among them are very small. Simulation results regarding the garnma distribution are shown in Fig. 5.13 and Fig. 5.16 for accuracy and group disagreement measurement, respectively. Contrary to the uniform and lognormal distribution cases, the MDM operated on both final priority vectors and pairwise comparison matrices gives better results for all the prioritization methods as indicated in Fig. 5.13 for accuracy measurement, while the group disagreement measurements are close to each other for all the aggregation methods. In general, if the input data have higher variance, the MDM gives better results than any other aggregation method. Otherwise, the arithmetic mean method gives slightly better results than the rest of the aggregation methods. MDM operated on the final prior- 
ity vectors does not give as accurate measurements as the other methods when the variations are low.

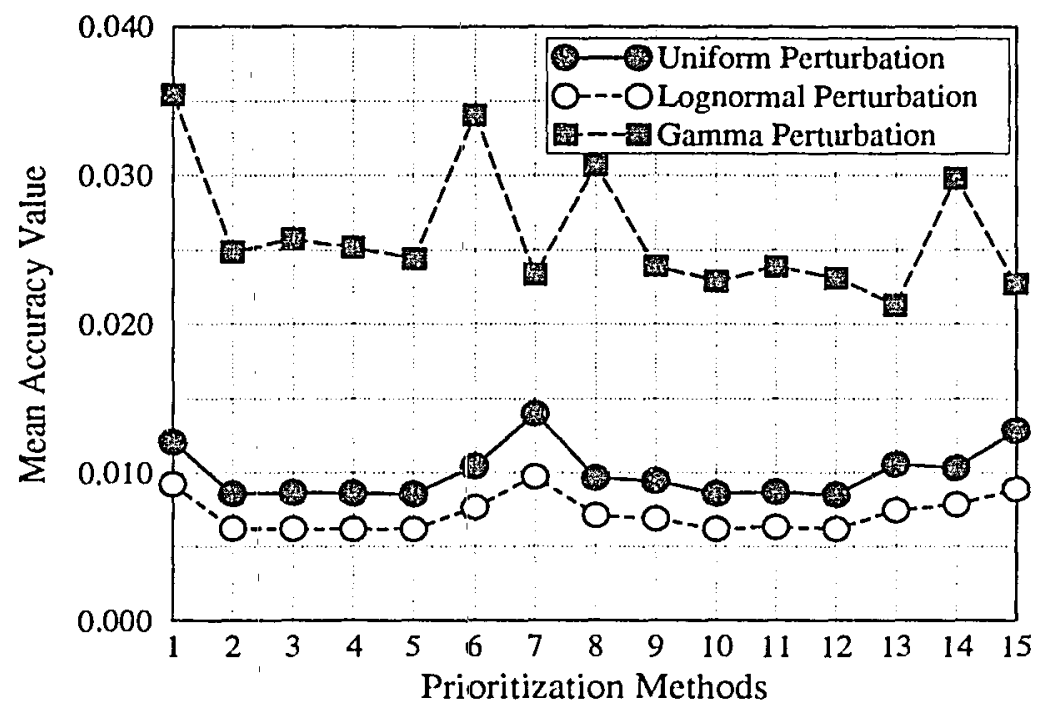

Figure 5.1: The Mean Accuracy of Geometric Mean Method Operated on Final Priority Vector $(N=8, M=3)$ 


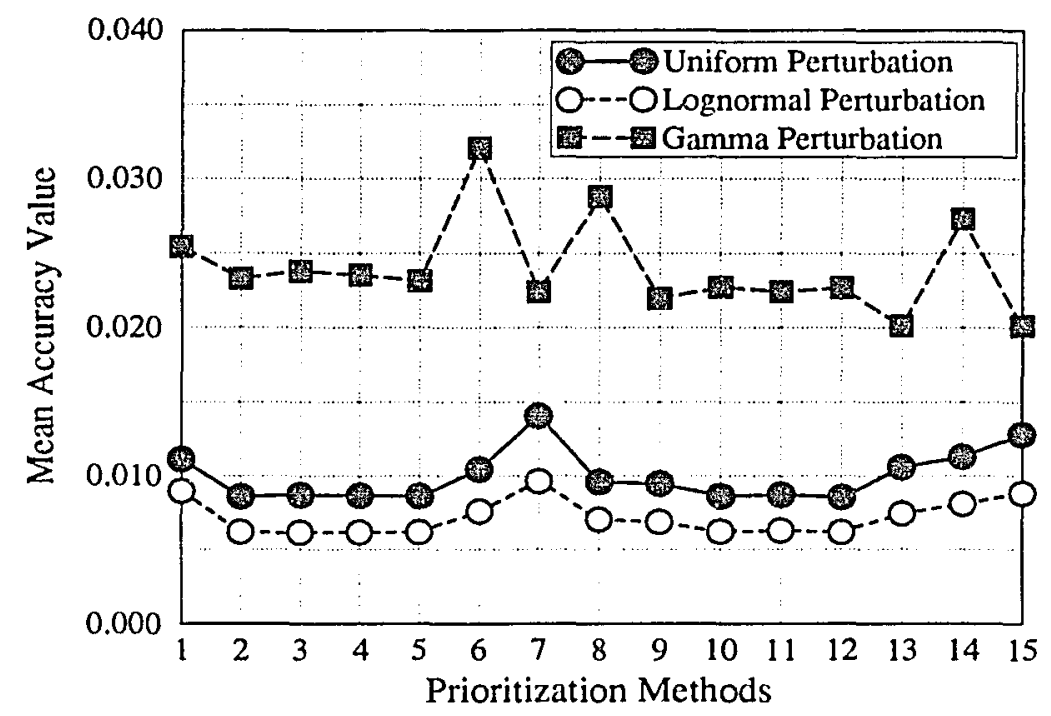

Figure 5.2: The Mean Accuracy of Geometric Mean Method Operated on Pairwise Comparison Matrix $(\mathrm{N}=8, \mathrm{M}=3$ )

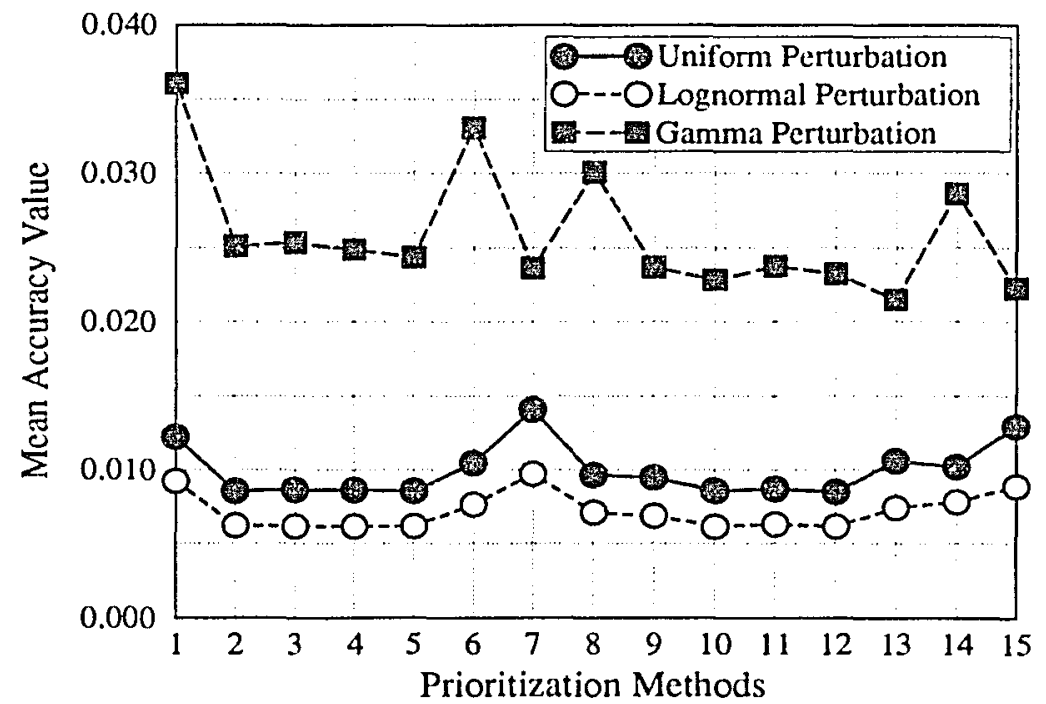

Figure 5.3: The Mean Accuracy of Arithmetic Mean Method Operated on Final Priority Vector $(N=8, M=3)$ 


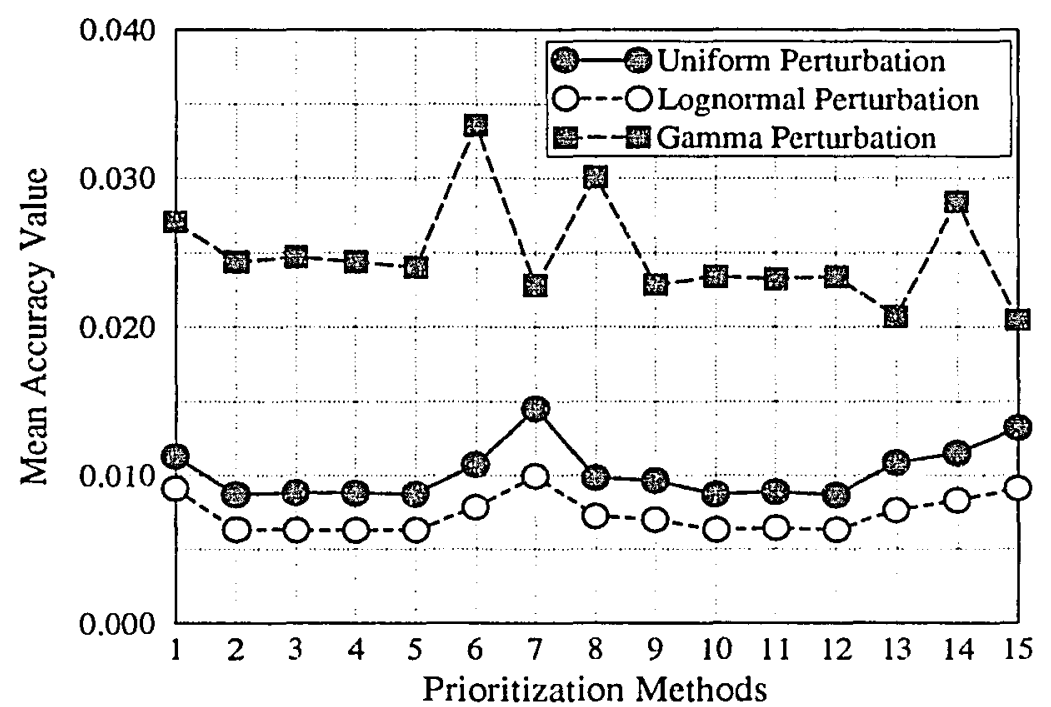

Figure 5.4: The Mean Accuracy of Minimum Distance Method Operated on Pairwise Comparison Matrix $(\mathrm{N}=8, \mathrm{M}=3)$

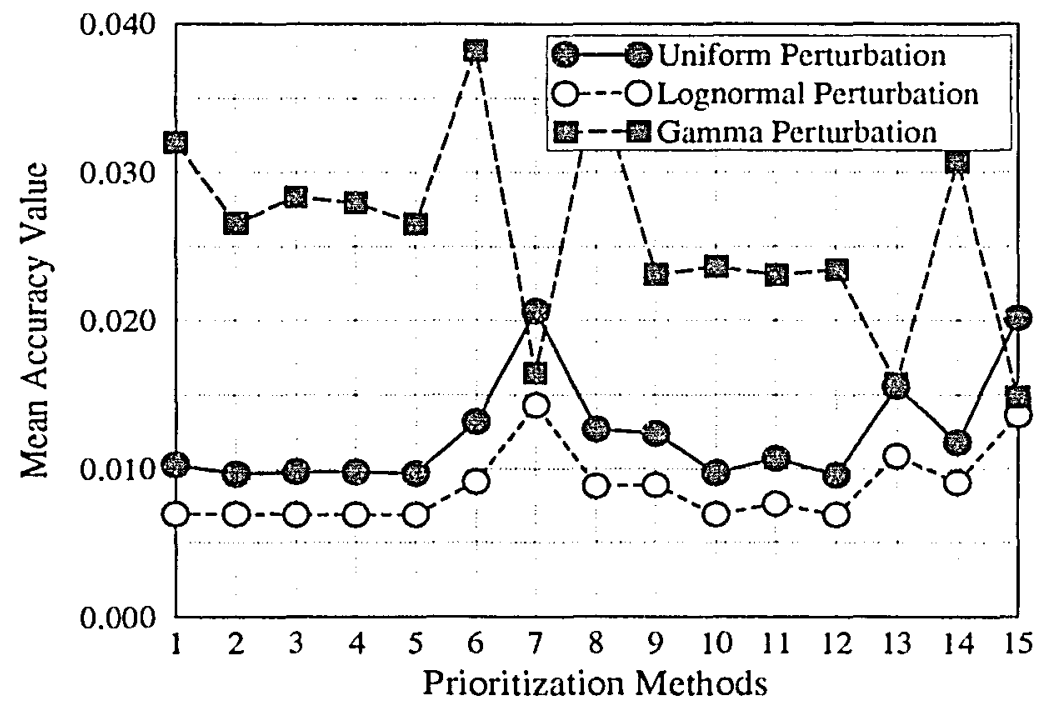

Figure 5.5: The Mean Accuracy of Minimum Distance Method Operated on Final Priority Vector $(\mathrm{N}=8, \mathrm{M}=3)$ 


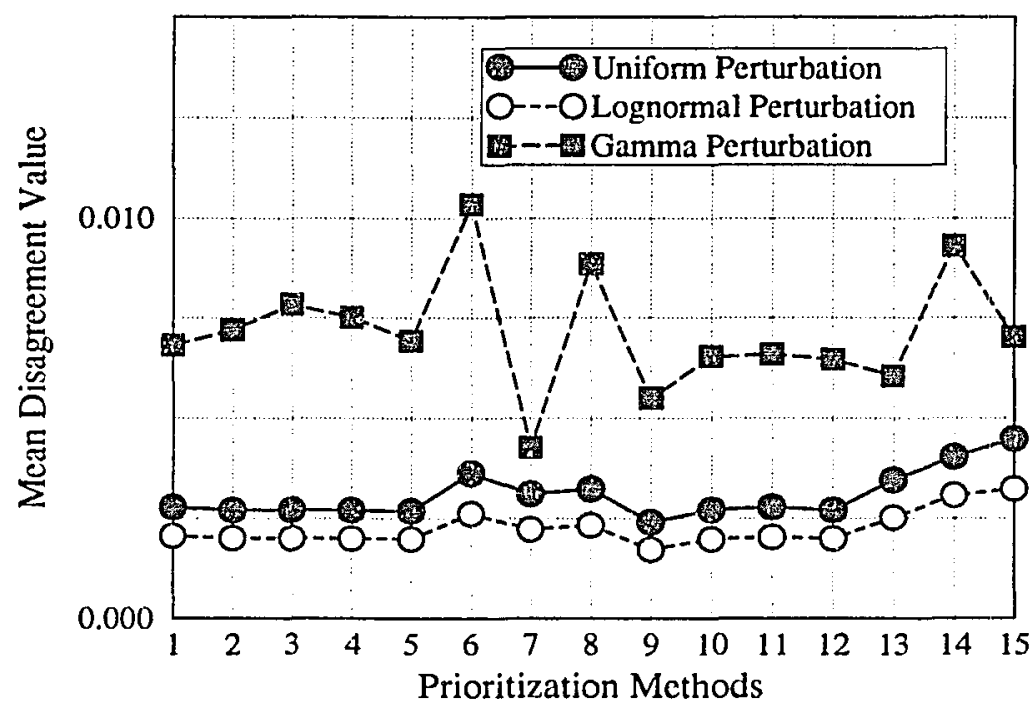

Figure 5.6: The Mean Disagreement of Geometric Mean Method Operated on Final Priority Vector $(\mathrm{N}=12, \mathrm{M}=7)$

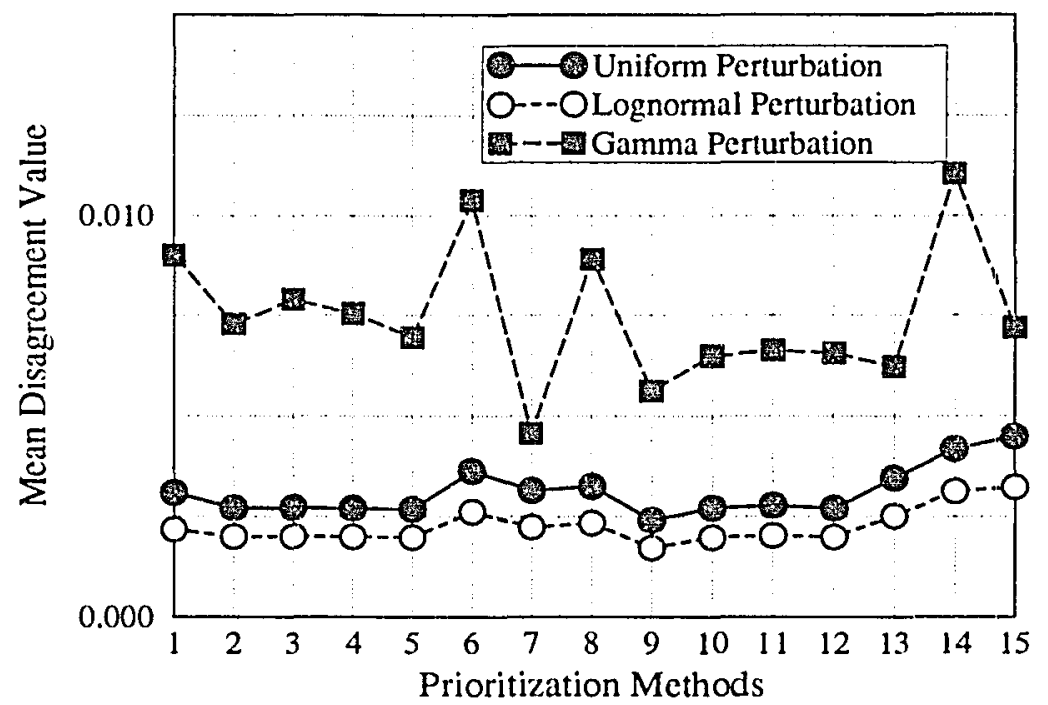

Figure 5.7: The Mean Disagreement of Geometric Mean Method Operated on Pairwise Comparison Matrix $(\mathrm{N}=12, \mathrm{M}=7)$ 


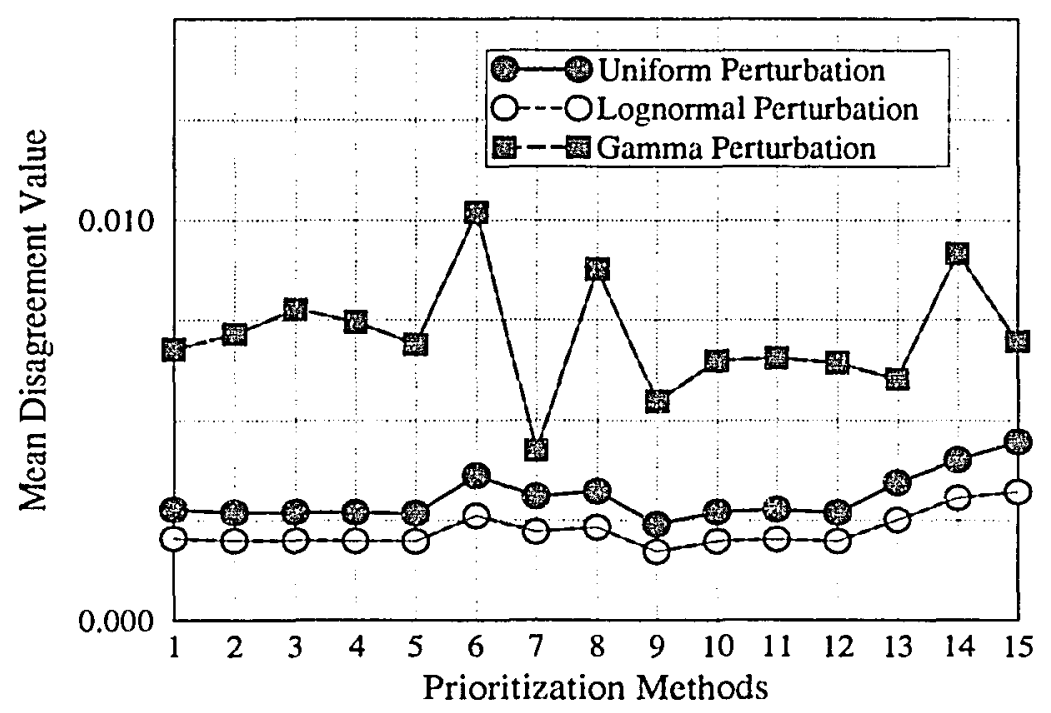

Figure 5.8: The Mean Disagreement of Arithmetic Mean Method Operated on Final Priority Vector $(N=12, M=7)$

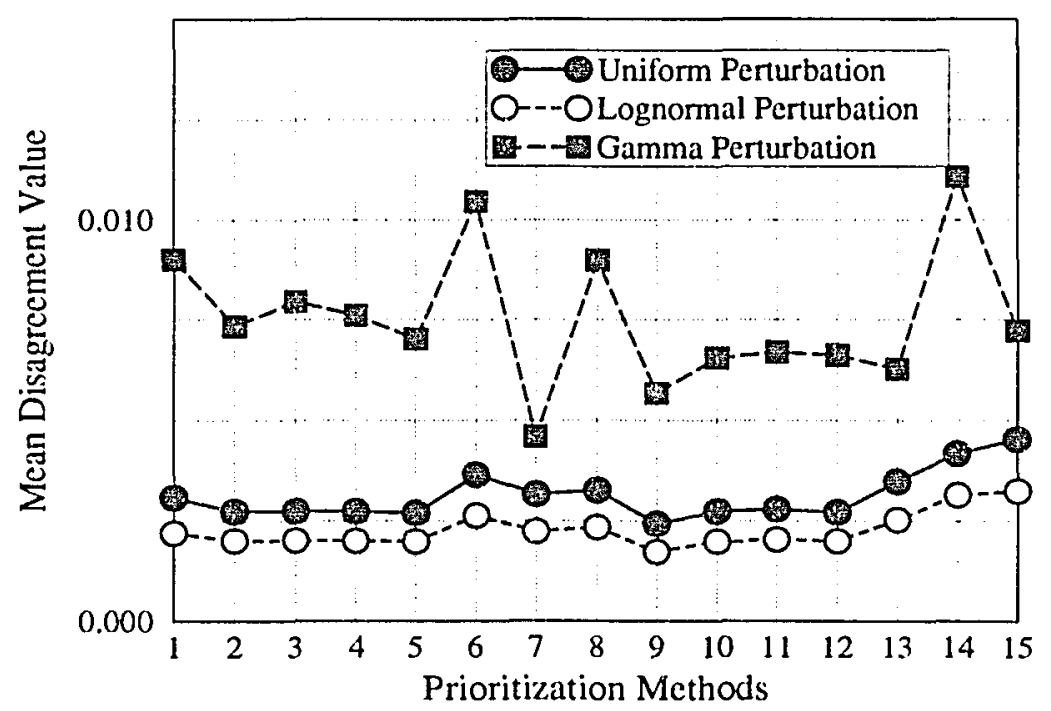

Figure 5.9: The Mean Disagreement of Minimum Distance Method Operated on Pairwise Comparison Matrix $(\mathrm{N}=12, \mathrm{M}=7)$ 


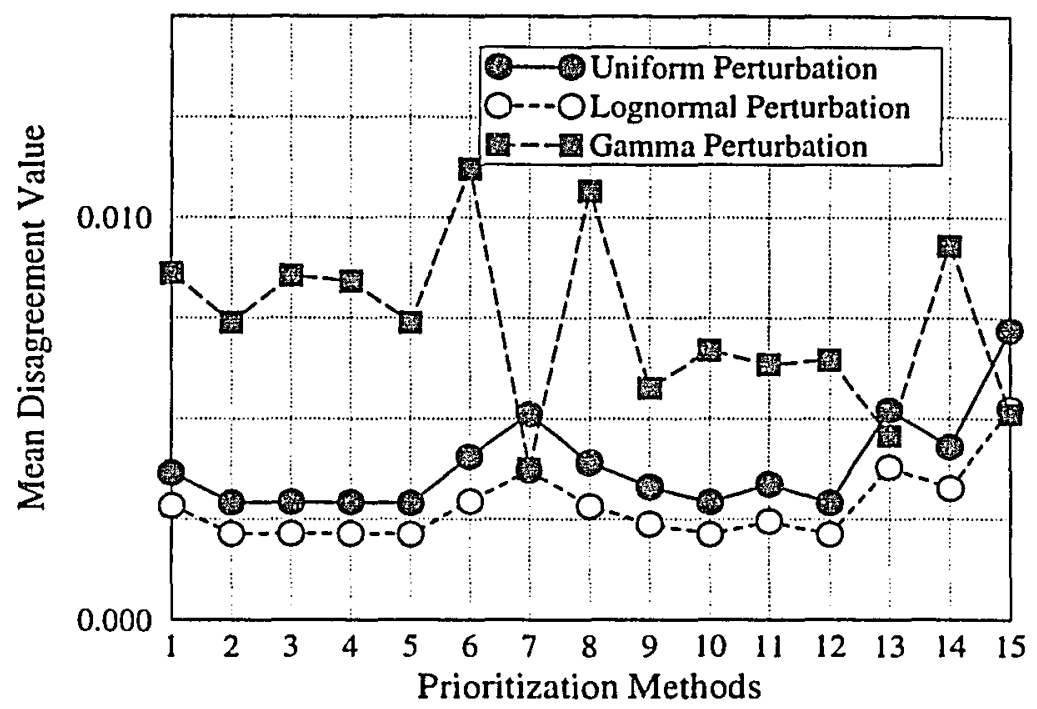

Figure 5.10: The Mean Disagreement of Minimum Distance Method Operated on Final Priority Vector $(\mathrm{N}=12, \mathrm{M}=7$ )

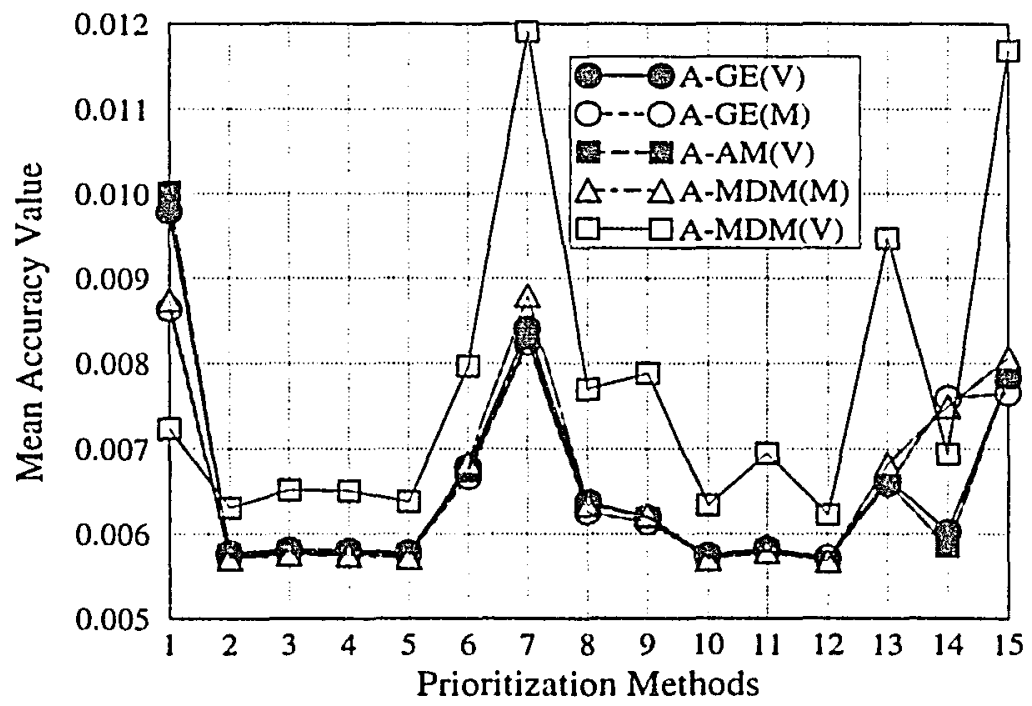

Figure 5.11: The Mean Accuracy of Uniform Distribution for All Aggregation Methods $(N=8, M=9)$ 


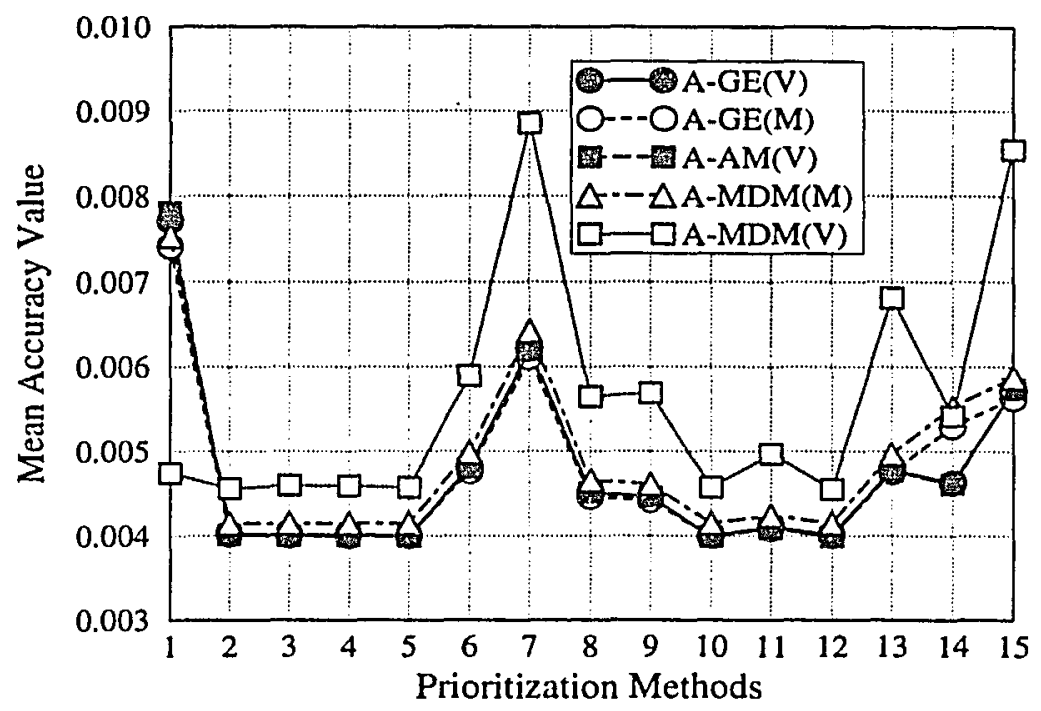

Figure 5.12: The Mean Accuracy of Lognormal Distribution for All Aggregation Methods $(\mathrm{N}=8, \mathrm{M}=9)$

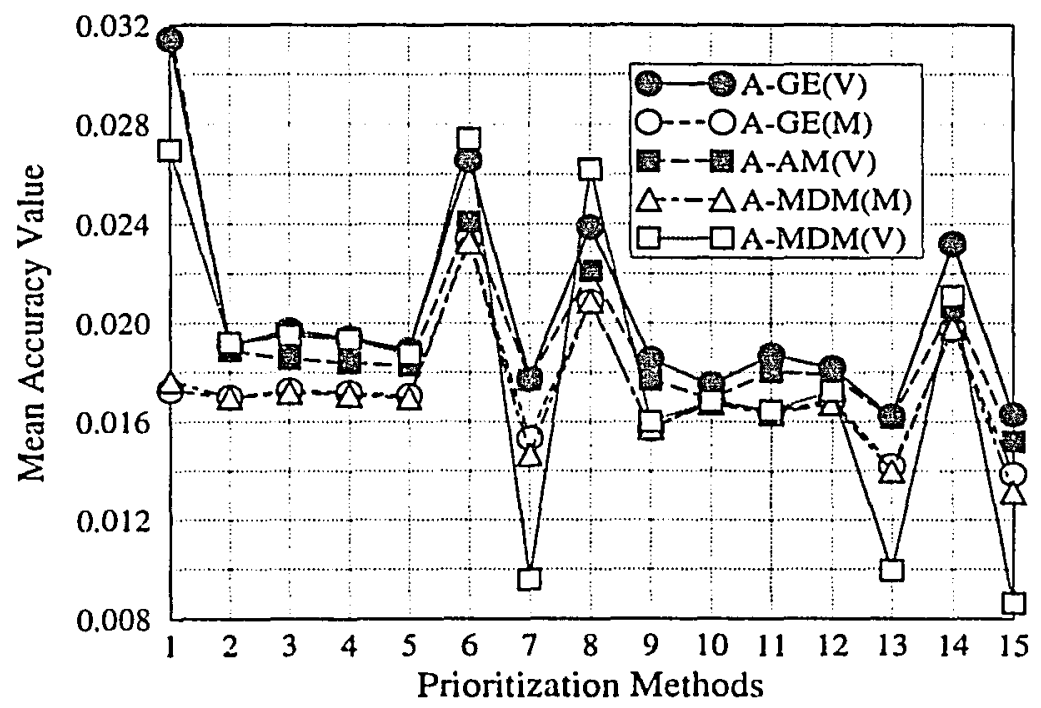

Figure 5.13: The Mean Accuracy of Gamma Distribution for All Aggregation Methods $(N=8, M=9)$ 


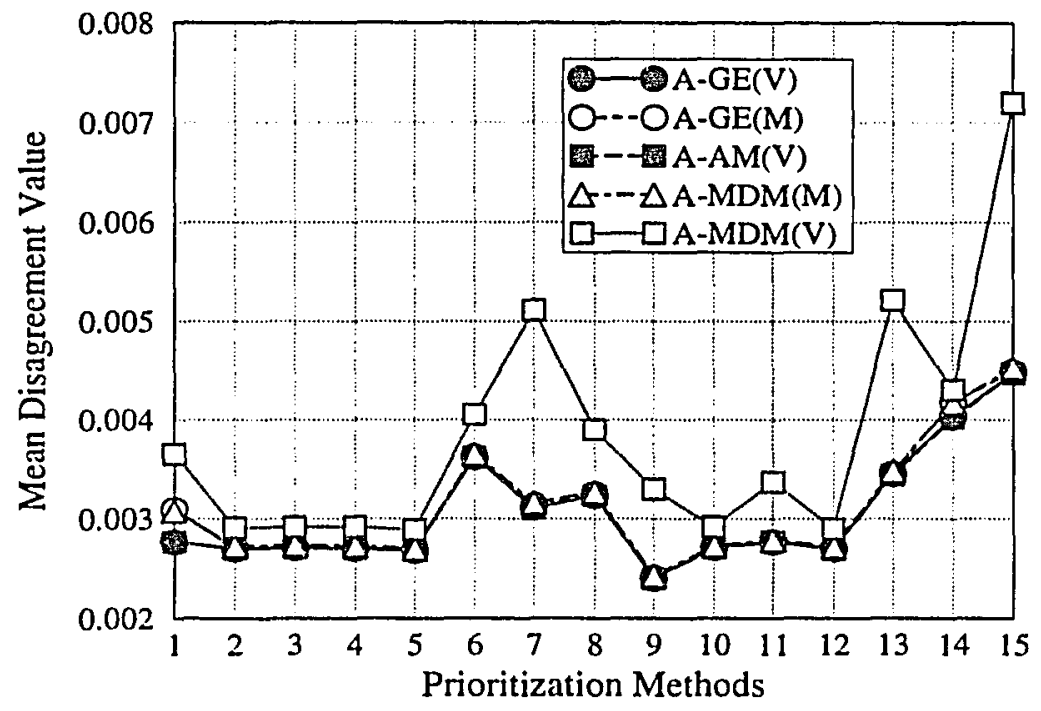

Figure 5.14: The Mean Disagreement of Uniform Distribution for All Aggregation Methods $(\mathrm{N}=12, \mathrm{M}=7)$

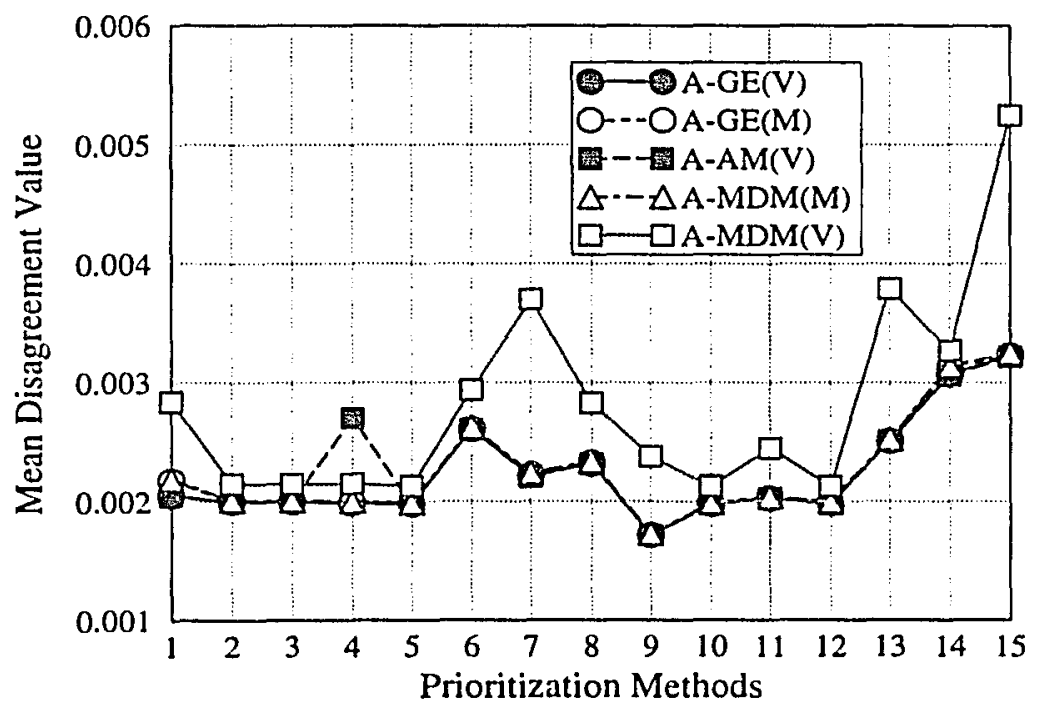

Figure 5.15: The Mean Disagreement of Lognormal Distribution for All Aggregation Methods $(N=12, M=7)$ 


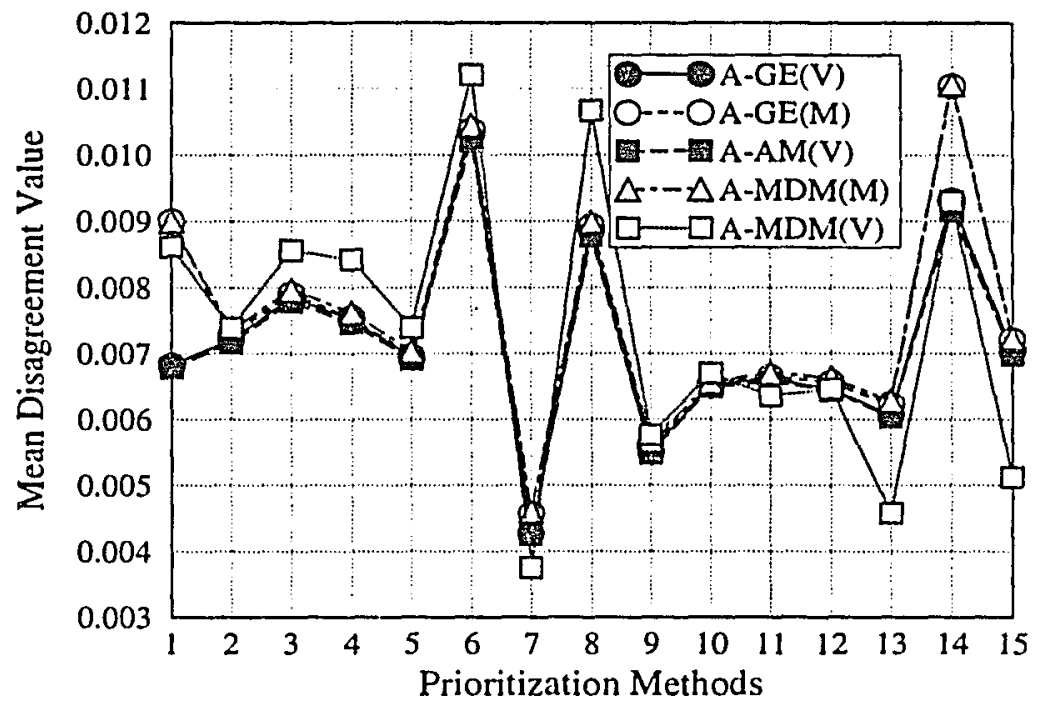

Figure 5.16: The Mean Disagreement of Gamma Distribution for All Aggregation Methods $(\mathrm{N}=12, \mathrm{M}=7)$ 


\subsection{Prioritization Methods vs. Aggregation Meth- ods}

Another dimension of our interests is the combination of different prioritization methods with aggregation methods. The key questions are: which combination will give us good results in terms of accuracy and group disagreement measurement, among all the combinations? Are there any differences among themselves? These questions are the focus of this section. As usual, all our discussions are in terms of accuracy and group disagreement measurements.

Accuracy: Simulation results for different combinations of prioritization methods and aggregation methods are illustrated in Fig. 5.11 through Fig. 5.13, which are classified by input data distribution. For given types of input data distribution and the simulated decision making environments (i.e. the number of decision makers and decision elements, etc.), different combinations result in different levels of the mean of accuracy over all 500 simulation runs for given types of input data and decision environments, i.e. the number of decision makers and decision elements, etc. There are differences between prioritization methods to prioritization method. Some of them are considerably large, and some of them small. For uniform input data distribution, the prioritization methods $2,3,4,5,10,11,12$ and 14 produce almost identical results over all aggregation methods except MDM operated on final priority vector as shown in Fig. 5.11. We also notice that some combinations generate far worse results, such as the combination of prioritization 1 with the geometric mean method or arithmetic mean. In general, the combination of aggregation 
methods with any prioritization method gives worse results. For lognormal input data distribution, the relationship between aggregation methods and prioritization methods is almost the same as the uniform case as shown in Fig. 5.12. For gamma distribution, the prioritization methods $1,2,3,4,5,10,11,12$ and 14 produce almost identical results over all aggregation methods, but also notice that prioritization methods 13 and 15 produce better results for all the aggregation methods. The results discussed above are summarized in Table 5.1.

Table 5.1: The prioritization methods with good mean accuracy and good mean disagreement over different input data type for all the aggregation methods

\begin{tabular}{||l||l|c|c|c||}
\hline ID NO. & ABBREVIATIONS & Uniform & Lognormal & Gamma \\
\hline \hline 1 & CSM & & & \\
\hline 2 & R-EV & $\mathrm{X}$ & $\mathrm{X}$ & $\mathrm{X}$ \\
\hline 3 & L-EV & $\mathrm{X}$ & $\mathrm{X}$ & $\mathrm{X}$ \\
\hline 4 & AM-EV & $\mathrm{X}$ & $\mathrm{X}$ & $\mathrm{X}$ \\
\hline 5 & GM-EV & $\mathrm{X}$ & $\mathrm{X}$ & $\mathrm{X}$ \\
\hline 6 & EV[AA'] & & & \\
\hline 7 & EV[A'A] & & & \\
\hline 8 & AM - EV $\left[\mathrm{AA}^{\prime}\right]$ & & & \\
\hline & AND EV $\left[\mathrm{A}^{\prime} \mathrm{A}\right]$ & & & \\
\hline 9 & GE - EV $\left[\mathrm{AA}^{\prime}\right]$ & & & \\
\hline & AND EV $\left[\mathrm{A}^{\prime} \mathrm{A}\right]$ & & & \\
\hline 10 & GE & $\mathrm{X}$ & $\mathrm{X}$ & $\mathrm{X}$ \\
\hline 11 & C-RSM & $\mathrm{X}$ & $\mathrm{X}$ & $\mathrm{X}$ \\
\hline 12 & MT & $\mathrm{X}$ & $\mathrm{X}$ & $\mathrm{X}$ \\
\hline 13 & SAV & & & $\mathrm{X}$ \\
\hline 14 & NEV & & & \\
\hline 15 & LSM & & & $\mathrm{X}$ \\
\hline
\end{tabular}

Group disagreement: The simulation results of group disagreement, which are represented by prioritization methods and aggregation methods, are shown in 
Fig. 5.14 through Fig. 5.16 according to the input data distribution. Different combinations of prioritization and aggregation methods result in different group disagreement as in Fig. 5.14 through Fig. 5.16, which are for a given type of input data distribution and decision environment, i.e the number of decision makers and decision elements, etc. The difference is very small for all aggregation methods even though the MDM operated on the final priority vector generates higher group disagreement for all input data distribution and prioritization methods. For uniform and lognormal input data distribution, prioritization methods 7,13 and 15 give higher group disagreement, especially for MDM approach. For gamma input data distribution, contrary to the uniform and lognormal cases, the prioritization methods 13 and 15 combined with MDM produce better results than any other prioritization methods, while for all other combinations of prioritization methods and aggregation methods, the group disagreement is very close as shown in Fig. 5.13 with prioritization methods $6,8,14$ giving the worse results.

Table 5.1 summarizes which prioritization methods yield very good agreement with respect to all given aggregation methods and input data distributions. All the prioritization methods marked with $\mathrm{X}$ in Table 5.1 generated very close mean of accuracy and group disagreement for all given aggregation methods. The difference of magnitude among all marked prioritization methods is less than $10 \%$. For input data distribution with large variance, we should notice that simple average prioritization methods (ID No. 13), which is combined with any aggregation method, create better results for both accuracy and group disagreement. Prioritization methods 7 and 9 in general, generate much worse results in terms of accuracy measurement, as 
we see in Fig. 5.11 and Fig. 5.12. For $N=10$ and $N=12$, those two prioritization methods are significantly worse (usually they are $200 \%$ to $50 \%$ worse; see Appendix $B$ for details) than the rest of the methods. Therefore, some of the figures in the following sections have omitted those two methods in order to better illustrate the rest of the prioritization methods.

\subsection{Aggregation Methods vs. Number of Deci- sion Makers}

The influence of the number of decision makers on the decision outcome, i.e. the accuracy and group disagreement, is the focus of this section. Specifically, we are concerned with how the aggregation methods function, and if there are differences among the aggregation methods with respect to an increase in the number of decision makers. The following discussions are also classified by accuracy and group disagreement measurements.

Accuracy: Fig. 5.17 through Fig. 5.31 summarize the simulation results for the $N=10$ case according to aggregation methods and input data distributions. In general, accuracy increases (small mean accuracy value) by increasing the number of decision makers $(M)$ under the condition that the type of input data and number of decision elements $(N)$ are given. But we should notice that the rate of improvement for accuracy measurement reduces significantly when you compare the change of $M=3$ to $M=5$ with the change of $M=5$ to $M=7$. These phenomena are true for all the combinations of input data distribution and aggregation methods as 
shown in Fig. 5.17 through Fig. 5.31. As a numerical example, look at Fig. 5.17 for prioritization method 10 (geometric mean approach). The mean accuracy for different $M$ s are as follows:

$$
\begin{array}{ll}
d_{1(M=3)}=0.00666, & d_{1(M=5)}=0.00551 \\
d_{1(M=7)}=0.00489, & d_{1(M=9)}=0.00453
\end{array}
$$

where $d_{1}$ stands for accuracy measurement. It can be seen that if decision makers increase from $M=3$ to $M=5$, the accuracy measurement improves $17 \%$, while the improvement for changing $M$ from 7 to 9 is $7 \%$. Therefore, further increases to the number of decision maker means the benefit of increasing the accuracy will be diminished. After all, we should keep in mind that this conclusion is a result of the fact that we assume that all decision makers' judgments are in the same interval $\left[0.5 a_{j k}, 1.5 a_{j k}\right]$ and have the same probability distribution of the judgments. In other words, theses results tell us that adding more decision makers to a decision making group will enforce the decision if the new members have the same knowledge or biases. But the improvement is limited as the number of decision makers increase.

Group disagreement: Fig. 5.32 through Fig. 5.46 are the simulation results for group disagreement with $N=10$ according to input data distributions and aggregation methods. Group disagreement decreases as the number of decision makers $(M)$ increases for a given type of input data and number of decision elements as indicated in Fig. 5.32 through Fig. 5.46. This may contrary to people's thinking about the increase in the number of decision makers. Usually, people think that 
group disagreement may become larger with an increasing number of decision makers in a decision making group. This may be true if the decision makers added to the decision making group have different knowledge and information. But in our simulation we assume that all decision makers' judgments are in the same interval $\left(\left[0.5 a_{j k}, 1.5 a_{j k}\right]\right)$ with the same knowledge level (or distribution) of a given subject; therefore, increasing the number of decision makers results in reinforcing the previous decision. Otherwise, this characteristic may not exist, which is obvious. Hence, the conclusion presented here is also valid. We also notice the same characteristics as accuracy measurement: the group disagreement decreases by increasing the number of decision makers in the decision making group. The improvement is decreases as the number of decision makers increase.

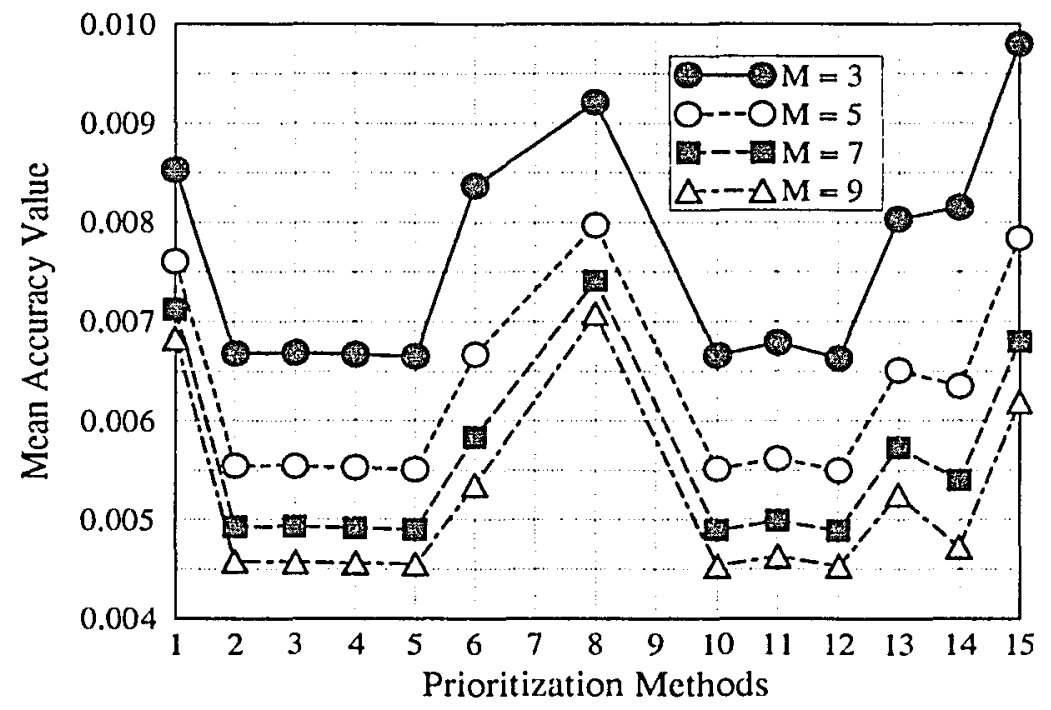

Figure 5.17: The Mean Accuracy of Geometric Mean Method with Uniform Distribution for $M=3,5,7,9(N=10)$, The Geometric Mean Method Operates on Final Priority Vector 


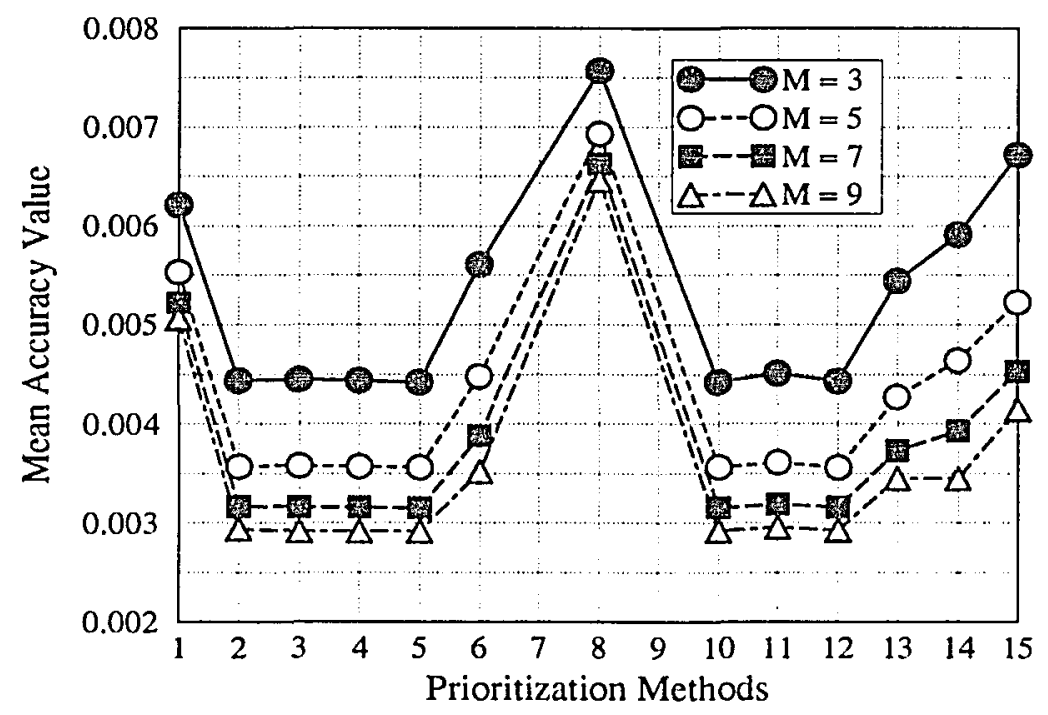

Figure 5.18: The Mean Accuracy of Geometric Mean Method with Lognormal Distribution for $\mathrm{M}=3,5,7,9(\mathrm{~N}=10)$, The Geometric Mean Method Operates on Final Priority Vector

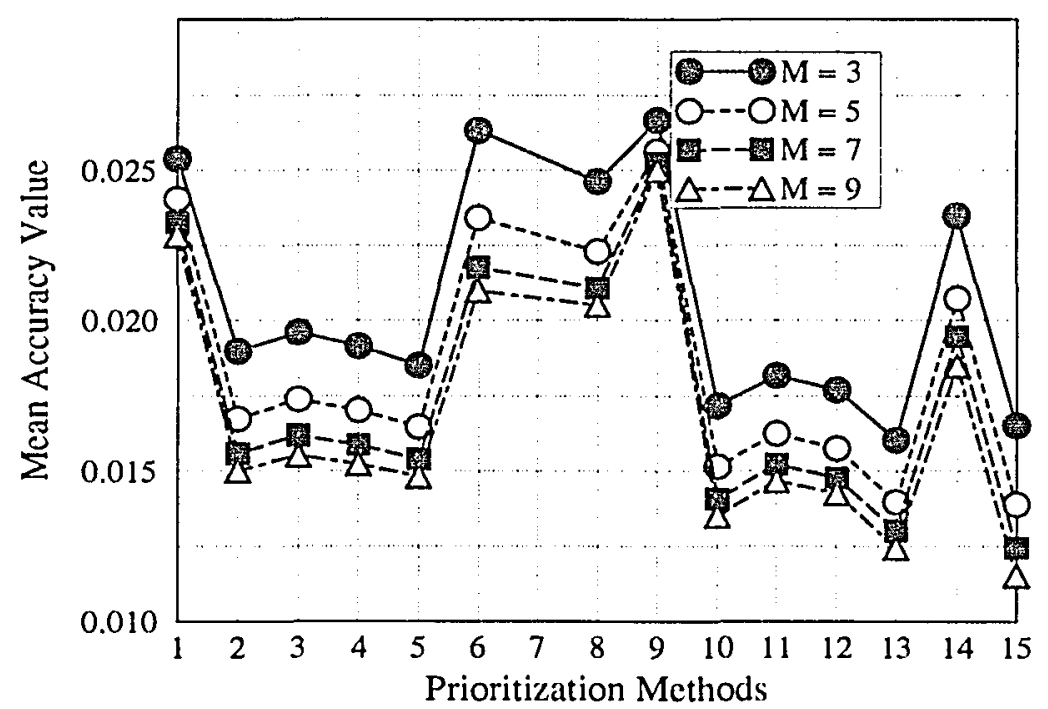

Figure 5.19: The Mean Accuracy of Geometric Mean Method with Gamma Distribution for $M=3,5,7,9(N=10)$, The Geometric Mean Method Operates on Final Priority Vector 


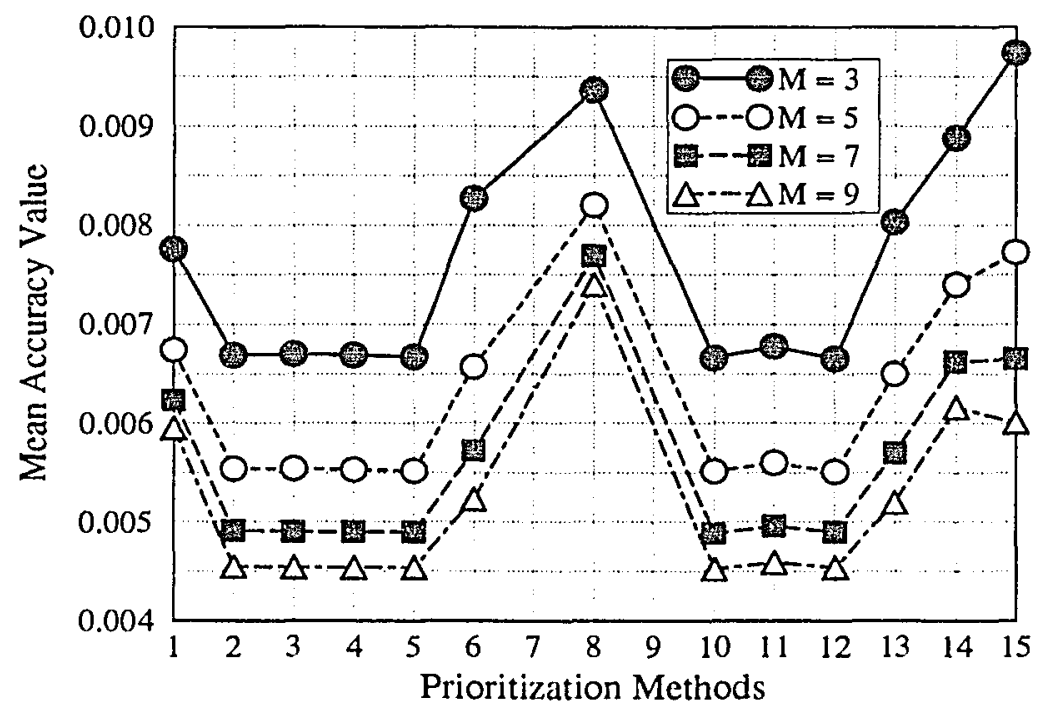

Figure 5.20: The Mean Accuracy of Geometric Mean Method with Uniform Distribution for $\mathrm{M}=3,5,7,9(\mathrm{~N}=10)$, The Geometric Mean Method Operates on Pairwise Comparison Matrix

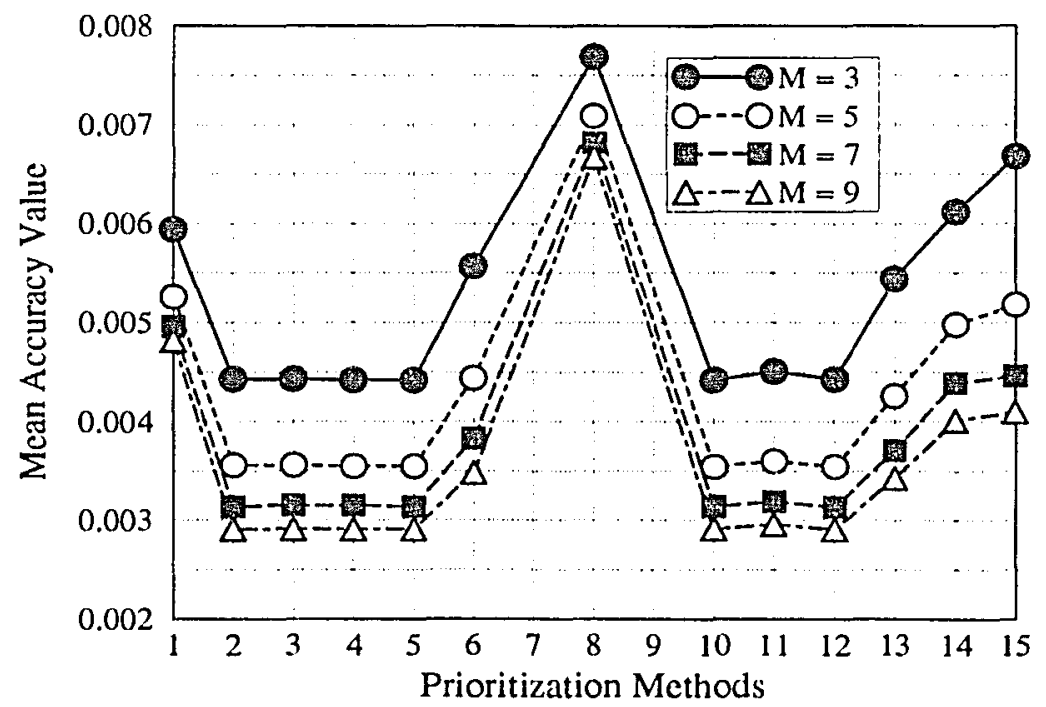

Figure 5.21: The Mean Accuracy of Geometric Mean Method with Lognormal Distribution for $M=3,5,7,9(N=10)$, The Geometric Mean Method Operates on Pairwise Comparison Matrix 


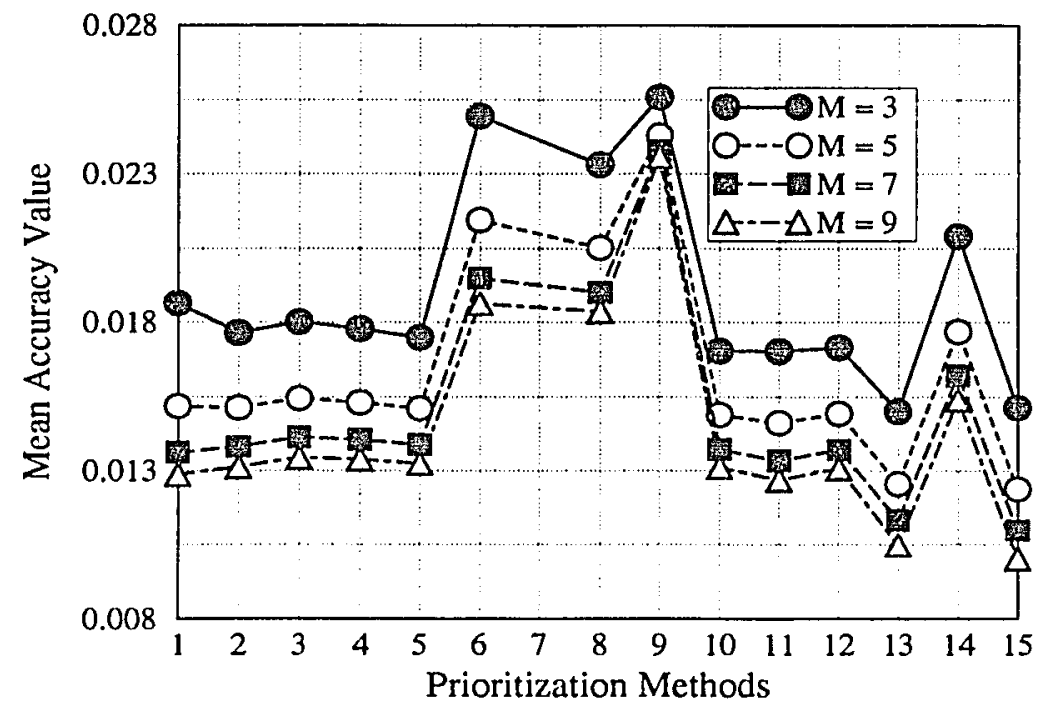

Figure 5.22: The Mean Accuracy of Geometric Mean Method with Gamma Distribution for $M=3,5,7,9(\mathrm{~N}=10)$, The Geometric Mean Method Operates on Pairwise Comparison Matrix

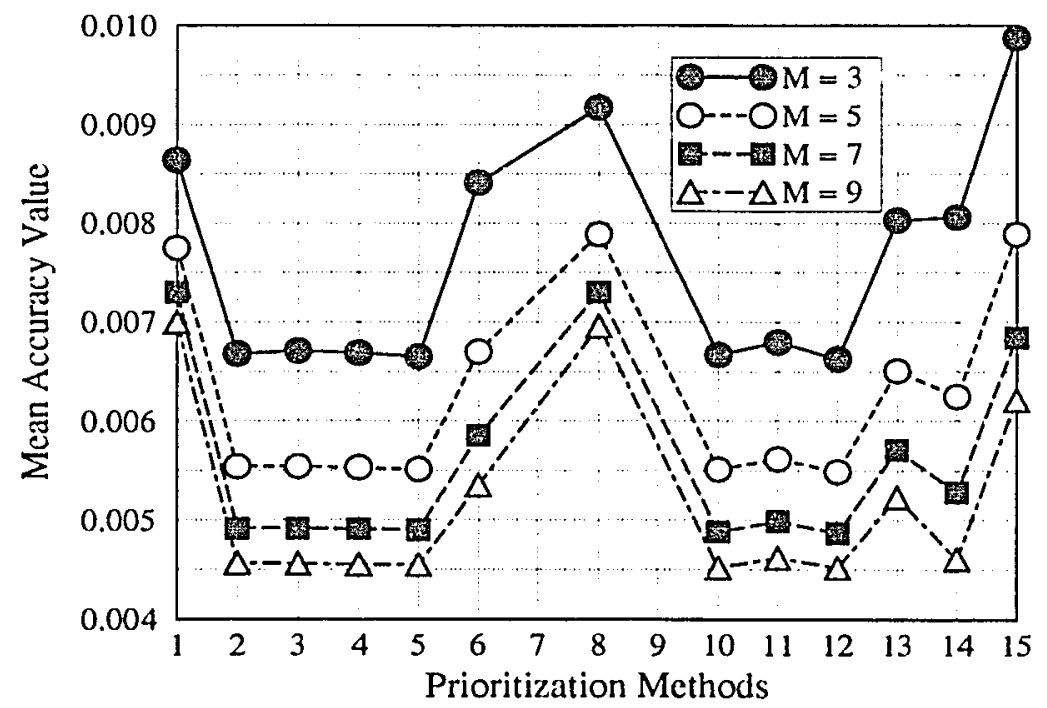

Figure 5.23: The Mean Accuracy of Arithmetic Mean Method with Uniform Distribution for $M=3,5,7,9(N=10)$, The Arithmetic Mean Method Operates on Final Priority Vector 


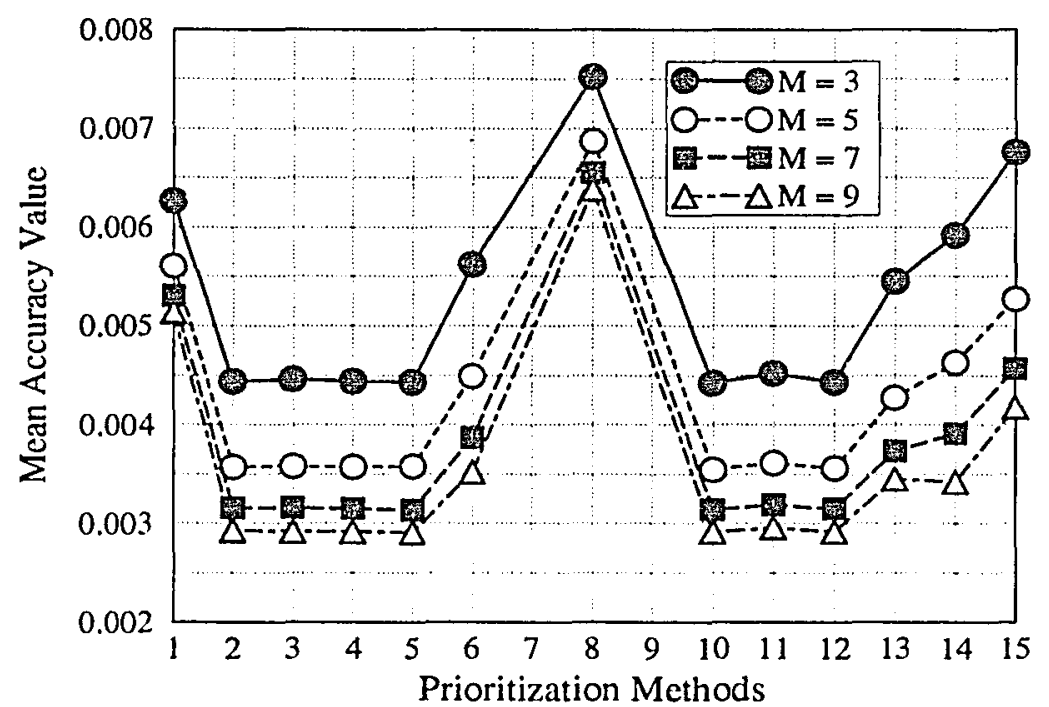

Figure 5.24: The Mean Accuracy of Arithmetic Mean Method with Lognormal Distribution for $\mathrm{M}=3,5,7,9(\mathrm{~N}=10)$, The Arithmetic Mean Method Operates on Final Priority Vector

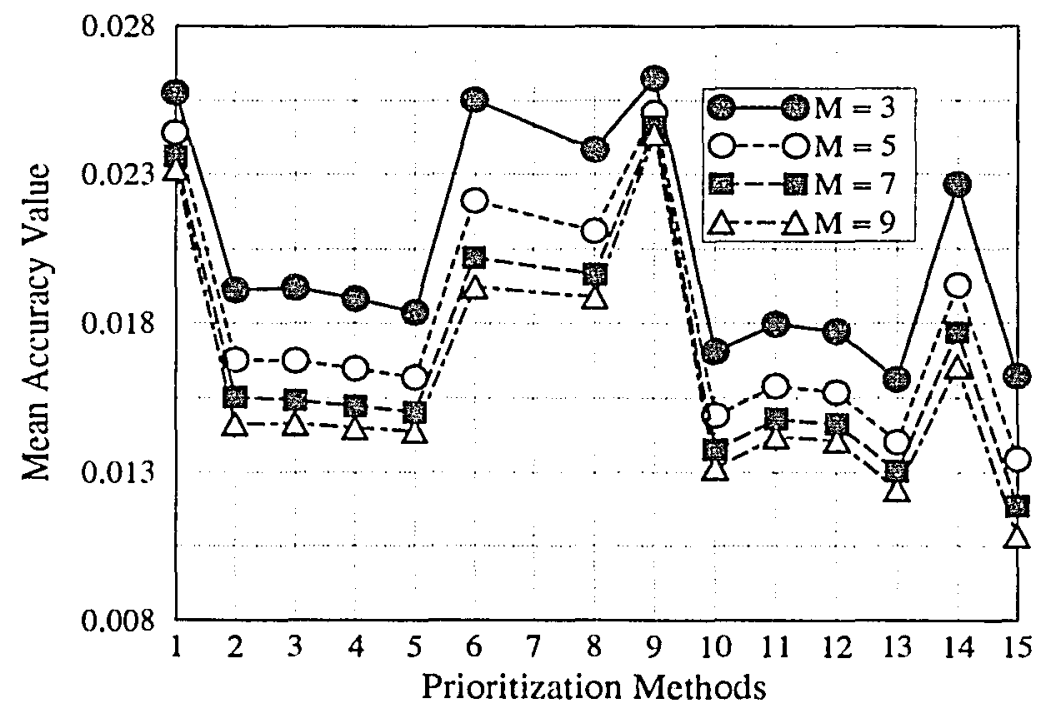

Figure 5.25: The Mean Accuracy of Arithmetic Mean Method with Gamma Distribution for $M=3,5,7,9(\mathrm{~N}=10)$, The Arithmetic Mean Method Operates on Final Priority Vector 


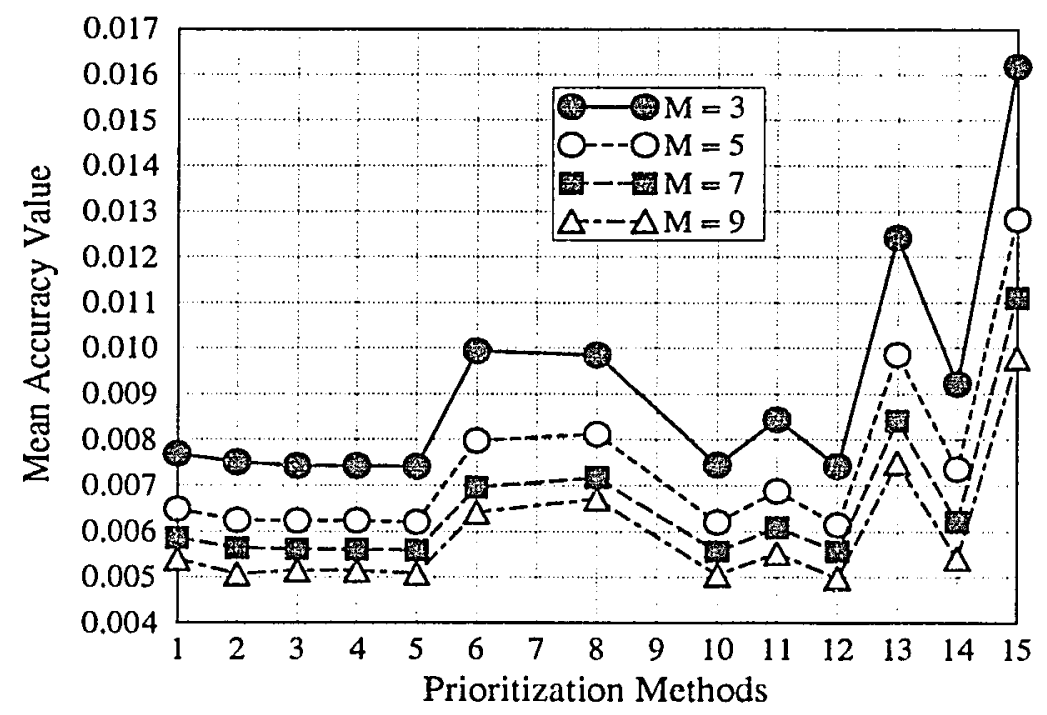

Figure 5.26: The Mean Accuracy of Minimum Distance Method with Uniform Distribution for $\mathrm{M}=3,5,7,9(\mathrm{~N}=10)$, The Minimum Distance Method Operates on Final Priority Vector

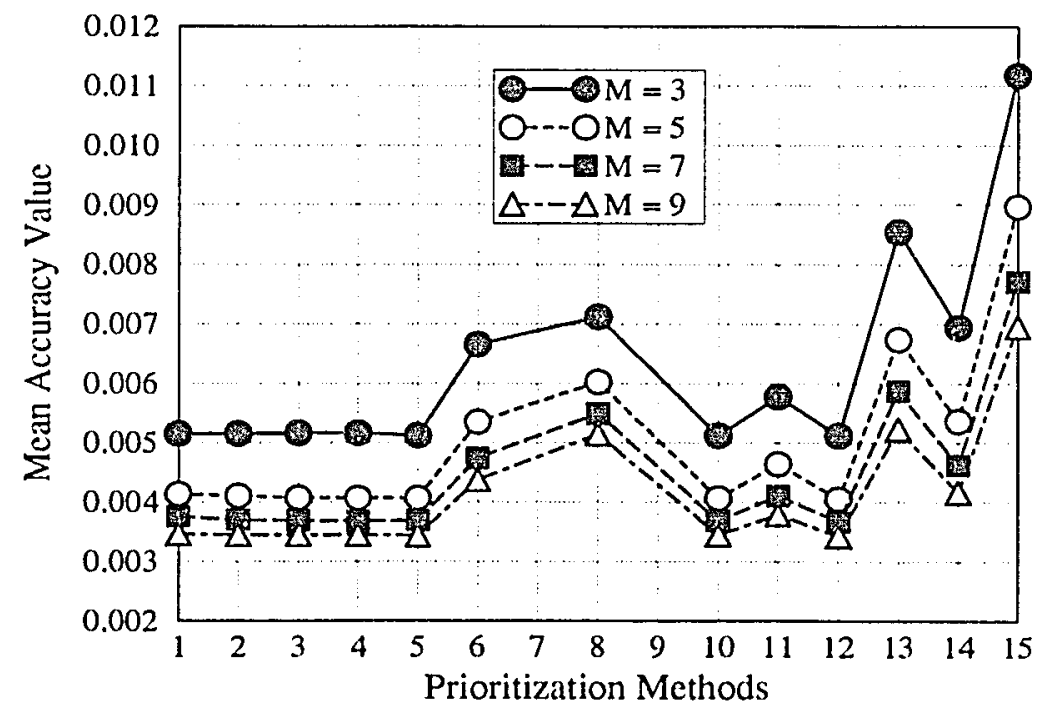

Figure 5.27: The Mean Accuracy of Minimum Distance Method with Lognormal Distribution for $M=3,5,7,9(\mathrm{~N}=10)$, The Minimum Distance Method Operates on Final Priority Vector 


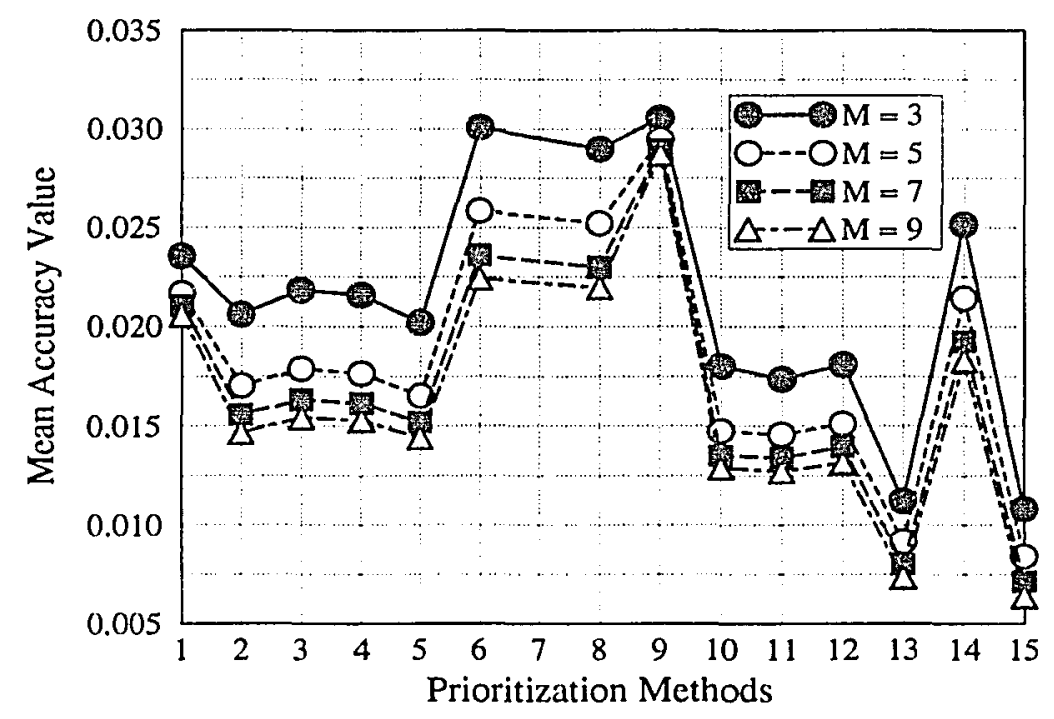

Figure 5.28: The Mean Accuracy of Minimum Distance Method with Gamma Distribution for $M=3,5,7,9(N=10)$, The Minimum Distance Method Operates on Final Priority Vector

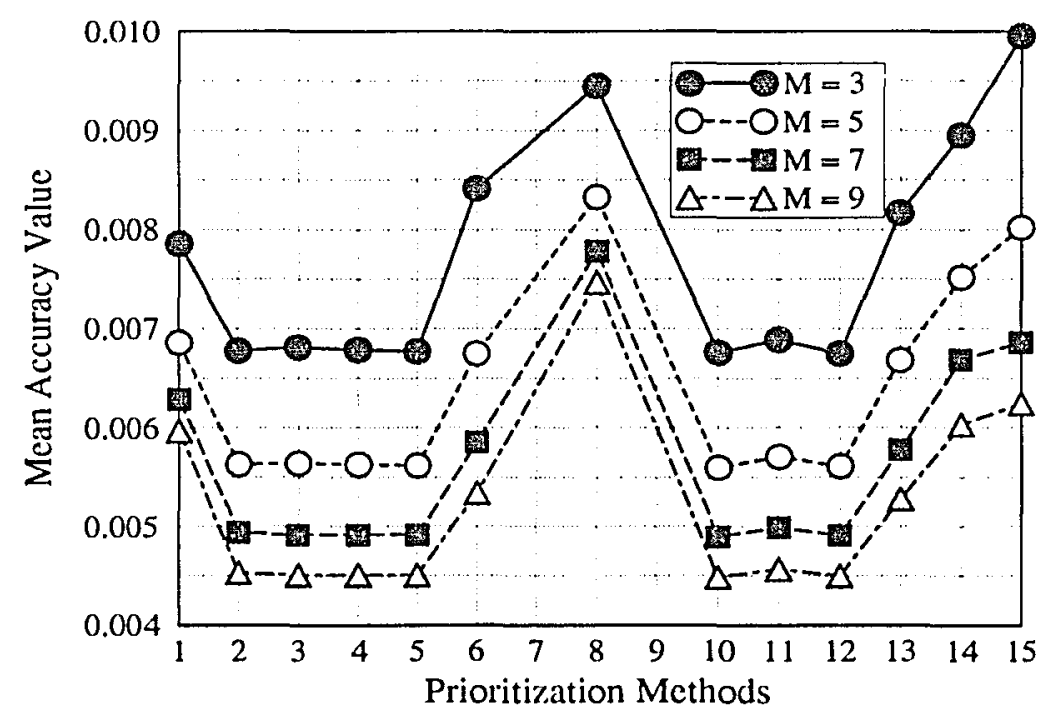

Figure 5.29: The Mean Accuracy of Minimum Distance Method with Uniform Distribution for $\mathrm{M}=3,5,7,9(\mathrm{~N}=10)$, The Minimum Distance Method Operates on Pairwise Comparison Matrix 


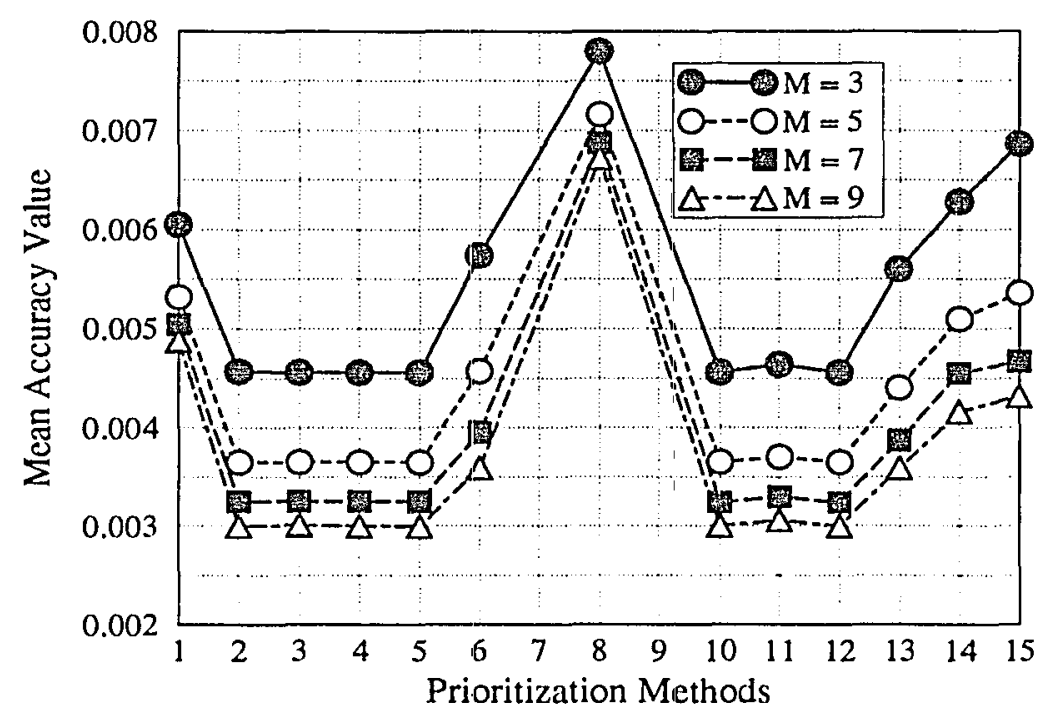

Figure 5.30: The Mean Accuracy of Minimum Distance Method with Lognormal Distribution for $\mathrm{M}=3,5,7,9(\mathrm{~N}=10)$, The Minimum Distance Method Operates on Pairwise Comparison Matrix

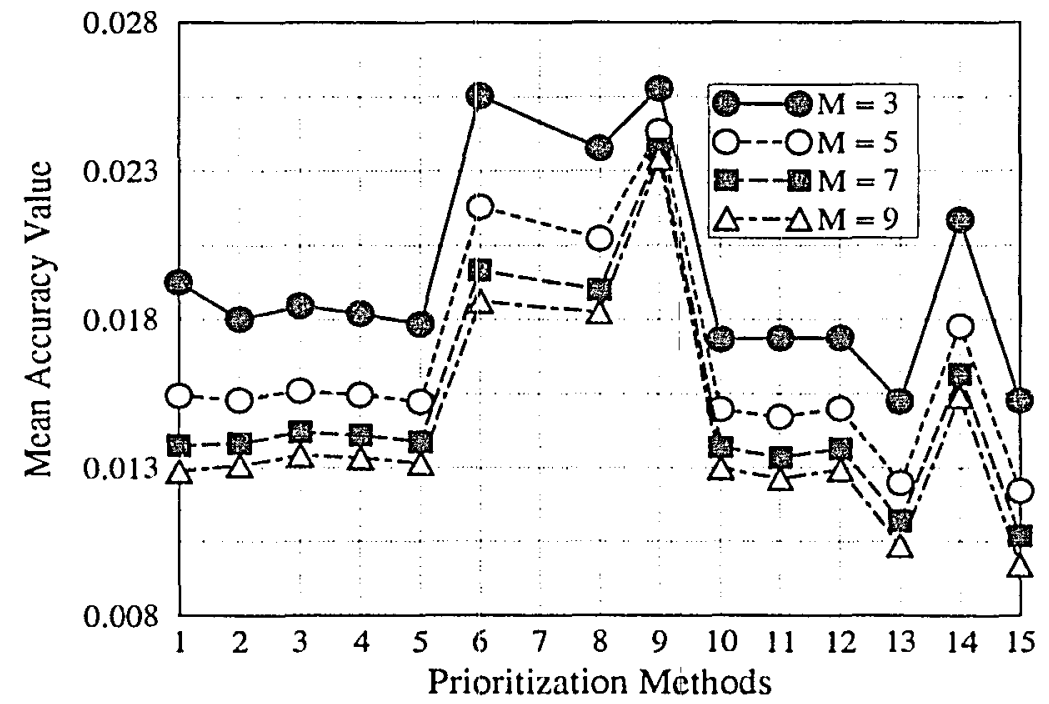

Figure 5.31: The Mean Accuracy of Minimum Distance Method with Gamma Distribution for $\mathrm{M}=3,5,7,9(\mathrm{~N}=10)$, The Minimum Distance Method Operates on Pairwise Comparison Matrix 


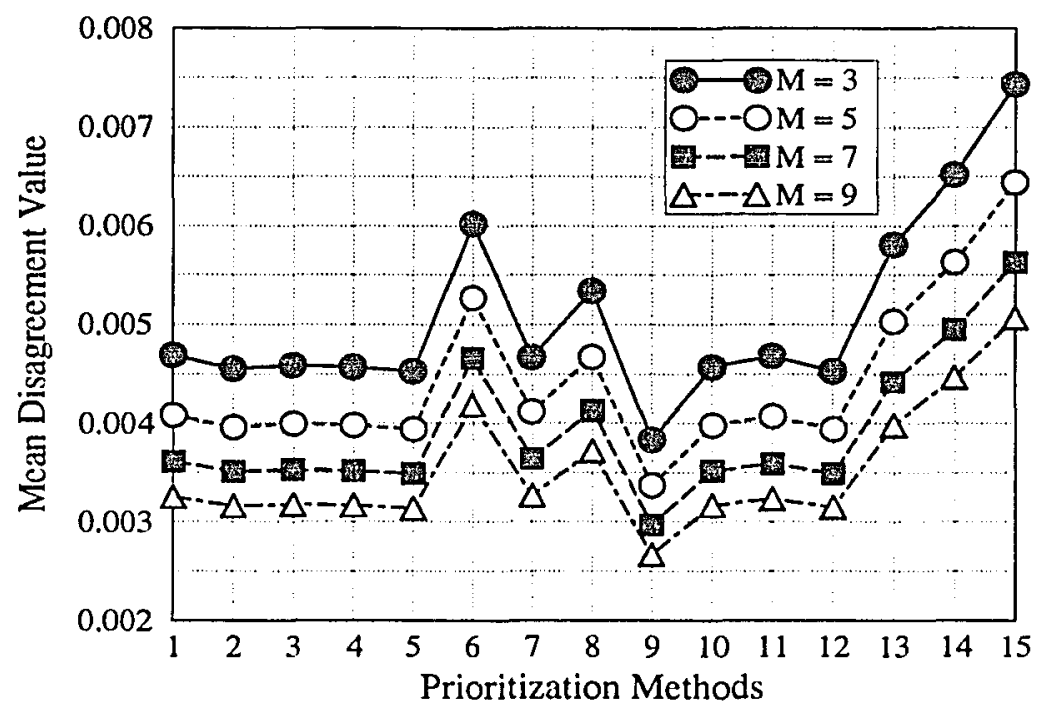

Figure 5.32: The Mean Disagreement of Geometric Mean Method with Uniform Distribution for $\mathrm{M}=3,5,7,9(\mathrm{~N}=10)$, The Geometric Mean Method Operates on Final Priority Vector

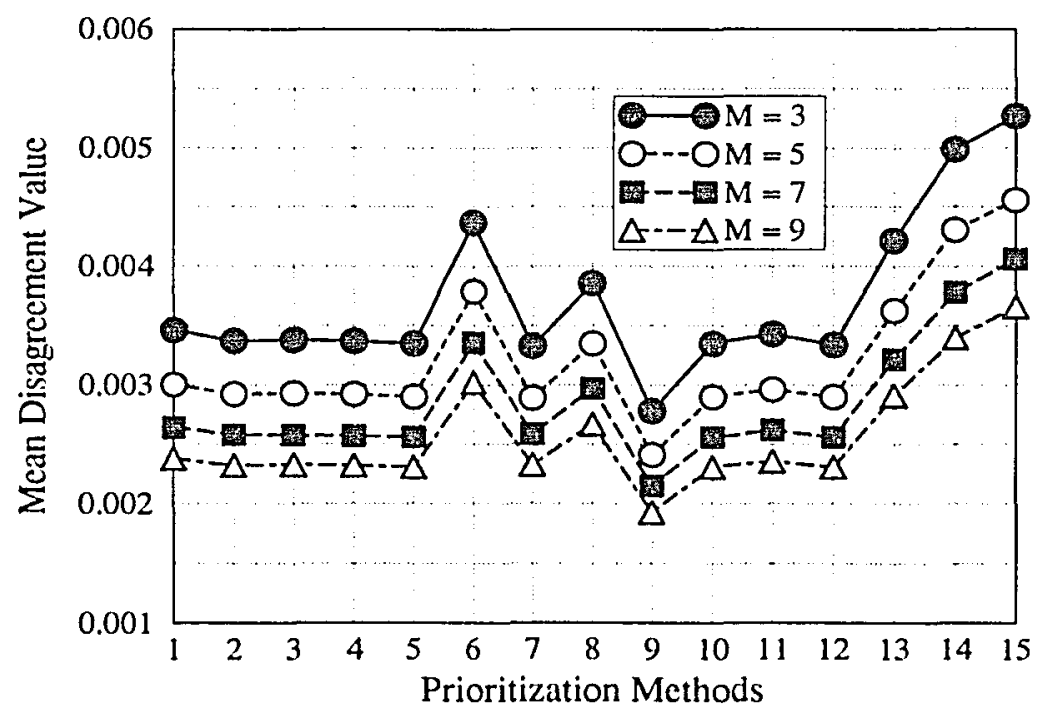

Figure 5.33: The Mean Disagreement of Geometric Mean Method with Lognormal Distribution for $M=3,5,7,9(N=10)$, The Geometric Mean Method Operates on Final Priority Vector 


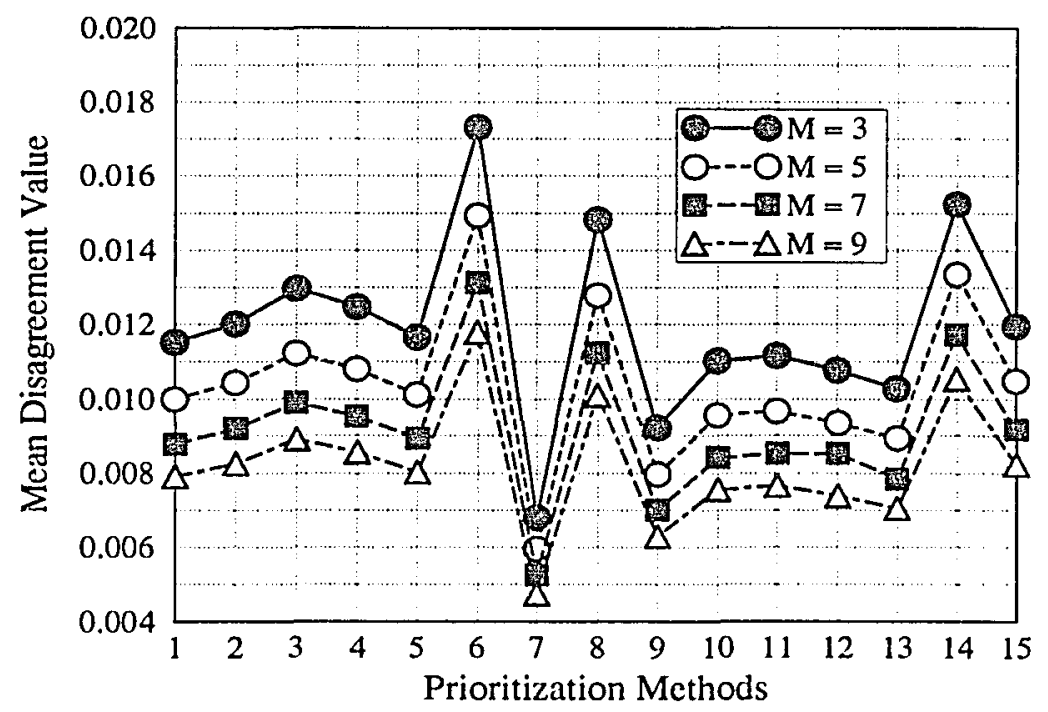

Figure 5.34: The Mean Disagreement of Geometric Mean Method with Gamma Distribution for $M=3,5,7,9(N=10)$, The Geometric Mean Method Operates on Final Priority Vector 


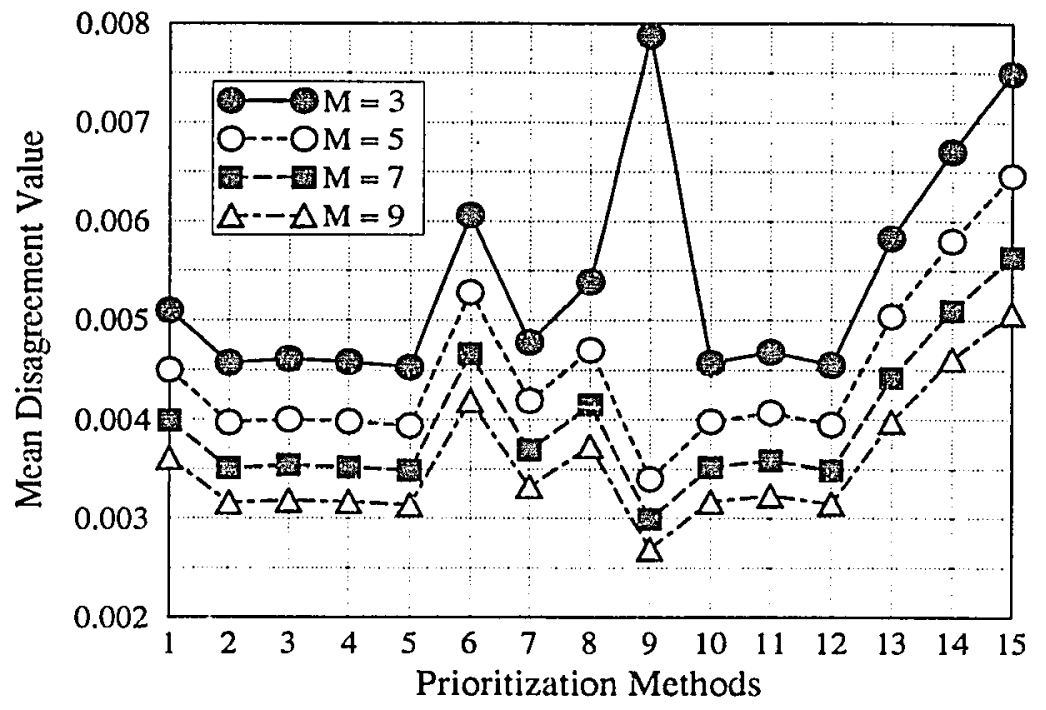

Figure 5.35: The Mean Disagreement of Geometric Mean Method with Uniform Distribution for $\mathrm{M}=3,5,7,9(\mathrm{~N}=10)$, The Geometric Mean Method Operates on Pairwise Comparison Matrix

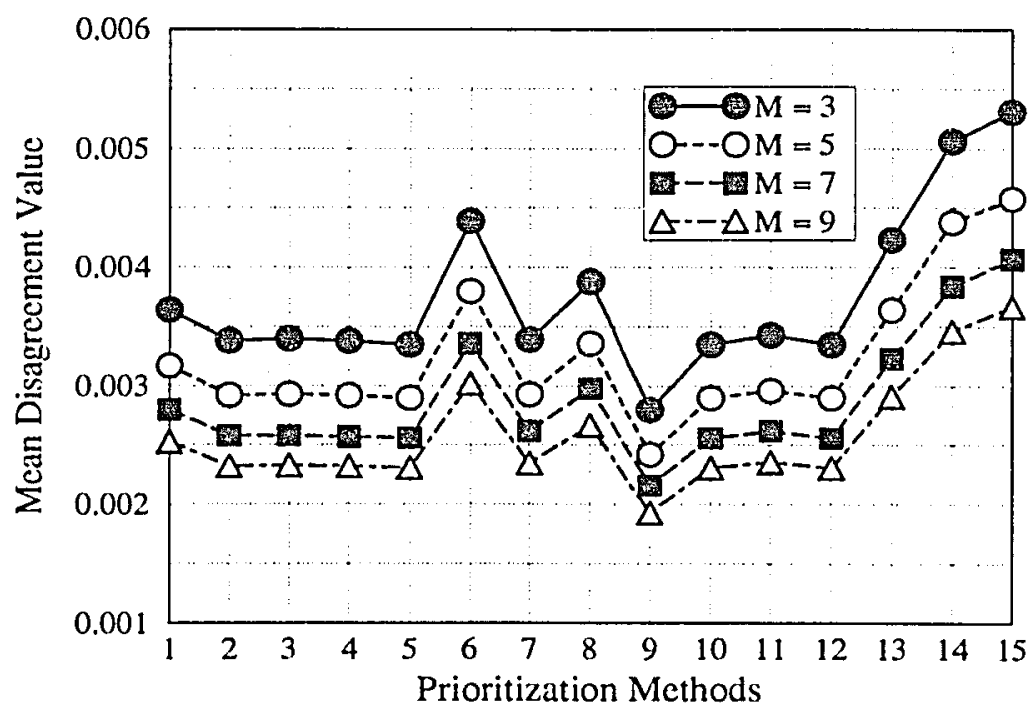

Figure 5.36: The Mean Disagreement of Geometric Mean Method with Lognormal Distribution for $M=3,5,7,9(N=10)$, The Geometric Mean Method Operates on Pairwise Comparison Matrix 


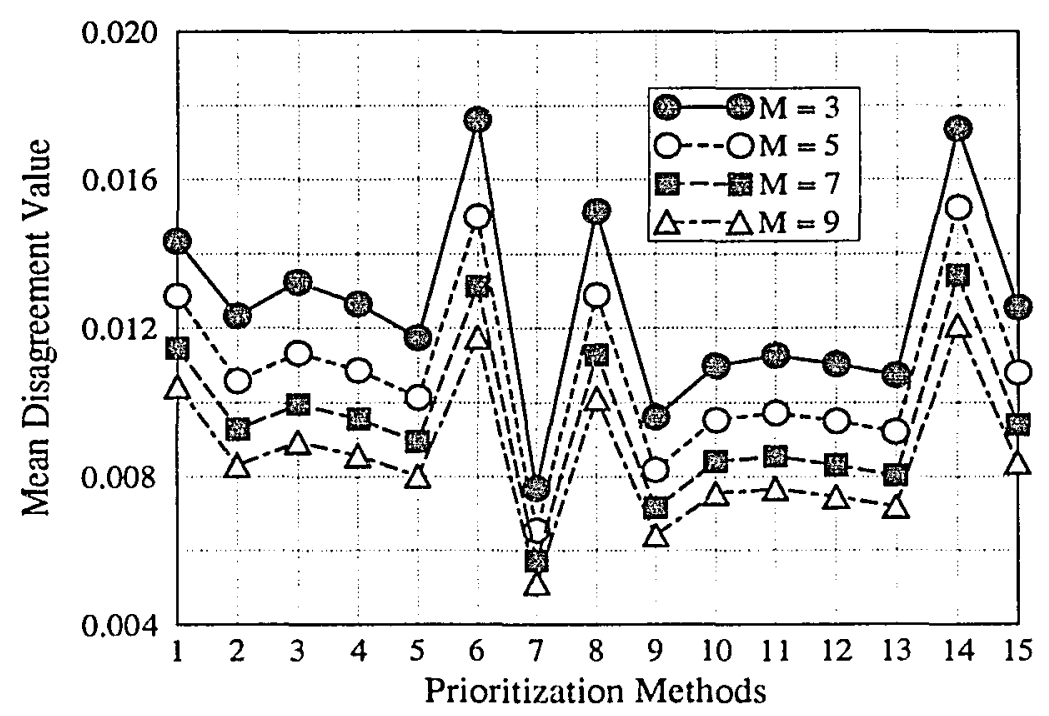

Figure 5.37: The Mean Disagreement of Geometric Mean Method with Gamma Distribution for $\mathrm{M}=3,5,7,9(\mathrm{~N}=10)$, The Geometric Mean Method Operates on Pairwise Comparison Matrix

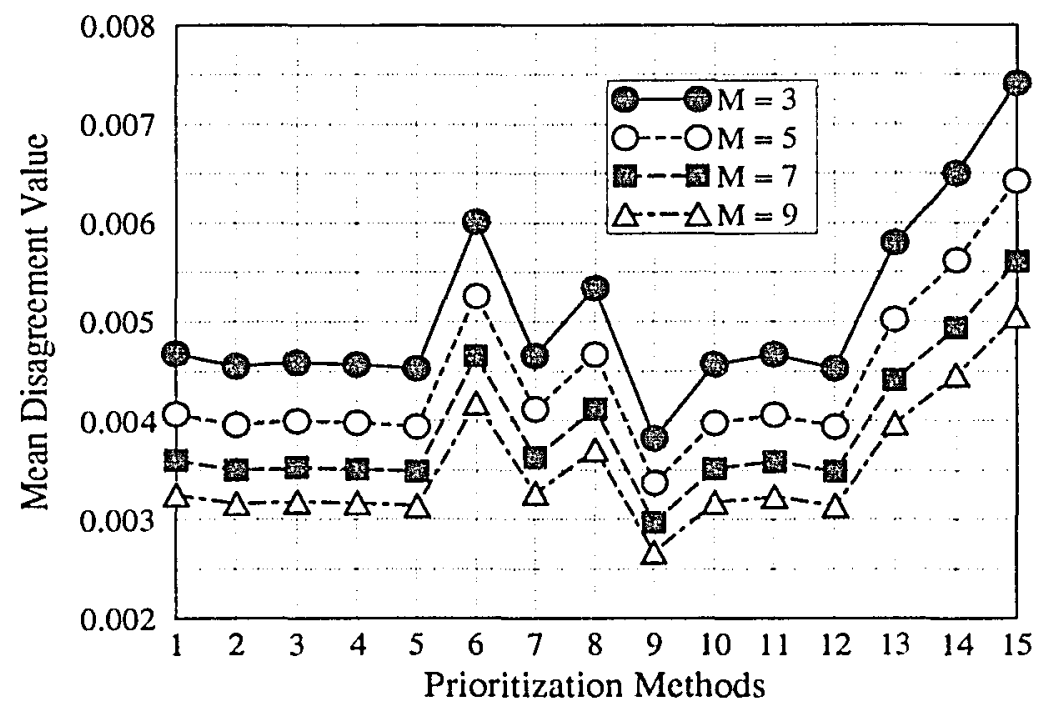

Figure 5.38: The Mean Disagreement of Arithmetic Mean Method with Uniform Distribution for $M=3,5,7,9(N=10)$, The Arithmetic Mean Method Operates on Final Priority Vector 


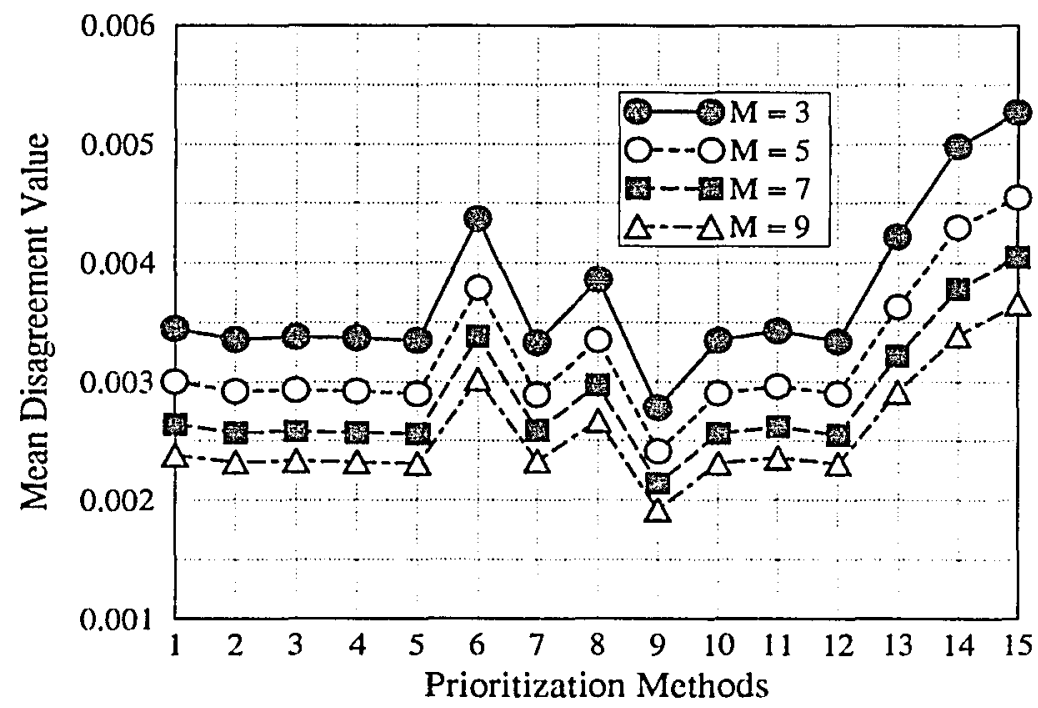

Figure 5.39: The Mean Disagreement of Arithmetic Mean Method with Lognormal Distribution for $\mathrm{M}=3,5,7,9(\mathrm{~N}=10)$, The Arithmetic Mean Method Operates on Final Priority Vector

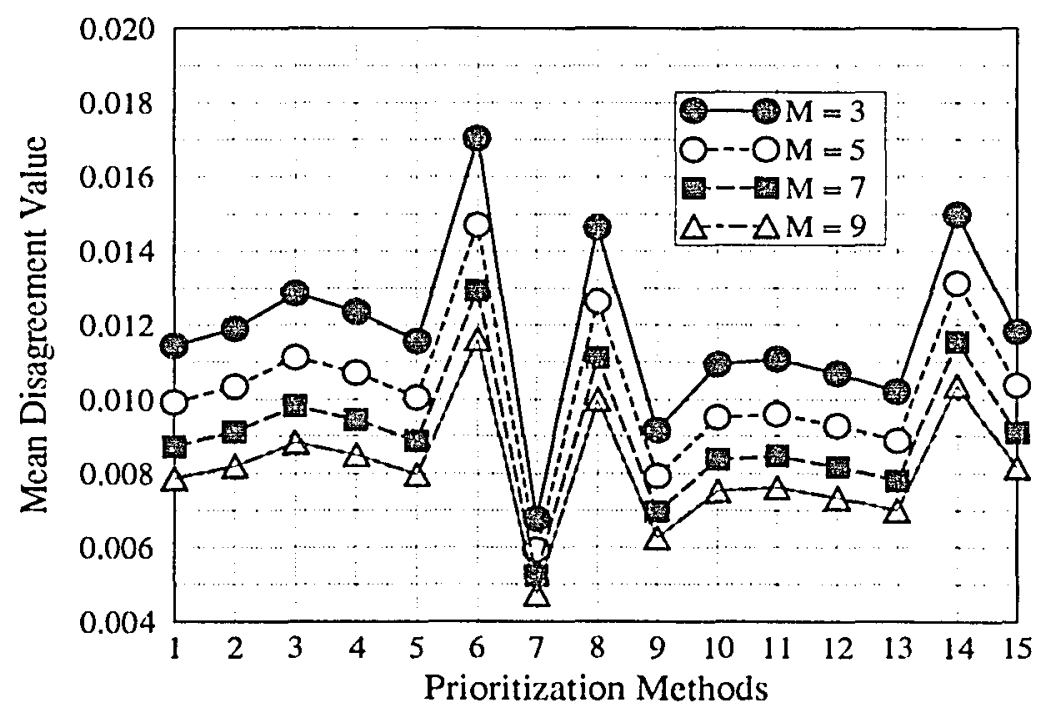

Figure 5.40: The Mean Disagreement of Arithmetic Mean Method with Gamma Distribution for $M=3,5,7,9(N=10)$, The Arithmetic Mean Method Operates on Final Priority Vector 


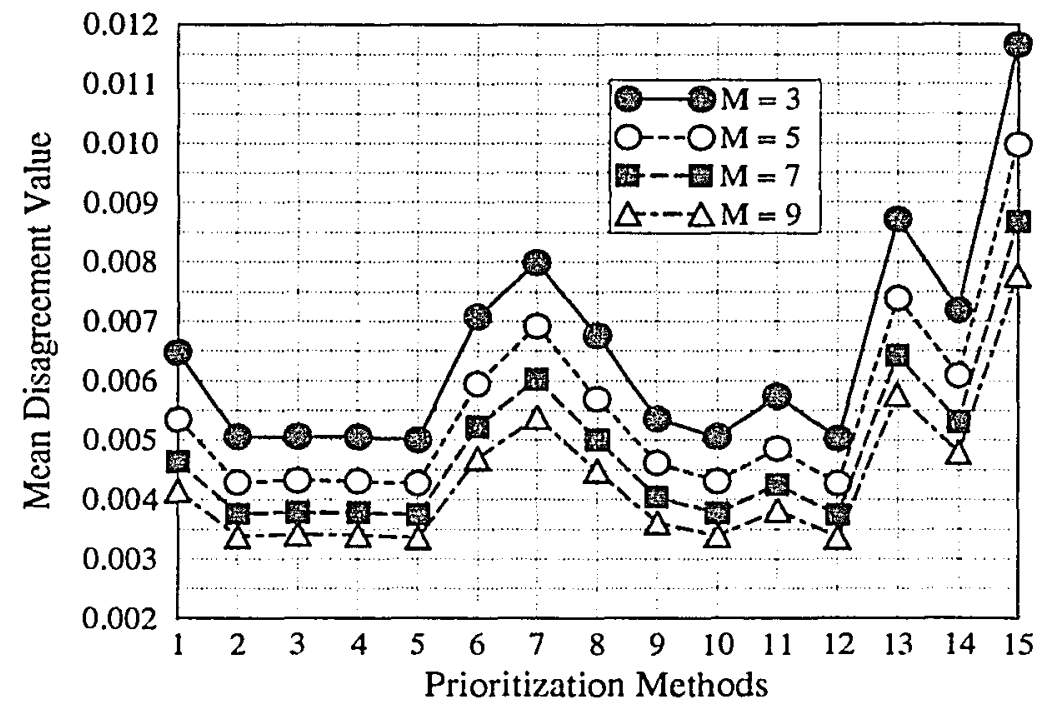

Figure 5.41: The Mean Disagreement of Minimum Distance Method with Uniform Distribution for $\mathrm{M}=3,5,7,9(\mathrm{~N}=10)$, The Minimum Distance Method Operates on Final Priority Vector

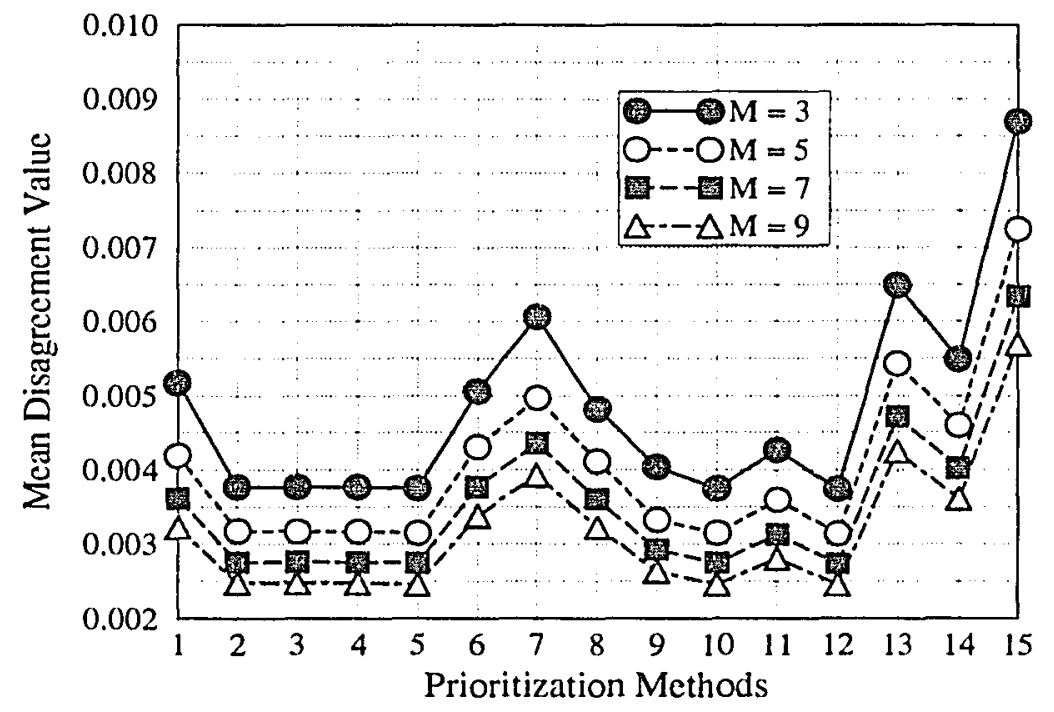

Figure 5.42: The Mean Disagreement of Minimum Distance Method with Lognormal Distribution for $M=3,5,7,9(N=10)$, The Minimum Distance Method Operates on Final Priority Vector 


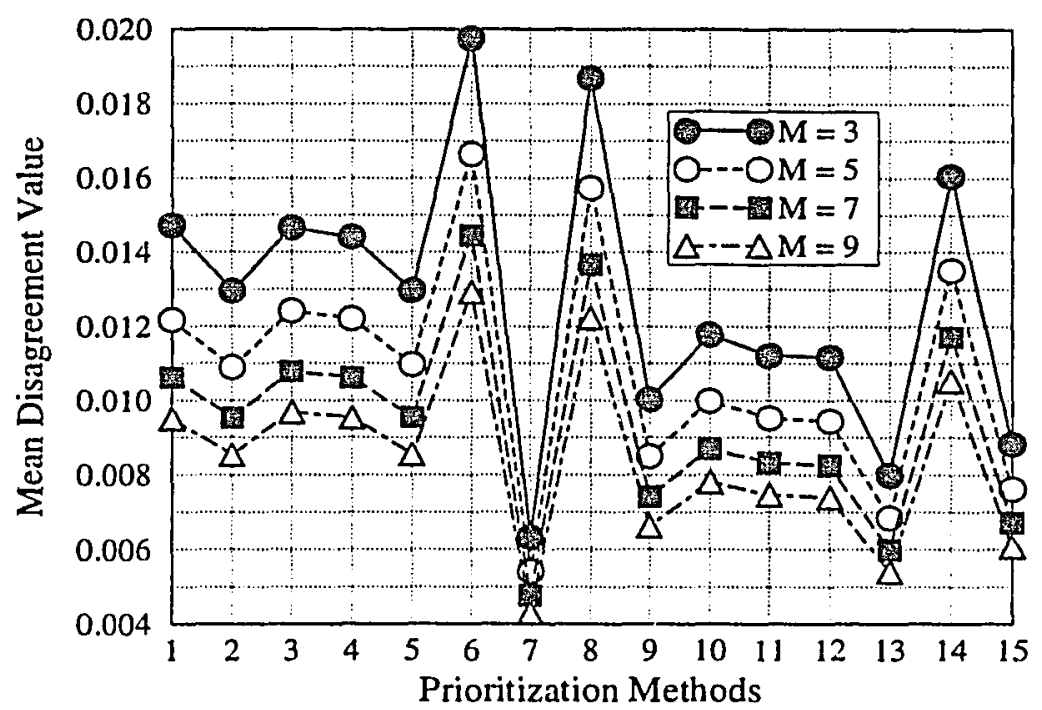

Figure 5.43: The Mean Disagreement of Minimum Distance Method with Gamma Distribution for $M=3,5,7,9(N=10)$, The Minimum Distance Method Operates on Final Priority Vector

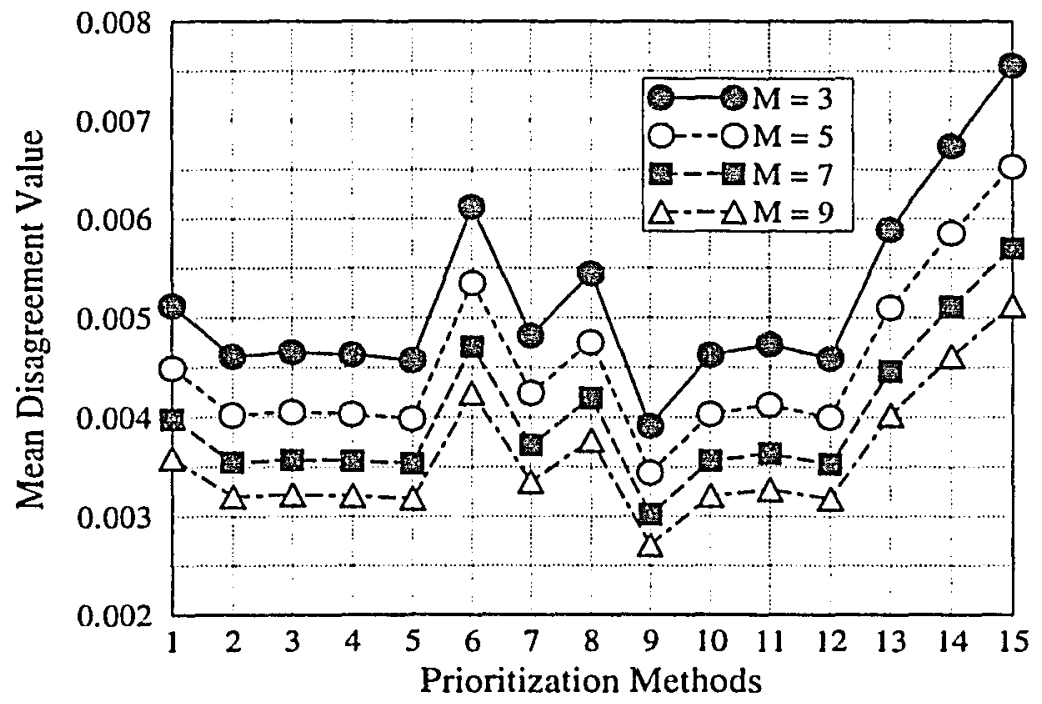

Figure 5.44: The Mean Disagreement of Minimum Distance Method with Uniform Distribution for $M=3,5,7,9(N=10)$, The Minimum Distance Method Operates on Pairwise Comparison Matrix 


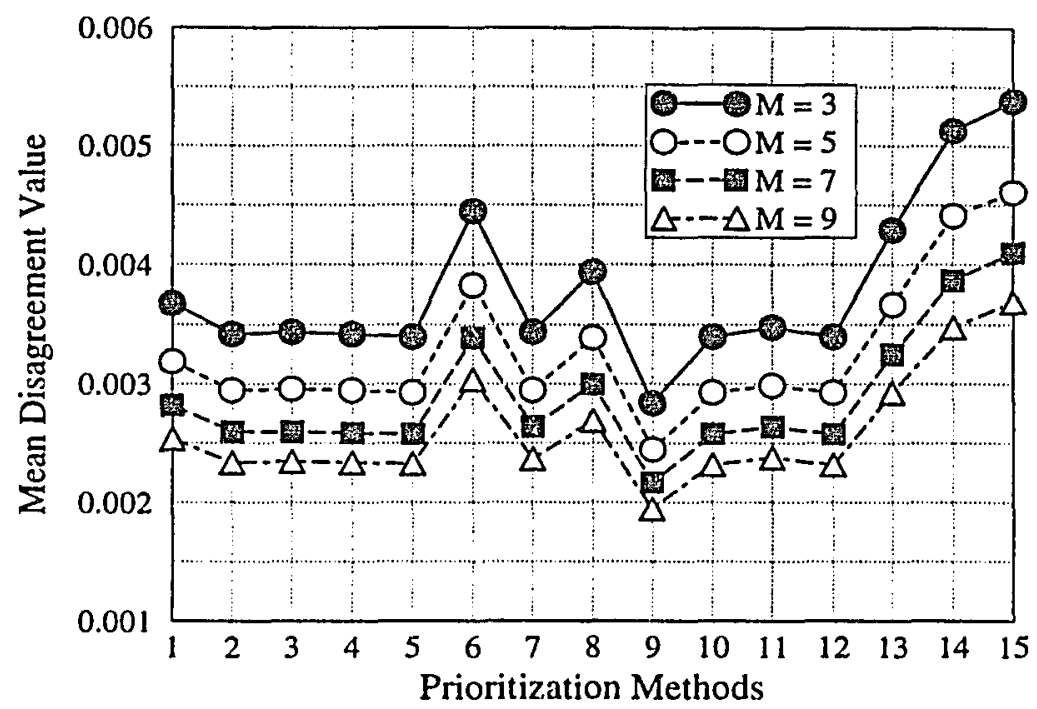

Figure 5.45: The Mean Disagreement of Minimum Distance Method with Lognormal Distribution for $M=3,5,7,9(N=10)$, The Minimum Distance Method Operates on Pairwise Comparison Matrix

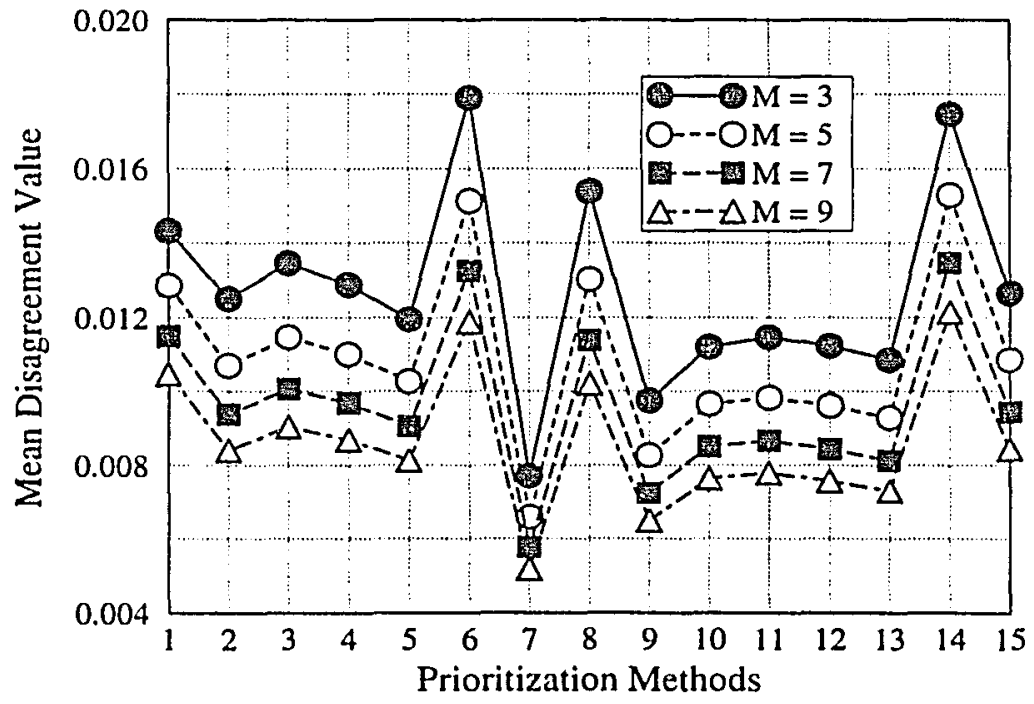

Figure 5.46: The Mean Disagreement of Minimum Distance Method with Gamma Distribution for $\mathrm{M}=3,5,7,9(\mathrm{~N}=10)$, The Minimum Distance Method Operates on Pairwise Comparison Matrix 


\subsection{Analysis of the empirical test of the aggrega- tion methods}

In previous sections of this chapter, the simulation results have been presented and discussed. As we mentioned before, the simulation approach is a fast way to reproduce or partially produce the real situation of a pairwise comparison. But there are limitations for the simulation approach, such as the capability limitation of each decision maker as we discussed at the beginning of this chapter. For this and other reasons, this dissertation also presents limited empirical test results for all the aggregation methods. The discussion of the empirical data sets have been presented in section 4.5. In this section, the results of this empirical test are analyzed. The discussion will focus on the accuracy and group disagreement measurements. The calculation results are presented in Appendix E. In general, the empirical test supports the results from the simulation study.

Accuracy: Fig. 5.47 through Fig. 5.53 are empirical testing results of accuracy measurements. The empirical test results are input data type dependent. For different categories, the distributions of judgments are different due to the knowledge level difference of each individual who gives the judgments. The influence of judgment distribution on accuracy is significant from category to category, which can be seen from the mean accuracy value of 0.006 for category one to 0.066 for category six in Fig. 5.47 and Fig. 5.52, respectively. In the empirical test, the aggregation method of the MDM produces better results overall than any other aggregation methods. This is consistent with the above simulation study with gamma 
input data distribution. The MDM operated on the pairwise comparison matrices (i.e. A-MDM(M)) outperforms other aggregation methods. For any given category (one through seven), the combination of an aggregation method with a prioritization method yields the same pattern for most cases. This means the relationship among aggregation methods in terms of accuracy measurement is nearly the same for all the prioritization methods. In general, the prioritization methods $1,2,3,4,5,6$, $10,11,12$ and 13 perform very consistently across all categories as indicated in the above simulation and empirical results.

Group disagreement: The simulation results for group disagreement are presented in Fig. 5.47 through Fig. 5.53. For any give category, all the combinations of aggregation methods with prioritization methods perform almost identically, which is also consistent with the simulation results. Across different categories, the higher the variance of input data, the higher the disagreements, which is expected. 


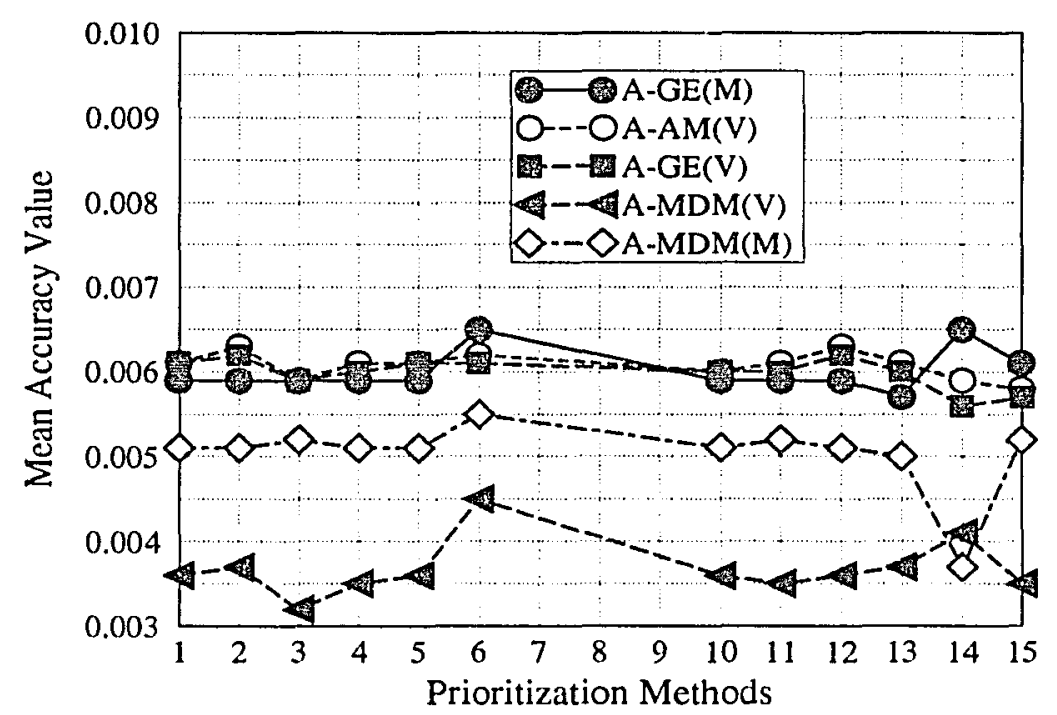

Figure 5.47: The Mean Accuracy of Aggregation Method for Category One Empirical Data

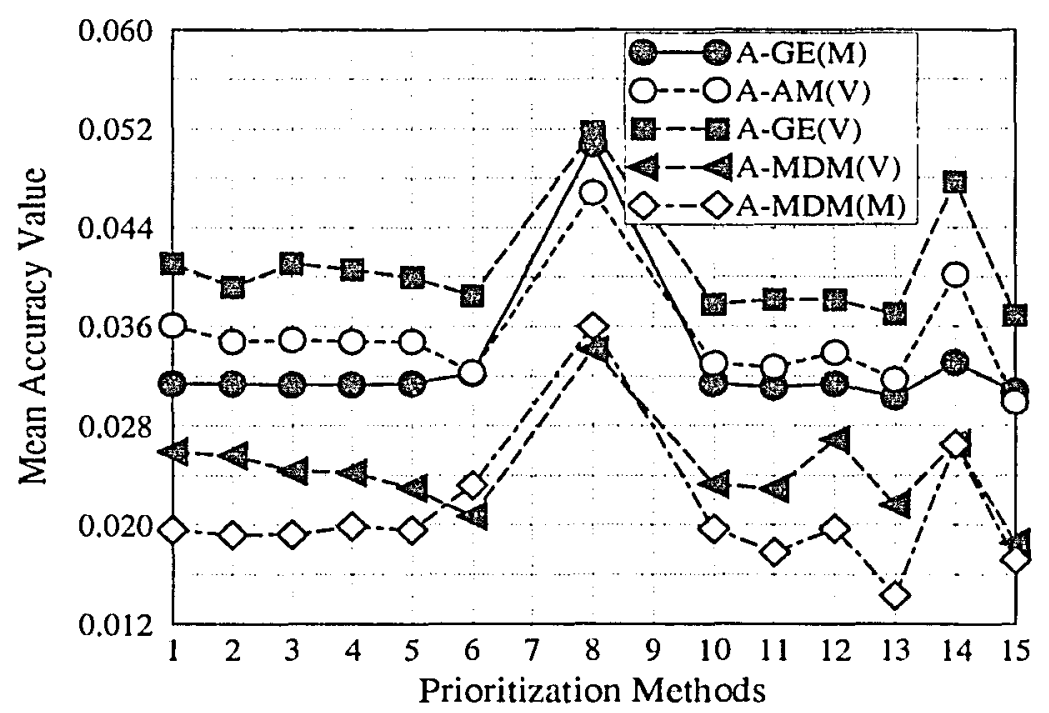

Figure 5.48: The Mean Accuracy of Aggregation Method for Category Two Empirical Data 


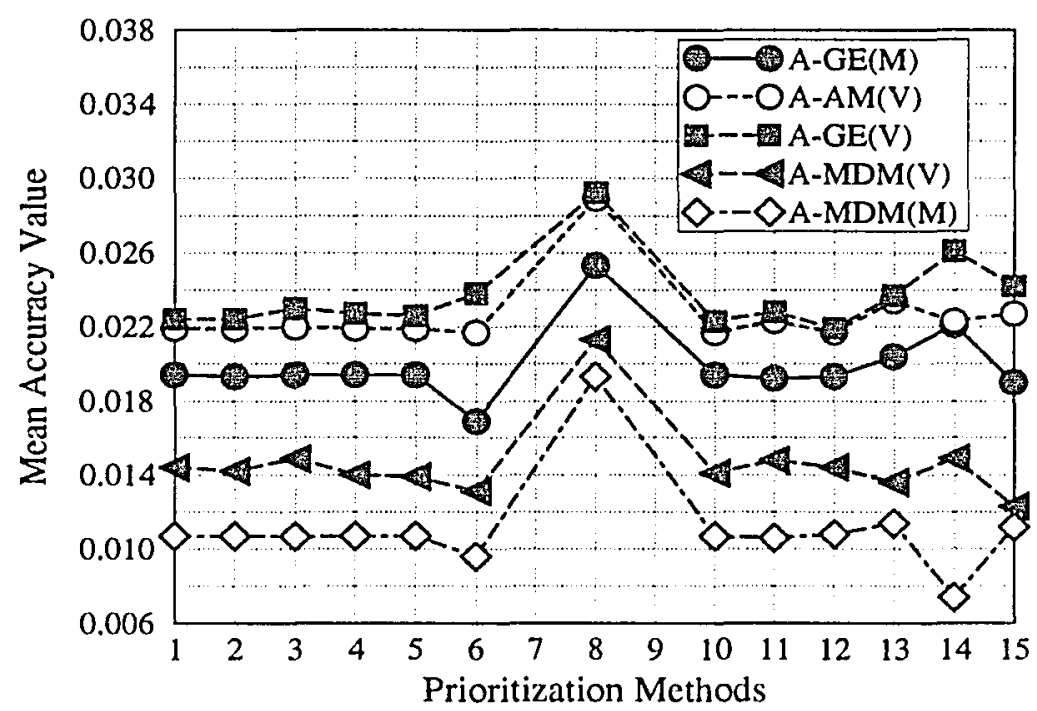

Figure 5.49: The Mean Accuracy of Aggregation Method for Category Three Empirical Data

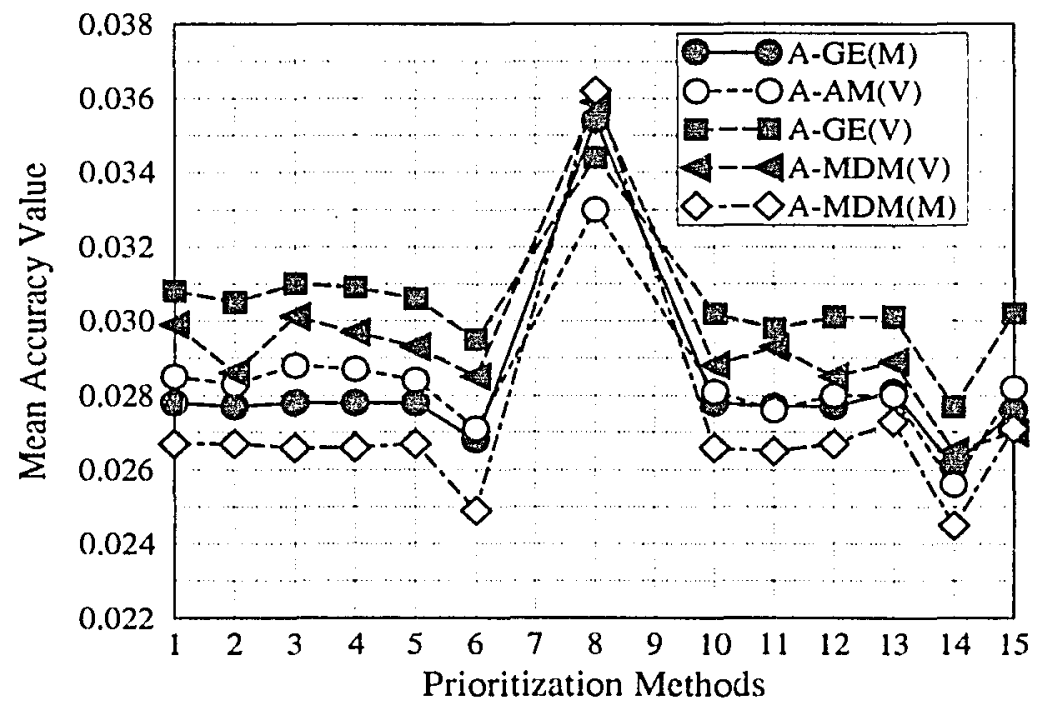

Figure 5.50: The Mean Accuracy of Aggregation Method for Category Four Empirical Data 


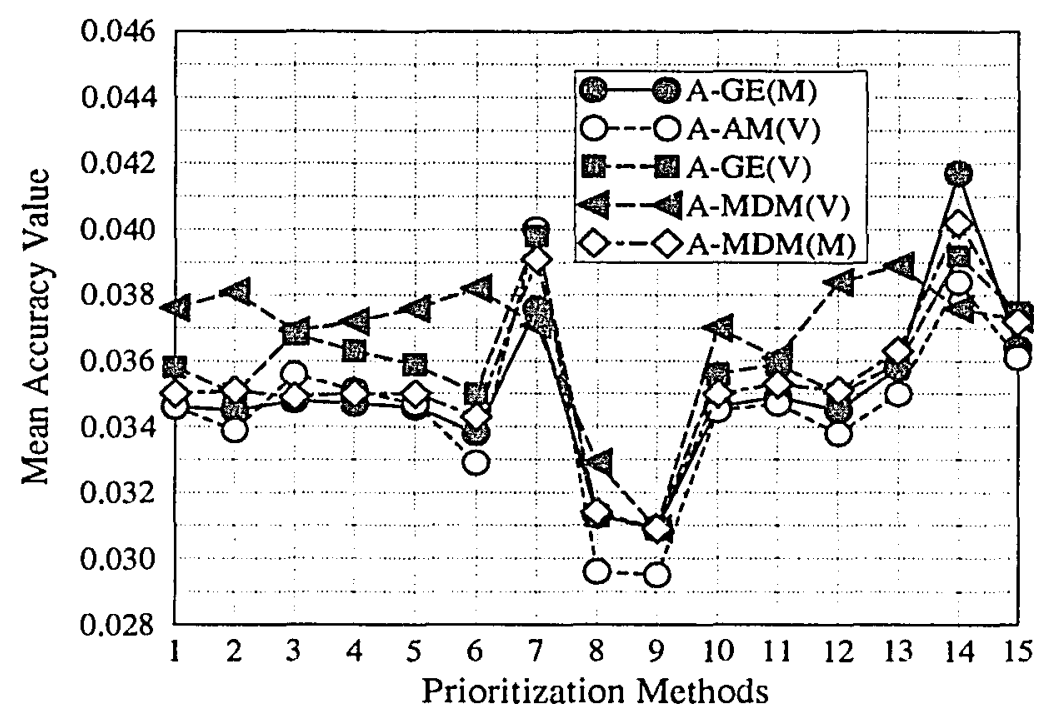

Figure 5.51: The Mean Accuracy of Aggregation Method for Category Five Empirical Data

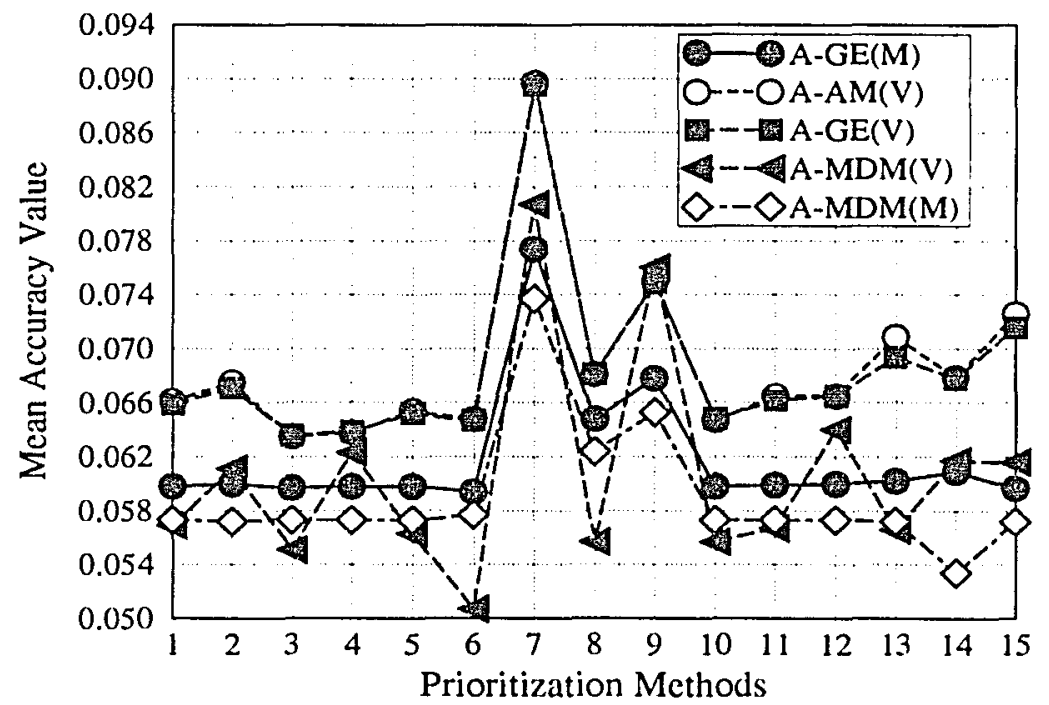

Figure 5.52: The Mean Accuracy of Aggregation Method for Category Six Empirical Data 


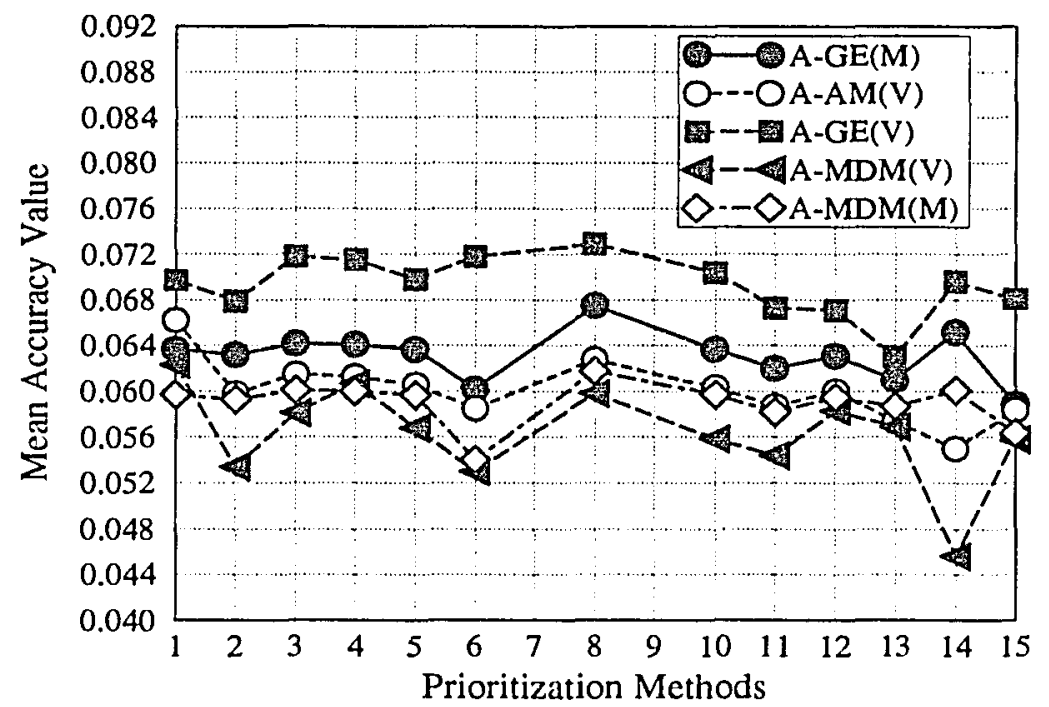

Figure 5.53: The Mean Accuracy of Aggregation Method for Category Seven Empirical Data

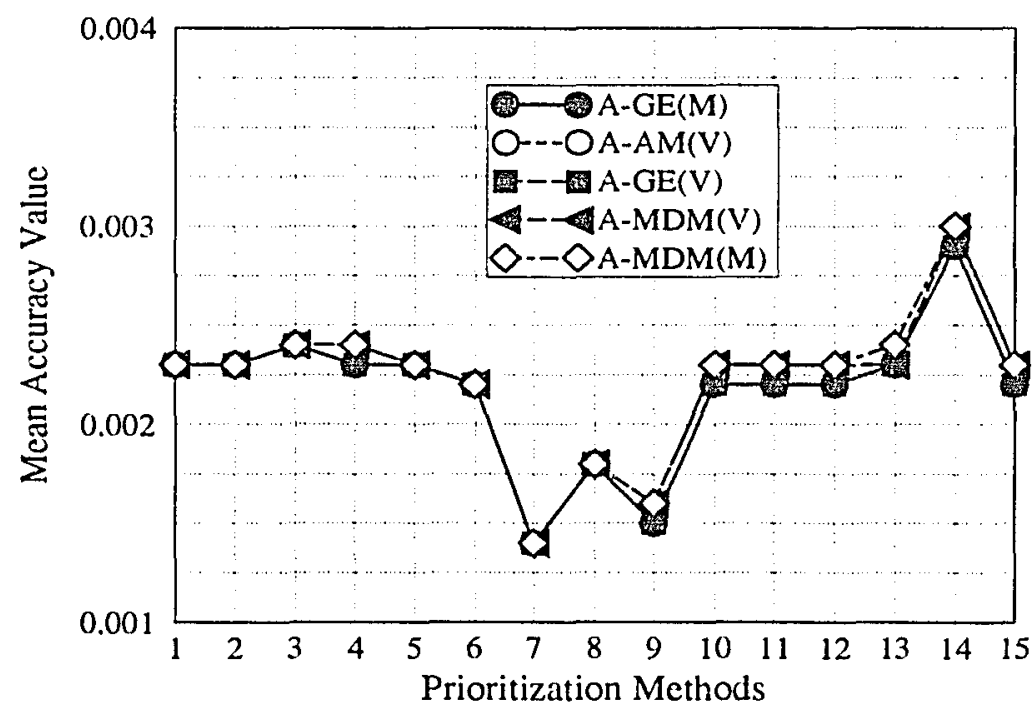

Figure 5.54: The Mean Disagreement of Aggregation Method for Category One Empirical Data 


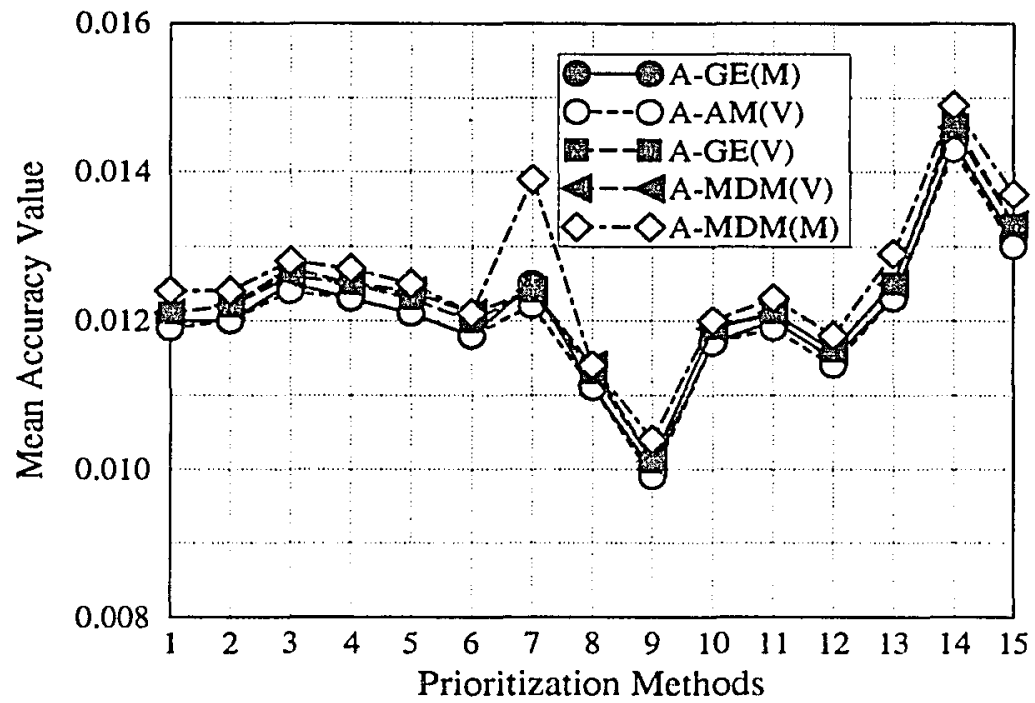

Figure 5.55: The Mean Disagreement of Aggregation Method for Category Two Empirical Data

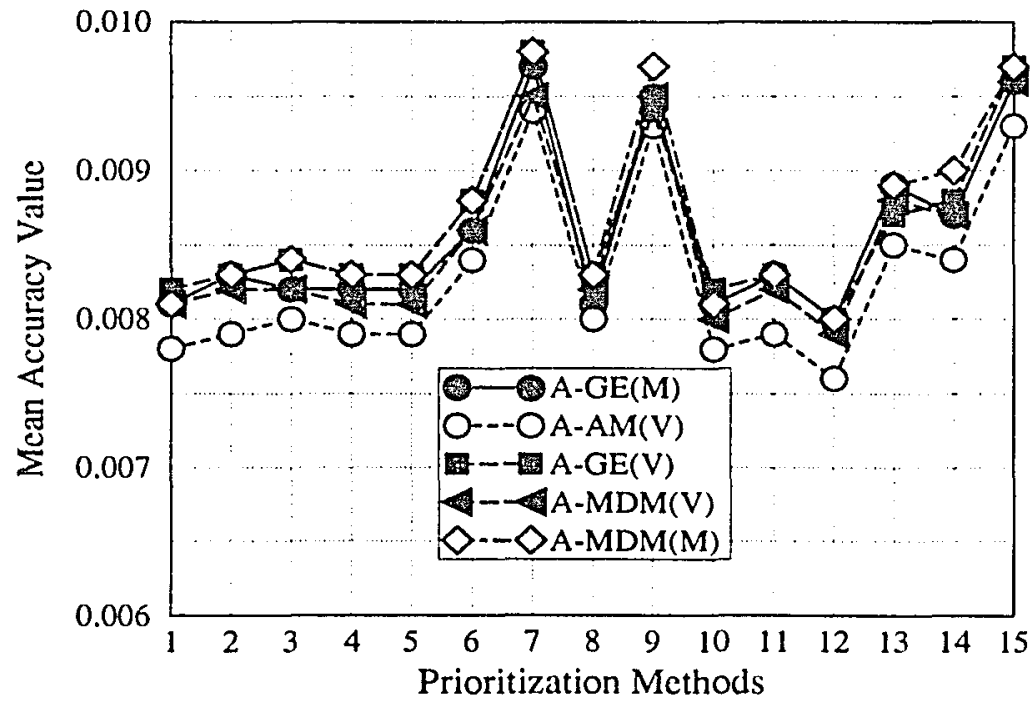

Figure 5.56: The Mean Disagreement of Aggregation Method for Category Three Empirical Data 


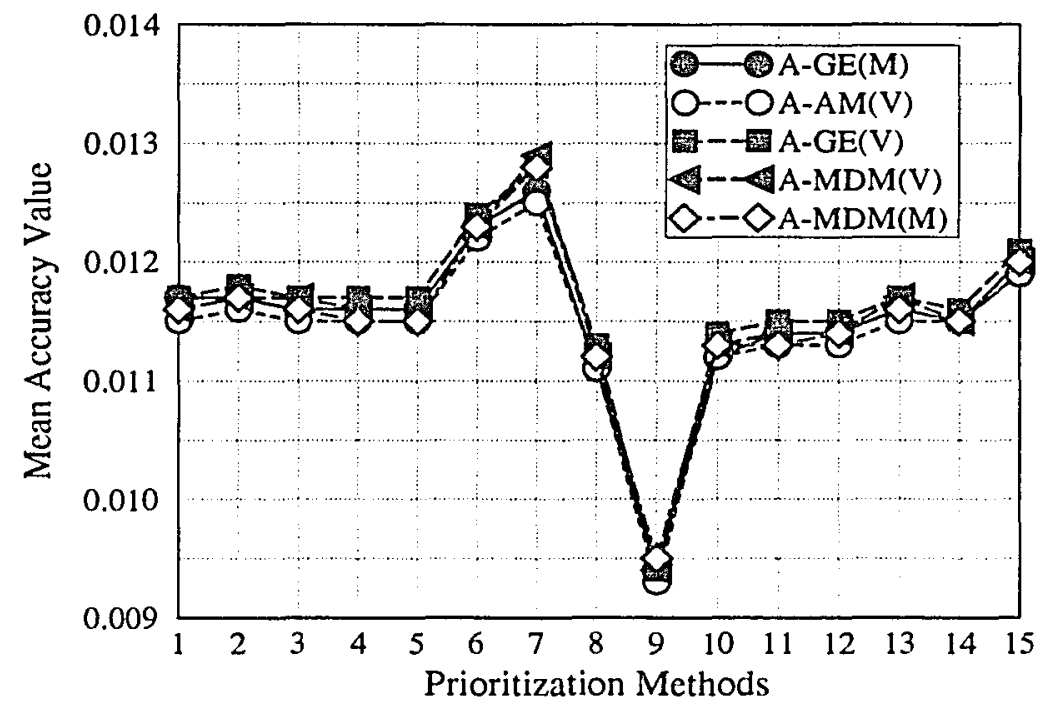

Figure 5.57: The Mean Disagreement of Aggregation Method for Category Four Empirical Data

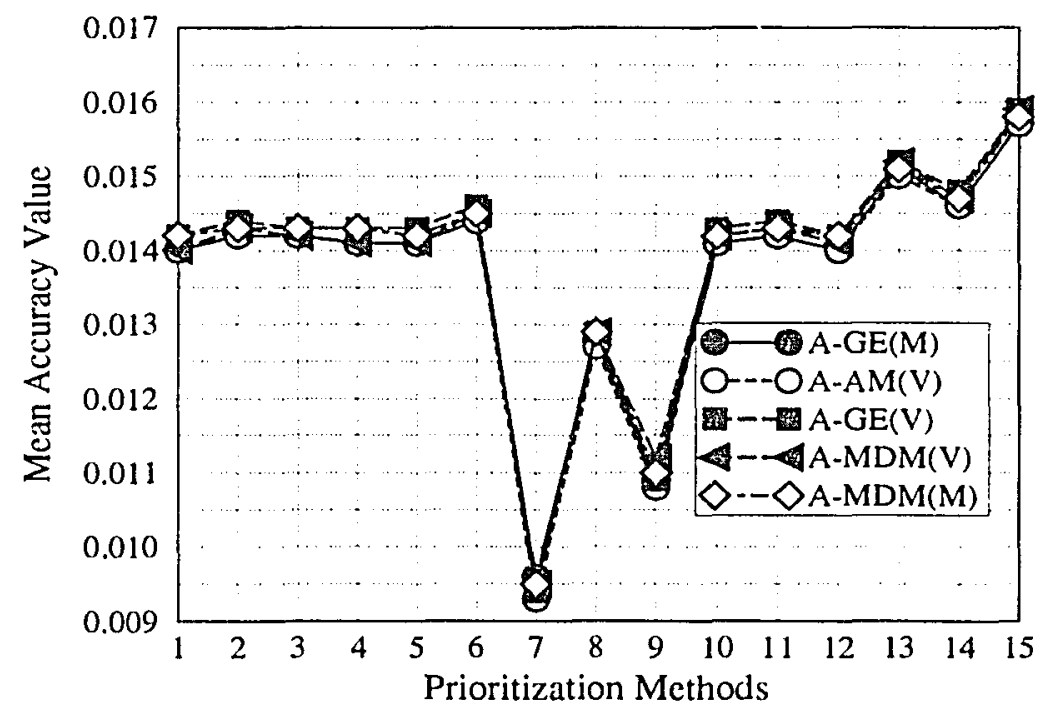

Figure 5.58: The Mean Disagreement of Aggregation Method for Category Five Empirical Data 


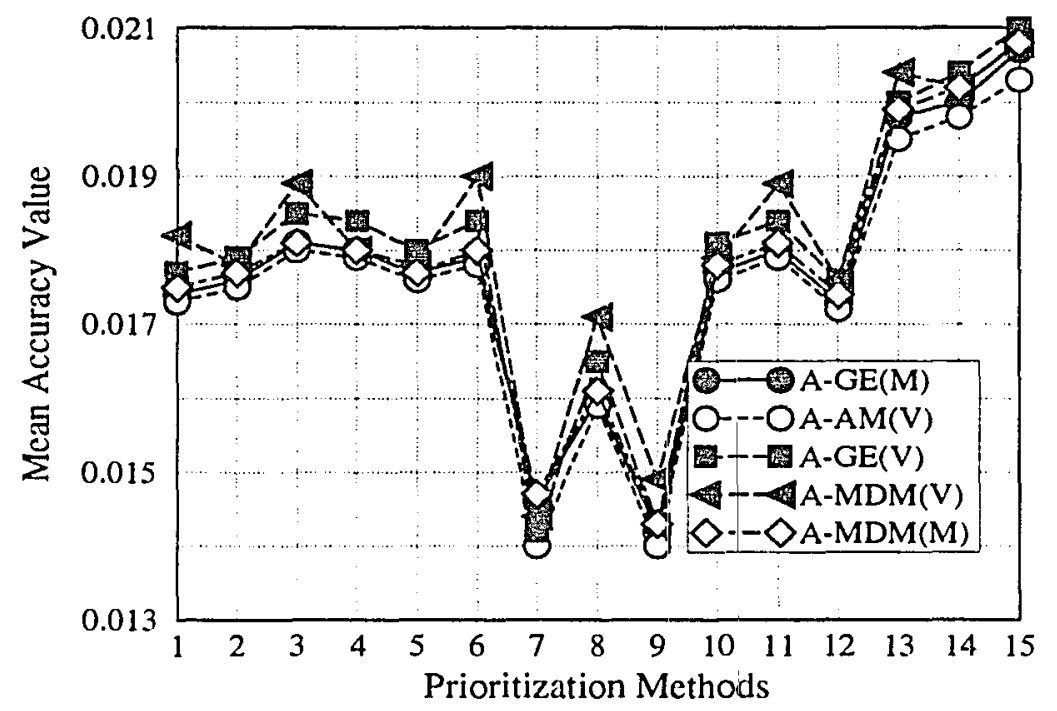

Figure 5.59: The Mean Disagreement of Aggregation Method for Category Six Empirical Data

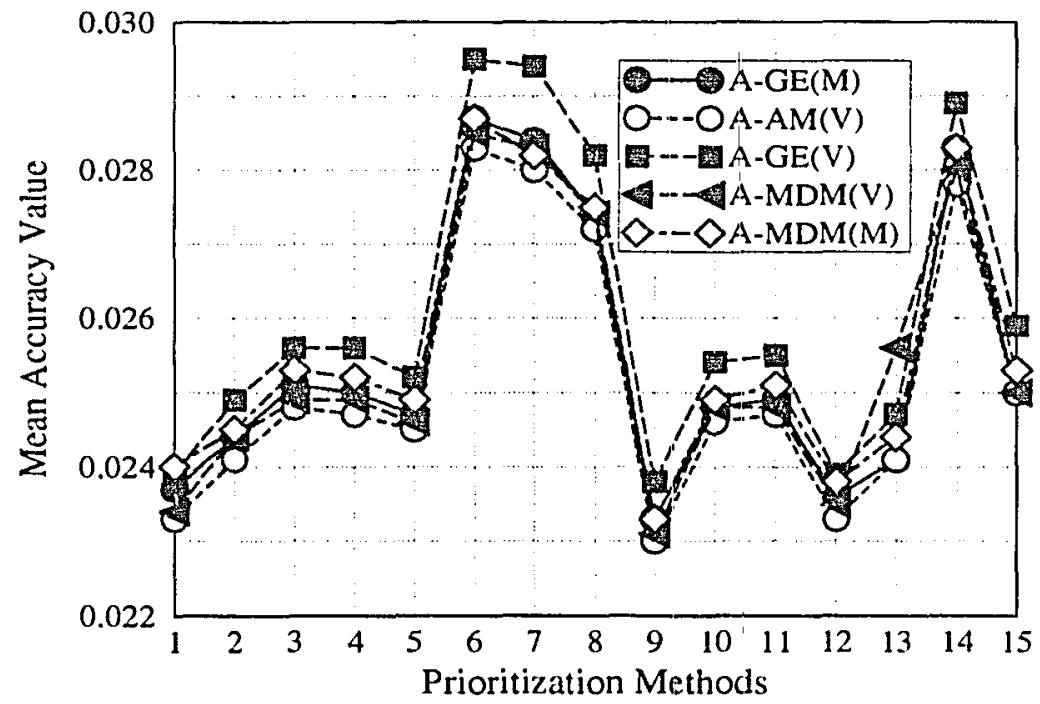

Figure 5.60: The Mean Disagreement of Aggregation Method for Category Seven Empirical Data 


\subsection{Summary of the Analysis}

So far in this chapter, the simulation results and empirical test results have been presented. We have discussed the following relationships:

- aggregation methods vs input data type

- aggregation methods vs prioritization methods

- aggregation methods vs number of decision makers

Those discussions dealt with different aspects of the performance of prioritization methods, aggregation methods and the relationship among them. In this section, the performances of prioritization methods and aggregation methods are summarized in tables $5.2,5.3,5.4$ and 5.5 according to the performance measurements. 
Table 5.2: The comparison of prioritization methods for accuracy

\begin{tabular}{||l|l||c||c||}
\hline ID NO. & ABBREVIATIONS & Empirical Data & Simulation Results \\
\hline \hline 1 & CSM & Very Good & Good \\
\hline 2 & R-EV & Very Good & Very Good \\
\hline 3 & L-EV & Very Good & Very Good \\
\hline 4 & AM-EV & Very Good & Very Good \\
\hline 5 & GM-EV & Very Good & Very Good \\
\hline 6 & EV[AA'] & Unstable & Unstable \\
\hline 7 & EV[A'A] & Unstable & Unstable \\
\hline 8 & AM - EV [AA'] & Unstable & Unstable \\
& AND EV[A'A] & & \\
\hline 9 & GE - EV [AA'] & Unstable & Unstable \\
\hline 10 & AND EV[A'A] & & \\
\hline 11 & GE & Very Good & Very Good \\
\hline 12 & MT & Very Good & Very Good \\
\hline 13 & SAV & Very Good & Very Good \\
\hline 14 & NEV & Unstable & Unstable \\
\hline 15 & LSM & Unstable & Unstable \\
\hline
\end{tabular}


Table 5.3: The comparison of prioritization methods for group disagreement

\begin{tabular}{||l|l||c||c||}
\hline ID NO. & ABBREVIATIONS & Empirical Data & Simulation Results \\
\hline \hline 1 & CSM & Very Good & Very Good \\
\hline 2 & R-EV & Very Good & Very Good \\
\hline 3 & L-EV & Very Good & Very Good \\
\hline 4 & AM-EV & Very Good & Very Good \\
\hline 5 & GM-EV & Very Good & Very Good \\
\hline 6 & EV[AA'] & Unstable & Unstable \\
\hline 7 & EV[A'A] & Unstable & Unstable \\
\hline 8 & AM - EV $[$ AA'] & Unstable & Unstable \\
& AND EV[A'A] & & \\
\hline 9 & GE - EV [AA'] & Unstable & Unstable \\
\hline 10 & AND EV[A'A] & & \\
\hline 11 & GE & Very Good & Very Good \\
\hline 12 & C-RSM & Very Good & Very Good \\
\hline 13 & SAV & Very Good & Very Good \\
\hline 14 & NEV & Unstable & Unstable \\
\hline 15 & LSM & Unstable & Unstable \\
\hline
\end{tabular}


Table 5.4: The comparison of aggregation methods for accuracy

\begin{tabular}{||l||c||c|c||}
\hline \multicolumn{1}{|c||}{$\begin{array}{c}\text { Aggregation } \\
\text { Methods }\end{array}$} & $\begin{array}{c}\text { Empirical } \\
\text { Data }\end{array}$ & Gamma & Uniform/Lognormal \\
\cline { 2 - 4 } $\begin{array}{l}\text { A-GE(V): The Geometric } \\
\text { Mean operates on the } \\
\text { priority vector }\end{array}$ & Good & Good & Very Good \\
\hline $\begin{array}{l}\text { A-GE(M): The Geometric } \\
\text { Mean operates on the } \\
\text { pairwise comparison matrix }\end{array}$ & Good & Good & Very Good \\
\hline $\begin{array}{l}\text { A-AM(V): The Arithmetic } \\
\text { Mean operates on the } \\
\text { priority vector }\end{array}$ & Good & Good & Very Good \\
\hline $\begin{array}{l}\text { A-MDM(M): The } \\
\text { Minimum Distance Method } \\
\text { operates on the pairwise } \\
\text { comparison matrix }\end{array}$ & Very Good & Very Good & Good \\
\hline $\begin{array}{l}\text { A-MDM(V): The } \\
\text { Minimum Distance Method } \\
\text { Operates on the } \\
\text { priority vector }\end{array}$ & Very Good & Very Good & Fairly Good \\
\hline
\end{tabular}


Table 5.5: The comparison of aggregation methods for group disagreement

\begin{tabular}{||l||c||c|c||}
\hline \multicolumn{1}{||c||}{$\begin{array}{l}\text { Aggregation } \\
\text { Methods }\end{array}$} & $\begin{array}{c}\text { Empirical } \\
\text { Data }\end{array}$ & Gamma & Uniform/Lognormal \\
\cline { 3 - 4 } & Good & Good & Good \\
\hline $\begin{array}{l}\text { A-GE(V): The Geometric } \\
\text { Mriority vector }\end{array}$ & Good & Good & Good \\
\hline $\begin{array}{l}\text { A-GE(M): The Geometric } \\
\text { Mean operates on the } \\
\text { pairwise comparison matrix }\end{array}$ & Very Good & Very Good & Very Good \\
\hline $\begin{array}{l}\text { A-AM(V): The Arithmetic } \\
\text { Mean operates on the } \\
\text { priority vector }\end{array}$ & Good & Good & Good \\
\hline $\begin{array}{l}\text { A-MDM(M): The } \\
\text { Minimum Distance Method } \\
\text { operates on the pairwise } \\
\text { comparison matrix }\end{array}$ & Good & Good & Good \\
\hline $\begin{array}{l}\text { A-MDM(V): The } \\
\text { Minimum Distance Method } \\
\text { Operates on the } \\
\text { priority vector }\end{array}$ & & & \\
\hline
\end{tabular}




\section{Chapter 6}

\section{CONCLUSIONS}

In this dissertation, we have fulfilled the following two objectives:

1. Using the general distance concept developed by $Y u$ [4] and Cook et al. [5], and the representation of group aggregated judgments $(\bar{A}$ or $\bar{V})$ as weighted geometric mean of group members' judgments $\left(\left\{A_{i}\right\}\right.$ or $\left\{V_{i}\right\}$, where $\left.i=1, \cdots, m\right)$, a new aggregation method -Minimum Distance Method (MDM)-was developed. Both approaches (i.e. Approach A and Approach B) were investigated for the MDM. Approach A stands for the MDM operated on pairwise comparison matrices. Approach B stands for the MDM operated on priority vectors.

2. Using the simulation method and empirical test approach, evaluation of the performance of aggregation methods was conducted. Two performance measurements were used for this purpose. Accuracy measurement was to measure how close the aggregated group judgments in terms of relative weights matched the "real" relative weights of decision elements. The group disagreement measurement was designed to measure the deviation between the group members' 
judgments and the aggregated group judgments. Aggregation methods that were under investigation are:

- geometric mean operated on pairwise comparison matrices

- geometric mean operated on priority vectors

- arithmetic mean operated on priority vectors

- MDM operated on pairwise comparison matrices

- MDM operated on priority vectors

All of these studies are under the framework of the Hierarchical Decision Model (HDM) via the Analytic Hierarchy Process (AHP) with emphasis on the pairwise comparison technique. In addition to the above two objectives, we surveyed the literature categorized and summarized research works in the AHP area, Group decision making characteristics and techniques are also discussed in chapter 2 . In the following sections, we conclude our research reported in this dissertation, which includes the findings of this research and future works.

\subsection{Main Results}

Based on our study, simulation results and empirical test results, we conclude that:

- The most important factors in the aggregation and estimation of pairwise comparison judgments are the probability distribution of error terms and the 
aggregation method. Using an appropriate aggregation method will result in significant impirovement of decision quality in terms of accuracy.

- MDM outperforms the other aggregation methods in terms of accuracy measurement when empirical daita are used.

- Simulation results also indicate that the MDM outperforms the other aggregation methods in term of accuracy measurement under certain distributions of the input data, such as the gamma distribution.

- MDM works best on pairwise comparison matrix vs. final priority vector.

- Geometric mean and arithmetic mean produce better results in terms of accuracy measurement when the simulated perturbations follow a uniform distribution or a lognormal distribution.

- The arithmetic mean aggregation method performs better than any other aggregation method in terms of group disagreement. But the difference among aggregation methods is very small, which has also been demonstrated in the empirical test.

- The influence of the prioritization method on the aggregation method is not significant. There is no combination of aggregation method and prioritization method that yields markedly different results. As indicated in the empirical test, for any given category, one aggregation method performs better than other aggregation method for all prioritization methods. 
- The simulation and empirical test results suggested that the following prioritization methods could be dropped out.

1. $\mathbf{E V}\left[\mathbf{A A}^{\prime}\right]$ - eigenvector of $\left[\mathrm{AA}^{\prime}\right]$ matrix

2. $\mathbf{E V}\left[A^{\prime} A\right]$ - eigenvector of $\left[A^{\prime} A\right]$ matrix

3. AM EV $\left[\mathrm{AA}^{\prime}\right]$ and $\operatorname{EV}\left[\mathrm{A}^{\prime} \mathrm{A}\right]$ - arithmetic mean of eigenvector of $\left[\mathrm{AA}^{\prime}\right]$ and $\left[A^{\prime} A\right]$ matrices

4. GM EV[AA'] and EV[A'A] - geometric mean eigenvector of [AA'] and $\left[A^{\prime} A\right]$ matrices

because they generally produce worse results than any other prioritization method in terms of accuracy, and they show the highest degree of sensitivity toward the underlying distribution of error terms.

- Simulation results also indicated that increasing the number of decision makers in a group will effectively increase the quality of the decision making. If all group members uniformly have one of the following input data types uniform distribution, lognormal distribution and gamma distribution. But the improvement diminish with further increase of number of group members.

\subsection{Contributions}

The major contributions of this dissertation are as follows:

- A new approach - Minimum Distance Method (MDM) - to aggregate group pairwise comparison judgments has been developed. 
- MDM, which employs the general distance concept, was proven to be very appealing to the compromise nature of group decision making.

- MDM preserves all characteristics of the functional approach (i.e. geometric mean approach), which was proposed by Aczel and Saaty $[6,7,8]$.

- MDM can aggregate not only the pairwise comparison matrices, but also the final priority vectors.

- The sensitivity analysis can be performed on MDM to investigate the effect of varying the decision makers' relative importance in terms of weights in the goal programming. Sensitivity analysis allows us to make robust decisions.

- A methodology has been developed and demonstrated for the evaluation of aggregation methods

\subsection{Suggested Future Work}

This study focus on aggregating the group pairwise comparison judgments as well as the performance issues of aggregation methods. These are only some of the aspects of decision analysis of HDM or AHP. There is much additional research to be done. The research areas listed below would enhance the findings of this dissertation.

1. Further Study of the Aggregation Methods: Further study of the aggregation methods with more complete and more readily available experi- 
mental data. Those experimental data should include the changes of decision elements, decision makers and decision makers' knowledge level of decision problem.

2. Sensitivity and Comparison Analysis in the Hierarchy: The pairwise comparison technique described in the dissertation is a building-block of the HDM or AHP, which has been developed for complex decision-making problems to select alternatives with respect to a specified objective through multiple criteria and multiple levels. There are several approaches for aggregating the vectors of relative weights under multiple criteria and multiple level. To put the group aggregation methods into the context of multiple criteria and multiple level is very important. The questions for this study would be what is the influence of the methods for aggregating the relative weights in hierarchy to the aggregation methods among decision makers. What is the best combinations of them to yield the best performance as regarding to accuracy and group disagreement, etc

3. Measurement to Test the Judgment Distribution: In the dissertation, the simulation study and empirical test have demonstrated that the performance of aggregation methods is highly dependent on the input data type. Therefore, it is highly desirable to have some kind of measurement to link the judgment distribution to the choice of aggregation methods.

4. Software and Field Testing Developing software to facilitate the usage of these methods in real situations, and also help the field testing of the software. 


\section{Appendix A}

\section{The Prioritization Methods in AHP}

The input matrix of pairwise comparisons shows the extent that one element is preferred over another in achieving an objective of one level higher in hierarchy. If there were no measurement errors in the input data (i.e. pairwise comparison matrix), the $n \times n$ square matrix of pairwise comparisons would be:

$$
A_{\mathrm{I}}=\left[\begin{array}{ccccc}
\left\{a_{11}\right\}_{1} & \left\{a_{12}\right\}_{1} & \ldots & \ldots & \left\{a_{1 n}\right\}_{1} \\
\left\{a_{21}\right\}_{1} & \left\{a_{22}\right\}_{1} & \ldots & \ldots & \left\{a_{2 n}\right\}_{1} \\
\cdot & \cdot & \ldots & \ldots & \cdot \\
\cdot & & \ldots & \ldots & \cdot \\
\cdot & \cdot & \ldots & \ldots & \cdot \\
\left\{a_{n 1}\right\}_{1} & \left\{a_{n 2}\right\}_{1} & \ldots & \ldots & \left\{a_{n n}\right\}_{1}
\end{array}\right]
$$

where $\mathrm{n}$ is the number of decision elements, $\left\{a_{j k}\right\}_{\imath}=\left\{v_{j}\right\}_{1} /\left\{v_{k}\right\}_{l}$ and $V_{\mathfrak{I}}=\left(\left\{v_{1}\right\}_{l}\right.$, $\left.\cdots,\left\{v_{n}\right\}_{1}\right)$ is the vector of actual relative weights of $n$ elemets. However, the pairwise comparison matrix $A=\left(a_{j k}\right)$, which are actual judgments by real people, contains measurement errors. Therefore, $a_{j k} \neq\left\{v_{j}\right\}_{1} /\left\{v_{k}\right\}_{l}$. Furthermore, in most decision cases, the value of $V_{\pi}$ is unknown, the estimation methods in the AHP attempt to estimate the vector of relative weights $V=\left(v_{1}, \cdots, v_{n}\right)$, which is the estimation of 
estimation of $V_{\mathbb{I}}$, from the pairwise comparison matrix $A$.

In developing the AHP approach, Saaty [2] was the first to suggest the eigenvalue method for deriving the $V$ from the pairwise comparison matrix $A$. Since then, a number of other estimation methods have been proposed in the literature. In supporting this dissertation, this appendix reviews these estimation methods briefly.

\section{A.1 The (Right) Eigenvalue Method}

The eigenvalue method is based on the following argument. If there were no errors in measurement, the relative weights (we also call priority vector) could be trivially obtained from each one of $n$ rows of matrix A. In other words, if matrix $A$ has rank 1, and then the following holds:

$$
A_{\mathfrak{T}} V_{\mathfrak{T}}=n V_{\mathfrak{I}}
$$

The AHP acknowledges that the matrix A, which is obtained from real people, contains inconsistencies. The estimation of priority vector $V$ could be obtained similar to expression (A.2) from:

$$
A V=\lambda_{\max } V
$$

where $\lambda_{\max }$ is the largest eigenvalue of $A$, and $V$ constitutes the estimation of $V_{\mathfrak{I}}$.

In expression (A.3), $\lambda_{\max }$ may be considered the estimation of $n$. Saaty [3] has shown that $\lambda_{\max }$ is always greater than or equal to $n$. The closer the value of $\lambda_{\max }$ is to $n$, the more consistent are the observed values of $A$. This property has led to the consistency index as:

$$
\mu=\frac{\lambda_{\max }-n}{n-1}
$$




\section{A.2 The Mean Transformation Method}

Zahedi [66] argues that enforcing the reciprocal condition on the input data ( i.e. pairwise comparison matrix) creates unnecessary dependency among observations and loses additional information contained in elements of the lower triangle of Matrix A. Hence, the data for all off-diagonal elements should be collected, which means to obtain a full input matrix; and the estimator should enforce consistency requirements. This estimator consists of:

$$
\operatorname{Min} \sum_{j} \sum_{k}\left(\hat{b}_{j k}-v_{k}\right)^{2} \quad \text { for } v_{j}>0
$$

where $\hat{b}_{j k}$ is the element of a matrix obtained from transposing Matrix A and dividing each of its row elements by the row sum. This transformation changes elements of Matrix A from pairwise preferences to relative weights, each observed $n$ times.

In other words, the mean transformation method minimizes the squared estimation error and enforces the constraint that each row of the input matrix should lead to the same estimation of relative weights, which is a strict form of the consistency requirement.

The solution of the above minimization problem lead to

$$
v_{j}=\sum_{j}^{n} \frac{\hat{b}_{j k}}{n}
$$

where $\hat{b}_{j k}$ is defined above. 


\section{A.3 Row Geometric Mean (or the Logarithmic Least Squares) Method}

This method was fully developed to the argument for this method by Crawford and Williams [27]. The estimation criterion in this method is the minimization of the sum of square distances of the natural logarithm of $a_{j k}$ from the logarithm of $v_{j} / v_{k}:$

$$
\operatorname{Min} \sum_{j \neq k}^{n}\left[\ln \left(a_{j k}\right)-\left(\ln \left(v_{j}\right)-\ln \left(v_{k}\right)\right] \quad v_{j}, v_{k}>0\right.
$$

This minimization lead to the estimation of relative weights as the geometric mean of the row elements of Matrix A:

$$
v_{j}=\left(\prod_{k=1}^{n} a_{j k}\right)^{\frac{1}{n}}
$$

\section{A.4 The Column Geometric Mean Method}

This method is similar to the row geometric method, except that the geometric mean is calculated over the columns of matrix A:

$$
v_{k}=\left(\prod_{j=1}^{n} a_{j k}\right)^{\frac{1}{n}}
$$

\section{A.5 The Harmonic Mean (the left eigenvector) method}

Johnson, Beine and Wang [18] presented the possibility of using the left eigenvector as an estimator of relative weights:

$$
V A=\lambda_{\max } V
$$


It has been shown that the left and right eigenvectors are asymmetric in ranking the elements.

\section{A.6 The Simple Row Average}

One of the most simplest methods of estimating relative weights is to compute the average of the row elements of Matrix A as shown in [3] by Saaty:

$$
v_{j}=\frac{\sum_{k=1}^{n} a_{j k}}{n}
$$

\section{A.7 Ordinary Least Squares}

This Least Squares Method (LSM), which was mentioned by Chu and et al. [65], determines the nearest (in the Euclidean metric) vector in $R_{n \times n}$ the elements of which have the form $v_{j} / v_{k}$ :

$$
\operatorname{Min} \sum_{j=1}^{n} \sum_{k=1}^{n}\left(a_{j k}-\frac{v_{j}}{v_{k}}\right)^{2}
$$

\section{A.8 Constant Sum Method}

This method, which is based on the work of Comery [37], was refined by Kocaoglu [1]. The term constant-sum refers to the procedure for expressing judgments as a total of 100 points which are divided between the two elements.

With the pairwise comparison Matrix $A$, the next step is to obtain the second matrix (call it Mat,rix $B$ ) by dividing each element in a row by the element in the next row:

$$
b_{j k}=\frac{a_{j k}}{a_{j+1, k}} \quad j=1, \cdots, n-1, j=1, \cdots, n
$$


Due to the inconsistency, the estimate for the ratio of the weight of jth element to that of its successor is obtained by taking arithmetic mean of the cells values in the jth row.

$$
\bar{b}_{j}=\frac{1}{n} \sum_{k=1}^{n} b_{j k}
$$

The relative values of the elements, $r_{j}$, are obtained by assigning a value of 1.0 to the element in the last row, calculating the other element values, and then normalizing them for the $n$ elements:

$$
\begin{aligned}
& e_{n}=1, \\
& e_{n-1}=1, \times \bar{b}_{n-1} \\
& \ldots \\
& e_{2}=e_{3} \bar{b}_{2}, \\
& e_{1}=e_{2} \bar{b}_{1} . \quad \text { therefore }: \\
& r_{j}=\frac{e_{j}}{\sum_{j=1}^{n}, \quad \sum_{j=1}^{n} r_{j}=1}
\end{aligned}
$$

So far, $r_{j}$ has been obtained from only one orientation of $\bar{b}_{j}$, that is the order in which the elements are arranged. In cases of inconsistency, $\bar{b}_{j}$ based on the other orientations shows perturbations. Hence, it is required to estimate $r_{j}$ from all possible orientations, that is, all permutations of the $\mathrm{n}$ elements $(n !)$. 
The final relative values of elements, $v_{j}$, are the means of the $n$ ! values obtained in $n$ ! orientations of the rows:

$$
\begin{aligned}
& v_{j}=\frac{1}{n !} \sum_{k=1}^{n !} r_{j k} \\
& \sum_{j=1}^{n} v_{j}=1
\end{aligned}
$$

where $r_{j k}$ is the relative value of element $j$ in the kth orientation.

\section{A.9 Column-Row Sums Method}

This technique developed by $\operatorname{Ra}[23,55]$ uses geometric means of normalized inverse column sums (NICS) and the normalized row sums (NRS).

In the column orientation, inverse of the sum of cell values in each column divided by the total sum gives the relative weight of element in that column to total elements' weights:

$$
N I C S_{j}=\frac{\frac{1}{\sum_{j=1}^{n} a_{j k}}}{\sum_{k=1}^{n}\left(\frac{1}{\sum_{j=1}^{n} a_{j k}}\right)}
$$

In the row orientation, the same relative weight is derived from the sum of the cell values in a row divided by the total sum:

$$
N R S_{j}=\frac{\sum_{k=1}^{n} a_{j k}}{\sum_{j=1}^{n} \sum_{k=1}^{n} a_{j k}}
$$

However, in a practical case, matrix $\mathrm{A}$ is inconsistent, and thus, the two ratios, NICS and NRS, are not always identical. 
The final relative weights of the jth elements, $v_{j}$, are obtained by taking the geometric mean of $N I C S_{j}$ and $N R S_{j}$, and normalizing them:

$$
v_{j}=\frac{\left(N I C S_{j} \times N R S_{j}\right)^{\frac{1}{2}}}{\sum_{j=1}^{n}\left(N I C S_{j} \times N I C S_{j}\right)^{\frac{1}{2}}}
$$

Simplifying the expression (A.25) (see [23] for detail), the relative weight of the jth element, $v_{j}$, is represented by row sums (RS) and column sums (CS):

$$
v_{j}=\frac{\left(\frac{R S_{j}}{C S_{j}}\right)^{\frac{1}{2}}}{\sum_{j=1}^{n}\left(\frac{R S_{j}}{C S_{j}}\right)^{\frac{1}{2}}}
$$




\section{Appendix B}

\section{The Mean and Standard Deviation of Accuracy Measurement from Simulation}

The entries of the following tables are the mean and standard deviation of accuracy measurement from simulation study. All of them are in pairs in each cell, the number inside the parenthesis is the standard deviation of accuracy measurement, and the number without parenthesis is the mean of accuracy measurement. All the notations in the tables follow the definition Table 4.1 and 4.2. Other notations such as $\mathrm{N}$ stands for number of decision elements simulated (i.e. the pairwise comparison matrix size). $M$ is the number of decision makers in the simulation process. UF stands for Uniform probability distribution. LN stands for lognormal probability distribution and GA is the gamma probability distribution. 
Table B.1: Mean and Standard Deviation of Accuracy Measurement $\left(d_{1}\right)$ from Simulation (With $\mathrm{N}=8, \mathrm{M}=3$, scale $[1 / 9,9], \mathrm{T}=500$ )

\begin{tabular}{||l||c|c|c||c|c|c||}
\hline \multicolumn{1}{|c||}{ M=3 } & \multicolumn{3}{c|}{ A-GE(V) } & \multicolumn{3}{c||}{ A-GE(M) } \\
\hline ABBREVIATIONS & UF & LN & GA & UF & LN & GA \\
\hline \hline CSM & 0.01206 & 0.00923 & 0.03542 & 0.01110 & 0.00896 & 0.02541 \\
& $(0.00424)$ & $(0.00393)$ & $(0.00955)$ & $(0.00401)$ & $(0.00384)$ & $(0.00790)$ \\
\hline R-EV & 0.00858 & 0.00622 & 0.02486 & 0.00858 & 0.00620 & 0.02329 \\
& $(0.00283)$ & $(0.00224)$ & $(0.00771)$ & $(0.00285)$ & $(0.00223)$ & $(0.00762)$ \\
\hline L-EV & 0.00866 & 0.00619 & 0.02571 & 0.00870 & 0.00617 & 0.02379 \\
& $(0.00287)$ & $(0.00221)$ & $(0.00748)$ & $(0.00289)$ & $(0.00220)$ & $(0.00748)$ \\
\hline AM-EV & 0.00862 & 0.00618 & 0.02516 & 0.00867 & 0.00617 & 0.02351 \\
& $(0.00286)$ & $(0.00221)$ & $(0.00735)$ & $(0.00288)$ & $(0.00221)$ & $(0.00739)$ \\
\hline GM-EV & 0.00857 & 0.00618 & 0.02439 & 0.00861 & 0.00618 & 0.02315 \\
& $(0.00283)$ & $(0.00221)$ & $(0.00737)$ & $(0.00285)$ & $(0.00221)$ & $(0.00738)$ \\
\hline EV[AA'] & 0.01043 & 0.00767 & 0.03406 & 0.01042 & 0.00760 & 0.03209 \\
& $(0.00384)$ & $(0.00283)$ & $(0.01113)$ & $(0.00388)$ & $(0.00281)$ & $(0.01153)$ \\
\hline EV[A'A] & 0.01398 & 0.00973 & 0.02334 & 0.01402 & 0.00963 & 0.02242 \\
& $(0.00513)$ & $(0.00371)$ & $(0.00821)$ & $(0.00528)$ & $(0.00376)$ & $(0.00797)$ \\
\hline AM - EV[AA'] & 0.00965 & 0.00712 & 0.03068 & 0.00959 & 0.00705 & 0.02880 \\
AND EV[A'A] & $(0.00358)$ & $(0.00266)$ & $(0.00984)$ & $(0.00359)$ & $(0.00265)$ & $(0.01011)$ \\
\hline GE- EV[AA'] & 0.00946 & 0.00689 & 0.02392 & 0.00945 & 0.00686 & 0.02194 \\
AND EV[A'A] & $(0.00336)$ & $(0.00256)$ & $(0.00729)$ & $(0.00335)$ & $(0.00255)$ & $(0.00712)$ \\
\hline GE & 0.00859 & 0.00618 & 0.02286 & 0.00860 & 0.00618 & 0.02272 \\
& $(0.00284)$ & $(0.00221)$ & $(0.00702)$ & $(0.00285)$ & $(0.00222)$ & $(0.00722)$ \\
\hline C-RSM & 0.00870 & 0.00632 & 0.02387 & 0.00872 & 0.00631 & 0.02241 \\
& $(0.00293)$ & $(0.00230)$ & $(0.00701)$ & $(0.00296)$ & $(0.00229)$ & $(0.00708)$ \\
\hline MT & 0.00852 & 0.00617 & 0.02315 & 0.00854 & 0.00618 & 0.02266 \\
& $(0.00280)$ & $(0.00221)$ & $(0.00678)$ & $(0.00283)$ & $(0.00222)$ & $(0.00705)$ \\
\hline SAV & 0.01057 & 0.00745 & 0.02130 & 0.01057 & 0.00743 & 0.02010 \\
. & $(0.00373)$ & $(0.00275)$ & $(0.00672)$ & $(0.00381)$ & $(0.00274)$ & $(0.00648)$ \\
\hline NEV & 0.01032 & 0.00785 & 0.02977 & 0.01126 & 0.00809 & 0.02728 \\
& $(0.00374)$ & $(0.00275)$ & $(0.01047)$ & $(0.00400)$ & $(0.00286)$ & $(0.00990)$ \\
\hline LSM & 0.01282 & 0.00887 & 0.02269 & 0.01278 & 0.00880 & 0.02010 \\
& $(0.00499)$ & $(0.00358)$ & $(0.00878)$ & $(0.00513)$ & $(0.00359)$ & $(0.00737)$ \\
\hline
\end{tabular}


Table B.2: Mean and Standard Deviation of Accuracy Measurement $\left(d_{1}\right)$ from Simulation (With $N=8, M=3$, scale $[1 / 9,9], T=500$ ) [Continued]

\begin{tabular}{|c|c|c|c|c|c|c|}
\hline $\mathrm{M}=3$ & \multicolumn{3}{|c|}{$\overline{A-A M(V)}$} & \multicolumn{3}{|c|}{$\overline{A-M D M(M)}$} \\
\hline ABBREVIATIONS & $\overline{U F}$ & $\mathrm{LN}$ & $\overline{\mathrm{GA}}$ & $\overline{\mathrm{UF}}$ & $\mathrm{IN}$ & $\overline{\mathrm{GA}}$ \\
\hline$\overline{\mathrm{CSM}}$ & $\begin{array}{c}0.01220 \\
(0.00433)\end{array}$ & $\begin{array}{c}0.00923 \\
(0.00399)\end{array}$ & $\begin{array}{c}0.03607 \\
(0.00941)\end{array}$ & $\begin{array}{c}0.01127 \\
(0.00407)\end{array}$ & $\begin{array}{c}0.00910 \\
(0.00382)\end{array}$ & $\begin{array}{c}0.02706 \\
(0.00869)\end{array}$ \\
\hline R-EV & $\begin{array}{c}0.00856 \\
(0.00281)\end{array}$ & $\begin{array}{c}0.00622 \\
(0.00225)\end{array}$ & $\begin{array}{c}0.02513 \\
(0.00797)\end{array}$ & $\begin{array}{c}0.00870 \\
(0.00297)\end{array}$ & $\begin{array}{c}0.00634 \\
(0.00224)\end{array}$ & $\begin{array}{c}0.02439 \\
(0.00834)\end{array}$ \\
\hline L-EV & $\begin{array}{c}0.00867 \\
(0.00287)\end{array}$ & $\begin{array}{c}0.00619 \\
(0.00221)\end{array}$ & $\begin{array}{c}0.02533 \\
(0.00771)\end{array}$ & $\begin{array}{c}0.00884 \\
(0.00300)\end{array}$ & $\begin{array}{c}0.00631 \\
(0.00222)\end{array}$ & $\begin{array}{c}0.02470 \\
(0.00805)\end{array}$ \\
\hline AM-EV & $\begin{array}{c}0.00863 \\
(0.00285)\end{array}$ & $\begin{array}{c}0.00618 \\
(0.00221)\end{array}$ & $\begin{array}{c}0.02488 \\
(0.00757)\end{array}$ & $\begin{array}{c}0.00880 \\
(0.00299)\end{array}$ & $\begin{array}{c}0.00631 \\
(0.00222)\end{array}$ & $\begin{array}{c}0.02439 \\
(0.00796)\end{array}$ \\
\hline GE-EV & $\begin{array}{c}0.00856 \\
(0.00282) \\
\end{array}$ & $\begin{array}{c}0.00618 \\
(0.00222)\end{array}$ & $\begin{array}{c}0.02434 \\
(0.00759)\end{array}$ & $\begin{array}{c}0.00874 \\
(0.00297)\end{array}$ & $\begin{array}{c}0.00632 \\
(0.00223)\end{array}$ & $\begin{array}{c}0.02404 \\
(0.00800)\end{array}$ \\
\hline$\overline{\mathrm{EV}}\left[\mathrm{AA}^{\prime}\right]$ & $\begin{array}{c}0.01045 \\
(0.00384)\end{array}$ & $\begin{array}{c}0.00767 \\
(0.00283)\end{array}$ & $\begin{array}{c}0.03310 \\
(0.01161)\end{array}$ & $\begin{array}{c}0.01071 \\
(0.00394)\end{array}$ & $\begin{array}{c}0.00783 \\
(0.00289)\end{array}$ & $\begin{array}{c}0.03354 \\
(0.01302)\end{array}$ \\
\hline $\mathrm{EV}\left[\mathrm{A}^{\prime} \mathrm{A}\right]$ & $\begin{array}{c}0.01404 \\
(0.00513) \\
\end{array}$ & $\begin{array}{l}0.00973 \\
(0.00371)\end{array}$ & $\begin{array}{c}0.02361 \\
(0.00845)\end{array}$ & $\begin{array}{c}0.01449 \\
(0.00531)\end{array}$ & $\begin{array}{c}0.00996 \\
(0.00390)\end{array}$ & $\begin{array}{c}0.02282 \\
(0.00791)\end{array}$ \\
\hline $\begin{array}{l}\text { AM - EV }\left[\mathrm{AA}^{\prime}\right] \\
\text { AND EV }\left[\mathrm{A}^{\prime} \mathrm{A}\right]\end{array}$ & $\begin{array}{c}0.00966 \\
(0.00357)\end{array}$ & $\begin{array}{c}0.00712 \\
(0.00266)\end{array}$ & $\begin{array}{c}0.03009 \\
(0.01027)\end{array}$ & $\begin{array}{c}0.00982 \\
(0.00364)\end{array}$ & $\begin{array}{c}0.00723 \\
(0.00271)\end{array}$ & $\begin{array}{c}0.03009 \\
(0.01140)\end{array}$ \\
\hline $\begin{array}{l}\text { GE - EV }\left[A^{\prime} A^{\prime}\right] \\
\text { AND EV }\left[A^{\prime} A\right]\end{array}$ & $\begin{array}{c}0.00948 \\
(0.00948) \\
\end{array}$ & $\begin{array}{c}0.00689 \\
(0.00256)\end{array}$ & $\begin{array}{c}0.02369 \\
(0.00743)\end{array}$ & $\begin{array}{c}0.00966 \\
(0.00341)\end{array}$ & $\begin{array}{c}0.00703 \\
(0.00252)\end{array}$ & $\begin{array}{c}0.02284 \\
(0.00756)\end{array}$ \\
\hline $\mathrm{GE}$ & $\begin{array}{c}0.00858 \\
(0.00283)\end{array}$ & $\begin{array}{c}0.00618 \\
(0.00221)\end{array}$ & $\begin{array}{c}0.02280 \\
(0.00721)\end{array}$ & $\begin{array}{c}0.00873 \\
(0.00297)\end{array}$ & $\begin{array}{c}0.00632 \\
(0.00223)\end{array}$ & $\begin{array}{c}0.02339 \\
(0.00776)\end{array}$ \\
\hline C-RSM & $\begin{array}{c}0.00870 \\
0.00292)\end{array}$ & $\begin{array}{c}0.00632 \\
(0.00230)\end{array}$ & $\begin{array}{c}0.02375 \\
(0.00723)\end{array}$ & $\begin{array}{c}0.00887 \\
(0.00305)\end{array}$ & $\begin{array}{c}0.00645 \\
(0.00227)\end{array}$ & $\begin{array}{c}0.02325 \\
(0.00761)\end{array}$ \\
\hline MT & $\begin{array}{c}0.00850 \\
(0.00279)\end{array}$ & $\begin{array}{c}0.00617 \\
(0.00221)\end{array}$ & $\begin{array}{c}0.02322 \\
(0.00696)\end{array}$ & $\begin{array}{c}0.00866 \\
(0.00296)\end{array}$ & $\begin{array}{c}0.00632 \\
(0.00223)\end{array}$ & $\begin{array}{c}0.02338 \\
(0.00746)\end{array}$ \\
\hline SAV & $\begin{array}{l}0.01059 \\
(0.00372)\end{array}$ & $\begin{array}{l}0.00745 \\
(0.00275)\end{array}$ & $\begin{array}{c}0.02149 \\
(0.00687)\end{array}$ & $\begin{array}{c}0.01085 \\
(0.00391)\end{array}$ & $\begin{array}{c}0.00765 \\
(0.00277)\end{array}$ & $\begin{array}{c}0.02070 \\
(0.00655)\end{array}$ \\
\hline NEV & $\begin{array}{c}0.01020 \\
(0.00370)\end{array}$ & $\begin{array}{c}0.00785 \\
(0.00275)\end{array}$ & $\begin{array}{c}0.02864 \\
(0.01026)\end{array}$ & $\begin{array}{c}0.01148 \\
(0.00420)\end{array}$ & $\begin{array}{c}0.00832 \\
(0.00297)\end{array}$ & $\begin{array}{c}0.02843 \\
(0.01068)\end{array}$ \\
\hline$\overline{\mathrm{LSM}}$ & $\begin{array}{c}0.01294 \\
(0.00499)\end{array}$ & $\begin{array}{c}0.00887 \\
(0.00358)\end{array}$ & $\begin{array}{c}0.02228 \\
(0.00882)\end{array}$ & $\begin{array}{c}0.01318 \\
(0.00522)\end{array}$ & $\begin{array}{c}0.00910 \\
(0.00370)\end{array}$ & $\begin{array}{c}0.02047 \\
(0.00761)\end{array}$ \\
\hline
\end{tabular}


Table B.3: Mean and Standard Deviation of Accuracy Measurement $\left(d_{1}\right)$ from Simulation (With $\mathrm{N}=8, \mathrm{M}=3$, scale $[1 / 9,9], \mathrm{T}=500$ ) [continued]

\begin{tabular}{||l||c|c|c||}
\hline \multicolumn{1}{||c||}{ M=3 } & \multicolumn{3}{c||}{ A-MDM(V) } \\
\hline ABBREVIATIONS & UF & LN & GA \\
\hline \hline CSM & 0.01027 & 0.00694 & 0.03204 \\
& $(0.00355)$ & $(0.00260)$ & $(0.01125)$ \\
\hline R-EV & 0.00964 & 0.00695 & 0.02656 \\
& $(0.00348)$ & $(0.00269)$ & $(0.00997)$ \\
\hline L-EV & 0.00980 & 0.00689 & 0.02833 \\
& $(0.00357)$ & $(0.00261)$ & $(0.01035)$ \\
\hline AM-EV & 0.00977 & 0.00689 & 0.02794 \\
& $(0.00357)$ & $(0.00261)$ & $(0.01020)$ \\
\hline GE-EV & 0.00967 & 0.00689 & 0.02655 \\
& $(0.00356)$ & $(0.00265)$ & $(0.00978)$ \\
\hline EV[AA'] & 0.01318 & 0.00913 & 0.03822 \\
& $(0.00486)$ & $(0.00360)$ & $(0.01524)$ \\
\hline EV[A'A] & 0.02067 & 0.01431 & 0.01647 \\
& $(0.00930)$ & $(0.00632)$ & $(0.01061)$ \\
\hline AM - EV[AA'] & 0.01266 & 0.00883 & 0.03623 \\
AND EV[A'A] & $(0.00471)$ & $(0.00346)$ & $(0.01453)$ \\
\hline GE - EV[AA'] & 0.01239 & 0.00891 & 0.02311 \\
AND EV[A'A] & $(0.00515)$ & $(0.00360)$ & $(0.00876)$ \\
\hline GE & 0.00973 & 0.00689 & 0.02361 \\
& $(0.00361)$ & $(0.00265)$ & $(0.00891)$ \\
\hline C-RSM & 0.01070 & 0.00764 & 0.02309 \\
& $(0.00396)$ & $(0.00287)$ & $(0.00875)$ \\
\hline MT & 0.00959 & 0.00690 & 0.02344 \\
& $(0.00346)$ & $(0.00261)$ & $(0.00844)$ \\
\hline SAV & 0.01556 & 0.01086 & 0.01574 \\
& $(0.00669)$ & $(0.00467)$ & $(0.00823)$ \\
\hline NEV & 0.01179 & 0.00909 & 0.03072 \\
& $(0.00476)$ & $(0.00365)$ & $(0.01309)$ \\
\hline LSM & 0.02015 & 0.01361 & 0.01484 \\
& $(0.00915)$ & $(0.00628)$ & $(0.00963)$ \\
\hline
\end{tabular}


Table B.4: Mean and Standard Deviation of Accuracy Measurement $\left(d_{1}\right)$ from Simulation (With $\mathrm{N}=8, \mathrm{M}=5$, scale $[1 / 9,9], \mathrm{T}=500$ )

\begin{tabular}{||l||c|c|c||c|c|c||}
\hline \multicolumn{1}{|c|}{ M=5 } & \multicolumn{3}{c|}{ A-GE(V) } & \multicolumn{3}{c|}{ A-GE(M) } \\
\hline ABBREVIATIONS & UF & LN & GA & UF & LN & GA \\
\hline \hline CSM & 0.01080 & 0.00835 & 0.03327 & 0.00971 & 0.00802 & 0.02058 \\
& $(0.00408)$ & $(0.00387)$ & $(0.00836)$ & $(0.00392)$ & $(0.00376)$ & $(0.00625)$ \\
\hline R-EV & 0.00703 & 0.00491 & 0.02161 & 0.00703 & 0.00490 & 0.01973 \\
& $(0.00247)$ & $(0.00180)$ & $(0.00636)$ & $(0.00250)$ & $(0.00179)$ & $(0.00623)$ \\
\hline L-EV & 0.00710 & 0.00490 & 0.02233 & 0.00711 & 0.00488 & 0.02016 \\
& $(0.00248)$ & $(0.00175)$ & $(0.00624)$ & $(0.00252)$ & $(0.00176)$ & $(0.00636)$ \\
\hline AM-EV & 0.00708 & 0.00489 & 0.02193 & 0.00709 & 0.00488 & 0.01999 \\
& $(0.00247)$ & $(0.00176)$ & $(0.00512)$ & $(0.00251)$ & $(0.00177)$ & $(0.00625)$ \\
\hline GM-EV & 0.00703 & 0.00489 & 0.02131 & 0.00705 & 0.00488 & 0.01974 \\
& $(0.00246)$ & $(0.00177)$ & $(0.00607)$ & $(0.00250)$ & $(0.00177)$ & $(0.00616)$ \\
\hline EV[AA'] & 0.00833 & 0.00597 & 0.02998 & 0.00825 & 0.00592 & 0.02720 \\
& $(0.00318)$ & $(0.00227)$ & $(0.00894)$ & $(0.00320)$ & $(0.00227)$ & $(0.00914)$ \\
\hline EV[A'A] & 0.01092 & 0.00777 & 0.02019 & 0.01093 & 0.00767 & 0.01859 \\
& $(0.00449)$ & $(0.00304)$ & $(0.00671)$ & $(0.00466)$ & $(0.00307)$ & $(0.00668)$ \\
\hline AM - EV[AA'] & 0.00775 & 0.00557 & 0.02694 & 0.00765 & 0.00552 & 0.02431 \\
AND EV[A'A] & $(0.00293)$ & $(0.00213)$ & $(0.00791)$ & $(0.00292)$ & $(0.00212)$ & $(0.00802)$ \\
\hline GE- EV[AA'] & 0.00761 & 0.00551 & 0.02093 & 0.00760 & 0.00546 & 0.01847 \\
AND EV[A'A] & $(0.00291)$ & $(0.00203)$ & $(0.00618)$ & $(0.00291)$ & $(0.00204)$ & $(0.00602)$ \\
\hline GE & 0.00703 & 0.00488 & 0.01986 & 0.00703 & 0.00488 & 0.01949 \\
& $(0.00247)$ & $(0.00177)$ & $(0.00579)$ & $(0.00248)$ & $(0.00177)$ & $(0.00601)$ \\
\hline C-RSM & 0.00709 & 0.00501 & 0.02099 & 0.00710 & 0.00500 & 0.01906 \\
& $(0.00254)$ & $(0.00182)$ & $(0.00578)$ & $(0.00257)$ & $(0.00182)$ & $(0.00590)$ \\
\hline M'T & 0.00700 & 0.00489 & 0.02034 & 0.00699 & 0.00589 & 0.01944 \\
& $(0.00244)$ & $(0.00178)$ & $(0.00572)$ & $(0.00248)$ & $(0.00178)$ & $(0.00588)$ \\
\hline SAV & 0.00840 & 0.00593 & 0.01836 & 0.00843 & 0.00590 & 0.01679 \\
& $(0.00327)$ & $(0.00221)$ & $(0.00572)$ & $(0.00333)$ & $(0.00220)$ & $(0.00547)$ \\
\hline NEV & 0.00821 & 0.00610 & 0.02597 & 0.00938 & 0.00646 & 0.02297 \\
& $(0.00302)$ & $(0.00217)$ & $(0.00900)$ & $(0.00339)$ & $(0.00237)$ & $(0.00807)$ \\
\hline LSM & 0.01007 & 0.00711 & 0.01923 & 0.01003 & 0.00700 & 0.01663 \\
& $(0.00434)$ & $(0.00287)$ & $(0.00758)$ & $(0.00451)$ & $(0.00291)$ & $(0.00619)$ \\
\hline
\end{tabular}


Table B.5: Mean and Standard Deviation of Accuracy Measurement $\left(d_{1}\right)$ from Simulation (With $N=8, M=5$, scale $[1 / 9,9], T=500$ ) [Continued]

\begin{tabular}{||l||c|c|c||c|c|c||}
\hline \multicolumn{1}{|c||}{ M=5 } & \multicolumn{3}{c|}{ A-AM(V) } & \multicolumn{3}{c||}{ A-MDM(M) } \\
\hline ABBREVIATIONS & UF & LN & GA & UF & LN & GA \\
\hline \hline CSM & 0.01099 & 0.00844 & 0.03390 & 0.00998 & 0.00819 & 0.02144 \\
& $(0.00421)$ & $(0.00394)$ & $(0.00813)$ & $(0.00401)$ & $(0.00376)$ & $(0.00685)$ \\
\hline R-EV & 0.00701 & 0.00491 & 0.02164 & 0.00723 & 0.00508 & 0.02013 \\
& $(0.00247)$ & $(0.00181)$ & $(0.00664)$ & $(0.00267)$ & $(0.00184)$ & $(0.00684)$ \\
\hline L-EV & 0.00710 & 0.00490 & 0.02153 & 0.00732 & 0.00507 & 0.02055 \\
& $(0.00248)$ & $(0.00176)$ & $(0.00661)$ & $(0.00269)$ & $(0.00183)$ & $(0.00684)$ \\
\hline AM-EV & 0.00708 & 0.00489 & 0.02126 & 0.00729 & 0.00507 & 0.02037 \\
& $(0.00248)$ & $(0.00177)$ & $(0.00648)$ & $(0.00268)$ & $(0.00183)$ & $(0.00670)$ \\
\hline GE-EV & 0.00702 & 0.00488 & 0.02096 & 0.00725 & 0.00507 & 0.02010 \\
& $(0.00246)$ & $(0.00178)$ & $(0.00640)$ & $(0.00267)$ & $(0.00184)$ & $(0.00664)$ \\
\hline EV[AA'] & 0.00833 & 0.00597 & 0.02817 & 0.00860 & 0.00620 & 0.02774 \\
& $(0.00320)$ & $(0.00226)$ & $(0.00953)$ & $(0.00337)$ & $(0.00239)$ & $(0.01033)$ \\
\hline EV[A'A] & 0.01086 & 0.00779 & 0.02033 & 0.01159 & 0.00797 & 0.01852 \\
& $(0.00453)$ & $(0.00306)$ & $(0.00700)$ & $(0.00495)$ & $(0.00313)$ & $(0.00639)$ \\
\hline AM-EV[AA'] & 0.00776 & 0.00557 & 0.02568 & 0.00793 & 0.00577 & 0.02482 \\
AND EV[A'A] & $(0.00294)$ & $(0.00212)$ & $(0.00843)$ & $(0.00310)$ & $(0.00221)$ & $(0.00907)$ \\
\hline GE - EV[AA'] & 0.00761 & 0.00551 & 0.02035 & 0.00790 & 0.00566 & 0.01882 \\
AND EV[A'A] & $(0.00291)$ & $(0.00204)$ & $(0.00636)$ & $(0.00306)$ & $(0.00206)$ & $(0.00631)$ \\
\hline GE & 0.00702 & 0.00488 & 0.01953 & 0.00723 & 0.00507 & 0.01975 \\
& $(0.00247)$ & $(0.00178)$ & $(0.00607)$ & $(0.00266)$ & $(0.00184)$ & $(0.00644)$ \\
\hline C-RSM & 0.00708 & 0.00501 & 0.02056 & 0.00733 & 0.00518 & 0.01941 \\
& $(0.00254)$ & $(0.00183)$ & $(0.00606)$ & $(0.00274)$ & $(0.00186)$ & $(0.00631)$ \\
\hline MT & 0.00695 & 0.00488 & 0.02020 & 0.00719 & 0.00507 & 0.01968 \\
& $(0.00244)$ & $(0.00179)$ & $(0.00589)$ & $(0.00265)$ & $(0.00184)$ & $(0.00636)$ \\
\hline SAV & 0.00838 & 0.00593 & 0.01840 & 0.00886 & 0.00611 & 0.01699 \\
& $(0.00328)$ & $(0.00223)$ & $(0.00588)$ & $(0.00357)$ & $(0.00224)$ & $(0.00553)$ \\
\hline NEV & 0.00806 & 0.00609 & 0.02418 & 0.00979 & 0.00679 & 0.02366 \\
& $(0.00303)$ & $(0.00218)$ & $(0.00858)$ & $(0.00374)$ & $(0.00247)$ & $(0.00886)$ \\
\hline LSM & 0.01011 & 0.07154 & 0.01847 & 0.01062 & 0.00728 & 0.01647 \\
& $(0.00436)$ & $(0.00289)$ & $(0.00752)$ & $(0.00482)$ & $(0.00295)$ & $(0.00607)$ \\
\hline
\end{tabular}


Table B.6: Mean and Standard Deviation of Accuracy Measurement $\left(d_{1}\right)$ from Simulation (With $N=8, M=5$, scale $[1 / 9,9], T=500$ ) [Continued]

\begin{tabular}{|c|c|c|c|}
\hline $\mathrm{M}=5$ & \multicolumn{3}{|c|}{$\mathrm{A}-\mathrm{MDM}(\mathrm{V})$} \\
\hline ABBREVIATIONS & UF & LN & GA \\
\hline CSM & $\begin{array}{c}0.00870 \\
(0.00316)\end{array}$ & $\begin{array}{c}0.00579 \\
(0.00214)\end{array}$ & $\begin{array}{c}0.02964 \\
(0.00979)\end{array}$ \\
\hline $\mathrm{R}-\mathrm{EV}$ & $\begin{array}{c}0.00787 \\
(0.00293) \\
\end{array}$ & $\begin{array}{c}0.00557 \\
(0.00209) \\
\end{array}$ & $\begin{array}{r}0.02263 \\
(0.00817) \\
\end{array}$ \\
\hline $\mathrm{L}-\mathrm{EV}$ & $\begin{array}{c}0.00802 \\
(0.00301)\end{array}$ & $\begin{array}{c}0.00564 \\
(0.00217)\end{array}$ & $\begin{array}{c}0.02397 \\
(0.00830)\end{array}$ \\
\hline AM-EV & $\begin{array}{c}0.00802 \\
(0.00299) \\
\end{array}$ & $\begin{array}{c}0.00562 \\
(0.00215) \\
\end{array}$ & $\begin{array}{r}0.02375 \\
(0.00825) \\
\end{array}$ \\
\hline GE-EV & $\begin{array}{c}0.00791 \\
(0.00296)\end{array}$ & $\begin{array}{c}0.00555 \\
(0.00213)\end{array}$ & $\begin{array}{c}0.02240 \\
(0.00793)\end{array}$ \\
\hline$E V\left[A^{\prime}\right]$ & $\begin{array}{c}0.01006 \\
(0.00389)\end{array}$ & $\begin{array}{c}0.00708 \\
(0.00279)\end{array}$ & $\begin{array}{c}0.03282 \\
(0.01355) \\
\end{array}$ \\
\hline $\mathrm{EV}\left[\mathrm{A}^{\prime} \mathrm{A}\right]$ & $\begin{array}{c}0.01616 \\
(0.00796)\end{array}$ & $\begin{array}{c}0.01104 \\
(0.00464)\end{array}$ & $\begin{array}{c}0.01262 \\
(0.00836)\end{array}$ \\
\hline $\begin{array}{l}A \mathrm{AM}-\mathrm{EV}\left[\mathrm{AA}^{\prime}\right] \\
\text { AND EV[A'A] }\end{array}$ & $\begin{array}{c}0.00968 \\
(0.00381) \\
\end{array}$ & $\begin{array}{c}0.00684 \\
(0.00268)\end{array}$ & $\begin{array}{c}0.03131 \\
(0.01296) \\
\end{array}$ \\
\hline $\mathrm{GE}-\mathrm{EV}\left[\mathrm{AA}^{\prime}\right]$ & $\begin{array}{c}0.01008 \\
(0.00410)\end{array}$ & $\begin{array}{c}0.00691 \\
(0.00267)\end{array}$ & $\begin{array}{c}0.01951 \\
(0.00727)\end{array}$ \\
\hline GE & $\begin{array}{c}0.00792 \\
(0.00297)\end{array}$ & $\begin{array}{c}0.00555 \\
(0.00211)\end{array}$ & $\begin{array}{c}0.02013 \\
(0.00736)\end{array}$ \\
\hline C-RSM & $\begin{array}{c}0.00875 \\
(0.00337) \\
\end{array}$ & $\begin{array}{c}0.00609 \\
(0.00230)\end{array}$ & $\begin{array}{c}0.01990 \\
(0.00717)\end{array}$ \\
\hline$\overline{\mathrm{MT}}$ & $\begin{array}{c}0.00784 \\
(0.00290) \\
\end{array}$ & $\begin{array}{c}0.00550 \\
(0.00205) \\
\end{array}$ & $\begin{array}{c}0.01998 \\
(0.00692) \\
\end{array}$ \\
\hline SAV & $\begin{array}{c}0.01237 \\
(0.00586)\end{array}$ & $\begin{array}{c}0.00838 \\
(0.00347)\end{array}$ & $\begin{array}{c}0.01250 \\
(0.00680)\end{array}$ \\
\hline NEV & $\begin{array}{c}0.00937 \\
(0.00376)\end{array}$ & $\begin{array}{c}0.00720 \\
(0.00296)\end{array}$ & $\begin{array}{c}0.02488 \\
(0.01044)\end{array}$ \\
\hline$\overline{\mathrm{LSM}}$ & $\begin{array}{c}0.01569 \\
(0.00796)\end{array}$ & $\begin{array}{c}0.01059 \\
(0.00473)\end{array}$ & $\begin{array}{c}0.01142 \\
(0.00767)\end{array}$ \\
\hline
\end{tabular}


Table B.7: Mean and Standard Deviation of Accuracy Measurement $\left(d_{1}\right)$ from Simulation (With $\mathrm{N}=8, \mathrm{M}=7$, scale $[1 / 9,9], \mathrm{T}=500$ )

\begin{tabular}{||l||c|c|c||c|c|c||}
\hline \multicolumn{1}{|c||}{ M=7 } & \multicolumn{3}{c||}{ A-GE(V) } & \multicolumn{3}{c||}{ A-GE(M) } \\
\hline ABBREVIATIONS & UF & LN & GA & UF & LN & GA \\
\hline \hline CSM & 0.01016 & 0.00798 & 0.03225 & 0.00903 & 0.00767 & 0.01851 \\
& $(0.00398)$ & $(0.00384)$ & $(0.00782)$ & $(0.00390)$ & $(0.00377)$ & $(0.00538)$ \\
\hline R-EV & 0.00625 & 0.00438 & 0.02010 & 0.00623 & 0.00436 & 0.01810 \\
& $(0.00214)$ & $(0.00158)$ & $(0.00558)$ & $(0.00216)$ & $(0.00158)$ & $(0.00559)$ \\
\hline L-EV & 0.00629 & 0.00437 & 0.02078 & 0.00627 & 0.00435 & 0.01838 \\
& $(0.00215)$ & $(0.00156)$ & $(0.00537)$ & $(0.00219)$ & $(0.00157)$ & $(0.00556)$ \\
\hline AM-EV & 0.00627 & 0.00436 & 0.02042 & 0.00626 & 0.00435 & 0.01826 \\
& $(0.00214)$ & $(0.00156)$ & $(0.00531)$ & $(0.00218)$ & $(0.00157)$ & $(0.00549)$ \\
\hline GM-EV & 0.00624 & 0.00436 & 0.01987 & 0.00623 & 0.00436 & 0.01811 \\
& $(0.00213)$ & $(0.00157)$ & $(0.00532)$ & $(0.00216)$ & $(0.00157)$ & $(0.00547)$ \\
\hline EV[AA'] & 0.00738 & 0.00533 & 0.02784 & 0.00726 & 0.00527 & 0.02488 \\
& $(0.00291)$ & $(0.00197)$ & $(0.00765)$ & $(0.00293)$ & $(0.00198)$ & $(0.00810)$ \\
\hline EV[A'A] & 0.00942 & 0.00681 & 0.01878 & 0.00930 & 0.00670 & 0.01668 \\
& $(0.00381)$ & $(0.00261)$ & $(0.00612)$ & $(0.00402)$ & $(0.00258)$ & $(0.00594)$ \\
\hline AM- EV[AA'] & 0.00690 & 0.00498 & 0.02502 & 0.00678 & 0.00492 & 0.02221 \\
AND EV[A'A] & $(0.00269)$ & $(0.00184)$ & $(0.00678)$ & $(0.00267)$ & $(0.00185)$ & $(0.00709)$ \\
\hline GE- EV[AA'] & 0.00677 & 0.00488 & 0.01943 & 0.00671 & 0.00482 & 0.01680 \\
AND EV[A'A] & $(0.00253)$ & $(0.00174)$ & $(0.00553)$ & $(0.00254)$ & $(0.00175)$ & $(0.00538)$ \\
\hline GE & 0.00622 & 0.00436 & 0.01845 & 0.00621 & 0.00435 & 0.01793 \\
& $(0.00214)$ & $(0.00157)$ & $(0.00512)$ & $(0.00215)$ & $(0.00157)$ & $(0.00537)$ \\
\hline C-RSM & 0.00631 & 0.00447 & 0.01960 & 0.00629 & 0.00445 & 0.01745 \\
& $(0.00219)$ & $(0.00160)$ & $(0.00509)$ & $(0.00222)$ & $(0.00160)$ & $(0.00528)$ \\
\hline MT & 0.00619 & 0.00437 & 0.01902 & 0.00619 & 0.00436 & 0.01793 \\
& $(0.00211)$ & $(0.00157)$ & $(0.00506)$ & $(0.00214)$ & $(0.00157)$ & $(0.00532)$ \\
\hline SAV & 0.00734 & 0.00523 & 0.01710 & 0.00731 & 0.00519 & 0.01529 \\
& $(0.00274)$ & $(0.00192)$ & $(0.00514)$ & $(0.00284)$ & $(0.00189)$ & $(0.00493)$ \\
\hline NEV & 0.00683 & 0.00521 & 0.02407 & 0.00825 & 0.00573 & 0.02080 \\
& $(0.00255)$ & $(0.00193)$ & $(0.00815)$ & $(0.00298)$ & $(0.00213)$ & $(0.00719)$ \\
\hline LSM & 0.00870 & 0.00625 & 0.01746 & 0.00857 & 0.00615 & 0.01499 \\
& $(0.00362)$ & $(0.00245)$ & $(0.00685)$ & $(0.00383)$ & $(0.00243)$ & $(0.00551)$ \\
\hline
\end{tabular}


Table B.8: Mean and Standard Deviation of Accuracy Measurement $\left(d_{1}\right)$ from Simulation (With $N=8, M=7$, scale $[1 / 9,9], T=500$ ) [Continued]

\begin{tabular}{||l||c|c|c||c|c|c||}
\hline \multicolumn{1}{|c||}{ M=7 } & \multicolumn{3}{c|}{ A-AM(V) } & \multicolumn{3}{c||}{ A-MDM(M) } \\
\hline ABBREVIATIONS & UF & LN & GA & UF & LN & GA \\
\hline \hline CSM & 0.01037 & 0.00807 & 0.03289 & 0.00924 & 0.00782 & 0.01904 \\
& $(0.00413)$ & $(0.00393)$ & $(0.00758)$ & $(0.00394)$ & $(0.00379)$ & $(0.00577)$ \\
\hline R-EV & 0.00622 & 0.00438 & 0.01995 & 0.00637 & 0.00453 & 0.01827 \\
& $(0.00215)$ & $(0.00159)$ & $(0.00592)$ & $(0.00230)$ & $(0.00173)$ & $(0.00611)$ \\
\hline L-EV & 0.00627 & 0.00437 & 0.01975 & 0.00641 & 0.00452 & 0.01851 \\
& $(0.00217)$ & $(0.00157)$ & $(0.00569)$ & $(0.00233)$ & $(0.00172)$ & $(0.00605)$ \\
\hline AM-EV & 0.00625 & 0.00436 & 0.01955 & 0.00640 & 0.00452 & 0.01839 \\
& $(0.00216)$ & $(0.00157)$ & $(0.00563)$ & $(0.00232)$ & $(0.00173)$ & $(0.00599)$ \\
\hline GE-EV & 0.00622 & 0.00435 & 0.01934 & 0.00637 & 0.00452 & 0.01823 \\
& $(0.00214)$ & $(0.00157)$ & $(0.00563)$ & $(0.00230)$ & $(0.00173)$ & $(0.00597)$ \\
\hline EV[AA'] & 0.00735 & 0.00532 & 0.02561 & 0.00757 & 0.00561 & 0.02488 \\
& $(0.00295)$ & $(0.00196)$ & $(0.00811)$ & $(0.00312)$ & $(0.00219)$ & $(0.00930)$ \\
\hline EV[A'A] & 0.00933 & 0.00681 & 0.01882 & 0.09880 & 0.00698 & 0.01621 \\
& $(0.00388)$ & $(0.00265)$ & $(0.00644)$ & $(0.00431)$ & $(0.00269)$ & $(0.00573)$ \\
\hline AM-EV[AA'] & 0.00688 & 0.00497 & 0.02344 & 0.00700 & 0.00521 & 0.02233 \\
AND EV[A'A] & $(0.00272)$ & $(0.00184)$ & $(0.00718)$ & $(0.00286)$ & $(0.00204)$ & $(0.00814)$ \\
\hline GE- EV[AA'] & 0.00676 & 0.00488 & 0.01869 & 0.00695 & 0.00502 & 0.01695 \\
AND EV[A'A] & $(0.00254)$ & $(0.00175)$ & $(0.00574)$ & $(0.00264)$ & $(0.00186)$ & $(0.00574)$ \\
\hline GE & 0.00620 & 0.00435 & 0.01794 & 0.00635 & 0.00452 & 0.01796 \\
& $(0.00215)$ & $(0.00157)$ & $(0.00541)$ & $(0.00229)$ & $(0.00173)$ & $(0.00583)$ \\
\hline C-RSM & 0.00629 & 0.00446 & 0.01900 & 0.00646 & 0.00462 & 0.01756 \\
& $(0.00220)$ & $(0.00160)$ & $(0.00537)$ & $(0.00232)$ & $(0.00174)$ & $(0.00575)$ \\
\hline MT & 0.00615 & 0.00436 & 0.01877 & 0.00633 & 0.00452 & 0.01796 \\
& $(0.00212)$ & $(0.00157)$ & $(0.00526)$ & $(0.00228)$ & $(0.00173)$ & $(0.00577)$ \\
\hline SAV & 0.00732 & 0.00523 & 0.01707 & 0.00767 & 0.00539 & 0.01527 \\
& $(0.00277)$ & $(0.00194)$ & $(0.00532)$ & $(0.00303)$ & $(0.00200)$ & $(0.00496)$ \\
\hline NEV & 0.00667 & 0.00519 & 0.02180 & 0.00841 & 0.00603 & 0.02114 \\
& $(0.00257)$ & $(0.00194)$ & $(0.00771)$ & $(0.00317)$ & $(0.00223)$ & $(0.00792)$ \\
\hline LSM & 0.00873 & 0.00628 & 0.01652 & 0.00909 & 0.00640 & 0.01445 \\
& $(0.00366)$ & $(0.00248)$ & $(0.00682)$ & $(0.00413)$ & $(0.00255)$ & $(0.00549)$ \\
\hline
\end{tabular}


Table B.9: Mean and Standard Deviation of Accuracy Measurement $\left(d_{1}\right)$ from Simulation (With $\mathrm{N}=8, \mathrm{M}=7$, scale $[1 / 9,9], \mathrm{T}=500$ ) [Continued]

\begin{tabular}{||l||c|c|c||}
\hline \multicolumn{1}{||c||}{ M=7 } & \multicolumn{3}{c||}{ A-MDM(V) } \\
\hline ABBREVIATIONS & UF & LN & GA \\
\hline \hline CSM & 0.00776 & 0.00512 & 0.02803 \\
& $(0.00295)$ & $(0.00188)$ & $(0.00911)$ \\
\hline R-EV & 0.00686 & 0.00488 & 0.02039 \\
& $(0.00253)$ & $(0.00180)$ & $(0.00692)$ \\
\hline L-EV & 0.00713 & 0.00492 & 0.02116 \\
& $(0.00257)$ & $(0.00184)$ & $(0.00715)$ \\
\hline AM-EV & 0.00712 & 0.00492 & 0.02100 \\
& $(0.00258)$ & $(0.00184)$ & $(0.00710)$ \\
\hline GE-EV & 0.00694 & 0.00490 & 0.02003 \\
& $(0.00253)$ & $(0.00182)$ & $(0.00660)$ \\
\hline EV[AA'] & 0.00880 & 0.00634 & 0.02933 \\
& $(0.00345)$ & $(0.00251)$ & $(0.01168)$ \\
\hline EV[A'A] & 0.01358 & 0.00962 & 0.01063 \\
& $(0.00686)$ & $(0.00409)$ & $(0.00703)$ \\
\hline AM - EV[AA'] & 0.00850 & 0.00614 & 0.02791 \\
AND EV[A'A] & $(0.00333)$ & $(0.00243)$ & $(0.01110)$ \\
\hline GE - EV[AA'] & 0.00868 & 0.00623 & 0.01740 \\
AND EV[A'A] & $(0.00354)$ & $(0.00244)$ & $(0.00632)$ \\
\hline GE & 0.00695 & 0.00491 & 0.01794 \\
& $(0.00254)$ & $(0.00182)$ & $(0.00603)$ \\
\hline C-RSM & 0.00760 & 0.00541 & 0.01768 \\
& $(0.00287)$ & $(0.00208)$ & $(0.00595)$ \\
\hline MT & 0.00683 & 0.00480 & 0.01825 \\
& $(0.00253)$ & $(0.00174)$ & $(0.00573)$ \\
\hline SAV & 0.01059 & 0.00739 & 0.01088 \\
& $(0.00507)$ & $(0.00302)$ & $(0.00587)$ \\
\hline NEV & 0.00775 & 0.00623 & 0.02236 \\
& $(0.00325)$ & $(0.00251)$ & $(0.00852)$ \\
\hline LSM & 0.01322 & 0.00925 & 0.00973 \\
& $(0.00669)$ & $(0.00408)$ & $(0.00648)$ \\
\hline
\end{tabular}


Table B.10: Mean and Standard Deviation of Accuracy Measurement $\left(d_{1}\right)$ from Simulation (With $N=8, M=9$, scale $[1 / 9,9], T=500$ )

\begin{tabular}{||l||c|c|c||c|c|c||}
\hline \multicolumn{1}{|c||}{ M=9 } & \multicolumn{3}{c|}{ A-GE(V) } & \multicolumn{3}{c||}{ A-GE(M) } \\
\hline ABBREVIATIONS & UF & LN & GA & UF & LN & GA \\
\hline \hline CSM & 0.00979 & 0.00771 & 0.03143 & 0.00864 & 0.00741 & 0.01727 \\
& $(0.00392)$ & $(0.00391)$ & $(0.00750)$ & $(0.00388)$ & $(0.00386)$ & $(0.00486)$ \\
\hline R-EV & 0.00578 & 0.00403 & 0.01912 & 0.00574 & 0.00401 & 0.01698 \\
& $(0.00186)$ & $(0.00143)$ & $(0.00494)$ & $(0.00189)$ & $(0.00142)$ & $(0.00496)$ \\
\hline L-EV & 0.00582 & 0.00401 & 0.01971 & 0.00579 & 0.00400 & 0.01726 \\
& $(0.00190)$ & $(0.00143)$ & $(0.00493)$ & $(0.00193)$ & $(0.00143)$ & $(0.00501)$ \\
\hline AM-EV & 0.00581 & 0.00401 & 0.01938 & 0.00578 & 0.00400 & 0.01717 \\
& $(0.00189)$ & $(0.00143)$ & $(0.00485)$ & $(0.00192)$ & $(0.00143)$ & $(0.00495)$ \\
\hline GM-EV & 0.00578 & 0.00400 & 0.01890 & 0.00576 & 0.00400 & 0.01704 \\
& $(0.00187)$ & $(0.00142)$ & $(0.00480)$ & $(0.00190)$ & $(0.00142)$ & $(0.00490)$ \\
\hline EV[AA'] & 0.00679 & 0.00481 & 0.02656 & 0.00667 & 0.00476 & 0.02339 \\
& $(0.00261)$ & $(0.00180)$ & $(0.00683)$ & $(0.00260)$ & $(0.00181)$ & $(0.00715)$ \\
\hline EV[A'A] & 0.00841 & 0.00622 & 0.01773 & 0.00824 & 0.00611 & 0.01532 \\
& $(0.00341)$ & $(0.00234)$ & $(0.00577)$ & $(0.00356)$ & $(0.00236)$ & $(0.00522)$ \\
\hline AM-EV[AA'] & 0.00638 & 0.00451 & 0.02389 & 0.00626 & 0.00446 & 0.02087 \\
AND EV[A'A] & $(0.00237)$ & $(0.00168)$ & $(0.00606)$ & $(0.00234)$ & $(0.00168)$ & $(0.00627)$ \\
\hline GE - EV[AA'] & 0.00620 & 0.00446 & 0.01853 & 0.00614 & 0.00442 & 0.01569 \\
AND EV[A'A] & $(0.00224)$ & $(0.00160)$ & $(0.01835)$ & $(0.00223)$ & $(0.00159)$ & $(0.00489)$ \\
\hline GE & 0.00575 & 0.00400 & 0.01751 & 0.00574 & 0.00400 & 0.01689 \\
& $(0.00188)$ & $(0.00142)$ & $(0.00460)$ & $(0.00189)$ & $(0.00142)$ & $(0.00483)$ \\
\hline C-RSM & 0.00583 & 0.00410 & 0.01870 & 0.00580 & 0.00408 & 0.01639 \\
& $(0.00192)$ & $(0.00145)$ & $(0.00460)$ & $(0.00195)$ & $(0.00145)$ & $(0.00476)$ \\
\hline MT & 0.00572 & 0.00401 & 0.01817 & 0.00572 & 0.00400 & 0.01688 \\
& $(0.00184)$ & $(0.00142)$ & $(0.00455)$ & $(0.00188)$ & $(0.00142)$ & $(0.00477)$ \\
\hline SAV & 0.00665 & 0.00478 & 0.01620 & 0.00659 & 0.00475 & 0.01420 \\
& $(0.00246)$ & $(0.00174)$ & $(0.00476)$ & $(0.00252)$ & $(0.00172)$ & $(0.00449)$ \\
\hline NEV & 0.00603 & 0.00463 & 0.02320 & 0.00760 & 0.00530 & 0.01974 \\
& $(0.00229)$ & $(0.00177)$ & $(0.00765)$ & $(0.00259)$ & $(0.00196)$ & $(0.00688)$ \\
\hline LSM & 0.00783 & 0.00572 & 0.01630 & 0.00766 & 0.00563 & 0.01386 \\
& $(0.00312)$ & $(0.00225)$ & $(0.00647)$ & $(0.00329)$ & $(0.00223)$ & $(0.00495)$ \\
\hline
\end{tabular}


Table B.11: Mean and Standard Deviation of Accuracy Measurement $\left(d_{1}\right)$ from Simulation (With $N=8, M=9$, scale $[1 / 9,9], T=500$ ) [Continued]

\begin{tabular}{||l||c|c|c||c|c|c||}
\hline \multicolumn{1}{|c||}{ M=9 } & \multicolumn{3}{c|}{ A-AM(V) } & \multicolumn{3}{c|}{ A-MDM(M) } \\
\hline ABBREVIATIONS & UF & LN & GA & UF & LN & GA \\
\hline \hline CSM & 0.01002 & 0.00781 & 0.03210 & 0.00874 & 0.00753 & 0.01763 \\
& $(0.00408)$ & $(0.00399)$ & $(0.00721)$ & $(0.00391)$ & $(0.00387)$ & $(0.00528)$ \\
\hline R-EV & 0.00576 & 0.00401 & 0.01888 & 0.00571 & 0.00415 & 0.01698 \\
& $(0.00187)$ & $(0.00144)$ & $(0.00524)$ & $(0.00204)$ & $(0.00153)$ & $(0.00548)$ \\
\hline L-EV & 0.00580 & 0.00400 & 0.01854 & 0.00576 & 0.00415 & 0.01719 \\
& $(0.00192)$ & $(0.00143)$ & $(0.00526)$ & $(0.00208)$ & $(0.00153)$ & $(0.00555)$ \\
\hline AM-EV & 0.00578 & 0.00400 & 0.01838 & 0.00575 & 0.00415 & 0.01709 \\
& $(0.00191)$ & $(0.00143)$ & $(0.00517)$ & $(0.00207)$ & $(0.00153)$ & $(0.00547)$ \\
\hline GE-EV & 0.00576 & 0.00399 & 0.01827 & 0.00572 & 0.00415 & 0.01696 \\
& $(0.00188)$ & $(0.00143)$ & $(0.00510)$ & $(0.00205)$ & $(0.00153)$ & $(0.00541)$ \\
\hline EV[AA'] & 0.00674 & 0.00480 & 0.02409 & 0.00684 & 0.00499 & 0.02326 \\
& $(0.00266)$ & $(0.00180)$ & $(0.00735)$ & $(0.00286)$ & $(0.00201)$ & $(0.00838)$ \\
\hline EV[A'A] & 0.00829 & 0.00619 & 0.01771 & 0.00880 & 0.00643 & 0.01465 \\
& $(0.00348)$ & $(0.00239)$ & $(0.00617)$ & $(0.00388)$ & $(0.00245)$ & $(0.00519)$ \\
\hline AM - EV[AA'] & 0.00635 & 0.00451 & 0.02211 & 0.00634 & 0.00466 & 0.02089 \\
AND EV[A'A] & $(0.00241)$ & $(0.00168)$ & $(0.00650)$ & $(0.00257)$ & $(0.00185)$ & $(0.00735)$ \\
\hline GE - EV[AA'] & 0.00619 & 0.00446 & 0.01770 & 0.00623 & 0.00461 & 0.01575 \\
AND EV[A'A] & $(0.00226)$ & $(0.00161)$ & $(0.00526)$ & $(0.00239)$ & $(0.00163)$ & $(0.00519)$ \\
\hline GE & 0.00573 & 0.00399 & 0.01693 & 0.00570 & 0.00415 & 0.01674 \\
& $(0.00189)$ & $(0.00143)$ & $(0.00487)$ & $(0.00204)$ & $(0.00153)$ & $(0.00528)$ \\
\hline C-RSM & 0.00581 & 0.00409 & 0.01802 & 0.00579 & 0.00424 & 0.01632 \\
& $(0.00194)$ & $(0.00146)$ & $(0.00485)$ & $(0.00208)$ & $(0.00153)$ & $(0.00521)$ \\
\hline MT & 0.00568 & 0.00399 & 0.01787 & 0.00568 & 0.00415 & 0.01673 \\
& $(0.00185)$ & $(0.00142)$ & $(0.00471)$ & $(0.00202)$ & $(0.00153)$ & $(0.00522)$ \\
\hline SAV & 0.00661 & 0.00477 & 0.01614 & 0.00683 & 0.00497 & 0.01399 \\
& $(0.00248)$ & $(0.00176)$ & $(0.00492)$ & $(0.00272)$ & $(0.00176)$ & $(0.00448)$ \\
\hline NEV & 0.00585 & 0.00461 & 0.02066 & 0.00750 & 0.00553 & 0.01979 \\
& $(0.00233)$ & $(0.00180)$ & $(0.00720)$ & $(0.00280)$ & $(0.00208)$ & $(0.00734)$ \\
\hline LSM & 0.00784 & 0.00574 & 0.01520 & 0.00807 & 0.00588 & 0.01314 \\
& $(0.00315)$ & $(0.00229)$ & $(0.00646)$ & $(0.00362)$ & $(0.00235)$ & $(0.00501)$ \\
\hline
\end{tabular}


Table B.12: Mean and Standard Deviation of Accuracy Measurement $\left(d_{1}\right)$ from Simulation (With $N=8, M=9$, scale $[1 / 9,9], T=500$ ) [Continued]

\begin{tabular}{||l||c|c|c||}
\hline \multicolumn{1}{|c||}{ M=9 } & \multicolumn{3}{c||}{ A-MDM(V) } \\
\hline ABBREVIATIONS & UF & LN & GA \\
\hline \hline CSM & 0.00724 & 0.00474 & 0.02697 \\
& $(0.00270)$ & $(0.00173)$ & $(0.00867)$ \\
\hline R-EV & 0.00631 & 0.00456 & 0.01919 \\
& $(0.00230)$ & $(0.00166)$ & $(0.00642)$ \\
\hline L-EV & 0.00652 & 0.00460 & 0.01949 \\
& $(0.00238)$ & $(0.00170)$ & $(0.00638)$ \\
\hline AM-EV & 0.00651 & 0.00459 & 0.01933 \\
& $(0.00238)$ & $(0.00169)$ & $(0.00638)$ \\
\hline GE-EV & 0.00638 & 0.00457 & 0.01872 \\
& $(0.00233)$ & $(0.00168)$ & $(0.00595)$ \\
\hline EV[AA'] & 0.00797 & 0.00590 & 0.02742 \\
& $(0.00322)$ & $(0.00217)$ & $(0.01053)$ \\
\hline EV[A'A] & 0.01191 & 0.00886 & 0.00959 \\
& $(0.00604)$ & $(0.00386)$ & $(0.00657)$ \\
\hline AM - EV[AA'] & 0.00770 & 0.00565 & 0.02620 \\
AND EV[A'A] & $(0.00310)$ & $(0.00212)$ & $(0.01002)$ \\
\hline GE - EV[AA'] & 0.00789 & 0.00569 & 0.01600 \\
AND EV[A'A] & $(0.00326)$ & $(0.00210)$ & $(0.00582)$ \\
\hline GE & 0.00634 & 0.00457 & 0.01682 \\
& $(0.00232)$ & $(0.00168)$ & $(0.00543)$ \\
\hline C-RSM & 0.00695 & 0.00497 & 0.01644 \\
& $(0.00264)$ & $(0.00179)$ & $(0.00548)$ \\
\hline MT & 0.00623 & 0.00455 & 0.01722 \\
& $(0.00227)$ & $(0.00162)$ & $(0.00524)$ \\
\hline SAV & 0.00947 & 0.00681 & 0.00994 \\
& $(0.00451)$ & $(0.00284)$ & $(0.00551)$ \\
\hline NEV & 0.00694 & 0.00542 & 0.02110 \\
& $(0.00277)$ & $(0.00218)$ & $(0.00807)$ \\
\hline LSM & 0.01169 & 0.00855 & 0.00864 \\
& $(0.00604)$ & $(0.00383)$ & $(0.00590)$ \\
\hline
\end{tabular}


Table B.13: Mean and Standard Deviation of Accuracy Measurement $\left(d_{1}\right)$ from Simulation (With $N=10, M=3$, scale $[1 / 9,9], T=500$ )

\begin{tabular}{||l||c|c|c||c|c|c||}
\hline \multicolumn{1}{|c||}{ M=3 } & \multicolumn{3}{c||}{ A-GE(V) } & \multicolumn{3}{c||}{ A-GE(M) } \\
\hline ABBREVIATIONS & UF & LN & GA & UF & LN & GA \\
\hline \hline CSM & 0.00853 & 0.00621 & 0.02539 & 0.00776 & 0.00594 & 0.01864 \\
& $(0.00268)$ & $(0.00236)$ & $(0.00670)$ & $(0.00253)$ & $(0.00227)$ & $(0.00533)$ \\
\hline R-EV & 0.00668 & 0.00444 & 0.01899 & 0.00669 & 0.00443 & 0.01764 \\
& $(0.00210)$ & $(0.00133)$ & $(0.00510)$ & $(0.00210)$ & $(0.00133)$ & $(0.00514)$ \\
\hline L-EV & 0.00669 & 0.00445 & 0.01962 & 0.00670 & 0.00443 & 0.01800 \\
& $(0.00211)$ & $(0.00133)$ & $(0.00535)$ & $(0.00213)$ & $(0.00132)$ & $(0.00522)$ \\
\hline AM-EV & 0.00667 & 0.00444 & 0.01916 & 0.00669 & 0.00442 & 0.01778 \\
& $(0.00211)$ & $(0.00132)$ & $(0.00519)$ & $(0.00213)$ & $(0.00132)$ & $(0.00512)$ \\
\hline GM-EV & 0.00665 & 0.00442 & 0.01852 & 0.00667 & 0.00442 & 0.01748 \\
& $(0.00209)$ & $(0.00132)$ & $(0.00494)$ & $(0.00211)$ & $(0.00132)$ & $(0.00501)$ \\
\hline EV[AA'] & 0.00837 & 0.00561 & 0.02633 & 0.00827 & 0.00557 & 0.02495 \\
& $(0.00259)$ & $(0.00177)$ & $(0.00742)$ & $(0.00257)$ & $(0.00174)$ & $(0.00764)$ \\
\hline EV[A'A] & 0.04485 & 0.04418 & 0.04474 & 0.04483 & 0.04420 & 0.04457 \\
& $(0.01588)$ & $(0.01552)$ & $(0.01393)$ & $(0.01590)$ & $(0.01553)$ & $(0.01474)$ \\
\hline AM-EV[AA'] & 0.00921 & 0.00757 & 0.02463 & 0.00936 & 0.00769 & 0.02331 \\
AND EV[A'A] & $(0.00251)$ & $(0.00230)$ & $(0.00621)$ & $(0.00254)$ & $(0.00233)$ & $(0.00632)$ \\
\hline GE- EV[AA'] & 0.02092 & 0.02047 & 0.02666 & 0.02102 & 0.02054 & 0.02558 \\
AND EV[A'A] & $(0.00725)$ & $(0.00711)$ & $(0.00696)$ & $(0.00724)$ & $(0.00711)$ & $(0.00714)$ \\
\hline GE & 0.00666 & 0.00442 & 0.01718 & 0.00666 & 0.00442 & 0.01704 \\
& $(0.00210)$ & $(0.00132)$ & $(0.00476)$ & $(0.00211)$ & $(0.00132)$ & $(0.00489)$ \\
\hline C-RSM & 0.00679 & 0.00451 & 0.01817 & 0.00678 & 0.00451 & 0.01703 \\
& $(0.00214)$ & $(0.00134)$ & $(0.00474)$ & $(0.00216)$ & $(0.00133)$ & $(0.00484)$ \\
\hline MT & 0.00663 & 0.00443 & 0.01770 & 0.00665 & 0.00443 & 0.01715 \\
& $(0.00207)$ & $(0.00131)$ & $(0.00453)$ & $(0.00209)$ & $(0.00132)$ & $(0.00482)$ \\
\hline SAV & 0.00803 & 0.00544 & 0.01603 & 0.00803 & 0.00544 & 0.01500 \\
& $(0.00265)$ & $(0.00164)$ & $(0.00438)$ & $(0.00268)$ & $(0.00163)$ & $(0.00437)$ \\
\hline NEV & 0.00815 & 0.00591 & 0.02348 & 0.00888 & 0.00612 & 0.02090 \\
& $(0.00288)$ & $(0.00189)$ & $(0.0074)$ & $(0.00312)$ & $(0.00195)$ & $(0.00705)$ \\
\hline LSM & 0.00980 & 0.00672 & 0.01652 & 0.00974 & 0.00668 & 0.01513 \\
& $(0.00357)$ & $(0.00229)$ & $(0.00602)$ & $(0.00367)$ & $(0.00227)$ & $(0.00491)$ \\
\hline
\end{tabular}


Table B.14: Mean and Standard Deviation of Accuracy Measurement $\left(d_{1}\right)$ from Simulation (With $\mathrm{N}=10, \mathrm{M}=3$, scale $[1 / 9,9], \mathrm{T}=500$ ) [Continued]

\begin{tabular}{||l||c|c|c||c|c|c||}
\hline \multicolumn{1}{|c||}{ M=3 } & \multicolumn{3}{c|}{ A-AM(V) } & \multicolumn{3}{c||}{ A-MDM(M) } \\
\hline ABBREVIATIONS & UF & LN & GA & UF & LN & GA \\
\hline \hline CSM & 0.00864 & 0.00626 & 0.02576 & 0.00786 & 0.00605 & 0.01926 \\
& $(0.00273)$ & $(0.00240)$ & $(0.00653)$ & $(0.00254)$ & $(0.00224)$ & $(0.00562)$ \\
\hline R-EV & 0.00668 & 0.00444 & 0.01912 & 0.00678 & 0.00457 & 0.01802 \\
& $(0.00210)$ & $(0.00134)$ & $(0.00528)$ & $(0.00213)$ & $(0.00135)$ & $(0.00535)$ \\
\hline L-EV & 0.00671 & 0.00446 & 0.01920 & 0.00681 & 0.00456 & 0.01846 \\
& $(0.00210)$ & $(0.00133)$ & $(0.00547)$ & $(0.00216)$ & $(0.00135)$ & $(0.00544)$ \\
\hline AM-EV & 0.00669 & 0.00444 & 0.01882 & 0.00679 & 0.00456 & 0.01821 \\
& $(0.00211)$ & $(0.00133)$ & $(0.00530)$ & $(0.00215)$ & $(0.00135)$ & $(0.00534)$ \\
\hline GE-EV & 0.00665 & 0.00443 & 0.01838 & 0.00677 & 0.00456 & 0.01785 \\
& $(0.00210)$ & $(0.00133)$ & $(0.00506)$ & $(0.00213)$ & $(0.00135)$ & $(0.00520)$ \\
\hline EV[AA'] & 0.00841 & 0.00562 & 0.02552 & 0.00841 & 0.00574 & 0.02554 \\
& $(0.00262)$ & $(0.00178)$ & $(0.00772)$ & $(0.00262)$ & $(0.00179)$ & $(0.00804)$ \\
\hline EV[A'A] & 0.04486 & 0.04419 & 0.04476 & 0.04483 & 0.04424 & 0.04470 \\
& $(0.01592)$ & $(0.01554)$ & $(0.01393)$ & $(0.01588)$ & $(0.01554)$ & $(0.01481)$ \\
\hline AM -EV[AA'] & 0.00917 & 0.00752 & 0.02384 & 0.00945 & 0.00780 & 0.02377 \\
AND EV[A'A] & $(0.00252)$ & $(0.00229)$ & $(0.00642)$ & $(0.00256)$ & $(0.00236)$ & $(0.00656)$ \\
\hline GE- EV[AA'] & 0.02090 & 0.02046 & 0.02625 & 0.02104 & 0.02058 & 0.02578 \\
AND EV[A'A] & $(0.00726)$ & $(0.00711)$ & $(0.00696)$ & $(0.00722)$ & $(0.00713)$ & $(0.00708)$ \\
\hline GE & 0.00667 & 0.00442 & 0.01705 & 0.00676 & 0.00456 & 0.01734 \\
& $(0.00211)$ & $(0.00132)$ & $(0.00487)$ & $(0.00213)$ & $(0.00135)$ & $(0.00508)$ \\
\hline C-RSM & 0.00680 & 0.00452 & 0.01797 & 0.00689 & 0.00464 & 0.01737 \\
& $(0.00215)$ & $(0.00134)$ & $(0.00487)$ & $(0.00218)$ & $(0.00135)$ & $(0.00501)$ \\
\hline MT & 0.00663 & 0.00443 & 0.01773 & 0.00675 & 0.00456 & 0.01739 \\
& $(0.00208)$ & $(0.00132)$ & $(0.00465)$ & $(0.00212)$ & $(0.00134)$ & $(0.00497)$ \\
\hline SAV & 0.00803 & 0.00545 & 0.01612 & 0.00817 & 0.00560 & 0.01524 \\
& $(0.00266)$ & $(0.00165)$ & $(0.00450)$ & $(0.00273)$ & $(0.00163)$ & $(0.00452)$ \\
\hline NEV & 0.00806 & 0.00592 & 0.02266 & 0.00895 & 0.00628 & 0.02136 \\
& $(0.00289)$ & $(0.00189)$ & $(0.00708)$ & $(0.00317)$ & $(0.00197)$ & $(0.00716)$ \\
\hline LSM & 0.00987 & 0.00676 & 0.01623 & 0.00995 & 0.00686 & 0.01526 \\
& $(0.00359)$ & $(0.00232)$ & $(0.00604)$ & $(0.00372)$ & $(0.00228)$ & $(0.00518)$ \\
\hline
\end{tabular}


Table B.15: Mean and Standard Deviation of Accuracy Measurement $\left(d_{1}\right)$ from Simulation (With $N=10, M=3$, scale $[1 / 9,9], T=500$ ) [Continued]

\begin{tabular}{||l||c|c|c||}
\hline \multicolumn{1}{|c||}{ M=3 } & \multicolumn{3}{c||}{ A-MDM(V) } \\
\hline ABBREVIATIONS & UF & LN & GA \\
\hline \hline CSM & 0.00769 & 0.00516 & 0.02355 \\
& $(0.00254)$ & $(0.00159)$ & $(0.00710)$ \\
\hline R-EV & 0.00751 & 0.00516 & 0.02061 \\
& $(0.00250)$ & $(0.00165)$ & $(0.00673)$ \\
\hline L-EV & 0.00743 & 0.00517 & 0.02184 \\
& $(0.00249)$ & $(0.00170)$ & $(0.00681)$ \\
\hline AM-EV & 0.00743 & 0.00517 & 0.02159 \\
& $(0.00249)$ & $(0.00170)$ & $(0.00676)$ \\
\hline GE-EV & 0.00741 & 0.00513 & 0.02021 \\
& $(0.00247)$ & $(0.00166)$ & $(0.00644)$ \\
\hline EV[AA'] & 0.00994 & 0.00666 & 0.03008 \\
& $(0.00330)$ & $(0.00228)$ & $(0.01024)$ \\
\hline EV[A'A] & 0.05774 & 0.05674 & 0.05603 \\
& $(0.02395)$ & $(0.02397)$ & $(0.02268)$ \\
\hline AM - EV[AA'] & 0.00985 & 0.00712 & 0.02897 \\
AND EV[A'A] & $(0.00319)$ & $(0.00232)$ & $(0.00968)$ \\
\hline GE - EV[AA'] & 0.02590 & 0.02543 & 0.03054 \\
AND EV[A'A] & $(0.01075)$ & $(0.01099)$ & $(0.01051)$ \\
\hline GE & 0.00745 & 0.00513 & 0.01801 \\
& $(0.00251)$ & $(0.00165)$ & $(0.00565)$ \\
\hline C-RSM & 0.00844 & 0.00578 & 0.01737 \\
& $(0.00283)$ & $(0.00191)$ & $(0.00567)$ \\
\hline MT & 0.00743 & 0.00513 & 0.01809 \\
& $(0.00251)$ & $(0.00162)$ & $(0.00539)$ \\
\hline SAV & 0.01242 & 0.00855 & 0.01124 \\
& $(0.00486)$ & $(0.00335)$ & $(0.00559)$ \\
\hline NEV & 0.00922 & 0.00695 & 0.02516 \\
& $(0.00324)$ & $(0.00251)$ & $(0.00931)$ \\
\hline LSM & 0.01619 & 0.01118 & 0.01082 \\
& $(0.00639)$ & $(0.00453)$ & $(0.00692)$ \\
\hline
\end{tabular}


Table B.16: Mean and Standard Deviation of Accuracy Measurement $\left(d_{1}\right)$ from Simulation (With $N=10, M=5$, scale $[1 / 9,9], T=500$ )

\begin{tabular}{||l||c|c|c||c|c|c||}
\hline \multicolumn{1}{|c||}{ M=5 } & \multicolumn{3}{c|}{ A-GE(V) } & \multicolumn{3}{c||}{ A-GE(M) } \\
\hline ABBREVIATIONS & UF & LN & GA & UF & LN & GA \\
\hline \hline CSM & 0.00761 & 0.00553 & 0.02404 & 0.00674 & 0.00526 & 0.01517 \\
& $(0.00254)$ & $(0.00240)$ & $(0.00580)$ & $(0.00238)$ & $(0.00228)$ & $(0.00414)$ \\
\hline R-EV & 0.00555 & 0.00357 & 0.01674 & 0.00554 & 0.00356 & 0.01512 \\
& $(0.00173)$ & $(0.00106)$ & $(0.00404)$ & $(0.00176)$ & $(0.00107)$ & $(0.00403)$ \\
\hline L-EV & 0.00554 & 0.00358 & 0.01739 & 0.00554 & 0.00356 & 0.01543 \\
& $(0.00177)$ & $(0.00107)$ & $(0.00442)$ & $(0.00178)$ & $(0.00106)$ & $(0.00427)$ \\
\hline AM-EV & 0.00553 & 0.00357 & 0.01702 & 0.00553 & 0.00355 & 0.01530 \\
& $(0.00176)$ & $(0.00107)$ & $(0.00429)$ & $(0.00178)$ & $(0.00106)$ & $(0.00420)$ \\
\hline GM-EV & 0.00551 & 0.00356 & 0.01646 & 0.00552 & 0.00355 & 0.01510 \\
& $(0.00174)$ & $(0.00106)$ & $(0.00404)$ & $(0.00176)$ & $(0.00106)$ & $(0.00407)$ \\
\hline EV[AA'] & 0.00667 & 0.00448 & 0.02342 & 0.00657 & 0.00444 & 0.02145 \\
& $(0.00221)$ & $(0.00141)$ & $(0.00585)$ & $(0.00223)$ & $(0.00140)$ & $(0.00609)$ \\
\hline EV[A'A] & 0.04455 & 0.04417 & 0.04427 & 0.04453 & 0.04418 & 0.04404 \\
& $(0.01583)$ & $(0.01557)$ & $(0.01381)$ & $(0.01587)$ & $(0.01558)$ & $(0.01466)$ \\
\hline AM - EV[AA'] & 0.00797 & 0.00693 & 0.02231 & 0.00820 & 0.00709 & 0.02051 \\
AND EV[A'A] & $(0.00227)$ & $(0.00225)$ & $(0.00491)$ & $(0.00235)$ & $(0.00232)$ & $(0.00500)$ \\
\hline GE - EV[AA'] & 0.02053 & 0.02040 & 0.02563 & 0.02064 & 0.02048 & 0.02429 \\
AND EV[A'A] & $(0.00713)$ & $(0.00713)$ & $(0.00682)$ & $(0.00714)$ & $(0.00714)$ & $(0.00708)$ \\
\hline GE & 0.00551 & 0.00356 & 0.01516 & 0.00551 & 0.00355 & 0.01489 \\
& $(0.00175)$ & $(0.00106)$ & $(0.00386)$ & $(0.00176)$ & $(0.00106)$ & $(0.00401)$ \\
\hline C-RSM & 0.00562 & 0.00361 & 0.01624 & 0.00560 & 0.00360 & 0.01461 \\
& $(0.00177)$ & $(0.00109)$ & $(0.00382)$ & $(0.00178)$ & $(0.00109)$ & $(0.00392)$ \\
\hline MT & 0.00550 & 0.00356 & 0.01575 & 0.00551 & 0.00355 & 0.01492 \\
& $(0.00171)$ & $(0.00106)$ & $(0.00362)$ & $(0.00174)$ & $(0.00107)$ & $(0.00389)$ \\
\hline SAV & 0.00651 & 0.00427 & 0.01397 & 0.00650 & 0.00426 & 0.01255 \\
& $(0.00209)$ & $(0.00134)$ & $(0.00366)$ & $(0.00211)$ & $(0.00134)$ & $(0.00357)$ \\
\hline NEV & 0.00635 & 0.00464 & 0.02074 & 0.00740 & 0.00498 & 0.01768 \\
& $(0.00255)$ & $(0.00153)$ & $(0.00637)$ & $(0.00276)$ & $(0.00162)$ & $(0.00579)$ \\
\hline LSM & 0.00784 & 0.00523 & 0.01390 & 0.00773 & 0.00518 & 0.01238 \\
& $(0.00281)$ & $(0.00185)$ & $(0.00519)$ & $(0.00288)$ & $(0.00184)$ & $(0.00392)$ \\
\hline
\end{tabular}


Table B.17: Mean and Standard Deviation of Accuracy Measurement $\left(d_{1}\right)$ from Simulation (With $N=10, M=5$, scale $[1 / 9,9], T=500$ ) [Continued]

\begin{tabular}{||l||c|c|c||c|c|c||}
\hline \multicolumn{1}{|c||}{ M=5 } & \multicolumn{3}{c|}{ A-AM(V) } & \multicolumn{3}{c||}{ A-MDM(M) } \\
\hline ABBREVIATIONS & UF & LN & GA & UF & LN & GA \\
\hline \hline CSM & 0.00775 & 0.00560 & 0.02440 & 0.00686 & 0.00532 & 0.01544 \\
& $(0.00261)$ & $(0.00245)$ & $(0.00565)$ & $(0.00239)$ & $(0.00228)$ & $(0.00428)$ \\
\hline R-EV & 0.00554 & 0.00357 & 0.01678 & 0.00563 & 0.00365 & 0.01528 \\
& $(0.00173)$ & $(0.00107)$ & $(0.00420)$ & $(0.00181)$ & $(0.00108)$ & $(0.00416)$ \\
\hline L-EV & 0.00554 & 0.00358 & 0.01675 & 0.00564 & 0.00365 & 0.01560 \\
& $(0.00177)$ & $(0.00108)$ & $(0.00459)$ & $(0.00183)$ & $(0.00107)$ & $(0.00441)$ \\
\hline AM-EV & 0.00553 & 0.00357 & 0.01648 & 0.00563 & 0.00365 & 0.01546 \\
& $(0.00177)$ & $(0.00107)$ & $(0.00444)$ & $(0.00182)$ & $(0.00107)$ & $(0.00433)$ \\
\hline GE-EV & 0.00551 & 0.00358 & 0.01618 & 0.00562 & 0.00365 & 0.01524 \\
& $(0.00174)$ & $(0.00106)$ & $(0.00417)$ & $(0.00181)$ & $(0.00107)$ & $(0.00419)$ \\
\hline EV[AA'] & 0.00670 & 0.00449 & 0.02212 & 0.00675 & 0.00457 & 0.02180 \\
& $(0.00225)$ & $(0.00142)$ & $(0.00618)$ & $(0.00232)$ & $(0.00147)$ & $(0.00630)$ \\
\hline EV[A'A] & 0.04456 & 0.04418 & 0.04428 & 0.04455 & 0.04421 & 0.04411 \\
& $(0.01586)$ & $(0.01559)$ & $(0.01384)$ & $(0.01582)$ & $(0.01558)$ & $(0.01474)$ \\
\hline AM - EV[AA'] & 0.00789 & 0.00687 & 0.0211. & 0.00833 & 0.00716 & 0.02073 \\
AND EV[A'A] & $(0.00225)$ & $(0.00223)$ & $(0.00508)$ & $(0.00243)$ & $(0.00233)$ & $(0.00523)$ \\
\hline GE - EV[AA'] & 0.02050 & 0.02038 & 0.02509 & 0.02071 & 0.02050 & 0.02434 \\
AND EV[A'A] & $(0.00713)$ & $(0.00713)$ & $(0.00681)$ & $(0.00715)$ & $(0.00713)$ & $(0.00718)$ \\
\hline GE & 0.00551 & 0.00355 & 0.01490 & 0.00560 & 0.00365 & 0.01496 \\
& $(0.00175)$ & $(0.00107)$ & $(0.00397)$ & $(0.00180)$ & $(0.00107)$ & $(0.00412)$ \\
\hline C-RSM & 0.00562 & 0.00361 & 0.01590 & 0.00571 & 0.00370 & 0.01472 \\
& $(0.00177)$ & $(0.00110)$ & $(0.00394)$ & $(0.00184)$ & $(0.00110)$ & $(0.00403)$ \\
\hline MT & 0.00549 & 0.00356 & 0.01568 & 0.00561 & 0.00365 & 0.01500 \\
& $(0.00171)$ & $(0.00106)$ & $(0.00372)$ & $(0.00179)$ & $(0.00107)$ & $(0.00400)$ \\
\hline SAV & 0.00651 & 0.00427 & 0.01401 & 0.00668 & 0.00440 & 0.01248 \\
& $(0.00209)$ & $(0.00136)$ & $(0.00379)$ & $(0.00220)$ & $(0.00135)$ & $(0.00357)$ \\
\hline NEV & 0.00625 & 0.00463 & 0.01929 & 0.00752 & 0.00510 & 0.01777 \\
& $(0.00256)$ & $(0.00153)$ & $(0.00596)$ & $(0.00287)$ & $(0.00161)$ & $(0.00613)$ \\
\hline LSM & 0.00789 & 0.00527 & 0.01343 & 0.00802 & 0.00536 & 0.01220 \\
& $(0.00283)$ & $(0.00188)$ & $(0.00514)$ & $(0.00303)$ & $(0.00188)$ & $(0.00394)$ \\
\hline
\end{tabular}


Table B.18: Mean and Standard Deviation of Accuracy Measurement $\left(d_{1}\right)$ from Simulation (With $N=10, M=5$, scale $[1 / 9,9], T=500$ ) [Continued]

\begin{tabular}{||l||c|c|c||}
\hline \multicolumn{1}{||c||}{ M=5 } & \multicolumn{3}{c||}{ A-MDM(V) } \\
\hline ABBREVIATIONS & UF & LN & GA \\
\hline \hline CSM & 0.00648 & 0.00414 & 0.02166 \\
& $(0.00216)$ & $(0.00133)$ & $(0.00635)$ \\
\hline R-EV & 0.00624 & 0.00411 & 0.01702 \\
& $(0.00624)$ & $(0.00136)$ & $(0.00527)$ \\
\hline L-EV & 0.00623 & 0.00408 & 0.01788 \\
& $(0.00204)$ & $(0.00135)$ & $(0.00558)$ \\
\hline AM-EV & 0.00623 & 0.00407 & 0.01765 \\
& $(0.00204)$ & $(0.00136)$ & $(0.00552)$ \\
\hline GE-EV & 0.00620 & 0.00407 & 0.01653 \\
& $(0.00205)$ & $(0.00134)$ & $(0.00502)$ \\
\hline EV[AA'] & 0.00798 & 0.00536 & 0.02584 \\
& $(0.00274)$ & $(0.00190)$ & $(0.00907)$ \\
\hline EV[A'A] & 0.05730 & 0.05647 & 0.05566 \\
& $(0.02389)$ & $(0.02363)$ & $(0.02280)$ \\
\hline AM - EV[AA'] & 0.00812 & 0.00603 & 0.02520 \\
AND EV[A'A] & $(0.00261)$ & $(0.00206)$ & $(0.00847)$ \\
\hline GE - EV[AA'] & 0.02546 & 0.02519 & 0.02945 \\
AND EV[A'A] & $(0.01068)$ & $(0.01087)$ & $(0.01059)$ \\
\hline GE & 0.00620 & 0.00407 & 0.01475 \\
& $(0.00207)$ & $(0.00135)$ & $(0.00463)$ \\
\hline C-RSM & 0.00688 & 0.00465 & 0.01456 \\
& $(0.00238)$ & $(0.00158)$ & $(0.00466)$ \\
\hline MT & 0.00614 & 0.00406 & 0.01511 \\
& $(0.00203)$ & $(0.00135)$ & $(0.00449)$ \\
\hline SAV & 0.00986 & 0.00674 & 0.00916 \\
& $(0.00395)$ & $(0.00275)$ & $(0.00440)$ \\
\hline NEV & 0.00736 & 0.00537 & 0.02143 \\
& $(0.00296)$ & $(0.00192)$ & $(0.00771)$ \\
\hline LSM & 0.01282 & 0.00897 & 0.00842 \\
& $(0.00546)$ & $(0.00380)$ & $(0.00506)$ \\
\hline
\end{tabular}


Table B.19: Mean and Standard Deviation of Accuracy Measurement $\left(d_{1}\right)$ from Simulation (With $N=10, M=7$, scale $[1 / 9,9], T=500$ )

\begin{tabular}{||l||c|c|c||c|c|c||}
\hline \multicolumn{1}{|c||}{ M=7 } & \multicolumn{3}{c|}{ A-GE(V) } & \multicolumn{3}{c||}{ A-GE(M) } \\
\hline ABBREVIATIONS & UF & LN & GA & UF & LN & GA \\
\hline \hline CSM & 0.00713 & 0.00522 & 0.02326 & 0.00623 & 0.00496 & 0.01361 \\
& $(0.00251)$ & $(0.00243)$ & $(0.00534)$ & $(0.00235)$ & $(0.00229)$ & $(0.00341)$ \\
\hline R-EV & 0.00493 & 0.00317 & 0.01559 & 0.00492 & 0.00314 & 0.01381 \\
& $(0.00153)$ & $(0.00094)$ & $(0.00353)$ & $(0.00156)$ & $(0.00095)$ & $(0.00350)$ \\
\hline L-EV & 0.00493 & 0.00316 & 0.01618 & 0.00490 & 0.00315 & 0.01413 \\
& $(0.00158)$ & $(0.00096)$ & $(0.00378)$ & $(0.00159)$ & $(0.00095)$ & $(0.00367)$ \\
\hline AM-EV & 0.00492 & 0.00316 & 0.01587 & 0.00490 & 0.00315 & 0.01404 \\
& $(0.00157)$ & $(0.00096)$ & $(0.00366)$ & $(0.00158)$ & $(0.00095)$ & $(0.00361)$ \\
\hline GM-EV & 0.00490 & 0.00315 & 0.01538 & 0.00490 & 0.00314 & 0.01387 \\
& $(0.00155)$ & $(0.00095)$ & $(0.00347)$ & $(0.00157)$ & $(0.00095)$ & $(0.00352)$ \\
\hline EV[AA'] & 0.00584 & 0.00388 & 0.02177 & 0.00572 & 0.00383 & 0.01948 \\
& $(0.00196)$ & $(0.00122)$ & $(0.00503)$ & $(0.00196)$ & $(0.00121)$ & $(0.00522)$ \\
\hline EV[A'A] & 0.04445 & 0.04416 & 0.04403 & 0.04441 & 0.04417 & 0.04380 \\
& $(0.01581)$ & $(0.01561)$ & $(0.01381)$ & $(0.01585)$ & $(0.01562)$ & $(0.01469)$ \\
\hline AM - EV[AA'] & 0.00741 & 0.00663 & 0.02109 & 0.00769 & 0.00682 & 0.01901 \\
AND EV[A'A] & $(0.00217)$ & $(0.00221)$ & $(0.00433)$ & $(0.00229)$ & $(0.00228)$ & $(0.00435)$ \\
\hline GE- EV[AA'] & 0.02043 & 0.02036 & 0.02523 & 0.02056 & 0.02045 & 0.02379 \\
& $(0.00713)$ & $(0.00712)$ & $(0.00677)$ & $(0.00714)$ & $(0.00714)$ & $(0.00709)$ \\
\hline GE & 0.00489 & 0.00315 & 0.01408 & 0.00488 & 0.00314 & 0.01372 \\
& $(0.00156)$ & $(0.00095)$ & $(0.00333)$ & $(0.00157)$ & $(0.00095)$ & $(0.00349)$ \\
\hline C-RSM & 0.00499 & 0.00319 & 0.01522 & 0.00496 & 0.00319 & 0.01334 \\
& $(0.00156)$ & $(0.00096)$ & $(0.00332)$ & $(0.00157)$ & $(0.00097)$ & $(0.00342)$ \\
\hline MT & 0.00489 & 0.00316 & 0.01476 & 0.00490 & 0.00314 & 0.01371 \\
& $(0.00151)$ & $(0.00094)$ & $(0.00320)$ & $(0.00155)$ & $(0.00095)$ & $(0.00342)$ \\
\hline SAV & 0.00573 & 0.00373 & 0.01302 & 0.00570 & 0.00371 & 0.01133 \\
& $(0.00181)$ & $(0.00119)$ & $(0.00326)$ & $(0.00184)$ & $(0.00120)$ & $(0.00312)$ \\
\hline NEV & 0.00540 & 0.00393 & 0.01948 & 0.00662 & 0.00439 & 0.01621 \\
& $(0.00225)$ & $(0.00132)$ & $(0.00560)$ & $(0.00239)$ & $(0.00147)$ & $(0.00495)$ \\
\hline LSM & 0.00680 & 0.00453 & 0.01246 & 0.00665 & 0.00446 & 0.01099 \\
& $(0.00248)$ & $(0.00157)$ & $(0.00463)$ & $(0.00254)$ & $(0.00159)$ & $(0.00332)$ \\
\hline
\end{tabular}


Table B.20: Mean and Standard Deviation of Accuracy Measurement $\left(d_{1}\right)$ from Simulation (With $N=10, M=7$, scale $[1 / 9,9], T=500$ ) [Continued]

\begin{tabular}{||l||c|c|c||c|c|c||}
\hline \multicolumn{1}{|c||}{ M=7 } & \multicolumn{3}{c|}{ A-AM(V) } & \multicolumn{3}{c||}{ A-MDM(M) } \\
\hline ABBREVIATIONS & UF & LN & GA & UF & LN & GA \\
\hline \hline CSM & 0.00730 & 0.00530 & 0.02360 & 0.00629 & 0.00504 & 0.01374 \\
& $(0.00261)$ & $(0.00249)$ & $(0.00519)$ & $(0.00236)$ & $(0.00227)$ & $(0.00358)$ \\
\hline R-EV & 0.00492 & 0.00316 & 0.01553 & 0.00494 & 0.00325 & 0.01382 \\
& $(0.00153)$ & $(0.00095)$ & $(0.00372)$ & $(0.00161)$ & $(0.00097)$ & $(0.00369)$ \\
\hline L-EV & 0.00492 & 0.00316 & 0.01541 & 0.00492 & 0.00325 & 0.01420 \\
& $(0.00159)$ & $(0.00097)$ & $(0.00393)$ & $(0.00163)$ & $(0.00097)$ & $(0.00381)$ \\
\hline AM-EV & 0.00491 & 0.00315 & 0.01522 & 0.00492 & 0.00325 & 0.01409 \\
& $(0.00158)$ & $(0.00096)$ & $(0.00381)$ & $(0.00163)$ & $(0.00097)$ & $(0.00375)$ \\
\hline GE-EV & 0.00490 & 0.00314 & 0.01502 & 0.00492 & 0.00325 & 0.01388 \\
& $(0.00156)$ & $(0.00095)$ & $(0.00362)$ & $(0.00161)$ & $(0.00097)$ & $(0.00367)$ \\
\hline EV[AA'] & 0.00585 & 0.00387 & 0.02020 & 0.00585 & 0.00395 & 0.01966 \\
& $(0.00199)$ & $(0.00123)$ & $(0.00531)$ & $(0.00211)$ & $(0.00124)$ & $(0.00548)$ \\
\hline EV[A'A] & 0.04446 & 0.04416 & 0.04405 & 0.04442 & 0.04420 & 0.04391 \\
& $(0.01585)$ & $(0.01563)$ & $(0.01383)$ & $(0.01581)$ & $(0.01562)$ & $(0.01487)$ \\
\hline AM - EV[AA'] & 0.00730 & 0.00655 & 0.01964 & 0.00778 & 0.00687 & 0.01901 \\
AND EV[A'A] & $(0.00215)$ & $(0.00219)$ & $(0.00445)$ & $(0.00233)$ & $(0.00229)$ & $(0.00464)$ \\
\hline GE - EV[AA'] & 0.02039 & 0.02034 & 0.02462 & 0.02059 & 0.02047 & 0.02372 \\
AND EV[A'A] & $(0.00713)$ & $(0.00712)$ & $(0.00676)$ & $(0.00714)$ & $(0.00713)$ & $(0.00715)$ \\
\hline GE & 0.00488 & 0.00314 & 0.01375 & 0.00490 & 0.00324 & 0.01368 \\
& $(0.00157)$ & $(0.00095)$ & $(0.00345)$ & $(0.00161)$ & $(0.00096)$ & $(0.00361)$ \\
\hline C-RSM & 0.00499 & 0.00319 & 0.01478 & 0.00499 & 0.00330 & 0.01335 \\
& $(0.00157)$ & $(0.00097)$ & $(0.00344)$ & $(0.00163)$ & $(0.00098)$ & $(0.00350)$ \\
\hline MT & 0.00487 & 0.00315 & 0.01461 & 0.00491 & 0.00324 & 0.01365 \\
& $(0.00152)$ & $(0.00094)$ & $(0.00332)$ & $(0.00160)$ & $(0.00096)$ & $(0.00357)$ \\
\hline SAV & 0.00571 & 0.00373 & 0.01303 & 0.00578 & 0.00387 & 0.01121 \\
& $(0.00183)$ & $(0.00121)$ & $(0.00337)$ & $(0.00194)$ & $(0.00134)$ & $(0.00316)$ \\
\hline NEV & 0.00528 & 0.00391 & 0.01770 & 0.00668 & 0.00455 & 0.01616 \\
& $(0.00226)$ & $(0.00132)$ & $(0.00523)$ & $(0.00251)$ & $(0.00146)$ & $(0.00502)$ \\
\hline LSM & 0.00684 & 0.00457 & 0.01186 & 0.00686 & 0.00467 & 0.01069 \\
& $(0.00251)$ & $(0.00160)$ & $(0.00458)$ & $(0.00271)$ & $(0.00164)$ & $(0.00336)$ \\
\hline
\end{tabular}


Table B.21: Mean and Standard Deviation of Accuracy Measurement $\left(d_{1}\right)$ from Simulation (With $N=10, M=7$, scale $[1 / 9,9], T=500$ ) [Continued]

\begin{tabular}{||l||c|c|c||}
\hline \multicolumn{1}{|c||}{ M=7 } & \multicolumn{3}{c|}{ A-MDM(V) } \\
\hline ABBREVIATIONS & UF & LN & GA \\
\hline \hline CSM & 0.00585 & 0.00376 & 0.02104 \\
& $(0.00187)$ & $(0.00125)$ & $(0.00596)$ \\
\hline R-EV & 0.00564 & 0.00370 & 0.01558 \\
& $(0.00178)$ & $(0.00125)$ & $(0.00504)$ \\
\hline L-EV & 0.00561 & 0.00369 & 0.01632 \\
& $(0.00180)$ & $(0.00126)$ & $(0.00494)$ \\
\hline AM-EV & 0.00560 & 0.00368 & 0.01614 \\
& $(0.00180)$ & $(0.00127)$ & $(0.00492)$ \\
\hline GE-EV & 0.00559 & 0.00369 & 0.01521 \\
& $(0.00176)$ & $(0.00127)$ & $(0.00485)$ \\
\hline EV[AA'] & 0.00696 & 0.00475 & 0.02360 \\
& $(0.00254)$ & $(0.00162)$ & $(0.00808)$ \\
\hline EV[A'A] & 0.05701 & 0.05620 & 0.05559 \\
& $(0.02376)$ & $(0.02353)$ & $(0.02277)$ \\
\hline AM - EV[AA'] & 0.00717 & 0.00550 & 0.02300 \\
AND EV[A'A] & $(0.00245)$ & $(0.00186)$ & $(0.00749)$ \\
\hline GE - EV[AA'] & 0.02524 & 0.02498 & 0.02896 \\
AND EV[A'A] & $(0.01074)$ & $(0.01077)$ & $(0.01066)$ \\
\hline GE & 0.00556 & 0.00369 & 0.01353 \\
& $(0.00179)$ & $(0.00126)$ & $(0.00435)$ \\
\hline C-RSM & 0.00608 & 0.00411 & 0.01339 \\
& $(0.00214)$ & $(0.00145)$ & $(0.00440)$ \\
\hline MT & 0.00557 & 0.00368 & 0.01395 \\
& $(0.00174)$ & $(0.00124)$ & $(0.00425)$ \\
\hline SAV & 0.00841 & 0.00587 & 0.00802 \\
& $(0.00353)$ & $(0.00239)$ & $(0.00693)$ \\
\hline NEV & 0.00622 & 0.00463 & 0.01926 \\
& $(0.00252)$ & $(0.00170)$ & $(0.00693)$ \\
\hline LSM & 0.01111 & 0.00772 & 0.00714 \\
& $(0.00495)$ & $(0.00334)$ & $(0.00423)$ \\
\hline
\end{tabular}


Table B.22: Mean and Standard Deviation of Accuracy Measurement $\left(d_{1}\right)$ from Simulation (With $N=10, M=9$, scale $[1 / 9,9], T=500$ )

\begin{tabular}{||l||c|c|c||c|c|c||}
\hline \multicolumn{1}{|c||}{ M=9 } & \multicolumn{3}{c|}{ A-GE(V) } & \multicolumn{3}{c||}{ A-GE(M) } \\
\hline ABBREVIATIONS & UF & LN & GA & UF & LN & GA \\
\hline \hline CSM & 0.00683 & 0.00506 & 0.02282 & 0.00595 & 0.00482 & 0.01286 \\
& $(0.00252)$ & $(0.00244)$ & $(0.00507)$ & $(0.00229)$ & $(0.00228)$ & $(0.00304)$ \\
\hline R-EV & 0.00458 & 0.00294 & 0.01501 & 0.00455 & 0.00291 & 0.01313 \\
& $(0.00133)$ & $(0.00085)$ & $(0.00331)$ & $(0.00137)$ & $(0.00084)$ & $(0.00325)$ \\
\hline L-EV & 0.00457 & 0.00292 & 0.01551 & 0.00454 & 0.00291 & 0.01345 \\
& $(0.00138)$ & $(0.00085)$ & $(0.00342)$ & $(0.00140)$ & $(0.00085)$ & $(0.00337)$ \\
\hline AM-EV & 0.00456 & 0.00292 & 0.01524 & 0.00454 & 0.00291 & 0.01338 \\
& $(0.00138)$ & $(0.00085)$ & $(0.00333)$ & $(0.00139)$ & $(0.00085)$ & $(0.00333)$ \\
\hline GM-EV & 0.00455 & 0.00292 & 0.01481 & 0.00454 & 0.00291 & 0.01323 \\
& $(0.00135)$ & $(0.00084)$ & $(0.00321)$ & $(0.00138)$ & $(0.00085)$ & $(0.00326)$ \\
\hline EV[AA'] & 0.00535 & 0.00352 & 0.02100 & 0.00523 & 0.00348 & 0.01863 \\
& $(0.00185)$ & $(0.00107)$ & $(0.00470)$ & $(0.00184)$ & $(0.00106)$ & $(0.00484)$ \\
\hline EV[A'A] & 0.04443 & 0.04419 & 0.04398 & 0.04439 & 0.04419 & 0.04377 \\
& $(0.01578)$ & $(0.01562)$ & $(0.01372)$ & $(0.01581)$ & $(0.01564)$ & $(0.01462)$ \\
\hline AM - EV[AA'] & 0.00708 & 0.00646 & 0.02051 & 0.00740 & 0.00667 & 0.01837 \\
AND EV[A'A] & $(0.00218)$ & $(0.00219)$ & $(0.00407)$ & $(0.00230)$ & $(0.00228)$ & $(0.00406)$ \\
\hline GE - EV[AA'] & 0.02038 & 0.02036 & 0.02500 & 0.02051 & 0.02045 & 0.02359 \\
AND EV[A'A] & $(0.00712)$ & $(0.00714)$ & $(0.00668)$ & $(0.00713)$ & $(0.00715)$ & $(0.00702)$ \\
\hline GE & 0.00453 & 0.00292 & 0.01352 & 0.00452 & 0.00291 & 0.01311 \\
& $(0.00136)$ & $(0.00084)$ & $(0.00309)$ & $(0.00137)$ & $(0.00085)$ & $(0.00323)$ \\
\hline C-RSM & 0.00463 & 0.00296 & 0.01468 & 0.00459 & 0.00296 & 0.01269 \\
& $(0.00136)$ & $(0.00085)$ & $(0.00309)$ & $(0.00137)$ & $(0.00085)$ & $(0.00317)$ \\
\hline MT & 0.00453 & 0.00293 & 0.01425 & 0.00454 & 0.00291 & 0.01307 \\
& $(0.00132)$ & $(0.00084)$ & $(0.00300)$ & $(0.00136)$ & $(0.00084)$ & $(0.00318)$ \\
\hline SAV & 0.00525 & 0.00345 & 0.01244 & 0.00520 & 0.00343 & 0.01049 \\
& $(0.00161)$ & $(0.00105)$ & $(0.00316)$ & $(0.00166)$ & $(0.00106)$ & $(0.00298)$ \\
\hline NEV & 0.00472 & 0.00345 & 0.01850 & 0.00616 & 0.00401 & 0.01539 \\
& $(0.00203)$ & $(0.00115)$ & $(0.00517)$ & $(0.00218)$ & $(0.00132)$ & $(0.00475)$ \\
\hline LSM & 0.00619 & 0.00415 & 0.01153 & 0.00601 & 0.00410 & 0.01003 \\
& $(0.00223)$ & $(0.00140)$ & $(0.00444)$ & $(0.00234)$ & $(0.00142)$ & $(0.00307)$ \\
\hline
\end{tabular}


Table B.23: Mean and Standard Deviation of Accuracy Measurement $\left(d_{1}\right)$ from Simulation (With $N=10, M=9$, scale $[1 / 9,9], T=500$ ) [Continued]

\begin{tabular}{||l||c|c|c||c|c|c||}
\hline \multicolumn{1}{|c||}{ M=9 } & \multicolumn{3}{c|}{ A-AM(V) } & \multicolumn{3}{c||}{ A-MDM(M) } \\
\hline ABBREVIATIONS & UF & LN & GA & UF & LN & GA \\
\hline \hline CSM & 0.00700 & 0.00514 & 0.02317 & 0.00597 & 0.00488 & 0.01288 \\
& $(0.00262)$ & $(0.00250)$ & $(0.00492)$ & $(0.00227)$ & $(0.00227)$ & $(0.00329)$ \\
\hline R-EV & 0.00457 & 0.00293 & 0.01463 & 0.00453 & 0.00300 & 0.01308 \\
& $(0.00134)$ & $(0.00085)$ & $(0.00348)$ & $(0.00141)$ & $(0.00087)$ & $(0.00349)$ \\
\hline L-EV & 0.00456 & 0.00292 & 0.01463 & 0.00451 & 0.00301 & 0.01342 \\
& $(0.00140)$ & $(0.00086)$ & $(0.00360)$ & $(0.00145)$ & $(0.00087)$ & $(0.00357)$ \\
\hline AM-EV & 0.00455 & 0.00292 & 0.01449 & 0.00451 & 0.00300 & 0.01333 \\
& $(0.00139)$ & $(0.00085)$ & $(0.00350)$ & $(0.00145)$ & $(0.00087)$ & $(0.00353)$ \\
\hline GE-EV & 0.00455 & 0.00291 & 0.01438 & 0.00451 & 0.00300 & 0.01315 \\
& $(0.00136)$ & $(0.00085)$ & $(0.00335)$ & $(0.00142)$ & $(0.00087)$ & $(0.00347)$ \\
\hline EV[AA'] & 0.00535 & 0.00352 & 0.01922 & 0.00534 & 0.00359 & 0.01862 \\
& $(0.00187)$ & $(0.00108)$ & $(0.00496)$ & $(0.00194)$ & $(0.00111)$ & $(0.00520)$ \\
\hline EV[A'A] & 0.04444 & 0.04419 & 0.0440 & 0.04438 & 0.04421 & 0.04383 \\
& $(0.01582)$ & $(0.01564)$ & $(0.01374)$ & $(0.01579)$ & $(0.01563)$ & $(0.01484)$ \\
\hline AM - EV[AA'] & 0.00696 & 0.00638 & 0.01889 & 0.00747 & 0.00672 & 0.01824 \\
AND EV[A'A] & $(0.00216)$ & $(0.00217)$ & $(0.00415)$ & $(0.00232)$ & $(0.00227)$ & $(0.00432)$ \\
\hline GE - EV[AA'] & 0.02034 & 0.02034 & 0.02437 & 0.02052 & 0.02046 & 0.02344 \\
AND EV[A'A] & $(0.00713)$ & $(0.00714)$ & $(0.00666)$ & $(0.00713)$ & $(0.00715)$ & $(0.00704)$ \\
\hline GE & 0.00452 & 0.00291 & 0.01313 & 0.00449 & 0.00300 & 0.01298 \\
& $(0.00137)$ & $(0.00085)$ & $(0.00320)$ & $(0.00142)$ & $(0.00087)$ & $(0.00341)$ \\
\hline C-RSM & 0.00462 & 0.00296 & 0.01419 & 0.00457 & 0.00306 & 0.01263 \\
& $(0.00137)$ & $(0.00086)$ & $(0.00319)$ & $(0.00144)$ & $(0.00088)$ & $(0.00330)$ \\
\hline MT & 0.00452 & 0.00292 & 0.01407 & 0.00450 & 0.00300 & 0.01294 \\
& $(0.00133)$ & $(0.00084)$ & $(0.00311)$ & $(0.00140)$ & $(0.00087)$ & $(0.00336)$ \\
\hline SAV & 0.00523 & 0.00345 & 0.01241 & 0.00528 & 0.00359 & 0.01037 \\
& $(0.00163)$ & $(0.00106)$ & $(0.00326)$ & $(0.00178)$ & $(0.00110)$ & $(0.00298)$ \\
\hline NEV & 0.00461 & 0.00342 & 0.01655 & 0.00603 & 0.00416 & 0.01540 \\
& $(0.00204)$ & $(0.00116)$ & $(0.00480)$ & $(0.00227)$ & $(0.00133)$ & $(0.00486)$ \\
\hline LSM & 0.00621 & 0.00418 & 0.01085 & 0.00624 & 0.00432 & 0.00971 \\
& $(0.00227)$ & $(0.00143)$ & $(0.00440)$ & $(0.00253)$ & $(0.00147)$ & $(0.00319)$ \\
\hline
\end{tabular}


Table B.24: Mean and Standard Deviation of Accuracy Measurement $\left(d_{1}\right)$ from Simulation (With $N=10, M=9$, scale $[1 / 9,9], T=500$ ) [Continued]

\begin{tabular}{||l||c|c|c||}
\hline \multicolumn{1}{|c||}{ M=9 } & \multicolumn{3}{c|}{ A-MDM(V) } \\
\hline ABBREVIATIONS & UF & LN & GA \\
\hline \hline CSM & 0.00539 & 0.00346 & 0.02056 \\
& $(0.00164)$ & $(0.00112)$ & $(0.00568)$ \\
\hline R-EV & 0.00507 & 0.00345 & 0.01466 \\
& $(0.00155)$ & $(0.00117)$ & $(0.00473)$ \\
\hline L-EV & 0.00515 & 0.00345 & 0.01542 \\
& $(0.00159)$ & $(0.00116)$ & $(0.00454)$ \\
\hline AM-EV & 0.00514 & 0.00345 & 0.01528 \\
& $(0.00159)$ & $(0.00116)$ & $(0.00448)$ \\
\hline GE-EV & 0.00508 & 0.00344 & 0.01441 \\
& $(0.00155)$ & $(0.00116)$ & $(0.00436)$ \\
\hline EV[AA'] & 0.00642 & 0.00438 & 0.02244 \\
& $(0.00235)$ & $(0.00149)$ & $(0.00729)$ \\
\hline EV[A'A] & 0.05681 & 0.05632 & 0.05564 \\
& $(0.02372)$ & $(0.02359)$ & $(0.02274)$ \\
\hline AM-EV[AA'] & 0.00671 & 0.00515 & 0.02196 \\
AND EV[A'A] & $(0.00232)$ & $(0.00184)$ & $(0.00679)$ \\
\hline GE - EV[AA'] & 0.02509 & 0.02501 & 0.02870 \\
AND EV[A'A] & $(0.01062)$ & $(0.01079)$ & $(0.01064)$ \\
\hline GE & 0.00505 & 0.00345 & 0.01290 \\
& $(0.00157)$ & $(0.00116)$ & $(0.00393)$ \\
\hline C-RSM & 0.00552 & 0.00379 & 0.01270 \\
& $(0.00190)$ & $(0.00130)$ & $(0.00389)$ \\
\hline MT & 0.00499 & 0.00343 & 0.01316 \\
& $(0.00151)$ & $(0.00117)$ & $(0.00397)$ \\
\hline SAV & 0.00750 & 0.00524 & 0.00735 \\
& $(0.00322)$ & $(0.00203)$ & $(0.00380)$ \\
\hline NEV & 0.00541 & 0.00417 & 0.01829 \\
& $(0.00222)$ & $(0.00150)$ & $(0.00651)$ \\
\hline LSM & 0.00979 & 0.00696 & 0.00637 \\
& $(0.00461)$ & $(0.00287)$ & $(0.00385)$ \\
\hline
\end{tabular}


Table B.25: Mean and Standard Deviation of Accuracy Measurement $\left(d_{1}\right)$ from Simulation (With $N=12, M=3$, scale $[1 / 9,9], T=500$ )

\begin{tabular}{||l||c|c|c||c|c|c||}
\hline \multicolumn{1}{|c||}{ M=3 } & \multicolumn{3}{c||}{ A-GE(V) } & \multicolumn{3}{c||}{ A-GE(M) } \\
\hline ABBREVIATIONS & UF & LN & GA & UF & LN & GA \\
\hline \hline CSM & 0.00650 & 0.00485 & 0.01931 & 0.00580 & 0.00455 & 0.01490 \\
& $(0.00194)$ & $(0.00171)$ & $(0.00436)$ & $(0.00181)$ & $(0.00159)$ & $(0.00367)$ \\
\hline R-EV & 0.00509 & 0.00354 & 0.01589 & 0.00505 & 0.00353 & 0.01470 \\
& $(0.00145)$ & $(0.00095)$ & $(0.00375)$ & $(0.00145)$ & $(0.00096)$ & $(0.00381)$ \\
\hline L-EV & 0.00505 & 0.00356 & 0.01653 & 0.00510 & 0.00354 & 0.01491 \\
& $(0.00143)$ & $(0.00095)$ & $(0.00397)$ & $(0.00146)$ & $(0.00095)$ & $(0.00381)$ \\
\hline AM-EV & 0.00504 & 0.00355 & 0.01613 & 0.00509 & 0.00353 & 0.01474 \\
& $(0.00143)$ & $(0.00095)$ & $(0.00384)$ & $(0.00145)$ & $(0.00095)$ & $(0.00374)$ \\
\hline GM-EV & 0.00504 & 0.00353 & 0.01548 & 0.00505 & 0.00353 & 0.01451 \\
& $(0.00143)$ & $(0.00095)$ & $(0.00361)$ & $(0.00145)$ & $(0.00095)$ & $(0.00365)$ \\
\hline EV[AA'] & 0.00631 & 0.00447 & 0.02170 & 0.00626 & 0.00443 & 0.02031 \\
& $(0.00185)$ & $(0.00123)$ & $(0.00575)$ & $(0.00185)$ & $(0.00123)$ & $(0.00589)$ \\
\hline EV[A'A] & 0.03796 & 0.03767 & 0.03792 & 0.03782 & 0.03762 & 0.03783 \\
& $(0.01067)$ & $(0.01063)$ & $(0.00898)$ & $(0.01067)$ & $(0.01063)$ & $(0.01006)$ \\
\hline AM-EV[AA'] & 0.00721 & 0.00625 & 0.02078 & 0.00734 & 0.00634 & 0.01941 \\
AND EV[A'A] & $(0.00192)$ & $(0.00169)$ & $(0.00479)$ & $(0.00200)$ & $(0.00172)$ & $(0.00486)$ \\
\hline GE - EV[AA'] & 0.01777 & 0.01766 & 0.02312 & 0.01779 & 0.01768 & 0.02206 \\
AND EV[A'A] & $(0.00481)$ & $(0.00469)$ & $(0.00498)$ & $(0.00483)$ & $(0.00469)$ & $(0.00494)$ \\
\hline GE & 0.00504 & 0.00352 & 0.01423 & 0.00505 & 0.00353 & 0.01417 \\
& $(0.00144)$ & $(0.00095)$ & $(0.00349)$ & $(0.00145)$ & $(0.00095)$ & $(0.00356)$ \\
\hline C-RSM & 0.00515 & 0.00356 & 0.01508 & 0.00515 & 0.00357 & 0.01401 \\
& $(0.00149)$ & $(0.00096)$ & $(0.00347)$ & $(0.00149)$ & $(0.00096)$ & $(0.00351)$ \\
\hline MT & 0.00505 & 0.00353 & 0.01483 & 0.00503 & 0.00352 & 0.01429 \\
& $(0.00144)$ & $(0.00095)$ & $(0.00337)$ & $(0.00144)$ & $(0.00095)$ & $(0.00356)$ \\
\hline SAV & 0.00617 & 0.00425 & 0.01314 & 0.00616 & 0.00425 & 0.01220 \\
& $(0.00188)$ & $(0.00116)$ & $(0.00321)$ & $(0.00187)$ & $(0.00117)$ & $(0.00315)$ \\
\hline NEV & 0.00616 & 0.00506 & 0.01998 & 0.00682 & 0.00521 & 0.01723 \\
& $(0.00179)$ & $(0.00159)$ & $(0.00547)$ & $(0.00196)$ & $(0.00166)$ & $(0.00525)$ \\
\hline LSM & 0.00769 & 0.00529 & 0.01298 & 0.00764 & 0.00524 & 0.01195 \\
& $(0.00257)$ & $(0.00157)$ & $(0.00434)$ & $(0.00260)$ & $(0.00159)$ & $(0.00341)$ \\
\hline
\end{tabular}


Table B.26: Mean and Standard Deviation of Accuracy Measurement $\left(d_{1}\right)$ from Simulation (With $N=12, M=3$, scale $[1 / 9,9], T=500$ ) [Continued]

\begin{tabular}{||l||c|c|c||c|c|c||}
\hline \multicolumn{1}{|c||}{ M=3 } & \multicolumn{3}{c|}{ A-AM(V) } & \multicolumn{3}{c||}{ A-MDM(M) } \\
\hline ABBREVIATIONS & UF & LN & GA & UF & LN & GA \\
\hline \hline CSM & 0.00660 & 0.00490 & 0.01967 & 0.00584 & 0.00462 & 0.01529 \\
& $(0.00197)$ & $(0.00173)$ & $(0.00443)$ & $(0.00183)$ & $(0.00160)$ & $(0.00381)$ \\
\hline R-EV & 0.00510 & 0.00354 & 0.01602 & 0.00508 & 0.00362 & 0.01498 \\
& $(0.00145)$ & $(0.00096)$ & $(0.00386)$ & $(0.00148)$ & $(0.00098)$ & $(0.00389)$ \\
\hline L-EV & 0.00507 & 0.00356 & 0.01627 & 0.00512 & 0.00363 & 0.01525 \\
& $(0.00143)$ & $(0.00096)$ & $(0.00408)$ & $(0.00149)$ & $(0.00097)$ & $(0.00389)$ \\
\hline AM-EV & 0.00506 & 0.00355 & 0.01592 & 0.00511 & 0.00362 & 0.01506 \\
& $(0.00143)$ & $(0.00095)$ & $(0.00395)$ & $(0.00149)$ & $(0.00097)$ & $(0.00382)$ \\
\hline GE-EV & 0.00505 & 0.00353 & 0.01541 & 0.00508 & 0.00361 & 0.01478 \\
& $(0.00143)$ & $(0.00095)$ & $(0.00372)$ & $(0.00148)$ & $(0.00098)$ & $(0.00374)$ \\
\hline EV[AA'] & 0.00634 & 0.00448 & 0.02125 & 0.00632 & 0.00455 & 0.02071 \\
& $(0.00186)$ & $(0.00123)$ & $(0.00597)$ & $(0.00191)$ & $(0.00128)$ & $(0.00600)$ \\
\hline EV[A'A] & 0.03798 & 0.03768 & 0.03795 & 0.03781 & 0.03763 & 0.03790 \\
& $(0.01068)$ & $(0.01064)$ & $(0.00988)$ & $(0.01067)$ & $(0.01063)$ & $(0.01010)$ \\
\hline AM - EV[AA'] & 0.00717 & 0.00621 & 0.02027 & 0.00737 & 0.00369 & 0.01972 \\
AND EV[A'A] & $(0.00192)$ & $(0.00168)$ & $(0.00497)$ & $(0.00202)$ & $(0.00173)$ & $(0.00497)$ \\
\hline GE - EV[AA'] & 0.01775 & 0.01765 & 0.02282 & 0.01777 & 0.01767 & 0.02219 \\
AND EV[A'A] & $(0.00480)$ & $(0.00468)$ & $(0.00496)$ & $(0.00483)$ & $(0.00469)$ & $(0.00499)$ \\
\hline GE & 0.00506 & 0.00353 & 0.01419 & 0.00507 & 0.00361 & 0.01437 \\
& $(0.00144)$ & $(0.00095)$ & $(0.00358)$ & $(0.00148)$ & $(0.00098)$ & $(0.00363)$ \\
\hline C-RSM & 0.00516 & 0.00357 & 0.01498 & 0.00517 & 0.00365 & 0.01423 \\
& $(0.00150)$ & $(0.00097)$ & $(0.00359)$ & $(0.00152)$ & $(0.00099)$ & $(0.00356)$ \\
\hline MT & 0.00505 & 0.00353 & 0.01487 & 0.00505 & 0.00361 & 0.01450 \\
& $(0.00144)$ & $(0.00095)$ & $(0.00346)$ & $(0.00147)$ & $(0.00098)$ & $(0.00361)$ \\
\hline SAV & 0.00619 & 0.00427 & 0.01321 & 0.00190 & 0.00433 & 0.01234 \\
& $(0.00188)$ & $(0.00117)$ & $(0.00327)$ & $(0.00604)$ & $(0.00122)$ & $(0.00361)$ \\
\hline NEV & 0.00612 & 0.00506 & 0.01924 & 0.00202 & 0.00535 & 0.01755 \\
& $(0.00180)$ & $(0.00159)$ & $(0.00546)$ & $(0.00663)$ & $(0.00170)$ & $(0.00536)$ \\
\hline LSM & 0.00778 & 0.00534 & 0.01276 & 0.00264 & 0.00534 & 0.01201 \\
& $(0.00259)$ & $(0.00158)$ & $(0.00427)$ & $(0.00729)$ & $(0.00165)$ & $(0.00349)$ \\
\hline
\end{tabular}


Table B.27: Mean and Standard Deviation of Accuracy Measurement $\left(d_{1}\right)$ from Simulation (With $N=12, M=3$, scale $[1 / 9,9], T=500$ ) [Continued]

\begin{tabular}{||l||c|c|c||}
\hline \multicolumn{1}{|c||}{ M=3 } & \multicolumn{3}{c||}{ A-MDM(V) } \\
\hline ABBREVIATIONS & UF & LN & GA \\
\hline \hline CSM & 0.00551 & 0.00411 & 0.01764 \\
& $(0.00162)$ & $(0.00137)$ & $(0.00515)$ \\
\hline R-EV & 0.00570 & 0.00406 & 0.01586 \\
& $(0.00166)$ & $(0.00127)$ & $(0.00460)$ \\
\hline L-EV & 0.00566 & 0.00403 & 0.01717 \\
& $(0.00164)$ & $(0.00125)$ & $(0.00519)$ \\
\hline AM-EV & 0.00565 & 0.00403 & 0.01699 \\
& $(0.00164)$ & $(0.00125)$ & $(0.00514)$ \\
\hline GE-EV & 0.00566 & 0.00402 & 0.01558 \\
& $(0.00163)$ & $(0.00127)$ & $(0.00436)$ \\
\hline EV[AA'] & 0.00742 & 0.00525 & 0.02378 \\
& $(0.00222)$ & $(0.00164)$ & $(0.00750)$ \\
\hline EV[A'A] & 0.04713 & 0.04700 & 0.04641 \\
& $(0.01551)$ & $(0.01547)$ & $(0.01527)$ \\
\hline AM-EV[AA'] & 0.00747 & 0.00558 & 0.02309 \\
AND EV[A'A] & $(0.00218)$ & $(0.00167)$ & $(0.00716)$ \\
\hline GE- EV[AA'] & 0.02106 & 0.02087 & 0.02526 \\
AND EV[A'A] & $(0.00708)$ & $(0.00709)$ & $(0.00713)$ \\
\hline GE & 0.00569 & 0.00402 & 0.01385 \\
& $(0.00164)$ & $(0.00127)$ & $(0.00396)$ \\
\hline C-RSM & 0.00638 & 0.00452 & 0.01340 \\
& $(0.00200)$ & $(0.00149)$ & $(0.00377)$ \\
\hline MT & 0.00564 & 0.00404 & 0.01412 \\
& $(0.00160)$ & $(0.00127)$ & $(0.00373)$ \\
\hline SAV & 0.00943 & 0.00671 & 0.00878 \\
& $(0.00350)$ & $(0.00250)$ & $(0.00375)$ \\
\hline NEV & 0.00719 & 0.00571 & 0.02051 \\
& $(0.00230)$ & $(0.00197)$ & $(0.00729)$ \\
\hline LSM & 0.01292 & 0.00912 & 0.00804 \\
& $(00501)$ & $(0.00348)$ & $(0.00458)$ \\
\hline
\end{tabular}


Table B.28: Mean and Standard Deviation of Accuracy Measurement $\left(d_{1}\right)$ from Simulation (With $N=12, M=5$, scale $[1 / 9,9], T=500$ )

\begin{tabular}{||l||c|c|c||c|c|c||}
\hline \multicolumn{1}{|c||}{ M=5 } & \multicolumn{3}{c|}{ A-GE(V) } & \multicolumn{3}{c||}{ A-GE(M) } \\
\hline ABBREVIATIONS & UF & LN & GA & UF & LN & GA \\
\hline \hline CSM & 0.00580 & 0.00431 & 0.001787 & 0.00499 & 0.00398 & 0.01206 \\
& $(0.00187)$ & $(0.00175)$ & $(0.00387)$ & $(0.00172)$ & $(0.00159)$ & $(0.00290)$ \\
\hline R-EV & 0.00422 & 0.00288 & 0.01372 & 0.00417 & 0.00287 & 0.01226 \\
& $(0.00122)$ & $(0.00076)$ & $(0.00311)$ & $(0.00122)$ & $(0.00075)$ & $(0.00307)$ \\
\hline L-EV & 0.00419 & 0.00288 & 0.01423 & 0.00422 & 0.00287 & 0.01248 \\
& $(0.00121)$ & $(0.00076)$ & $(0.00318)$ & $(0.00123)$ & $(0.00076)$ & $(0.00313)$ \\
\hline AM-EV & 0.00418 & 0.00288 & 0.01392 & 0.00420 & 0.00287 & 0.01238 \\
& $(0.00121)$ & $(0.00076)$ & $(0.00309)$ & $(0.00123)$ & $(0.00076)$ & $(0.00309)$ \\
\hline GM-EV & 0.00418 & 0.00287 & 0.01340 & 0.00418 & 0.00287 & 0.01222 \\
& $(0.00121)$ & $(0.00075)$ & $(0.00296)$ & $(0.00122)$ & $(0.00075)$ & $(0.00303)$ \\
\hline EV[AA'] & 0.00516 & 0.00353 & 0.001889 & 0.00510 & 0.00250 & 0.01702 \\
& $(0.00155)$ & $(0.00098)$ & $(0.00443)$ & $(0.00154)$ & $(0.00097)$ & $(0.00454)$ \\
\hline EV[A'A] & 0.03778 & 0.03757 & 0.03763 & 0.03763 & 0.03752 & 0.03752 \\
& $(0.01066)$ & $(0.01061)$ & $(0.00984)$ & $(0.01065)$ & $(0.01060)$ & $(0.01010)$ \\
\hline AM - EV[AA'] & 0.00647 & 0.00574 & 0.01861 & 0.00667 & 0.00588 & 0.01681 \\
AND EV[A'A] & $(0.00176)$ & $(0.00171)$ & $(0.00374)$ & $(0.00186)$ & $(0.00176)$ & $(0.00379)$ \\
\hline GE - EV[AA'] & 0.01759 & 0.01755 & 0.02226 & 0.01761 & 0.01757 & 0.02100 \\
AND EV[A'A] & $(0.00482)$ & $(0.00472)$ & $(0.00492)$ & $(0.00483)$ & $(0.00473)$ & $(0.00497)$ \\
\hline GE & 0.00417 & 0.00287 & 0.01223 & 0.00417 & 0.00287 & 0.01205 \\
& $(0.00121)$ & $(0.00075)$ & $(0.00289)$ & $(0.00122)$ & $(0.00075)$ & $(0.00299)$ \\
\hline C-RSM & 0.00425 & 0.00290 & 0.01319 & 0.00423 & 0.00290 & 0.01174 \\
& $(0.00126)$ & $(0.00075)$ & $(0.00280)$ & $(0.00126)$ & $(0.00075)$ & $(0.00290)$ \\
\hline MT & 0.00418 & 0.00288 & 0.01294 & 0.00416 & 0.00287 & 0.01210 \\
& $(0.00120)$ & $(0.00075)$ & $(0.00285)$ & $(0.00121)$ & $(0.00075)$ & $(0.00296)$ \\
\hline SAV & 0.00495 & 0.00341 & 0.01141 & 0.00493 & 0.00339 & 0.01011 \\
& $(0.00152)$ & $(0.00093)$ & $(0.00283)$ & $(0.00155)$ & $(0.00093)$ & $(0.00263)$ \\
\hline NEV & 0.00494 & 0.00390 & 0.01769 & 0.00580 & 0.00420 & 0.01436 \\
& $(0.00163)$ & $(0.00123)$ & $(0.00463)$ & $(0.00181)$ & $(0.00128)$ & $(0.00448)$ \\
\hline LSM & 0.00607 & 0.00416 & 0.01069 & 0.00600 & 0.00410 & 0.00895 \\
& $(0.00208)$ & $(0.00128)$ & $(0.00354)$ & $(0.00214)$ & $(0.00130)$ & $(0.00273)$ \\
\hline
\end{tabular}


Table B.29: Mean and Standard Deviation of Accuracy Measurement $\left(d_{1}\right)$ from Simulation (With $N=12, M=5$, scale $[1 / 9,9], T=500$ ) [Continued]

\begin{tabular}{||l||c|c|c||c|c|c||}
\hline \multicolumn{1}{|c||}{ M=5 } & \multicolumn{3}{c|}{ A-AM(V) } & \multicolumn{3}{c||}{ A-MDM(M) } \\
\hline ABBREVIATIONS & UF & LN & GA & UF & LN & GA \\
\hline \hline CSM & 0.00593 & 0.00437 & 0.01826 & 0.00508 & 0.00402 & 0.01222 \\
& $(0.00193)$ & $(0.00178)$ & $(0.00384)$ & $(0.00175)$ & $(0.00159)$ & $(0.00297)$ \\
\hline R-EV & 0.00422 & 0.00289 & 0.01379 & 0.00425 & 0.00292 & 0.01238 \\
& $(0.00122)$ & $(0.00076)$ & $(0.00322)$ & $(0.00128)$ & $(0.00077)$ & $(0.00312)$ \\
\hline L-EV & 0.00420 & 0.00289 & 0.01379 & 0.00428 & 0.00292 & 0.01261 \\
& $(0.00122)$ & $(0.00076)$ & $(0.00333)$ & $(0.00130)$ & $(0.00078)$ & $(0.00316)$ \\
\hline AM-EV & 0.00419 & 0.00288 & 0.01355 & 0.00427 & 0.00292 & 0.01250 \\
& $(0.00122)$ & $(0.00076)$ & $(0.00324)$ & $(0.00130)$ & $(0.00078)$ & $(0.00312)$ \\
\hline GE-EV & 0.00419 & 0.00287 & 0.01323 & 0.00425 & 0.00292 & 0.01233 \\
& $(0.00121)$ & $(0.00075)$ & $(0.00309)$ & $(0.00129)$ & $(0.00077)$ & $(0.00305)$ \\
\hline EV[AA'] & 0.00519 & 0.00353 & 0.01802 & 0.00527 & 0.00357 & 0.01717 \\
& $(0.00157)$ & $(0.00098)$ & $(0.00474)$ & $(0.00167)$ & $(0.00098)$ & $(0.00457)$ \\
\hline EV[A'A] & 0.03782 & 0.03759 & 0.03766 & 0.03765 & 0.03753 & 0.03759 \\
& $(0.01067)$ & $(0.01062)$ & $(0.00984)$ & $(0.01063)$ & $(0.01062)$ & $(0.01012)$ \\
\hline AM- EV[AA'] & 0.00640 & 0.00569 & 0.01775 & 0.00677 & 0.00590 & 0.01689 \\
AND EV[A'A] & $(0.00176)$ & $(0.00170)$ & $(0.00393)$ & $(0.00193)$ & $(0.00177)$ & $(0.00382)$ \\
\hline GE- EV[AA'] & 0.01756 & 0.01753 & 0.02188 & 0.01763 & 0.01757 & 0.02101 \\
AND EV[A'A] & $(0.00482)$ & $(0.00472)$ & $(0.00490)$ & $(0.00483)$ & $(0.00473)$ & $(0.00467)$ \\
\hline GE & 0.00418 & 0.00287 & 0.01208 & 0.00424 & 0.00292 & 0.01213 \\
& $(0.00122)$ & $(0.00075)$ & $(0.00301)$ & $(0.00128)$ & $(0.00077)$ & $(0.00300)$ \\
\hline C-RSM & 0.00426 & 0.00291 & 0.01297 & 0.00431 & 0.00295 & 0.01184 \\
& $(0.00127)$ & $(0.00075)$ & $(0.00291)$ & $(0.00132)$ & $(0.00077)$ & $(0.00291)$ \\
\hline MT & 0.00418 & 0.00287 & 0.01293 & 0.00424 & 0.00292 & 0.01219 \\
& $(0.00120)$ & $(0.00075)$ & $(0.00293)$ & $(0.00128)$ & $(0.00077)$ & $(0.00299)$ \\
\hline SAV & 0.00497 & 0.00342 & 0.01146 & 0.00504 & 0.00347 & 0.01017 \\
& $(0.00154)$ & $(0.00093)$ & $(0.00288)$ & $(0.00157)$ & $(0.00096)$ & $(0.00264)$ \\
\hline NEV & 0.00488 & 0.00389 & 0.01655 & 0.00585 & 0.00427 & 0.01458 \\
& $(0.00165)$ & $(0.00123)$ & $(0.00450)$ & $(0.00181)$ & $(0.00131)$ & $(0.00460)$ \\
\hline LSM & 0.00616 & 0.00420 & 0.01039 & 0.00617 & 0.00422 & 0.00978 \\
& $(0.00212)$ & $(0.00130)$ & $(0.00350)$ & $(0.00219)$ & $(0.00136)$ & $(0.00275)$ \\
\hline
\end{tabular}


Table B.30: Mean and Standard Deviation of Accuracy Measurement $\left(d_{1}\right)$ from Simulation (With $N=12, M=5$, scale $[1 / 9,9], T=500$ ) [Continued]

\begin{tabular}{||l||c|c|c||}
\hline \multicolumn{1}{||}{ M=5 } & \multicolumn{3}{c|}{ A-MDM(V) } \\
\hline ABBREVIATIONS & UF & LN & GA \\
\hline \hline CSM & 0.00472 & 0.00331 & 0.01610 \\
& $(0.00139)$ & $(0.00111)$ & $(0.00431)$ \\
\hline R-EV & 0.00476 & 0.00323 & 0.01356 \\
& $(0.00147)$ & $(0.00099)$ & $(0.00358)$ \\
\hline L-EV & 0.00473 & 0.00326 & 0.01459 \\
& $(0.00147)$ & $(0.00099)$ & $(0.00392)$ \\
\hline AM-EV & 0.00472 & 0.00326 & 0.01443 \\
& $(0.00147)$ & $(0.00099)$ & $(0.00389)$ \\
\hline GE-EV & 0.00471 & 0.00322 & 0.01327 \\
& $(0.00146)$ & $(0.00097)$ & $(0.00355)$ \\
\hline EV[AA'] & 0.00616 & 0.00422 & 0.02059 \\
& $(0.00193)$ & $(0.00131)$ & $(0.00614)$ \\
\hline EV[A'A] & 0.04683 & 0.04674 & 0.04629 \\
& $(0.01553)$ & $(0.01542)$ & $(0.01523)$ \\
\hline AM - EV[AA'] & 0.00619 & 0.00462 & 0.02022 \\
AND EV[A'A] & $(0.00190)$ & $(0.00145)$ & $(0.00576)$ \\
\hline GE - EV[AA'] & 0.02076 & 0.02067 & 0.02436 \\
AND EV[A'A] & $(0.00709)$ & $(0.00698)$ & $(0.00715)$ \\
\hline GE & 0.00471 & 0.00323 & 0.01175 \\
& $(0.00147)$ & $(0.00097)$ & $(0.00330)$ \\
\hline C-RSM & 0.00526 & 0.00363 & 0.01156 \\
& $(0.00166)$ & $(0.00112)$ & $(0.00312)$ \\
\hline MT & 0.00469 & 0.00320 & 0.01213 \\
& $(0.00145)$ & $(0.00096)$ & $(0.00305)$ \\
\hline SAV & 0.00753 & 0.00534 & 0.00710 \\
& $(0.00280)$ & $(0.00178)$ & $(0.00300)$ \\
\hline NEV & 0.00559 & 0.00447 & 0.01773 \\
& $(0.00198)$ & $(0.00139)$ & $(0.00623)$ \\
\hline LSM & 0.01037 & 0.00731 & 0.00645 \\
& $(0.00410)$ & $(0.00277)$ & $(0.00347)$ \\
\hline
\end{tabular}


Table B.31: Mean and Standard Deviation of Accuracy Measurement $\left(d_{1}\right)$ from Simulation (With $N=12, M=7$, scale $[1 / 9,9], T=500$ )

\begin{tabular}{||l||c|c|c||c|c|c||}
\hline \multicolumn{1}{||c||}{ M=7 } & \multicolumn{3}{c|}{ A-GE(V) } & \multicolumn{3}{c||}{ A-GE $(\mathrm{M})$} \\
\hline ABBREVIATIONS & UF & LN & GA & UF & LN & GA \\
\hline \hline CSM & 0.00544 & 0.00405 & 0.01723 & 0.00463 & 0.00371 & 0.01096 \\
& $(0.00188)$ & $(0.00178)$ & $(0.00356)$ & $(0.00165)$ & $(0.00159)$ & $(0.00250)$ \\
\hline R-EV & 0.03812 & 0.00253 & 0.01283 & 0.00376 & 0.00252 & 0.01130 \\
& $(0.00101)$ & $(0.00067)$ & $(0.00274)$ & $(0.00102)$ & $(0.00066)$ & $(0.00265)$ \\
\hline L-EV & 0.00379 & 0.00253 & 0.01325 & 0.00380 & 0.00251 & 0.01148 \\
& $(0.00102)$ & $(0.00066)$ & $(0.00275)$ & $(0.00102)$ & $(0.00066)$ & $(0.00272)$ \\
\hline AM-EV & 0.00379 & 0.00252 & 0.01299 & 0.00379 & 0.00251 & 0.01141 \\
& $(0.00101)$ & $(0.00066)$ & $(0.00268)$ & $(0.00102)$ & $(0.00066)$ & $(0.00269)$ \\
\hline GM-EV & 0.00378 & 0.00252 & 0.01256 & 0.00377 & 0.00251 & 0.01130 \\
& $(0.00101)$ & $(0.00066)$ & $(0.00261)$ & $(0.00102)$ & $(0.00066)$ & $(0.00265)$ \\
\hline EV[AA'] & 0.00456 & 0.00303 & 0.01784 & 0.00449 & 0.00300 & 0.01583 \\
& $(0.00132)$ & $(0.00084)$ & $(0.00370)$ & $(0.00133)$ & $(0.00082)$ & $(0.00390)$ \\
\hline EV[A'A] & 0.03771 & 0.03755 & 0.03749 & 0.03755 & 0.03750 & 0.03736 \\
& $(0.01064)$ & $(0.01059)$ & $(0.00986)$ & $(0.01062)$ & $(0.01059)$ & $(0.01017)$ \\
\hline AM-EV[AA'] & 0.00607 & 0.00548 & 0.01782 & 0.00631 & 0.00565 & 0.01587 \\
AND EV[A'A] & $(0.00172)$ & $(0.00548)$ & $(0.00325)$ & $(0.00185)$ & $(0.00179)$ & $(0.00335)$ \\
\hline GE- EV[AA'] & 0.01752 & 0.01750 & 0.02198 & 0.01754 & 0.01753 & 0.02065 \\
AND EV[A'A] & $(0.00482)$ & $(0.00471)$ & $(0.00487)$ & $(0.00483)$ & $(0.00473)$ & $(0.00493)$ \\
\hline GE & 0.00376 & 0.00251 & 0.01142 & 0.00376 & 0.00251 & 0.01119 \\
& $(0.00101)$ & $(0.00066)$ & $(0.00253)$ & $(0.00101)$ & $(0.00066)$ & $(0.00262)$ \\
\hline C-RSM & 0.00384 & 0.00255 & 0.01246 & 0.00382 & 0.00254 & 0.01084 \\
& $(0.00105)$ & $(0.00066)$ & $(0.00241)$ & $(0.00106)$ & $(0.00066)$ & $(0.00252)$ \\
\hline MT & 0.00377 & 0.00252 & 0.01218 & 0.00375 & 0.00251 & 0.01123 \\
& $(0.00100)$ & $(0.00066)$ & $(0.00252)$ & $(0.00101)$ & $(0.00066)$ & $(0.00258)$ \\
\hline SAV & 0.00440 & 0.00300 & 0.01065 & 0.00437 & 0.00298 & 0.00916 \\
' & $(0.00130)$ & $(0.00081)$ & $(0.00253)$ & $(0.00132)$ & $(0.00080)$ & $(0.00235)$ \\
\hline NEV & 0.00412 & 0.00327 & 0.01673 & 0.00517 & 0.00367 & 0.01335 \\
& $(0.00136)$ & $(0.00102)$ & $(0.00436)$ & $(0.00160)$ & $(0.00110)$ & $(0.00417)$ \\
\hline LSM & 0.00533 & 0.00364 & 0.00969 & 0.00524 & 0.00358 & 0.00890 \\
& $(0.00182)$ & $(0.00113)$ & $(0.00318)$ & $(0.00188)$ & $(0.00112)$ & $(0.00243)$ \\
\hline
\end{tabular}


Table B.32: Mean and Standard Deviation of Accuracy Measurement $\left(d_{1}\right)$ from Simulation (With $N=12, M=7$, scale $[1 / 9,9], T=500$ ) [Continued]

\begin{tabular}{||l||c|c|c||c|c|c||}
\hline \multicolumn{1}{|c||}{ M=7 } & \multicolumn{3}{c|}{ A-AM(V) } & \multicolumn{3}{c||}{ A-MDM(M) } \\
\hline ABBREVIATIONS & UF & LN & GA & UF & LN & GA \\
\hline \hline CSM & 0.00559 & 0.00411 & 0.01766 & 0.00464 & 0.00375 & 0.01107 \\
& $(0.00195)$ & $(0.00183)$ & $(0.00350)$ & $(0.00169)$ & $(0.00159)$ & $(0.00262)$ \\
\hline R-EV & 0.00382 & 0.00253 & 0.01285 & 0.00376 & 0.00257 & 0.01136 \\
& $(0.00102)$ & $(0.00067)$ & $(0.00287)$ & $(0.00107)$ & $(0.00069)$ & $(0.00275)$ \\
\hline L-EV & 0.00380 & 0.00253 & 0.01269 & 0.00378 & 0.00257 & 0.01155 \\
& $(0.00102)$ & $(0.00066)$ & $(0.00290)$ & $(0.00108)$ & $(0.00068)$ & $(0.00281)$ \\
\hline AM-EV & 0.00379 & 0.00252 & 0.01253 & 0.00377 & 0.00257 & 0.01147 \\
& $(0.00102)$ & $(0.00066)$ & $(0.00283)$ & $(0.00108)$ & $(0.00068)$ & $(0.00278)$ \\
\hline GE-EV & 0.00389 & 0.00251 & 0.01233 & 0.00376 & 0.00257 & 0.001135 \\
& $(0.00101)$ & $(0.00066)$ & $(0.00275)$ & $(0.00107)$ & $(0.00069)$ & $(0.00273)$ \\
\hline EV[AA'] & 0.00457 & 0.00303 & 0.01673 & 0.00454 & 0.00307 & 0.01594 \\
& $(0.00133)$ & $(0.00084)$ & $(0.00398)$ & $(0.00145)$ & $(0.00086)$ & $(0.00410)$ \\
\hline EV[A'A] & 0.03774 & 0.03756 & 0.03752 & 0.03755 & 0.03751 & 0.03743 \\
& $(0.01066)$ & $(0.01059)$ & $(0.00986)$ & $(0.01061)$ & $(0.01059)$ & $(0.01016)$ \\
\hline AM-EV[AA'] & 0.00598 & 0.00543 & 0.01677 & 0.00633 & 0.00568 & 0.01590 \\
AND EV[A'A] & $(0.00170)$ & $(0.00170)$ & $(0.00336)$ & $(0.00188)$ & $(0.00180)$ & $(0.00351)$ \\
\hline GE- EV[AA'] & 0.01749 & 0.01748 & 0.02156 & 0.01753 & 0.01754 & 0.02062 \\
AND EV[A'A] & $(0.00481)$ & $(0.00471)$ & $(0.00483)$ & $(0.00483)$ & $(0.00474)$ & $(0.00493)$ \\
\hline GE & 0.00376 & 0.00251 & 0.01122 & 0.00375 & 0.00257 & 0.01112 \\
& $(0.00101)$ & $(0.00066)$ & $(0.00264)$ & $(0.00107)$ & $(0.00069)$ & $(0.00270)$ \\
\hline C-RSM & 0.00385 & 0.00255 & 0.01217 & 0.00382 & 0.00260 & 0.01089 \\
& $(0.00106)$ & $(0.00066)$ & $(0.00252)$ & $(0.00111)$ & $(0.00068)$ & $(0.00260)$ \\
\hline MT & 0.00377 & 0.00252 & 0.01212 & 0.00374 & 0.00257 & 0.01124 \\
& $(0.00100)$ & $(0.00066)$ & $(0.00261)$ & $(0.00107)$ & $(0.00069)$ & $(0.00267)$ \\
\hline SAV & 0.00441 & 0.00300 & 0.01068 & 0.00444 & 0.00308 & 0.00913 \\
& $(0.00132)$ & $(0.00081)$ & $(0.00260)$ & $(0.00134)$ & $(0.00084)$ & $(0.00244)$ \\
\hline NEV & 0.00404 & 0.00325 & 0.01533 & 0.00514 & 0.00375 & 0.01348 \\
& $(0.00140)$ & $(0.00101)$ & $(0.00416)$ & $(0.00162)$ & $(0.00114)$ & $(0.00437)$ \\
\hline LSM & 0.00541 & 0.00367 & 0.00932 & 0.00537 & 0.00371 & 0.00874 \\
& $(0.00185)$ & $(0.00114)$ & $(0.00316)$ & $(0.00195)$ & $(0.00120)$ & $(0.00254)$ \\
\hline
\end{tabular}


Table B.33: Mean and Standard Deviation of Accuracy Measurement $\left(d_{1}\right)$ from Simulation (With $N=12, M=7$, scale $[1 / 9,9], T=500$ ) [Continued]

\begin{tabular}{||l||c|c|c||}
\hline \multicolumn{1}{|c||}{ M=7 } & \multicolumn{3}{c||}{ A-MDM(V) } \\
\hline ABBREVIATIONS & UF & LN & GA \\
\hline \hline CSM & 0.00420 & 0.00291 & 0.01541 \\
& $(0.00123)$ & $(0.00098)$ & $(0.00417)$ \\
\hline R-EV & 0.00426 & 0.00290 & 0.01253 \\
& $(0.00127)$ & $(0.00084)$ & $(0.00340)$ \\
\hline L-EV & 0.00427 & 0.00288 & 0.01344 \\
& $(0.00127)$ & $(0.00084)$ & $(0.00365)$ \\
\hline AM-EV & 0.00426 & 0.00288 & 0.01330 \\
& $(0.00127)$ & $(0.00084)$ & $(0.00359)$ \\
\hline GE-EV & 0.00422 & 0.00288 & 0.01224 \\
& $(0.00125)$ & $(0.00083)$ & $(0.00331)$ \\
\hline EV[AA'] & 0.00537 & 0.00366 & 0.01901 \\
& $(0.00167)$ & $(0.00110)$ & $(0.00538)$ \\
\hline EV[A'A] & 0.04678 & 0.04665 & 0.04621 \\
& $(0.01555)$ & $(0.01542)$ & $(0.01525)$ \\
\hline AM - EV[AA'] & 0.00550 & 0.00417 & 0.01884 \\
AND EV[A'A] & $(0.00167)$ & $(0.00131)$ & $(0.00499)$ \\
\hline GE - EV[AA'] & 0.02064 & 0.02061 & 0.02405 \\
AND EV[A'A] & $(0.00704)$ & $(0.00703)$ & $(0.00724)$ \\
\hline GE & 0.00420 & 0.00288 & 0.01086 \\
& $(0.00125)$ & $(0.00084)$ & $(0.00297)$ \\
\hline C-RSM & 0.00472 & 0.00320 & 0.01074 \\
& $(0.00145)$ & $(0.00097)$ & $(0.00287)$ \\
\hline MT & 0.00418 & 0.00289 & 0.01132 \\
& $(0.00125)$ & $(0.00084)$ & $(0.00293)$ \\
\hline SAV & 0.00659 & 0.00462 & 0.00629 \\
& $(0.00255)$ & $(0.00158)$ & $(0.00283)$ \\
\hline NEV & 0.00478 & 0.00388 & 0.01630 \\
& $(0.00166)$ & $(0.00120)$ & $(0.00552)$ \\
\hline LSM & 0.00877 & 0.00632 & 0.00541 \\
& $(0.00366)$ & $(0.00232)$ & $(0.00305)$ \\
\hline
\end{tabular}


Table B.34: Mean and Standard Deviation of Accuracy Measurement $\left(d_{1}\right)$ from Simulation (With $\mathrm{N}=12, \mathrm{M}=9$, scale $[1 / 9,9], \mathrm{T}=500$ )

\begin{tabular}{||l||c|c|c||c|c|c||}
\hline \multicolumn{1}{|c||}{ M=9 } & \multicolumn{3}{c|}{ A-GE(V) } & \multicolumn{3}{c||}{ A-GE(M) } \\
\hline ABBREVIATIONS & UF & LN & GA & UF & LN & GA \\
\hline \hline CSM & 0.00522 & 0.00390 & 0.01695 & 0.00439 & 0.00357 & 0.01041 \\
& $(0.00190)$ & $(0.00180)$ & $(0.00338)$ & $(0.00165)$ & $(0.00160)$ & $(0.00233)$ \\
\hline R-EV & 0.00353 & 0.00236 & 0.01236 & 0.00347 & 0.00235 & 0.01074 \\
& $(0.00094)$ & $(0.00060)$ & $(0.00246)$ & $(0.00095)$ & $(0.00060)$ & $(0.00243)$ \\
\hline L-EV & 0.00351 & 0.00236 & 0.01273 & 0.00350 & 0.00234 & 0.01096 \\
& $(0.00094)$ & $(0.00060)$ & $(0.00261)$ & $(0.00095)$ & $(0.00060)$ & $(0.00254)$ \\
\hline AM-EV & 0.00351 & 0.00235 & 0.01251 & 0.00350 & 0.00234 & 0.01090 \\
& $(0.00094)$ & $(0.00060)$ & $(0.00254)$ & $(0.00095)$ & $(0.00060)$ & $(0.00251)$ \\
\hline GM-EV & 0.00350 & 0.00235 & 0.01212 & 0.00348 & 0.00234 & 0.01079 \\
& $(0.00093)$ & $(0.00060)$ & $(0.00243)$ & $(0.00094)$ & $(0.00060)$ & $(0.00246)$ \\
\hline EV[AA'] & 0.00414 & 0.00283 & 0.01722 & 0.00406 & 0.00280 & 0.01508 \\
& $(0.00123)$ & $(0.00077)$ & $(0.00340)$ & $(0.00125)$ & $(0.00075)$ & $(0.00349)$ \\
\hline EV[A'A] & 0.03770 & 0.03754 & 0.03745 & 0.03754 & 0.03748 & 0.03730 \\
& $(0.01061)$ & $(0.01058)$ & $(0.00986)$ & $(0.01060)$ & $(0.01058)$ & $(0.01020)$ \\
\hline AM - EV[AA'] & 0.00584 & 0.00539 & 0.01737 & 0.00610 & 0.00557 & 0.01531 \\
AND EV[A'A] & $(0.00173)$ & $(0.00173)$ & $(0.00304)$ & $(0.00186)$ & $(0.00182)$ & $(0.00308)$ \\
\hline GE - EV[AA'] & 0.01749 & 0.01748 & 0.02183 & 0.01752 & 0.01751 & 0.02044 \\
AND EV[A'A] & $(0.00480)$ & $(0.00474)$ & $(0.00484)$ & $(0.00482)$ & $(0.00476)$ & $(0.00492)$ \\
\hline GE & 0.00347 & 0.00235 & 0.01098 & 0.00347 & 0.00234 & 0.01070 \\
& $(0.00093)$ & $(0.00060)$ & $(0.00234)$ & $(0.00094)$ & $(0.00060)$ & $(0.00244)$ \\
\hline C-RSM & 0.00355 & 0.00238 & 0.01205 & 0.00353 & 0.00237 & 0.01032 \\
& $(0.00097)$ & $(0.00061)$ & $(0.00226)$ & $(0.00098)$ & $(0.00061)$ & $(0.00234)$ \\
\hline MT & 0.00349 & 0.00236 & 0.01176 & 0.00346 & 0.00234 & 0.01071 \\
& $(0.00092)$ & $(0.00060)$ & $(0.00226)$ & $(0.00094)$ & $(0.00060)$ & $(0.00238)$ \\
\hline SAV & 0.00401 & 0.00276 & 0.01022 & 0.00397 & 0.00274 & 0.00858 \\
& $(0.00118)$ & $(0.00075)$ & $(0.00239)$ & $(0.00120)$ & $(0.00074)$ & $(0.00219)$ \\
\hline NEV & 0.00363 & 0.00292 & 0.01621 & 0.00485 & 0.00339 & 0.01266 \\
& $(0.00124)$ & $(0.00091)$ & $(0.00413)$ & $(0.00149)$ & $(0.00102)$ & $(0.00395)$ \\
\hline LSM & 0.03481 & 0.00331 & 0.00905 & 0.00469 & 0.00325 & 0.00821 \\
& $(0.00164)$ & $(0.00099)$ & $(0.00303)$ & $(0.00170)$ & $(0.00100)$ & $(0.00229)$ \\
\hline
\end{tabular}


Table B.35: Mean and Standard Deviation of Accuracy Measurement $\left(d_{1}\right)$ from Simulation (With $N=12, M=9$, scale $[1 / 9,9], T=500$ ) [Continued]

\begin{tabular}{||l||c|c|c||c|c|c||}
\hline \multicolumn{1}{|c||}{ M=9 } & \multicolumn{3}{c|}{ A-AM(V) } & \multicolumn{3}{c||}{ A-MDM(M) } \\
\hline ABBREVIATIONS & UF & LN & GA & UF & LN & GA \\
\hline \hline CSM & 0.00539 & 0.00398 & 0.01739 & 0.00597 & 0.00360 & 0.01048 \\
& $(0.00197)$ & $(0.00184)$ & $(0.00331)$ & $(0.00227)$ & $(0.00161)$ & $(0.00242)$ \\
\hline R-EV & 0.00353 & 0.00236 & 0.01233 & 0.00453 & 0.00240 & 0.01075 \\
& $(0.00094)$ & $(0.00061)$ & $(0.00259)$ & $(0.00141)$ & $(0.00063)$ & $(0.00250)$ \\
\hline L-EV & 0.00351 & 0.00236 & 0.01211 & 0.00451 & 0.00240 & 0.01097 \\
& $(0.00094)$ & $(0.00061)$ & $(0.00276)$ & $(0.00145)$ & $(0.00063)$ & $(0.00260)$ \\
\hline AM-EV & 0.00351 & 0.00235 & 0.01198 & 0.00451 & 0.00240 & 0.01090 \\
& $(0.00094)$ & $(0.00061)$ & $(0.00269)$ & $(0.00144)$ & $(0.00063)$ & $(0.00257)$ \\
\hline GE-EV & 0.00351 & 0.00234 & 0.01184 & 0.00451 & 0.00240 & 0.01078 \\
& $(0.00093)$ & $(0.00060)$ & $(0.00257)$ & $(0.00142)$ & $(0.00063)$ & $(0.00251)$ \\
\hline EV[AA'] & 0.00415 & 0.00283 & 0.01598 & 0.00534 & 0.00288 & 0.01501 \\
& $(0.00124)$ & $(0.00077)$ & $(0.00370)$ & $(0.00194)$ & $(0.00078)$ & $(0.00375)$ \\
\hline EV[A'A] & 0.03773 & 0.03755 & 0.03748 & 0.04438 & 0.03748 & 0.03737 \\
& $(0.01063)$ & $(0.01058)$ & $(0.00968)$ & $(0.01579)$ & $(0.01058)$ & $(0.01022)$ \\
\hline AM-EV[AA'] & 0.00574 & 0.00533 & 0.01622 & 0.00747 & 0.00559 & 0.01518 \\
AND EV[A'A] & $(0.00171)$ & $(0.00171)$ & $(0.00314)$ & $(0.00232)$ & $(0.00182)$ & $(0.00324)$ \\
\hline GE- EV[AA'] & 0.01747 & 0.01747 & 0.02139 & 0.02052 & 0.01751 & 0.02035 \\
AND EV[A'A] & $(0.00480)$ & $(0.00474)$ & $(0.00480)$ & $(0.00713)$ & $(0.00476)$ & $(0.00488)$ \\
\hline GE & 0.00347 & 0.00234 & 0.01073 & 0.00449 & 0.00240 & 0.01066 \\
& $(0.00094)$ & $(0.00060)$ & $(0.00245)$ & $(0.00142)$ & $(0.00063)$ & $(0.00248)$ \\
\hline C-RSM & 0.00356 & 0.00237 & 0.01172 & 0.00457 & 0.00242 & 0.01032 \\
& $(0.00097)$ & $(0.00061)$ & $(0.00236)$ & $(0.00144)$ & $(0.00063)$ & $(0.00238)$ \\
\hline MT & 0.00349 & 0.00235 & 0.01167 & 0.00450 & 0.00240 & 0.01067 \\
& $(0.00093)$ & $(0.00060)$ & $(0.00235)$ & $(0.00140)$ & $(0.00063)$ & $(0.00243)$ \\
\hline SAV & 0.00402 & 0.00276 & 0.01022 & 0.00528 & 0.00281 & 0.00853 \\
& $(0.00120)$ & $(0.00075)$ & $(0.00247)$ & $(0.00178)$ & $(0.00078)$ & $(0.00217)$ \\
\hline NEV & 0.00355 & 0.00290 & 0.01462 & 0.00603 & 0.00346 & 0.01269 \\
& $(0.00127)$ & $(0.00091)$ & $(0.00389)$ & $(0.00227)$ & $(0.00105)$ & $(0.00399)$ \\
\hline LSM & 0.00488 & 0.00334 & 0.00862 & 0.00624 & 0.00334 & 0.00804 \\
& $(0.00168)$ & $(0.00101)$ & $(0.00300)$ & $(0.00253)$ & $(0.00105)$ & $(0.00223)$ \\
\hline
\end{tabular}


Table B.36: Mean and Standard Deviation of Accuracy Measurement $\left(d_{1}\right)$ from Simulation (With $N=12, M=9$, scale $[1 / 9,9], T=500$ ) [Continued]

\begin{tabular}{||l||c|c|c||}
\hline \multicolumn{1}{|c|}{ M=9 } & \multicolumn{3}{c||}{ A-MDM(V) } \\
\hline ABBREVIATIONS & UF & LN & GA \\
\hline \hline CSM & 0.00397 & 0.00269 & 0.01503 \\
& $(0.00115)$ & $(0.00088)$ & $(0.00411)$ \\
\hline R-EV & 0.00394 & 0.00268 & 0.01181 \\
& $(0.00115)$ & $(0.00077)$ & $(0.00312)$ \\
\hline L-EV & 0.00394 & 0.00266 & 0.01254 \\
& $(0.00116)$ & $(0.00077)$ & $(0.00333)$ \\
\hline AM-EV & 0.00393 & 0.00266 & 0.01244 \\
& $(0.00116)$ & $(0.00077)$ & $(0.00331)$ \\
\hline GE-EV & 0.00389 & 0.00266 & 0.01160 \\
& $(0.00115)$ & $(0.00075)$ & $(0.00305)$ \\
\hline EV[AA'] & 0.00485 & 0.00337 & 0.01799 \\
& $(0.00153)$ & $(0.00106)$ & $(0.00486)$ \\
\hline EV[A'A] & 0.04670 & 0.04659 & 0.04623 \\
& $(0.01549)$ & $(0.01547)$ & $(0.01518)$ \\
\hline AM - EV[AA'] & 0.00507 & 0.00388 & 0.01778 \\
AND EV[A'A] & $(0.00157)$ & $(0.00130)$ & $(0.00454)$ \\
\hline GE - EV[AA'] & 0.02061 & 0.02053 & 0.02386 \\
AND EV[A'A] & $(0.00697)$ & $(0.00705)$ & $(0.00705)$ \\
\hline GE & 0.00388 & 0.00266 & 0.01025 \\
& $(0.00116)$ & $(0.00076)$ & $(0.00273)$ \\
\hline C-RSM & 0.00433 & 0.00297 & 0.01018 \\
& $(0.00138)$ & $(0.00085)$ & $(0.00268)$ \\
\hline MT & 0.00389 & 0.00266 & 0.01068 \\
& $(0.00113)$ & $(0.00076)$ & $(0.00267)$ \\
\hline SAV & 0.00591 & 0.00420 & 0.00578 \\
& $(0.00245)$ & $(0.00144)$ & $(0.00269)$ \\
\hline NEV & 0.00425 & 0.00342 & 0.01556 \\
& $(0.00159)$ & $(0.00114)$ & $(0.00502)$ \\
\hline LSM & 0.00794 & 0.00570 & 0.00487 \\
& $(0.00361)$ & $(0.00209)$ & $(0.00279)$ \\
\hline
\end{tabular}




\section{Appendix $\mathbb{C}$}

\section{The Mean and Standard Deviation of Group Disagreement Measurement from Simulation}

The entries of the following tables are the mean and standard deviation of group disagreement measurement from simulation study. All of them are in pairs in each cell, the number inside the parenthesis is the standard deviation of group disagreement measurement, and the number without parenthesis is the mean of group disagreement measurement. All the notations in the tables follow the definition Table 4.1 and 4.2. Other notations such as $\mathrm{N}$ stands for number of decision elements simulated (i.e. the pairwise comparison matrix size). $M$ is the number of decision makers in the simulation process. UF stands for Uniform probability distribution. LN stands for lognormal probability distribution and GA is the gamma probability distribution. 
Table C.1: Mean and Standard Deviation of Group Disagreement $\left(d_{2}\right)$ from Simulation (With $N=8, M=3$, scale $[1 / 9,9], T=500$ )

\begin{tabular}{||l||c|c|c||c|c|c||}
\hline \multicolumn{1}{|c||}{ M=3 } & \multicolumn{3}{c|}{ A-GE(V) } & \multicolumn{3}{c||}{ A-GE(M) } \\
\hline ABBREVIATIONS & UF & LN & GA & UF & LN & GA \\
\hline \hline CSM & 0.00648 & 0.00475 & 0.01638 & 0.00704 & 0.00500 & 0.01989 \\
& $(0.00162)$ & $(0.00114)$ & $(0.00413)$ & $(0.00151)$ & $(0.00110)$ & $(0.00392)$ \\
\hline R-EV & 0.00620 & 0.00460 & 0.01623 & 0.00622 & 0.00460 & 0.01655 \\
& $(0.00154)$ & $(0.00107)$ & $(0.00387)$ & $(0.00153)$ & $(0.00107)$ & $(0.00385)$ \\
\hline L-EV & 0.00627 & 0.00458 & 0.01701 & 0.00629 & 0.00459 & 0.01731 \\
& $(0.00156)$ & $(0.00108)$ & $(0.00424)$ & $(0.00156)$ & $(0.00108)$ & $(0.00406)$ \\
\hline AM-EV & 0.00624 & 0.00457 & 0.01645 & 0.00625 & 0.00457 & 0.01664 \\
& $(0.00156)$ & $(0.00108)$ & $(0.00407)$ & $(0.00155)$ & $(0.00108)$ & $(0.00393)$ \\
\hline GM-EV & 0.00619 & 0.00456 & 0.01572 & 0.00618 & 0.00456 & 0.01579 \\
& $(0.00154)$ & $(0.00107)$ & $(0.00382)$ & $(0.00154)$ & $(0.00107)$ & $(0.00373)$ \\
\hline EV[AA'] & 0.00831 & 0.00596 & 0.02291 & 0.00836 & 0.00598 & 0.02326 \\
& $(0.00214)$ & $(0.00155)$ & $(0.00609)$ & $(0.00212)$ & $(0.00154)$ & $(0.00582)$ \\
\hline EV[A'A] & 0.01052 & 0.00784 & 0.01599 & 0.01059 & 0.00789 & 0.01705 \\
& $(0.00303)$ & $(0.00247)$ & $(0.00408)$ & $(0.00298)$ & $(0.00245)$ & $(0.00415)$ \\
\hline AM - EV[AA'] & 0.00763 & 0.00542 & 0.02047 & 0.00767 & 0.00544 & 0.02081 \\
AND EV[A'A] & $(0.00197)$ & $(0.00141)$ & $(0.00546)$ & $(0.00196)$ & $(0.00141)$ & $(0.00527)$ \\
\hline GE - EV[AA'] & 0.00715 & 0.00523 & 0.01550 & 0.00719 & 0.00525 & 0.01589 \\
AND EV[A'A] & $(0.00178)$ & $(0.00136)$ & $(0.00389)$ & $(0.00178)$ & $(0.00136)$ & $(0.0038)$ \\
\hline GE & 0.00624 & 0.00456 & 0.01491 & 0.00624 & 0.00456 & 0.01484 \\
& $(0.00154)$ & $(0.00107)$ & $(0.00371)$ & $(0.00154)$ & $(0.00107)$ & $(0.00366)$ \\
\hline C-RSM & 0.00636 & 0.00469 & 0.01506 & 0.00637 & 0.00469 & 0.01515 \\
& $(0.00157)$ & $(0.00112)$ & $(0.00376)$ & $(0.00156)$ & $(0.00112)$ & $(0.00368)$ \\
\hline MT & 0.00617 & 0.00455 & 0.01439 & 0.00619 & 0.00456 & 0.01466 \\
& $(0.00152)$ & $(0.00106)$ & $(0.00340)$ & $(0.00151)$ & $(0.00105)$ & $(0.00328)$ \\
\hline SAV & 0.00773 & 0.00579 & 0.01395 & 0.00775 & 0.00580 & 0.01446 \\
& $(0.00206)$ & $(0.00156)$ & $(0.00339)$ & $(0.00204)$ & $(0.00156)$ & $(0.00331)$ \\
\hline NEV & 0.00870 & 0.00649 & 0.01971 & 0.00890 & 0.00658 & 0.02192 \\
& $(0.00224)$ & $(0.00173)$ & $(0.00526)$ & $(0.00218)$ & $(0.00171)$ & $(0.00508)$ \\
\hline LSM & 0.00956 & 0.00705 & 0.01635 & 0.00963 & 0.00709 & 0.01723 \\
& $(0.00277)$ & $(0.00229)$ & $(0.00515)$ & $(0.00274)$ & $(0.00228)$ & $(0.00533)$ \\
\hline
\end{tabular}


Table C.2: Mean and Standard Deviation of Group Disagreement $\left(d_{2}\right)$ from Simulation (With $\mathrm{N}=8, \mathrm{M}=3$, scale $[1 / 9,9], \mathrm{T}=500$ ) [Continued]

\begin{tabular}{||l||c|c|c||c|c|c||}
\hline \multicolumn{1}{|c||}{ M=3 } & \multicolumn{3}{c|}{ A-AM(V) } & \multicolumn{3}{c||}{ A-MDM(M) } \\
\hline ABBREVIATIONS & UF & LN & GA & UF & LN & GA \\
\hline \hline CSM & 0.00647 & 0.00474 & 0.01622 & 0.00705 & 0.00507 & 0.01985 \\
& $(0.00162)$ & $(0.00113)$ & $(0.00402)$ & $(0.00153)$ & $(0.00112)$ & $(0.00422)$ \\
\hline R-EV & 0.00619 & 0.00459 & 0.01608 & 0.00629 & 0.00471 & 0.01693 \\
& $(0.00153)$ & $(0.00107)$ & $(0.00388)$ & $(0.00154)$ & $(0.00109)$ & $(0.00399)$ \\
\hline L-EV & 0.00626 & 0.00458 & 0.01683 & 0.00636 & 0.00469 & 0.01775 \\
& $(0.00156)$ & $(0.00108)$ & $(0.00413)$ & $(0.00157)$ & $(0.00110)$ & $(0.00423)$ \\
\hline AM-EV & 0.00623 & 0.00457 & 0.01629 & 0.00632 & 0.00468 & 0.01708 \\
& $(0.00155)$ & $(0.00108)$ & $(0.00397)$ & $(0.00157)$ & $(0.00110)$ & $(0.00407)$ \\
\hline GE-EV & 0.00618 & 0.00456 & 0.01559 & 0.00625 & 0.00467 & 0.01623 \\
& $(0.00154)$ & $(0.00107)$ & $(0.00374)$ & $(0.00155)$ & $(0.00109)$ & $(0.00384)$ \\
\hline EV[AA'] & 0.00829 & 0.00595 & 0.02250 & 0.00845 & 0.00611 & 0.02385 \\
& $(0.00213)$ & $(0.00154)$ & $(0.00585)$ & $(0.00214)$ & $(0.00158)$ & $(0.00603)$ \\
\hline EV[A'A] & 0.01048 & 0.00783 & 0.01580 & 0.01071 & 0.00806 & 0.01731 \\
& $(0.00300)$ & $(0.00245)$ & $(0.00397)$ & $(0.00301)$ & $(0.00252)$ & $(0.00426)$ \\
\hline AM - EV[AA'] & 0.00761 & 0.00542 & 0.02018 & 0.00775 & 0.00556 & 0.02133 \\
AND EV[A'A] & $(0.00196)$ & $(0.00141)$ & $(0.00528)$ & $(0.00198)$ & $(0.00145)$ & $(0.00547)$ \\
\hline GE - EV[AA'] & 0.00714 & 0.00522 & 0.01536 & 0.00727 & 0.00536 & 0.01624 \\
AND EV[A'A] & $(0.00178)$ & $(0.00136)$ & $(0.00381)$ & $(0.00179)$ & $(0.00139)$ & $(0.00390)$ \\
\hline GE & 0.00623 & 0.00455 & 0.01479 & 0.00631 & 0.00466 & 0.01527 \\
& $(0.00154)$ & $(0.00107)$ & $(0.00363)$ & $(0.00155)$ & $(0.00109)$ & $(0.00373)$ \\
\hline C-RSM & 0.00636 & 0.00469 & 0.01494 & 0.00644 & 0.00479 & 0.01556 \\
& $(0.00156)$ & $(0.00112)$ & $(0.00369)$ & $(0.00157)$ & $(0.00114)$ & $(0.00377)$ \\
\hline MT & 0.00616 & 0.00455 & 0.01427 & 0.00626 & 0.00467 & 0.01505 \\
& $(0.00152)$ & $(0.00105)$ & $(0.00333)$ & $(0.00153)$ & $(0.00107)$ & $(0.00340)$ \\
\hline SAV & 0.00771 & 0.00578 & 0.01383 & 0.00784 & 0.00593 & 0.01473 \\
& $(0.00205)$ & $(0.00156)$ & $(0.00332)$ & $(0.00206)$ & $(0.00159)$ & $(0.00339)$ \\
\hline NEV & 0.00867 & 0.00645 & 0.01935 & 0.00896 & 0.00673 & 0.02211 \\
& $(0.00222)$ & $(0.00172)$ & $(0.00501)$ & $(0.00220)$ & $(0.00177)$ & $(0.00538)$ \\
\hline LSM & 0.00953 & 0.00704 & 0.01614 & 0.00974 & 0.00725 & 0.01745 \\
& $(0.00274)$ & $(0.00228)$ & $(0.00497)$ & $(0.00277)$ & $(0.00235)$ & $(0.00539)$ \\
\hline
\end{tabular}


Table C.3: Mean and Standard Deviation of Group Disagreement $\left(d_{2}\right)$ from Simulation (With $\mathrm{N}=8, \mathrm{M}=3$, scale $[1 / 9,9], \mathrm{T}=500$ ) [Continued]

\begin{tabular}{|c|c|c|c|}
\hline$M=3$ & \multicolumn{3}{|c|}{ A-MDM(V) } \\
\hline ABBREVIATIONS & $\overline{U F}$ & $\mathrm{LN}$ & GA \\
\hline$\overline{\mathrm{CSM}}$ & $\begin{array}{c}0.00921 \\
(0.00306)\end{array}$ & $\begin{array}{c}0.00736 \\
(0.00288)\end{array}$ & $\begin{array}{c}0.02054 \\
(0.00576)\end{array}$ \\
\hline R-EV & $\begin{array}{c}0.00680 \\
(0.00185)\end{array}$ & $\begin{array}{c}0.00505 \\
(0.00138)\end{array}$ & $\begin{array}{c}0.01705 \\
(0.00470)\end{array}$ \\
\hline $\mathrm{L}-\mathrm{EV}$ & $\begin{array}{c}0.00687 \\
(0.00189)\end{array}$ & $\begin{array}{c}0.00503 \\
(0.00136)\end{array}$ & $\begin{array}{c}0.01893 \\
(0.00550)\end{array}$ \\
\hline AM-EV & $\begin{array}{c}0.00685 \\
(0.00189)\end{array}$ & $\begin{array}{c}0.00502 \\
(0.00135)\end{array}$ & $\begin{array}{c}0.01862 \\
(0.00540)\end{array}$ \\
\hline GE-EV & $\begin{array}{c}0.00677 \\
(0.00186)\end{array}$ & $\begin{array}{c}0.00500 \\
(0.00136)\end{array}$ & $\begin{array}{c}0.01713 \\
(0.00470)\end{array}$ \\
\hline$\overline{E V}\left[\mathrm{AA}^{\prime}\right]$ & $\begin{array}{c}0.00969 \\
(0.00253)\end{array}$ & $\begin{array}{c}0.00689 \\
(0.00193)\end{array}$ & $\begin{array}{c}0.02566 \\
(0.00721)\end{array}$ \\
\hline $\mathrm{EV}\left[\mathrm{A}^{\prime} \mathrm{A}\right]$ & $\begin{array}{c}0.01513 \\
(0.00520)\end{array}$ & $\begin{array}{c}0.01112 \\
(0.00365)\end{array}$ & $\begin{array}{c}0.01307 \\
(0.00629)\end{array}$ \\
\hline $\begin{array}{l}\mathrm{AM}-\mathrm{EV}\left[\overline{\mathrm{AA}} \mathrm{A}^{\prime}\right] \\
\mathrm{AND} \mathrm{EV}\left[\mathrm{A}^{\prime} \mathrm{A}\right]\end{array}$ & $\begin{array}{c}0.00928 \\
(0.00249)\end{array}$ & $\begin{array}{c}0.00662 \\
(0.00191)\end{array}$ & $\begin{array}{c}0.02439 \\
(0.00698)\end{array}$ \\
\hline $\begin{array}{l}\text { GE - EV[AA'] } \\
\text { AND EV }\left[A^{\prime} A\right]\end{array}$ & $\begin{array}{c}0.00907 \\
(0.00267)\end{array}$ & $\begin{array}{c}0.00662 \\
(0.00206)\end{array}$ & $\begin{array}{r}0.01587 \\
(0.00449)\end{array}$ \\
\hline $\mathrm{GE}$ & $\begin{array}{c}0.00683 \\
(0.00188)\end{array}$ & $\begin{array}{c}0.00500 \\
(0.00136)\end{array}$ & $\begin{array}{c}0.01580 \\
(0.00437)\end{array}$ \\
\hline C-RSM & $\begin{array}{c}0.00758 \\
(0.00216)\end{array}$ & $\begin{array}{c}0.00559 \\
(0.00164)\end{array}$ & $\begin{array}{c}0.01526 \\
(0.00426)\end{array}$ \\
\hline MT & $\begin{array}{c}0.00675 \\
(0.00182)\end{array}$ & $\begin{array}{c}0.00499 \\
(0.00136)\end{array}$ & $\begin{array}{c}0.01482 \\
(0.00390)\end{array}$ \\
\hline SAV & $\begin{array}{c}0.01127 \\
(0.00396)\end{array}$ & $\begin{array}{c}0.00833 \\
(0.00283)\end{array}$ & $\begin{array}{c}0.01128 \\
(0.00452)\end{array}$ \\
\hline NEV & $\begin{array}{c}0.00947 \\
(0.00257)\end{array}$ & $\begin{array}{c}0.00705 \\
(0.00215)\end{array}$ & $\begin{array}{r}0.01997 \\
(0.00580)\end{array}$ \\
\hline LSM & $\begin{array}{c}0.01453 \\
(0.00526)\end{array}$ & $\begin{array}{c}0.01065 \\
(0.00376)\end{array}$ & $\begin{array}{c}0.01216 \\
(0.00632)\end{array}$ \\
\hline
\end{tabular}


Table C.4: Mean and Standard Deviation of Group Disagreement $\left(d_{2}\right)$ from Simulation (With $\mathrm{N}=8, \mathrm{M}=5$, scale $[1 / 9,9], \mathrm{T}=500$ )

\begin{tabular}{|c|c|c|c|c|c|c|}
\hline$M=5$ & \multicolumn{3}{|c|}{ A-GE(V) } & \multicolumn{3}{|c|}{$\mathrm{A}-\mathrm{GE}(\mathrm{M})$} \\
\hline ABBREVIATIONS & UF & LN & $\overline{\mathrm{GA}}$ & UF & LN & GA \\
\hline CSM & $\begin{array}{c}0.00559 \\
(0.00098) \\
\end{array}$ & $\begin{array}{c}0.00410 \\
(0.00075)\end{array}$ & $\begin{array}{c}0.01420 \\
(0.00263)\end{array}$ & $\begin{array}{c}0.00614 \\
(0.00094)\end{array}$ & $\begin{array}{c}0.00433 \\
(0.00072) \\
\end{array}$ & $\begin{array}{c}0.01786 \\
(0.00261) \\
\end{array}$ \\
\hline R-EV & $\begin{array}{c}0.00534 \\
(0.00090)\end{array}$ & $\begin{array}{c}0.00397 \\
(0.00070)\end{array}$ & $\begin{array}{c}0.01410 \\
(0.00248)\end{array}$ & $\begin{array}{c}0.00534 \\
(0.00090)\end{array}$ & $\begin{array}{c}0.00397 \\
(0.00070)\end{array}$ & $\begin{array}{c}0.01421 \\
(0.00243)\end{array}$ \\
\hline L-EV & $\begin{array}{c}0.00542 \\
(0.00092) \\
\end{array}$ & $\begin{array}{c}0.00396 \\
(0.00070) \\
\end{array}$ & $\begin{array}{c}0.01480 \\
(0.00269) \\
\end{array}$ & $\begin{array}{c}0.00543 \\
(0.00092) \\
\end{array}$ & $\begin{array}{c}0.00396 \\
(0.00070) \\
\end{array}$ & $\begin{array}{r}0.01488 \\
(0.00263) \\
\end{array}$ \\
\hline AM-EV & $\begin{array}{c}0.00539 \\
(0.00092) \\
\end{array}$ & $\begin{array}{c}0.00395 \\
(0.00070) \\
\end{array}$ & $\begin{array}{c}0.01432 \\
(0.00258) \\
\end{array}$ & $\begin{array}{c}0.00539 \\
(0.00092) \\
\end{array}$ & $\begin{array}{c}0.00395 \\
(0.00070) \\
\end{array}$ & $\begin{array}{c}0.01436 \\
(0.00252) \\
\end{array}$ \\
\hline GM-EV & $\begin{array}{c}0.00534 \\
(0.00090) \\
\end{array}$ & $\begin{array}{c}0.00394 \\
(0.00070) \\
\end{array}$ & $\begin{array}{c}0.01370 \\
(0.00241) \\
\end{array}$ & $\begin{array}{c}0.00533 \\
(0.00090) \\
\end{array}$ & $\begin{array}{c}0.00394 \\
(0.00070) \\
\end{array}$ & $\begin{array}{c}0.01369 \\
(0.00236) \\
\end{array}$ \\
\hline$\overline{\mathrm{EV}}\left[\mathrm{AA}^{\prime}\right]$ & $\begin{array}{c}0.00712 \\
(0.00131)\end{array}$ & $\begin{array}{c}0.00513 \\
(0.00102)\end{array}$ & $\begin{array}{c}0.01993 \\
(0.00395)\end{array}$ & $\begin{array}{c}0.00714 \\
(0.00130)\end{array}$ & $\begin{array}{c}0.00514 \\
(0.00102)\end{array}$ & $\begin{array}{c}0.01995 \\
(0.00377)\end{array}$ \\
\hline $\mathrm{EV}\left[\mathrm{A}^{\prime} \mathrm{A}\right]$ & $\begin{array}{c}0.00919 \\
(0.00197)\end{array}$ & $\begin{array}{c}0.00674 \\
(0.00162)\end{array}$ & $\begin{array}{c}0.01403 \\
(0.00283)\end{array}$ & $\begin{array}{c}0.00922 \\
(0.00194)\end{array}$ & $\begin{array}{c}0.00676 \\
(0.00161)\end{array}$ & $\begin{array}{c}0.01473 \\
(0.00285)\end{array}$ \\
\hline $\begin{array}{l}\text { AM - EV }\left[\mathrm{AA}^{\prime}\right] \\
\text { AND EV }\left[\mathrm{A}^{\prime} \mathrm{A}\right]\end{array}$ & $\begin{array}{c}0.00653 \\
(0.00121)\end{array}$ & $\begin{array}{c}0.00468 \\
(0.00093)\end{array}$ & $\begin{array}{c}0.01782 \\
(0.00353)\end{array}$ & $\begin{array}{c}0.00655 \\
(0.00120)\end{array}$ & $\begin{array}{c}0.00469 \\
(0.00093)\end{array}$ & $\begin{array}{c}0.01788 \\
(0.00341)\end{array}$ \\
\hline $\begin{array}{l}\text { GE - EV[AA'] } \\
\text { AND EV[A'A] }\end{array}$ & $\begin{array}{c}0.00618 \\
(0.00107) \\
\end{array}$ & $\begin{array}{c}0.00450 \\
(0.00085) \\
\end{array}$ & $\begin{array}{c}0.01358 \\
(0.00246) \\
\end{array}$ & $\begin{array}{c}0.00620 \\
(0.00106)\end{array}$ & $\begin{array}{c}0.00451 \\
(0.00085)\end{array}$ & $\begin{array}{c}0.01377 \\
(0.00241) \\
\end{array}$ \\
\hline$\overline{G E}$ & $\begin{array}{c}0.00538 \\
(0.00090)\end{array}$ & $\begin{array}{c}0.00394 \\
(0.00070)\end{array}$ & $\begin{array}{c}0.01299 \\
(0.00236)\end{array}$ & $\begin{array}{c}0.00538 \\
(0.00090)\end{array}$ & $\begin{array}{c}0.00394 \\
(0.00070)\end{array}$ & $\begin{array}{c}0.01291 \\
(0.00233\end{array}$ \\
\hline C-RSM & $\begin{array}{c}0.00550 \\
(0.00092)\end{array}$ & $\begin{array}{c}0.00404 \\
(0.00071)\end{array}$ & $\begin{array}{c}0.01315 \\
(0.00237)\end{array}$ & $\begin{array}{c}0.00550 \\
(0.00092)\end{array}$ & $\begin{array}{c}0.00404 \\
(0.00071)\end{array}$ & $\begin{array}{c}0.01317 \\
(0.00233)\end{array}$ \\
\hline$\overline{\mathrm{MT}}$ & $\begin{array}{c}0.00531 \\
(0.00089)\end{array}$ & $\begin{array}{c}0.00393 \\
(0.00069)\end{array}$ & $\begin{array}{c}0.01248 \\
(0.00214)\end{array}$ & $\begin{array}{c}0.00532 \\
(0.00089)\end{array}$ & $\begin{array}{c}0.00394 \\
(0.00069)\end{array}$ & $\begin{array}{c}0.01262 \\
(0.00208)\end{array}$ \\
\hline SAV & $\begin{array}{c}0.00673 \\
(0.00127)\end{array}$ & $\begin{array}{c}0.00498 \\
(0.00099)\end{array}$ & $\begin{array}{c}0.01220 \\
(0.00225)\end{array}$ & $\begin{array}{c}0.00673 \\
(0.00126)\end{array}$ & $\begin{array}{c}0.00499 \\
(0.00099)\end{array}$ & $\begin{array}{c}0.01247 \\
(0.00221)\end{array}$ \\
\hline NEV & $\begin{array}{c}0.00739 \\
(0.00139)\end{array}$ & $\begin{array}{c}0.00558 \\
(0.00107)\end{array}$ & $\begin{array}{c}0.01712 \\
(0.00336)\end{array}$ & $\begin{array}{c}0.00757 \\
(0.00136)\end{array}$ & $\begin{array}{c}0.00565 \\
(0.00106)\end{array}$ & $\begin{array}{c}0.01902 \\
(0.00328)\end{array}$ \\
\hline LSM & $\begin{array}{c}0.00834 \\
(0.00181)\end{array}$ & $\begin{array}{c}0.00610 \\
(0.00149)\end{array}$ & $\begin{array}{c}0.01429 \\
(0.00365)\end{array}$ & $\begin{array}{c}0.00836 \\
(0.00179)\end{array}$ & $\begin{array}{c}0.00612 \\
(0.00148)\end{array}$ & $\begin{array}{c}0.01475 \\
(0.00371)\end{array}$ \\
\hline
\end{tabular}


Table C.5: Mean and Standard Deviation of Group Disagreement $\left(d_{2}\right)$ from Simulation (With $N=8, M=5$, scale $[1 / 9,9], T=500$ ) [Continued]

\begin{tabular}{||l||c|c|c||c|c|c||}
\hline \multicolumn{1}{|c||}{ M=5 } & \multicolumn{3}{c|}{ A-AM(V) } & \multicolumn{3}{c||}{ A-MDM(M) } \\
\hline ABBREVIATIONS & UF & LN & GA & UF & LN & GA \\
\hline \hline CSM & 0.00558 & 0.00410 & 0.01407 & 0.00613 & 0.00437 & 0.01781 \\
& $(0.00097)$ & $(0.00075)$ & $(0.00256)$ & $(0.00094)$ & $(0.00074)$ & $(0.00284)$ \\
\hline R-EV & 0.00533 & 0.00396 & 0.01397 & 0.00543 & 0.00403 & 0.01448 \\
& $(0.00090)$ & $(0.00070)$ & $(0.00243)$ & $(0.00092)$ & $(0.00071)$ & $(0.00247)$ \\
\hline L-EV & 0.00542 & 0.00396 & 0.01465 & 0.00552 & 0.00401 & 0.01516 \\
& $(0.00092)$ & $(0.00070)$ & $(0.00263)$ & $(0.00094)$ & $(0.00071)$ & $(0.00268)$ \\
\hline AM-EV & 0.00539 & 0.00395 & 0.01419 & 0.00549 & 0.00400 & 0.01464 \\
& $(0.00092)$ & $(0.00070)$ & $(0.00251)$ & $(0.00094)$ & $(0.00071)$ & $(0.00257)$ \\
\hline GE-EV & 0.00533 & 0.00394 & 0.01359 & 0.00542 & 0.00399 & 0.01397 \\
& $(0.00090)$ & $(0.00070)$ & $(0.00235)$ & $(0.00092)$ & $(0.00071)$ & $(0.00240)$ \\
\hline EV[AA'] & 0.00711 & 0.00512 & 0.01958 & 0.00725 & 0.00521 & 0.02026 \\
& $(0.00131)$ & $(0.00102)$ & $(0.00378)$ & $(0.00132)$ & $(0.00103)$ & $(0.00385)$ \\
\hline EV[A'A] & 0.00916 & 0.00673 & 0.01386 & 0.00937 & 0.00685 & 0.01492 \\
& $(0.00195)$ & $(0.00161)$ & $(0.00274)$ & $(0.00199)$ & $(0.00163)$ & $(0.00288)$ \\
\hline AM - EV[AA'] & 0.00652 & 0.00468 & 0.01757 & 0.00665 & 0.00475 & 0.01816 \\
AND EV[A'A] & $(0.00121)$ & $(0.00093)$ & $(0.00341)$ & $(0.00123)$ & $(0.00086)$ & $(0.00349)$ \\
\hline GE - EV[AA'] & 0.00617 & 0.00450 & 0.01346 & 0.00630 & 0.00457 & 0.01401 \\
AND EV[A'A] & $(0.00106)$ & $(0.00085)$ & $(0.00241)$ & $(0.00109)$ & $(0.00086)$ & $(0.00244)$ \\
\hline GE & 0.00538 & 0.00394 & 0.01289 & 0.00547 & 0.00399 & 0.01317 \\
& $(0.00090)$ & $(0.00070)$ & $(0.00230)$ & $(0.00092)$ & $(0.00071)$ & $(0.00236)$ \\
\hline C-RSM & 0.00549 & 0.00404 & 0.01304 & 0.00559 & 0.00410 & 0.01343 \\
& $(0.00092)$ & $(0.00071)$ & $(0.00232)$ & $(0.00094)$ & $(0.00072)$ & $(0.00236)$ \\
\hline MT & 0.00531 & 0.00393 & 0.01238 & 0.00541 & 0.00399 & 0.01288 \\
& $(0.00089)$ & $(0.00069)$ & $(0.00210)$ & $(0.00091)$ & $(0.00070)$ & $(0.00213)$ \\
\hline SAV & 0.00671 & 0.00498 & 0.00684 & 0.00506 & 0.01269 & \\
& $(0.00126)$ & $(0.00220)$ & $(0.00129)$ & $(0.00100)$ & $(0.00225)$ & \\
\hline NEV & 0.00737 & 0.00557 & 0.01683 & 0.00765 & 0.00573 & 0.01917 \\
& $(0.00138)$ & $(0.00107)$ & $(0.00321)$ & $(0.00139)$ & $(0.00108)$ & $(0.00350)$ \\
\hline LSM & 0.00831 & 0.00609 & 0.01412 & 0.00850 & 0.00620 & 0.01489 \\
& $(0.00180)$ & $(0.00148)$ & $(0.00353)$ & $(0.00183)$ & $(0.00150)$ & $(0.00373)$ \\
\hline
\end{tabular}


Table C.6: Mean and Standard Deviation of Group Disagreement $\left(d_{2}\right)$ from Simulation (With $\mathrm{N}=8, \mathrm{M}=5$, scale $[1 / 9,9], \mathrm{T}=500$ ) [Continued]

\begin{tabular}{|c|c|c|c|}
\hline$M=5$ & \multicolumn{3}{|c|}{$\mathrm{A}-\mathrm{MDM}(\mathrm{V})$} \\
\hline ABBREVIATIONS & UF & LN & GA \\
\hline CSM & $\begin{array}{c}0.00751 \\
(0.00223)\end{array}$ & $\begin{array}{c}0.00597 \\
(0.00215) \\
\end{array}$ & $\begin{array}{r}0.01684 \\
(0.00375) \\
\end{array}$ \\
\hline R-EV & $\begin{array}{r}0.00571 \\
(0.00105) \\
\end{array}$ & $\begin{array}{c}0.00425 \\
(0.00081) \\
\end{array}$ & $\begin{array}{c}0.01441 \\
(0.00285) \\
\end{array}$ \\
\hline L-EV & $\begin{array}{c}0.00580 \\
(0.00108) \\
\end{array}$ & $\begin{array}{c}0.00425 \\
(0.00084) \\
\end{array}$ & $\begin{array}{c}0.01592 \\
(0.00336) \\
\end{array}$ \\
\hline $\mathrm{AM}-\mathrm{EV}$ & $\begin{array}{c}0.00579 \\
(0.00108)\end{array}$ & $\begin{array}{c}0.00424 \\
(0.00084)\end{array}$ & $\begin{array}{c}0.01567 \\
(0.00331)\end{array}$ \\
\hline GE-EV & $\begin{array}{c}0.00571 \\
(0.00105) \\
\end{array}$ & $\begin{array}{r}0.00422 \\
(0.00083) \\
\end{array}$ & $\begin{array}{r}0.01442 \\
(0.00289) \\
\end{array}$ \\
\hline $\mathrm{EV}\left[\overline{\mathrm{AA}} \bar{A}^{\prime}\right]$ & $\begin{array}{c}0.00813 \\
(0.00157)\end{array}$ & $\begin{array}{c}0.00576 \\
(0.00122)\end{array}$ & $\begin{array}{c}0.02173 \\
(0.00458) \\
\end{array}$ \\
\hline$E V\left[A^{\prime} A\right]$ & $\begin{array}{r}0.01273 \\
(0.00339) \\
\end{array}$ & $\begin{array}{c}0.00928 \\
(0.00244) \\
\end{array}$ & $\begin{array}{r}0.01094 \\
(0.00436) \\
\end{array}$ \\
\hline $\begin{array}{l}A M-E V\left[A A^{\prime}\right] \\
A N D E V\left[A^{\prime} A\right]\end{array}$ & $\begin{array}{c}0.00780 \\
(0.00159)\end{array}$ & $\begin{array}{c}0.00553 \\
(0.00123)\end{array}$ & $\begin{array}{c}0.02066 \\
(0.00450)\end{array}$ \\
\hline $\begin{array}{l}\text { GE - EV }\left[\mathrm{AA}^{\prime}\right] \\
\text { AND EV }\left[\mathrm{A}^{\prime} \mathrm{A}\right]\end{array}$ & $\begin{array}{c}0.00760 \\
(0.00172)\end{array}$ & $\begin{array}{c}0.00557 \\
(0.00127) \\
\end{array}$ & $\begin{array}{c}0.01330 \\
(0.00275) \\
\end{array}$ \\
\hline GE & $\begin{array}{c}0.00576 \\
(0.00106) \\
\end{array}$ & $\begin{array}{r}0.00422 \\
(0.00083) \\
\end{array}$ & $\begin{array}{c}0.01334 \\
(0.00268) \\
\end{array}$ \\
\hline C-RSM & $\begin{array}{c}0.00638 \\
(0.00133)\end{array}$ & $\begin{array}{c}0.00471 \\
(0.00100)\end{array}$ & $\begin{array}{c}0.01282 \\
(0.00265)\end{array}$ \\
\hline MT & $\begin{array}{c}0.00568 \\
(0.00104) \\
\end{array}$ & $\begin{array}{c}0.00421 \\
(0.00081) \\
\end{array}$ & $\begin{array}{c}0.01246 \\
(0.00235) \\
\end{array}$ \\
\hline SAV & $\begin{array}{r}0.00947 \\
(0.00262) \\
\end{array}$ & $\begin{array}{r}0.00694 \\
(0.00188) \\
\end{array}$ & $\begin{array}{r}0.00944 \\
(0.00318) \\
\end{array}$ \\
\hline NEV & $\begin{array}{c}0.00788 \\
(0.00164) \\
\end{array}$ & $\begin{array}{c}0.00594 \\
(0.00130)\end{array}$ & $\begin{array}{c}0.01691 \\
(0.00370)\end{array}$ \\
\hline LSM & $\begin{array}{c}0.01226 \\
(0.00348)\end{array}$ & $\begin{array}{c}0.00890 \\
(0.00253)\end{array}$ & $\begin{array}{c}0.01037 \\
(0.00452)\end{array}$ \\
\hline
\end{tabular}


Table C.7: Mean and Standard Deviation of Group Disagreement $\left(d_{2}\right)$ from Simulation (With $\mathrm{N}=8, \mathrm{M}=7$, scale $[1 / 9,9], \mathrm{T}=500$ )

\begin{tabular}{||l||c|c|c||c|c|c||}
\hline \multicolumn{1}{|c||}{ M=7 } & \multicolumn{3}{c|}{ A-GE(V) } & \multicolumn{3}{c||}{ A-GE(M) } \\
\hline ABBREVIATIONS & UF & LN & GA & UF & LN & GA \\
\hline \hline CSM & 0.00491 & 0.00360 & 0.01248 & 0.00543 & 0.00381 & 0.01589 \\
& $(0.00071)$ & $(0.00055)$ & $(0.00190)$ & $(0.00068)$ & $(0.00054)$ & $(0.00205)$ \\
\hline R-EV & 0.00470 & 0.00347 & 0.01236 & 0.00470 & 0.00347 & 0.01240 \\
& $(0.00063)$ & $(0.00051)$ & $(0.00178)$ & $(0.00063)$ & $(0.00051)$ & $(0.00174)$ \\
\hline L-EV & 0.00475 & 0.00347 & 0.01296 & 0.00476 & 0.00347 & 0.01298 \\
& $(0.00065)$ & $(0.00051)$ & $(0.00192)$ & $(0.00065)$ & $(0.00051)$ & $(0.00188)$ \\
\hline AM-EV & 0.00473 & 0.00346 & 0.01255 & 0.00473 & 0.00346 & 0.01254 \\
& $(0.00065)$ & $(0.00051)$ & $(0.00183)$ & $(0.00064)$ & $(0.00051)$ & $(0.00179)$ \\
\hline GM-EV & 0.00469 & 0.00345 & 0.01201 & 0.00468 & 0.00345 & 0.01198 \\
& $(0.00063)$ & $(0.00051)$ & $(0.00170)$ & $(0.00063)$ & $(0.00051)$ & $(0.00166)$ \\
\hline EV[AA'] & 0.00623 & 0.00450 & 0.01744 & 0.00624 & 0.00451 & 0.01738 \\
& $(0.00098)$ & $(0.00076)$ & $(0.00277)$ & $(0.00097)$ & $(0.00076)$ & $(0.00265)$ \\
\hline EV[A'A] & 0.00808 & 0.00589 & 0.01243 & 0.00810 & 0.00590 & 0.01295 \\
& $(0.00154)$ & $(0.00128)$ & $(0.00219)$ & $(0.00152)$ & $(0.00127)$ & $(0.00221)$ \\
\hline AM - EV[AA'] & 0.00571 & 0.00411 & 0.01560 & 0.00572 & 0.00411 & 0.01559 \\
AND EV[A'A] & $(0.00090)$ & $(0.00070)$ & $(0.00248)$ & $(0.00090)$ & $(0.00070)$ & $(0.00240)$ \\
\hline GE - EV[AA'] & 0.00541 & 0.00393 & 0.01193 & 0.00543 & 0.00394 & 0.01205 \\
AND EV[A'A] & $(0.00078)$ & $(0.00065)$ & $(0.00179)$ & $(0.00077)$ & $(0.00065)$ & $(0.00176)$ \\
\hline GE & 0.00473 & 0.00345 & 0.01139 & 0.00473 & 0.00345 & 0.01132 \\
& $(0.00063)$ & $(0.00051)$ & $(0.00168)$ & $(0.00063)$ & $(0.00051)$ & $(0.00165)$ \\
\hline C-RSM & 0.00483 & 0.00354 & 0.01153 & 0.00482 & 0.00354 & 0.01152 \\
& $(0.00064)$ & $(0.00053)$ & $(0.00169)$ & $(0.00064)$ & $(0.00052)$ & $(0.00165)$ \\
\hline MT & 0.00468 & 0.00344 & 0.01096 & 0.00468 & 0.00344 & 0.01104 \\
& $(0.00062)$ & $(0.00050)$ & $(0.00153)$ & $(0.00062)$ & $(0.00050)$ & $(0.00149)$ \\
\hline SAV & 0.00593 & 0.00435 & 0.01073 & 0.00593 & 0.00436 & 0.01092 \\
& $(0.00093)$ & $(0.00077)$ & $(0.00171)$ & $(0.00092)$ & $(0.00077)$ & $(0.00167)$ \\
\hline NEV & 0.00652 & 0.00491 & 0.01504 & 0.00668 & 0.00498 & 0.01671 \\
& $(0.00102)$ & $(0.00079)$ & $(0.00265)$ & $(0.00010)$ & $(0.00078)$ & $(0.00260)$ \\
\hline LSM & 0.00735 & 0.00534 & 0.01261 & 0.00737 & 0.00535 & 0.01290 \\
& $(0.00143)$ & $(0.00117)$ & $(0.00291)$ & $(0.00141)$ & $(0.00117)$ & $(0.00295)$ \\
\hline
\end{tabular}


Table C.8: Mean and Standard Deviation of Group Disagreement $\left(d_{2}\right)$ from Simulation (With $\mathrm{N}=8, \mathrm{M}=7$, scale $[1 / 9,9], \mathrm{T}=500$ ) [Continued]

\begin{tabular}{||l||c|c|c||c|c|c||}
\hline \multicolumn{1}{|c|}{ M=7 } & \multicolumn{3}{c|}{ A-AM(V) } & \multicolumn{3}{c||}{ A-MDM(M) } \\
\hline ABBREVIATIONS & UF & LN & GA & UF & LN & GA \\
\hline \hline CSM & 0.00490 & 0.00359 & 0.01237 & 0.00540 & 0.00384 & 0.01591 \\
& $(0.00071)$ & $(0.00055)$ & $(0.00186)$ & $(0.00069)$ & $(0.00055)$ & $(0.00223)$ \\
\hline R-EV & 0.00469 & 0.00347 & 0.01225 & 0.00477 & 0.00352 & 0.01263 \\
& $(0.00063)$ & $(0.00051)$ & $(0.00174)$ & $(0.00061)$ & $(0.00052)$ & $(0.00174)$ \\
\hline L-EV & 0.00475 & 0.00347 & 0.01283 & 0.00482 & 0.00352 & 0.01321 \\
& $(0.00065)$ & $(0.00051)$ & $(0.00187)$ & $(0.00066)$ & $(0.00052)$ & $(0.00193)$ \\
\hline AM-EV & 0.00472 & 0.00346 & 0.01243 & 0.00480 & 0.00350 & 0.01278 \\
& $(0.00064)$ & $(0.00051)$ & $(0.00179)$ & $(0.00066)$ & $(0.00052)$ & $(0.00184)$ \\
\hline GE-EV & 0.00468 & 0.00345 & 0.01191 & 0.00475 & 0.00349 & 0.01222 \\
& $(0.00063)$ & $(0.00051)$ & $(0.00166)$ & $(0.00065)$ & $(0.00051)$ & $(0.00168)$ \\
\hline EV[AA'] & 0.00622 & 0.00450 & 0.01714 & 0.00632 & 0.00456 & 0.01762 \\
& $(0.00097)$ & $(0.00076)$ & $(0.00266)$ & $(0.00099)$ & $(0.00077)$ & $(0.00272)$ \\
\hline EV[A'A] & 0.00805 & 0.00588 & 0.01229 & 0.00821 & 0.00597 & 0.01314 \\
& $(0.00153)$ & $(0.00127)$ & $(0.00213)$ & $(0.00155)$ & $(0.00130)$ & $(0.00227)$ \\
\hline AM - EV[AA'] & 0.00570 & 0.00411 & 0.01539 & 0.00579 & 0.00416 & 0.01582 \\
AND EV[A'A] & $(0.00090)$ & $(0.00070)$ & $(0.00240)$ & $(0.00091)$ & $(0.00071)$ & $(0.00245)$ \\
\hline GE- EV[AA'] & 0.00540 & 0.00393 & 0.01183 & 0.00550 & 0.00399 & 0.01226 \\
AND EV[A'A] & $(0.00077)$ & $(0.00065)$ & $(0.00175)$ & $(0.00078)$ & $(0.00066)$ & $(0.00180)$ \\
\hline GE & 0.00472 & 0.00345 & 0.01131 & 0.00479 & 0.00349 & 0.01154 \\
& $(0.00063)$ & $(0.00051)$ & $(0.00165)$ & $(0.00064)$ & $(0.00051)$ & $(0.00166)$ \\
\hline C-RSM & 0.00482 & 0.00354 & 0.01144 & 0.00489 & 0.00358 & 0.01175 \\
& $(0.00064)$ & $(0.00052)$ & $(0.00165)$ & $(0.00065)$ & $(0.00053)$ & $(0.00167)$ \\
\hline MT & 0.00467 & 0.00344 & 0.01088 & 0.00475 & 0.00349 & 0.01128 \\
& $(0.00062)$ & $(0.00050)$ & $(0.00150)$ & $(0.00063)$ & $(0.00051)$ & $(0.00150)$ \\
\hline SAV & 0.00591 & 0.00435 & 0.01065 & 0.00601 & 0.00441 & 0.01112 \\
& $(0.00092)$ & $(0.00077)$ & $(0.00167)$ & $(0.00094)$ & $(0.00078)$ & $(0.00170)$ \\
\hline NEV & 0.00650 & 0.00491 & 0.01479 & 0.00670 & 0.00504 & 0.01689 \\
& $(0.00101)$ & $(0.00079)$ & $(0.00253)$ & $(0.00101)$ & $(0.00079)$ & $(0.00276)$ \\
\hline LSM & 0.00733 & 0.00533 & 0.01246 & 0.00747 & 0.00541 & 0.01302 \\
& $(0.00142)$ & $(0.00117)$ & $(0.00282)$ & $(0.00144)$ & $(0.00119)$ & $(0.00298)$ \\
\hline
\end{tabular}


Table C.9: Mean and Standard Deviation of Group Disagreement $\left(d_{2}\right)$ from Simulation (With $\mathrm{N}=8, \mathrm{M}=7$, scale $[1 / 9,9], \mathrm{T}=500$ ) [Continued]

\begin{tabular}{||l||c|c|c||}
\hline \multicolumn{1}{|c||}{ M=7 } & \multicolumn{3}{c||}{ A-MDM(V) } \\
\hline ABBREVIATIONS & UF & LN & GA \\
\hline \hline CSM & 0.00647 & 0.00513 & 0.01473 \\
& $(0.00179)$ & $(0.00177)$ & $(0.00294)$ \\
\hline R-EV & 0.00500 & 0.00370 & 0.01262 \\
& $(0.00077)$ & $(0.00060)$ & $(0.00202)$ \\
\hline L-EV & 0.00505 & 0.00369 & 0.01400 \\
& $(0.00079)$ & $(0.00061)$ & $(0.00253)$ \\
\hline AM-EV & 0.00504 & 0.00369 & 0.01376 \\
& $(0.00078)$ & $(0.00061)$ & $(0.00249)$ \\
\hline GE-EV & 0.00498 & 0.00368 & 0.01267 \\
& $(0.00077)$ & $(0.00060)$ & $(0.00209)$ \\
\hline EV[AA'] & 0.00705 & 0.00505 & 0.01901 \\
& $(0.00113)$ & $(0.00096)$ & $(0.00331)$ \\
\hline EV[A'A] & 0.01109 & 0.00814 & 0.00965 \\
& $(0.00273)$ & $(0.00199)$ & $(0.00343)$ \\
\hline AM - EV[AA'] & 0.00675 & 0.00484 & 0.01806 \\
AND EV[A'A] & $(0.00116)$ & $(0.00097)$ & $(0.00332)$ \\
\hline GE - EV[AA'] & 0.00662 & 0.00489 & 0.01161 \\
AND EV[A'A] & $(0.00129)$ & $(0.00099)$ & $(0.00198)$ \\
\hline GE & 0.00503 & 0.00368 & 0.01169 \\
& $(0.00077)$ & $(0.00060)$ & $(0.00192)$ \\
\hline C-RSM & 0.00556 & 0.00411 & 0.01123 \\
& $(0.00097)$ & $(0.00076)$ & $(0.00192)$ \\
\hline MT & 0.00497 & 0.00367 & 0.01091 \\
& $(0.00076)$ & $(0.00059)$ & $(0.00166)$ \\
\hline SAV & 0.00826 & 0.00608 & 0.00826 \\
& $(0.00212)$ & $(0.00156)$ & $(0.00251)$ \\
\hline NEV & 0.00690 & 0.00520 & 0.01479 \\
& $(0.00122)$ & $(0.00098)$ & $(0.00283)$ \\
\hline LSM & 0.01068 & 0.00783 & 0.00907 \\
& $(0.00281)$ & $(0.00209)$ & $(0.00352)$ \\
\hline
\end{tabular}


Table C.10: Mean and Standard Deviation of Group Disagreement $\left(d_{2}\right)$ from Simulation (With $\mathrm{N}=8, \mathrm{M}=9$, scale $[1 / 9,9], \mathrm{T}=500$ )

\begin{tabular}{||l||c|c|c||c|c|c||}
\hline \multicolumn{1}{|c||}{ M=9 } & \multicolumn{3}{c|}{ A-GE(V) } & \multicolumn{3}{c||}{ A-GE(M) } \\
\hline ABBREVIATIONS & UF & LN & GA & UF & LN & GA \\
\hline \hline CSM & 0.00442 & 0.00324 & 0.01125 & 0.00490 & 0.00343 & 0.01445 \\
& $(0.00058)$ & $(0.00045)$ & $(0.00158)$ & $(0.00056)$ & $(0.00044)$ & $(0.00178)$ \\
\hline R-EV & 0.00423 & 0.00313 & 0.01112 & 0.00423 & 0.00313 & 0.01114 \\
& $(0.00050)$ & $(0.00040)$ & $(0.00146)$ & $(0.00050)$ & $(0.00040)$ & $(0.00143)$ \\
\hline L-EV & 0.00428 & 0.00312 & 0.01170 & 0.00428 & 0.00313 & 0.01169 \\
& $(0.00051)$ & $(0.00040)$ & $(0.00155)$ & $(0.00051)$ & $(0.00040)$ & $(0.00151)$ \\
\hline AM-EV & 0.00426 & 0.00312 & 0.01133 & 0.00426 & 0.00312 & 0.01130 \\
& $(0.00051)$ & $(0.00040)$ & $(0.00149)$ & $(0.00051)$ & $(0.00040)$ & $(0.00145)$ \\
\hline GM-EV & 0.00422 & 0.00311 & 0.01083 & 0.00422 & 0.00311 & 0.01079 \\
& $(0.00050)$ & $(0.00040)$ & $(0.00139)$ & $(0.00050)$ & $(0.00040)$ & $(0.00137)$ \\
\hline EV[AA'] & 0.00561 & 0.00406 & 0.01569 & 0.00561 & 0.00407 & 0.01559 \\
& $(0.00081)$ & $(0.00063)$ & $(0.00220)$ & $(0.00080)$ & $(0.00063)$ & $(0.00210)$ \\
\hline EV[A'A] & 0.00727 & 0.00531 & 0.01122 & 0.00728 & 0.00532 & 0.01163 \\
& $(0.00128)$ & $(0.00106)$ & $(0.00181)$ & $(0.00126)$ & $(0.00105)$ & $(0.00183)$ \\
\hline AM - EV[AA'] & 0.00514 & 0.00371 & 0.01405 & 0.00514 & 0.00371 & 0.01399 \\
AND EV[A'A] & $(0.00074)$ & $(0.00058)$ & $(0.00197)$ & $(0.00074)$ & $(0.00058)$ & $(0.00191)$ \\
\hline GE - EV[AA'] & 0.00487 & 0.00354 & 0.01075 & 0.00488 & 0.00354 & 0.01082 \\
AND EV[A'A] & $(0.00060)$ & $(0.00052)$ & $(0.00148)$ & $(0.00060)$ & $(0.00051)$ & $(0.00145)$ \\
\hline GE & 0.00426 & 0.00311 & 0.01027 & 0.00425 & 0.00311 & 0.01020 \\
& $(0.00050)$ & $(0.00040)$ & $(0.00139)$ & $(0.00050)$ & $(0.00040)$ & $(0.00137)$ \\
\hline C-RSM & 0.00434 & 0.00319 & 0.01040 & 0.00434 & 0.00318 & 0.01039 \\
& $(0.00050)$ & $(0.00041)$ & $(0.00139)$ & $(0.00050)$ & $(0.00041)$ & $(0.00136)$ \\
\hline MT & 0.00421 & 0.00310 & 0.00986 & 0.00421 & 0.00310 & 0.00992 \\
& $(0.00049)$ & $(0.00040)$ & $(0.00127)$ & $(0.00049)$ & $(0.00039)$ & $(0.00123)$ \\
\hline SAV & 0.00532 & 0.00392 & 0.00968 & 0.00532 & 0.00392 & 0.00982 \\
& $(0.00073)$ & $(0.00061)$ & $(0.00144)$ & $(0.00073)$ & $(0.00061)$ & $(0.00141)$ \\
\hline NEV & 0.00588 & 0.00443 & 0.01355 & 0.00602 & 0.00449 & 0.01507 \\
& $(0.00080)$ & $(0.00063)$ & $(0.00215)$ & $(0.00078)$ & $(0.00062)$ & $(0.00213)$ \\
\hline LSM & 0.00662 & 0.00481 & 0.01142 & 0.00663 & 0.00481 & 0.01160 \\
& $(0.00118)$ & $(0.00097)$ & $(0.00248)$ & $(0.00117)$ & $(0.00096)$ & $(0.00249)$ \\
\hline
\end{tabular}


Table C.11: Mean and Standard Deviation of Group Disagreement $\left(d_{2}\right)$ from Simulation (With $\mathrm{N}=8, \mathrm{M}=9$, scale $[1 / 9,9], \mathrm{T}=500$ ) [Continued]

\begin{tabular}{||l||c|c|c||c|c|c||}
\hline \multicolumn{1}{|c||}{ M=9 } & \multicolumn{3}{c|}{ A-AM(V) } & \multicolumn{3}{c||}{ A-MDM(M) } \\
\hline ABBREVIATIONS & UF & LN & GA & UF & LN & GA \\
\hline \hline CSM & 0.00441 & 0.00324 & 0.01115 & 0.00485 & 0.00346 & 0.01449 \\
& $(0.00058)$ & $(0.00045)$ & $(0.00154)$ & $(0.00056)$ & $(0.00045)$ & $(0.00195)$ \\
\hline R-EV & 0.00423 & 0.00313 & 0.01102 & 0.00429 & 0.00317 & 0.01134 \\
& $(0.00050)$ & $(0.00040)$ & $(0.00143)$ & $(0.00051)$ & $(0.00040)$ & $(0.00144)$ \\
\hline L-EV & 0.00427 & 0.00312 & 0.01159 & 0.00434 & 0.00316 & 0.01190 \\
& $(0.00051)$ & $(0.00040)$ & $(0.00151)$ & $(0.00052)$ & $(0.00041)$ & $(0.00155)$ \\
\hline AM-EV & 0.00425 & 0.00312 & 0.01123 & 0.00432 & 0.00315 & 0.01151 \\
& $(0.00051)$ & $(0.00040)$ & $(0.00145)$ & $(0.00052)$ & $(0.00041)$ & $(0.00149)$ \\
\hline GE-EV & 0.00422 & 0.00311 & 0.01075 & 0.00428 & 0.00314 & 0.01100 \\
& $(0.00050)$ & $(0.00040)$ & $(0.00136)$ & $(0.00051)$ & $(0.00040)$ & $(0.00139)$ \\
\hline EV[AA'] & 0.00559 & 0.00406 & 0.01543 & 0.00568 & 0.00411 & 0.01583 \\
& $(0.00080)$ & $(0.00063)$ & $(0.00211)$ & $(0.00082)$ & $(0.00063)$ & $(0.00217)$ \\
\hline EV[A'A] & 0.00724 & 0.00530 & 0.01109 & 0.00737 & 0.00538 & 0.01183 \\
& $(0.00127)$ & $(0.00105)$ & $(0.00176)$ & $(0.00128)$ & $(0.00107)$ & $(0.00189)$ \\
\hline AM-EV[AA'] & 0.00513 & 0.00370 & 0.01386 & 0.00521 & 0.00375 & 0.01421 \\
AND EV[A'A] & $(0.00074)$ & $(0.00058)$ & $(0.00191)$ & $(0.00075)$ & $(0.00058)$ & $(0.00197)$ \\
\hline GE- EV[AA'] & 0.00486 & 0.00354 & 0.01066 & 0.00494 & 0.00358 & 0.01102 \\
AND EV[A'A] & $(0.00060)$ & $(0.00051)$ & $(0.00145)$ & $(0.00061)$ & $(0.00052)$ & $(0.00149)$ \\
\hline GE & 0.00425 & 0.00311 & 0.01019 & 0.00431 & 0.00314 & 0.01039 \\
& $(0.00050)$ & $(0.00040)$ & $(0.00136)$ & $(0.00051)$ & $(0.0040)$ & $(0.00138)$ \\
\hline C-RSM & 0.00434 & 0.00318 & 0.01032 & 0.00440 & 0.00322 & 0.01060 \\
& $(0.00050)$ & $(0.00041)$ & $(0.00136)$ & $(0.00051)$ & $(0.00042)$ & $(0.00137)$ \\
\hline MT & 0.00420 & 0.00310 & 0.00979 & 0.00427 & 0.00314 & 0.01014 \\
& $(0.00049)$ & $(0.00039)$ & $(0.00125)$ & $(0.00050)$ & $(0.00040)$ & $(0.00125)$ \\
\hline SAV & 0.00531 & 0.00392 & 0.00960 & 0.00540 & 0.00397 & 0.01100 \\
& $(0.00073)$ & $(0.00061)$ & $(0.00141)$ & $(0.00073)$ & $(0.00062)$ & $(0.00142)$ \\
\hline NEV & 0.00586 & 0.00443 & 0.01333 & 0.00602 & 0.00454 & 0.01522 \\
& $(0.00080)$ & $(0.00062)$ & $(0.00205)$ & $(0.00080)$ & $(0.00062)$ & $(0.00225)$ \\
\hline LSM & 0.00660 & 0.00480 & 0.01129 & 0.00671 & 0.00487 & 0.01172 \\
& $(0.00117)$ & $(0.00096)$ & $(0.00240)$ & $(0.00118)$ & $(0.00097)$ & $(0.00251)$ \\
\hline
\end{tabular}


Table C.12: Mean and Standard Deviation of Group Disagreement $\left(d_{2}\right)$ from Simulation (With $\mathrm{N}=8, \mathrm{M}=9$, scale $[1 / 9,9], \mathrm{T}=500$ ) [Continued]

\begin{tabular}{||l||c|c|c||}
\hline \multicolumn{1}{|c||}{ M=9 } & \multicolumn{3}{c||}{ A-MDM(V) } \\
\hline ABBREVIATIONS & UF & LN & GA \\
\hline \hline CSM & 0.00576 & 0.00457 & 0.01321 \\
& $(0.00153)$ & $(0.00154)$ & $(0.00248)$ \\
\hline R-EV & 0.00448 & 0.00332 & 0.01134 \\
& $(0.00061)$ & $(0.00047)$ & $(0.00157)$ \\
\hline L-EV & 0.00452 & 0.00331 & 0.01259 \\
& $(0.00061)$ & $(0.00047)$ & $(0.00199)$ \\
\hline AM-EV & 0.00451 & 0.00331 & 0.01237 \\
& $(0.00061)$ & $(0.00047)$ & $(0.00197)$ \\
\hline GE-EV & 0.00447 & 0.00330 & 0.01139 \\
& $(0.00061)$ & $(0.00047)$ & $(0.00162)$ \\
\hline EV[AA'] & 0.00628 & 0.00454 & 0.01701 \\
& $(0.00094)$ & $(0.00078)$ & $(0.00277)$ \\
\hline EV[A'A] & 0.00990 & 0.00729 & 0.00868 \\
& $(0.00239)$ & $(0.00167)$ & $(0.00285)$ \\
\hline AM - EV[AA'] & 0.00602 & 0.00435 & 0.01617 \\
AND EV[A'A] & $(0.00096)$ & $(0.00080)$ & $(0.00282)$ \\
\hline GE- EV[AA'] & 0.00595 & 0.00437 & 0.01041 \\
AND EV[A'A] & $(0.00110)$ & $(0.00080)$ & $(0.00159)$ \\
\hline GE & 0.00450 & 0.00330 & 0.01051 \\
& $(0.00060)$ & $(0.00047)$ & $(0.00149)$ \\
\hline C-RSM & 0.00499 & 0.00369 & 0.01007 \\
& $(0.00081)$ & $(0.00061)$ & $(0.00151)$ \\
\hline MT & 0.00445 & 0.00329 & 0.00980 \\
& $(0.00060)$ & $(0.00047)$ & $(0.00127)$ \\
\hline SAV & 0.00739 & 0.00545 & 0.00742 \\
& $(0.00186)$ & $(0.00130)$ & $(0.00212)$ \\
\hline NEV & 0.00619 & 0.00468 & 0.01335 \\
& $(0.00095)$ & $(0.00076)$ & $(0.00230)$ \\
\hline LSM & 0.00954 & 0.00701 & 0.00819 \\
& $(0.00247)$ & $(0.00174)$ & $(0.00297)$ \\
\hline
\end{tabular}


Table C.13: Mean and Standard Deviation of Group Disagreement $\left(d_{2}\right)$ from Simulation (With $\mathrm{N}=10, \mathrm{M}=3$, scale $[1 / 9,9], \mathrm{T}=500$ )

\begin{tabular}{||l||c|c|c||c|c|c||}
\hline \multicolumn{1}{|c||}{ M=3 } & \multicolumn{3}{c|}{ A-GE(V) } & \multicolumn{3}{c||}{ A-GE(M) } \\
\hline ABBREVIATIONS & UF & LN & GA & UF & LN & GA \\
\hline \hline CSM & 0.00469 & 0.00346 & 0.01152 & 0.00510 & 0.00364 & 0.01434 \\
& $(0.00098)$ & $(0.00075)$ & $(0.00278)$ & $(0.00091)$ & $(0.00072)$ & $(0.00265)$ \\
\hline R-EV & 0.00455 & 0.00337 & 0.01201 & 0.00457 & 0.00338 & 0.01232 \\
& $(0.00095)$ & $(0.00072)$ & $(0.00274)$ & $(0.00095)$ & $(0.00071)$ & $(0.00266)$ \\
\hline L-EV & 0.00459 & 0.00338 & 0.01297 & 0.00451 & 0.00340 & 0.01324 \\
& $(0.00094)$ & $(0.00072)$ & $(0.00288)$ & $(0.00093)$ & $(0.00072)$ & $(0.00277)$ \\
\hline AM-EV & 0.00457 & 0.00337 & 0.01247 & 0.00458 & 0.00338 & 0.01265 \\
& $(0.00093)$ & $(0.00072)$ & $(0.00277)$ & $(0.00093)$ & $(0.00072)$ & $(0.00268)$ \\
\hline GM-EV & 0.00453 & 0.00335 & 0.01166 & 0.00453 & 0.00335 & 0.01173 \\
& $(0.00093)$ & $(0.00072)$ & $(0.00258)$ & $(0.00093)$ & $(0.00071)$ & $(0.00252)$ \\
\hline EV[AA'] & 0.00602 & 0.00437 & 0.01731 & 0.00606 & 0.00439 & 0.01761 \\
& $(0.00133)$ & $(0.00104)$ & $(0.00406)$ & $(0.00132)$ & $(0.00103)$ & $(0.00391)$ \\
\hline EV[A'A] & 0.00467 & 0.00333 & 0.00681 & 0.00478 & 0.00339 & 0.00768 \\
& $(0.00165)$ & $(0.00116)$ & $(0.00206)$ & $(0.00165)$ & $(0.00117)$ & $(0.00214)$ \\
\hline AM-EV[AA'] & 0.00534 & 0.00386 & 0.01482 & 0.00539 & 0.00388 & 0.01515 \\
AND EV[A'A] & $(0.00121)$ & $(0.00092)$ & $(0.00358)$ & $(0.00120)$ & $(0.00092)$ & $(0.00346)$ \\
\hline GE-EV[AA'] & 0.00383 & 0.00278 & 0.00922 & 0.00788 & 0.00280 & 0.00960 \\
AND EV[A'A] & $(0.00091)$ & $(0.00064)$ & $(0.00199)$ & $(0.00090)$ & $(0.00064)$ & $(0.00195)$ \\
\hline GE & 0.00457 & 0.00335 & 0.01102 & 0.00457 & 0.00335 & 0.01098 \\
& $(0.00094)$ & $(0.00071)$ & $(0.00245)$ & $(0.00093)$ & $(0.00071)$ & $(0.00242)$ \\
\hline C-RSM & 0.00468 & 0.00343 & 0.01116 & 0.00468 & 0.00343 & 0.01126 \\
& $(0.00098)$ & $(0.00074)$ & $(0.00244)$ & $(0.00097)$ & $(0.00074)$ & $(0.00240)$ \\
\hline MT & 0.00453 & 0.00334 & 0.01077 & 0.00455 & 0.00335 & 0.01102 \\
& $(0.00094)$ & $(0.00071)$ & $(0.00232)$ & $(0.00093)$ & $(0.00071)$ & $(0.00224)$ \\
\hline SAV & 0.00581 & 0.00422 & 0.01028 & 0.00583 & 0.00423 & 0.01072 \\
& $(0.00132)$ & $(0.00100)$ & $(0.00226)$ & $(0.00132)$ & $(0.00100)$ & $(0.00219)$ \\
\hline NEV & 0.00652 & 0.00499 & 0.01523 & 0.00670 & 0.00506 & 0.01736 \\
& $(0.00159)$ & $(0.00118)$ & $(0.00354)$ & $(0.001550$ & $(0.00116)$ & $(0.00352)$ \\
\hline LSM & 0.00743 & 0.00527 & 0.01196 & 0.00749 & 0.00531 & 0.01255 \\
& $(0.00192)$ & $(0.00154)$ & $(0.00342)$ & $(0.00190)$ & $(0.00154)$ & $(0.00351)$ \\
\hline
\end{tabular}


Table C.14: Mean and Standard Deviation of Group Disagreement $\left(d_{2}\right)$ from Simulation (With $\mathrm{N}=10, \mathrm{M}=3$, scale $[1 / 9,9], \mathrm{T}=500$ ) [Continued]

\begin{tabular}{||l||c|c|c||c|c|c||}
\hline \multicolumn{1}{|c||}{ M=3 } & \multicolumn{3}{c|}{ A-AM(V) } & \multicolumn{3}{c||}{ A-MDM(M) } \\
\hline ABBREVIATIONS & UF & LN & GA & UF & LN & GA \\
\hline \hline CSM & 0.00468 & 0.00345 & 0.01144 & 0.00512 & 0.00368 & 0.01434 \\
& $(0.00098)$ & $(0.00074)$ & $(0.00271)$ & $(0.00093)$ & $(0.00074)$ & $(0.00275)$ \\
\hline R-EV & 0.00455 & 0.00336 & 0.01191 & 0.00461 & 0.00342 & 0.01250 \\
& $(0.00095)$ & $(0.00072)$ & $(0.00269)$ & $(0.00096)$ & $(0.00073)$ & $(0.00274)$ \\
\hline L-EV & 0.00459 & 0.00338 & 0.01286 & 0.00465 & 0.00344 & 0.01347 \\
& $(0.00093)$ & $(0.00072)$ & $(0.00281)$ & $(0.00094)$ & $(0.00073)$ & $(0.00287)$ \\
\hline AM-EV & 0.00457 & 0.00337 & 0.01236 & 0.00463 & 0.00342 & 0.01288 \\
& $(0.00093)$ & $(0.00072)$ & $(0.00270)$ & $(0.00094)$ & $(0.00073)$ & $(0.00277)$ \\
\hline GE-EV & 0.00453 & 0.00335 & 0.01157 & 0.00457 & 0.00340 & 0.01195 \\
& $(0.00093)$ & $(0.00071)$ & $(0.00252)$ & $(0.00094)$ & $(0.00073)$ & $(0.00259)$ \\
\hline EV[AA'] & 0.00601 & 0.00437 & 0.01703 & 0.00612 & 0.00445 & 0.01790 \\
& $(0.00133)$ & $(0.00104)$ & $(0.00390)$ & $(0.00134)$ & $(0.00105)$ & $(0.00403)$ \\
\hline EV[A'A] & 0.00466 & 0.00333 & 0.00678 & 0.00482 & 0.00344 & 0.00771 \\
& $(0.00164)$ & $(0.00116)$ & $(0.00204)$ & $(0.00167)$ & $(0.00119)$ & $(0.00215)$ \\
\hline AM- EV[AA'] & 0.00534 & 0.00386 & 0.01463 & 0.00544 & 0.00394 & 0.01539 \\
AND EV[A'A] & $(0.00121)$ & $(0.00092)$ & $(0.00347)$ & $(0.00122)$ & $(0.00094)$ & $(0.00357)$ \\
\hline GE - EV[AA'] & 0.00382 & 0.00278 & 0.00917 & 0.00391 & 0.00284 & 0.00975 \\
AND EV[A'A] & $(0.00090)$ & $(0.00064)$ & $(0.00196)$ & $(0.00091)$ & $(0.00066)$ & $(0.00200)$ \\
\hline GE & 0.00457 & 0.00335 & 0.01094 & 0.00462 & 0.00340 & 0.01120 \\
& $(0.00093)$ & $(0.00071)$ & $(0.00240)$ & $(0.00094)$ & $(0.00072)$ & $(0.00248)$ \\
\hline C-RSM & 0.00467 & 0.00343 & 0.01108 & 0.00473 & 0.00348 & 0.01147 \\
& $(0.00097)$ & $(0.00074)$ & $(0.00240)$ & $(0.00098)$ & $(0.00075)$ & $(0.00246)$ \\
\hline MT & 0.00453 & 0.00334 & 0.01069 & 0.00459 & 0.00340 & 0.01123 \\
& $(0.00094)$ & $(0.00071)$ & $(0.00228)$ & $(0.00094)$ & $(0.00072)$ & $(0.00231)$ \\
\hline SAV & 0.00580 & 0.00422 & 0.01021 & 0.00589 & 0.00429 & 0.01085 \\
& $(0.00132)$ & $(0.00100)$ & $(0.00222)$ & $(0.00133)$ & $(0.00102)$ & $(0.00231)$ \\
\hline NEV & 0.00650 & 0.00498 & 0.01498 & 0.00674 & 0.00513 & 0.01745 \\
& $(0.00158)$ & $(0.00117)$ & $(0.00339)$ & $(0.00158)$ & $(0.00118)$ & $(0.00365)$ \\
\hline LSM & 0.00741 & 0.00527 & 0.01183 & 0.00756 & 0.00538 & 0.01265 \\
& $(0.00190)$ & $(0.00153)$ & $(0.00333)$ & $(0.00191)$ & $(0.00156)$ & $(0.00356)$ \\
\hline
\end{tabular}


Table C.15: Mean and Standard Deviation of Group Disagreement $\left(d_{2}\right)$ from Simulation (With $\mathrm{N}=10, \mathrm{M}=3$, scale $[1 / 9,9], \mathrm{T}=500$ ) [Continued]

\begin{tabular}{||l||c|c|c||}
\hline \multicolumn{1}{|c||}{ M=3 } & \multicolumn{3}{c||}{ A-MDM(V) } \\
\hline ABBREVIATIONS & UF & LN & GA \\
\hline \hline CSM & 0.00648 & 0.00518 & 0.01472 \\
& $(0.00189)$ & $(0.00169)$ & $(0.0040)$ \\
\hline R-EV & 0.00506 & 0.00377 & 0.01295 \\
& $(0.00114)$ & $(0.00088)$ & $(0.00309)$ \\
\hline L-EV & 0.00506 & 0.00377 & 0.01466 \\
& $(0.00117)$ & $(0.00086)$ & $(0.00385)$ \\
\hline AM-EV & 0.00505 & 0.00377 & 0.01441 \\
& $(0.00117)$ & $(0.00086)$ & $(0.00378)$ \\
\hline GE-EV & 0.00500 & 0.00375 & 0.01297 \\
& $(0.00113)$ & $(0.00087)$ & $(0.00308)$ \\
\hline EV[AA'] & 0.00708 & 0.00506 & 0.01976 \\
& $(0.00167)$ & $(0.00125)$ & $(0.00505)$ \\
\hline EV[A'A] & 0.00799 & 0.00606 & 0.00632 \\
& $(0.00340)$ & $(0.00286)$ & $(0.00322)$ \\
\hline AM - EV[AA'] & 0.00676 & 0.00482 & 0.01868 \\
AND EV[A'A] & $(0.00165)$ & $(0.00123)$ & $(0.00499)$ \\
\hline GE - EV[AA'] & 0.00536 & 0.00404 & 0.01003 \\
AND EV[A'A] & $(0.00180)$ & $(0.00143)$ & $(0.00262)$ \\
\hline GE & 0.00506 & 0.00375 & 0.01178 \\
& $(0.00114)$ & $(0.00087)$ & $(0.00282)$ \\
\hline C-RSM & 0.00574 & 0.00427 & 0.01122 \\
& $(0.00140)$ & $(0.00112)$ & $(0.00271)$ \\
\hline MT & 0.00503 & 0.00374 & 0.01117 \\
& $(0.00112)$ & $(0.00087)$ & $(0.00257)$ \\
\hline SAV & 0.00872 & 0.00649 & 0.00799 \\
& $(0.00261)$ & $(0.00213)$ & $(0.00293)$ \\
\hline NEV & 0.00719 & 0.00549 & 0.01605 \\
& $(0.00192)$ & $(0.00137)$ & $(0.00435)$ \\
\hline LSM & 0.01166 & 0.00870 & 0.00882 \\
& $(0.00347)$ & $(0.00299)$ & $(0.00410)$ \\
\hline
\end{tabular}


Table C.16: Mean and Standard Deviation of Group Disagreement $\left(d_{2}\right)$ from Simulation (With $\mathrm{N}=10, \mathrm{M}=5$, scale $[1 / 9,9], \mathrm{T}=500$ )

\begin{tabular}{||l||c|c|c||c|c|c||}
\hline \multicolumn{1}{|c||}{ M=5 } & \multicolumn{3}{c|}{ A-GE(V) } & \multicolumn{3}{c||}{ A-GE(M) } \\
\hline ABBREVIATIONS & UF & LN & GA & UF & LN & GA \\
\hline \hline CSM & 0.00408 & 0.00300 & 0.00999 & 0.00450 & 0.00317 & 0.01287 \\
& $(0.00065)$ & $(0.00048)$ & $(0.00175)$ & $(0.00059)$ & $(0.00047)$ & $(0.00183)$ \\
\hline R-EV & 0.00396 & 0.00292 & 0.01043 & 0.00397 & 0.00292 & 0.01057 \\
& $(0.00061)$ & $(0.00047)$ & $(0.00169)$ & $(0.00060)$ & $(0.00046)$ & $(0.00165)$ \\
\hline L-EV & 0.00400 & 0.00293 & 0.01122 & 0.00400 & 0.00293 & 0.01133 \\
& $(0.00061)$ & $(0.00045)$ & $(0.00187)$ & $(0.00061)$ & $(0.00045)$ & $(0.00182)$ \\
\hline AM-EV & 0.00398 & 0.00292 & 0.01080 & 0.00398 & 0.00292 & 0.01086 \\
& $(0.00061)$ & $(0.00045)$ & $(0.00179)$ & $(0.00060)$ & $(0.00045)$ & $(0.00175)$ \\
\hline GM-EV & 0.00394 & 0.00290 & 0.01012 & 0.00394 & 0.00290 & 0.01013 \\
& $(0.00060)$ & $(0.00045)$ & $(0.00164)$ & $(0.00060)$ & $(0.00045)$ & $(0.00161)$ \\
\hline EV[AA'] & 0.00527 & 0.00379 & 0.01493 & 0.00528 & 0.00380 & 0.01500 \\
& $(0.00085)$ & $(0.00066)$ & $(0.00257)$ & $(0.00085)$ & $(0.00066)$ & $(0.00246)$ \\
\hline EV[A'A] & 0.00412 & 0.00289 & 0.00596 & 0.00419 & 0.00293 & 0.00655 \\
& $(0.00119)$ & $(0.00083)$ & $(0.00140)$ & $(0.00119)$ & $(0.00083)$ & $(0.00146)$ \\
\hline AM-EV[AA'] & 0.00467 & 0.00335 & 0.01278 & 0.00470 & 0.00336 & 0.01289 \\
AND EV[A'A] & $(0.00079)$ & $(0.00060)$ & $(0.00229)$ & $(0.00079)$ & $(0.00060)$ & $(0.00221)$ \\
\hline GE- EV[AA'] & 0.00337 & 0.00241 & 0.00797 & 0.00340 & 0.00242 & 0.00818 \\
AND EV[A'A] & $(0.00060)$ & $(0.00045)$ & $(0.00129)$ & $(0.000600$ & $(0.00045)$ & $(0.00128)$ \\
\hline GE & 0.00398 & 0.00290 & 0.00957 & 0.00398 & 0.00290 & 0.00953 \\
& $(0.00060)$ & $(0.00045)$ & $(0.00158)$ & $(0.00060)$ & $(0.00045)$ & $(0.00156)$ \\
\hline C-RSM & 0.00407 & 0.00296 & 0.00967 & 0.00407 & 0.00296 & 0.00971 \\
& $(0.00062)$ & $(0.00046)$ & $(0.00157)$ & $(0.00061)$ & $(0.00046)$ & $(0.00154)$ \\
\hline MT & 0.00394 & 0.00290 & 0.00935 & 0.00395 & 0.00290 & 0.00949 \\
& $(0.00060)$ & $(0.00045)$ & $(0.00145)$ & $(0.00060)$ & $(0.00045)$ & $(0.00141)$ \\
\hline SAV & 0.00503 & 0.00363 & 0.00894 & 0.00504 & 0.00364 & 0.00920 \\
& $(0.00084)$ & $(0.00065)$ & $(0.00146)$ & $(0.00084)$ & $(0.00065)$ & $(0.00142)$ \\
\hline NEV & 0.00563 & 0.00431 & 0.01335 & 0.00580 & 0.00438 & 0.01523 \\
& $(0.00099)$ & $(0.00071)$ & $(0.00243)$ & $(0.00096)$ & $(0.00070)$ & $(0.00240)$ \\
\hline LSM & 0.00644 & 0.00456 & 0.01048 & 0.00646 & 0.00458 & 0.01079 \\
& $(0.00127)$ & $(0.00101)$ & $(0.00231)$ & $(0.00125)$ & $(0.00100)$ & $(0.00233)$ \\
\hline
\end{tabular}


Table C.17: Mean and Standard Deviation of Group Disagreement $\left(d_{2}\right)$ from Simulation (With $\mathrm{N}=10, \mathrm{M}=5$, scale $[1 / 9,9], \mathrm{T}=500$ ) [Continued]

\begin{tabular}{||l||c|c|c||c|c|c||}
\hline \multicolumn{1}{|c||}{ M=5 } & \multicolumn{3}{c|}{ A-AM(V) } & \multicolumn{3}{c||}{ A-MDM(M) } \\
\hline ABBREVIATIONS & UF & LN & GA & UF & LN & GA \\
\hline \hline CSM & 0.00407 & 0.00300 & 0.00992 & 0.00449 & 0.00319 & 0.01286 \\
& $(0.00065)$ & $(0.00048)$ & $(0.00171)$ & $(0.00061)$ & $(0.00048)$ & $(0.00192)$ \\
\hline R-EV & 0.00396 & 0.00292 & 0.01035 & 0.00402 & 0.00295 & 0.01069 \\
& $(0.00061)$ & $(0.00046)$ & $(0.00165)$ & $(0.00061)$ & $(0.00046)$ & $(0.00166)$ \\
\hline L-EV & 0.00400 & 0.00293 & 0.01112 & 0.00405 & 0.00296 & 0.01147 \\
& $(0.00061)$ & $(0.00045)$ & $(0.00183)$ & $(0.00061)$ & $(0.00046)$ & $(0.00185)$ \\
\hline AM-EV & 0.00398 & 0.00292 & 0.01071 & 0.00403 & 0.00295 & 0.01100 \\
& $(0.00060)$ & $(0.00045)$ & $(0.00175)$ & $(0.00061)$ & $(0.00046)$ & $(0.00178)$ \\
\hline GE-EV & 0.00394 & 0.00290 & 0.01005 & 0.00399 & 0.00293 & 0.01027 \\
& $(0.00060)$ & $(0.00045)$ & $(0.00161)$ & $(0.00061)$ & $(0.00046)$ & $(0.00163)$ \\
\hline EV[AA'] & 0.00526 & 0.00379 & 0.01470 & 0.00535 & 0.00383 & 0.01514 \\
& $(0.00085)$ & $(0.00066)$ & $(0.00247)$ & $(0.00086)$ & $(0.00066)$ & $(0.00249)$ \\
\hline EV[A'A] & 0.00412 & 0.00289 & 0.00594 & 0.00424 & 0.00295 & 0.00659 \\
& $(0.00118)$ & $(0.00083)$ & $(0.00138)$ & $(0.00121)$ & $(0.00084)$ & $(0.00150)$ \\
\hline AM - EV[AA'] & 0.00467 & 0.00335 & 0.01263 & 0.00475 & 0.00339 & 0.01302 \\
AND EV[A'A] & $(0.00079)$ & $(0.00060)$ & $(0.00222)$ & $(0.00080)$ & $(0.00061)$ & $(0.00224)$ \\
\hline GE - EV[AA'] & 0.00337 & 0.00241 & 0.00793 & 0.00344 & 0.00245 & 0.00827 \\
AND EV[A'A] & $(0.00060)$ & $(0.00045)$ & $(0.00127)$ & $(0.00061)$ & $(0.00045)$ & $(0.00128)$ \\
\hline GE & 0.00398 & 0.00290 & 0.00951 & 0.00403 & 0.00293 & 0.00966 \\
& $(0.00060)$ & $(0.00045)$ & $(0.00156)$ & $(0.00061)$ & $(0.00046)$ & $(0.00158)$ \\
\hline C-RSM & 0.00406 & 0.00296 & 0.00960 & 0.00412 & 0.00299 & 0.00983 \\
& $(0.00061)$ & $(0.00046)$ & $(0.00154)$ & $(0.00062)$ & $(0.00047)$ & $(0.00156)$ \\
\hline MT & 0.00394 & 0.00290 & 0.00929 & 0.00400 & 0.00293 & 0.00961 \\
& $(0.00060)$ & $(0.00045)$ & $(0.00143)$ & $(0.00061)$ & $(0.00045)$ & $(0.00143)$ \\
\hline SAV & 0.00502 & 0.00363 & 0.00888 & 0.00510 & 0.00367 & 0.00929 \\
& $(0.00084)$ & $(0.00065)$ & $(0.00143)$ & $(0.00085)$ & $(0.00065)$ & $(0.00145)$ \\
\hline NEV & 0.00562 & 0.00430 & 0.01313 & 0.00585 & 0.00441 & 0.01528 \\
& $(0.00098)$ & $(0.00071)$ & $(0.00231)$ & $(0.00098)$ & $(0.00071)$ & $(0.00250)$ \\
\hline LSM & 0.00642 & 0.00455 & 0.01038 & 0.00653 & 0.00461 & 0.01085 \\
& $(0.00126)$ & $(0.00100)$ & $(0.00224)$ & $(0.00127)$ & $(0.00101)$ & $(0.00236)$ \\
\hline
\end{tabular}


Table C.18: Mean and Standard Deviation of Group Disagreement $\left(d_{2}\right)$ from Simulation (With $N=10, M=5$, scale $[1 / 9,9], T=500$ ) [Continued]

\begin{tabular}{||l||c|c|c||}
\hline \multicolumn{1}{|c||}{ M=5 } & \multicolumn{3}{c||}{ A-MDM(V) } \\
\hline ABBREVIATIONS & UF & LN & GA \\
\hline \hline CSM & 0.00535 & 0.00420 & 0.01216 \\
& $(0.00138)$ & $(0.00126)$ & $(0.00263)$ \\
\hline R-EV & 0.00429 & 0.00318 & 0.01089 \\
& $(0.00070)$ & $(0.00051)$ & $(0.00187)$ \\
\hline L-EV & 0.00432 & 0.00317 & 0.01241 \\
& $(0.00070)$ & $(0.00050)$ & $(0.00236)$ \\
\hline AM-EV & 0.00431 & 0.00317 & 0.01220 \\
& $(0.00070)$ & $(0.00050)$ & $(0.00234)$ \\
\hline GE-EV & 0.00427 & 0.00315 & 0.01096 \\
& $(0.00070)$ & $(0.00050)$ & $(0.00193)$ \\
\hline EV[AA'] & 0.00595 & 0.00431 & 0.01665 \\
& $(0.00108)$ & $(0.00078)$ & $(0.00334)$ \\
\hline EV[A'A] & 0.00692 & 0.00497 & 0.00541 \\
& $(0.00253)$ & $(0.00189)$ & $(0.00222)$ \\
\hline AM - EV[AA'] & 0.00570 & 0.00412 & 0.01573 \\
AND EV[A'A] & $(0.00109)$ & $(0.00078)$ & $(0.00337)$ \\
\hline GE - EV[AA'] & 0.00462 & 0.00333 & 0.00850 \\
AND EV[A'A] & $(0.00127)$ & $(0.00093)$ & $(0.00165)$ \\
\hline GE & 0.00431 & 0.00315 & 0.00999 \\
& $(0.00070)$ & $(0.00050)$ & $(0.00178)$ \\
\hline C-RSM & 0.00486 & 0.00360 & 0.00952 \\
& $(0.00090)$ & $(0.00069)$ & $(0.00172)$ \\
\hline MT & 0.00427 & 0.00314 & 0.00945 \\
& $(0.00069)$ & $(0.00050)$ & $(0.00160)$ \\
\hline SAV & 0.00739 & 0.00543 & 0.00685 \\
& $(0.00183)$ & $(0.00140)$ & $(0.00208)$ \\
\hline NEV & 0.00609 & 0.00460 & 0.01351 \\
& $(0.00116)$ & $(0.00083)$ & $(0.00273)$ \\
\hline LSM & 0.00998 & 0.00725 & 0.00762 \\
& $(0.00248)$ & $(0.00196)$ & $(0.00284)$ \\
\hline
\end{tabular}


Table C.19: Mean and Standard Deviation of Group Disagreement $\left(d_{2}\right)$ from Simulation (With $\mathrm{N}=10, \mathrm{M}=7$, scale $[1 / 9,9], \mathrm{T}=500$ )

\begin{tabular}{|c|c|c|c|c|c|c|}
\hline$M=7$ & \multicolumn{3}{|c|}{$\mathrm{A}-\mathrm{GE}(\mathrm{V})$} & \multicolumn{3}{|c|}{ A-GE(M) } \\
\hline ABBREVIATIONS & $\overline{\mathrm{UF}}$ & LN & GA & $\overline{\mathrm{UF}}$ & LN & GA \\
\hline CSM & $\begin{array}{c}0.00361 \\
(0.00049)\end{array}$ & $\begin{array}{c}0.00264 \\
(0.00035)\end{array}$ & $\begin{array}{c}0.00879 \\
(0.00127)\end{array}$ & $\begin{array}{c}0.00399 \\
(0.00045)\end{array}$ & $\begin{array}{c}0.00280 \\
(0.00034)\end{array}$ & $\begin{array}{c}0.01146 \\
(0.00143)\end{array}$ \\
\hline R-EV & $\begin{array}{c}0.00351 \\
(0.00045)\end{array}$ & $\begin{array}{c}0.00258 \\
(0.00033)\end{array}$ & $\begin{array}{c}0.00920 \\
(0.00119)\end{array}$ & $\begin{array}{c}0.00351 \\
(0.00045)\end{array}$ & $\begin{array}{c}0.00258 \\
(0.00033)\end{array}$ & $\begin{array}{c}0.00927 \\
(0.00116)\end{array}$ \\
\hline L-EV & $\begin{array}{c}0.00353 \\
(0.00046)\end{array}$ & $\begin{array}{c}0.00258 \\
(0.00033)\end{array}$ & $\begin{array}{c}0.00990 \\
(0.00134)\end{array}$ & $\begin{array}{c}0.00354 \\
(0.00045)\end{array}$ & $\begin{array}{c}0.00258 \\
(0.00033)\end{array}$ & $\begin{array}{c}0.00995 \\
(0.00130)\end{array}$ \\
\hline AM-EV & $\begin{array}{c}0.00352 \\
(0.00045)\end{array}$ & $\begin{array}{c}0.00257 \\
(0.00033)\end{array}$ & $\begin{array}{c}0.00953 \\
(0.00129)\end{array}$ & $\begin{array}{c}0.00352 \\
(0.00045)\end{array}$ & $\begin{array}{c}0.00257 \\
(0.00033)\end{array}$ & $\begin{array}{c}0.00955 \\
(0.00126)\end{array}$ \\
\hline GM-EV & $\begin{array}{c}0.00349 \\
(0.00045)\end{array}$ & $\begin{array}{c}0.00256 \\
(0.00032)\end{array}$ & $\begin{array}{c}0.00893 \\
(0.00116\end{array}$ & $\begin{array}{c}0.00349 \\
(0.00045)\end{array}$ & $\begin{array}{c}0.00256 \\
(0.00032)\end{array}$ & $\begin{array}{c}0.00893 \\
(0.00114)\end{array}$ \\
\hline $\mathrm{EV}\left[\mathrm{AA}^{\prime}\right]$ & $\begin{array}{c}0.00466 \\
(0.00066)\end{array}$ & $\begin{array}{c}0.00336 \\
(0.00049)\end{array}$ & $\begin{array}{c}0.01313 \\
(0.00183)\end{array}$ & $\begin{array}{c}0.00466 \\
(0.00065)\end{array}$ & $\begin{array}{c}0.00336 \\
(0.00049)\end{array}$ & $\begin{array}{c}0.01312 \\
(0.00175)\end{array}$ \\
\hline$\overline{E V}\left[A^{\prime} A\right]$ & $\begin{array}{c}0.00364 \\
(0.00094)\end{array}$ & $\begin{array}{c}0.00259 \\
(0.00067)\end{array}$ & $\begin{array}{c}0.00527 \\
(0.00107)\end{array}$ & $\begin{array}{c}0.00369 \\
(0.00094)\end{array}$ & $\begin{array}{c}0.00262 \\
(0.00067)\end{array}$ & $\begin{array}{c}0.00572 \\
(0.00111)\end{array}$ \\
\hline $\begin{array}{l}\text { AM - EV [AA'] } \\
\text { AND EV }\left[A^{\prime} A\right]\end{array}$ & $\begin{array}{c}0.00413 \\
(0.00062)\end{array}$ & $\begin{array}{c}0.00297 \\
(0.00046)\end{array}$ & $\begin{array}{c}0.01124 \\
(0.00165)\end{array}$ & $\begin{array}{c}0.00415 \\
(0.00062)\end{array}$ & $\begin{array}{c}0.00298 \\
(0.00046)\end{array}$ & $\begin{array}{c}0.01128 \\
(0.00159)\end{array}$ \\
\hline $\begin{array}{l}\text { GE - EV }\left[A A^{\prime}\right] \\
\text { AND EV }\left[A^{\prime} A\right]\end{array}$ & $\begin{array}{c}0.00297 \\
(0.00046)\end{array}$ & $\begin{array}{c}0.00215 \\
(0.00034)\end{array}$ & $\begin{array}{c}0.00701 \\
(0.00094)\end{array}$ & $\begin{array}{c}0.00299 \\
(0.00046)\end{array}$ & $\begin{array}{c}0.00216 \\
(0.00034)\end{array}$ & $\begin{array}{c}0.00715 \\
(0.00092)\end{array}$ \\
\hline GE & $\begin{array}{c}0.00352 \\
(0.00045)\end{array}$ & $\begin{array}{c}0.00256 \\
(0.00032)\end{array}$ & $\begin{array}{c}0.00843 \\
(0.00114)\end{array}$ & $\begin{array}{c}0.00352 \\
(0.00045)\end{array}$ & $\begin{array}{c}0.00256 \\
(0.00032)\end{array}$ & $\begin{array}{c}0.00839 \\
(0.00113)\end{array}$ \\
\hline C-RSM & $\begin{array}{c}0.00359 \\
(0.00045)\end{array}$ & $\begin{array}{c}0.00262 \\
(0.00034)\end{array}$ & $\begin{array}{c}0.00853 \\
(0.00112)\end{array}$ & $\begin{array}{c}0.00359 \\
(0.00045)\end{array}$ & $\begin{array}{c}0.00262 \\
(0.00034)\end{array}$ & $\begin{array}{c}0.00855 \\
(0.00110)\end{array}$ \\
\hline$\overline{M T}$ & $\begin{array}{c}0.00349 \\
(0.00044)\end{array}$ & $\begin{array}{c}0.00256 \\
(0.00032)\end{array}$ & $\begin{array}{c}0.00853 \\
(0.00105)\end{array}$ & $\begin{array}{c}0.00349 \\
(0.00044)\end{array}$ & $\begin{array}{c}0.00256 \\
(0.00032)\end{array}$ & $\begin{array}{c}0.00831 \\
(0.00102)\end{array}$ \\
\hline $\mathrm{SAV}$ & $\begin{array}{c}0.00442 \\
(0.00063)\end{array}$ & $\begin{array}{c}0.00322 \\
(0.00049)\end{array}$ & $\begin{array}{c}0.00785 \\
(0.00110)\end{array}$ & $\begin{array}{c}0.00442 \\
(0.00062)\end{array}$ & $\begin{array}{c}0.00323 \\
(0.00049)\end{array}$ & $\begin{array}{c}0.00802 \\
(0.00107)\end{array}$ \\
\hline NEV & $\begin{array}{c}0.00495 \\
(0.00072)\end{array}$ & $\begin{array}{c}0.00378 \\
(0.00051)\end{array}$ & $\begin{array}{c}0.01173 \\
(0.00185)\end{array}$ & $\begin{array}{c}0.00510 \\
(0.00069)\end{array}$ & $\begin{array}{c}0.00384 \\
(0.00050)\end{array}$ & $\begin{array}{c}0.01341 \\
(0.00186)\end{array}$ \\
\hline$\overline{\text { LSM }}$ & $\begin{array}{c}0.00563 \\
(0.00098)\end{array}$ & $\begin{array}{c}0.00406 \\
(0.00078)\end{array}$ & $\begin{array}{c}0.00919 \\
(0.00182)\end{array}$ & $\begin{array}{c}0.00564 \\
(0.00097)\end{array}$ & $\begin{array}{c}0.00407 \\
(0.00078)\end{array}$ & $\begin{array}{c}0.00939 \\
(0.00183)\end{array}$ \\
\hline
\end{tabular}


Table C.20: Mean and Standard Deviation of Group Disagreement $\left(d_{2}\right)$ from Simulation (With $N=10, M=7$, scale $[1 / 9,9], T=500$ ) [Continued]

\begin{tabular}{|c|c|c|c|c|c|c|}
\hline$M=7$ & \multicolumn{3}{|c|}{$\mathrm{A}-\mathrm{AM}(\mathrm{V})$} & \multicolumn{3}{|c|}{$\mathrm{A}-\mathrm{MDM}(\mathrm{M})$} \\
\hline ABBREVIATIONS & UF & LN & GA & $\mathrm{UF}$ & LN & $\mathrm{GA}$ \\
\hline CSM & $\begin{array}{c}0.00360 \\
(0.00049)\end{array}$ & $\begin{array}{c}0.00264 \\
(0.00035)\end{array}$ & $\begin{array}{c}0.00873 \\
(0.001251)\end{array}$ & $\begin{array}{c}0.00398 \\
(0.00046)\end{array}$ & $\begin{array}{c}0.00282 \\
(0.00035)\end{array}$ & $\begin{array}{c}0.01150 \\
(0.00149)\end{array}$ \\
\hline R-EV & $\begin{array}{r}0.00350 \\
(0.00045) \\
\end{array}$ & $\begin{array}{c}0.00257 \\
(0.00033) \\
\end{array}$ & $\begin{array}{r}0.00913 \\
(0.00116) \\
\end{array}$ & $\begin{array}{r}0.00355 \\
(0.00045) \\
\end{array}$ & $\begin{array}{c}0.00260 \\
(0.00033) \\
\end{array}$ & $\begin{array}{c}0.00938 \\
(0.00117) \\
\end{array}$ \\
\hline $\mathrm{L}-\mathrm{EV}$ & $\begin{array}{c}0.00353 \\
(0.00046)\end{array}$ & $\begin{array}{c}0.00258 \\
(0.00033)\end{array}$ & $\begin{array}{c}0.00981 \\
(0.00131)\end{array}$ & $\begin{array}{c}0.00357 \\
(0.00046)\end{array}$ & $\begin{array}{c}0.00260 \\
(0.00033)\end{array}$ & $\begin{array}{c}0.01007 \\
(0.00131)\end{array}$ \\
\hline AM-EV & $\begin{array}{c}0.00351 \\
(0.00045) \\
\end{array}$ & $\begin{array}{c}0.00257 \\
(0.00033) \\
\end{array}$ & $\begin{array}{c}0.00945 \\
(0.00128) \\
\end{array}$ & $\begin{array}{r}0.00356 \\
(0.00046) \\
\end{array}$ & $\begin{array}{c}0.00259 \\
(0.00033) \\
\end{array}$ & $\begin{array}{c}0.00968 \\
(0.00126) \\
\end{array}$ \\
\hline GE-EV & $\begin{array}{c}0.00349 \\
(0.00048)\end{array}$ & $\begin{array}{c}0.00256 \\
(0.00032)\end{array}$ & $\begin{array}{r}0.00887 \\
(0.00114)\end{array}$ & $\begin{array}{c}0.00353 \\
(0.00046)\end{array}$ & $\begin{array}{c}0.00258 \\
(0.00033)\end{array}$ & $\begin{array}{c}0.00905 \\
(0.00115)\end{array}$ \\
\hline $\mathrm{EV}\left[\mathrm{AA}^{\prime}\right]$ & $\begin{array}{c}0.00465 \\
(0.00066)\end{array}$ & $\begin{array}{c}0.00338 \\
(0.00049)\end{array}$ & $\begin{array}{c}0.01293 \\
(0.00176)\end{array}$ & $\begin{array}{c}0.00471 \\
(0.00066)\end{array}$ & $\begin{array}{c}0.00339 \\
(0.00049)\end{array}$ & $\begin{array}{c}0.01326 \\
(0.00176)\end{array}$ \\
\hline$\overline{\mathrm{EV}}\left[\mathrm{A}^{\prime} \mathrm{A}\right]$ & $\begin{array}{c}0.00363 \\
(0.00093)\end{array}$ & $\begin{array}{c}0.00259 \\
(0.00067)\end{array}$ & $\begin{array}{r}0.00525 \\
(0.00106)\end{array}$ & $\begin{array}{c}0.00372 \\
(0.00095)\end{array}$ & $\begin{array}{c}0.00264 \\
(0.00068)\end{array}$ & $\begin{array}{c}0.00577 \\
(0.00113)\end{array}$ \\
\hline $\begin{array}{l}A M-E V\left[A^{\prime}\right] \\
A N D E V\left[A^{\prime} A\right]\end{array}$ & $\begin{array}{c}0.00412 \\
(0.00062)\end{array}$ & $\begin{array}{c}0.00297 \\
(0.00046)\end{array}$ & $\begin{array}{r}0.01111 \\
(0.00160)\end{array}$ & $\begin{array}{c}0.00419 \\
(0.00063)\end{array}$ & $\begin{array}{c}0.00300 \\
(0.00046)\end{array}$ & $\begin{array}{c}0.01140 \\
(0.00160)\end{array}$ \\
\hline $\begin{array}{l}\text { GE - EV }\left[A^{\prime}\right] \\
\text { AND EV[A'A] }\end{array}$ & $\begin{array}{c}0.00297 \\
(0.00046)\end{array}$ & $\begin{array}{c}0.00214 \\
(0.00034)\end{array}$ & $\begin{array}{r}0.00697 \\
(0.00092)\end{array}$ & $\begin{array}{c}0.00302 \\
(0.00047)\end{array}$ & $\begin{array}{c}0.00217 \\
(0.00034)\end{array}$ & $\begin{array}{c}0.00724 \\
(0.00093)\end{array}$ \\
\hline $\mathrm{GE}$ & $\begin{array}{c}0.00352 \\
(0.00045)\end{array}$ & $\begin{array}{c}0.00256 \\
(0.00032)\end{array}$ & $\begin{array}{r}0.00838 \\
(0.00113)\end{array}$ & $\begin{array}{c}0.00356 \\
(0.00046)\end{array}$ & $\begin{array}{c}0.00258 \\
(0.00033)\end{array}$ & $\begin{array}{c}0.00851 \\
(0.00113)\end{array}$ \\
\hline C-RSM & $\begin{array}{c}0.00359 \\
(0.00045)\end{array}$ & $\begin{array}{c}0.00262 \\
(0.00034)\end{array}$ & $\begin{array}{r}0.00847 \\
(0.010110)\end{array}$ & $\begin{array}{c}0.00363 \\
(0.00046)\end{array}$ & $\begin{array}{c}0.00264 \\
(0.00034)\end{array}$ & $\begin{array}{c}0.00867 \\
(0.00110)\end{array}$ \\
\hline$\overline{\mathrm{MT}}$ & $\begin{array}{c}0.00349 \\
(0.00044)\end{array}$ & $\begin{array}{c}0.00255 \\
(0.00032)\end{array}$ & $\begin{array}{r}0.010818 \\
(0.010103)\end{array}$ & $\begin{array}{c}0.00353 \\
(0.00045)\end{array}$ & $\begin{array}{c}0.00258 \\
(0.00032)\end{array}$ & $\begin{array}{c}0.00844 \\
(0.00102)\end{array}$ \\
\hline SAV & $\begin{array}{c}0.00441 \\
(0.00062)\end{array}$ & $\begin{array}{c}0.00322 \\
(0.00049)\end{array}$ & $\begin{array}{r}0.010780 \\
(0.010108)\end{array}$ & $\begin{array}{c}0.00446 \\
(0.00063)\end{array}$ & $\begin{array}{c}0.00325 \\
(0.00049)\end{array}$ & $\begin{array}{c}0.00813 \\
(0.00107)\end{array}$ \\
\hline NEV & $\begin{array}{c}0.00494 \\
(0.00071)\end{array}$ & $\begin{array}{c}0.00378 \\
(0.00051)\end{array}$ & $\begin{array}{r}0.01154 \\
(0.00176)\end{array}$ & $\begin{array}{c}0.00511 \\
(0.00071)\end{array}$ & $\begin{array}{c}0.00387 \\
(0.00051)\end{array}$ & $\begin{array}{c}0.01347 \\
(0.00192)\end{array}$ \\
\hline$\overline{\mathrm{LSM}}$ & $\begin{array}{c}0.00561 \\
(0.00097)\end{array}$ & $\begin{array}{c}0.00405 \\
(0.00078)\end{array}$ & $\begin{array}{r}0.00910 \\
(0.00177)\end{array}$ & $\begin{array}{c}0.00570 \\
(0.00098)\end{array}$ & $\begin{array}{c}0.00410 \\
(0.00079)\end{array}$ & $\begin{array}{c}0.00944 \\
(0.00185)\end{array}$ \\
\hline
\end{tabular}


Table C.21: Mean and Standard Deviation of Group Disagreement $\left(d_{2}\right)$ from Simulation (With $\mathrm{N}=10, \mathrm{M}=7$, scale $[1 / 9,9], \mathrm{T}=500$ ) [Continued]

\begin{tabular}{||l||c|c|c||}
\hline \multicolumn{1}{|c||}{ M=7 } & \multicolumn{3}{c||}{ A-MDM(V) } \\
\hline ABBREVIATIONS & UF & LN & GA \\
\hline \hline CSM & 0.00464 & 0.00362 & 0.01061 \\
& $(0.00111)$ & $(0.00105)$ & $(0.00260)$ \\
\hline R-EV & 0.00376 & 0.00276 & 0.00953 \\
& $(0.00052)$ & $(0.00037)$ & $(0.00132)$ \\
\hline L-EV & 0.00378 & 0.00277 & 0.01078 \\
& $(0.00052)$ & $(0.00037)$ & $(0.00169)$ \\
\hline AM-EV & 0.00378 & 0.00276 & 0.01061 \\
& $(0.00052)$ & $(0.00037)$ & $(0.00169)$ \\
\hline GE-EV & 0.00374 & 0.00275 & 0.00956 \\
& $(0.00051)$ & $(0.00037)$ & $(0.00137)$ \\
\hline EV[AA'] & 0.00523 & 0.00377 & 0.01445 \\
& $(0.00082)$ & $(0.00058)$ & $(0.00249)$ \\
\hline EV[A'A] & 0.00603 & 0.00436 & 0.00477 \\
& $(0.00210)$ & $(0.00157)$ & $(0.00185)$ \\
\hline AM - EV[AA'] & 0.00501 & 0.00361 & 0.01366 \\
AND EV[A'A] & $(0.00084)$ & $(0.00059)$ & $(0.00259)$ \\
\hline GE - EV[AA'] & 0.00404 & 0.00293 & 0.00741 \\
AND EV[A'A] & $(0.00101)$ & $(0.00074)$ & $(0.00122)$ \\
\hline GE & 0.00377 & 0.00275 & 0.00870 \\
& $(0.00052)$ & $(0.00037)$ & $(0.00127)$ \\
\hline C-RSM & 0.00425 & 0.00313 & 0.00832 \\
& $(0.00069)$ & $(0.00053)$ & $(0.00124)$ \\
\hline MT & 0.00374 & 0.00274 & 0.00826 \\
& $(0.00051)$ & $(0.00036)$ & $(0.00113)$ \\
\hline SAV & 0.00642 & 0.00472 & 0.00600 \\
& $(0.00152)$ & $(0.00117)$ & $(0.00168)$ \\
\hline NEV & 0.00531 & 0.00402 & 0.01172 \\
& $(0.00083)$ & $(0.00062)$ & $(0.00208)$ \\
\hline LSM & 0.00868 & 0.00634 & 0.00672 \\
& $(0.00210)$ & $(0.00164)$ & $(0.00230)$ \\
\hline
\end{tabular}


Table C.22: Mean and Standard Deviation of Group Disagreement $\left(d_{2}\right)$ from Simulation (With $\mathrm{N}=10, \mathrm{M}=9$, scale $[1 / 9,9], \mathrm{T}=500$ )

\begin{tabular}{||l||c|c|c||c|c|c||}
\hline \multicolumn{1}{|c||}{ M=9 } & \multicolumn{3}{c|}{ A-GE(V) } & \multicolumn{3}{c||}{ A-GE(M) } \\
\hline ABBREVIATIONS & UF & LN & GA & UF & LN & GA \\
\hline \hline CSM & 0.00325 & 0.00238 & 0.00791 & 0.00361 & 0.00253 & 0.01042 \\
& $(0.00038)$ & $(0.00028)$ & $(0.00100)$ & $(0.00036)$ & $(0.00028)$ & $(0.00122)$ \\
\hline R-EV & 0.00316 & 0.00232 & 0.00826 & 0.00316 & 0.00232 & 0.00830 \\
& $(0.00034)$ & $(0.00026$ & $(0.00093)$ & $(0.00034)$ & $(0.00026)$ & $(0.00091)$ \\
\hline L-EV & 0.00318 & 0.00233 & 0.00891 & 0.00318 & 0.00233 & 0.00893 \\
& $(0.00035)$ & $(0.00026)$ & $(0.00106)$ & $(0.00035)$ & $(0.00026)$ & $(0.00103)$ \\
\hline AM-EV & 0.00317 & 0.00232 & 0.00857 & 0.00317 & 0.00232 & 0.00857 \\
& $(0.00035)$ & $(0.00026)$ & $(0.00102)$ & $(0.00035)$ & $(0.00026)$ & $(0.00099)$ \\
\hline GM-EV & 0.00314 & 0.00231 & 0.00803 & 0.00314 & 0.00231 & 0.00801 \\
& $(0.00034)$ & $(0.00026)$ & $(0.00092)$ & $(0.00034)$ & $(0.00026)$ & $(0.00090)$ \\
\hline EV[AA'] & 0.00419 & 0.00302 & 0.01179 & 0.00419 & 0.00302 & 0.01175 \\
& $(0.00052)$ & $(0.00040)$ & $(0.00149)$ & $(0.00052)$ & $(0.00040)$ & $(0.00143)$ \\
\hline EV[A'A] & 0.00327 & 0.00233 & 0.00475 & 0.00332 & 0.00235 & 0.00513 \\
& $(0.00080)$ & $(0.00057)$ & $(0.00090)$ & $(0.00080)$ & $(0.00057)$ & $(0.00094)$ \\
\hline AM - EV[AA'] & 0.00372 & 0.00267 & 0.01010 & 0.00373 & 0.00267 & 0.01011 \\
AND EV[A'A] & $(0.00050)$ & $(0.00038)$ & $(0.00136)$ & $(0.00050)$ & $(0.00038)$ & $(0.00131)$ \\
\hline GE - EV[AA'] & 0.00267 & 0.00193 & 0.00630 & 0.00269 & 0.00193 & 0.00642 \\
AND EV[A'A] & $(0.00037)$ & $(0.00028)$ & $(0.00074)$ & $(0.00037)$ & $(0.00028)$ & $(0.00074)$ \\
\hline GE & 0.00317 & 0.00231 & 0.00757 & 0.00317 & 0.00231 & 0.00753 \\
& $(0.00034)$ & $(0.00026)$ & $(0.00090)$ & $(0.00034)$ & $(0.00026)$ & $(0.00088)$ \\
\hline C-RSM & 0.00323 & 0.00236 & 0.00766 & 0.00323 & 0.00236 & 0.00767 \\
& $(0.00035)$ & $(0.00027)$ & $(0.00090)$ & $(0.00035)$ & $(0.00027)$ & $(0.00088)$ \\
\hline M'T & 0.00315 & 0.00231 & 0.00739 & 0.00315 & 0.00231 & 0.00745 \\
& $(0.00034)$ & $(0.00026)$ & $(0.00081)$ & $(0.00034)$ & $(0.00026)$ & $(0.00079)$ \\
\hline SAV & 0.00398 & 0.00291 & 0.00706 & 0.00398 & 0.00291 & 0.00719 \\
& $(0.00052)$ & $(0.00039)$ & $(0.00089)$ & $(0.00051)$ & $(0.00039)$ & $(0.00087)$ \\
\hline NEV & 0.00447 & 0.00340 & 0.01054 & 0.00461 & 0.00346 & 0.01206 \\
& $(0.00056)$ & $(0.00042)$ & $(0.00154)$ & $(0.00054)$ & $(0.00041)$ & $(0.00156)$ \\
\hline LSM & 0.00507 & 0.00366 & 0.00824 & 0.00507 & 0.00367 & 0.00838 \\
& $(0.00083)$ & $(0.00066)$ & $(0.00157)$ & $(0.00082)$ & $(0.00065)$ & $(0.00157)$ \\
\hline
\end{tabular}


Table C.23: Mean and Standard Deviation of Group Disagreement $\left(d_{2}\right)$ from Simulation (With $N=10, M=9$, scale $[1 / 9,9], T=500$ ) [Continued]

\begin{tabular}{||l||c|c|c||c|c|c||}
\hline \multicolumn{1}{|c||}{ M=9 } & \multicolumn{3}{c|}{ A-AM(V) } & \multicolumn{3}{c||}{ A-MDM(M) } \\
\hline ABBREVIATIONS & UF & LN & GA & UF & LN & GA \\
\hline \hline CSM & 0.00325 & 0.00238 & 0.00786 & 0.00359 & 0.00254 & 0.01046 \\
& $(0.00038)$ & $(0.00028)$ & $(0.00099)$ & $(0.00036)$ & $(0.00028)$ & $(0.00127)$ \\
\hline R-EV & 0.00316 & 0.00232 & 0.00820 & 0.00320 & 0.00234 & 0.00841 \\
& $(0.00034)$ & $(0.00026)$ & $(0.00091)$ & $(0.00035)$ & $(0.00026)$ & $(0.00092)$ \\
\hline L-EV & 0.00318 & 0.00233 & 0.00883 & 0.00322 & 0.00235 & 0.00904 \\
& $(0.00035)$ & $(0.00026)$ & $(0.00103)$ & $(0.00035)$ & $(0.00026)$ & $(0.00104)$ \\
\hline AM-EV & 0.00317 & 0.00232 & 0.00850 & 0.00321 & 0.00234 & 0.00869 \\
& $(0.00035)$ & $(0.00026)$ & $(0.00100)$ & $(0.00035)$ & $(0.00026)$ & $(0.00101)$ \\
\hline GE-EV & 0.00314 & 0.00231 & 0.00797 & 0.00318 & 0.00233 & 0.00813 \\
& $(0.00034)$ & $(0.00026)$ & $(0.00090)$ & $(0.00035)$ & $(0.00026)$ & $(0.00091)$ \\
\hline EV[AA'] & 0.00418 & 0.00302 & 0.01161 & 0.00424 & 0.00304 & 0.01188 \\
& $(0.00052)$ & $(0.00040)$ & $(0.00144)$ & $(0.00053)$ & $(0.00040)$ & $(0.00146)$ \\
\hline EV[A'A] & 0.00327 & 0.00233 & 0.00474 & 0.00335 & 0.00237 & 0.00519 \\
& $(0.00080)$ & $(0.00057)$ & $(0.00089)$ & $(0.00081)$ & $(0.00058)$ & $(0.00096)$ \\
\hline AM - EV[AA'] & 0.00371 & 0.00267 & 0.00999 & 0.00377 & 0.00269 & 0.01022 \\
AND EV[A'A] & $(0.00050)$ & $(0.00038)$ & $(0.00132)$ & $(0.00051)$ & $(0.00038)$ & $(0.00138)$ \\
\hline GE- EV[AA'] & 0.00267 & 0.00192 & 0.00627 & 0.00271 & 0.00195 & 0.00650 \\
AND EV[A'A] & $(0.00037)$ & $(0.00028)$ & $(0.00073)$ & $(0.00037)$ & $(0.00028)$ & $(0.00074)$ \\
\hline GE & 0.00317 & 0.00231 & 0.00753 & 0.00321 & 0.00232 & 0.00764 \\
& $(0.00034)$ & $(0.00026)$ & $(0.00088)$ & $(0.00035)$ & $(0.00026)$ & $(0.00089)$ \\
\hline C-RSM & 0.00323 & 0.00236 & 0.00762 & 0.00327 & 0.00238 & 0.00779 \\
& $(0.00035)$ & $(0.00027)$ & $(0.00088)$ & $(0.00036)$ & $(0.00027)$ & $(0.00088)$ \\
\hline MT & 0.00314 & 0.00231 & 0.00734 & 0.00318 & 0.00232 & 0.00757 \\
& $(0.00034)$ & $(0.00026)$ & $(0.00080$ & $(0.00034)$ & $(0.00026)$ & $(0.00079)$ \\
\hline SAV & 0.00397 & 0.00291 & 0.00701 & 0.00402 & 0.00293 & 0.00730 \\
& $(0.00051)$ & $(0.00039)$ & $(0.00088)$ & $(0.00052)$ & $(0.00039)$ & $(0.00086)$ \\
\hline NEV & 0.00446 & 0.00339 & 0.01037 & 0.00461 & 0.00348 & 0.01213 \\
& $(0.00055)$ & $(0.00042)$ & $(0.00146)$ & $(0.00055)$ & $(0.00066)$ & $(0.00143)$ \\
\hline LSM & 0.00505 & 0.00366 & 0.00816 & 0.00512 & 0.00369 & 0.00844 \\
& $(0.00082)$ & $(0.00065)$ & $(0.00153)$ & $(0.00083)$ & $(0.00066)$ & $(0.00158)$ \\
\hline
\end{tabular}


Table C.24: Mean and Standard Deviation of Group Disagreement $\left(d_{2}\right)$ from Simulation (With $\mathrm{N}=10, \mathrm{M}=9$, scale $[1 / 9,9], \mathrm{T}=500$ ) [Continued]

\begin{tabular}{||l||c|c|c||}
\hline \multicolumn{1}{|c||}{ M=9 } & \multicolumn{3}{c||}{ A-MDM(V) } \\
\hline ABBREVIATIONS & UF & LN & GA \\
\hline \hline CSM & 0.00415 & 0.00323 & 0.00950 \\
& $(0.00097)$ & $(0.00092)$ & $(0.00214)$ \\
\hline R-EV & 0.00338 & 0.00248 & 0.00854 \\
& $(0.00041)$ & $(0.00030)$ & $(0.00106)$ \\
\hline L-EV & 0.00341 & 0.00248 & 0.00969 \\
& $(0.00041)$ & $(0.00030)$ & $(0.00138)$ \\
\hline AM-EV & 0.00340 & 0.00248 & 0.00953 \\
& $(0.00042)$ & $(0.00030)$ & $(0.00138)$ \\
\hline GE-EV & 0.00336 & 0.00246 & 0.00857 \\
& $(0.00040)$ & $(0.00029)$ & $(0.00108)$ \\
\hline EV[AA'] & 0.00469 & 0.00338 & 0.01292 \\
& $(0.00066)$ & $(0.00046)$ & $(0.00205)$ \\
\hline EV[A'A] & 0.00538 & 0.00393 & 0.00430 \\
& $(0.00177)$ & $(0.00137)$ & $(0.00152)$ \\
\hline AM - EV[AA'] & 0.00449 & 0.00323 & 0.01222 \\
AND EV[A'A] & $(0.00069)$ & $(0.00048)$ & $(0.00216)$ \\
\hline GE- EV[AA'] & 0.00361 & 0.00264 & 0.00663 \\
AND EV[A'A] & $(0.00083)$ & $(0.00065)$ & $(0.00098)$ \\
\hline GE & 0.00339 & 0.00246 & 0.00781 \\
& $(0.00040)$ & $(0.00029)$ & $(0.00099)$ \\
\hline C-RSM & 0.00382 & 0.00281 & 0.00746 \\
& $(0.00057)$ & $(0.00044)$ & $(0.00100)$ \\
\hline MT & 0.00336 & 0.00246 & 0.00740 \\
& $(0.00040)$ & $(0.00029)$ & $(0.00090)$ \\
\hline SAV & 0.00577 & 0.00425 & 0.00541 \\
& $(0.00132)$ & $(0.00102)$ & $(0.00143)$ \\
\hline NEV & 0.00479 & 0.00361 & 0.01053 \\
& $(0.00068)$ & $(0.00050)$ & $(0.00174)$ \\
\hline LSM & 0.00778 & 0.00570 & 0.00608 \\
& $(0.00184)$ & $(0.00141)$ & $(0.00197)$ \\
\hline
\end{tabular}


Table C.25: Mean and Standard Deviation of Group Disagreement $\left(d_{2}\right)$ from Simulation (With $\mathrm{N}=12, \mathrm{M}=3$, scale $[1 / 9,9], \mathrm{T}=500$ )

\begin{tabular}{||l||c|c|c||c|c|c||}
\hline \multicolumn{1}{|c||}{ M=3 } & \multicolumn{3}{c|}{ A-GE(V) } & \multicolumn{3}{c||}{ A-GE(M) } \\
\hline ABBREVIATIONS & UF & LN & GA & UF & LN & GA \\
\hline \hline CSM & 0.00367 & 0.00273 & 0.00902 & 0.00400 & 0.00288 & 0.01150 \\
& $(0.00071)$ & $(0.00060)$ & $(0.00234)$ & $(0.00066)$ & $(0.00058)$ & $(0.00236)$ \\
\hline R-EV & 0.00358 & 0.00267 & 0.00958 & 0.00360 & 0.00268 & 0.00989 \\
& $(0.00069)$ & $(0.00058)$ & $(0.00187)$ & $(0.00069)$ & $(0.00058)$ & $(0.00181)$ \\
\hline L-EV & 0.00358 & 0.00267 & 0.01029 & 0.00360 & 0.00268 & 0.01058 \\
& $(0.00069)$ & $(0.00059)$ & $(0.00209)$ & $(0.00069)$ & $(0.00058)$ & $(0.00200)$ \\
\hline AM-EV & 0.00357 & 0.00266 & 0.00988 & 0.00358 & 0.00267 & 0.01009 \\
& $(0.00069)$ & $(0.00058)$ & $(0.00200)$ & $(0.00069)$ & $(0.00058)$ & $(0.00192)$ \\
\hline GM-EV & 0.00355 & 0.00265 & 0.00920 & 0.00355 & 0.00265 & 0.00928 \\
& $(0.00068)$ & $(0.00058)$ & $(0.00180)$ & $(0.00068)$ & $(0.00058)$ & $(0.00175)$ \\
\hline EV[AA'] & 0.00477 & 0.00348 & 0.01366 & 0.00480 & 0.00349 & 0.01399 \\
& $(0.00093)$ & $(0.00078)$ & $(0.00289)$ & $(0.00092)$ & $(0.00078)$ & $(0.00277)$ \\
\hline EV[A'A] & 0.00411 & 0.00285 & 0.00557 & 0.00418 & 0.00289 & 0.00616 \\
& $(0.00130)$ & $(0.00092)$ & $(0.00149)$ & $(0.00129)$ & $(0.00091)$ & $(0.00147)$ \\
\hline AM - EV[AA'] & 0.00426 & 0.00309 & 0.01169 & 0.00430 & 0.00311 & 0.01201 \\
AND EV[A'A] & $(0.00084)$ & $(0.00071)$ & $(0.00252)$ & $(0.00083)$ & $(0.00070)$ & $(0.00243)$ \\
\hline GE- EV[AA'] & 0.00318 & 0.00227 & 0.00726 & 0.00321 & 0.00229 & 0.00756 \\
AND EV[A'A] & $(0.00070)$ & $(0.00051)$ & $(0.00145)$ & $(0.00069)$ & $(0.00051)$ & $(0.00140)$ \\
\hline GE & 0.00359 & 0.00265 & 0.00864 & 0.00358 & 0.00265 & 0.00861 \\
& $(0.00069)$ & $(0.00058)$ & $(0.00169)$ & $(0.00069)$ & $(0.00058)$ & $(0.00168)$ \\
\hline C-RSM & 0.00365 & 0.00272 & 0.00872 & 0.00366 & 0.00272 & 0.00884 \\
& $(0.00070)$ & $(0.00058)$ & $(0.00169)$ & $(0.00070)$ & $(0.00058)$ & $(0.00165)$ \\
\hline MT & 0.00357 & 0.00265 & 0.00860 & 0.00358 & 0.00266 & 0.00884 \\
& $(0.00069)$ & $(0.00058)$ & $(0.00159)$ & $(0.00068)$ & $(0.00057)$ & $(0.00154)$ \\
\hline SAV & 0.00457 & 0.00335 & 0.00809 & 0.00458 & 0.00336 & 0.00847 \\
& $(0.00094)$ & $(0.00071)$ & $(0.00150)$ & $(0.00094)$ & $(0.00071)$ & $(0.00146)$ \\
\hline NEV & 0.00542 & 0.00408 & 0.01227 & 0.00561 & 0.00416 & 0.01442 \\
& $(0.00119)$ & $(0.00093)$ & $(0.00282)$ & $(0.00115)$ & $(0.00091)$ & $(0.00278)$ \\
\hline LSM & 0.00595 & 0.00428 & 0.00940 & 0.00600 & 0.00430 & 0.00986 \\
& $(0.00140)$ & $(0.00105)$ & $(0.00240)$ & $(0.00138)$ & $(0.00105)$ & $(0.00247)$ \\
\hline
\end{tabular}


Table C.26: Mean and Standard Deviation of Group Disagreement $\left(d_{2}\right)$ from Simulation (With $\mathrm{N}=12, \mathrm{M}=3$, scale $[1 / 9,9], \mathrm{T}=500$ ) [Continued]

\begin{tabular}{|c|c|c|c|c|c|c|}
\hline$M=3$ & \multicolumn{3}{|c|}{$\mathrm{A}-\mathrm{AM}(\mathrm{V})$} & \multicolumn{3}{|c|}{ A-MDM(M) } \\
\hline ABBREVIATIONS & $\mathrm{UF}$ & LN & $\mathrm{GA}$ & UF & LN & GA \\
\hline$\overline{\mathrm{CSM}}$ & $\begin{array}{c}0.00366 \\
(0.00071)\end{array}$ & $\begin{array}{c}0.00273 \\
(0.00060)\end{array}$ & $\begin{array}{c}0.00896 \\
(0.00227) \\
\end{array}$ & $\begin{array}{c}0.0040 \\
(0.00067)\end{array}$ & $\begin{array}{c}0.00291 \\
(0.00059)\end{array}$ & $\begin{array}{c}0.01147 \\
(0.00240) \\
\end{array}$ \\
\hline R-EV & $\begin{array}{c}0.00358 \\
(0.00069)\end{array}$ & $\begin{array}{c}0.00267 \\
(0.00058)\end{array}$ & $\begin{array}{c}0.00950 \\
(0.00183)\end{array}$ & $\begin{array}{c}0.00362 \\
(0.00069)\end{array}$ & $\begin{array}{c}0.00270 \\
(0.00059)\end{array}$ & $\begin{array}{c}0.01000 \\
(0.00184)\end{array}$ \\
\hline L-EV & $\begin{array}{c}0.00358 \\
(0.00069)\end{array}$ & $\begin{array}{c}0.00267 \\
(0.00058)\end{array}$ & $\begin{array}{c}0.01021 \\
(0.00205) \\
\end{array}$ & $\begin{array}{c}0.00362 \\
(0.00069)\end{array}$ & $\begin{array}{c}0.00270 \\
(0.00059)\end{array}$ & $\begin{array}{c}0.01070 \\
(0.00204)\end{array}$ \\
\hline AM-EV & $\begin{array}{c}0.00356 \\
(0.00069)\end{array}$ & $\begin{array}{c}0.00266 \\
(0.00058)\end{array}$ & $\begin{array}{c}0.00981 \\
(0.00196)\end{array}$ & $\begin{array}{c}0.00360 \\
(0.00069)\end{array}$ & $\begin{array}{c}0.00269 \\
(0.00059)\end{array}$ & $\begin{array}{c}0.01021 \\
(0.00196)\end{array}$ \\
\hline GE-EV & $\begin{array}{c}0.00354 \\
(0.00068)\end{array}$ & $\begin{array}{c}0.00265 \\
(0.00058)\end{array}$ & $\begin{array}{c}0.00913 \\
(0.00176) \\
\end{array}$ & $\begin{array}{c}0.00357 \\
(0.00069)\end{array}$ & $\begin{array}{c}0.00267 \\
(0.00059)\end{array}$ & $\begin{array}{c}0.00940 \\
(0.00179)\end{array}$ \\
\hline$\overline{E V}\left[\mathrm{AA}^{\prime}\right]$ & $\begin{array}{c}0.00476 \\
(0.00092)\end{array}$ & $\begin{array}{c}0.00348 \\
(0.00078)\end{array}$ & $\begin{array}{c}0.01345 \\
(0.00278)\end{array}$ & $\begin{array}{c}0.00483 \\
(0.00092)\end{array}$ & $\begin{array}{c}0.00353 \\
(0.00079)\end{array}$ & $\begin{array}{c}0.01412 \\
(0.00281)\end{array}$ \\
\hline $\operatorname{EV}\left[A^{\prime} A\right]$ & $\begin{array}{c}0.00410 \\
(0.00129)\end{array}$ & $\begin{array}{c}0.00285 \\
(0.00091)\end{array}$ & $\begin{array}{c}0.00555 \\
(0.00147) \\
\end{array}$ & $\begin{array}{c}0.00420 \\
(0.00130)\end{array}$ & $\begin{array}{c}0.00292 \\
(0.00092)\end{array}$ & $\begin{array}{c}0.00619 \\
(0.00152)\end{array}$ \\
\hline $\begin{array}{l}\overline{A M}-E V\left[A A^{\prime}\right] \\
A N D \text { EV }\left[A^{\prime} A\right]\end{array}$ & $\begin{array}{c}0.00425 \\
(0.00084)\end{array}$ & $\begin{array}{c}0.00309 \\
(0.00071)\end{array}$ & $\begin{array}{c}0.01156 \\
(0.00245)\end{array}$ & $\begin{array}{c}0.00432 \\
(0.00084)\end{array}$ & $\begin{array}{c}0.00314 \\
(0.00072)\end{array}$ & $\begin{array}{c}0.01213 \\
(0.00247)\end{array}$ \\
\hline $\begin{array}{l}\text { GE - EV[AA'] } \\
\text { AND EV[A'A] }\end{array}$ & $\begin{array}{c}0.00318 \\
(0.00069)\end{array}$ & $\begin{array}{c}0.00227 \\
(0.00051)\end{array}$ & $\begin{array}{c}0.00723 \\
(0.00143) \\
\end{array}$ & $\begin{array}{c}0.00323 \\
(0.00070)\end{array}$ & $\begin{array}{c}0.00231 \\
(0.00052)\end{array}$ & $\begin{array}{c}0.00764 \\
(0.00144)\end{array}$ \\
\hline GE & $\begin{array}{c}0.00358 \\
(0.00069)\end{array}$ & $\begin{array}{c}0.00265 \\
(0.00059)\end{array}$ & $\begin{array}{c}0.00859 \\
(0.00166)\end{array}$ & $\begin{array}{c}0.00361 \\
(0.00069)\end{array}$ & $\begin{array}{c}0.00267 \\
(0.00059)\end{array}$ & $\begin{array}{c}0.00873 \\
(0.00171)\end{array}$ \\
\hline C-RSM & $\begin{array}{c}0.00365 \\
(0.00070)\end{array}$ & $\begin{array}{c}0.00272 \\
(0.00058)\end{array}$ & $\begin{array}{c}0.00867 \\
(0.00167) \\
\end{array}$ & $\begin{array}{c}0.00368 \\
(0.00070)\end{array}$ & $\begin{array}{c}0.00275 \\
(0.00059)\end{array}$ & $\begin{array}{c}0.00895 \\
(0.00169)\end{array}$ \\
\hline MT & $\begin{array}{c}0.00357 \\
(0.00069)\end{array}$ & $\begin{array}{c}0.00265 \\
(0.00057)\end{array}$ & $\begin{array}{c}0.00854 \\
(0.00157)\end{array}$ & $\begin{array}{c}0.00361 \\
(0.00069)\end{array}$ & $\begin{array}{c}0.00268 \\
(0.00058)\end{array}$ & $\begin{array}{c}0.00895 \\
(0.00157)\end{array}$ \\
\hline$\overline{\mathrm{SAV}}$ & $\begin{array}{c}0.00456 \\
(0.00094)\end{array}$ & $\begin{array}{c}0.00335 \\
(0.00071)\end{array}$ & $\begin{array}{c}0.00804 \\
(0.00148)\end{array}$ & $\begin{array}{c}0.00462 \\
(0.00094)\end{array}$ & $\begin{array}{c}0.00339 \\
(0.00072)\end{array}$ & $\begin{array}{c}0.00855 \\
(0.00150)\end{array}$ \\
\hline NEV & $\begin{array}{c}0.00541 \\
(0.00118)\end{array}$ & $\begin{array}{c}0.00407 \\
(0.00093)\end{array}$ & $\begin{array}{c}0.01207 \\
(0.00270)\end{array}$ & $\begin{array}{c}0.00563 \\
(0.00116)\end{array}$ & $\begin{array}{c}0.00419 \\
(0.00093)\end{array}$ & $\begin{array}{c}0.01441 \\
(0.00282)\end{array}$ \\
\hline LSM & $\begin{array}{c}0.00593 \\
(0.00139) \\
\end{array}$ & $\begin{array}{c}0.00427 \\
(0.00104)\end{array}$ & $\begin{array}{c}0.00931 \\
(0.00233)\end{array}$ & $\begin{array}{c}0.00603 \\
(0.00140) \\
\end{array}$ & $\begin{array}{c}0.00434 \\
(0.00106)\end{array}$ & $\begin{array}{c}0.00993 \\
(0.00249)\end{array}$ \\
\hline
\end{tabular}


Table C.27: Mean and Standard Deviation of Group Disagreement $\left(d_{2}\right)$ from Simulation (With $\mathrm{N}=12, \mathrm{M}=3$, scale $[1 / 9,9], \mathrm{T}=500$ ) [Continued]

\begin{tabular}{||l||c|c|c||}
\hline \multicolumn{1}{|c||}{ M=3 } & \multicolumn{3}{c||}{ A-MDM(V) } \\
\hline ABBREVIATIONS & UF & LN & GA \\
\hline \hline CSM & 0.00515 & 0.00405 & 0.01201 \\
& $(0.00159)$ & $(0.00146)$ & $(0.00423)$ \\
\hline R-EV & 0.00395 & 0.00293 & 0.00997 \\
& $(0.00089)$ & $(0.00065)$ & $(0.00217)$ \\
\hline L-EV & 0.00397 & 0.00293 & 0.01168 \\
& $(0.00090)$ & $(0.00064)$ & $(0.00286)$ \\
\hline AM-EV & 0.00397 & 0.00292 & 0.01148 \\
& $(0.00090)$ & $(0.00064)$ & $(0.00282)$ \\
\hline GE-EV & 0.00393 & 0.00290 & 0.01005 \\
& $(0.00089)$ & $(0.00064)$ & $(0.00225)$ \\
\hline EV[AA'] & 0.00556 & 0.00399 & 0.01532 \\
& $(0.00127)$ & $(0.00093)$ & $(0.00348)$ \\
\hline EV[A'A] & 0.00698 & 0.00516 & 0.00510 \\
& $(0.00269)$ & $(0.00198)$ & $(0.00253)$ \\
\hline AM - EV[AA'] & 0.00535 & 0.00384 & 0.01458 \\
AND EV[A'A] & $(0.00124)$ & $(0.00092)$ & $(0.00346)$ \\
\hline GE - EV[AA'] & 0.00450 & 0.00326 & 0.00791 \\
AND EV[A'A] & $(0.00132)$ & $(0.00101)$ & $(0.00188)$ \\
\hline GE & 0.00397 & 0.00290 & 0.00908 \\
& $(0.00090)$ & $(0.00064)$ & $(0.00203)$ \\
\hline C-RSM & 0.00457 & 0.00335 & 0.00862 \\
& $(0.00111)$ & $(0.00077)$ & $(0.00189)$ \\
\hline MT & 0.00394 & 0.00291 & 0.00872 \\
& $(0.00088)$ & $(0.00064)$ & $(0.00176)$ \\
\hline SAV & 0.00707 & 0.00520 & 0.00627 \\
& $(0.00213)$ & $(0.00146)$ & $(0.00208)$ \\
\hline NEV & 0.00589 & 0.00445 & 0.01256 \\
& $(0.00146)$ & $(0.00109)$ & $(0.00334)$ \\
\hline LSM & 0.00977 & 0.00721 & 0.00685 \\
& $(0.00299)$ & $(0.00218)$ & $(0.00311)$ \\
\hline
\end{tabular}


Table C.28: Mean and Standard Deviation of Group Disagreement $\left(d_{2}\right)$ from Simulation (With $\mathrm{N}=12, \mathrm{M}=5$, scale $[1 / 9,9], \mathrm{T}=500$ )

\begin{tabular}{||l||c|c|c||c|c|c||}
\hline \multicolumn{1}{|c||}{ M=5 } & \multicolumn{3}{c|}{ A-GE(V) } & \multicolumn{3}{c||}{ A-GE(M) } \\
\hline ABBREVIATIONS & UF & LN & GA & UF & LN & GA \\
\hline \hline CSM & 0.00316 & 0.00233 & 0.00775 & 0.00349 & 0.00248 & 0.01012 \\
& $(0.00044)$ & $(0.00035)$ & $(0.00143)$ & $(0.00040)$ & $(0.00034)$ & $(0.00148)$ \\
\hline R-EV & 0.00308 & 0.00228 & 0.00820 & 0.00309 & 0.00228 & 0.00835 \\
& $(0.00042)$ & $(0.00034)$ & $(0.00117)$ & $(0.00041)$ & $(0.00034)$ & $(0.00115)$ \\
\hline L-EV & 0.00309 & 0.00228 & 0.00887 & 0.00309 & 0.00229 & 0.00898 \\
& $(0.00043)$ & $(0.00034)$ & $(0.00126)$ & $(0.00042)$ & $(0.00034)$ & $(0.00123)$ \\
\hline AM-EV & 0.00308 & 0.00228 & 0.00850 & 0.00308 & 0.00228 & 0.00858 \\
& $(0.00042)$ & $(0.00034)$ & $(0.00122)$ & $(0.00042)$ & $(0.00034)$ & $(0.00119)$ \\
\hline GM-EV & 0.00305 & 0.00226 & 0.00789 & 0.00305 & 0.00226 & 0.00791 \\
& $(0.00042)$ & $(0.00034)$ & $(0.00111)$ & $(0.00041)$ & $(0.00034)$ & $(0.00109)$ \\
\hline EV[AA'] & 0.00411 & 0.00298 & 0.01172 & 0.00413 & 0.00299 & 0.01182 \\
& $(0.00060)$ & $(0.00048)$ & $(0.00180)$ & $(0.00059)$ & $(0.00048)$ & $(0.00173)$ \\
\hline EV[A'A] & 0.00353 & 0.00250 & 0.00482 & 0.00357 & 0.00252 & 0.00521 \\
& $(0.00085)$ & $(0.00062)$ & $(0.00098)$ & $(0.00085)$ & $(0.00062)$ & $(0.00099)$ \\
\hline AM- EV [AA'] & 0.00367 & 0.00265 & 0.01005 & 0.00369 & 0.00265 & 0.01017 \\
AND EV[A'A] & $(0.00055)$ & $(0.00045)$ & $(0.00163)$ & $(0.00055)$ & $(0.00045)$ & $(0.00158)$ \\
\hline GE - EV[A.A'] & 0.00273 & 0.00195 & 0.00624 & 0.00275 & 0.00196 & 0.00641 \\
AND EV[A'A] & $(0.00043)$ & $(0.00033)$ & $(0.00093)$ & $(0.00043)$ & $(0.00034)$ & $(0.00091)$ \\
\hline GE & 0.00309 & 0.00226 & 0.00741 & 0.00309 & 0.00226 & 0.00738 \\
& $(0.00042)$ & $(0.00034)$ & $(0.00105)$ & $(0.00042)$ & $(0.00034)$ & $(0.00104)$ \\
\hline C-RSM & 0.00315 & 0.00231 & 0.00748 & 0.00315 & 0.00231 & 0.00754 \\
& $(0.00043)$ & $(0.00034)$ & $(0.00105)$ & $(0.00042)$ & $(0.00034)$ & $(0.00103)$ \\
\hline MT & 0.00307 & 0.00226 & 0.00737 & 0.00308 & 0.00227 & 0.00750 \\
& $(0.00041)$ & $(0.00033)$ & $(0.00103)$ & $(0.00041)$ & $(0.00033)$ & $(0.00100)$ \\
\hline SAV & 0.00393 & 0.00285 & 0.00690 & 0.00393 & 0.00285 & 0.00713 \\
& $(0.00058)$ & $(0.00045)$ & $(0.00100)$ & $(0.00058)$ & $(0.00045)$ & $(0.00098)$ \\
\hline NEV & 0.00457 & 0.00348 & 0.01058 & 0.00474 & 0.00355 & 0.01253 \\
& $(0.00073)$ & $(0.00058)$ & $(0.00187)$ & $(0.00070)$ & $(0.00057)$ & $(0.00186)$ \\
\hline LSM & 0.00511 & 0.00365 & 0.00799 & 0.00513 & 0.00366 & 0.00825 \\
& $(0.00092)$ & $(0.00071)$ & $(0.00165)$ & $(0.00091)$ & $(0.00070)$ & $(0.00166)$ \\
\hline
\end{tabular}


Table C.29: Mean and Standard Deviation of Group Disagreement $\left(d_{2}\right)$ from Simulation (With $\mathrm{N}=12, \mathrm{M}=5$, scale $[1 / 9,9], \mathrm{T}=500$ ) [Continued]

\begin{tabular}{||l||c|c|c||c|c|c||}
\hline \multicolumn{1}{|c||}{ M=5 } & \multicolumn{3}{c|}{ A-AM(V) } & \multicolumn{3}{c||}{ A-MDM(M) } \\
\hline ABBREVIATIONS & UF & LN & GA & UF & LN & GA \\
\hline \hline CSM & 0.00315 & 0.00233 & 0.00770 & 0.00350 & 0.00249 & 0.01012 \\
& $(0.00044)$ & $(0.00035)$ & $(0.00140)$ & $(0.00041)$ & $(0.00034)$ & $(0.00151)$ \\
\hline R-EV & 0.00308 & 0.00228 & 0.00815 & 0.00312 & 0.00230 & 0.00842 \\
& $(0.00042)$ & $(0.00034)$ & $(0.00115)$ & $(0.00042)$ & $(0.00034)$ & $(0.00115)$ \\
\hline L-EV & 0.00309 & 0.00228 & 0.00880 & 0.00313 & 0.00230 & 0.00905 \\
& $(0.00042)$ & $(0.00034)$ & $(0.00124)$ & $(0.00043)$ & $(0.00034)$ & $(0.00124)$ \\
\hline AM-EV & 0.00307 & 0.00227 & 0.00844 & 0.00311 & 0.00229 & 0.00865 \\
& $(0.00042)$ & $(0.00034)$ & $(0.00120)$ & $(0.00043)$ & $(0.00034)$ & $(0.00120)$ \\
\hline GE-EV & 0.00305 & 0.00226 & 0.00784 & 0.00309 & 0.00228 & 0.00798 \\
& $(0.00041)$ & $(0.00034)$ & $(0.00110)$ & $(0.00042)$ & $(0.00034)$ & $(0.00110)$ \\
\hline EV[AA'] & 0.00410 & 0.00298 & 0.01156 & 0.00417 & 0.00300 & 0.01190 \\
& $(0.00060)$ & $(0.00048)$ & $(0.00174)$ & $(0.00060)$ & $(0.00048)$ & $(0.00175)$ \\
\hline EV[A'A] & 0.00352 & 0.00249 & 0.00480 & 0.00360 & 0.00253 & 0.00524 \\
& $(0.00085)$ & $(0.00062)$ & $(0.00097)$ & $(0.00086)$ & $(0.00062)$ & $(0.00101)$ \\
\hline AM - EV[AA'] & 0.00366 & 0.00264 & 0.00994 & 0.00373 & 0.00267 & 0.01023 \\
AND EV[A'A] & $(0.00055)$ & $(0.00045)$ & $(0.00159)$ & $(0.00055)$ & $(0.00045)$ & $(0.00160)$ \\
\hline GE - EV[AA'] & 0.00272 & 0.00195 & 0.00621 & 0.00278 & 0.00197 & 0.00646 \\
AND EV[A'A] & $(0.00043)$ & $(0.00033)$ & $(0.00092)$ & $(0.00044)$ & $(0.00034)$ & $(0.00092)$ \\
\hline GE & 0.00309 & 0.00226 & 0.00737 & 0.00312 & 0.00227 & 0.00745 \\
& $(0.00042)$ & $(0.00034)$ & $(0.00103)$ & $(0.00043)$ & $(0.00034)$ & $(0.00104)$ \\
\hline C-RSM & 0.00315 & 0.00231 & 0.00744 & 0.00319 & 0.00233 & 0.00761 \\
& $(0.00042)$ & $(0.00034)$ & $(0.00104)$ & $(0.00043)$ & $(0.00034)$ & $(0.00104)$ \\
\hline MT & 0.00307 & 0.00226 & 0.00733 & 0.00311 & 0.00228 & 0.00758 \\
& $(0.00041)$ & $(0.00033)$ & $(0.00101)$ & $(0.00042)$ & $(0.00033)$ & $(0.00101)$ \\
\hline SAV & 0.00392 & 0.00285 & 0.00686 & 0.00398 & 0.00287 & 0.00719 \\
& $(0.00058)$ & $(0.00045)$ & $(0.00098)$ & $(0.00059)$ & $(0.00045)$ & $(0.00099)$ \\
\hline NEV & 0.00455 & 0.00348 & 0.01042 & 0.00476 & 0.00357 & 0.01257 \\
& $(0.00072)$ & $(0.00058)$ & $(0.00179)$ & $(0.00072)$ & $(0.00058)$ & $(0.00190)$ \\
\hline LSM & 0.00509 & 0.00364 & 0.00792 & 0.00518 & 0.00368 & 0.00828 \\
& $(0.00091)$ & $(0.00070)$ & $(0.00160)$ & $(0.00092)$ & $(0.00071)$ & $(0.00167)$ \\
\hline
\end{tabular}


Table C.30: Mean and Standard Deviation of Group Disagreement $\left(d_{2}\right)$ from Simulation (With $\mathrm{N}=12, \mathrm{M}=5$, scale $[1 / 9,9], \mathrm{T}=500$ ) [Continued]

\begin{tabular}{||l||c|c|c||}
\hline \multicolumn{1}{|c||}{ M=5 } & \multicolumn{3}{c||}{ A-MDM(V) } \\
\hline ABBREVIATIONS & UF & LN & GA \\
\hline \hline CSM & 0.00422 & 0.00328 & 0.00992 \\
& $(0.00112)$ & $(0.00106)$ & $(0.00271)$ \\
\hline R-EV & 0.00332 & 0.00246 & 0.00844 \\
& $(0.00051)$ & $(0.00037)$ & $(0.00125)$ \\
\hline L-EV & 0.00333 & 0.00245 & 0.00979 \\
& $(0.00054)$ & $(0.00037)$ & $(0.00166)$ \\
\hline AM-EV & 0.00333 & 0.00245 & 0.00963 \\
& $(0.00054)$ & $(0.00037)$ & $(0.00165)$ \\
\hline GE-EV & 0.00330 & 0.00243 & 0.00845 \\
& $(0.00051)$ & $(0.00037)$ & $(0.00130)$ \\
\hline EV[AA'] & 0.00464 & 0.00335 & 0.01289 \\
& $(0.00078)$ & $(0.00058)$ & $(0.00213)$ \\
\hline EV[A'A] & 0.00590 & 0.00423 & 0.00429 \\
& $(0.00186)$ & $(0.00135)$ & $(0.00169)$ \\
\hline AM - EV[AA'] & 0.00447 & 0.00322 & 0.01226 \\
AND EV[A'A] & $(0.00078)$ & $(0.00059)$ & $(0.00219)$ \\
\hline GE - EV[AA'] & 0.00379 & 0.00271 & 0.00662 \\
AND EV[A'A] & $(0.00089)$ & $(0.00066)$ & $(0.00108)$ \\
\hline GE & 0.00333 & 0.00243 & 0.00763 \\
& $(0.00052)$ & $(0.00037)$ & $(0.00117)$ \\
\hline C-RSM & 0.00386 & 0.00280 & 0.00725 \\
& $(0.00069)$ & $(0.00049)$ & $(0.00111)$ \\
\hline MT & 0.00331 & 0.00244 & 0.00736 \\
& $(0.00051)$ & $(0.00037)$ & $(0.00105)$ \\
\hline SAV & 0.00598 & 0.00433 & 0.00525 \\
& $(0.00151)$ & $(0.00105)$ & $(0.00146)$ \\
\hline NEV & 0.00491 & 0.00374 & 0.01062 \\
& $(0.00086)$ & $(0.00065)$ & $(0.00208)$ \\
\hline LSM & 0.00827 & 0.00601 & 0.00583 \\
& $(0.00213)$ & $(0.00156)$ & $(0.00215)$ \\
\hline
\end{tabular}


Table C.31: Mean and Standard Deviation of Group Disagreement $\left(d_{2}\right)$ from Simulation (With $\mathrm{N}=12, \mathrm{M}=7$, scale $[1 / 9,9], \mathrm{T}=500$ )

\begin{tabular}{||l||c|c|c||c|c|c||}
\hline \multicolumn{1}{|c||}{ M=7 } & \multicolumn{3}{c|}{ A-GE(V) } & \multicolumn{3}{c||}{ A-GE(M) } \\
\hline ABBREVIATIONS & UF & LN & GA & UF & LN & GA \\
\hline \hline CSM & 0.00277 & 0.00205 & 0.00682 & 0.00309 & 0.00218 & 0.00900 \\
& $(0.00033)$ & $(0.00027)$ & $(0.00105)$ & $(0.00030)$ & $(0.00026)$ & $(0.00110)$ \\
\hline R-EV & 0.00270 & 0.00200 & 0.00721 & 0.00271 & 0.00200 & 0.00729 \\
& $(0.00031)$ & $(0.00025)$ & $(0.00082)$ & $(0.00031)$ & $(0.00025)$ & $(0.00080)$ \\
\hline L-EV & 0.00271 & 0.00200 & 0.00784 & 0.00272 & 0.00200 & 0.00790 \\
& $(0.00031)$ & $(0.00025)$ & $(0.00093)$ & $(0.00031)$ & $(0.00025)$ & $(0.00090)$ \\
\hline AM-EV & 0.00270 & 0.00199 & 0.00752 & 0.00270 & 0.00199 & 0.00755 \\
& $(0.00031)$ & $(0.00025)$ & $(0.00090)$ & $(0.00031)$ & $(0.00025)$ & $(0.00087)$ \\
\hline GM-EV & 0.00268 & 0.00198 & 0.00695 & 0.00268 & 0.00198 & 0.00696 \\
& $(0.00030)$ & $(0.00025)$ & $(0.00080)$ & $(0.00030)$ & $(0.00025)$ & $(0.00078)$ \\
\hline EV[AA'] & 0.00362 & 0.00261 & 0.01034 & 0.00363 & 0.00261 & 0.01037 \\
& $(0.00045)$ & $(0.00036)$ & $(0.00134)$ & $(0.00045)$ & $(0.00036)$ & $(0.00128)$ \\
\hline EV[A'A] & 0.00311 & 0.00222 & 0.00427 & 0.00314 & 0.00223 & 0.00457 \\
& $(0.00066)$ & $(0.00048)$ & $(0.00073)$ & $(0.00066)$ & $(0.00048)$ & $(0.00076)$ \\
\hline AM - EV[AA'] & 0.00323 & 0.00232 & 0.00886 & 0.00325 & 0.00233 & 0.00892 \\
AND EV[A'A] & $(0.00042)$ & $(0.00034)$ & $(0.00122)$ & $(0.00042)$ & $(0.00034)$ & $(0.00118)$ \\
\hline GE-EV[AA'] & 0.00240 & 0.00172 & 0.00550 & 0.00241 & 0.00172 & 0.00562 \\
AND EV[A'A] & $(0.00033)$ & $(0.00026)$ & $(0.00067)$ & $(0.00033)$ & $(0.00026)$ & $(0.00066)$ \\
\hline GE & 0.00271 & 0.00198 & 0.00653 & 0.00271 & 0.00198 & 0.00650 \\
& $(0.00031)$ & $(0.00025)$ & $(0.00077)$ & $(0.00031)$ & $(0.00025)$ & $(0.00076)$ \\
\hline C-RSM & 0.00277 & 0.00203 & 0.00660 & 0.00277 & 0.00203 & 0.00664 \\
& $(0.00031)$ & $(0.00025)$ & $(0.00076)$ & $(0.00031)$ & $(0.00025)$ & $(0.00074)$ \\
\hline MT & 0.00270 & 0.00199 & 0.00647 & 0.00270 & 0.00199 & 0.00656 \\
& $(0.00031)$ & $(0.00025)$ & $(0.00072)$ & $(0.00030)$ & $(0.00025)$ & $(0.00070)$ \\
\hline SAV & 0.00345 & 0.00251 & 0.00606 & 0.00345 & 0.00251 & 0.00623 \\
& $(0.00043)$ & $(0.00034)$ & $(0.00077)$ & $(0.00043)$ & $(0.00034)$ & $(0.00075)$ \\
\hline NEV & 0.00403 & 0.00307 & 0.00931 & 0.00418 & 0.00313 & 0.01104 \\
& $(0.00053)$ & $(0.00043)$ & $(0.00140)$ & $(0.00051)$ & $(0.00043)$ & $(0.00143)$ \\
\hline LSM & 0.00448 & 0.00323 & 0.00704 & 0.00449 & 0.00323 & 0.00721 \\
& $(0.00071)$ & $(0.00055)$ & $(0.00132)$ & $(0.00070)$ & $(0.00055)$ & $(0.00133)$ \\
\hline
\end{tabular}


Table C.32: Mean and Standard Deviation of Group Disagreement $\left(d_{2}\right)$ from Simulation (With $\mathrm{N}=12, \mathrm{M}=7$, scale $[1 / 9,9], \mathrm{T}=500$ ) [Continued]

\begin{tabular}{||l||c|c|c||c|c|c||}
\hline \multicolumn{1}{|c|}{ M=7 } & \multicolumn{3}{c|}{ A-AM(V) } & \multicolumn{3}{c||}{ A-MDM(M) } \\
\hline ABBREVIATIONS & UF & LN & GA & UF & LN & GA \\
\hline \hline CSM & 0.00277 & 0.00205 & 0.00678 & 0.00308 & 0.00219 & 0.00900 \\
& $(0.00033)$ & $(0.00027)$ & $(0.00103)$ & $(0.00031)$ & $(0.00026)$ & $(0.00111)$ \\
\hline R-EV & 0.00270 & 0.00200 & 0.00716 & 0.00273 & 0.00201 & 0.00736 \\
& $(0.00031)$ & $(0.00025)$ & $(0.00081)$ & $(0.00031)$ & $(0.00025)$ & $(0.00081)$ \\
\hline L-EV & 0.00271 & 0.00200 & 0.00778 & 0.00274 & 0.00201 & 0.00796 \\
& $(0.00031)$ & $(0.00025)$ & $(0.00091)$ & $(0.00031)$ & $(0.00026)$ & $(0.00092)$ \\
\hline AM-EV & 0.00270 & 0.00199 & 0.00746 & 0.00273 & 0.00200 & 0.00762 \\
& $(0.00031)$ & $(0.00025)$ & $(0.00088)$ & $(0.00031)$ & $(0.00025)$ & $(0.00088)$ \\
\hline GE-EV & 0.00268 & 0.00198 & 0.00691 & 0.00270 & 0.00199 & 0.00703 \\
& $(0.00030)$ & $(0.00025)$ & $(0.00079)$ & $(0.00031)$ & $(0.00025)$ & $(0.00079)$ \\
\hline EV[AA'] & 0.00362 & 0.00261 & 0.01020 & 0.00365 & 0.00263 & 0.01045 \\
& $(0.00045)$ & $(0.00036)$ & $(0.00129)$ & $(0.00045)$ & $(0.00036)$ & $(0.00131)$ \\
\hline EV[A'A] & 0.00310 & 0.00221 & 0.00425 & 0.00316 & 0.00224 & 0.00460 \\
& $(0.00066)$ & $(0.00047)$ & $(0.00073)$ & $(0.00066)$ & $(0.00048)$ & $(0.00078)$ \\
\hline AM - EV[AA'] & 0.00323 & 0.00232 & 0.00877 & 0.00327 & 0.00234 & 0.00899 \\
AND EV[A'A] & $(0.00042)$ & $(0.00034)$ & $(0.00119)$ & $(0.00042)$ & $(0.00034)$ & $(0.00120)$ \\
\hline GE- EV[AA'] & 0.00240 & 0.00172 & 0.00548 & 0.00243 & 0.00173 & 0.00568 \\
AND EV[A'A] & $(0.00033)$ & $(0.00026)$ & $(0.00066)$ & $(0.00033)$ & $(0.00026)$ & $(0.00068)$ \\
\hline GE & 0.00271 & 0.00198 & 0.00649 & 0.00273 & 0.00199 & 0.00656 \\
& $(0.00031)$ & $(0.00025)$ & $(0.00076)$ & $(0.00031)$ & $(0.00025)$ & $(0.00076)$ \\
\hline C-RSM & 0.00277 & 0.00203 & 0.00656 & 0.00279 & 0.00204 & 0.00670 \\
& $(0.00031)$ & $(0.00025)$ & $(0.00075)$ & $(0.00031)$ & $(0.00026)$ & $(0.00075)$ \\
\hline MT & 0.00269 & 0.00198 & 0.00644 & 0.00272 & 0.00200 & 0.00663 \\
& $(0.00030)$ & $(0.00025)$ & $(0.00071)$ & $(0.00031)$ & $(0.00025)$ & $(0.00071)$ \\
\hline SAV & 0.00344 & 0.00251 & 0.00603 & 0.00348 & 0.00253 & 0.00628 \\
& $(0.00043)$ & $(0.00034)$ & $(0.00076)$ & $(0.00043)$ & $(0.00034)$ & $(0.00076)$ \\
\hline NEV & 0.00401 & 0.00306 & 0.00916 & 0.00417 & 0.00314 & 0.01107 \\
& $(0.00053)$ & $(0.00043)$ & $(0.00134)$ & $(0.00052)$ & $(0.00043)$ & $(0.00146)$ \\
\hline LSM & 0.00447 & 0.00322 & 0.00698 & 0.00453 & 0.00325 & 0.00724 \\
& $(0.00070)$ & $(0.00055)$ & $(0.00129)$ & $(0.00070)$ & $(0.00055)$ & $(0.00134)$ \\
\hline
\end{tabular}


Table C.33: Mean and Standard Deviation of Group Disagreement $\left(d_{2}\right)$ from Simulation (With $\mathrm{N}=12, \mathrm{M}=7$, scale $[1 / 9,9], \mathrm{T}=500$ ) [Continued]

\begin{tabular}{|c|c|c|c|}
\hline$M=7$ & \multicolumn{3}{|c|}{$\mathrm{A}-\mathrm{MDM}(\mathrm{V})$} \\
\hline ABBREVIATIONS & $\mathrm{UF}$ & LN & GA \\
\hline CSM & $\begin{array}{c}0.00365 \\
(0.00092)\end{array}$ & $\begin{array}{c}0.00284 \\
(0.00087)\end{array}$ & $\begin{array}{c}0.00862 \\
(0.00240)\end{array}$ \\
\hline R-EV & $\begin{array}{c}0.00291 \\
(0.00036) \\
\end{array}$ & $\begin{array}{c}0.00215 \\
(0.00029) \\
\end{array}$ & $\begin{array}{c}0.00739 \\
(0.00088) \\
\end{array}$ \\
\hline $\mathrm{L}-\mathrm{EV}$ & $\begin{array}{c}0.00292 \\
(0.00038)\end{array}$ & $\begin{array}{c}0.00215 \\
(0.00028)\end{array}$ & $\begin{array}{c}0.00856 \\
(0.00119)\end{array}$ \\
\hline AM-EV & $\begin{array}{c}0.00292 \\
(0.00038) \\
\end{array}$ & $\begin{array}{c}0.00215 \\
(0.00028) \\
\end{array}$ & $\begin{array}{c}0.00842 \\
(0.00119) \\
\end{array}$ \\
\hline GE-EV & $\begin{array}{r}0.00289 \\
(0.00037) \\
\end{array}$ & $\begin{array}{c}0.00213 \\
(0.00028) \\
\end{array}$ & $\begin{array}{c}0.00739 \\
(0.00091) \\
\end{array}$ \\
\hline $\mathrm{EV}\left[\mathrm{AA}^{\prime}\right]$ & $\begin{array}{c}0.00405 \\
(0.00057)\end{array}$ & $\begin{array}{c}0.00294 \\
(0.00043)\end{array}$ & $\begin{array}{c}0.01121 \\
(0.00162)\end{array}$ \\
\hline $\mathrm{EV}\left[\mathrm{A}^{\prime} \mathrm{A}\right]$ & $\begin{array}{c}0.00511 \\
(0.00153)\end{array}$ & $\begin{array}{c}0.00370 \\
(0.00109)\end{array}$ & $\begin{array}{c}0.00375 \\
(0.00127)\end{array}$ \\
\hline $\begin{array}{l}A M-\operatorname{EV}\left[A^{\prime} A^{\prime}\right] \\
A N D \text { EV }\left[A^{\prime} A\right]\end{array}$ & $\begin{array}{r}0.00390 \\
(0.00058) \\
\end{array}$ & $\begin{array}{c}0.00283 \\
(0.00044) \\
\end{array}$ & $\begin{array}{c}0.01067 \\
(0.00167) \\
\end{array}$ \\
\hline $\begin{array}{l}\text { GE - EV }\left[\mathrm{AA}^{\prime}\right] \\
\text { AND EV[A'A] }\end{array}$ & $\begin{array}{c}0.00330 \\
(0.00072) \\
\end{array}$ & $\begin{array}{c}0.00238 \\
(0.00052) \\
\end{array}$ & $\begin{array}{c}0.00576 \\
(0.00079) \\
\end{array}$ \\
\hline $\mathrm{GE}$ & $\begin{array}{c}0.00292 \\
(0.00037) \\
\end{array}$ & $\begin{array}{c}0.00213 \\
(0.00028)\end{array}$ & $\begin{array}{c}0.00670 \\
(0.00082) \\
\end{array}$ \\
\hline C-RSM & $\begin{array}{c}0.00337 \\
(0.00053) \\
\end{array}$ & $\begin{array}{c}0.00245 \\
(0.00038) \\
\end{array}$ & $\begin{array}{c}0.00635 \\
(0.00081) \\
\end{array}$ \\
\hline$\overline{M T}$ & $\begin{array}{c}0.00290 \\
(0.00036)\end{array}$ & $\begin{array}{c}0.00213 \\
(0.00028)\end{array}$ & $\begin{array}{c}0.00646 \\
(0.00074) \\
\end{array}$ \\
\hline SAV & $\begin{array}{c}0.00521 \\
(0.00123) \\
\end{array}$ & $\begin{array}{c}0.00379 \\
(0.00086) \\
\end{array}$ & $\begin{array}{c}0.00458 \\
(0.00119) \\
\end{array}$ \\
\hline $\mathrm{NEV}$ & $\begin{array}{r}0.00430 \\
(0.00063) \\
\end{array}$ & $\begin{array}{c}0.00327 \\
(0.00048) \\
\end{array}$ & $\begin{array}{c}0.00930 \\
(0.00155) \\
\end{array}$ \\
\hline LSM & $\begin{array}{c}0.00720 \\
(0.00174)\end{array}$ & $\begin{array}{c}0.00525 \\
(0.00126)\end{array}$ & $\begin{array}{c}0.00512 \\
(0.00168)\end{array}$ \\
\hline
\end{tabular}


Table C.34: Mean and Standard Deviation of Group Disagreement $\left(d_{2}\right)$ from Simulation (With $\mathrm{N}=12, \mathrm{M}=9$, scale $[1 / 9,9], \mathrm{T}=500$ )

\begin{tabular}{||l||c|c|c||c|c|c||}
\hline \multicolumn{1}{|c|}{ M=9 } & \multicolumn{3}{c|}{ A-GE(V) } & \multicolumn{3}{c||}{ A-GE(M) } \\
\hline ABBREVIATIONS & UF & LN & GA & UF & LN & GA \\
\hline \hline CSM & 0.00250 & 0.00184 & 0.00614 & 0.00279 & 0.00196 & 0.00815 \\
& $(0.00026)$ & $(0.00021)$ & $(0.00083)$ & $(0.00024)$ & $(0.00020)$ & $(0.00089)$ \\
\hline R-EV & 0.00244 & 0.00179 & 0.00649 & 0.00244 & 0.00179 & 0.00655 \\
& $(0.00024)$ & $(0.00019)$ & $(0.00068)$ & $(0.00024)$ & $(0.00019)$ & $(0.00066)$ \\
\hline L-EV & 0.00244 & 0.00179 & 0.00706 & 0.00244 & 0.00179 & 0.00709 \\
& $(0.00024)$ & $(0.00019)$ & $(0.00072)$ & $(0.00024)$ & $(0.00019)$ & $(0.00070)$ \\
\hline AM-EV & 0.00243 & 0.00179 & 0.00677 & 0.00243 & 0.00179 & 0.00678 \\
& $(0.00024)$ & $(0.00019)$ & $(0.00069)$ & $(0.00023)$ & $(0.00019)$ & $(0.00067)$ \\
\hline GM-EV & 0.00242 & 0.00178 & 0.00626 & 0.00242 & 0.00178 & 0.00626 \\
& $(0.00023)$ & $(0.00019)$ & $(0.00063)$ & $(0.00023)$ & $(0.00019)$ & $(0.00062)$ \\
\hline EV[AA'] & 0.00325 & 0.00234 & 0.00930 & 0.00326 & 0.00235 & 0.00930 \\
& $(0.00037)$ & $(0.00030)$ & $(0.00108)$ & $(0.00036)$ & $(0.00030)$ & $(0.00104)$ \\
\hline EV[A'A] & 0.00278 & 0.00201 & 0.00385 & 0.00280 & 0.00202 & 0.00409 \\
& $(0.00055)$ & $(0.00039)$ & $(0.00060)$ & $(0.00055)$ & $(0.00039)$ & $(0.00062)$ \\
\hline AM-EV[AA'] & 0.00290 & 0.00208 & 0.00797 & 0.00291 & 0.00209 & 0.00800 \\
AND EV[A'A] & $(0.00034)$ & $(0.00028)$ & $(0.00100)$ & $(0.00034)$ & $(0.00028)$ & $(0.00097)$ \\
\hline GE - EV[AA'] & 0.00215 & 0.00155 & 0.00495 & 0.00216 & 0.00155 & 0.00504 \\
AND EV[A'A] & $(0.00027)$ & $(0.00021)$ & $(0.00054)$ & $(0.00027)$ & $(0.00021)$ & $(0.00053)$ \\
\hline GE & 0.00244 & 0.00178 & 0.00588 & 0.00244 & 0.00155 & 0.00586 \\
& $(0.00023)$ & $(0.00019)$ & $(0.00062)$ & $(0.00023)$ & $(0.00021)$ & $(0.00062)$ \\
\hline C-RSM & 0.00249 & 0.00182 & 0.00593 & 0.00249 & 0.00182 & 0.00596 \\
& $(0.00024)$ & $(0.00019)$ & $(0.00060)$ & $(0.00024)$ & $(0.00019)$ & $(0.00059)$ \\
\hline MT & 0.00243 & 0.00178 & 0.00584 & 0.00243 & 0.00178 & 0.00590 \\
& $(0.00024)$ & $(0.00019)$ & $(0.00060)$ & $(0.00023)$ & $(0.00019)$ & $(0.00058)$ \\
\hline SAV & 0.00310 & 0.00226 & 0.00547 & 0.00310 & 0.00226 & 0.00560 \\
& $(0.00036)$ & $(0.00027)$ & $(0.00064)$ & $(0.00040)$ & $(0.00027)$ & $(0.00062)$ \\
\hline NEV & 0.00361 & 0.00275 & 0.00840 & 0.00375 & 0.00281 & 0.00997 \\
& $(0.00041)$ & $(0.00035)$ & $(0.00114)$ & $(0.00040)$ & $(0.00034)$ & $(0.00118)$ \\
\hline LSM & 0.00402 & 0.00291 & 0.00635 & 0.00403 & 0.00291 & 0.00647 \\
& $(0.00061)$ & $(0.00046)$ & $(0.00112)$ & $(0.00060)$ & $(0.00046)$ & $(0.00113)$ \\
\hline
\end{tabular}


Table C.35: Mean and Standard Deviation of Group Disagreement $\left(d_{2}\right)$ from Simulation (With $\mathrm{N}=12, \mathrm{M}=9$, scale $[1 / 9,9], \mathrm{T}=500$ ) [Continued]

\begin{tabular}{||l||c|c|c||c|c|c||}
\hline \multicolumn{1}{|c||}{ M=9 } & \multicolumn{3}{c|}{ A-AM(V) } & \multicolumn{3}{c||}{ A-MDM(M) } \\
\hline ABBREVIATIONS & UF & LN & GA & UF & LN & GA \\
\hline \hline CSM & 0.00250 & 0.00183 & 0.00610 & 0.00359 & 0.00197 & 0.00816 \\
& $(0.00026)$ & $(0.00021)$ & $(0.00082)$ & $(0.00036)$ & $(0.00020)$ & $(0.00092)$ \\
\hline R-EV & 0.00244 & 0.00179 & 0.00645 & 0.00320 & 0.00180 & 0.00661 \\
& $(0.00024)$ & $(0.00019)$ & $(0.00066)$ & $(0.00035)$ & $(0.00019)$ & $(0.00066)$ \\
\hline L-EV & 0.00244 & 0.00179 & 0.00701 & 0.00322 & 0.00180 & 0.00715 \\
& $(0.00024)$ & $(0.00019)$ & $(0.00070)$ & $(0.00035)$ & $(0.00019)$ & $(0.00071)$ \\
\hline AM-EV & 0.00243 & 0.00179 & 0.00672 & 0.00321 & 0.00180 & 0.00685 \\
& $(0.00023)$ & $(0.00019)$ & $(0.00068)$ & $(0.00035)$ & $(0.00019)$ & $(0.00069)$ \\
\hline GE-EV & 0.00241 & 0.00178 & 0.00622 & 0.00318 & 0.00178 & 0.00632 \\
& $(0.00023)$ & $(0.00019)$ & $(0.00062)$ & $(0.00035)$ & $(0.00019)$ & $(0.00063)$ \\
\hline EV[AA'] & 0.00325 & 0.00234 & 0.00918 & 0.00424 & 0.00236 & 0.00937 \\
& $(0.00036)$ & $(0.00030)$ & $(0.00106)$ & $(0.00053)$ & $(0.00030)$ & $(0.00106)$ \\
\hline EV[A'A] & 0.00278 & 0.00201 & 0.00384 & 0.00335 & 0.00203 & 0.00413 \\
& $(0.00054)$ & $(0.00039)$ & $(0.00059)$ & $(0.00081)$ & $(0.00039)$ & $(0.00063)$ \\
\hline AM - EV[AA'] & 0.00290 & 0.00208 & 0.00789 & 0.00377 & 0.00210 & 0.00807 \\
AND EV[A'A] & $(0.00034)$ & $(0.00028)$ & $(0.00098)$ & $(0.00051)$ & $(0.00028)$ & $(0.00098)$ \\
\hline GE - EV[AA'] & 0.00215 & 0.00155 & 0.00493 & 0.00271 & 0.00156 & 0.00510 \\
AND EV[A'A] & $(0.00027)$ & $(0.00021)$ & $(0.00053)$ & $(0.00037)$ & $(0.00021)$ & $(0.00054)$ \\
\hline GE & 0.00244 & 0.00178 & 0.00585 & 0.00321 & 0.00179 & 0.00591 \\
& $(0.00023)$ & $(0.00019)$ & $(0.00062)$ & $(0.00035)$ & $(0.00019)$ & $(0.00062)$ \\
\hline C-RSM & 0.00249 & 0.00182 & 0.00590 & 0.00327 & 0.00183 & 0.00603 \\
& $(0.00024)$ & $(0.00019)$ & $(0.00060)$ & $(0.00036)$ & $(0.00020)$ & $(0.00060)$ \\
\hline MT & 0.00243 & 0.00178 & 0.00580 & 0.00318 & 0.00179 & 0.00597 \\
& $(0.00024)$ & $(0.00019)$ & $(0.00059)$ & $(0.00034)$ & $(0.00019)$ & $(0.00058)$ \\
\hline SAV & 0.00309 & 0.00226 & 0.00544 & 0.00402 & 0.00227 & 0.00566 \\
& $(0.00036)$ & $(0.00027)$ & $(0.00063)$ & $(0.00052)$ & $(0.00027)$ & $(0.00063)$ \\
\hline NEV & 0.00360 & 0.00275 & 0.00828 & 0.00461 & 0.00282 & 0.01001 \\
& $(0.00040)$ & $(0.00034)$ & $(0.00109)$ & $(0.00055)$ & $(0.00035)$ & $(0.00122)$ \\
\hline LSM & 0.00401 & 0.00290 & 0.00629 & 0.00512 & 0.00293 & 0.00650 \\
& $(0.00061)$ & $(0.00046)$ & $(0.00109)$ & $(0.00083)$ & $(0.00046)$ & $(0.00113)$ \\
\hline
\end{tabular}


Table C.36: Mean and Standard Deviation of Group Disagreement $\left(d_{2}\right)$ from Simulation (With $N=12, \mathrm{M}=9$, scale $[1 / 9,9], \mathrm{T}=500$ ) [Continued]

\begin{tabular}{||l||c|c|c||}
\hline \multicolumn{1}{|c||}{ M=9 } & \multicolumn{3}{c|}{ A-MDM(V) } \\
\hline ABBREVIATIONS & UF & LN & GA \\
\hline \hline CSM & 0.00326 & 0.00252 & 0.00779 \\
& $(0.00080)$ & $(0.00076)$ & $(0.00234)$ \\
\hline R-EV & 0.00261 & 0.00192 & 0.00665 \\
& $(0.00028)$ & $(0.00023)$ & $(0.00070)$ \\
\hline L-EV & 0.00262 & 0.00192 & 0.00769 \\
& $(0.00028)$ & $(0.00022)$ & $(0.00096)$ \\
\hline AM-EV & 0.00262 & 0.00191 & 0.00756 \\
& $(0.00028)$ & $(0.00022)$ & $(0.00096)$ \\
\hline GE-EV & 0.00259 & 0.00190 & 0.00665 \\
& $(0.00027)$ & $(0.00022)$ & $(0.00073)$ \\
\hline EV[AA'] & 0.00363 & 0.00263 & 0.01007 \\
& $(0.00047)$ & $(0.00035)$ & $(0.00131)$ \\
\hline EV[A'A] & 0.00458 & 0.00330 & 0.00334 \\
& $(0.00129)$ & $(0.00090)$ & $(0.00101)$ \\
\hline AM - EV[AA'] & 0.00350 & 0.00253 & 0.00958 \\
AND EV[A'A] & $(0.00048)$ & $(0.00037)$ & $(0.00138)$ \\
\hline GE - EV[AA'] & 0.00296 & 0.00213 & 0.00517 \\
AND EV[A'A] & $(0.00061)$ & $(0.00043)$ & $(0.00063)$ \\
\hline GE & 0.00262 & 0.00190 & 0.00601 \\
& $(0.00028)$ & $(0.00022)$ & $(0.00065)$ \\
\hline C-RSM & 0.00303 & 0.00219 & 0.00570 \\
& $(0.00044)$ & $(0.00032)$ & $(0.00065)$ \\
\hline MT & 0.00260 & 0.00191 & 0.00581 \\
& $(0.00027)$ & $(0.00022)$ & $(0.00058)$ \\
\hline SAV & 0.00469 & 0.00338 & 0.00410 \\
& $(0.00107)$ & $(0.00074)$ & $(0.00101)$ \\
\hline NEV & 0.00384 & 0.00292 & 0.00832 \\
& $(0.00049)$ & $(0.00038)$ & $(0.00124)$ \\
\hline LSM & 0.00647 & 0.00469 & 0.00460 \\
& $(0.00152)$ & $(0.00108)$ & $(0.00140)$ \\
\hline
\end{tabular}




\section{Appendix $\mathbb{D}$}

\section{Paired Comparison Data for Empirical Test}

The entries of the following tables are the assigned values to the elements of pairs placed on the first row. These values are expressed on the constant-sum measurement scale ( 1 to 100). Indices of pairs indicate two of six decision elements. 
Table D.1: Category 1 - Lengths of Straight Lines

\begin{tabular}{|c|c|c|c|c|c|c|c|c|c|c|c|c|c|c|c|}
\hline Student/Pair & 12 & 13 & 14 & 15 & 16 & 23 & 24 & 25 & 26 & 34 & 35 & 36 & 45 & 46 & 56 \\
\hline$\overline{1}$ & 65 & 55 & 45 & $\overline{\overline{60}}$ & $\overline{40}$ & 40 & $\overline{30}$ & 45 & 25 & 40 & 55 & 35 & 65 & 45 & $\overline{\overline{30}}$ \\
\hline$\overline{2}$ & 60 & 55 & 45 & 55 & 40 & 40 & 35 & 45 & $\overline{35}$ & 45 & 60 & 35 & 60 & 48 & 40 \\
\hline 3 & 60 & 52 & 48 & 58 & 45 & 40 & 35 & 48 & 33 & 45 & 52 & 42 & 60 & 48 & 40 \\
\hline 4 & 50 & 50 & 40 & 50 & 40 & 50 & 40 & 50 & 35 & 40 & 50 & 35 & 55 & 50 & 35 \\
\hline 5 & 65 & $\overline{55}$ & 45 & 60 & 40 & 40 & 35 & 45 & 30 & 45 & 60 & 40 & 35 & 45 & 35 \\
\hline 6 & 60 & 52 & 49 & 60 & 45 & 45 & 40 & 48 & 40 & 45 & 55 & 42 & 60 & 48 & 60 \\
\hline 7 & 60 & 53 & 46 & 55 & 45 & 44 & 40 & 46 & 35 & 44 & 52 & 41 & 58 & 47 & 39 \\
\hline 8 & 35 & 55 & 45 & 60 & 40 & 60 & 30 & 45 & 30 & 40 & $\overline{55}$ & 35 & 65 & 45 & 33 \\
\hline 9 & 60 & 54 & 47 & 56 & 47 & 43 & 40 & 44 & 33 & 44 & $\overline{53}$ & 43 & 43 & 47 & 40 \\
\hline 10 & 60 & 55 & 45 & 57 & 42 & 40 & 35 & 45 & 33 & 45 & $\overline{53}$ & 40 & 60 & 47 & 40 \\
\hline 11 & 62 & 55 & 48 & 60 & 45 & 61 & 34 & 45 & 35 & 45 & 55 & 40 & 60 & 47 & 37 \\
\hline 12 & 60 & 55 & 45 & $\overline{55}$ & 40 & 40 & 35 & 45 & 35 & 45 & 60 & 35 & 60 & 48 & 40 \\
\hline 13 & 61 & 48 & 47 & 61 & 46 & 53 & 35 & 45 & 34 & 41 & 53 & 42 & 60 & 48 & 38 \\
\hline 14 & 62 & 54 & 47 & 57 & 45 & 42 & 36 & 46 & 33 & 43 & 54 & 41 & 60 & 47 & 37 \\
\hline 15 & 62 & 55 & 48 & 57 & 45 & 43 & 35 & 43 & 33 & 45 & 55 & 43 & 60 & 48 & 38 \\
\hline 16 & 62 & 55 & 47 & 60 & 45 & 40 & 33 & 45 & 33 & 45 & 55 & 40 & 60 & 47 & 40 \\
\hline 17 & 65 & 55 & 45 & 42 & 40 & 35 & 30 & 45 & 30 & 40 & 55 & 35 & 65 & 47 & 30 \\
\hline 18 & 60 & 47 & 46 & 55 & 43 & 43 & 36 & 44 & 33 & 43 & 52 & 40 & 42 & 47 & 39 \\
\hline 19 & 65 & 53 & 45 & 60 & 45 & 35 & 35 & 45 & 33 & 47 & 60 & 45 & 60 & 45 & 40 \\
\hline 20 & 60 & 55 & 47 & 48 & 45 & 45 & 30 & 45 & 35 & 45 & 57 & 42 & 60 & 49 & 40 \\
\hline 21 & 61 & 59 & 45 & 56 & 45 & 43 & 40 & 48 & 33 & 43 & 57 & 42 & 54 & 48 & 35 \\
\hline 22 & 38 & 55 & 47 & 57 & 44 & 44 & 36 & 46 & 33 & 42 & 52 & 40 & 60 & 47 & 37 \\
\hline 23 & 60 & 53 & 45 & 55 & 40 & 44 & 40 & 45 & 33 & 45 & 52 & 45 & 60 & 48 & 40 \\
\hline 24 & 63 & 62 & 38 & 60 & 40 & 37 & 33 & 40 & 33 & 37 & 60 & 37 & 63 & 40 & 28 \\
\hline 25 & $\overline{0} 0$ & 45 & 45 & 60 & 45 & 40 & 35 & 45 & 20 & 45 & 55 & 45 & 40 & 45 & 35 \\
\hline 26 & 70 & 55 & 40 & 70 & 40 & 40 & 30 & 45 & 30 & 40 & 55 & 30 & 70 & 45 & 30 \\
\hline 27 & 59 & 55 & 47 & 56 & 48 & 45 & 43 & 45 & 34 & 42 & 54 & 44 & 58 & 49 & 40 \\
\hline 28 & 65 & 55 & 45 & 60 & 40 & 40 & 30 & 45 & 35 & 40 & 60 & 35 & 60 & 45 & 35 \\
\hline 29 & 60 & 55 & 47 & 55 & 44 & 45 & 40 & 45 & 33 & 45 & 55 & 35 & 40 & 53 & 35 \\
\hline 30 & 46 & 43 & 40 & 37 & 35 & 47 & 44 & 41 & 39 & 48 & 45 & 42 & 47 & 45 & 48 \\
\hline 31 & 65 & 55 & 45 & 60 & 40 & 40 & 37 & 45 & 35 & 44 & 52 & 41 & 58 & 49 & 40 \\
\hline$\overline{32}$ & 59 & 53 & 52 & 55 & 45 & 40 & 38 & 47 & 37 & 45 & 53 & 43 & 58 & 48 & 40 \\
\hline 33 & 66 & 60 & 45 & 60 & 40 & 40 & 30 & 45 & 66 & 45 & 60 & 35 & 40 & 48 & 35 \\
\hline 34 & 60 & 52 & 48 & 57 & 45 & 46 & 35 & 47 & 33 & 53 & 53 & 42 & 60 & 48 & 35 \\
\hline 35 & 60 & 47 & 47 & 57 & 43 & 45 & 35 & 45 & 35 & 47 & 55 & 40 & 62 & 48 & 40 \\
\hline 36 & 62 & 55 & 48 & 58 & 46 & 43 & 36 & 46 & 34 & 43 & 53 & 40 & 61 & 48 & 37 \\
\hline 37 & 60 & 55 & 45 & 57 & 45 & 40 & 35 & 45 & 33 & 43 & 55 & 40 & 60 & 47 & 37 \\
\hline 38 & 62 & 55 & 45 & 57 & 40 & 45 & 70 & 45 & 33 & 45 & 60 & 40 & 65 & 48 & 33 \\
\hline 39 & 60 & 55 & 45 & 60 & 40 & 40 & 40 & 40 & 35 & 40 & 60 & 45 & 60 & 45 & 40 \\
\hline
\end{tabular}


Table D.2: Category 2 - Air Distance between Pittsburgh and Other Cities

\begin{tabular}{||l||l|l|l|l|l|l|l|l|l|l|l|l|l|l|l||}
\hline Student/Pair & 12 & 13 & 14 & 15 & 16 & 23 & 24 & 25 & 26 & 34 & 35 & 36 & 45 & 46 & 56 \\
\hline \hline 1 & 20 & 20 & 10 & 5 & 45 & 50 & 20 & 10 & 20 & 20 & 10 & 70 & 45 & 90 & 95 \\
\hline 2 & 50 & 50 & 45 & 20 & 45 & 50 & 40 & 20 & 40 & 40 & 30 & 50 & 30 & 60 & 80 \\
\hline 3 & 40 & 33 & 10 & 25 & 25 & 45 & 20 & 30 & 35 & 35 & 40 & 50 & 50 & 65 & 65 \\
\hline 4 & 50 & 33 & 25 & 25 & 50 & 33 & 25 & 25 & 50 & 40 & 40 & 67 & 50 & 75 & 75 \\
\hline 5 & 33 & 25 & 10 & 11 & 30 & 40 & 20 & 20 & 44 & 25 & 27 & 55 & 53 & 78 & 76 \\
\hline 6 & 25 & 33 & 10 & 11 & 33 & 60 & 17 & 20 & 50 & 17 & 20 & 50 & 53 & 83 & 80 \\
\hline 7 & 33 & 30 & 10 & 15 & 33 & 45 & 20 & 23 & 50 & 23 & 25 & 53 & 55 & 20 & 75 \\
\hline 8 & 35 & 40 & 10 & 20 & 35 & 45 & 20 & 25 & 45 & 25 & 25 & 50 & 55 & 70 & 70 \\
\hline 9 & 36 & 26 & 10 & 12 & 26 & 29 & 18 & 19 & 28 & 24 & 27 & 49 & 53 & 72 & 74 \\
\hline 10 & 40 & 25 & 15 & 15 & 33 & 50 & 20 & 20 & 40 & 25 & 25 & 50 & 50 & 75 & 75 \\
\hline 11 & 75 & 70 & 90 & 95 & 80 & 55 & 90 & 65 & 65 & 65 & 55 & 40 & 50 & 30 & 30 \\
\hline 12 & 65 & 35 & 10 & 10 & 30 & 50 & 20 & 30 & 45 & 35 & 40 & 55 & 50 & 60 & 25 \\
\hline 13 & 25 & 15 & 7 & 5 & 20 & 40 & 25 & 15 & 45 & 30 & 25 & 55 & 47 & 75 & 75 \\
\hline 14 & 35 & 40 & 15 & 30 & 40 & 55 & 35 & 40 & 55 & 30 & 15 & 50 & 55 & 70 & 70 \\
\hline 15 & 33 & 22 & 25 & 10 & 23 & 36 & 17 & 18 & 37 & 26 & 28 & 52 & 53 & 75 & 27 \\
\hline 16 & 33 & 35 & 20 & 25 & 40 & 40 & 30 & 33 & 50 & 33 & 40 & 50 & 60 & 75 & 70 \\
\hline 17 & 33 & 20 & 15 & 10 & 25 & 33 & 20 & 85 & 40 & 33 & 30 & 37 & 40 & 75 & 78 \\
\hline 18 & 25 & 33 & 20 & 17 & 14 & 60 & 43 & 38 & 33 & 33 & 29 & 25 & 44 & 60 & 45 \\
\hline 19 & 45 & 20 & 30 & 10 & 20 & 30 & 20 & 20 & 40 & 30 & 30 & 50 & 50 & 70 & 70 \\
\hline 20 & 45 & 40 & 10 & 20 & 35 & 50 & 20 & 30 & 45 & 30 & 40 & 50 & 50 & 70 & 80 \\
\hline 21 & 40 & 40 & 10 & 20 & 45 & 50 & 25 & 30 & 50 & 20 & 30 & 50 & 55 & 70 & 20 \\
\hline 22 & 33 & 25 & 8 & 8 & 20 & 40 & 42 & 14 & 33 & 21 & 20 & 43 & 48 & 73 & 75 \\
\hline 23 & 20 & 17 & 27 & 8 & 30 & 44 & 24 & 26 & 50 & 28 & 31 & 56 & 54 & 76 & 73 \\
\hline 24 & 37 & 27 & 9 & 6 & 26 & 39 & 14 & 10 & 37 & 21 & 15 & 48 & 40 & 78 & 84 \\
\hline 25 & 33 & 30 & 15 & 20 & 33 & 45 & 25 & 33 & 45 & 25 & 40 & 50 & 60 & 66 & 67 \\
\hline 26 & 38 & 47 & 20 & 25 & 38 & 50 & 25 & 30 & 30 & 75 & 20 & 45 & 52 & 70 & 67 \\
\hline 27 & 40 & 40 & 10 & 10 & 40 & 50 & 30 & 30 & 50 & 30 & 35 & 50 & 45 & 65 & 65 \\
\hline
\end{tabular}


Table D.3: Category 3 - Number of Times Football Teams Have Won the Super Bowl

\begin{tabular}{||l||c|c|c|c|c|c|c|c|c|c|c|c|c|c|c||}
\hline Student/Pair & 12 & 13 & 14 & 15 & 16 & 23 & 24 & 25 & 26 & 34 & 35 & 36 & 45 & 46 & 56 \\
\hline \hline 1 & 80 & 80 & 70 & 60 & 60 & 50 & 40 & 30 & 30 & 40 & 30 & 30 & 40 & 40 & 50 \\
\hline 2 & 55 & 80 & 65 & 55 & 65 & 75 & 60 & 50 & 60 & 35 & 25 & 35 & 40 & 50 & 60 \\
\hline 3 & 65 & 70 & 65 & 65 & 65 & 60 & 50 & 50 & 50 & 40 & 40 & 40 & 50 & 50 & 50 \\
\hline 4 & 65 & 80 & 65 & 55 & 65 & 65 & 50 & 40 & 60 & 35 & 25 & 40 & 50 & 50 & 60 \\
\hline 5 & 67 & 67 & 67 & 57 & 67 & 50 & 50 & 40 & 50 & 50 & 40 & 50 & 40 & 50 & 60 \\
\hline 6 & 67 & 80 & 67 & 57 & 67 & 67 & 50 & 60 & 50 & 33 & 25 & 33 & 40 & 50 & 60 \\
\hline 7 & 60 & 75 & 60 & 55 & 60 & 25 & 50 & 50 & 66 & 40 & 25 & 34 & 50 & 60 & 70 \\
\hline 8 & 67 & 80 & 67 & 57 & 67 & 67 & 50 & 40 & 50 & 33 & 25 & 33 & 40 & 50 & 60 \\
\hline 9 & 30 & 75 & 60 & 65 & 75 & 50 & 40 & 45 & 40 & 35 & 50 & 50 & 55 & 55 & 45 \\
\hline 10 & 70 & 90 & 55 & 70 & 55 & 50 & 20 & 50 & 50 & 35 & 40 & 40 & 70 & 50 & 35 \\
\hline 11 & 57 & 99 & 57 & 67 & 67 & 99 & 50 & 60 & 60 & 1 & 1 & 1 & 60 & 60 & 50 \\
\hline 12 & 67 & 67 & 67 & 55 & 67 & 50 & 50 & 40 & 50 & 50 & 40 & 50 & 40 & 50 & 60 \\
\hline 13 & 66 & 80 & 67 & 57 & 66 & 67 & 50 & 40 & 50 & 33 & 40 & 34 & 40 & 50 & 60 \\
\hline 14 & 60 & 67 & 60 & 67 & 80 & 60 & 50 & 60 & 75 & 40 & 50 & 33 & 60 & 75 & 67 \\
\hline 15 & 57 & 66 & 66 & 66 & 80 & 40 & 60 & 60 & 75 & 50 & 50 & 66 & 50 & 66 & 66 \\
\hline 16 & 67 & 80 & 67 & 56 & 67 & 67 & 50 & 40 & 50 & 33 & 25 & 33 & 40 & 50 & 60 \\
\hline 17 & 33 & 42 & 65 & 50 & 40 & 59 & 55 & 25 & 54 & 51 & 80 & 50 & 47 & 68 & 10 \\
\hline 18 & 99 & 80 & 67 & 57 & 67 & 1 & 1 & 1 & 1 & 33 & 25 & 33 & 40 & 50 & 60 \\
\hline 19 & 80 & 80 & 67 & 67 & 67 & 50 & 40 & 33 & 33 & 33 & 40 & 34 & 50 & 50 & 50 \\
\hline 20 & 57 & 67 & 67 & 57 & 57 & 60 & 60 & 50 & 50 & 50 & 40 & 40 & 40 & 40 & 50 \\
\hline 21 & 60 & 80 & 67 & 60 & 66 & 75 & 40 & 50 & 60 & 33 & 25 & 34 & 40 & 50 & 60 \\
\hline 22 & 57 & 80 & 67 & 57 & 67 & 75 & 60 & 50 & 40 & 33 & 25 & 33 & 40 & 50 & 60 \\
\hline 23 & 70 & 90 & 65 & 60 & 70 & 50 & 30 & 20 & 20 & 20 & 30 & 50 & 50 & 70 & 60 \\
\hline
\end{tabular}


Table D.4: Category 4 - Metropolitan Area Population in 1980

\begin{tabular}{||l||l|l|l|l|l|l|l|l|l|l|l|l|l|l|l||}
\hline Student/Pair & 12 & 13 & 14 & 15 & 16 & 23 & 24 & 25 & 26 & 34 & 35 & 36 & 45 & 46 & 56 \\
\hline \hline 1 & 60 & 30 & 33 & 75 & 70 & 60 & 35 & 40 & 70 & 20 & 55 & 50 & 80 & 75 & 60 \\
\hline 2 & 30 & 45 & 30 & 50 & 45 & 60 & 45 & 60 & 60 & 35 & 50 & 50 & 65 & 65 & 50 \\
\hline 3 & 40 & 50 & 30 & 55 & 50 & 50 & 30 & 70 & 50 & 30 & 60 & 40 & 80 & 70 & 40 \\
\hline 4 & 15 & 25 & 10 & 60 & 45 & 65 & 30 & 15 & 80 & 15 & 80 & 70 & 95 & 10 & 40 \\
\hline 5 & 35 & 55 & 30 & 60 & 75 & 60 & 45 & 70 & 85 & 35 & 60 & 70 & 75 & 85 & 60 \\
\hline 6 & 50 & 40 & 11 & 60 & 67 & 50 & 10 & 60 & 58 & 11 & 53 & 73 & 92 & 92 & 52 \\
\hline 7 & 40 & 50 & 33 & 67 & 67 & 60 & 40 & 75 & 75 & 33 & 67 & 67 & 80 & 80 & 50 \\
\hline 8 & 34 & 50 & 34 & 66 & 60 & 67 & 55 & 85 & 70 & 25 & 75 & 60 & 90 & 75 & 33 \\
\hline 9 & 40 & 50 & 25 & 60 & 65 & 55 & 35 & 75 & 50 & 30 & 60 & 40 & 80 & 65 & 25 \\
\hline 10 & 26 & 46 & 19 & 72 & 47 & 70 & 39 & 87 & 71 & 21 & 75 & 52 & 92 & 80 & 26 \\
\hline 12 & 47 & 54 & 41 & 58 & 52 & 57 & 56 & 61 & 55 & 37 & 54 & 48 & 67 & 61 & 55 \\
\hline 13 & 40 & 40 & 30 & 60 & 45 & 65 & 40 & 75 & 65 & 35 & 65 & 55 & 80 & 70 & 35 \\
\hline 14 & 35 & 67 & 25 & 50 & 33 & 60 & 35 & 70 & 60 & 25 & 60 & 50 & 80 & 67 & 35 \\
\hline 15 & 70 & 55 & 20 & 52 & 47 & 25 & 38 & 72 & 67 & 16 & 49 & 40 & 18 & 75 & 42 \\
\hline 16 & 35 & 40 & 30 & 52 & 50 & 50 & 40 & 70 & 65 & 35 & 60 & 40 & 80 & 70 & 50 \\
\hline 17 & 45 & 41 & 20 & 59 & 58 & 45 & 25 & 66 & 60 & 40 & 70 & 58 & 80 & 75 & 45 \\
\hline 18 & 10 & 39 & 7 & 56 & 47 & 86 & 43 & 92 & 90 & 11 & 67 & 59 & 94 & 92 & 42 \\
\hline 19 & 38 & 38 & 27 & 26 & 42 & 50 & 38 & 38 & 55 & 38 & 37 & 66 & 49 & 66 & 33 \\
\hline 20 & 20 & 40 & 10 & 50 & 45 & 60 & 40 & 90 & 80 & 30 & 60 & 60 & 90 & 90 & 50 \\
\hline 21 & 50 & 38 & 25 & 75 & 50 & 38 & 25 & 75 & 50 & 35 & 84 & 62 & 90 & 75 & 25 \\
\hline 22 & 30 & 40 & 30 & 70 & 30 & 35 & 30 & 70 & 40 & 20 & 70 & 35 & 80 & 60 & 30 \\
\hline 23 & 33 & 33 & 25 & 20 & 33 & 50 & 40 & 33 & 50 & 40 & 33 & 50 & 57 & 60 & 33 \\
\hline 24 & 17 & 34 & 7 & 55 & 47 & 75 & 34 & 85 & 82 & 13 & 68 & 64 & 94 & 91 & 49 \\
\hline 25 & 40 & 50 & 25 & 50 & 30 & 65 & 40 & 67 & 55 & 30 & 45 & 35 & 70 & 55 & 35 \\
\hline 26 & 45 & 42 & 40 & 51 & 45 & 51 & 45 & 67 & 65 & 46 & 70 & 55 & 52 & 55 & 42 \\
\hline 27 & 10 & 21 & 9 & 70 & 14 & 71 & 47 & 96 & 60 & 26 & 90 & 38 & 96 & 63 & 7 \\
\hline 28 & 50 & 45 & 40 & 58 & 50 & 50 & 40 & 57 & 55 & 42 & 60 & 50 & 65 & 60 & 50 \\
\hline
\end{tabular}


Table D.5: Category 5 - Annual Number of Air Passengers in Airports

\begin{tabular}{||l||l|l|l|l|l|l|l|l|l|l|l|l|l|l|l||}
\hline Student/Pair & 12 & 13 & 14 & 15 & 16 & 23 & 24 & 25 & 26 & 34 & 35 & 36 & 45 & 46 & 56 \\
\hline \hline 1 & 45 & 55 & 50 & 45 & 80 & 60 & 45 & 60 & 80 & 50 & 50 & 75 & 50 & 75 & 75 \\
\hline 2 & 40 & 60 & 50 & 30 & 60 & 25 & 60 & 40 & 80 & 40 & 30 & 50 & 40 & 70 & 80 \\
\hline 3 & 45 & 75 & 63 & 63 & 65 & 30 & 65 & 65 & 67 & 40 & 40 & 45 & 50 & 55 & 55 \\
\hline 4 & 55 & 75 & 33 & 33 & 80 & 75 & 33 & 33 & 75 & 15 & 25 & 67 & 50 & 90 & 90 \\
\hline 5 & 40 & 45 & 40 & 40 & 60 & 55 & 50 & 45 & 70 & 40 & 45 & 65 & 45 & 65 & 70 \\
\hline 6 & 33 & 50 & 25 & 20 & 60 & 50 & 60 & 35 & 60 & 42 & 36 & 63 & 33 & 30 & 75 \\
\hline 7 & 33 & 55 & 56 & 42 & 83 & 71 & 71 & 59 & 91 & 51 & 37 & 80 & 36 & 80 & 87 \\
\hline 8 & 43 & 60 & 54 & 48 & 62 & 67 & 61 & 55 & 69 & 44 & 39 & 53 & 45 & 59 & 64 \\
\hline 9 & 50 & 70 & 50 & 65 & 75 & 65 & 50 & 60 & 60 & 35 & 50 & 50 & 50 & 50 & 65 \\
\hline 10 & 38 & 58 & 52 & 43 & 70 & 70 & 60 & 55 & 70 & 40 & 39 & 54 & 36 & 60 & 80 \\
\hline 11 & 75 & 65 & 75 & 60 & 90 & 38 & 50 & 34 & 75 & 62 & 45 & 84 & 34 & 75 & 83 \\
\hline 12 & 70 & 60 & 70 & 65 & 60 & 10 & 35 & 48 & 50 & 67 & 80 & 80 & 45 & 45 & 44 \\
\hline 13 & 50 & 75 & 50 & 45 & 70 & 80 & 50 & 49 & 78 & 20 & 17 & 50 & 48 & 80 & 90 \\
\hline 14 & 62 & 71 & 56 & 56 & 83 & 60 & 43 & 43 & 75 & 33 & 33 & 67 & 50 & 80 & 80 \\
\hline 15 & 45 & 48 & 42 & 43 & 60 & 53 & 48 & 49 & 63 & 47 & 47 & 41 & 51 & 49 & 62 \\
\hline
\end{tabular}


Table D.6: Category 6 - Number of Professionals in Major Occupation in the Unite State

\begin{tabular}{||l||l|l|l|l|l|l|l|l|l|l|l|l|l|l|l||}
\hline Student/Pair & 12 & 13 & 14 & 15 & 16 & 23 & 24 & 25 & 26 & 34 & 35 & 36 & 45 & 46 & 56 \\
\hline \hline 1 & 50 & 40 & 65 & 60 & 33 & 30 & 30 & 50 & 40 & 60 & 80 & 40 & 80 & 55 & 20 \\
\hline 2 & 50 & 25 & 50 & 33 & 67 & 25 & 33 & 33 & 40 & 75 & 75 & 85 & 40 & 50 & 67 \\
\hline 3 & 60 & 55 & 75 & 85 & 80 & 45 & 60 & 70 & 65 & 62 & 70 & 65 & 60 & 55 & 45 \\
\hline 4 & 50 & 20 & 14 & 17 & 33 & 25 & 20 & 25 & 33 & 33 & 50 & 60 & 67 & 60 & 45 \\
\hline 5 & 66 & 66 & 80 & 60 & 95 & 30 & 25 & 50 & 98 & 75 & 65 & 80 & 40 & 70 & 35 \\
\hline 6 & 50 & 65 & 35 & 80 & 75 & 50 & 75 & 80 & 75 & 60 & 65 & 70 & 55 & 50 & 50 \\
\hline 7 & 65 & 45 & 60 & 70 & 60 & 30 & 45 & 53 & 45 & 60 & 80 & 60 & 60 & 47 & 45 \\
\hline 8 & 50 & 65 & 75 & 70 & 80 & 60 & 75 & 75 & 80 & 65 & 65 & 75 & 50 & 65 & 60 \\
\hline 9 & 60 & 60 & 33 & 75 & 33 & 50 & 25 & 67 & 25 & 25 & 67 & 25 & 85 & 50 & 14 \\
\hline 10 & 80 & 57 & 56 & 67 & 40 & 25 & 14 & 33 & 14 & 43 & 60 & 33 & 71 & 45 & 25 \\
\hline 11 & 70 & 50 & 50 & 80 & 40 & 30 & 40 & 60 & 40 & 70 & 70 & 70 & 70 & 50 & 40 \\
\hline 12 & 60 & 50 & 70 & 70 & 60 & 40 & 40 & 60 & 40 & 65 & 70 & 70 & 45 & 60 & 30 \\
\hline 13 & 57 & 44 & 62 & 80 & 73 & 37 & 55 & 75 & 67 & 67 & 83 & 77 & 71 & 63 & 40 \\
\hline 14 & 50 & 40 & 75 & 75 & 75 & 40 & 75 & 75 & 75 & 80 & 80 & 80 & 50 & 50 & 50 \\
\hline 15 & 50 & 34 & 34 & 66 & 20 & 25 & 50 & 66 & 30 & 50 & 75 & 34 & 67 & 33 & 20 \\
\hline 16 & 65 & 60 & 80 & 65 & 80 & 40 & 70 & 60 & 70 & 60 & 60 & 70 & 40 & 55 & 70 \\
\hline
\end{tabular}


Table D.7: Category 7 - Country Population

\begin{tabular}{||l||c|c|c|c|c|c|c|c|c|c|c|c|c|c|c||}
\hline Student/Pair & 12 & 13 & 14 & 15 & 16 & 23 & 24 & 25 & 26 & 34 & 35 & 36 & 45 & 46 & 56 \\
\hline \hline 1 & 10 & 35 & 5 & 20 & 25 & 80 & 55 & 70 & 75 & 10 & 35 & 45 & 80 & 90 & 55 \\
\hline 2 & 25 & 45 & 10 & 35 & 20 & 70 & 40 & 60 & 45 & 20 & 25 & 20 & 70 & 60 & 40 \\
\hline 3 & 5 & 71 & 6 & 9 & 14 & 50 & 5 & 17 & 6 & 2 & 7 & 3 & 91 & 86 & 20 \\
\hline 4 & 25 & 35 & 5 & 15 & 15 & 65 & 35 & 35 & 35 & 15 & 25 & 10 & 65 & 35 & 40 \\
\hline 5 & 33 & 50 & 5 & 20 & 16 & 60 & 5 & 25 & 10 & 3 & 25 & 15 & 90 & 67 & 33 \\
\hline 6 & 10 & 50 & 3 & 40 & 10 & 90 & 40 & 70 & 50 & 4 & 35 & 25 & 75 & 70 & 45 \\
\hline 7 & 25 & 40 & 10 & 40 & 33 & 55 & 35 & 65 & 45 & 22 & 55 & 30 & 80 & 60 & 34 \\
\hline 8 & 15 & 49 & 31 & 30 & 28 & 85 & 41 & 71 & 31 & 11 & 31 & 29 & 78 & 76 & 47 \\
\hline 9 & 20 & 50 & 30 & 30 & 20 & 80 & 80 & 70 & 60 & 20 & 40 & 40 & 60 & 60 & 45 \\
\hline 10 & 50 & 40 & 70 & 35 & 80 & 35 & 30 & 20 & 90 & 50 & 30 & 70 & 40 & 75 & 95 \\
\hline 11 & 20 & 50 & 12 & 25 & 25 & 83 & 45 & 62 & 63 & 13 & 25 & 25 & 67 & 70 & 50 \\
\hline 12 & 39 & 60 & 72 & 40 & 38 & 56 & 30 & 52 & 40 & 19 & 35 & 71 & 62 & 59 & 45 \\
\hline
\end{tabular}




\section{Appendix $\mathbb{E}$}

\section{The Results of Empirical Test for Accuracy and Group Disagreement}

The entries of the following tables are the results of empirical test. for the accuracy measurement and group disagreement measurement. All them are in pairs, the numbers inside the parenthesis is the results of group disagreement, and the number without parenthesis is the the results of accuracy measurement. All the notations in tables follow the definition of Table 4.1 and 4.2. The C1 to C7 indicate the category 1 to category 7 in this Appendix. 
Table E.1: The Results of Empirical Test for Accuracy and Group Disagreement with Geometric Mean Operated on Pairwise Comparison Matrix

\begin{tabular}{|c|c|c|c|c|c|c|c|}
\hline \multicolumn{8}{|c|}{$\mathrm{A}-\mathrm{GE}(\mathrm{M})$} \\
\hline ABBR. & C1 & $\mathrm{C} 2$ & $\mathrm{C} 3$ & $\mathrm{C4}$ & $\mathrm{C5}$ & C6 & $\mathrm{C7}$ \\
\hline CSM & $\begin{array}{l}0.00590 \\
(0.0023)\end{array}$ & $\begin{array}{c}0.0314 \\
(0.0120)\end{array}$ & $\begin{array}{c}0.0194 \\
(0.0081)\end{array}$ & $\begin{array}{c}0.0278 \\
(0.0117)\end{array}$ & $\begin{array}{c}0.0346 \\
(0.0140)\end{array}$ & $\begin{array}{c}0.0598 \\
(0.0174)\end{array}$ & $\begin{array}{c}0.0637 \\
(0.0237)\end{array}$ \\
\hline R-EV & $\begin{array}{c}0.0059 \\
(0.0023)\end{array}$ & $\begin{array}{c}0.0314 \\
(0.0120)\end{array}$ & $\begin{array}{c}0.0193 \\
(0.0083)\end{array}$ & $\begin{array}{c}0.0277 \\
(0.0117)\end{array}$ & $\begin{array}{c}0.0345 \\
(0.0142)\end{array}$ & $\begin{array}{c}0.0600 \\
(0.0176)\end{array}$ & $\begin{array}{c}0.0632 \\
(0.0244)\end{array}$ \\
\hline L-EV & $\begin{array}{c}0.0059 \\
(0.0024)\end{array}$ & $\begin{array}{c}0.0313 \\
(0.0125)\end{array}$ & $\begin{array}{c}0.0194 \\
(0.0082)\end{array}$ & $\begin{array}{c}0.0278 \\
(0.0116)\end{array}$ & $\begin{array}{c}0.0348 \\
(0.0142)\end{array}$ & $\begin{array}{c}0.0597 \\
(0.0181)\end{array}$ & $\begin{array}{c}0.0642 \\
(0.0251)\end{array}$ \\
\hline AM-EV & $\begin{array}{c}0.0059 \\
(0.0023)\end{array}$ & $\begin{array}{c}0.0313 \\
(0.0123)\end{array}$ & $\begin{array}{c}0.0194 \\
(0.0082)\end{array}$ & $\begin{array}{c}0.0278 \\
(0.0116)\end{array}$ & $\begin{array}{c}0.0347 \\
(0.0141)\end{array}$ & $\begin{array}{c}0.0598 \\
(0.0180)\end{array}$ & $\begin{array}{c}0.0641 \\
(0.0250)\end{array}$ \\
\hline GM-EV & $\begin{array}{c}0.0059 \\
(0.0023)\end{array}$ & $\begin{array}{c}0.0314 \\
(0.0121)\end{array}$ & $\begin{array}{c}0.0194 \\
(0.0082)\end{array}$ & $\begin{array}{c}0.0278 \\
(0.0116)\end{array}$ & $\begin{array}{c}0.0346 \\
(0.0141)\end{array}$ & $\begin{array}{c}0.0598 \\
(0.0177)\end{array}$ & $\begin{array}{c}0.0637 \\
(0.0247)\end{array}$ \\
\hline$\overline{\mathrm{EV}}\left[\mathrm{AA}^{\prime}\right]$ & $\begin{array}{c}0.0065 \\
(0.0022)\end{array}$ & $\begin{array}{c}0.0322 \\
(0.0118) \\
\end{array}$ & $\begin{array}{c}0.0169 \\
(0.0086)\end{array}$ & $\begin{array}{c}0.0268 \\
(0.0123)\end{array}$ & $\begin{array}{c}0.0338 \\
(0.0144)\end{array}$ & $\begin{array}{c}0.0594 \\
(0.0179) \\
\end{array}$ & $\begin{array}{c}0.0602 \\
(0.0287)\end{array}$ \\
\hline $\mathrm{EV}\left[\mathrm{A}^{\prime} \mathrm{A}\right]$ & $\begin{array}{c}0.0467 \\
(0.0014)\end{array}$ & $\begin{array}{c}0.1340 \\
(0.0125)\end{array}$ & $\begin{array}{c}0.0561 \\
(0.0097)\end{array}$ & $\begin{array}{c}0.0953 \\
(0.0126)\end{array}$ & $\begin{array}{c}0.0376 \\
(0.0096)\end{array}$ & $\begin{array}{c}0.0774 \\
(0.0146)\end{array}$ & $\begin{array}{c}0.1500 \\
(0.0284)\end{array}$ \\
\hline $\begin{array}{l}\mathrm{AM}-\mathrm{EV}\left[\mathrm{AA} A^{\prime}\right] \\
\mathrm{AND} \text { EV }\left[\mathrm{A}^{\prime} \mathrm{A}\right]\end{array}$ & $\begin{array}{c}0.0167 \\
(0.0018)\end{array}$ & $\begin{array}{c}0.0507 \\
(0.0111)\end{array}$ & $\begin{array}{c}0.0253 \\
(0.0081)\end{array}$ & $\begin{array}{c}0.0354 \\
(0.0111)\end{array}$ & $\begin{array}{c}0.0313 \\
(0.0128)\end{array}$ & $\begin{array}{c}0.0648 \\
(0.0160)\end{array}$ & $\begin{array}{c}0.0675 \\
(0.0274)\end{array}$ \\
\hline $\begin{array}{l}\text { GE - EV }\left[A A^{\prime}\right] \\
\text { AND EV }\left[A^{\prime} A\right]\end{array}$ & $\begin{array}{c}0.0224 \\
(0.0015)\end{array}$ & $\begin{array}{c}0.0834 \\
(0.0100)\end{array}$ & $\begin{array}{c}0.0335 \\
(0.0095)\end{array}$ & $\begin{array}{c}0.0508 \\
(0.0094)\end{array}$ & $\begin{array}{c}0.0309 \\
(0.0109)\end{array}$ & $\begin{array}{c}0.0678 \\
(0.0142)\end{array}$ & $\begin{array}{c}0.0936 \\
(0.0233)\end{array}$ \\
\hline GE & $\begin{array}{c}0.0059 \\
(0.0022)\end{array}$ & $\begin{array}{c}0.0314 \\
(0.0117)\end{array}$ & $\begin{array}{c}0.0194 \\
(0.0081)\end{array}$ & $\begin{array}{c}0.0278 \\
(0.0112)\end{array}$ & $\begin{array}{c}0.0346 \\
(0.0141)\end{array}$ & $\begin{array}{c}0.0598 \\
(0.0177)\end{array}$ & $\begin{array}{c}0.0637 \\
(0.0248)\end{array}$ \\
\hline C-RSM & $\begin{array}{c}0.0059 \\
(0.0022)\end{array}$ & $\begin{array}{c}0.0311 \\
(0.0120)\end{array}$ & $\begin{array}{c}0.0192 \\
(0.0083)\end{array}$ & $\begin{array}{c}0.0277 \\
(0.0114)\end{array}$ & $\begin{array}{c}0.0349 \\
(0.0142)\end{array}$ & $\begin{array}{c}0.0599 \\
(0.0180)\end{array}$ & $\begin{array}{c}0.0620 \\
(0.0249)\end{array}$ \\
\hline $\mathrm{MT}$ & $\begin{array}{c}0.0059 \\
(0.0022) \\
\end{array}$ & $\begin{array}{c}0.0314 \\
(0.0115) \\
\end{array}$ & $\begin{array}{c}0.0193 \\
(0.0080)\end{array}$ & $\begin{array}{c}0.0277 \\
(0.0114) \\
\end{array}$ & $\begin{array}{c}0.0345 \\
(0.0140) \\
\end{array}$ & $\begin{array}{c}0.0600 \\
(0.0173) \\
\end{array}$ & $\begin{array}{c}0.0631 \\
(0.0236) \\
\end{array}$ \\
\hline$\overline{\mathrm{SAV}}$ & $\begin{array}{c}0.0057 \\
(0.0023)\end{array}$ & $\begin{array}{c}0.0303 \\
(0.0123)\end{array}$ & $\begin{array}{c}0.0204 \\
(0.0089)\end{array}$ & $\begin{array}{c}0.0281 \\
(0.0116)\end{array}$ & $\begin{array}{c}0.0358 \\
(0.0151)\end{array}$ & $\begin{array}{c}0.0602 \\
(0.0198)\end{array}$ & $\begin{array}{c}0.0610 \\
(0.0241)\end{array}$ \\
\hline NEV & $\begin{array}{c}0.0065 \\
(0.0029) \\
\end{array}$ & $\begin{array}{c}0.0331 \\
(0.0144) \\
\end{array}$ & $\begin{array}{c}0.0221 \\
(0.0087) \\
\end{array}$ & $\begin{array}{c}0.0261 \\
(0.0115) \\
\end{array}$ & $\begin{array}{c}0.0417 \\
(0.0146) \\
\end{array}$ & $\begin{array}{c}0.0609 \\
(0.0200) \\
\end{array}$ & $\begin{array}{c}0.0652 \\
(0.0283) \\
\end{array}$ \\
\hline LSM & $\begin{array}{c}0.0061 \\
(0.0022)\end{array}$ & $\begin{array}{c}0.0308 \\
(0.0131)\end{array}$ & $\begin{array}{c}0.0190 \\
(0.0096)\end{array}$ & $\begin{array}{c}0.0276 \\
(0.0119)\end{array}$ & $\begin{array}{c}0.0364 \\
(0.0157)\end{array}$ & $\begin{array}{c}0.0597 \\
(0.0207)\end{array}$ & $\begin{array}{c}0.0589 \\
(0.0250)\end{array}$ \\
\hline
\end{tabular}


Table E.2: The Results of Empirical Test for Accuracy and Group Disagreement with Arithmetic Mean Operated on Priority Vector

\begin{tabular}{|c|c|c|c|c|c|c|c|}
\hline \multicolumn{8}{|c|}{$A-A M(V)$} \\
\hline ABBR. & C1 & C2 & $\mathrm{C3}$ & $\mathrm{C} 4$ & C5 & C6 & $\mathrm{C} 7$ \\
\hline CSM & $\begin{array}{c}0.0061 \\
(0.0023)\end{array}$ & $\begin{array}{c}0.0361 \\
(0.0119)\end{array}$ & $\begin{array}{c}0.0219 \\
(0.0078)\end{array}$ & $\begin{array}{c}0.0285 \\
(0.0115)\end{array}$ & $\begin{array}{c}0.0346 \\
(0.0140)\end{array}$ & $\begin{array}{c}0.0661 \\
(0.0173)\end{array}$ & $\begin{array}{c}0.0662 \\
(0.0233)\end{array}$ \\
\hline$\overline{R-E V}$ & $\begin{array}{c}0.0063 \\
(0.0023)\end{array}$ & $\begin{array}{c}0.0348 \\
(0.0120)\end{array}$ & $\begin{array}{c}0.0219 \\
(0.0079)\end{array}$ & $\begin{array}{c}0.0283 \\
(0.0116)\end{array}$ & $\begin{array}{c}0.0339 \\
(0.0142)\end{array}$ & $\begin{array}{c}0.0675 \\
(0.0175)\end{array}$ & $\begin{array}{c}0.0598 \\
(0.0241)\end{array}$ \\
\hline L-EV & $\begin{array}{c}0.0059 \\
(0.0024) \\
\end{array}$ & $\begin{array}{c}0.0349 \\
(0.0124) \\
\end{array}$ & $\begin{array}{c}0.0220 \\
(0.0080)\end{array}$ & $\begin{array}{c}0.0288 \\
(0.0115) \\
\end{array}$ & $\begin{array}{c}0.0356 \\
(0.0142) \\
\end{array}$ & $\begin{array}{c}0.0635 \\
(0.0180) \\
\end{array}$ & $\begin{array}{c}0.0615 \\
(0.0248)\end{array}$ \\
\hline AM-EV & $\begin{array}{c}0.0061 \\
(0.0023) \\
\end{array}$ & $\begin{array}{c}0.0348 \\
(0.0123) \\
\end{array}$ & $\begin{array}{c}0.0219 \\
(0.0079) \\
\end{array}$ & $\begin{array}{c}0.0287 \\
(0.0115) \\
\end{array}$ & $\begin{array}{c}0.0351 \\
(0.0141) \\
\end{array}$ & $\begin{array}{c}0.0638 \\
(0.0179) \\
\end{array}$ & $\begin{array}{c}0.0613 \\
(0.0247) \\
\end{array}$ \\
\hline$\overline{G M}-\mathrm{EV}$ & $\begin{array}{c}0.0061 \\
(0.0023) \\
\end{array}$ & $\begin{array}{c}0.0348 \\
(0.0121) \\
\end{array}$ & $\begin{array}{c}0.0219 \\
(0.0079) \\
\end{array}$ & $\begin{array}{c}0.0284 \\
(0.0115) \\
\end{array}$ & $\begin{array}{c}0.0347 \\
(0.0141)\end{array}$ & $\begin{array}{c}0.0654 \\
(0.0176) \\
\end{array}$ & $\begin{array}{c}0.0606 \\
(0.0245)\end{array}$ \\
\hline$\overline{E V}\left[\overline{A A}^{\prime}\right]$ & $\begin{array}{c}0.0062 \\
(0.0022)\end{array}$ & $\begin{array}{c}0.0323 \\
(0.0118)\end{array}$ & $\begin{array}{c}0.0217 \\
(0.0084)\end{array}$ & $\begin{array}{c}0.0271 \\
(0.0122)\end{array}$ & $\begin{array}{c}0.0329 \\
(0.0144)\end{array}$ & $\begin{array}{c}0.0648 \\
(0.0178)\end{array}$ & $\begin{array}{c}0.0585 \\
(0.0283)\end{array}$ \\
\hline$\overline{\mathrm{EV}\left[\mathrm{A}^{\prime} \mathrm{A}\right]}$ & $\begin{array}{c}0.0460 \\
(0.0014)\end{array}$ & $\begin{array}{c}0.1440 \\
(0.0122)\end{array}$ & $\begin{array}{c}0.0616 \\
(0.0094)\end{array}$ & $\begin{array}{c}0.0987 \\
(0.0125)\end{array}$ & $\begin{array}{c}0.0400 \\
(0.0093)\end{array}$ & $\begin{array}{c}0.0897 \\
(0.0140)\end{array}$ & $\begin{array}{c}0.1630 \\
(0.0280)\end{array}$ \\
\hline $\begin{array}{l}A M-E V\left[A A^{\prime}\right] \\
A N D E V\left[A^{\prime} A\right]\end{array}$ & $\begin{array}{c}0.0156 \\
(0.0018)\end{array}$ & $\begin{array}{c}0.0468 \\
(0.0111)\end{array}$ & $\begin{array}{c}0.0289 \\
(0.0080)\end{array}$ & $\begin{array}{c}0.0330 \\
(0.0111)\end{array}$ & $\begin{array}{c}0.0296 \\
(0.0127)\end{array}$ & $\begin{array}{c}0.0681 \\
(0.0159)\end{array}$ & $\begin{array}{c}0.0628 \\
(0.0272)\end{array}$ \\
\hline $\begin{array}{l}\mathrm{GE}-\mathrm{EV}\left[\overline{\mathrm{AA}} \mathrm{A}^{\prime}\right] \\
\mathrm{AND} \mathrm{EV}\left[\mathrm{A}^{\prime} \mathrm{A}\right]\end{array}$ & $\begin{array}{c}0.0217 \\
(0.0015) \\
\end{array}$ & $\begin{array}{c}0.0870 \\
(0.0099)\end{array}$ & $\begin{array}{c}0.0400 \\
(0.0093)\end{array}$ & $\begin{array}{c}0.0533 \\
(0.0093) \\
\end{array}$ & $\begin{array}{c}0.0295 \\
(0.0108) \\
\end{array}$ & $\begin{array}{c}0.0750 \\
(0.0140)\end{array}$ & $\begin{array}{c}0.1050 \\
(0.0230)\end{array}$ \\
\hline$G E$ & $\begin{array}{c}0.0060 \\
(0.0022)\end{array}$ & $\begin{array}{c}0.0330 \\
(0.0117) \\
\end{array}$ & $\begin{array}{r}0.0217 \\
(0.0078) \\
\end{array}$ & $\begin{array}{r}0.0281 \\
(0.0112) \\
\end{array}$ & $\begin{array}{c}0.0345 \\
(0.0141) \\
\end{array}$ & $\begin{array}{c}0.0647 \\
(0.0176) \\
\end{array}$ & $\begin{array}{c}0.0603 \\
(0.0246) \\
\end{array}$ \\
\hline C-RSM & $\begin{array}{c}0.0061 \\
(0.0022) \\
\end{array}$ & $\begin{array}{c}0.0327 \\
(0.0119) \\
\end{array}$ & $\begin{array}{c}0.0223 \\
(0.0079) \\
\end{array}$ & $\begin{array}{c}0.0276 \\
(0.0113) \\
\end{array}$ & $\begin{array}{c}0.0347 \\
(0.0142) \\
\end{array}$ & $\begin{array}{c}0.0665 \\
(0.0179)\end{array}$ & $\begin{array}{c}0.0587 \\
(0.0247)\end{array}$ \\
\hline $\mathrm{MT}$ & $\begin{array}{c}0.0063 \\
(0.0022) \\
\end{array}$ & $\begin{array}{c}0.0339 \\
(0.0114) \\
\end{array}$ & $\begin{array}{c}0.0217 \\
(0.0076) \\
\end{array}$ & $\begin{array}{c}0.0280 \\
(0.0113) \\
\end{array}$ & $\begin{array}{c}0.0338 \\
(0.0140) \\
\end{array}$ & $\begin{array}{c}0.0665 \\
(0.0172) \\
\end{array}$ & $\begin{array}{c}0.0600 \\
(0.0233) \\
\end{array}$ \\
\hline$\overline{\mathrm{SAV}}$ & $\begin{array}{c}0.0061 \\
(0.0023)\end{array}$ & $\begin{array}{c}0.0317 \\
(0.0123)\end{array}$ & $\begin{array}{c}0.0234 \\
(0.0085) \\
\end{array}$ & $\begin{array}{c}0.0280 \\
(0.0115)\end{array}$ & $\begin{array}{c}0.0350 \\
(0.0150)\end{array}$ & $\begin{array}{c}0.0709 \\
(0.0195)\end{array}$ & $\begin{array}{c}0.0574 \\
(0.0241)\end{array}$ \\
\hline NEV & $\begin{array}{c}0.0059 \\
(0.0029)\end{array}$ & $\begin{array}{c}0.0402 \\
(0.0143)\end{array}$ & $\begin{array}{c}0.0223 \\
(0.0084)\end{array}$ & $\begin{array}{c}0.0256 \\
(0.0115)\end{array}$ & $\begin{array}{c}0.0384 \\
(0.0146)\end{array}$ & $\begin{array}{c}0.0679 \\
(0.0198)\end{array}$ & $\begin{array}{c}0.0550 \\
(0.0278)\end{array}$ \\
\hline$\overline{\text { LSM }}$ & $\begin{array}{c}0.0058 \\
(0.0022)\end{array}$ & $\begin{array}{c}0.0299 \\
(0.0130)\end{array}$ & $\begin{array}{c}0.0227 \\
(0.0093)\end{array}$ & $\begin{array}{c}0.0282 \\
(0.0119)\end{array}$ & $\begin{array}{c}0.0361 \\
(0.0157)\end{array}$ & $\begin{array}{c}0.0726 \\
(0.0203)\end{array}$ & $\begin{array}{c}0.0584 \\
(0.0250)\end{array}$ \\
\hline
\end{tabular}


Table E.3: The Results of Empirical Test for Accuracy and Group Disagreement with Geometric Mean Operated on Priority Vector

\begin{tabular}{|c|c|c|c|c|c|c|c|}
\hline \multicolumn{8}{|c|}{ A-GE(V) } \\
\hline ABBR. & $\mathrm{C1}$ & $\mathrm{C} 2$ & $\mathrm{C} 3$ & $\mathrm{C} 4$ & $\mathbf{C 5}$ & $\mathrm{C} 6$ & $\overline{\mathrm{C} 7}$ \\
\hline$\overline{\mathrm{CSM}}$ & $\begin{array}{c}0.0061 \\
(0.0023) \\
\end{array}$ & $\begin{array}{c}0.0411 \\
(0.0121) \\
(\end{array}$ & $\begin{array}{c}0.0224 \\
(0.0082) \\
\end{array}$ & $\begin{array}{c}0.0308 \\
(0.0117) \\
\end{array}$ & $\begin{array}{c}0.0358 \\
(0.0142)\end{array}$ & $\begin{array}{c}0.0659 \\
(0.0177)\end{array}$ & $\begin{array}{c}0.0697 \\
(0.0239)\end{array}$ \\
\hline R-EV & $\begin{array}{c}0.0062 \\
(0.0023)\end{array}$ & $\begin{array}{c}0.0392 \\
(0.0122)\end{array}$ & $\begin{array}{c}0.0224 \\
(0.0083)\end{array}$ & $\begin{array}{c}0.0305 \\
(0.0118)\end{array}$ & $\begin{array}{c}0.0350 \\
(0.0144)\end{array}$ & $\begin{array}{c}0.0671 \\
(0.0179)\end{array}$ & $\begin{array}{c}0.0679 \\
(0.0249)\end{array}$ \\
\hline$\overline{\mathrm{L}-\mathrm{EV}}$ & $\begin{array}{c}0.0059 \\
(0.0024)\end{array}$ & $\begin{array}{c}0.0411 \\
(0.0127)\end{array}$ & $\begin{array}{c}0.0230 \\
(0.0084)\end{array}$ & $\begin{array}{c}0.0310 \\
(0.0117)\end{array}$ & $\begin{array}{c}0.0368 \\
(0.0143)\end{array}$ & $\begin{array}{c}0.0636 \\
(0.0185)\end{array}$ & $\begin{array}{c}0.0718 \\
(0.0256)\end{array}$ \\
\hline AM-EV & $\begin{array}{c}0.0060 \\
(0.0023)\end{array}$ & $\begin{array}{c}0.0406 \\
(0.0125)\end{array}$ & $\begin{array}{c}0.0227 \\
(0.0083)\end{array}$ & $\begin{array}{c}0.0309 \\
(0.0117)\end{array}$ & $\begin{array}{c}0.0363 \\
(0.0143)\end{array}$ & $\begin{array}{c}0.0639 \\
(0.0184)\end{array}$ & $\begin{array}{c}0.0715 \\
(0.0256)\end{array}$ \\
\hline GM-EV & $\begin{array}{c}0.0061 \\
(0.0023)\end{array}$ & $\begin{array}{c}0.0400 \\
(0.0123)\end{array}$ & $\begin{array}{c}0.0226 \\
(0.0083)\end{array}$ & $\begin{array}{c}0.0306 \\
(0.0117)\end{array}$ & $\begin{array}{c}0.0359 \\
(0.0143)\end{array}$ & $\begin{array}{c}0.0652 \\
(0.0180)\end{array}$ & $\begin{array}{c}0.0698 \\
(0.0252)\end{array}$ \\
\hline$\overline{\mathrm{EV}}\left[\mathrm{AA}^{\prime}\right]$ & $\begin{array}{c}0.0061 \\
(0.0022)\end{array}$ & $\begin{array}{c}0.0385 \\
(0.0120)\end{array}$ & $\begin{array}{c}0.0238 \\
(0.0088)\end{array}$ & $\begin{array}{c}0.0295 \\
(0.0124)\end{array}$ & $\begin{array}{c}0.0350 \\
(0.0146)\end{array}$ & $\begin{array}{c}0.0647 \\
(0.0184)\end{array}$ & $\begin{array}{c}0.0718 \\
(0.0295)\end{array}$ \\
\hline$\overline{\mathrm{EV}}\left[\mathrm{A}^{\prime} \mathrm{A}\right]$ & $\begin{array}{c}0.0461 \\
(0.0014) \\
\end{array}$ & $\begin{array}{c}0.1400 \\
(0.0124) \\
\end{array}$ & $\begin{array}{c}0.0678 \\
(0.0098)\end{array}$ & $\begin{array}{c}0.0954 \\
(0.0128) \\
\end{array}$ & $\begin{array}{c}0.0398 \\
(0.0094)\end{array}$ & $\begin{array}{c}0.0895 \\
(0.0142)\end{array}$ & $\begin{array}{c}0.1480 \\
(0.0294)\end{array}$ \\
\hline $\begin{array}{l}\text { AM - EV }\left[A A^{\prime}\right] \\
\text { AND EV }\left[A^{\prime} A\right]\end{array}$ & $\begin{array}{c}0.0157 \\
(0.0018)\end{array}$ & $\begin{array}{c}0.0517 \\
(0.0113)\end{array}$ & $\begin{array}{c}0.0292 \\
(0.0083)\end{array}$ & $\begin{array}{c}0.0344 \\
(0.0113)\end{array}$ & $\begin{array}{c}0.0313 \\
(0.0129)\end{array}$ & $\begin{array}{c}0.0681 \\
(0.0165)\end{array}$ & $\begin{array}{c}0.0729 \\
(0.0282)\end{array}$ \\
\hline $\begin{array}{l}\text { GE - EV }\left[A A^{\prime}\right] \\
\text { AND EV[A'A] }\end{array}$ & $\begin{array}{c}0.0218 \\
(0.0015)\end{array}$ & $\begin{array}{c}0.0883 \\
(0.0101)\end{array}$ & $\begin{array}{c}0.0418 \\
(0.0094)\end{array}$ & $\begin{array}{c}0.0528 \\
(0.0094)\end{array}$ & $\begin{array}{c}0.0309 \\
(0.0109)\end{array}$ & $\begin{array}{c}0.0749 \\
(0.0143)\end{array}$ & $\begin{array}{c}0.0971 \\
(0.0238)\end{array}$ \\
\hline$\overline{\mathrm{GE}}$ & $\begin{array}{c}0.0060 \\
(0.0022)\end{array}$ & $\begin{array}{c}0.0378 \\
(0.0119)\end{array}$ & $\begin{array}{c}0.0223 \\
(0.0082)\end{array}$ & $\begin{array}{c}0.0302 \\
(0.0114)\end{array}$ & $\begin{array}{c}0.0356 \\
(0.0143)\end{array}$ & $\begin{array}{c}0.0648 \\
(0.0181)\end{array}$ & $\begin{array}{c}0.0704 \\
(0.0254)\end{array}$ \\
\hline C-RSM & $\begin{array}{c}0.0060 \\
(0.0022)\end{array}$ & $\begin{array}{c}0.0382 \\
(0.0121)\end{array}$ & $\begin{array}{c}0.0228 \\
(0.0083)\end{array}$ & $\begin{array}{c}0.0298 \\
(0.0115)\end{array}$ & $\begin{array}{c}0.0359 \\
(0.0144)\end{array}$ & $\begin{array}{c}0.0662 \\
(0.0184)\end{array}$ & $\begin{array}{c}0.0673 \\
(0.0255)\end{array}$ \\
\hline $\mathrm{MT}$ & $\begin{array}{c}0.0062 \\
(0.0022)\end{array}$ & $\begin{array}{c}0.0382 \\
(0.0116)\end{array}$ & $\begin{array}{c}0.0219 \\
(0.0080)\end{array}$ & $\begin{array}{c}0.0301 \\
(0.0115)\end{array}$ & $\begin{array}{c}0.0350 \\
(0.0142)\end{array}$ & $\begin{array}{c}0.0665 \\
(0.0176)\end{array}$ & $\begin{array}{c}0.0671 \\
(0.0239)\end{array}$ \\
\hline SAV & $\begin{array}{c}0.0060 \\
(0.0023)\end{array}$ & $\begin{array}{c}0.0370 \\
(0.0125)\end{array}$ & $\begin{array}{c}0.0237 \\
(0.0087)\end{array}$ & $\begin{array}{c}0.0301 \\
(0.0117)\end{array}$ & $\begin{array}{c}0.0359 \\
(0.0152)\end{array}$ & $\begin{array}{c}0.0604 \\
(0.0200)\end{array}$ & $\begin{array}{c}0.0630 \\
(0.0247)\end{array}$ \\
\hline NEV & $\begin{array}{c}0.0056 \\
(0.0029) \\
\end{array}$ & $\begin{array}{c}0.0477 \\
(0.0147) \\
\left(\begin{array}{c}0 \\
0\end{array}\right)\end{array}$ & $\begin{array}{c}0.0261 \\
(0.0088)\end{array}$ & $\begin{array}{c}0.0277 \\
(0.0116) \\
\end{array}$ & $\begin{array}{c}0.0392 \\
(0.0148) \\
\end{array}$ & $\begin{array}{c}0.0678 \\
(0.0204)\end{array}$ & $\begin{array}{c}0.0696 \\
(0.0289)\end{array}$ \\
\hline$\overline{\mathrm{LSM}}$ & $\begin{array}{c}0.0057 \\
(0.0022)\end{array}$ & $\begin{array}{c}0.0369 \\
(0.0133)\end{array}$ & $\begin{array}{c}0.0242 \\
(0.0097)\end{array}$ & $\begin{array}{c}0.0302 \\
(0.0121)\end{array}$ & $\begin{array}{c}0.0375 \\
(0.0159)\end{array}$ & $\begin{array}{c}0.0716 \\
(0.0210)\end{array}$ & $\begin{array}{c}0.0681 \\
(0.0259)\end{array}$ \\
\hline
\end{tabular}


Table E.4: The Results of Empirical Test for Accuracy and Group Disagreement with MDM Operated on Priority Vector

\begin{tabular}{|c|c|c|c|c|c|c|c|}
\hline \multicolumn{8}{|c|}{$\mathrm{A}-\mathrm{MDM}(\mathrm{V})$} \\
\hline ABBR. & C1 & $\mathrm{C} 2$ & C3 & $\mathrm{C} 4$ & $\mathrm{C5}$ & $\mathrm{C6}$ & $\overline{C 7}$ \\
\hline$\overline{\mathrm{CSM}}$ & $\begin{array}{c}0.00360 \\
(0.0023)\end{array}$ & $\begin{array}{c}0.0259 \\
(0.0121)\end{array}$ & $\begin{array}{c}0.0144 \\
(0.0081)\end{array}$ & $\begin{array}{c}0.0299 \\
(0.0116)\end{array}$ & $\begin{array}{c}0.0376 \\
(0.0140)\end{array}$ & $\begin{array}{c}0.0569 \\
(0.0182)\end{array}$ & $\begin{array}{c}0.0623 \\
(0.0234)\end{array}$ \\
\hline R-EV & $\begin{array}{c}0.0037 \\
(0.0023)\end{array}$ & $\begin{array}{c}0.0256 \\
(0.0122)\end{array}$ & $\begin{array}{c}0.0142 \\
(0.0082)\end{array}$ & $\begin{array}{c}0.0286 \\
(0.0117)\end{array}$ & $\begin{array}{c}0.0381 \\
(0.0143)\end{array}$ & $\begin{array}{c}0.0611 \\
(0.0178)\end{array}$ & $\begin{array}{c}0.0534 \\
(0.0244)\end{array}$ \\
\hline L-EV & $\begin{array}{c}0.0032 \\
(0.0024)\end{array}$ & $\begin{array}{c}0.0244 \\
(0.0126)\end{array}$ & $\begin{array}{c}0.0149 \\
(0.0082)\end{array}$ & $\begin{array}{c}0.0301 \\
(0.0117)\end{array}$ & $\begin{array}{c}0.0369 \\
(0.0142)\end{array}$ & $\begin{array}{c}0.0551 \\
(0.0189)\end{array}$ & $\begin{array}{c}0.0581 \\
(0.0249)\end{array}$ \\
\hline AM-EV & $\begin{array}{c}0.0035 \\
(0.0024)\end{array}$ & $\begin{array}{c}0.0242 \\
(0.0125)\end{array}$ & $\begin{array}{c}0.0140 \\
(0.0081)\end{array}$ & $\begin{array}{c}0.0297 \\
(0.0116)\end{array}$ & $\begin{array}{c}0.0372 \\
(0.0141)\end{array}$ & $\begin{array}{c}0.0623 \\
(0.0180)\end{array}$ & $\begin{array}{c}0.0607 \\
(0.0249)\end{array}$ \\
\hline GM-EV & $\begin{array}{c}0.0036 \\
(0.0023)\end{array}$ & $\begin{array}{c}0.0230 \\
(0.0124)\end{array}$ & $\begin{array}{c}0.0139 \\
(0.0081)\end{array}$ & $\begin{array}{c}0.0293 \\
(0.0116)\end{array}$ & $\begin{array}{c}0.0376 \\
(0.0141)\end{array}$ & $\begin{array}{c}0.0563 \\
(0.0178)\end{array}$ & $\begin{array}{c}0.0568 \\
(0.0246)\end{array}$ \\
\hline$\overline{\mathrm{EV}}\left[\mathrm{AA}^{\prime}\right]$ & $\begin{array}{c}0.0045 \\
(0.0022)\end{array}$ & $\begin{array}{c}0.0207 \\
(0.0121)\end{array}$ & $\begin{array}{c}0.0131 \\
(0.0086)\end{array}$ & $\begin{array}{c}0.0285 \\
(0.0123)\end{array}$ & $\begin{array}{c}0.0382 \\
(0.0145)\end{array}$ & $\begin{array}{c}0.0507 \\
(0.0190)\end{array}$ & $\begin{array}{c}0.0530 \\
(0.0285)\end{array}$ \\
\hline $\mathrm{EV}\left[\mathrm{A}^{\prime} \mathrm{A}\right]$ & $\begin{array}{c}0.0046 \\
(0.0014)\end{array}$ & $\begin{array}{c}0.0147 \\
(0.0124)\end{array}$ & $\begin{array}{c}0.0564 \\
(0.0095)\end{array}$ & $\begin{array}{c}0.0856 \\
(0.0129)\end{array}$ & $\begin{array}{c}0.0371 \\
(0.0095)\end{array}$ & $\begin{array}{c}0.0807 \\
(0.0144)\end{array}$ & $\begin{array}{c}0.0153 \\
(0.0283)\end{array}$ \\
\hline $\begin{array}{l}A M-E V\left[A A^{\prime}\right] \\
A N D E V\left[A^{\prime} A\right]\end{array}$ & $\begin{array}{c}0.0166 \\
(0.0018) \\
\end{array}$ & $\begin{array}{c}0.0341 \\
(0.0114) \\
\end{array}$ & $\begin{array}{c}0.0213 \\
(0.0082) \\
\end{array}$ & $\begin{array}{c}0.0359 \\
(0.0112) \\
\end{array}$ & $\begin{array}{c}0.0329 \\
(0.0129)\end{array}$ & $\begin{array}{c}0.0557 \\
(0.0171)\end{array}$ & $\begin{array}{c}0.0598 \\
(0.0274)\end{array}$ \\
\hline $\begin{array}{l}\text { GE - EV }\left[A A^{\prime}\right] \\
\text { AND EV }\left[A^{\prime} A\right]\end{array}$ & $\begin{array}{c}0.0218 \\
(0.0016)\end{array}$ & $\begin{array}{l}0.0820 \\
(0.0101)\end{array}$ & $\begin{array}{c}0.0306 \\
(0.0095)\end{array}$ & $\begin{array}{c}0.0526 \\
(0.0094)\end{array}$ & $\begin{array}{c}0.0309 \\
(0.0112)\end{array}$ & $\begin{array}{c}0.0760 \\
(0.0149)\end{array}$ & $\begin{array}{c}0.1010 \\
(0.0231)\end{array}$ \\
\hline $\mathrm{GE}$ & $\begin{array}{c}0.0036 \\
(0.0023)\end{array}$ & $\begin{array}{l}0.0233 \\
(0.0119)\end{array}$ & $\begin{array}{c}0.0141 \\
(0.0080)\end{array}$ & $\begin{array}{c}0.0288 \\
(0.0113)\end{array}$ & $\begin{array}{c}0.0370 \\
(0.0142)\end{array}$ & $\begin{array}{c}0.0557 \\
(0.0179)\end{array}$ & $\begin{array}{c}0.0558 \\
(0.0248)\end{array}$ \\
\hline C-RSM & $\begin{array}{c}0.0035 \\
(0.0023)\end{array}$ & $\begin{array}{c}0.0229 \\
(0.0121)\end{array}$ & $\begin{array}{c}0.0148 \\
(0.0082)\end{array}$ & $\begin{array}{c}0.0293 \\
(0.0114)\end{array}$ & $\begin{array}{c}0.0361 \\
(0.0143)\end{array}$ & $\begin{array}{c}0.0567 \\
(0.0189)\end{array}$ & $\begin{array}{c}0.0544 \\
(0.0248)\end{array}$ \\
\hline $\mathrm{MT}$ & $\begin{array}{c}0.0036 \\
(0.0023)\end{array}$ & $\begin{array}{c}0.0269 \\
(0.0116)\end{array}$ & $\begin{array}{c}0.0144 \\
(0.0079)\end{array}$ & $\begin{array}{c}0.0285 \\
(0.0114)\end{array}$ & $\begin{array}{c}0.0384 \\
(0.0141)\end{array}$ & $\begin{array}{c}0.0640 \\
(0.0176)\end{array}$ & $\begin{array}{c}0.0583 \\
(0.0235)\end{array}$ \\
\hline$\overline{\mathrm{SAV}}$ & $\begin{array}{c}0.0037 \\
(0.0023)\end{array}$ & $\begin{array}{c}0.0216 \\
(0.0125)\end{array}$ & $\begin{array}{c}0.0136 \\
(0.0088)\end{array}$ & $\begin{array}{c}0.0289 \\
(0.0117)\end{array}$ & $\begin{array}{c}0.0389 \\
(0.0152)\end{array}$ & $\begin{array}{c}0.0566 \\
(0.0204)\end{array}$ & $\begin{array}{c}0.0569 \\
(0.0256)\end{array}$ \\
\hline NEV & $\begin{array}{c}0.0041 \\
(0.0030)\end{array}$ & $\begin{array}{c}0.0266 \\
(0.0146)\end{array}$ & $\begin{array}{c}0.0149 \\
(0.0087)\end{array}$ & $\begin{array}{c}0.0265 \\
(0.0115)\end{array}$ & $\begin{array}{c}0.0376 \\
(0.0147)\end{array}$ & $\begin{array}{c}0.0617 \\
(0.0202)\end{array}$ & $\begin{array}{c}0.0456 \\
(0.0280)\end{array}$ \\
\hline LSM & $\begin{array}{c}0.0035 \\
(0.0023)\end{array}$ & $\begin{array}{c}0.0186 \\
(0.0133)\end{array}$ & $\begin{array}{c}0.0123 \\
(0.0096)\end{array}$ & $\begin{array}{c}0.0270 \\
(0.0120)\end{array}$ & $\begin{array}{c}0.0373 \\
(0.0159)\end{array}$ & $\begin{array}{c}0.0616 \\
(0.0208)\end{array}$ & $\begin{array}{c}0.0558 \\
(0.0250)\end{array}$ \\
\hline
\end{tabular}


Table E.5: The Results of Empirical Test for Accuracy and Group Disagreement with MDM Operated on Pairwise Comparison Matrix

\begin{tabular}{|c|c|c|c|c|c|c|c|}
\hline \multicolumn{8}{|c|}{$A-M D M(M)$} \\
\hline ABBR. & $\mathrm{C} 1$ & $\mathrm{C} 2$ & C3 & $\mathrm{C} 4$ & $\mathrm{C5}$ & C6 & C7 \\
\hline$\overline{\mathrm{CSM}}$ & $\begin{array}{l}0.00510 \\
(0.0023)\end{array}$ & $\begin{array}{c}0.0196 \\
(0.0124)\end{array}$ & $\begin{array}{c}0.0107 \\
(0.0081)\end{array}$ & $\begin{array}{c}0.0267 \\
(0.0116)\end{array}$ & $\begin{array}{c}0.0350 \\
(0.0142)\end{array}$ & $\begin{array}{c}0.0573 \\
(0.0175)\end{array}$ & $\begin{array}{c}0.0597 \\
(0.0240)\end{array}$ \\
\hline R-EV & $\begin{array}{c}0.0051 \\
(0.0023)\end{array}$ & $\begin{array}{c}0.0192 \\
(0.0124)\end{array}$ & $\begin{array}{c}0.0107 \\
(0.0083)\end{array}$ & $\begin{array}{c}0.0267 \\
(0.0117)\end{array}$ & $\begin{array}{c}0.0351 \\
(0.0143)\end{array}$ & $\begin{array}{c}0.0572 \\
(0.0177)\end{array}$ & $\begin{array}{c}0.0593 \\
(0.0245)\end{array}$ \\
\hline L-EV & $\begin{array}{c}0.0052 \\
(0.0024)\end{array}$ & $\begin{array}{c}0.0192 \\
(0.0128)\end{array}$ & $\begin{array}{c}0.0107 \\
(0.0084)\end{array}$ & $\begin{array}{c}0.0266 \\
(0.0116)\end{array}$ & $\begin{array}{c}0.0349 \\
(0.0143)\end{array}$ & $\begin{array}{c}0.0573 \\
(0.0181)\end{array}$ & $\begin{array}{c}0.0601 \\
(0.0253)\end{array}$ \\
\hline AM-EV & $\begin{array}{c}0.0051 \\
(0.0024)\end{array}$ & $\begin{array}{c}0.0199 \\
(0.0127) \\
\end{array}$ & $\begin{array}{c}0.0107 \\
(0.0083)\end{array}$ & $\begin{array}{c}0.0266 \\
(0.0115) \\
\end{array}$ & $\begin{array}{c}0.0350 \\
(0.0143) \\
\end{array}$ & $\begin{array}{c}0.0573 \\
(0.0180)\end{array}$ & $\begin{array}{c}0.0601 \\
(0.0252)\end{array}$ \\
\hline GM-EV & $\begin{array}{c}0.0051 \\
(0.0023)\end{array}$ & $\begin{array}{c}0.0196 \\
(0.0125)\end{array}$ & $\begin{array}{c}0.0107 \\
(0.0083)\end{array}$ & $\begin{array}{c}0.0267 \\
(0.0115)\end{array}$ & $\begin{array}{c}0.0350 \\
(0.0142)\end{array}$ & $\begin{array}{c}0.0573 \\
(0.0177)\end{array}$ & $\begin{array}{c}0.0597 \\
(0.0249)\end{array}$ \\
\hline $\mathrm{EV}\left[\mathrm{AA}^{\prime}\right]$ & $\begin{array}{c}0.0055 \\
(0.0022)\end{array}$ & $\begin{array}{c}0.0232 \\
(0.0121) \\
\end{array}$ & $\begin{array}{c}0.0096 \\
(0.0088) \\
\end{array}$ & $\begin{array}{c}0.0249 \\
(0.0123) \\
\end{array}$ & $\begin{array}{c}0.0343 \\
(0.0145) \\
\end{array}$ & $\begin{array}{c}0.0577 \\
(0.0180)\end{array}$ & $\begin{array}{c}0.0540 \\
(0.0287)\end{array}$ \\
\hline$\overline{E V}\left[A^{\prime} A\right]$ & $\begin{array}{c}0.0459 \\
(0.0014)\end{array}$ & $\begin{array}{c}0.1530 \\
(0.0139)\end{array}$ & $\begin{array}{c}0.0519 \\
(0.0098)\end{array}$ & $\begin{array}{c}0.0886 \\
(0.0128)\end{array}$ & $\begin{array}{c}0.0391 \\
(0.0095)\end{array}$ & $\begin{array}{c}0.0737 \\
(0.0147)\end{array}$ & $\begin{array}{c}0.1630 \\
(0.0282)\end{array}$ \\
\hline $\begin{array}{l}\overline{A M}-\mathrm{EV}[\overline{\mathrm{AA}}] \\
\mathrm{AND} \text { EV }\left[\mathrm{A}^{\prime} \mathrm{A}\right]\end{array}$ & $\begin{array}{c}0.0156 \\
(0.0018)\end{array}$ & $\begin{array}{c}0.0360 \\
(0.0114)\end{array}$ & $\begin{array}{c}0.0193 \\
(0.0083)\end{array}$ & $\begin{array}{c}0.0362 \\
(0.0112)\end{array}$ & $\begin{array}{c}0.0314 \\
(0.0129)\end{array}$ & $\begin{array}{c}0.0624 \\
(0.0161)\end{array}$ & $\begin{array}{c}0.0618 \\
(0.0275)\end{array}$ \\
\hline $\begin{array}{l}\text { GE - EV }\left[A A^{\prime}\right] \\
\text { AND EV }\left[A^{\prime} A\right]\end{array}$ & $\begin{array}{c}0.0215 \\
(0.0016)\end{array}$ & $\begin{array}{c}0.0849 \\
(0.0104)\end{array}$ & $\begin{array}{c}0.0284 \\
(0.0097)\end{array}$ & $\begin{array}{c}0.0504 \\
(0.0095)\end{array}$ & $\begin{array}{c}0.0309 \\
(0.0110)\end{array}$ & $\begin{array}{c}0.0653 \\
(0.0143)\end{array}$ & $\begin{array}{c}0.0980 \\
(0.0233)\end{array}$ \\
\hline $\mathrm{GE}$ & $\begin{array}{c}0.0051 \\
(0.0023)\end{array}$ & $\begin{array}{c}0.0197 \\
(0.0120)\end{array}$ & $\begin{array}{c}0.0107 \\
(0.0081)\end{array}$ & $\begin{array}{c}0.0266 \\
(0.0113)\end{array}$ & $\begin{array}{c}0.0350 \\
(0.0142)\end{array}$ & $\begin{array}{c}0.0573 \\
(0.0178)\end{array}$ & $\begin{array}{c}0.0597 \\
(0.0249)\end{array}$ \\
\hline C-RSM & $\begin{array}{c}0.0052 \\
(0.0023)\end{array}$ & $\begin{array}{c}0.0178 \\
(0.0123)\end{array}$ & $\begin{array}{c}0.0106 \\
(0.0083)\end{array}$ & $\begin{array}{c}0.0265 \\
(0.0113)\end{array}$ & $\begin{array}{c}0.0353 \\
(0.0143)\end{array}$ & $\begin{array}{c}0.0573 \\
(0.0181)\end{array}$ & $\begin{array}{c}0.0582 \\
(0.0251)\end{array}$ \\
\hline$\overline{\mathrm{MT}}$ & $\begin{array}{c}0.0051 \\
(0.0023)\end{array}$ & $\begin{array}{c}0.0197 \\
(0.0118)\end{array}$ & $\begin{array}{c}0.0108 \\
(0.0080)\end{array}$ & $\begin{array}{c}0.0267 \\
(0.0114)\end{array}$ & $\begin{array}{c}0.0351 \\
(0.0142)\end{array}$ & $\begin{array}{c}0.0573 \\
(0.0174)\end{array}$ & $\begin{array}{c}0.0595 \\
(0.0238)\end{array}$ \\
\hline$\overline{\mathrm{SAV}}$ & $\begin{array}{c}0.0050 \\
(0.0024) \\
\end{array}$ & $\begin{array}{c}0.0143 \\
(0.0129) \\
\end{array}$ & $\begin{array}{c}0.0114 \\
(0.0089) \\
\end{array}$ & $\begin{array}{c}0.0273 \\
(0.0116) \\
\end{array}$ & $\begin{array}{c}0.0363 \\
(0.0151) \\
\end{array}$ & $\begin{array}{c}0.0572 \\
(0.0199) \\
\end{array}$ & $\begin{array}{r}0.0587 \\
(0.0244) \\
(\end{array}$ \\
\hline NEV & $\begin{array}{c}0.0037 \\
(0.0030) \\
\end{array}$ & $\begin{array}{c}0.0265 \\
(0.0149) \\
\end{array}$ & $\begin{array}{c}0.0074 \\
(0.0090) \\
\end{array}$ & $\begin{array}{r}0.0245 \\
(0.0115) \\
\end{array}$ & $\begin{array}{c}0.0402 \\
(0.0147) \\
\end{array}$ & $\begin{array}{c}0.0534 \\
(0.0202) \\
\end{array}$ & $\begin{array}{r}0.0601 \\
(0.0283) \\
\end{array}$ \\
\hline LSM & $\begin{array}{c}0.0052 \\
(0.0023)\end{array}$ & $\begin{array}{c}0.0172 \\
(0.0137)\end{array}$ & $\begin{array}{c}0.0112 \\
(0.0097)\end{array}$ & $\begin{array}{c}0.0271 \\
(0.0120)\end{array}$ & $\begin{array}{c}0.0372 \\
(0.0158)\end{array}$ & $\begin{array}{c}0.0572 \\
(0.0208)\end{array}$ & $\begin{array}{c}0.0564 \\
(0.0253)\end{array}$ \\
\hline
\end{tabular}




\section{Bibliography}

[1] D. F. Kocaoglu, "A participative approach to program evaluation," IEEE Transactions on Engineering Management, vol. 30, 1983.

[2] T. L. Saaty, "A scaling method for priorities in hierarchical structures," Journal of Mathematical Psychology, vol. 15, pp. 234-281, 1977.

[3] T. L. Saaty, The Analytic Hierarchal Process. McGraw-Hill, New York, 1980.

[4] P. L. Yu, "A class of solutions for group decision problems," Management Science, vol. 19, no. 8, pp. 936-946, 1973.

[5] W. D. Cook and K. Moshe, "Deriving weights from pairwise comparison ratio matrices: An axiomatic approach," European Journal of Operational Research, vol. 37, pp. 355-362, 1988.

[6] C. Aczel and T. L. Saaty, "Procedures for synthesizing ratio judgments," Journal of Mathematical Psychology, vol. 27, pp. 93-102, 1983.

[7] C. Aczel and T. L. Saaty, "Synthesizing judgments: A functional equations approach," Math. Modeling, vol. 9, pp. 311-320, 1987.

[8] C. Aczel and T. L. Saaty, "On synthesis of judgments," Socio-Econ. Plann. Sci., vol. 20, pp. 333-339, 1986.

[9] C.-L. Hwang and M.-J. Lin, Group Decision Making under Multiple Criteria. Spring-Verlag, 1987.

[10] G. DeSanctis and R. B. Gallupe, "A foundation for the study of group decision support systems," Management Science, vol. 33, no. 5, pp. 589-605, 1987.

[11] R. F. Dyer and E. H. Forman, "Group decision support with the analytic hierarchy process," Decision Support Systems, vol. 8, pp. 99-124, 1992.

[12] T. L. Saaty, "Scenarios and priorities in transport planning: Application to Sudan," Transport Research, vol. 11, no. 3, pp. 343-350, 1977.

[13] T. L. Saaty, "The sudan transport study," Interfaces, vol. 8, no. 1, pp. 37-57, 1977. 
[14] T. L. Saaty, "Modeling unstructured decision problems: The theory of analytical hierarchies," Mathematics and Computers in Simulation, vol. 20, no. 3, pp. 147-157, 1978.

[15] T. L. Saaty, "Mathematical modeling of dynamic decisions: Priorities and hierarchies with time dependence," Mathematics and Computers in Simulation, vol. 21, no. 4, pp. 352-358, 1979.

[16] T. L. Saaty, "The Sudan transport study," Interfaces, vol. 8, no. 1, pp. 37-57, 1977.

[17] T. L. Saaty, "Exploring the interface between hierarchies, multiple objective and fuzzy sets," Fuzzy Sets and Systems, Junuary 1978.

[18] C. R. Johnson et al., "A note on right-left asymmetry in eigenvector ranking procedure," Journal of Mathematical Psychology, vol. 19, no. 1, pp. 61-64, 1979.

[19] J. M. Alexander and T. L. Saaty, "Stability analysis of the forward-backward process: Northern Ireland case study," Behavioral Science, vol. 22, no. 6, pp. 375-382, 1977.

[20] T. L. Saaty, "Axiomatic foundation of the analytic hierarchy process," Mgmt Sci., vol. 32, pp. 841-855, 1986.

[21] L. Vargas, "Priority theory and utility theory," Math. Modeling, vol. 9, pp. 381$385,1987$.

[22] R. D. Kamenetzky, "The relationship between the analytic hierarchy process and the additive value function," Decision Science, vol. 13, pp. 702-713, 1982.

[23] J. W. Ra, "Analysis of expert judgments in hierarchical decision process," Ph.D Dissertation, University of Pittsburgh, 1988.

[24] D. F. Kocaoglu, "A system approach to the resource allocation process in police patrol," Ph.D Dissertation, School of Engineering, University of Pittsburgh, 1976.

[25] J. Fichtner, "On deriving priority vectors from matrices of pairwise comparisons," Socio-Econ Plann. Sci., vol. 20, pp. 341-345, 1986.

[26] R. E. Jensen, "An alternative scaling method for priorities in hierarchical structures," Journal of Mathematical Psychology, vol. 28, pp. 317-332, 1984.

[27] G. Crawford and C. Williams, "A note on the analysis of subjective judgment matrices," Journal of Mathematical Psychology, vol. 29, pp. 387-405, 1988. 
[28] G. Crawford and C. Williams, "The geometric mean procedure for estimating the scale of a judgment matrix," Mathematical Modeling, vol. 9, pp. 327-334, 1987.

[29] P. T. Harker, "Incomplete pairwise comparisons in the analytic hierarchy process," Mathematical Modeling.

[30] P. T. Harker, "Alternative modes of questioning in the analytic hierarchy process," Math Modeling, vol. 9, pp. 353-360, 1987.

[31] E. N. Weiss, "Using the analytic hierarchy process in a dynamic environment," Math. Modeling, vol. 9, pp. 211-216, 1987.

[32] I. Millet and P. T. Harker, "Globally effective questioning in the analytic hierarchy process," European Journal of Operational Research, vol. 48, pp. 88-97, 1990.

[33] T. L. Saaty, "How to handle dependence with the analytic hierarchy process," Math. Modeling, vol. 9, pp. 369-376, 1987.

[34] C. R. Johnson, "Constructive critique of a hierarchical prioritization scheme employing paired comparisons," Proceedings of the International Conference of Cybernetics and Society of the IEE, IEE, Cambridge, Massachusetts, pp. 373378,1980 .

[35] M. F. Shipley, "A hierarchical strategic planning and 0-1 integer programming resource allocation decision support system for a university school of engineering," Ph.D dissertation of University of Pittsburgh, 1989.

[36] B. G. Mirkin, Group Choice. Winston, Washington, DC, 1979.

[37] A. L. Comrey, "A proposed approach for absolute ratio scaling," Psychometrika, vol. 15, pp. 317-325, 1950.

[38] F. Zahedi, "The analytic hierarchy process - a survey of the method and its applications," Interface, vol. 16, pp. 96-108, 1986.

[39] J. Barzilai and B. Golany, "AHP rank reversal, normalization and aggregation rules," INFOR, vol. 32, no. 2, pp. 57-64, 1994.

[40] K. J. Radford, Complex Decision Problems - An Integrated Strategy for Resolution. Reston Publishing Company, 1977.

[41] L. R. Hoffman, "Improving the problem-solving process in managerial groups," in Improving Group Decision Making in Organization (R. A. Guzzo, ed.), Academic Press, 1982. 
[42] A. Osborn, Applied Imagination. Chaeles Scribner's Son, New York., 1963.

[43] N. C. Dalkey, The Delphi Method: An Experimental Study of Group Opinion. The Rand Corp: Santa Monica, Calif., 1969.

[44] D. W. Malone, "An introduction to the application of interpretive structural modeling," in Portraits of Complexity: Applications of Systems Methodologies to societal Prob̆lems (M. M. Baldwin, ed.), Battelle, 1975.

[45] J. N. Warfield, Structuring Complex Systems. Battelle, 1974.

[46] D. W. Malone, "Applications of interpretive structural modeling: Relating factors for urban success," in Portraits of Complexity: Applications of Systems Methodologies to societal Problems (M. M. Baldwin, ed.), Battelle, 1975.

[47] R. A. Guzzo, "The study of group decision making approaches and applications," in Improving Group Decision Making in Organization (R. A. Guzzo, ed.), Academic Press, 1982.

[48] R. Khorramshahgol et al., "Delphic hierarchy process (dhp): A methodology for priority setting derived from the delphi method and analytical hierarchy process," European Journal of Operational Research, vol. 37, pp. 347-354, 1988.

[49] L. Vargas, "Analysis of sensitivity of reciprocal matrices,"

[50] S. Y. Dennis, "A probabilistic model for assignment of priorities in hierarchically structured decision problems," Mathematical Modeling, vol. 9, pp. 335$344,1987$.

[51] M. S. Zahir, "Incorporating the uncertainty of decision judgments in the analytic hierarchy process," European Journal of Operational Research, vol. 53, pp. 206-216, 1991.

[52] T. L. Saaty, "The analytic hierarchy process - what it is and how it is used," Math. Modeling, vol. 9, pp. 161-176, 1987.

[53] L. Vargas, "Reciprocal matrices with random coefficients," Math. Modeling, vol. 3, pp. 69-81, 1982.

[54] U. R. R. Uppuluri, "Logarithmic least-squares approach to Saaty's decision problem," in Mathematics and Statistics Research Department Progress Report (W. E. Lever et al., eds.), pp. 19-21, Oak Ridge National Laboratory, Oak Ridge, TN37830, 1978.

[55] J. W. Ra, "Analysis of the 'column - row sums' approach for pairwise comparisons," (unpublished M.S. Thesis, School of Engineering, University of Pittsburgh), 1987. 
[56] T. L. Saaty and L. G. Vargas, "Comparison of eigenvalue, logarithmic least squares and least squares methods in estimating ratios," Math. Modeling, vol. 5, pp. 309-324, 1984.

[57] L. Vargas, "An overview of the analytic hierarchy process and its applications," European Journal of Operational Research, vol. 48, pp. 2-8, 1990.

[58] J. G. Kemeny and L. J. Snell, Preference Ranking: Axiomatic Approach. Ginn and Co., Boston, 1962.

[59] R. L. Keeney and H. Raiffa, Decisions with Multiple Objectives. Wiley, New York, 1977.

[60] D. I. Cleland and D. F. Kocaoglu, Engineering Management. McGraw-Hill, Inc., 1981.

[61] L. L. Thurstone, "Psychophysical analysis," American Journal of Psyology, vol. 38 , pp. $368-389,1927$.

[62] L. L. Thurstone, "A law of comparative judgment," Psychological Review, vol. 34, pp. 273-286, 1927.

[63] D. V. Budescu et al., "A comparison of the eigenvalue method and the geometric mean procedure for ratio scaling," Applied Psychological Measurement, vol. 10, pp. 69-78, March 1986.

[64] C. D. Eckart and G. Young, "The approximation of one matrix by another of lower rank," Psychometrika, vol. 1, pp. 211-217, 1936.

[65] A. T. W. Chu et al., "A comparison of two methods for determining the weights of belonging to fuzzy sets," Journal of Optimization Theory and Applications, vol. 27, pp. 531-538, 1979.

[66] F. Zahedi, "A simulation study of estimation methods in the analytic hierarchy process," Socio-Economic Planning Sciences, vol. 20, pp. 347-354, 1986. 